\title{
NUCLEAR TECHNOLOGY DIVISION ANNUAL PROGRESS REPORT FOR PERIOD ENDING SEPTEMBER 30, 1976
}

April 1977

\author{
R. L. HEATH et al
}

\section{April 1977}

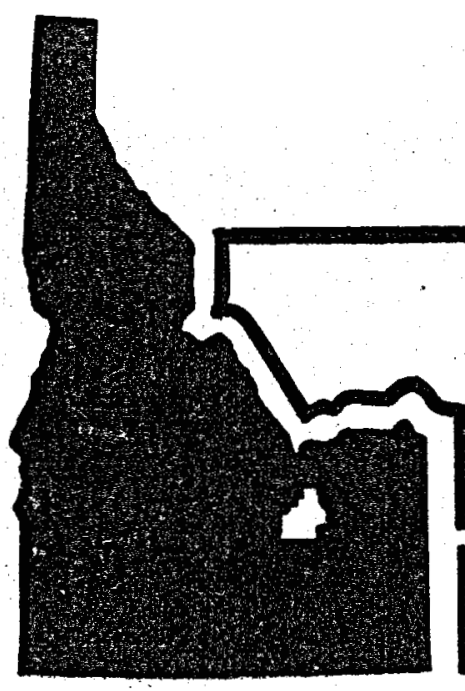

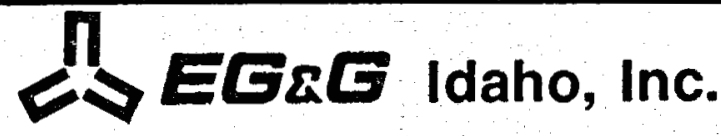

IDAHO NATIONAL ENGINEERING LABORATORY

ENERGY RESEARCH AND DEVELOPMENT ADMINISTRATION

IDAHO OPERATIONS OFFICE UNDER CONTRACT EY-76-C-07-1570 
TREE-1116

DISTRIBUTION UNDER CATEGORY:

$U C-2$

General, Miscellaneous, and

Progress Reports (Nuclear)

TID-4500, R65

NUCLEAR TECHNOLOGY DIVISION ANNUAL PROGRESS REPORT

FOR PERIOD ENDING SEPTEMBER 30, 1976

\author{
R. L. Heath et al \\ Nuclear Technology \\ Division
}

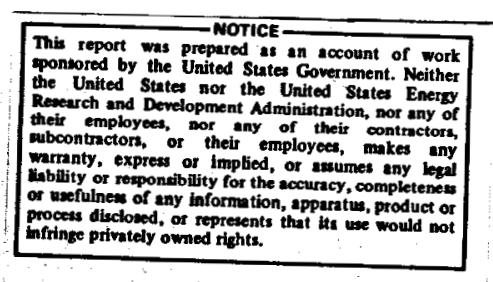

EG\&G IDAHO, INC.

Date Published - April 1977

PREPARED FOR THE ENERGY RESEARCH \& DEVELOPMENT ADMINISTRATION IDAHO OPERATIONS OFFICE

UNDER CONTRACT NO. EY-76-C-07-1570 

FOREWORD.....................

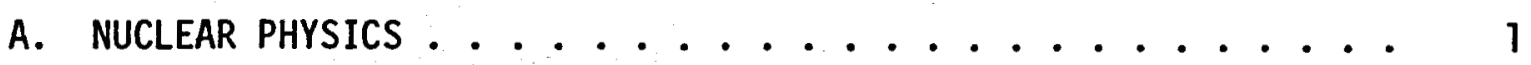

Nuclear structure. . . . . . . . . . . . . 3

Energy Levels of ${ }^{126} \mathrm{Te}$ Populated in the ${ }^{126} \mathrm{Te}(n, \gamma)$ Reaction .. 5

Decay of ${ }^{128 \mathrm{Ba}}$. . . . . . . . . . . . . 11

Decay of ${ }^{128} \mathrm{Cs} . \ldots \ldots 12$

Level Structure of $131 \mathrm{Cs}$ and the Decay Energy of $131 \mathrm{Ba} . \ldots \ldots 16$

Half-Lives of ${ }^{134} \mathrm{La}$ and ${ }^{132}{ }^{135} \mathrm{Ce} \ldots \ldots \ldots \ldots . . . . . .17$

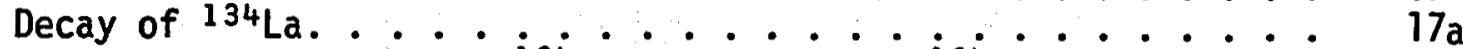

Gamma-Ray Emission From ${ }^{3}{ }^{4} \mathrm{Ce}$ and Leveis in ${ }^{134} \mathrm{La} \ldots{ }_{32}$

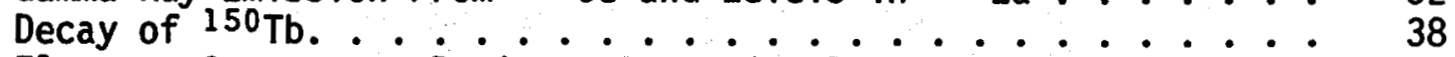

Electron-Capture to Positron Intensity Ratios and the Decay Energy of $131 \mathrm{Ba} . \ldots \ldots 45$

$K$ Conversion Coefficients of $\gamma$-Rays above $800 \mathrm{keV}$ from 182 Ta Decay.................. 48

Coriolis-Mixing Analys is of the $\dot{K}^{\pi}=0^{-}, \gamma^{-}$and $\dot{2}^{-}$octupoleVibrational Bands in ${ }^{156} \mathrm{Gd} . \ldots 49$

Features of the Low-Energy Level Scheme of 229 Th as Observed in the $\alpha$-Decay of $233 \mathrm{U} \ldots 54$

Level Structure of ${ }^{233} \mathrm{Th}$ From the ${ }^{232} \mathrm{Th}(\dot{n}, \dot{\gamma})$ Reaction Using 2- and 24-keV Neutrons ......... 56

Absolute $\gamma$-Ray Branching and Half-Life of ${ }^{250_{B K}} \ldots 62$

Determining the Negative Energy Resonance Parameters for the Four Exit Channels of ${ }^{35} \mathrm{C} l$..............

Radiation Measurements. ..............

Filtered Reactor Beams for Fast Neutron Capture $\gamma$-Ray

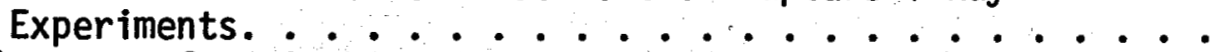

Development of an On-Line Isotope Separator System for FissionProduct Decay Studies Using a ${ }^{252} \mathrm{Cf}$ Spontaneous Fission

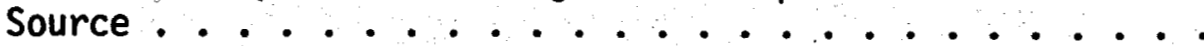

Development of a System for Fission Product Studies Using a ${ }^{252} \mathrm{Cf}$ Spontaneous Source, Helium-Jet Transfer and Fast Radiochemistry .................

Precise Relative $\gamma$-Ray Intensity Measurements . . . . . 98

A Shielded Cell for ${ }^{252} \mathrm{Cf} . \ldots \ldots \ldots 10 . \ldots . \ldots 1$

Isotope Separator Laboratory Development. ........ 108

ATR Experiment Loop Radiochemistry - Monitoring and Reporting . 116

ATR Fuel Element Fission Leak Test. . . . . . . . . . 117

NASA Combines Pulsed Neutron Experiment for Bulk Experimentai

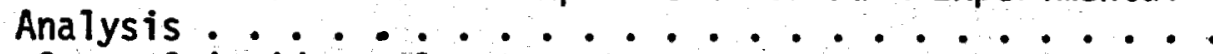

Gamma-Gamma Coincidence Spectrometer. . . . . . . . 121

Nuclear Data Compilation and Evaluation ......... 123

A Computerized File of Evaluated Radionuclide Decay Data. . . 124

A File of Actinide-Isotope Decay Data for ENDF/B. . . . . . 127

Evaluated Decay-Scheme Data for ILRR. .......... 128 
A File of Evaluated Decay-Scheme Data for NRC. . . . . 131

B. APPLIED REACTOR PHYSICS. . . . . . . . . . . 133

Experimental Measurements. . . . . . . . . . 135

EBR-II Irradiation of Highly-Enriched Isotopic Samples . . . . 137

Measurement of the Capture Cross Section of ${ }^{137} \mathrm{Cs}$ in the CFRMF . 143

Integral Cross-Section Measurements on ${ }^{242} \mathrm{Pu}$ in the CFRMF.... 146

${ }^{94} \mathrm{Nb}$ Cross-Section Measurements for CTR Technology ...... . 148

Ion. Exchange Separation of Tracer Amounts of $244 \mathrm{Cm}$ from

Milligram Amounts of ${ }^{241} \mathrm{Am}$. . . . . . . . . . 149

Cross Section of Np-237 in the CFRMF ........... 152

On-Line Reactivity Measurements at the ARMF and CFRMF. ..... 154

Neutron Activation Analysis at INEL. ......... 155

The Ratio of the Hydrogen and Manganese Cross Sections .... 158

Determination of Manganese Content in Aqueous Solutions. . . 161

Transieñt Reactivity Meter ............. 163

Model and Code Development ... . . . . . . . 167

Transformation of Histogram Type Differential Data ..... 168

Wigner - Seitz Approximation in PBF Reload Core Cell Calculations 173

ANS 5.1 Decay Heat Standard. ............ 178

The Cinder-10 Burnup and Fission-Product Buildup Code. . . . 180

Multi-Isotope Resonance Libraries for the PHROG Fast-

Spectrum Code ................... 181

ENDF/B Cross-Section Libraries for the RAFFLE Monte Carlo Code - 182

Auxiliary Computer Codes for Processing PDQ Data Files . . . 184

GOPP - A General One-Dimensional Plot Program for PDQ Flux

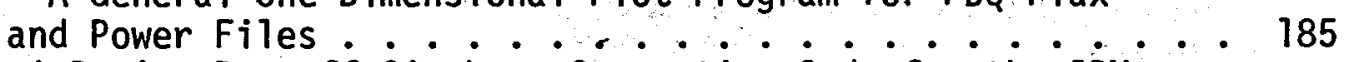

A. Multi-Region Dancoff-Ginsburg Correction Code for the IBM

360/75 System ............. 186

A Comparison of RELAP/4 and PARET Kinetics Calculations. ... 187

Development of a RELAP/4 Model for PBF . . . . . . . 189

Heat Transfer Models for Upper Head Injection. ........ 190

Development of a RELAP/4 Model for Reactivity Induced

Accident Analysis in ATR. . . . . . . . . . . 191

Implementation of KENO IV Code at INEL .......... 193

Remote Hybrid Computer Effectiveness . . . . . . . . . . . 194

Reactor Experiment Design Studies. . . . . . . . . . . 197

GRIST Conceptual Design System Analysis. . . . . . . . 199

Design of the Thermal Neutron Filter on the GRIST Loop in the

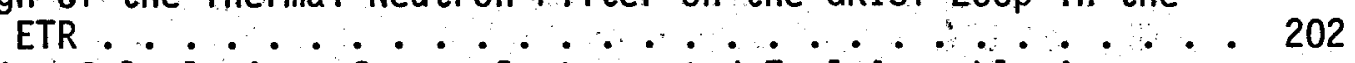

Physics Calculations for an Instrumented Fuel Assembly in
Halden HBWR

Reactor Physics Studies for an AHTGR Experiment in the ETR . . 208

Calculations . . . . . . . . . . . . 213

Study of Expanded Xenon and Samarium Decay Chàin Information . . 214

Data Testing ENDF/B IV Dosimetry Data.\%. . . . . . . 217 
Upgrade of Nuclear Criticality Safety Evaluation. . . . . . .

Determination of Minimum Critical Mass for Flourinel

Dissolvers . . . . . . . . . . . . . . . .

Criticality and Shielding Evaluation of the PBF Pool Storage

Racks for the PBF Reload Core. . . . . . . . . . .

Criticality Evaluation of PBF 5-Ton Cask. . . . . . . . . .

Criticality Evaluation of Overloaded Rocky Flats Waste Drum . . Cask (FMIRC)

Criticality Evaluation for the UFSF Rover Rack. . . . . . . 233

HTGR Dissolver Criticality Scoping Calculation. . . . . . . 234

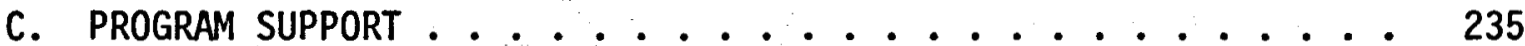

Test Reactors. . . . . . . . . . . . . . 237

ATR and SLSF Data Acquisition Systems Upgrades. . . . . . . 239

On-Line Testing of the Advanced Test Reactor Plant Protection System . . . . . . . . . . .......

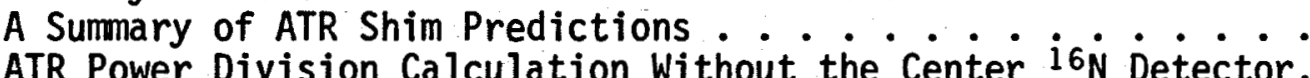

ATR Power Division Calculation Without the Center $16 \mathrm{~N}$ Detector.

Void Analysis for the ATR Experiments . - . . . . . . .

Fast Fluence of ATR Safety Rods . . . . . . . . . . . .

Nuclear Surveillance Program.

that Source Information for ATR Safety Analys is

Use of the DWARF Code to Predict ETR Rod Positions at Startup : 249

ETR SPND Computer Program . . . . . . . . . . . . . 250

Reactivity Worth of Mixed-0xide Fuel Rods . . . . . . . . . 251

Gamma Heating from ETR Mixed-0xide Fuel Experiment. . . . . . . 252

Reactor Gamma-Heating Measurements Using Thermoluminescent Dosimeters . . . . . . . . . . . . . . . 254

Review of Physics Parameters Used for ATR/ETR Burnup

Calculations . ................... . .

Evaluation of Current MeV-Per-Fission Data: Dependence on

Fissioning Isotope and Reactor Composition . . . . . .

Effects of Uncorrelated Noise on ETR $B / \ell$. Calculations from

Neutron Noise. ...................

Power Burst Facility. . . . . . . . . . . . . . .

Irradiated Fuel Rod Scanning. . . . . . . . . . . 266

Physics Calculations for Irradiation Effects Test in PBF. . . $\quad 267$

Physics Calculations for a 16-Pin LOCA Test Design in the PBF - 270

Computed Gamma-Ray Energy Desposition During PCM-20

Power Calibration Test in PBF. . . . . . . . 271

PBF GAP Conductance Experiments and Data Analys is . . . . 272

Energy Desposition Calculations for Lead Rod Tests in PBF . . . 273

Computed Reactivity Requirement for Operation of PBF with a

Large, Reactive Experiment . . . . . . . . . 274

PBF Reload Core Physics Design. . . . . . . . . 276

PBF Reload Core Reactivity Evaluation . . . . . . . . 277

Evaluation of Physics Methods and Data for the PBF Reload

Core Using PBF Critical Experiments. . . . . . . . 
Test Loop Axial Power Shaping for the PBF Reload Core. . . . 282

PBF Reload Core -- Rod Bowing Study. ............. 284

Alternate Control Rod Design for the PBF Reload Core ...... 289

Loss of Fluid Test . . . . . . . . . . . . . . 291

A Hybrid Computer Simulation for the Analysis of a Main Steam

Line Break in the LOFT Plant. ............... 293

An Analog Computer Simulation for the Loft Blowdown Suppression

Tank and Pressure Control System. .................. 295

Calculation of Critical Boron Concentration Versus Control Rod Position Using XYZ Synthesis in PDQ-7......... 298

Calculated Worths for the LOFT-1 Ag-Alloy Control Rod

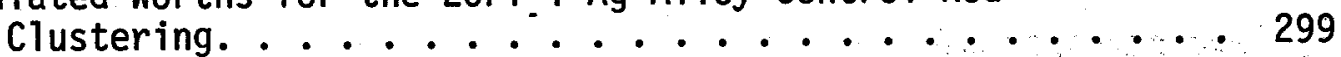

Reactivity Transient Following Shutdown and on Recovery at

Full Power in LOFT-I. . . . . . . . . . . . 302

LOFT Low-Power Physics Program . . . . . . . . . . 304

Power Distribution Calculations to Support LOFT-I Low-Power

Reactor Physics Measurements. .......... 305

LOFT Core Load Reactivity Surveillance Program . . . . . 306

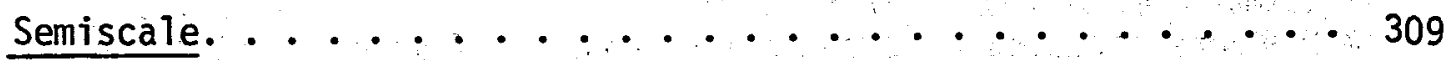

Computerized Core Power Control System ........... 310

Non-Nuclear Programs . . . . . . . . . . . . . . 313

The Clarendon Community: Mixed Energy Application for Human

Living. ............................... 315

A Guide for the Conversion to and Maintenance of Hydrogen-

Fueled, Spark-Ignited Engines . . . . . . . 316

Modification Techniques and Performance Characteristics of

Hydrogen-Powered IC Engines . . . . . . . . . . . 318

Energy Systems Network Simulator ............ 320

The Use of Relevance Trees in Geothermal Planning. . ..... 321

Wind-Augmented Geothermal Power Plant Conceptual Study . . . 326

Transient Modeling and Simulation of the Geothermal Electric

Plant ...................... 327

Geothermal Steam Plant Modeling and Power Trade-Off Studies. . . 331

Geothermal Floating Power Plant and Phase Cooling Studies... . 332

Development of an Evaluation Methodology for the Geothermal Loan Guaranty Program .............. 333

INEL Solar cost Benefit Study. .......................... 335

A Look at the Status of the Topolotron Project and INEL's Role - 336

Waste Management $\ldots \ldots \ldots 337$

Technical Feasibility Studies of Bulk Soil Monitoring for

Transuranic Contamination at $10 \mathrm{nCi} / \mathrm{g} . \ldots 339$

Experimental Studies for Large Boxes of Transuranic Waste. . . 344

Transuranic Waste Monitor for Large Drums. . . . . . . 352

Calibration of a Stabtlized Assay Meter for Waste Drum Assay. . 353

NRC Special Safeguards Study ........................ 357

Uranium Measurements Assessment. . . . . . . . . . . 359 
Source Term Measurement Program at Operating LWR's . . . . . . 360

Remote Gamma-Ray Spectrometer Systems for Facility Monitoring. • 365 
A. NUCLEAR PHYSICS

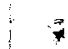

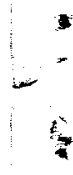

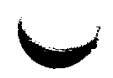





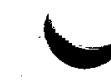

3

$\approx$

NUCLEAR STRUCTURE

条

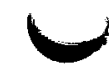





\section{ENERGY LEVELS OF ${ }^{126} \mathrm{Te}$ POPULATED IN THE ${ }^{126} \mathrm{Te}(n, \gamma)$ REACTION}

R. C. Greenwood and R. E. Chrien*

The experimental information available for even-even nuclei with $Z>50, N<82$ tencs to indicate that the $0^{+}$member of the two-phonon triplet is absent, or at least displaced to higher energies, in these nuclei. However for some nuclei, such as $126 \mathrm{Te}$, there is some experimental evidence for the existence of this close spaced triplet. Furthermore, recent calculations by Habs et al. $(2,3)$ based upon a generalized collective model inticate that $\overline{12} 6 \mathrm{Te}$ is a relatively well-behaved vibrational nucleus.

The 126 Te nucleus proves to be an ideal case to search for spin-0 states using the averaged neutron capture technique. The compound nucleus states resulting from s-curve neutron capture in $126 \mathrm{Te}$ have $\mathrm{I}^{\pi}=0^{+}$ and $1^{+}$and hence primary dipole transitions populate final states in ${ }^{126}$ Te with spin values of 0,1 and 2 . Furthermore, the prompt $\gamma$-ray spectra which result from neutron capture using the 2- and 24-keV neutron beams from the SC and Fe/Al filters in the HFBR at Brookhaven represent averages over $\sim 25$ and $\sim 50$ s-wave compound nucleus states, respectively. Hence with this degree of averaging we would expect primary transitions to all states of the same parity and having spin values 0,1 or 2 to have comparable reduced transition intensities. That this is qualitatively the case is immediately apparent from the portions of the 2- and 24-keV neutron capture spectra illustrated in Fig. 1.

In the analysis of these primary $\gamma$-ray spectra to obtain $1^{\pi}$ information on states in ${ }^{126} \mathrm{Te}$ it is important to recognize that while $1=0$ capture predominates for $2-\mathrm{keV}$ neutrons, $1=0$ and $1=1$ capture are comparable for 24-keV neutrons. A consequence of this is that the 24-keV neutron capture data by itself, provides little parity information on final states in ${ }^{126} \mathrm{Te}$ (except for these with $I^{\pi}=3^{+}$). These data do however provide additional confirmation as to the existence of specific final states in ${ }^{126} \mathrm{Te}$ through their population by primary transitions. Also, direct comparison of the 2- and 24-keV intensity ratios do provide unique parity assignments on final states, as do the reduced transition intensities of the primary transitions following 2-keV neutron capture. The results of a partial analysis of these 2- and 24-keV neutron capture spectra to determine level energies in ${ }^{126} \mathrm{Te}$, together with $I^{\pi}$ assignments, is given in Table 1.

More specific information on $I^{\pi}$ for these states is obtained from their de-excitation modes. Partial analysis of the secondary prompt $\gamma$ ray spectra following $2-\mathrm{keV}$ neutron capture in ${ }^{125} \mathrm{Te}$ has allowed us to construct the level scheme shown in Fig. 2 for states in $126 \mathrm{Te}$.

While the complete analysis of the ${ }^{125} \mathrm{Te}(n, \gamma)$ reaction data is as yet unfinished we are nevertheless able, at this point, to draw specific conclusions concerning the structure of the lower excitation states of

* Brookhaven National Laboratory 
${ }^{126} \mathrm{Te}$. Most notable of these being that the $0^{+}$state is not present in an approximately degenerate two-phonon triplet. The lowest excited $0+$ state is conclusively established in this work to lie $\sim 0.5 \mathrm{MeV}$ above the two phonon excitation energy, i.e., at $1873 \mathrm{keV}$. Comparison of the present experimental level information for ${ }^{126} \mathrm{Te}$ is compared with that predicted by Habs et al. in Fig. 3 .

(1) Z. T. Zhelev, N. G. Zaitseva, S. S. Sabirov, Izv. Akad, Nauk SSSR Ser. Fiz. 35, 43 (1971).

(2) D. Habsk H. Klewe-Nebenius, K. Wisshak, R. Lohken, G. Nowicki and H. Rebel, Z. Physik 267, 149 (1974).

(3) D. Habs, H. Klewe-Nebenius, R. Lohken, G. Nowicki, H, Rebel and K. Wisshak, Karlsruhe Report No. KfK-1715 (1972). 
TABLE I

ASSIGNMENT OF $I^{\pi}$ TO STATES IN 126 Te BASED ON PRIMARY $\gamma$-RAY

TRANSITION INTENSITIES FOLLOWING 2- AND 24-keV

NEUTRON CAPTURE IN ${ }^{125} \mathrm{Te}$

Final-state $I^{\pi}$ assignment

\begin{tabular}{|c|c|c|c|c|c|}
\hline $\begin{array}{r}\text { energy } \\
(\mathrm{keV})\end{array}$ & $\begin{array}{l}\text { Previous } \\
\text { work }\end{array}$ & $I_{\gamma}(2) E_{\gamma}^{-3}$ & I $(24) \mathrm{E}^{-3}$ & $\frac{I_{\gamma}(2)}{I_{\gamma}(24)}$ & Adopted \\
\hline 0.0 . & $0^{+}$ & $0^{+}, 2^{+}$ & 0,2 & + & $0^{+}, 2^{+}$ \\
\hline 666.3 & $2^{+}$ & $0^{+}, 1^{+}, 2^{+}$ & 0,2 & + & $0^{+}, 2^{+}$ \\
\hline 1419.9 & $2^{+}$ & $0^{+}, 1^{+}, 2^{+}$ & 0,2 & + & $0^{+}, 2^{+}$ \\
\hline 1873.3 & $0^{+}$ & $0^{+}$ & 0,2 & + & $0^{+}, 2^{+}$ \\
\hline 2044.9 & & $0^{+}$ & 0,2 & + & $0^{+}, 2^{+}$ \\
\hline 2113.1 & & $0^{+}, 2^{+}$ & $0,2,3^{+}$ & + & $0^{+}, 2^{+}$ \\
\hline 2127.7 & & $3^{+}$ & $3^{+}$ & + & $3^{+}$ \\
\hline 2182.2 & $(2)^{+}$ & $0^{+}, 1^{+}, 2^{+}$ & (1) & + & $0^{+}, 1^{+}, 2^{+}$ \\
\hline 2420.4 & & $0^{+}, 2^{+}$ & $0,1,2$ & + & $0^{+}, 1^{+}, 2^{+}$ \\
\hline 2503.3 & $=(2)^{+}$ & $0^{+}, 2^{+}$ & 0,2 & + & $0^{+}, 2^{+}$ \\
\hline 2578.1 & $(0)^{+}$ & $0^{+}, 2^{+}, 3^{+}$ & 0,2 & + & $0^{+}, 2^{+}$ \\
\hline 2638.3 & & $3^{+}$ & $3^{+}$ & + & $3^{+}$ \\
\hline 2680.9 & $(2)^{+}$ & $0^{+}, 1^{+}, 2^{+}$ & $0,1,2$ & + & $0^{+}, 1^{+}, 2^{+}$ \\
\hline
\end{tabular}




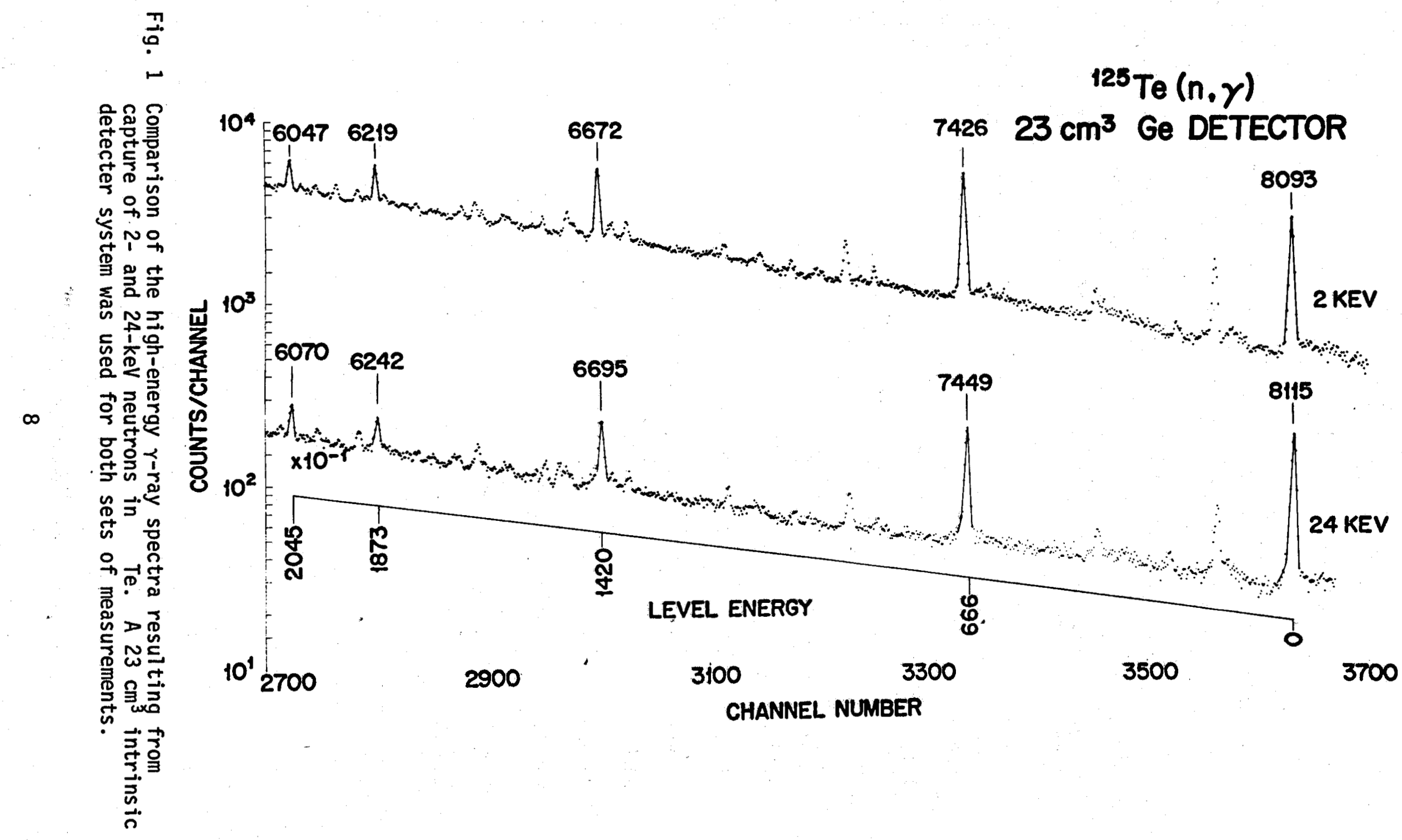

C. 


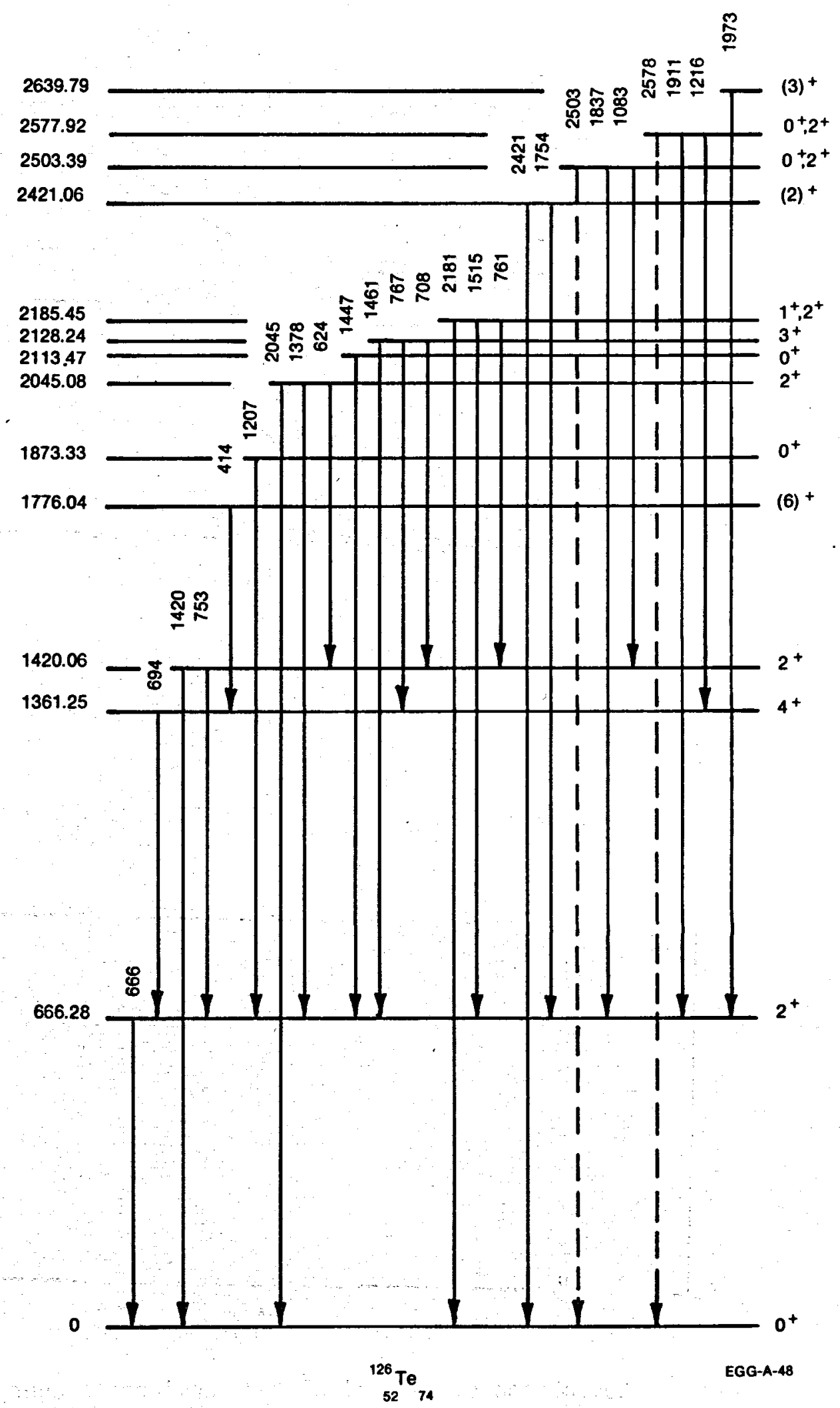

Fig. 2 Proposed level scheme for ${ }^{126} \mathrm{Te}$. 


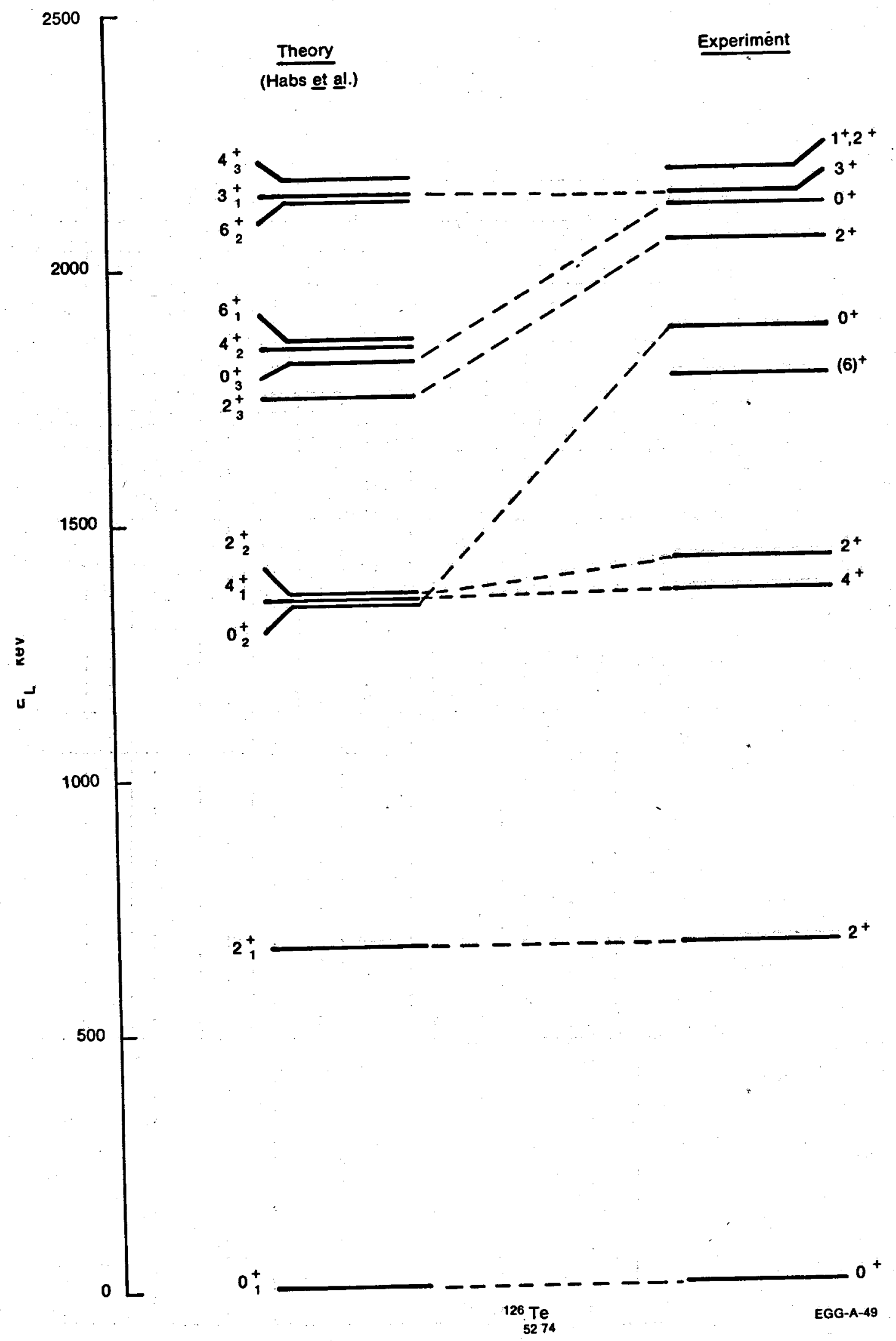

Fig. 3 Comparison of the calculated ${ }^{3}$ collective energy-level spectrum of ${ }^{126} \mathrm{Te}$ and the experimentally determined level scheme. 


\section{DECAY OF ${ }^{128} \mathrm{Ba}$}

R. G. Helmer, R. J, Gehrke,

R. C. Greenwood, C. $\mathbb{W}$. Reich, L. D. McIsaac*

The following is the abstract of a paper published in Nuclear Physics A258, 83-90 (1976).

Samples of ${ }^{128} \mathrm{Ba}$ have been produced by $\approx 800 \mathrm{MeV}$ proton-induced spallation in praseodymium metal foils at LAMPF. Chemical purification and isotope separation have been performed to produce pure samples of ${ }^{128} \mathrm{Ba}(2.4 \mathrm{~d})-{ }^{128} \mathrm{Cs}(3.9 \mathrm{~min})$. Some of the $\gamma$-ray and $\gamma-\gamma$ coincidence measurements were made during continuous $\mathrm{Ba}-\mathrm{Cs}$ separations which increased the $\mathrm{Ba} / \mathrm{Cs}$ ratio by factors of between two and six from that at secular equilibrium. These measurements improved the sensitivity for detecting weak transitions from the ${ }^{128} \mathrm{Ba}$ decay and allowed isotopic assignment of several $\gamma$-rays. From the experimental results, twelve $\gamma$-ray transitions have been assigned to the ${ }^{128} \mathrm{Ba}$ decay, compared to only one previously, and they have all been placed in a level scheme with excited states in ${ }^{128} \mathrm{Cs}$ at $187,215,229,273,317,359$ and $374 \mathrm{keV}$. By comparison with the daughter decay, absolute $\gamma$-ray intensities are determined.

\footnotetext{
*Allied Chemical Company, Idaho National Engineering Laboratory
} 
DECAY OF ${ }^{128} \mathrm{Cs}$

R. G. Helmer, C. W. Reich, R. J. Gehrke

R. C. Greenwood, R. A. Anderl

As discussed previously(1) as part of our program of nuclear structure studies of neutron-deficient isotopes off the line-of-stability, samples of ${ }^{128} \mathrm{Ba}-\mathrm{Cs}$ have been produced at the C. P. Anderson Meson Physics Facility (LAMPF) by irradiation of praseodymium metal with $\sim 800$ MeV protons. These targets were shipped to the INEL where the barium fraction was chemically extracted and the mass-128 fraction was obtained from an isotope separator. The resulting samples included only the radioactive isotopes ${ }^{128} \mathrm{Ba}$ and the daughter ${ }^{128} \mathrm{Cs}$.

Gamma-ray spectra obtained during a continuous Ba-Cs chemical separation were used $(2)$ to identify twelve $\gamma$ rays associated with the ${ }^{128 \mathrm{Ba}}(2.4 \mathrm{~d})$ activity. The remaining $\gamma$ rays are given in Table I and are associated with the ${ }^{128} \mathrm{Cs}(4 \mathrm{~m})$ decay.

Measurements were made of the $\gamma-\gamma$ coincidence spectra with gates on the peaks at $442,511,526,590,1030$ and $1140 \mathrm{keV}$. In addition to establishing many $\gamma-\gamma$ coincidence relationships, these spectra can be used to determine the absence of many other $\gamma-\gamma$ coincidences. For this particular decay scheme, the "no coincidence" result does really mean that the $\gamma$ ray does not populate any level at, or above, that depopulated by the gating $\gamma$ ray. From this fact, the $\gamma-\gamma$ coincidence data can be used to place many $r$ rays in the level scheme.

The $\gamma-\gamma$ coincidence results are also used to determine the absolute $\beta^{+}$branching ratios to the levels at 969 and $1999 \mathrm{keV}$, and the relative $\beta^{+}$intensities to the levels at 442 and $1582 \mathrm{keV}$.

The decay scheme deduced from these data is shown in Fig. 1. The seven transitions at the left-hand side of the figure are placed tentatively since only one transition is seen from each level. The remainder of the scheme is quite well determined. The spins and parities of the states below $1200 \mathrm{keV}$ are known from previous experiments and those for the states above $1200 \mathrm{keV}$ are deducted from the electroncapture population and $\gamma$-ray depopulation of the levels.

(1) R. G. Helmer, R. C. Greenwood, R. J. Gehrke and C. W. Reich, Nuclear Technology Division Annual Progress Report for Period Ending June 30, 1974, ANCR-1177 (1975) p.44.

(2) R. G. Helmer, R. J. Gehrke, R. C. Greenwood, C. W. Reich and L. D. McIsaac, Nucl. Phys. A258, 83 (1976). 
Table 1

${ }^{128} \mathrm{Cs} \gamma$-ray energies and intensities

Energy (keV) $\quad$ Relative intensity ${ }^{a}$ Placement in scheme

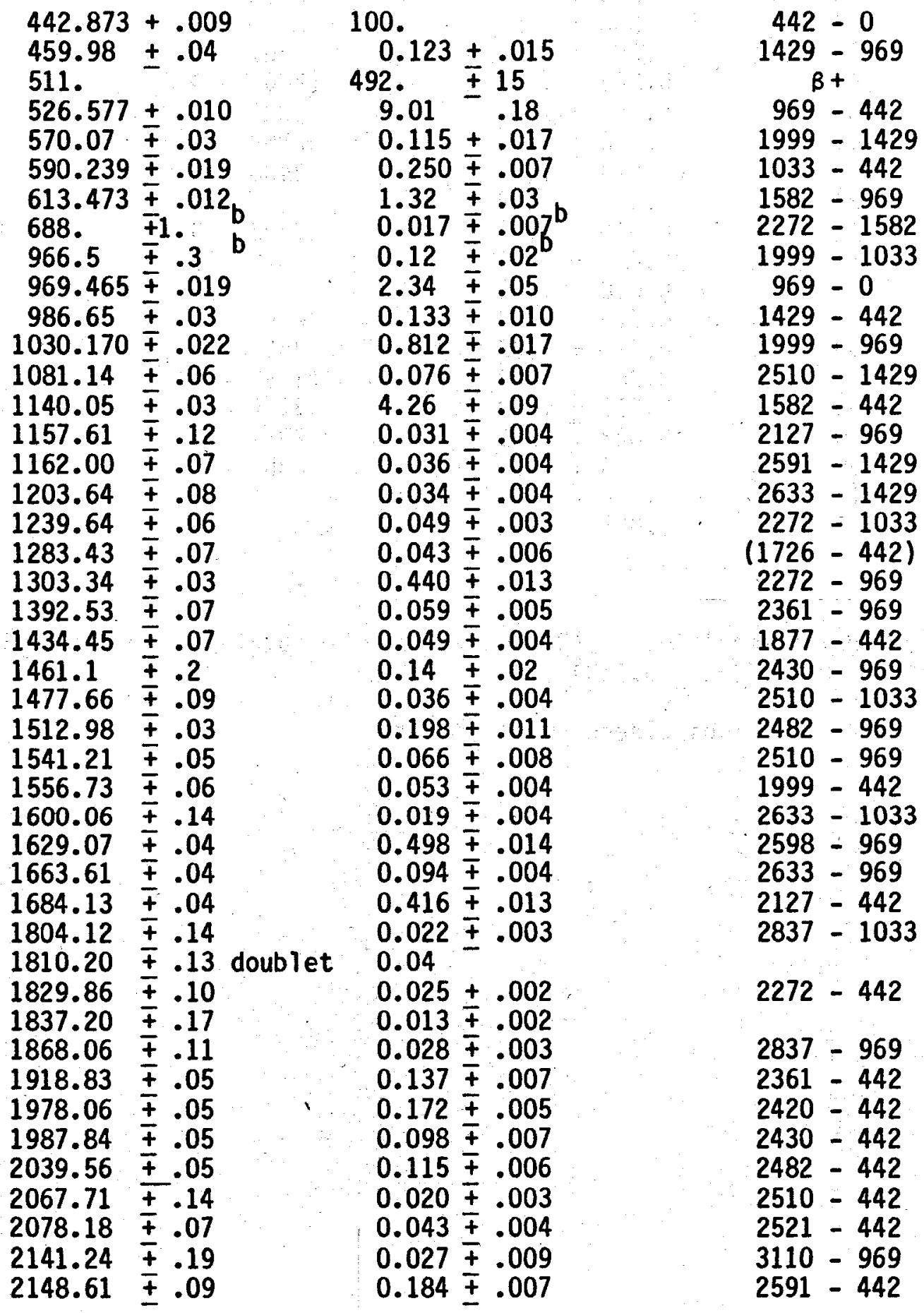


Table 1 (continued)

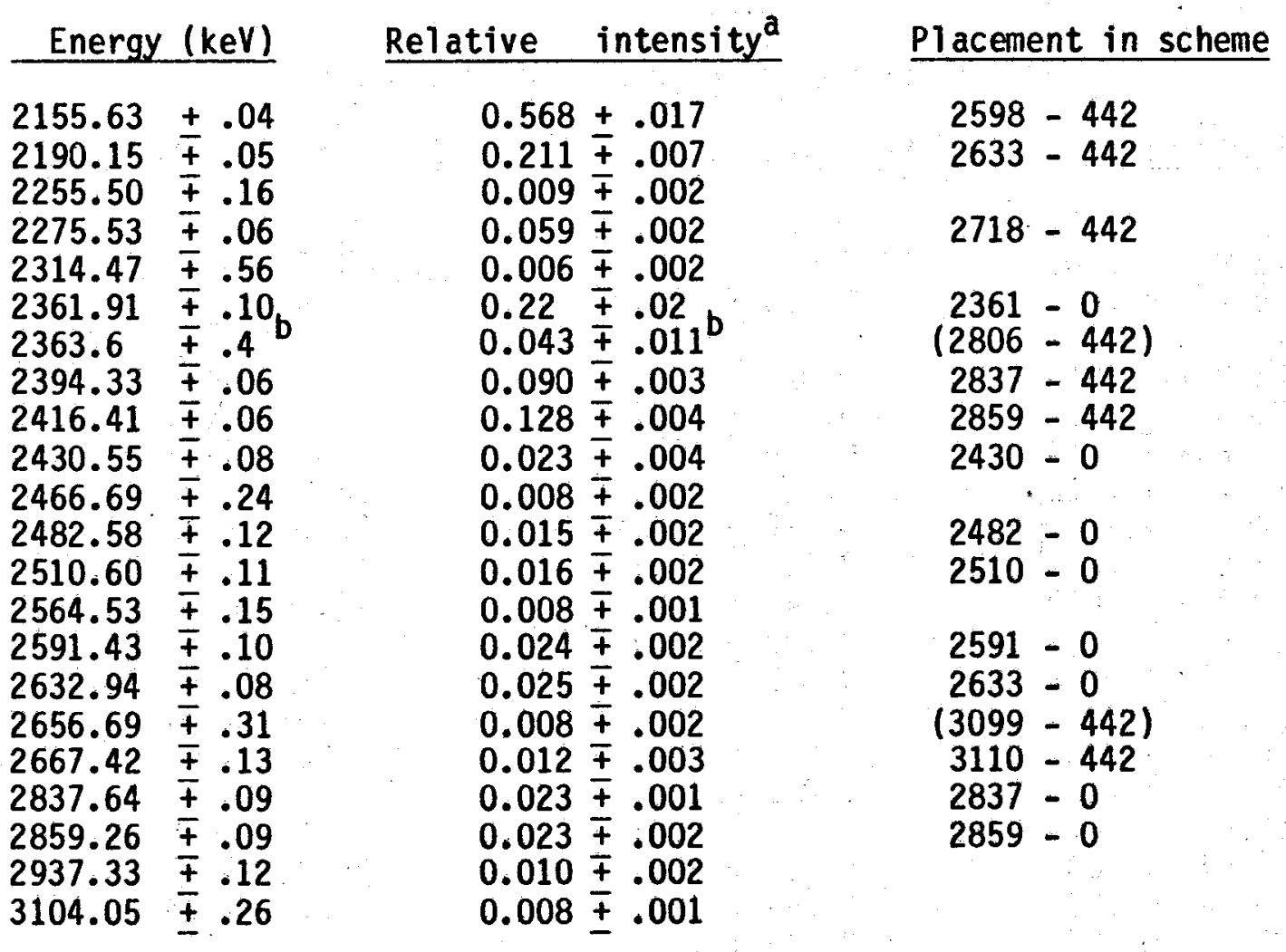

\footnotetext{
$a_{\text {Absolute }} \gamma$-ray intensities, $\gamma / 100$ decays, can be obtained by multiplying these values by $(0.258 \pm 0.013)$

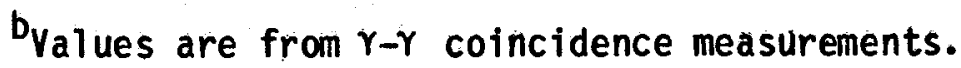



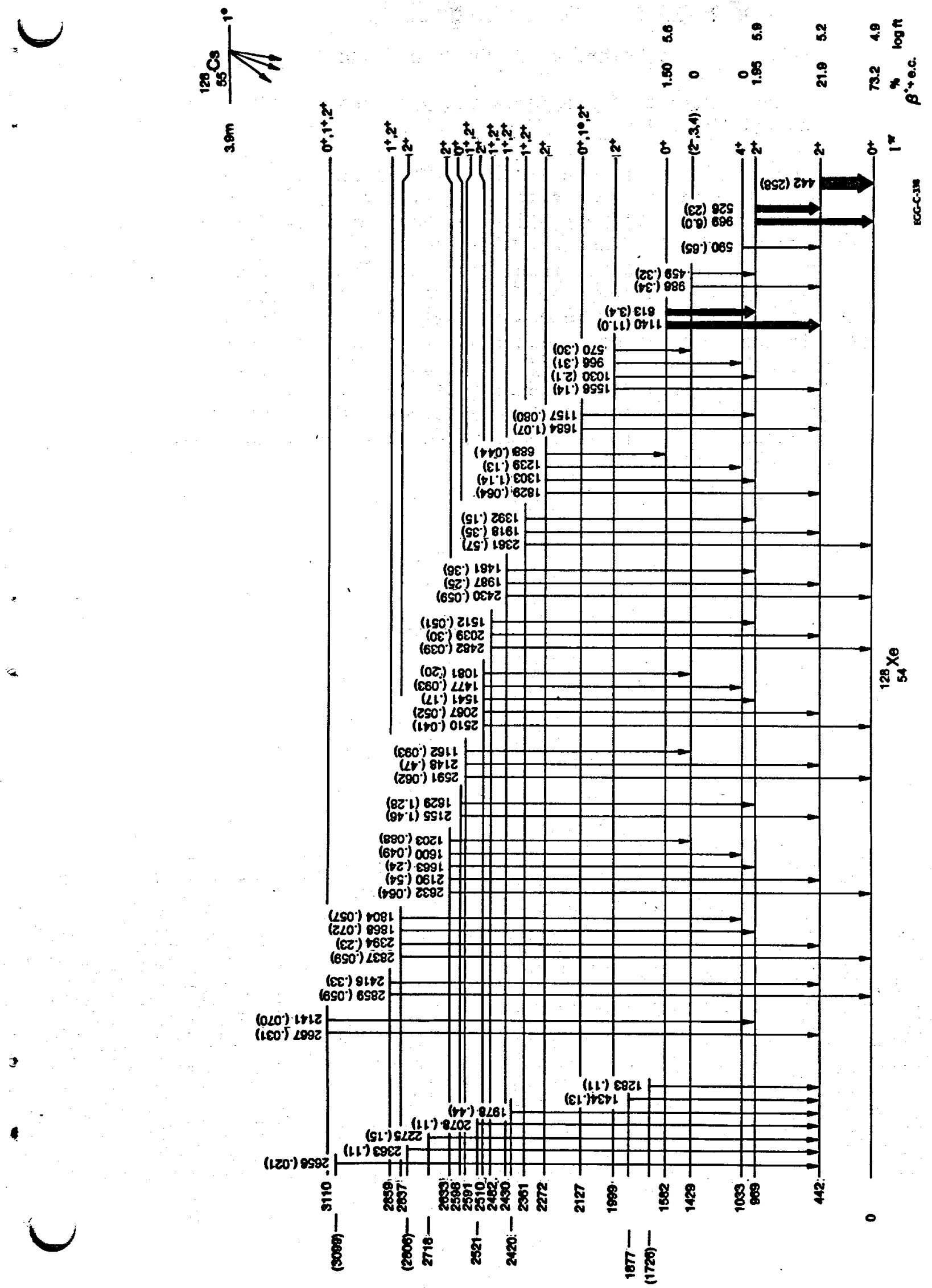

(ez'ㄴ) 629

(880') coz! 
LEVEL STRUCTURE OF ${ }^{131} \mathrm{CS}$ AND THE DECAY ENERGY OF $131 \mathrm{Ba}$

R. J. Gehrke, R. G. Helmer, C. W. Reich, R. C. Greenwood and R. A. Anderl

The following is the abstract of a paper which has been accepted for publication in the journal Phys. Rev. C.

The decay of $131 \mathrm{Ba}(11.7$ d) has been studied with Ge(Li) spectrometers and with a $\mathrm{Ge}(\mathrm{L} i) \gamma-\gamma$ coincidence system. Twelve $\gamma$ transitions and three levels in ${ }^{13} \mathrm{C}$ Cs at 919,1170 and $1342 \mathrm{keV}$ have been identified which were not reported previousiy. Upper limits are given for the $\gamma$-ray intensities of previously reported transitions at 323 and $508 \mathrm{keV}$ which were not observed in this experiment. The $Q$ value for the decay of ${ }^{131} \mathrm{Ba}$ was determined from the $\mathrm{ec} / \mathrm{B}^{+}$intensity ratio to be $1372 \pm 20 \mathrm{keV}$. This value agrees with the latest Atomic Mass Evaluation, but disagrees with the only previous measurement by $207 \mathrm{keV}$. Our ec/ $\beta^{+}$intensity ratio and the $Q$ value deduced from a reaction study suggest that the $\mathrm{ec} / \mathrm{\beta}^{+}$ratio is not anomalous, in contrast to some recent predictions. The ${ }^{131}$ Cs level structure and $r$ transition probabilities are compared to the results of an intermediate-coupling calculation. 


\section{HALF-LIVES OF $134 \mathrm{La}$ AND $132-135 \mathrm{Ce}$}

R. J. Gehrke and R. G. Helmer

The half-lives of 134 La and 132-135Ce have been measured by means of $\mathrm{r}$-ray spectrometry with $\mathrm{Ge}(\mathrm{Li})$ detectors. All samples were chemically and isotopically separated from material produced by proton spallation jn praseodymium metal. The measured half-lives are $134 \mathrm{La}(6.45+0.16)$ min, $132 \mathrm{Ce}(3.51+0.11) \mathrm{hr},{ }^{133} \mathrm{Ce}(4.93+0.39) \mathrm{hr},{ }^{134} \mathrm{Ce}(75.9+0.9) \mathrm{hr}$ and ${ }^{135} \mathrm{Ce}(17 . \overline{6}+0.31) \mathrm{hr}$. The errors include an estimated systematic contribution as well as the statistical contribution from the analysis of the data. The present measurements are compared to previously reported half-life values. 
R. C. Greenwood, C. W. Reich, R. G. Helmer

R. J. Gehrke, R. A. Anderl and J. D. Baker*

Here, we present the results of an investigation of the ${ }^{134} \mathrm{Ba}$ level scheme as observed in the decay of $134 \mathrm{La}$. The properties of nuclides in this mass region $(50<Z, N<82)$ are of considerable current interest because of the information which they provide concerning the collective potential-energy surfaces and how these surfaces vary with both proton and neutron number.

The sources of ${ }^{134} \mathrm{Ce}-134 \mathrm{La}$ used in these experiments were produced by spallation in Pr-metal targets using $-800-\mathrm{MeV}$ protons from the Clinton P. Anderson Meson Physics Facility (LAMPF). Typically, samples of praseodymium foils having a total thickness of $0.030 \mathrm{~cm}$ were irradiated in an integrated beam flux of -15 $\mu \mathrm{A}-\mathrm{h}$. After bombardment, the irradiated foils were transported to the INEL for study.

The Ce fraction was separated from the other spallation products and the $\operatorname{Pr}$ using the following procedure. The $\operatorname{Pr}$ foils were dissolved in $5 \mathrm{M} \mathrm{HCl}$. Following dissolution, $1 \mathrm{mg} \mathrm{Ce}{ }^{+3}$ carrier was added and $\mathrm{Ce}(\mathrm{OH})_{3}$ precipitated. This precipitate was washed with $\mathrm{H}_{2} \mathrm{O}$ and converted to the nitrate by thrice heating to near dryness with conc. $\mathrm{HNO}_{3}$. The $\mathrm{Pr}$ and $\mathrm{Ce}$ salts were dissolved in a $10 \mathrm{M} \mathrm{HNO}{ }_{3}-0.1 \mathrm{M} \mathrm{NaBrO}$ solution and the oxidized $\mathrm{Ce}^{+4}$ was extracted into $0.3 \mathrm{M}$ di-2ethylhexylphosphoric acid (HDEHP) in heptane. Cerium was further purified by twice washing the HDEHP with $10 \mathrm{M} \mathrm{HNO}{ }_{3}-0.1 \mathrm{M} \mathrm{NaBrO}_{3}$, back extracting with $6 \mathrm{M} \mathrm{HNO}_{3}$ containing several drops of $30 \% \mathrm{H}_{2} \mathrm{O}_{2}$, and finally scrubbing the strip solution with heptane. $\mathrm{Ce}(\mathrm{OH})_{3}$ was precipitated from the back extractant and washed twice with $\mathrm{H}_{2} \mathrm{O}$ and twice with ethyl alcohol. Following the chemical separation, the residual fraction of Ce was mass separated. A surface-ionization-type ion source was employed in these separations and a complete separation typically took $\sim 4-6$ hours.

A typical $\gamma$-ray spectrum of a mass-separated ${ }^{134} \mathrm{Ce}-{ }^{134} \mathrm{La}$ sample is shown in Fig. 1. A listing of the averaged energies and intensities (relative) of the $\gamma$-ray transitions which could be associated with the ${ }^{134}$ La decay in the present work is given in Table I.

To assist in the determination of absolute photon emission rates in the ${ }^{134} \mathrm{Ce}+{ }^{134} \mathrm{La} \rightarrow 134 \mathrm{Ba}$ decay chain, it is necessary to know the relative intensities of the $K x$-radiation from $L a$ and $B a$. The $S i(L i)$ photon spectrum from which these data were determined is shown in Figure 2.

Gamma-ray coincidence spectra were measured for several of the strongest $\gamma$-ray lines in the ${ }^{134} \mathrm{La}$ spectrum. These lines were those at

*Allied Chemical Corporation 
$563,604,1211,1424,1483,1554$ and $1732 \mathrm{keV}$ (in a $180^{\circ}$ geometry), as well as the 517-keV annihilation-radiation peak (in a $90^{\circ}$ geometry). A typical $\gamma-\gamma$ coincidence spectrum, that is coincident with the 604-keV $\left(2^{+} \rightarrow 0^{+}\right)$transition, is shown in Figure 3 . The $\gamma$-ray coincidence relationships which have been established from these data are summarized in Table II.

A decay scheme for ${ }^{134} \mathrm{La}$ is quite readily constructed from the data obtained in the present study. The extensive $\gamma-\gamma$ coincidence relationships, summarized in Table II, generally give quite unambiguous evidence as to the placement of $\gamma$-ray transitions in the $134 \mathrm{Ba}$ level scheme. These coincidence data are further supplemented by the noncoincident $\gamma$-ray transitions, which are therefore inferred to be ground-state transitions. Since the decay energy of ${ }^{134} \mathrm{La}$ is quite well known $(Q=3720 \pm 25 \mathrm{keV})$ we can also infer that those $\gamma$-ray transitions with energies $\geq 3140 \mathrm{keV}$ must populate the ${ }^{134} \mathrm{Ba}$ ground state, while those transitions with energies $z 2580 \mathrm{keV}$ must populate either the ground state or the 604-keV first excited state (assuming that the second excited state in ${ }^{134} \mathrm{Ba}$ is at $1167 \mathrm{keV}$ ).

The placement of other $\gamma$-ray transitions, which were not sufficiently intense to be observed in the coincidence spectra or by the energy limits noted above, was facilitated by the high precision. attained for the $\gamma$-ray energy measurements. Such $\gamma$-ray transitions could then generalily be placed in the level scheme from the Ritz combination principle.

The decay scheme of $134 \mathrm{La}$ which was proposed from the present data, using the considerations cited above, is shown in Fig. 4.

In Table III are summarized the reduced transition probabilities of the electric-multipole transitions involving those $134 \mathrm{Ba}$ states whose $I^{\pi}$ assignments seem reasonably firmly established at present. Also included in Table III are the results of two recent theoretical calculations $(1-3)$ in which certain of the properties of the lighter-mass even-even $\mathrm{Xe}, \mathrm{Ba}, \mathrm{Ce}$ and $\mathrm{Nd}$ nuclides were treated. The approach employed by Habs et al. (1) is based on the generalized collectlive model of Gneuss and Greiner. $(4)$. For ${ }^{134 \mathrm{Ba}}$, the resulting E2 transition data are given in Table III, and the predicted collective energy level spectrum is shown in Fig. 5. Using a somewhat different approach, Rohoziniski et al. (2) employed a Wilets-Jean(5) Hamiltonian to which they added a term producing a small oblate-prolate asymmetry. Although they give no level energies, they do 1ist the calculated EO and E2 transition probabilities. For ${ }^{134} \mathrm{Ba}$, they present these transition probabilities calculated for two different assumptions concerning the choice of parameters of the Hamiltonian. Both sets of calculated values are summarized in Table III. These authors treat two types of $0^{+}$states: a " $\beta$-vibration," with quantum numbers (2) $n_{\beta}=1, \lambda=0$; and a state which, in a spherical-nucleus coupling scheme, corresponds to the $0^{+}$ member of the three-phonon muitiplet, with quantum numbers $n_{B}=0, \lambda=3$. In Table III, the first value listed is that for the " $\beta$-vibration" and the second, listed immediately below, is that for the $0^{+}$state with $n_{\beta}=0, \lambda=3$. 
${ }^{134} \mathrm{Ba}$ exhibits an interesting spectrum of excited $0^{+}$states (see, e.g., Fig. 5). The decay of the $0^{+}$state at $1760 \mathrm{keV}$ is characterized by a very large $B(E 0) / B(E 2)$ (i.e., $X$ ) value. This is suggestive of $\beta$-vibrational states in strongly deformed nuclei, and indeed this state has been classified( 6 ) as a "quasi-beta" vibrational band head. However, its E2 decay, which shows a marked preference for decay to the second rather than to the first excited $2^{+}$state, suggests that another configuration assignment for this state is more appropriate. The three $0^{+}$states clustered near $-2.4 \mathrm{MeV}$ have quite different E0 and E2 decay patterns. The collective-model calculations reported by Habs et al. $(1,3)$ do not predict any $0^{+}$states in this energy region (the next excited $0^{+}$state above that at $\sim 1.7 \mathrm{MeV}$ is predicted to occur at $-6.2 \mathrm{MeV}$ ). The number and spacing of these three $0^{+}$states suggests that types of nuclear excitations such as, e.g., two-quasiparticle excitations in addition to those of collective character may play a role in their make-up. The fact that they are fed by beta transitions with comparable $\log \mathrm{ft}$ values suggests the presence of a common two-quasiparticle component, with a comparable amplitude, in each of these states. However, additional information is required before definitive statements can be made concerning the detailed wave functions of these states.

(1) D. Habs, H. Klewe-Nebenius, K. Wisshak; R. Lohken, G. Nowicki and H. Rebe1, Z. Physik 267, 149 (1974).

(2) S. G. Rohoziński, J. Srebrny and K. Horbaczewska, Z. Physik 268, 401 (1974).

(3) D. Habs, H. Klewe-Nebenius, R. Lohken, G. Nowicki, H. Rebel and K Wisshak, Karlsruhe Report No. KfK-1715, 1972.

(4) G. Gneuss and W. Greiner, Nucl. Phys. A171, 449 (1971).

(5) L. Wilets and M. Jean, Phys. Rev. 102, 788 (1956).

(6) M. Sakai, At. Data and Nucl. Data Tables 15, 513 (1975).

(7) J. R. Kerns and J. X. Saladin, Phys. Rev. C 6, 1016 (1972).

(8) M. Behar, R. M. Steffen and C. Telesco, Nucl. Phys. A192, 218 (1972).

(9) J. Burde, S. Eshhar, A. Ginzburg and A. Molchadzki, Nuc1. Phys. A250, 141 (1975).

(10) E. A. Henry, Nucl. Data Sheets 15, 203 (1975). 
TABLE I. $\gamma$-ray energies and intensities from ${ }^{134}$ La decay.

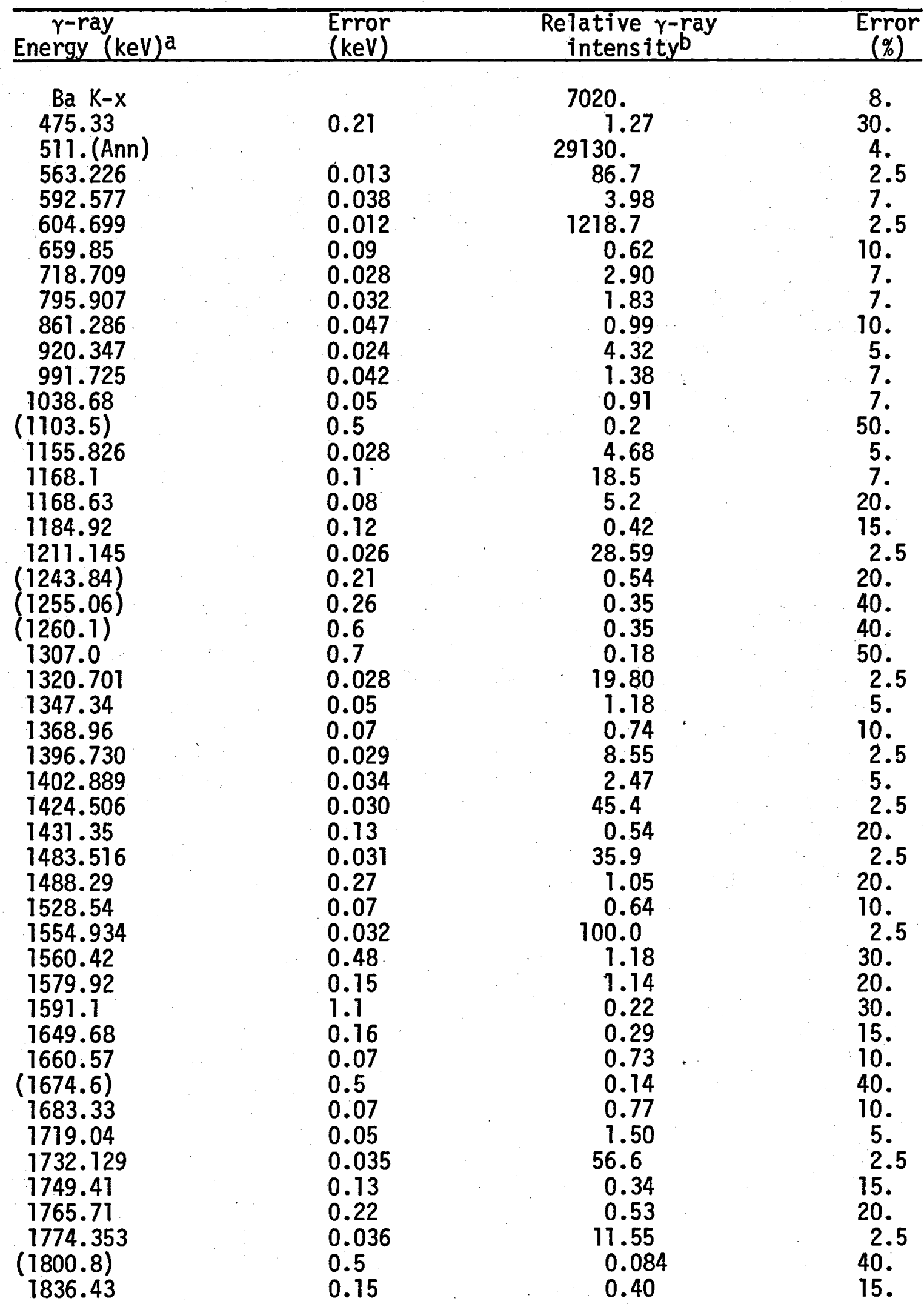


TABLE I. (Continued)

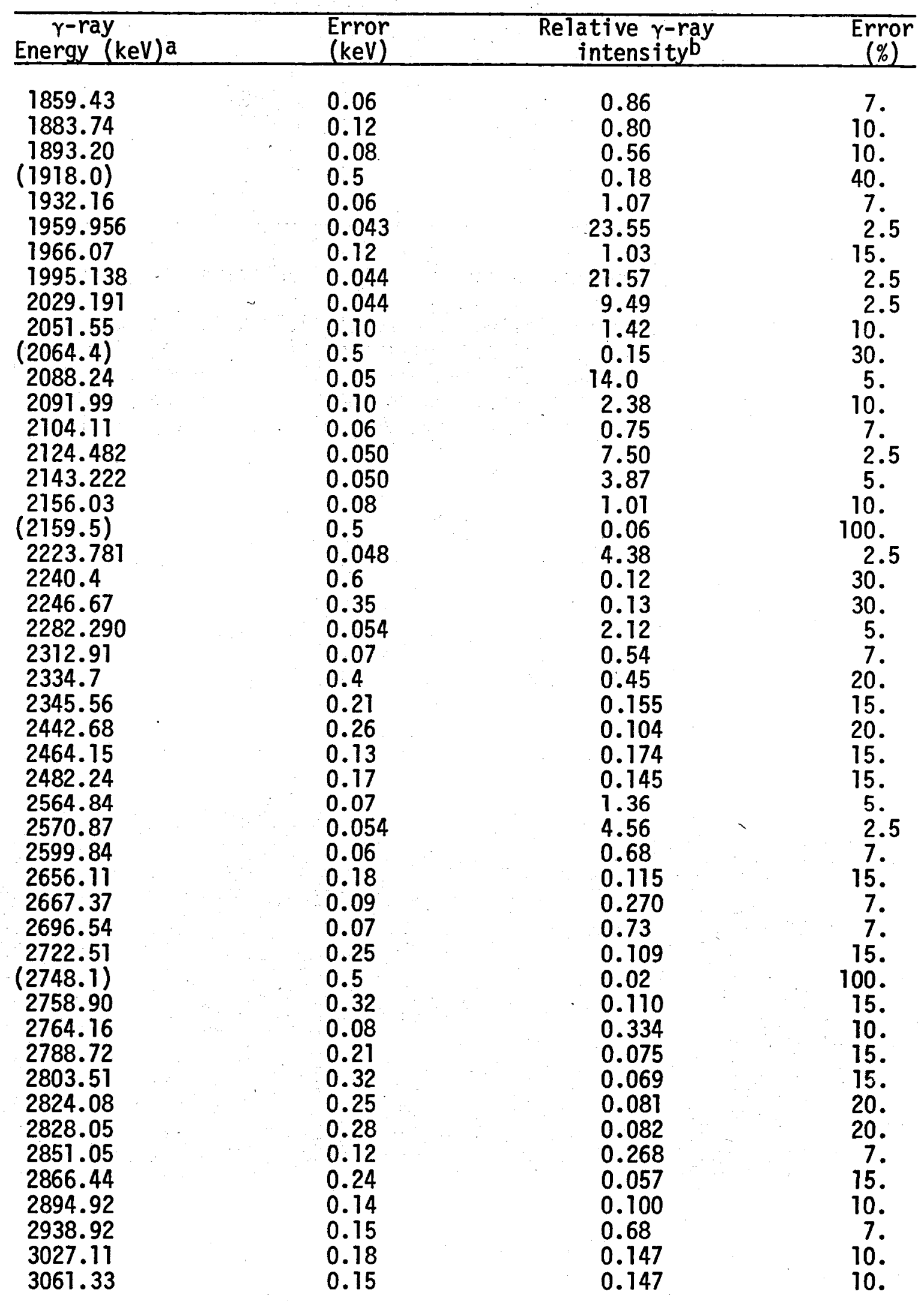


TABLE I. (Continued)

\begin{tabular}{cccc}
\hline $\begin{array}{c}\gamma-\text { ray } \\
\text { Energy (keV)a }\end{array}$ & $\begin{array}{c}\text { Error } \\
(\mathrm{keV})\end{array}$ & $\begin{array}{c}\text { Relative } \gamma \text {-ray } \\
\text { intensityb }\end{array}$ & $\begin{array}{c}\text { Error } \\
(\%)\end{array}$ \\
\hline 3086.59 & 0.15 & 0.082 & 15. \\
3160.04 & 0.15 & 0.135 & 10. \\
3245.84 & 0.19 & 0.055 & 20. \\
3327.18 & 0.15 & 0.139 & 10. \\
3449.46 & 0.18 & 0.143 & 10. \\
& & & \\
\hline
\end{tabular}

a Parentheses around a $\gamma$-ray energy indicate a tentative assignment.

b These values must be multiplied by a factor $4.14 \times 10^{-3}$ to convert to absolute intensities (in $\gamma / 100$ disintegrations). Since the uncertainty in this conversion factor is $\pm 4.1 \%$, this should be added in quadrature to the error assigned to the relative intensities to obtain the total error assigned to the absolute intensity values. 
TABLE II. $\gamma-\gamma$ coincidence relationships

\begin{tabular}{ll}
\hline $\begin{array}{c}\text { Gate energy } \\
(\mathrm{keV})\end{array}$ & \multicolumn{1}{c}{$\begin{array}{c}\text { Coincident } \gamma \text {-ray energies } \\
(\mathrm{keV})\end{array}$} \\
\hline 563 & $475,511,592,604,861,920,991,1168,1211,1320,1368,1396,1402,1528,1579$, \\
& $1660,1683,1719,1749,1836,1859,1893,1918,2104$ \\
604 & $511,563,592,718,861,920,1155,1168,1211,1320,1347,1396,1402,1424,1483$, \\
& $1528,1554,1732,1774,1883,1918,1959,1995,2051,2091,2104,2124,2143,2156$, \\
1211 & $2223,2282,2312,(2482), 2764,(2894)$ \\
1424 & $563,604,1168$ \\
1483 & 604,718 \\
1554 & 604,659 \\
1732 & 604 \\
& 604 \\
\hline
\end{tabular}

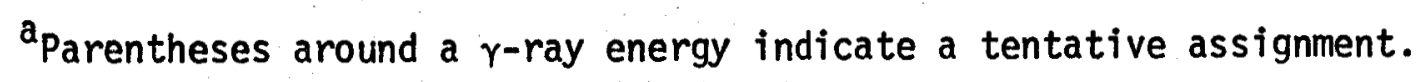


TABLE III. Summary of the E2 and EO branching-ratio data from the ${ }^{134} \mathrm{Ba}$ states whose spin and parity values appear to be unambiguously established. Also included are the relevant theoretical values from the calculations presented in Refs. 1-3. The $B(E 2)$ values are expressed in units of $e^{2} \cdot b^{2}$. The quantities in parentheses following the experimental values represent the uncertainties in the least significant figure (or figures) of the associated values.

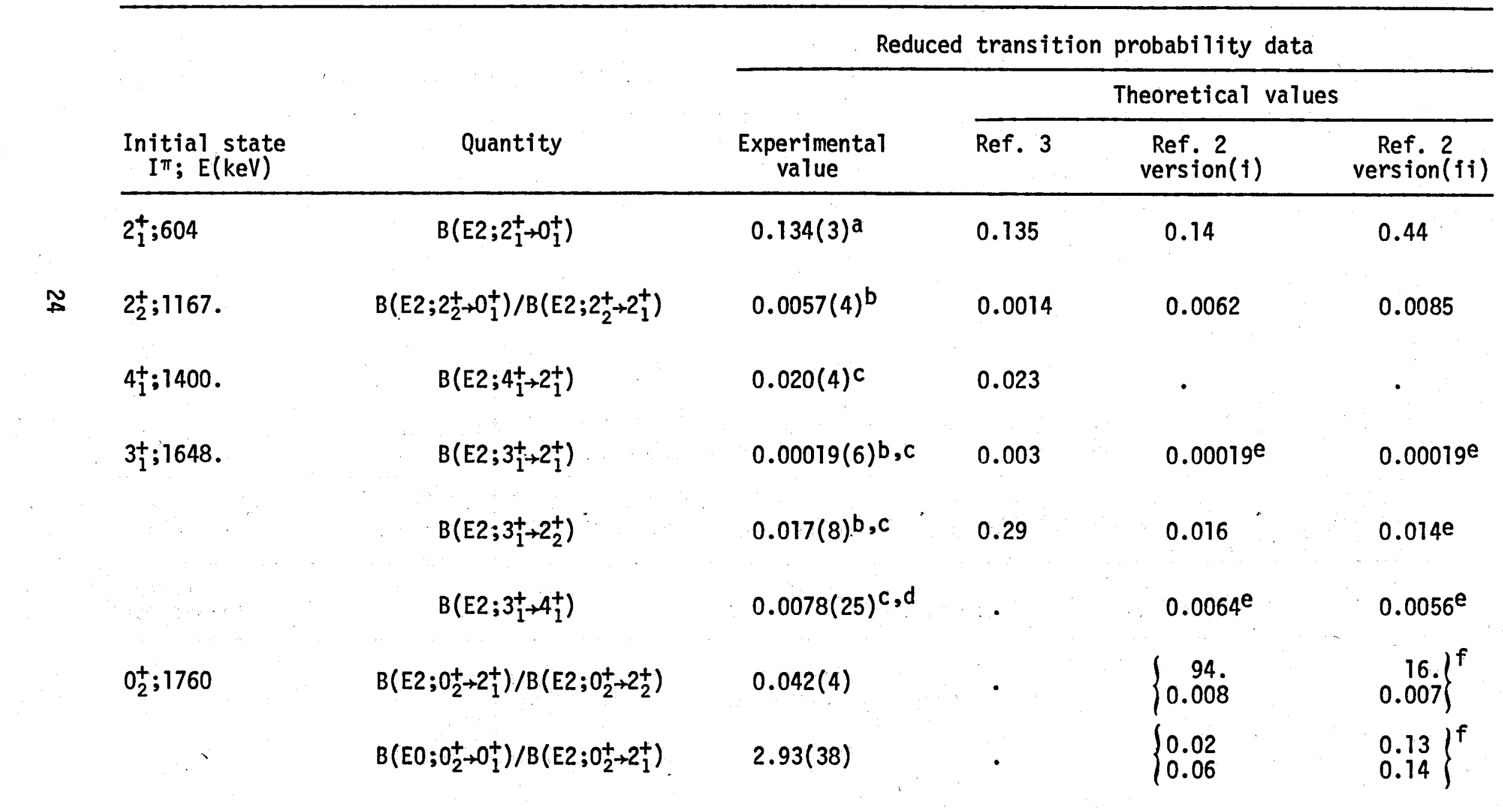




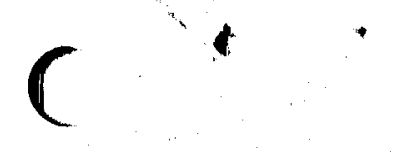

TABLE III. (Continued)

\begin{tabular}{|c|c|c|c|c|c|}
\hline \multirow[b]{3}{*}{$\begin{array}{c}\text { Initial state } \\
I^{\pi} ; E(\mathrm{keV})\end{array}$} & \multirow[b]{3}{*}{ Quantity } & \multicolumn{4}{|c|}{ Reduced transition probability data } \\
\hline & & \multirow[b]{2}{*}{$\begin{array}{l}\text { Experimental } \\
\text { value }\end{array}$} & \multicolumn{3}{|c|}{ Theoretical values } \\
\hline & & & Ref. 3 & $\begin{array}{l}\text { Ref. } 2 \\
\text { version( } i)\end{array}$ & $\begin{array}{l}\text { Ref. } 2 \\
\text { version( }(i i)\end{array}$ \\
\hline \multirow[t]{3}{*}{$4_{2}^{+} ; 1969.9$} & $B\left(E 2 ; 4_{2}^{+}+22_{1}^{+}\right) / B\left(E 2 ; 4_{2}^{+}+2_{1}^{+}\right)$ & $0.0243(3)$ & 0.01 & 0.014 & 0.012 \\
\hline & $B\left(E 2 ; 4_{2}^{+} \rightarrow 3_{1}^{+}\right) / B\left(E 2 ; 4_{2}^{+}+2_{2}^{+}\right)$ & $0.147(6)^{h}$ & 0.3 & 0.010 & 0.0037 \\
\hline & $\mathrm{B}\left(\mathrm{E} 2 ; 44_{2}^{+} \rightarrow 4_{1}^{+}\right) / \mathrm{B}\left(\mathrm{E} 2 ; 4_{2}^{+} \rightarrow 2_{2}^{+}\right)$ & $0.76(11)$ & 0.6 & 0.91 & 0.91 \\
\hline$\left(0_{3}^{+}\right) ; 2159$. & $\mathrm{B}\left(\mathrm{E} 2 ; 0_{3}^{+} \rightarrow 2_{1}^{+}\right) / \mathrm{B}\left(\mathrm{E} 2 ; 0_{3}^{+} \rightarrow 2_{2}^{+}\right)$ & $7.65(57)$ & - & $\left\{\begin{array}{c}94 \\
0.008\end{array}\right.$ & $\left.\begin{array}{c}16 \\
0.000\end{array}\right\}^{f}$ \\
\hline \multirow[t]{2}{*}{$0_{4}^{+} ; 2336$} & $B\left(E 2 ; 0_{4}^{+}+2_{1}^{+}\right) / B\left(E 2 ; 0_{4}^{+} \rightarrow 2_{2}^{+}\right)$ & $1.52(30)$ & - & $\left\{\begin{array}{c}94 \\
0.008\end{array}\right.$ & $\left.\begin{array}{c}16 \\
0.007\end{array}\right\}^{f}$ \\
\hline & $\mathrm{B}\left(\mathrm{E} 0 ; 0_{4}^{+} \rightarrow 0_{1}^{+}\right) / \mathrm{B}\left(\mathrm{E} 2 ; 0_{4}^{+} \rightarrow 2_{1}^{+}\right)$ & 0.015 & - & $\left\{\begin{array}{l}0.02 \\
0.06\end{array}\right.$ & $\left.\begin{array}{l}0.13 \\
0.14\end{array}\right\}^{f}$ \\
\hline \multirow[t]{2}{*}{0.5379} & $B\left(E 2 ; 05_{5} \rightarrow 2_{1}^{+}\right) / B\left(E 2 ; 0_{5}^{+} \rightarrow 2_{2}^{+}\right)$ & 0.060 & - & $\left\{\begin{array}{c}94 \\
0.008\end{array}\right.$ & $\left.\begin{array}{c}16 \\
0.007\end{array}\right\}$ \\
\hline & $B\left(E 0 ; 0_{5}^{+} \rightarrow 0_{1}^{+}\right) / B\left(E 2 ; 0_{5}^{+}+2_{2}^{+}\right)$ & $0.0010(2)$ & - & $\left\{\begin{array}{l}0.02 \\
0.06\end{array}\right.$ & $\left.\begin{array}{l}0.13 \\
0.14\end{array}\right\}^{f}$ \\
\hline
\end{tabular}


TABLE III. (Continued)

\begin{tabular}{|c|c|c|c|c|c|}
\hline \multirow[b]{3}{*}{$\begin{array}{l}\text { Initial state } \\
\quad I \pi ; E(\mathrm{keV})\end{array}$} & \multirow[b]{3}{*}{ Quantity } & \multicolumn{4}{|c|}{ Reduced transition probability data } \\
\hline & & \multirow[b]{2}{*}{$\begin{array}{l}\text { Experimental } \\
\text { value }\end{array}$} & \multicolumn{3}{|c|}{ Theoretical values } \\
\hline & & & Ref. 3 & $\begin{array}{l}\operatorname{Ref} .2 \\
\text { version }(i)\end{array}$ & $\begin{array}{l}\text { Ref. } 2 \\
\text { version( }(i i)\end{array}$ \\
\hline \multirow[t]{2}{*}{$0_{6}^{+} ; 2488$} & $B\left(E 2 ; 0_{6}^{+}+2_{1}^{+}\right) / B\left(E 2 ; 0_{6}^{+}+2_{2}^{+}\right)$ & $0.0068(10)$ & - & $\left\{\begin{array}{c}94 \\
0.008\end{array}\right.$ & $0.007\}^{f}$ \\
\hline & $B\left(E 0 ; 0_{6}^{+} \rightarrow 0_{1}^{+}\right) / B\left(E 2 ; 0_{6}^{+} \rightarrow 2_{1}^{+}\right)$ & $0.103(22)$ & • & $\left\{\begin{array}{l}0.02 \\
0.06\end{array}\right.$ & $\left.\begin{array}{l}0.13 \\
0.14\end{array}\right\}^{f}$ \\
\hline
\end{tabular}

$\approx \quad$ a Value from Ref. 7.

b E2 content of the transition derived from the mixing-ratio data of Ref. 8.

c Based on the measured $(9)$ initial state lifetime and the observed $\gamma$-ray branching.

d Calculated assuming a pure E2 multipolarity for this transition and the relative $\gamma$-ray intensity data summarized in Ref. 10.

e Only relative $B(E 2)$ values are quoted for these transitions. These theoretical relative values have been normalized to the experimental value for the $3_{1}^{+} \rightarrow 2_{1}^{+}$transition;

The two values listed correspond to the two different assumptions made concerning the make-up of the excited $0^{+}$state. For further discussion, see the text.

9 This state not observed in the ${ }^{134} \mathrm{La}$ decay. The experimental data listed are those from the 134 Cs decay, as summarized in Ref. 10.

$h$ The multipolarity of the $4_{2}^{+} \rightarrow 3_{1}^{+}$transition is not measured. The listed value has been calculated assuming a pure E2 multipolarity for this transition. 


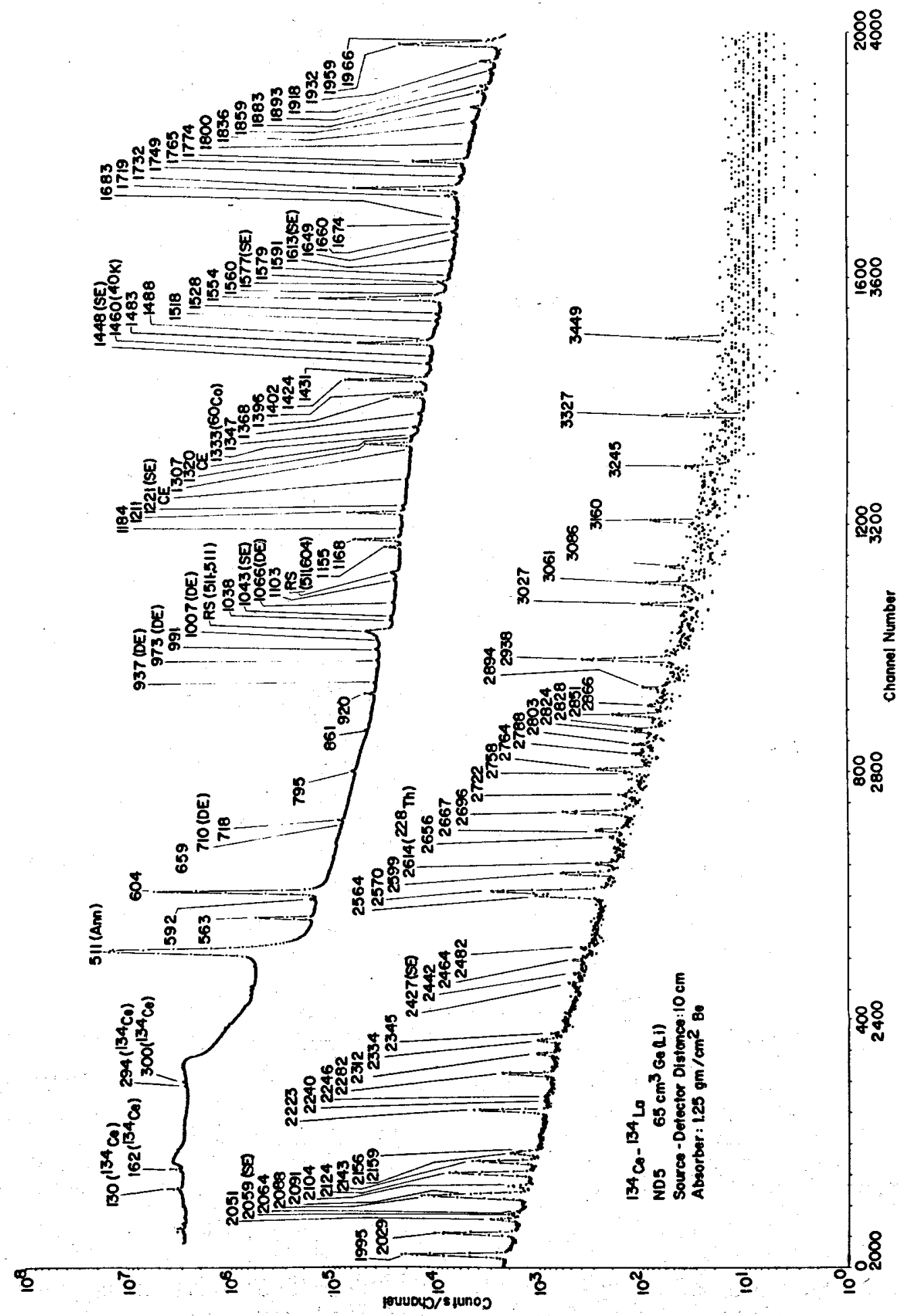

Fig. 1 r-ray spectrum of ${ }^{134} \mathrm{Ce}-13^{4} \mathrm{La}$ equilibrium source obtained using a $65-\mathrm{cm}^{3} \mathrm{Ge}(\mathrm{Li})$ detector. In this spectrum it should be noted that channels at the peak of the $511-\mathrm{keV}$ annihilation radiation have overflowed (maximum channel capacity $2^{24}$ ). The lowest energy peaks ( $<511$ ) identified in this spectrum are all associated with the ${ }^{134} \mathrm{Ce}$ decay while at higher energies the peaks result from the ${ }^{134}$ La decay. 


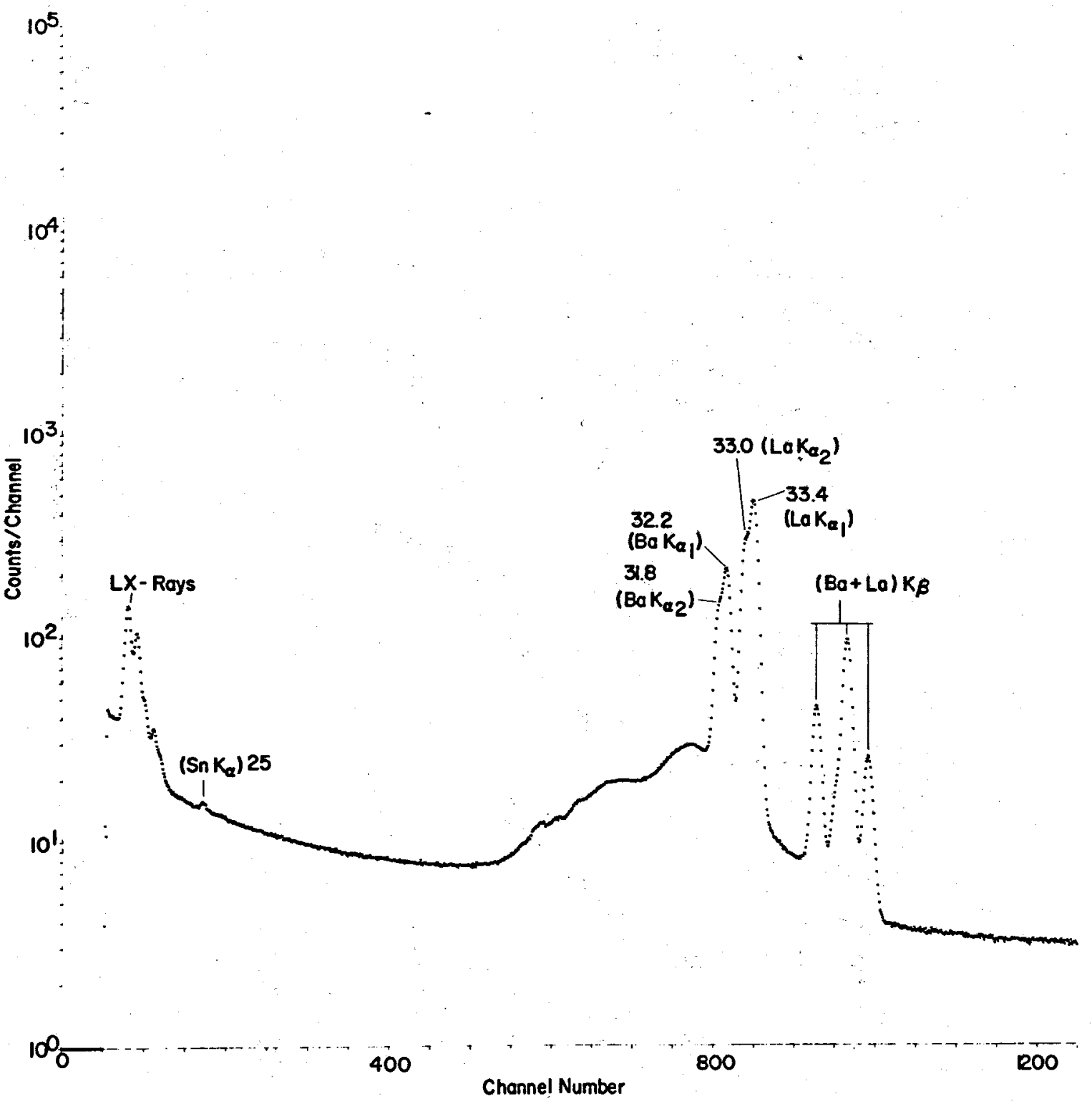

Fig. 2 Spectrum of $x$-rays emitted by ${ }^{134} \mathrm{Ce}-134 \mathrm{La}$ equilibrium source obtained using a $30-\mathrm{mm}^{2} \times 3-\mathrm{mm} \mathrm{Si}(\mathrm{Li})$ detector. 


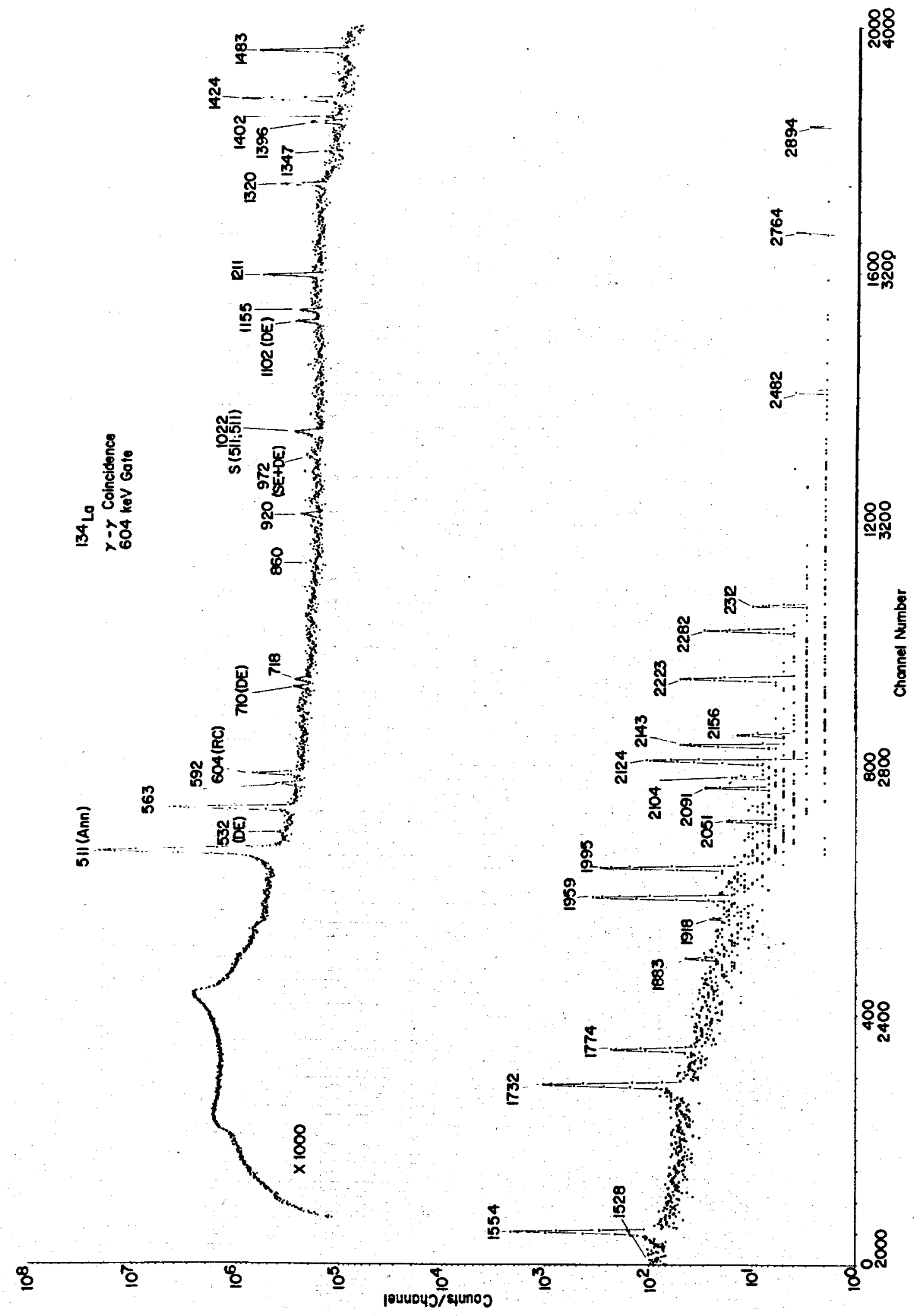

Fig. 3 Spectrum of $\gamma$ rays in coincidence with the 604-keV $\gamma$ ray. The symbol (RC) associated with some of the peaks in this spectrum and in Figs. 4 and 5 indicates that they result from random coincidence summing. 


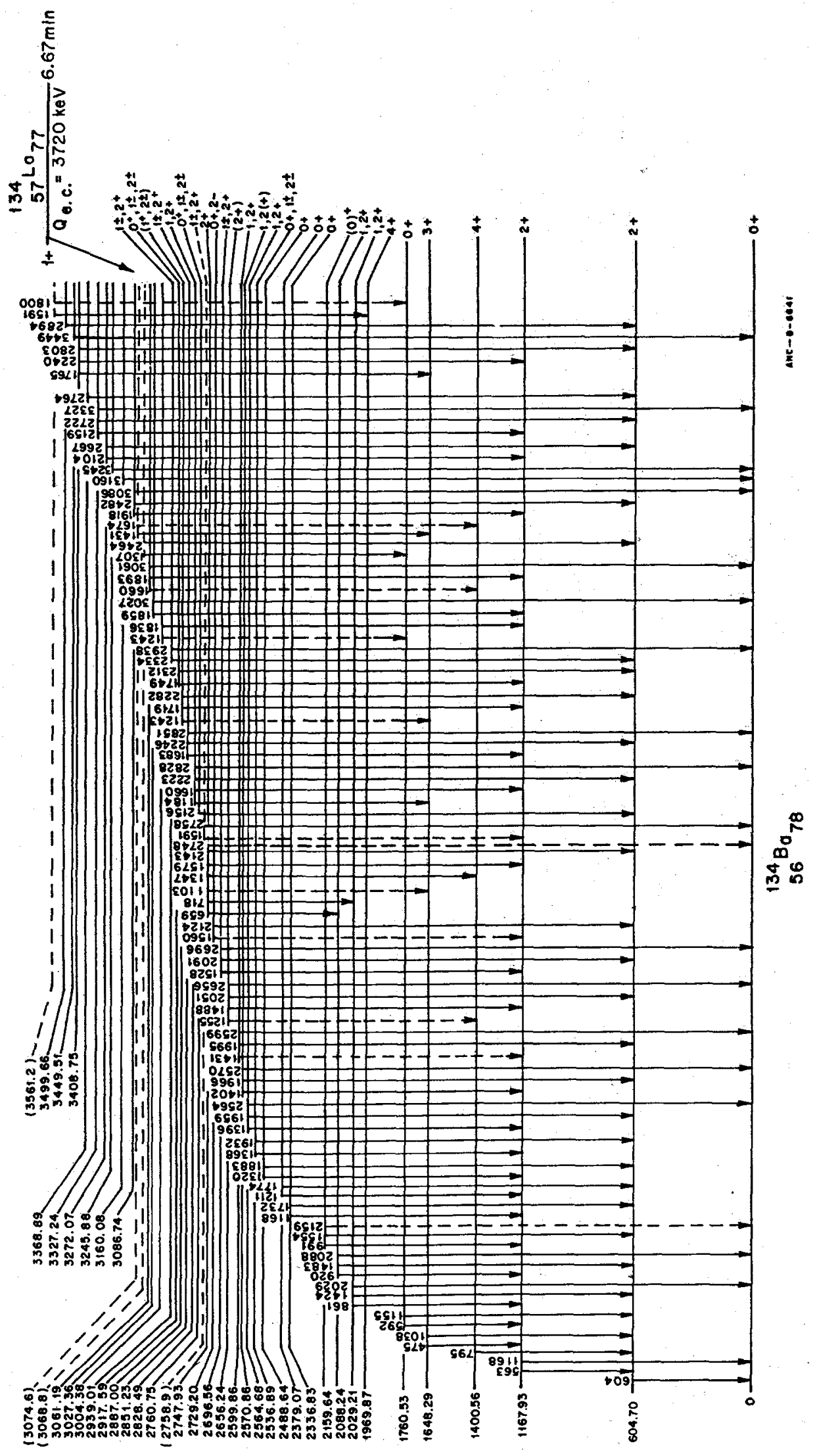

Fig. 4 Proposed decay scheme for ${ }^{134} \mathrm{La}$. Spin and parity assignments are listed only for those states below 3.0 MeV. 

(MeV)
THEORY
EXPERIMENT

$\cup$

${ }^{4.0}$

$6_{2}^{+}-$

$4_{3}^{+}$
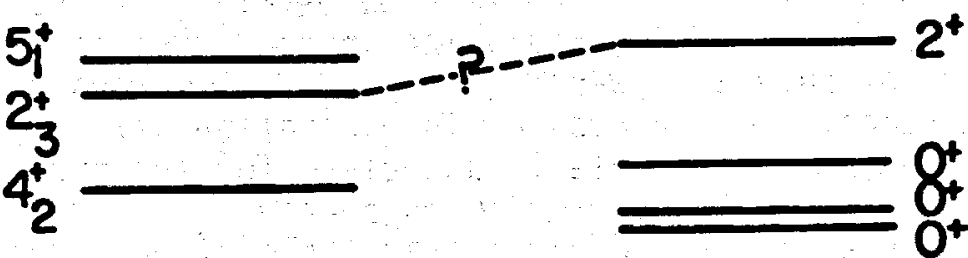

$6_{1}^{+}=\left(0^{+}\right)$
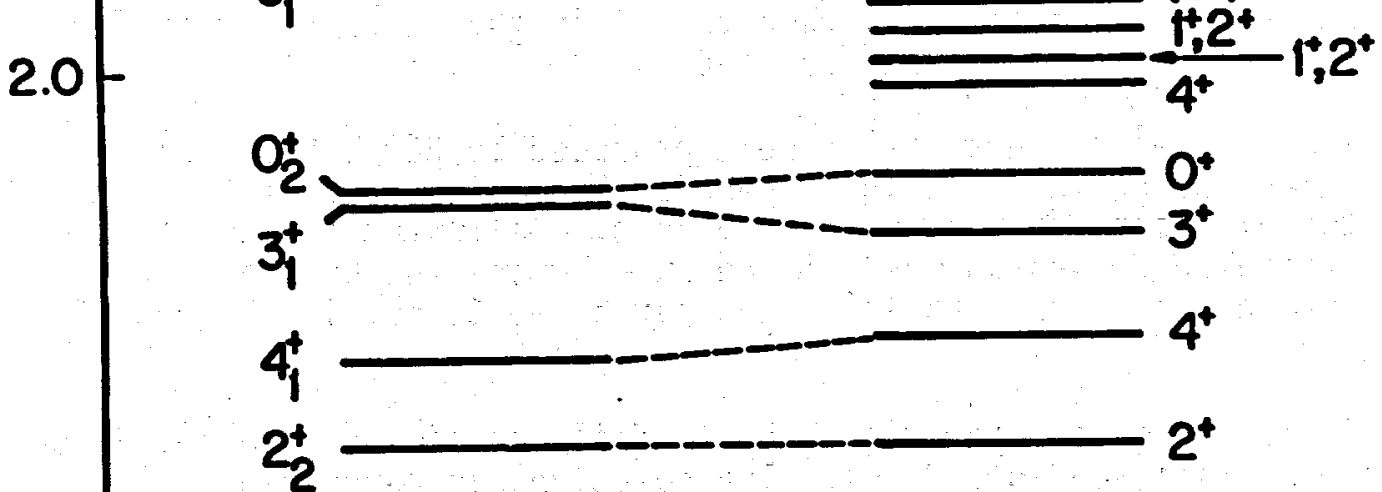

1.0
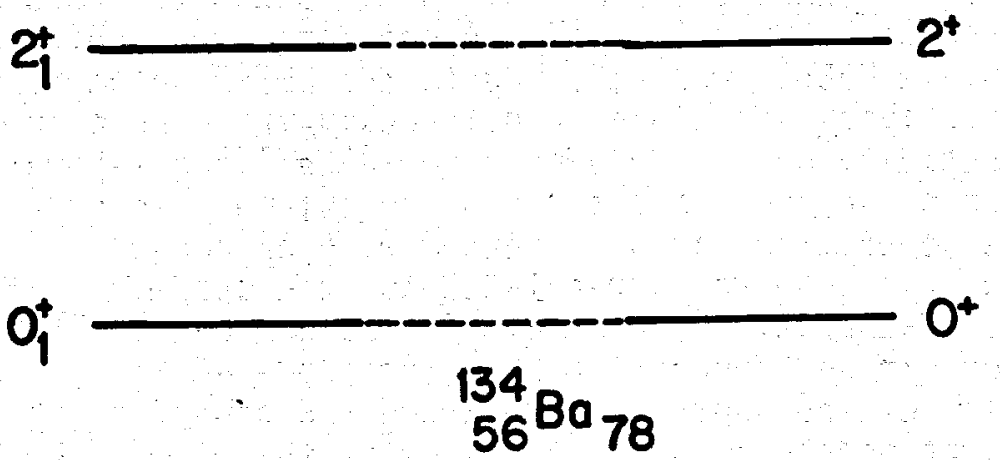

Fig. 5 Comparison of the calculated ${ }^{(3)}$ collective energy - level spectrum of $134 \mathrm{Ba}$ and the experimentally determined level scheme. While all the experimental levels below $2.0 \mathrm{MeV}$ are shown, for simplicity in comparison only a few known levels above this energy are included. (for more details, see Fig. 4.) 
At the present time no information exists concerning excited states in ${ }^{134} \mathrm{La}$. Although ${ }^{134} \mathrm{Ce}\left(T_{\frac{1}{2}}=75.9 \mathrm{~h}\right)$ decays to $1{ }^{34} \mathrm{La}$, no $\gamma$ radiation has to date been reported to be associated with its decay. Furthermore, values of $120_{-20}^{+60}$ and $110_{-38}^{+9} \mathrm{keV}$, respectively, have been reported in refs. (1) and (2) for the decay energy of ${ }^{134} \mathrm{Ce}$. The study of the ${ }^{134} \mathrm{Ce}$ decay is made difficult by the short half-life $(6.67 \mathrm{~min})$, large decay energy $(3720 \mathrm{keV})$ and the resulting complexity of the ${ }^{134} \mathrm{La} \gamma$-ray spectrum [see ref. (3)]. In view of the short half-life of 134La, it has generally proven convenient to study the ${ }^{134} \mathrm{La}$ decay scheme by using as a source the $134 \mathrm{La}$ in equilibrium with its $134 \mathrm{Ce}$ parent. This procedure, together with the reported low Q-value for the ${ }^{134} \mathrm{Ce}$ decay and the reported absence of $\gamma$ radiation in the ${ }^{134} \mathrm{Ce}$ decay, has led to the assignment of several low-energy $(\leqslant 0.3 \mathrm{MeV}) \gamma$ rays to the ${ }^{134} \mathrm{La}$ decay in the recent $A=134$ mass-chain evaluation. (4) However, in the present study we have been able to associate for the first time a number of $\gamma$-ray transitions, ranging in energy up to $-355 \mathrm{keV}$, with the ${ }_{134} \mathrm{Ce}$ decay. This result indicates that the previously reported values $(1,2)$ for the ${ }^{134} \mathrm{Ce}$ decay energy are incorrect and that the three low-energy $(<0.3 \mathrm{MeV}) \gamma$ rays reported $(4)$ in the ${ }^{134 \mathrm{La} \text { decay in fact }}$ are associated with the decay of ${ }^{134} \mathrm{Ce}$.

The ${ }^{134} \mathrm{Ce}-134 \mathrm{La}$ activities were produced by $800-\mathrm{MeV}$ proton bombardment of Pr-metal foils in LAMPF. Typical bombardments involved an integrated beam current of $\sim 10-15 \mu A-h$. Following the bombardment, the irradiated $\operatorname{Pr}$ was shipped to the INEL, where the Ce fraction was extracted and isotope separation was performed to obtain the mass-134 fraction.

Following mass separation, the $\mathrm{Al}$ foil containing imbedded ${ }^{134} \mathrm{Ce}-$ ${ }^{134} \mathrm{La}$ was dissolved in $5 \mathrm{M} \mathrm{HCl}$. The solution was converted to the nitrate, and the Ce was oxidized, extracted into HDEHP, stripped and washed as discussed elsewhere. (5) The aqueous phase containing ${ }_{134} \mathrm{Ce}^{-134} \mathrm{La}$ was heated to dryness twice with concentrated $\mathrm{HNO}_{3}$, dissolved in $0.5 \mathrm{ml}$ of $10 \mathrm{M} \mathrm{HNO}_{3}-0.1 \mathrm{M} \mathrm{NaBrO}_{3}$ and loaded onto a $0.5 \times 0.5 \mathrm{~cm}$ stationary phase HDEHP column. (6) A one-liter reservoir containing $10 \mathrm{M} \mathrm{HNO}_{3}-0.1 \mathrm{M} \mathrm{NaBrO}_{3}$ was attached to the HDEHP column and the $134 \mathrm{La}$ (III) activity. was eluted continuously from the column, leaving the ${ }^{134} \mathrm{Ce}$ (IV) activity on the column. The ${ }^{134} \mathrm{Ce} / 134 \mathrm{La}$ separation ratio was dependent upon the flow rate through the column. In order to achieve an adequate separation ratio, together with an adequate column life-time, the flow rate was generally regulated to $\sim 4 \mathrm{drops} / \mathrm{min}$. At this flow rate a ${ }^{134} \mathrm{Ce} /{ }^{134} \mathrm{La}$ separation factor of $\sim 40$ was achieved. It was found that, if the reservoir and connecting hose were covered with aluminum foil, decomposition of the $\mathrm{NaBrO}_{3}$ was retarded and good, continuous separation from ${ }^{134} \mathrm{La}$ was possible up to $36 \mathrm{~h}$. After that time, the ${ }^{134} \mathrm{Ce}-134 \mathrm{La}$ was reclaimed from eluate and the column, oxidized and reloaded onto a fresh HDEHP column.

*Allied Chemical Corporation, Idaho National Engineering Laboratory. 
A typical $\gamma$-ray spectrum of the ${ }^{134} \mathrm{Ce}$ "column" source is given in Figure 1. The presence of these $\gamma$ rays in the "Ce-enhanced" $\gamma$-ray spectra and their absence (at least for the stronger transitions) in spectra from ${ }^{134} \mathrm{La}$ (with the ${ }^{134} \mathrm{Ce}$ removed) establish that they are in fact associated with the Ce decay rather than with the ${ }^{134} \mathrm{La}$ decay. Analysis of this and similar spectra has established the existence of some $32 \gamma$ rays in the decay of ${ }^{134} \mathrm{Ce}$. The energies and intensities of these $\gamma$ rays are summarized in Table I.

Coincident $\gamma$-ray spectra were measured for a number of the strongest $\gamma$-ray peaks in the ${ }^{134} \mathrm{Ce}$ spectrum. Gates were set so as to encompass peaks with energies of $90,102,130,131,158,162,168,187$, 193,197 , and $300 \mathrm{keV}$. Coincidence running times ranged from -2 to -16 hours. At the shorter running times only the principal coincidence relationships could be delineated, and even these were often characterized by as few as 20-30 counts in the coincident peak. [Since all of the coincidence gates used had energies < $511 \mathrm{keV}$ it was necessary to utilize a 90-degree detector geometry in order that the coincident $\gamma$ spectra not be dominated by the 511-511 keV annihilation photons resulting from positron emission from the ${ }^{134} \mathrm{La}$ activity still remaining in the separator column (2-3\% of the equilibrium level).] The complete set of coincidence relationships determined in this work is summarized in Table 2 .

The level scheme which we have constructed for the doubly odd nucleus ${ }^{134} \mathrm{La}$ is based principally on the $\gamma-\gamma$ coincidence data. Cascade-crossover energy-sum relationships have also proven useful in supplementing these coincidence data. There is presently no information about excited states in ${ }^{134} \mathrm{La}$, and thus all the features of the level scheme proposed in Fig. 2 are not unambiguously derived, even with the aid of the $\gamma-\gamma$ coincidence data.

(1) E. I. Biryukov, V. T. Novikov and N. S. Shimanskaya, Izv. Akad. Nauk SSSR, Ser. Fiz. 29 (1965) 151; Bul1. Acad. Sci. USSR, Phys. Ser. 29 (1966) 148.

(2) V. S. Aleksandrov, V. S. Buttsev, T. Vylov, K. Ya. Gromov and V. G. Kalinnikov, Izv. Akad. Nauk SSSR, Ser. Fiz. 37 (1973) 948; Bul1. Acad. Sci. USSR, Phys. Ser. 37, No. 5 (1974) 34.

(3) R. C. Greenwood et a1.., "Decay of ${ }^{134}$ La," this report.

(4) E. A. Henry, NucTear Data Sheets 15, No. 2 (1975) 203.

(5) R. C. Greenwood, C. W. Reich, R. G. Helmer, R. J. Gehrke and R. A. Anderl, "134 Ba Level Scheme as Observed in the Decay of $1{ }^{34} \mathrm{La}, "$ this report.

(6) R. J. Sochacka and S. Sierkierski, J. Chromatog, 16, 316 (1964). 
TABLE I

Gamma-ray energies and intensities from ${ }^{134} \mathrm{Ce}$ decay

\begin{tabular}{|c|c|c|c|}
\hline $\begin{array}{c}\text { Gamma-ray } \\
\text { energy a) } \\
(\mathrm{keV})\end{array}$ & $\begin{array}{c}\text { Gamma-ray } \\
\text { intensity } \\
\left(\mathrm{r} / 10_{5}\right. \\
\text { disintegrations }) \\
\end{array}$ & $\begin{array}{c}\text { Gamma-ray } \\
\text { energy a) } \\
\text { (keV) } \\
\end{array}$ & $\begin{array}{r}\text { Gamma-ray } \\
\text { intensity } \\
\left(\gamma / 10^{5}\right. \\
\text { disintegrations }) \\
\end{array}$ \\
\hline 22.7 & $<22$ & $158.785+0.010$ & $39.1 \pm 2.7$ \\
\hline La K $\times$ ray & $72300 \pm 3600$ & $162.306 \pm 0.010$ & $230 \pm 16$ \\
\hline 31.89 & $<50$ & $168.453 \pm 0.025$ & $12.2 \pm 0.9$ \\
\hline 39.08 & $<150$ & $187.013 \pm 0.012$ & $21.8 \pm 1.5$ \\
\hline $54.65 \pm 0.06$ & $16.9 \pm 2.4$ & $193.157 \pm 0.012$ & $40.4 \pm 2.8$ \\
\hline $59.04 \pm 0.20$ & $0.21 \pm 0.10$ & $197.891 \pm 0.025$ & $13.6 \pm 1.0$ \\
\hline $61.88 \pm 0.14$ & $3.6 \pm 0.8$ & $200.60 \pm 0.08$ & $1.61 \pm 0.24$ \\
\hline$(66.26 \pm 0.20)$ & $0.12 \pm 0.06$ & $205.27 \pm 0.06$ & $4.2 \pm 0.05$ \\
\hline $68.55 \pm 0.16$ & $0.76 \pm 0.25$ & $220.56 \pm 0.05$ & $4.6 \pm 0.6$ \\
\hline $70.85 \pm 0.12$ & $1.21 \pm 0.20$ & $239.650 \pm 0.020$ & $13.1 \pm 1.0$ \\
\hline $90.18 \pm 0.06$ & $5.5 \pm 0.5$ & $252.54 \pm 0.07$ & $3.7 \pm 0.5$ \\
\hline $93.47 \pm 0.16$ & $0.93 \pm 0.25$ & $262.26 \pm 0.10$ & $3.4 \pm 0.5$ \\
\hline $102.998+0.010$ & $25.3 \pm 2.5$ & $265.54 \pm 0.07$ & $3.9 \pm 0.5$ \\
\hline $104.53 \pm 0.08$ & $2.14 \pm 0.28$ & $294.264 \pm 0.015$ & $54 \pm 4$ \\
\hline $107.34 \pm 0.04$ & $6.1 \pm 0.6$ & $300.884 \pm 0.015$ & $88 \pm 7$ \\
\hline $116.19 \pm 0.04$ & $2.68 \pm 0.27$ & $323.59 \pm 0.05$ & $15.6 \pm 1.6$ \\
\hline $130.414+0.015$ & $209 \pm 15$ & $355.54 \pm 0.05$ & $8.8 \pm 0.9$ \\
\hline $131.93 \pm 0.05$ & $17.1 \pm 1.7$ & & \\
\hline $150.20 \pm 0.04$ & $3.76 \pm 0.40$ & & \\
\hline
\end{tabular}

a) Parentheses around a $\gamma$-ray energy indicate a tentative assignment.

b) The uncertainties in these intensities include a $7 \%$ contribution from the normalization to absolute intensities. 
TABLE II

$\gamma-\gamma$ coincidence relationships for ${ }^{134}$ Ce decay

\begin{tabular}{|c|c|}
\hline $\begin{array}{l}\text { Gate energy } \\
(\mathrm{keV})\end{array}$ & Coincident $\gamma$-ray energies $(\mathrm{keV})$ a) \\
\hline 90 & $102 ;(130),(162),(193)$ \\
\hline 102 & $\begin{array}{l}54,90,104,116,158,197,220 ;(102),(130), \\
(162),(252)\end{array}$ \\
\hline 130 & $90,102,131,193 ;(68)$ \\
\hline 131 & 130,162 \\
\hline 158 & 54,$102 ;(61)$ \\
\hline 162 & $90,102,131,193 ;(187)$ \\
\hline 168 & $187 ;(61)$ \\
\hline 187 & 107,168 \\
\hline 193 & 130,162 \\
\hline 197 & $102 ;(54),(75)$ \\
\hline 300 & 54 \\
\hline
\end{tabular}

a) Parentheses around a $\gamma$-ray energy indicate a tentative coincident relationship. 


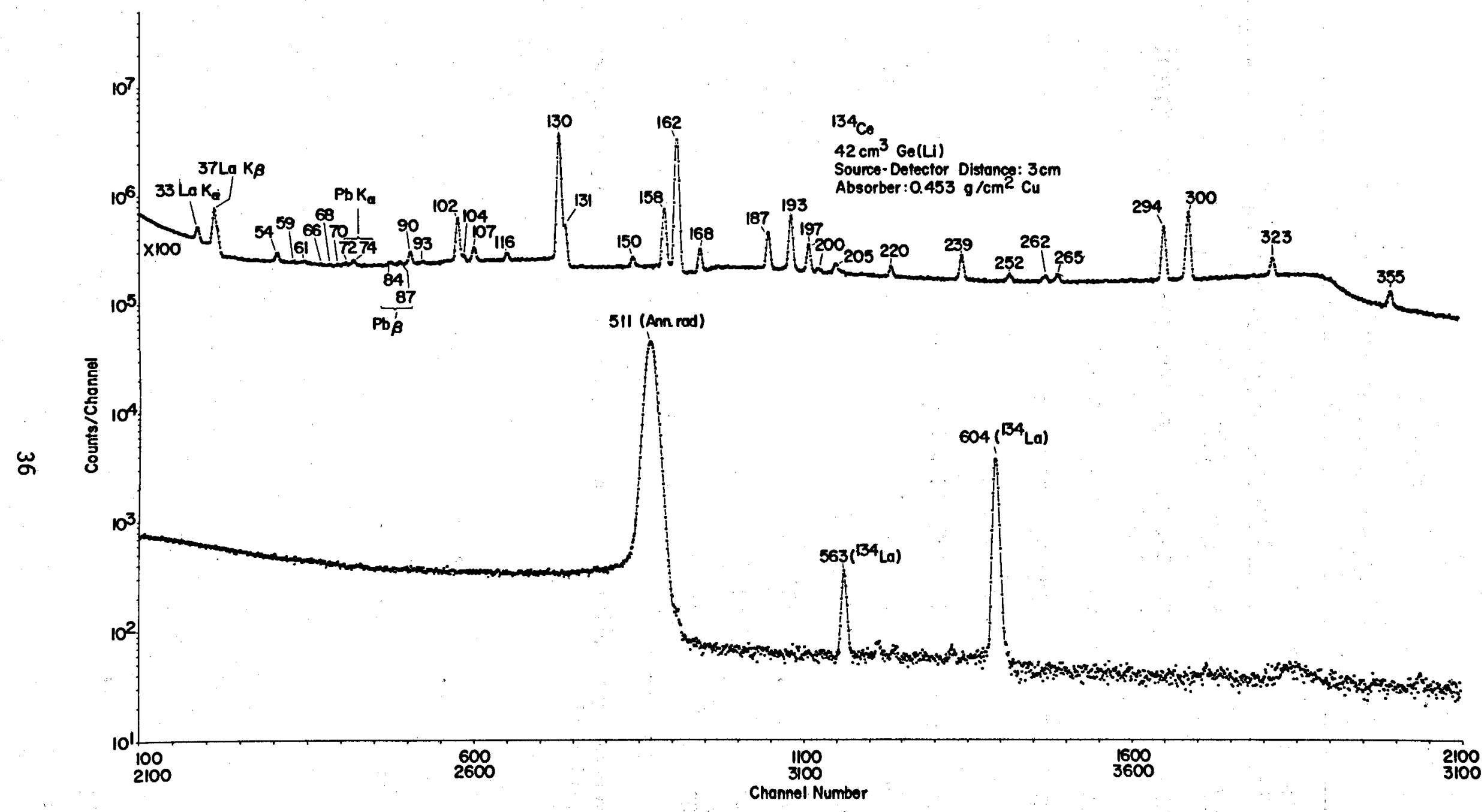

Figure 1. Gamma-ray spectrum of the ${ }^{134} \mathrm{Ce}$ activity in the separation column measured with a $42-\mathrm{cm}^{3}$ coaxial $\mathrm{Ge}(\mathrm{Li})$ detector. The ${ }^{134} \mathrm{Ce}-\mathrm{to}^{-134} \mathrm{La}$ enhancement, relative to the equilibrium source condition, is $\sim 41$ in this spectrum. 


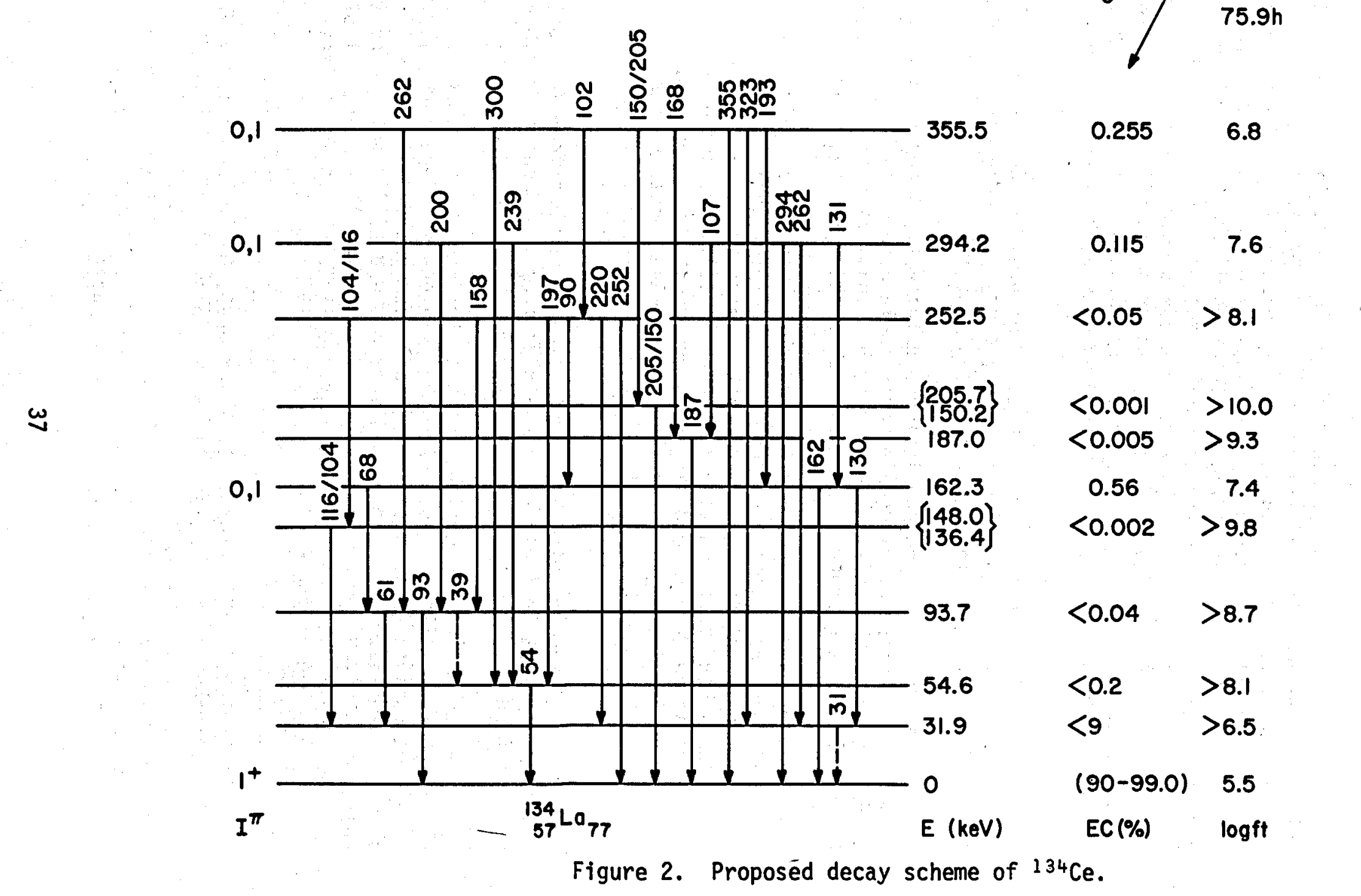


DECAY OF $150 \mathrm{~Tb}$

R. G. Helmer

As part of a program to study the nuclear structure of neutrondeficient isotopes off the line-of-stability, two samples of ${ }^{150} \mathrm{~Tb} / 3.1$ hr) were produced from tantalum metal irradiated wi th $\sim 800$ MeV protons in the C. P. Anderson Meson Physics Facility (LAMPF) at LASL. These samples were prepared by chemical extraction of the rare earth el ements and isotope separation of the mass 150 fraction. In addition to ${ }^{150} \mathrm{~Tb}$ $(3.1 \mathrm{hr})$, the only isotope observed in these samples was ${ }^{151} \mathrm{~Tb}(17.6$ $\mathrm{hr}$ ). The samples were available about seven hours after the completion of the irradiation. All of the sample preparation was done by LASL personnel and the measurements were made on equipment provided by the LAMPF staff.

Several $\gamma$-ray spectra were obtained with $\mathrm{Ge}(\mathrm{L} i)$ detectors including some energy calibration spectra. About $450 \gamma$ rays were observed from 153 to $4562 \mathrm{keV}$ with an intensity range of almost $10^{5}$.

Since the $\gamma$-ray spectrum includes relatively strong $\gamma$ rays of up to $4500 \mathrm{keV}$, the presence of double- and single-escape peaks at $E_{\gamma}-1022$ and $E_{\gamma}-511 \mathrm{keV}$ made the analysis very complex. One spectrum, which covered the complete energy range, was analyzed in the following manner.

(1) A preliminary analysis was done to obtain the energy and area of each peak and each visible component of a complex peak.

(2) Starting with the high energy peaks (which had to be full-energy peaks), the energies (and the channel positions) and areas of the associated double- and single-escape peaks were computed.

(3) In the final analysis of any section of the spectrum, the contribution of each escape peak was taken into account explicitly but with its area and position fixed at the computed values.

The list of $\gamma$-ray energies and intensities determined from this one spectrum is given in Table $I$. The $\gamma$-rays from the decay of ${ }^{151} \mathrm{~Tb}$ have been omitted.

The most complete published work on this decay is that of Vylov et al. which reports $93 \gamma$ rays. From the previous work and the relative $\gamma$-ray intensities it is clear that the lowest excited states in $150 \mathrm{Gd}$ are at 637.5 and $1133.3 \mathrm{keV}$ and probably 1206.5 and $1287.7 \mathrm{keV}$. From the $Q$ value of $4664 \mathrm{keV}$ and the energy of the first excited state, all of the $r$ rays above $4027 \mathrm{keV}$ must go to the ground state. This establishes the existence of 29 levels between 4100 and $4570 \mathrm{keV}$.

(1) Ts. Vylov, K. Ya. Gromov, I. I. Gromova, G. I. Iskhakov. V. V. Kuznetsov, M. Ya. Kuznetsova, A. V. Potempa and M. I. Fominykh, Bul1. Acad. Sci. USSR, Phys. Ser. 37, No. 1, 36 (1973).

(2) Ts. Vylov, K. Ya.Gromov, I. I. Gromova, G. I. Iskhakov, V. V. Kuznetsov, M. Ya. Kuznetsova, A. V. Potempa and M. I. Fominykh, Bul1. Acad. Sci. USSR, Phys. Ser. 37, No. 1, 41 (1973). 
TABLE I

GAMMA RAYS FROM THE DECAY OF 150 Tb

$(\mathrm{EeV})$

$$
\begin{aligned}
& 153.87(28) \\
& 225.05(22) \\
& 227.26(18) \\
& 256.93(4) \\
& 321.85(35) \\
& 326.02(25) \\
& 338.23(65) \\
& 344.20(35) \\
& 352.22(17) \\
& 378.33(30)
\end{aligned}
$$

$384.00(7)$

$387.80(85)$

411.38(4)

436.85(1)

472.06(43)

495.79

510.47

$524.94(2)$

$557.24(1)$

$565.58(5)$

$568.93(3)$

$573.24(12)$

$587.16(12)$

$602.70(2)$

$609.16(5)$

616.34(18)

$626.52(22)$

637.55

$650.14(5)$

661.03(19)

666.19(44)

$680.56(18)$

$685.98(29)$

$691.71(60)$

699.24(7)

$714.31(32)$

$723.29(53)$

$727.78(44)$

$731.20(19)$

$743.71(10)$
$I_{\gamma}$

$1.18(25)$

$1.33(28)$

$1.81(27)$

$0.86(25)$

$2.03(50)$

2.87(53)

$1.96(92)$

$1.83(46)$

$1.38(17)$

$2.17(50)$

9.53(60)

$0.81(50)$

$18.5(5)$

$19.3(2)$

$0.78(24)$

311.

1028.

15.1(3)

$6.57(9)$

$26.0(8)$

$53.5(10)$

$8.9(7)$

2.5

$3.47(9)$

$2.01(9)$

$0.83(10)$

$1.61(23)$

1590.

$91.7(39)$

$2.57(36)$

$1.14(33)$

$1.38(17)$

$0.81(16)$

0.2

$6.21(43)$

$0.59(13)$

$0.49(17)$

$0.62(18)$

0.6

$3.54(22)$ (kev)

$748.00(4)$

$753.01(90)$

755.76(90)

$766.10(32)$

$772.34(8)$

$778.88(5)$

$792.18(2)$

$807.61(13)$

$812.88(2)$

$820.86(2)$

$826.39(30)$

$831.39(25)$

$838.76(48)$

$846.54(24)$

853.46(45)

$861.08(78)$

$864.43(24)$

$871.53(16)$

$880.08(4)$

884.33(29)

890.83(42)

$895.71(10)$

$907.76(22)$

$910.62(13)$

915.60(12)

919.11(60)

$921.48(36)$

924.76(18)

928.28(55)

935.09(16)

945.38(36)

949.73(6)

$954.22(6)$

$957.12(8)$

$961.53(65)$

967.84(16)

972.39(43)

977.52(19)

986.85(28)

995.07(7)
$I_{\gamma}$

$11.4(3)$

$0.43(22)$

$1.05(22)$

$1.05(22)$

5.15(31)

$6.03(28)$

$100.0(24)$

$1.34(3)$

$10.6(2)$

$29.2(6)$

$1.80(36)$

$2.16(35)$

$0.35(11)$

$0.72(11)$

$0.25(24)$

$0.50(24)$

$1.65(23)$

$0.62(7)$

$63.9(17)$

$5.19(95)$

$1.00(17)$

4.07(33)

$0.93(11)$

$1.44(11)$

$1.37(10)$

$0.45(16)$

$0.81(16)$

$1.19(12)$

$0.35(12)$

$0.78(8)$

$2.40(54)$

$21.8(9)$

26.7(13)

$17.9(10)$

$1.26(52)$

4.70(51)

$1.42(37)$

$3.02(45)$

$0.47(8)$

$4.26(18)$ 
Table I (continued)

$$
\begin{gathered}
E_{\gamma} \\
(\mathrm{keV})
\end{gathered}
$$

$997.36(13)$
$1000.84(22)$
$1003.52(41)$
$1006.79(48)$
$1008.93(60)$

$1013.49(16)$

$1018.44(41)$

$1021.72(90)$

$1028.62(13)$

$1035.26(22)$

$1037.65(16)$

1045.41(01)

$1049.06(31)$

$1052.82(43)$

$1061.27(6)$

$1069.43(24)$

$1075.05(4)$

$1081.05(25)$

$1086.75(31)$

$1090.80(11)$

$1094.18(7)$

$1101.38(37)$

$1109.91(23)$

$1119.79(13)$

$1127.35(16)$

$1130.16(65)$

$1157.75(8)$

$1161.10(50)$

$1164.16(55)$

$1168.45(7)$

$1175.86(7)$

$1185.32(18)$

$1188.84(90)$

$1193.64(13)$

$1203.52(22)$

$1206.90(6)$

$1223.89(30)$

1232.71(17)

$1247.88(36)$

$1252.90(25)$
$I_{\gamma}$

$2.41(18)$
$3.02(34)$
$1.72(34)$
$1.79(47)$
$1.24(50)$

$0.90(8)$

$0.36(9)$

$0.15(9)$

$1.04(11)$

1.08(14)

$1.48(14)$

$27.4(3)$

$1.10(19)$

$0.68(18)$

$4.26(18)$

$1.85(28)$

$14.0(4)$

$1.28(20)$

$1.20(23)$

$3.76(24)$

$6.21(26)$

$0.38(9)$

$0.75(11)$

$3.21(32)$

$3.82(34)$

$1.03(32)$

$3.45(17)$

$0.68(17)$

$0.58(17)$

9.44(54)

$10.7(5)$

$1.97(20)$

$0.91(20)$

$2.57(20)$

$1.07(14)$

1.2

$1.19(22)$

$3.29(35)$

$1.12(25)$

$1.65(24)$
${ }_{(k e V)}^{E_{\gamma}}$

$1256.34(48)$
$1259.96(70)$
$1264.89(40)$
$1274.32(12)$
$1291.48(3)$

1296.29(80)

$1299.35(90)$

$1310.20(32)$

$1317.27(2)$

1326.90(29)

$1331.87(15)$

$1343.05(20)$

$1349.81(4)$

$1355.68(16)$.

$1363.92(31$ )

$1367.00(50)$

$1373.85(35)$

$1376.53(55)$

$1387.28(11)$

1389.76(16)

$1392.54(16)$

$1397.53(16)$

$1403.54(10)$

$1414.78(6)$

$1430.31(2)$

$1443.12(12)$

$1445.84(11)$

$1453.39(2)$

$1460.57(25)$

$1466.42(8)$

$1484.43(18)$

$1490.18(28)$

$1493.53(8)$

$1505.08(25)$

$1509.31(50)$

$1530.53(33)$

$1535.44(55)$

1541.73(11)

$1543.86(35)$

$1547.34(42)$
I

$0.94(24)$

$0.60(24)$

$0.95(23)$

$5.49(42)$

$37.3(10)$

$1.40(60)$

$1.37(64)$

$0.58(12)$

$9.83(15)$

$0.91(15)$

$1.78(16)$

$3.25(40)$

23.3(6)

4.36(40)

$1.21(19)$

$0.73(20)$

$1.31(24)$

$0.77(23)$

2.54(16)

$2.40(15)$

$1.56(13)$

$1.44(14)$

2.35(13)

$7.98(30)$

59.5(13)

$11.2(7)$

$12.5(7)$

86.9(17)

$7.21(110)$

$1.92(10)$

$1.01(11)$

$1.11(16)$

4.03(17)

$1.22(17)$

$0.57(17)$

$1.87(40)$

$1.20(40)$

$12.0(8)$

$2.67(56)$

$0.77(15)$ 
Table I (continued)

$$
\begin{gathered}
E \gamma \\
(\mathrm{keV})
\end{gathered}
$$

$1551.99(14)$
$1554.25(7)$
$1563.87(7)$
$1571.28(28)$
$1579.72(13)$

$1585.01(26)$

$1592.37(4)$

$1599.61(22)$

$1605.40(16)$

$1615.12(12)$

$1620.21(13)$

$1624.09(06)$

$1631.41(23)$

$1637.97(10)$

$1644.96(12)$

$1652.31(7)$

$1659.96(8)$

$1668.53(17)$

$1683.72(30)$

$1688.21(5)$

$1694.41(35)$

$1696.88(43)$

$1702.40(35)$

$1713.71(8)$

$1726.73(4)$

$1733.58(20)$

$1737.36(55)$

$1747.40(46)$

1751.94(11)

$1770.34(2)$

1778.39(2)

$1787.77(5)$

$1796.16(5)$

$1802.77(46)$

$1807.02(73)$

$1811.89(18)$

$1826.30(25)$

1843.66(30)

$1852.88(13)$

$1855.69(33)$
$I_{\gamma}$

$2.30(20)$

$4.54(20)$

$2.80(12)$

$2.20(35)$

$3.42(25)$

$1.65(25)$

45.7(13)

2.82(31)

$3.10(26)$

2.61(18)

$3.12(22)$

$7.83(28)$

$1.39(18)$

$3.55(19)$

1.99(13)

4.19(22)

$3.90(21)$

$1.06(10)$

$1.47(24)$

$11.1(3)$

$1.40(28)$

$1.15(27)$

$1.41(28)$

$2.81(13)$

6.19(13)

$3.30(34)$

1.21(34)

$0.69(17)$

$3.19(18)$

$13.4(2)$

$14.2(3)$
$43.1(13)$
$4.97(16)$
$0.57(14)$
$0.36(14)$

$1.47(14)$

$2.18(30)$

$0.62(10)$

2.76(19)

$1.08(18)$ (kef)

$1860.73(20)$

$1865.96(36)$

1876.40 (38)

$1883.52(7)$

1894.56(32)

$1901.56(5)$

$1908.42(35)$

$1914.07(7)$

$1926.40(12)$

$1938.83(36)$

1949.09(8)

$1955.12(14)$

1963.86(50)

1971.13(19)

1984.69(10)

1989.42(35)

$1993.62(38)$

$1997.52(20)$

2005.81(30)

$2009.68(40)$

2016.19(2)

2025.44(33)

2030.21(28)

2040.15(5)

2043.50(23)

2047.92(70)

2056.14(17)

2064.40(60)

$2076.79(80)$

2080.31(27)

2091.42(4)

2104.32(30)

$2116.61(2)$

$2136.52(35)$

2148.32(4)

2164.10(18)

2168.95(13)

2173.23(13)

$2179.74(6)$

2189.71(32)
$I_{\gamma}$

$1.62(16)$

$0.67(13)$

$0.92(19)$

$6.07(26)$

$0.64(11)$

$15.1(5)$

$1.72(32)$

$9.16(40)$

$3.44(22)$

$0.68(14)$

4.65(23)

$2.12(14)$

$1.07(29)$

$1.02(10)$

$3.60(19)$

$1.69(27)$

$0.63(11)$

$1.06(10)$

$1.52(23)$

$1.17(21)$

23.3(4)

$1.59(28)$

$1.96(29)$

8.64(20)

1.73(18)

$0.44(15)$

1.94(17)

$0.54(16)$

$0.40(15)$

$1.13(14)$

40.3(10)

$1.13(18)$

$10.17(14)$

$1.02(18)$

$25.5(5)$

2.09(19)

3.03(19)

$3.18(19)$

9.23(33)

0.91 (14) 
Table I (continued)<smiles>[AsH2]</smiles>

$$
\begin{aligned}
& 2194.36(6) \\
& 2201.15(55) \\
& 2207.43(6) \\
& 2229.94(22) \\
& 2233.59(3)
\end{aligned}
$$

2241.07(8)

$2253.48(40)$

$2259.22(36)$

2262.52(11)

2275.14(40)

2296.72(42)

$2317.86(6)$

2328.61(30)

$2333.74(25)$

$2340.82(31)$

2346.63(6)

$2359.49(16)$

2364.81(4)

$2376.36(6)$

2396.99(4)

$2403.69(30)$

2409.29(6)

$2425.84(5)$

2442.71(25)

2445.88(18)

2449.97(25)

2459.33(10)

2464.32(60)

2473.52(20)

2479.87(16)

2489.75(27)

2492.05(43)

$245 \therefore .50(26)$

$2501.47(50)$

2511.31(50)

2516.70(55)

2522.18(25)

2532.20(10)

$2539.53(2)$

$2552.68(25)$
$I_{\gamma}$

$6.46(21)$

$2.18(60)$

28.5(10)

$1.80(18)$

$1.26(18)$

$3.56(20)$

$0.48(10)$

$0.70(12)$

$2.36(12)$

$0.86(17)$

$0.48(15)$

$9.77(38)$

$1.00(15)$

$0.64(15)$

$1.01(16)$

4.23(14)

$1.74(14)$

$25.5(2)$

5.16(17)

15.52(32)

1.45(19)

$9.49(32)$

26.6(7)

$1.55(17)$

2.42(17)

$1.30(15)$

$2.24(10)$

$0.20(9)$

$1.02(10)$

$1.21(9)$

$2.09(25)$

2.65(53)

$4.03(50)$

$0.57(14)$

$0.49(12)$

$0.49(12)$

$0.84(10)$

2.78(15)

$12.42(15)$

$1.59(20)$
$E_{\gamma}$
$(\mathrm{keV})$

$I_{\gamma}$

$\begin{array}{ll}2558.36(6) & 6.55(26) \\ 2565.16(13) & 1.75(12) \\ 2579.29(31) & 1.21(18) \\ 2587.41(45) & 0.89(18) \\ 2592.00(6) & 7.28(21)\end{array}$

$2595.74(80) \quad 0.53(16)$

$2599.99(50) \quad 0.68(15)$

$2603.66(50) \quad 0.76(15)$

$2621.50(25) \quad 2.35(25)$

$2624.90(37)$. $1.42(24)$

$2636.50(22) \quad 0.85(9)$

$2646.51(24) \quad 0.75(8)$

2655.56(20)

$2660.73(7)$

2669.16(6)

2678.34(7)

$2690.78(7)$

$2701.56(45)$

$2706.54(4)$

2726.93(80)

$2732.47(36)$

2737.00(18)

2739.76(17)

$2750.73(25)$

2754.38(17)

$2769.36(100)$

2773.98(19)

2785.39(55)

$2788.08(38)$

$2796.28(32)$

2803.35(44)

$2808.13(8)$

2812.94(90)

2822.45(35)

$2828.20(38)$

$2832.30(70)$

$2839.60(42)$

2845.16(12)

2848.56(36)

2871.88(11)
$1.29(12)$

$3.78(16)$

$3.33(12)$

3.45(16)

$2.85(11)$

$0.51(9)$

8.20(14)

$0.42(15)$

$1.19(20)$

$6.72(64)$

$7.46(65)$

$0.78(17)$

2.46(18)

$0.32(17)$

$2.96(25)$

$0.73(19)$

$1.00(20)$

$0.66(10)$

$0.42(8)$

2.48(9)

$0.32(13)$

$0.70(12)$

0.89 (15)

$0.49(15)$

$0.98(19)$

$5.51(30)$

$1.73(26)$

$6.03(40)$ 
Table I (continued)

$$
\begin{gathered}
E_{\gamma} \\
(k e V)
\end{gathered}
$$

$$
\begin{aligned}
& 2876.34(20) \\
& 2894.15(25) \\
& 2898.14(25) \\
& 2909.65(40) \\
& 2913.45(14) \\
& 2935.27(25) \\
& 2938.65(60) \\
& 2944.61(28) \\
& 2952.32(13) \\
& 2956.56(43)
\end{aligned}
$$

$2971.41(60)$

$2975.57(32)$

$2984.62(5)$

2992.92(16)

$3008.57(23)$

3024.19(19)

$3034.68(5)$

$3042.07(8)$

$3055.59(45)$

$3082.06(29)$

$3087.44(45)$

$3095.84(15)$

$3101.97(20)$

$3107.43(72)$

$3123.73(13)$

$3134.00(12)$

$3152.05(11)$

$3168.26(11)$

$3191.23(16)$

$3197.14(22)$

3202.12(11)

$3211.79(60)$

$3217.11(30)$

$3225.08(50)$

$3238.53(8)$

$3250.50(25)$

$3255.94(50)$

$3261.88(14)$

$3268.84(16)$

$3273.08(55)$
$\mathrm{I}_{\gamma}$

$3.36(38)$

$0.89(9)$

$0.82(8)$

$0.58(10)$

$1.56(9)$

$1.59(16)$

$0.60(15)$

$0.61(8)$

$1.55(9)$

$0.44(8)$

$0.53(13)$

$0.97(13)$

$11.26(26)$

$1.63(15)$

$0.96(10)$

$0.86(8)$

$8.10(18)$

3.44(16)

$0.59(12)$

$0.97(12)$

$0.60(12)$

$3.0(3)$

$1.77(17)$

$0.46(15)$

2.37(19)

4.0(2)

$2.11(10)$

$1.80(13)$

$1.20(8)$

$0.90(8)$

$1.85(9)$

$0.58(15)$

$1.18(13)$

$0.51(10)$

$1.62(9)$

$0.94(9)$

$0.48(10)$

$1.65(11)$

$1.29(8)$

$0.47(10)$
$\left(k^{E} V_{)}\right)$

$I_{\gamma}$

$\begin{array}{ll}3287.92(37) & 0.44(6) \\ 3314.21(24) & 0.58(7) \\ 3320.80(33) & 0.57(8) \\ 3344.00(19) & 1.20(10) \\ 3351.44(24) & 1.02(10)\end{array}$

$3354.90(48)$

$3371.83(26)$

$3375.20(28)$

$3383.06(11)$

$3388.89(13)$

$3408.34(55)$

$3411.40(80)$

$3420.05(32)$

$3425.67(37)$

$3440.48(23)$

3448.05(80)

$3454.93(100)$

$3460.01(30)$

$3471.17(30)$

$3484.19(11)$

$3489.21(11)$

$3500.48(75)$

$3508.28(28)$

$3511.78(42)$

$3522.12(17)$

$3525.37(37)$

$3534.22(127)$

$3540.34(95)$

$3544.61(65)$

$3549.59(50)$

$3552.98(40)$

$3556.36(90)$

$3570.25(20)$

$3609.06(29)$

3623.34(190)

$3627.02(60)$

$3633.90(110)$

$3647.66(72)$

$3657.46(6)$

$3671.98(45)$
$0.50(10)$

$0.91(10)$

$0.93(10)$

$1.54(9)$

$1.32(9)$

$0.45(11)$

$0.34(12)$

$0.54(7)$

$0.48(7)$

$1.11(11)$

$0.44(15)$

$0.17(8)$

$0.62(8)$

$0.69(9)$

$1.58(7)$

$1.57(7)$

$0.18(6)$

$1.44(17)$

$0.93(16)$

$1.87(14)$

$0.84(14)$

$0.12(8)$

$0.26(9)$

$0.42(9)$

$0.72(12)$

$1.06(12)$

$0.33(12)$

$1.12(9)$

$0.59(7)$

$0.13(9)$

$0.39(10)$

$0.16(8)$

$0.20(6)$

$2.83(9)$

$0.83(19)$ 
Table I (continued)<smiles>[3H][AsH2]</smiles>

$3674.93(28)$
$3683.89(30)$
$3733.62(7)$
$3756.98(65)$
$3768.05(23)$

$3772.59(18)$

$3782.25(80)$

$3787.13(55)$

$3797.05(39)$

$3816.48(17)$

$3827.62(18)$

$3835.04(7)$

$3844.34(19)$

$3854.10(60)$

$3884.30(39)$

3907.07(51)

$3925.94(14)$

3964.89(110)

$3967.68(120)$

$3977.51(55)$

4004.74(41)

$4020.44(13)$

$4106.50(8)$

$4110.81(12)$

4116.39(85)

4145.03(23)

$4150.81(35)$

$4162.87(25)$

$4178.08(37)$

$4206.18(6)$

$4234.65(50)$

$4245.38(12)$

4256.03(28)

$4264.12(8)$

$4282.56(22)$

$4288.96(5)$

$4296.19(53)$

$4301.95(34)$

$4313.47(10)$

$4321.30(7)$
1.43(19)

$0.98(15)$

2.53(10)

$0.35(9)$

$0.86(7)$

$1.33(8)$

$0.24(6)$

$0.27(5)$

$0.33(5)$

$0.75(6)$

$0.80(6)$

$1.92(8)$

$0.79(6)$

$0.26(6)$

$0.39(6)$

$0.25(5)$

$0.79(6)$

$0.22(12)$

$0.20(12)$

0.19 (4)

\section{$0.26(4)$}

$0.89(5)$

$3.36(11)$

2.29(10)

$0.16(5)$

$0.63(6)$

$0.37(5)$

$0.47(5)$

$0.38(6)$

$2.09(6)$

$0.19(4)$

$1.47(8)$

$0.33(4)$

$1.24(6)$

$0.45(4)$

3.34(7)

$0.15(3)$

$0.22(3)$

$0.99(4)$

$1.46(5)$ $\underset{(k e V)}{E_{y}}$

$\begin{array}{ll}4342.94(33) & 0.16(2) \\ 4378.09(39) & 0.15(2) \\ 4404.78(7) & 1.42(4) \\ 4434.00(85) & 0.066(20) \\ 4445.22(8) & 2.55(10) \\ 4461.76(60) & 0.067(15) \\ 4492.88(110) & 0.036(14) \\ 4499.30(72) & 0.055(14) \\ 4531.02(32) & 0.144(20) \\ 4556.69(82) & 0.055(16) \\ & \\ 4562.74(80) & 0.057(17)\end{array}$

$I_{\gamma}$

$0.16(2)$

$0.15(2)$

$0.066(20)$

$0.067(15)$

$0.055(14)$

$0.144(20)$

$0.057(17)$ 


\section{ELECTRON-CAPTURE TO POSITRON INTENSITY RATIOS AND THE DECAY ENERGY OF ${ }^{131} \mathrm{Ba}$}

C. W. Reich, R. J. Gehrke, R. G. Helmer, R. C. Greenwood, R. A. Anderl

Evidence has recently been reported (see Ref. (1) and references contained therein)which suggests the existence of cases where the experimental electron-capture (e.c.) to positron $\left(\beta^{+}\right)$intensity ratios are "anomalously" large, in some instances by quite large factors. Since e.c. $/ \beta^{+}$ratios are sometimes used to determine decay energies (Q-values), such a possibility casts doubt on the validity of Q-values which are based on such measurements.

In a recent study ${ }^{(2)}$ of the decay of ${ }^{131} \mathrm{Ba}$ (see the proposed decay scheme, shown in Fig. 1), the e.c. $/ \beta^{+}$ratio of the transition to the 216-keV state was measured, Comparison of this measured value with the theoretical calculations $(3)$ yielded a value of $1372_{-20}^{+10} \mathrm{keV}$ for the beta-decay energy. The existence of the effect described in Ref. (1), however, would call into question the reliability of this value. From the figures given in Ref. (1), it is difficult to extrapolate to the situation in ${ }^{131} \mathrm{Ba}$, but a crude estimate suggests that the $\mathrm{Q}$-value deduced from the measured e.c. $/ \beta^{+}$ratio may be low by $\sim 100 \mathrm{keV}$.

Additional information exists which can shed light on the question of the ${ }^{131} \mathrm{Ba}$ decay energy and its relation to the e.c./ $\beta^{+}$ratio. From the measured $Q$-value of the $130 \mathrm{Ba}(\mathrm{d}, \mathrm{p})$ reaction $(4)$ a value of $1348+7$

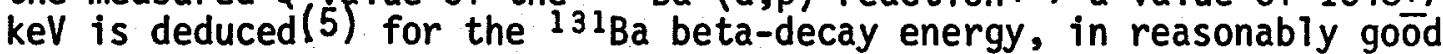
agreement with our value. The lower limit on this value must, in fact, be increased somewhat, since we observe e.c. feeding of a level at $1342 \mathrm{keV}$ (see Fig. 1). From the $Q$-value as determined from the $(d, p)$ results, the "skew ratio" (the ratio of our experimental e.c./ $\beta^{+}$ ratio to the theoretical value) (1) is $0.38_{-0}^{+0.7}$. This skew ratio is thus compatible with the conventional theory $\{$ i.e., a skew ratio $=1.0$ is possible). Further, most of the calculated range of the skew ratio is less than unity, whereas the situations illustrated in Fig. 1 of Ref. (1) all have ratios larger than unity. We thus conclude that the use of our e.c. $/ \beta^{+}$ratio to deduce the Q-value for the ${ }^{13} 1_{\mathrm{Ba}}$ decay is justified and that the type of anomaly discussed in Ref. (1) does not occur here.

(1) R. B. Firestone, R. A. Warner, W. C. McHarris and W. H. Kelly, Phys. Rev. Letters 35, 713 (1975).

(2) R. J. Gehrke, R. G. Helmer, C. W. Reich, R. C. Greenwood and R. A. Anderl, Annual Progress Report, Nuclear Structure Studies Utilizing LAMPF, ANCR-1283 (March 1976) 19-23 
(3). N. B. Gove and M. J. Martin, Nucl. Data Tables A 1, No. 3, 206, (1971).

(4) D. von Ehrenstein, G. C. Morrison, J. A. Nolen, Jr., and N. Williams, Phys. Rev. C 12066 (1970).

(5) R. L. Auble, H. R. Hiddleston and C. P. Browne, Nucl. Data Sheets 17, No. 4,573 (1976). 


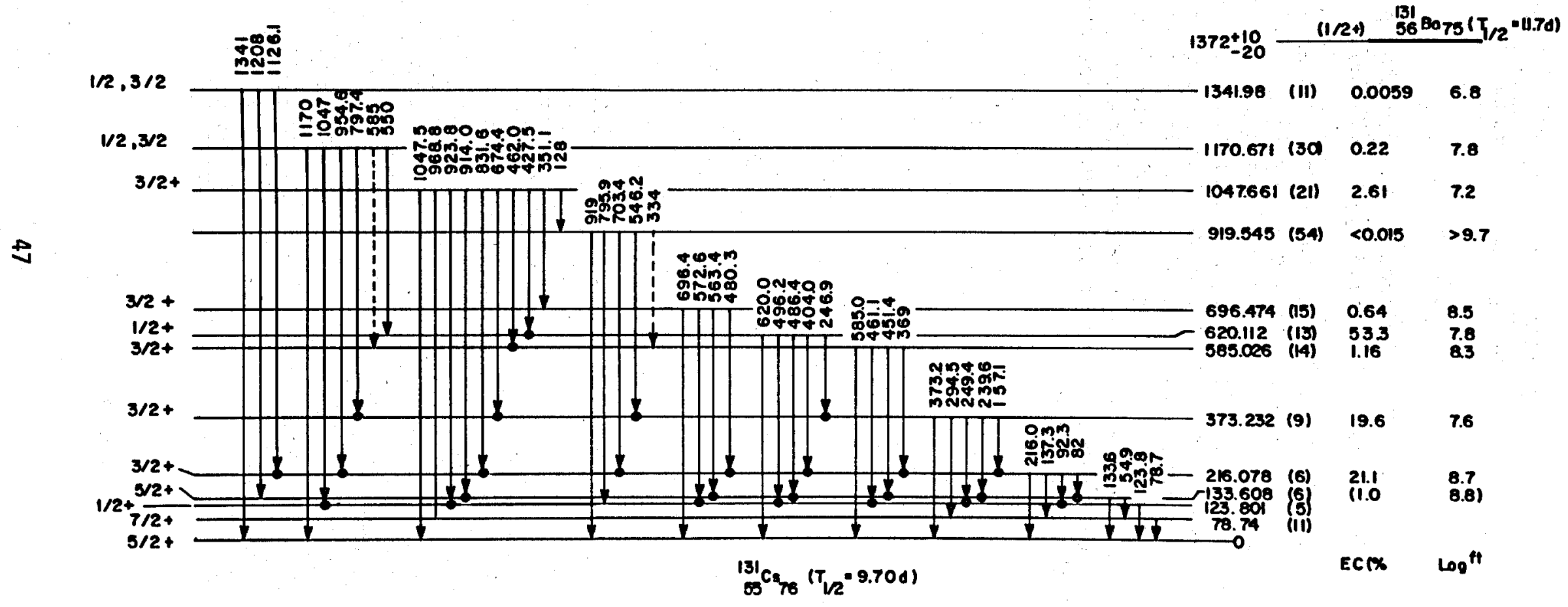

Fig. 1 Decay scheme proposed for ${ }^{131} \mathrm{Ba}$ on the basis of the data of Ref. (2). 


\title{
$K$ CONVERSION COEFFICIENTS OF $\gamma$-RAYS
} ABOVE $800 \mathrm{keV}$ FROM ${ }^{182} \mathrm{Ta}$ DECAY

\author{
R. G. Helmer
}

The following is the abstract of a paper that has been accepted for publication in Nuclear Physics A272, 269 (1976).

The relative intensities of the K-shell internal conversion electrons above $800 \mathrm{keV}$ from the decay of $182 \mathrm{Ta}(115 \mathrm{~d}$ ) have been studied. These measurements were made with a $\pi \sqrt{2}$ iron-free, magnetic, electron spectrometer with observed resolutions of 0.075 to $0.20 \%$. Relative $\gamma$-ray intensities were also determined on $\mathrm{Ge}(L i)$ detector systems. These data were combined to obtain K-shell conversion coefficients for 21 transitions. The estimated uncertainties are as small as $0.5 \%$ for the relative electron intensities and $0.8 \%$ for the conversion coefficients. Multipole mixing ratios are determined for a number of the transitions. 
CORIOLIS-MIXING ANALYSIS OF THE $K^{\pi}=0^{-}, 1^{-}$AND $2^{-}$OCTUPOLE-VIBRATIONAL

BANDS IN ${ }^{156} \mathrm{Gd}$

C. W. Reich

For some time, we have been involved with several European laboratories in a cooperative project to study the energy-level structure of the doubly-even deformed nucleus $156 \mathrm{Gd}$ by employing various facets of neutron-capture gamma-ray spectroscopy (see e.g., Ref. (1)). This study has produced a considerably more detailed picture of the $156 \mathrm{Gd}$ level scheme below $22.5 \mathrm{MeV}$ than was available previously. One interesting result, to be discussed here, is the identification of a number of negative-parity states in the region between 1.2 and $2.0 \mathrm{MeV}$ which can be interpreted as members of $\mathrm{K}^{\pi}=0^{-}, 1^{-}$and $2^{-}$octupolevibrational bands. These states are shown at the right in Fig. 1. As is evident from Fig. 1, the ordering of the seven lowest-7ying states deviates markedly from that of simple $I(I+1)$ patterns, indicative of the presence of strong coupling effects.

To explore this mixing and to understand various of the properties of these states, we have carried out a Coriolis-mixing analysis of their level-energy and El transition-probability data. The approach employed has been described elsewhere $(2-4)$. Briefiy, the approach was as follows. The Coriolis matrix el ement between two states of spin I, originating from rotational bands having quantum numbers $k$ and $k+1$, is given by the expression

$$
\left\langle H_{C}\right\rangle_{K, K+1}^{I}=-\frac{\hbar^{2}}{2 \Im}\left[\left(1+\delta_{K, 0}\right)(I-K)(I+K+1)\right]^{\frac{3}{2}}\left\langle K+1\left|J_{+}\right| K\right\rangle .
$$

In this analysis, the coriol is matrix elements between the bands, as well as the band-head energies and moments of inertia, were treated as variable parameters. Three bands, with $\mathrm{K}^{\pi}=0^{-}, \mathrm{1}^{-}$and $2^{-}$, were considered. The $\hbar^{2} / 2 \xi$ value of the unperturbed $2^{-}$band was fixed, and values of the remaining parameters were adjusted until a good fit to the level spectrum was obtained. This procedure was carried out for a number of different assumed values of this inertial parameter of the $2^{-}$band.

The results of a typical fit are illustrated in Fig. 1 and are summarized in Table I. Of particular importance is the fact that the admixed amplitudes of $\mathrm{K}=0$ and $\mathrm{K}=1$ in the mixed $\mathrm{O}^{-}$and $1^{-}$bands are rather insensitive to the choice of values for the inertial parameter of the $2^{-}$band (over the reasonable range from $\sim 10 \mathrm{keV}$ to $\sim 12 \mathrm{keV}$, these amplitudes varied only by a few percent). The values listed for these quantities in Table I are thus regarded as being realistic.

Using these values, we have analyzed the branching ratios of the E1 transitions between these Coriolis-mixed states and the ground-state band. The $B(E 1)$ values can be expressed in the form $(2-4)$

$$
B\left(E 1 ; I \rightarrow I^{\prime}\right)=\left\langle 0^{+}\left|M^{\prime}(1,0)\right| 0^{-}\right\rangle^{2} \quad\left\{a_{0}\left(I 010 \mid I^{\prime} 0\right)+\sqrt{2} a_{1}\left(I 11-1 \mid I^{\prime} 0\right) Z\right\}^{2} \text {, }
$$


where $\left\langle 0^{+}\left|M^{\prime}(1,0)\right| 0^{-}\right\rangle$is the intrinsic El matrix element connecting the $K^{\pi}=0^{-}$band and the ground-state band $\left(0^{+}\right), a_{0}$ and $a_{1}$ are the admixed amplitudes of $\mathrm{K}^{\pi}=\mathrm{O}^{-}$and $1^{-}$, respectively, in the initial state, the quantities in parentheses are Clebsch-Gordan coefficients, and the parameter $Z$ is defined by the expression

$$
Z=\left\langle 0^{+}\left|M^{\prime}(1,-1)\right| 1^{-}\right\rangle /\left\langle 0^{+}\left|M^{\prime}(1,0)\right| 0^{-}\right\rangle \text {. }
$$

Since all quantities except $Z$ are presumed to be known, the E1 branching-ratio data can be used to determine it. The results of this analys is are summarized in Table II.

The fact that a consistent value of $Z$ is obtained from the data lends support to the validity of the analysis. We find that, for ${ }^{156} \mathrm{Gd}$, the El matrix element with $\Delta K=I$ is much smaller than that with $\Delta K=0$, in agreement with the findings in other deformed nuclei.

(1) A. Bäcklin, B. Fogelberg, G. Hedin, M. Saraceno, R. C. Greenwood, C. W. Reich, H. R. Koch, H. A. Baader, H. D. Breitig and O. W. B. Schult, Neutron Capture Gamma-Ray Spectroscopy, (IAEA, Vienna, 1969) 147-154.

(2) C. W. Reich, Nuclear Technology Division Annual Progress Report for Period Ending June 30, 1972, ANCR-1088 (October 1972), 69-72.

(3) R. C. Greenwood, C. W. Reich and S. H. Vegors, Jr., Nucl. Phys. A252, (1975) 260-292.

(4) C. W. Reich and R. C. Greenwood, Nuclear Technology Division Annual Progress Report for Period Ending June 30, 1974, ANCR-1177 (January 1976) 3-13. 
Summary of the results of one of the Coriolis-mixing analyses of the energy-level structure of the octupole-vibrational bands in ${ }^{156} \mathrm{Gd}$.

$\underline{I}$

Level Energy (keV)

Admixed Ampl i tude

$\begin{array}{lcc} & \text { calculated } & \text { experimental } \\ 1 & 1243.1 & 1242.4 \\ 1 & 1363.4 & 1366.4 \\ 2 & 1316.7 & 1319.7 \\ 2 & 1778.7 & 1780.4 \\ 3 & 1281.2 & 1276.1 \\ 3 & 1541.0 & 1538.9 \\ 3 & 1853.7 & 1851.8 \\ 4 & 1469.4 & 1468.6 \\ 4 & 1952.1 & 1952.3 \\ 5 & 1405.4 & 1408.0 \\ 5 & 1803.4 & (2066.7)^{\mathrm{a}} \\ 5 & 2079.7 & \end{array}$

Input Parameters

Band-head Energy ( $k e V$ )

Inertial Parameter, $\hbar^{2} / 2 \xi(\mathrm{keV})$
$1273.2 \quad 1333.3$

10.74

11.55
$\underline{K=0}$

0.500

0.866

$\begin{array}{lll}. & 0.997 & 0.076\end{array}$

$\begin{array}{lll}. & 0.0976 & -0.997\end{array}$

0.623

0.781

0.040

0.779

$-0.614$

$-0.128$

$\begin{array}{lll}. & 0.988 & 0.155\end{array}$

0.155

0.746

$-0.626$

$-0.228$

0.076

$-0.111$

0.991

•

0.746

0.103
$-0.988$

0.105

$-0.227$

0.968

Coupl ing Matrix El ements, $A_{K, K+1}(k e V)$ 26.05

1776.0

$$
\begin{aligned}
& \left\langle H_{\text {Cor. }}\right\rangle_{K, K+1}^{I}=-A_{K, K+1} \quad\left[\left(1+\delta_{K, 0}\right) \quad(I-K) \quad(I+K+1)\right]^{\frac{1}{2}} \text {, } \\
& A_{K, K+1}={\left(\hbar^{2} / 2 \Im\right)}_{\text {Cor. }}\left\langle K+1\left|J_{+}\right| K\right\rangle .
\end{aligned}
$$

Tentative assignment

b Value held fixed during the analysis 


\section{TABLE II}

Summary of the results of the coriolis-mixing analysis of the transition probabilities of the El transitions between the octupole-vibrational states and the ground-state band of ${ }^{156} \mathrm{Gd}$.

Initial State

$\underline{I^{\pi} ; \mathrm{E}(\mathrm{keV})}$

$1^{-} ; 1242$.

$1+; 1366$.

$3^{-} ; 1276$.

$3^{-} ; 1538$.

$5^{-} ; 1408$.
$B(E 1 ; I \rightarrow I-1)$

B(E); $1+1+1)$

$0.754(83)^{a, b}$

$0.428(33)$

$1.34(10) \mathrm{c}$
$1.10(17) \mathrm{c}$

$0.598(51)$

$1.34(12)$ $\mathrm{z}$

$-0.058(17)$

$-0.062(31)$

$-0.117(18)$

$-0.074(33)^{c}$

$-0.071(25)$

$-0.045(9)$

$-0.057(8)^{d}$

Quantities in parentheses give the errors in the least significant figure (or figures) of the associated value.

b From the relative gamma-ray intensities as observed in ${ }^{156}$ Eu decay.

c From the relative gamma-ray intensities as observed in ${ }^{156} \mathrm{~Tb}$ decay.

d Based on the five values, excluding that determined from the present data for the 1276-keV state. 


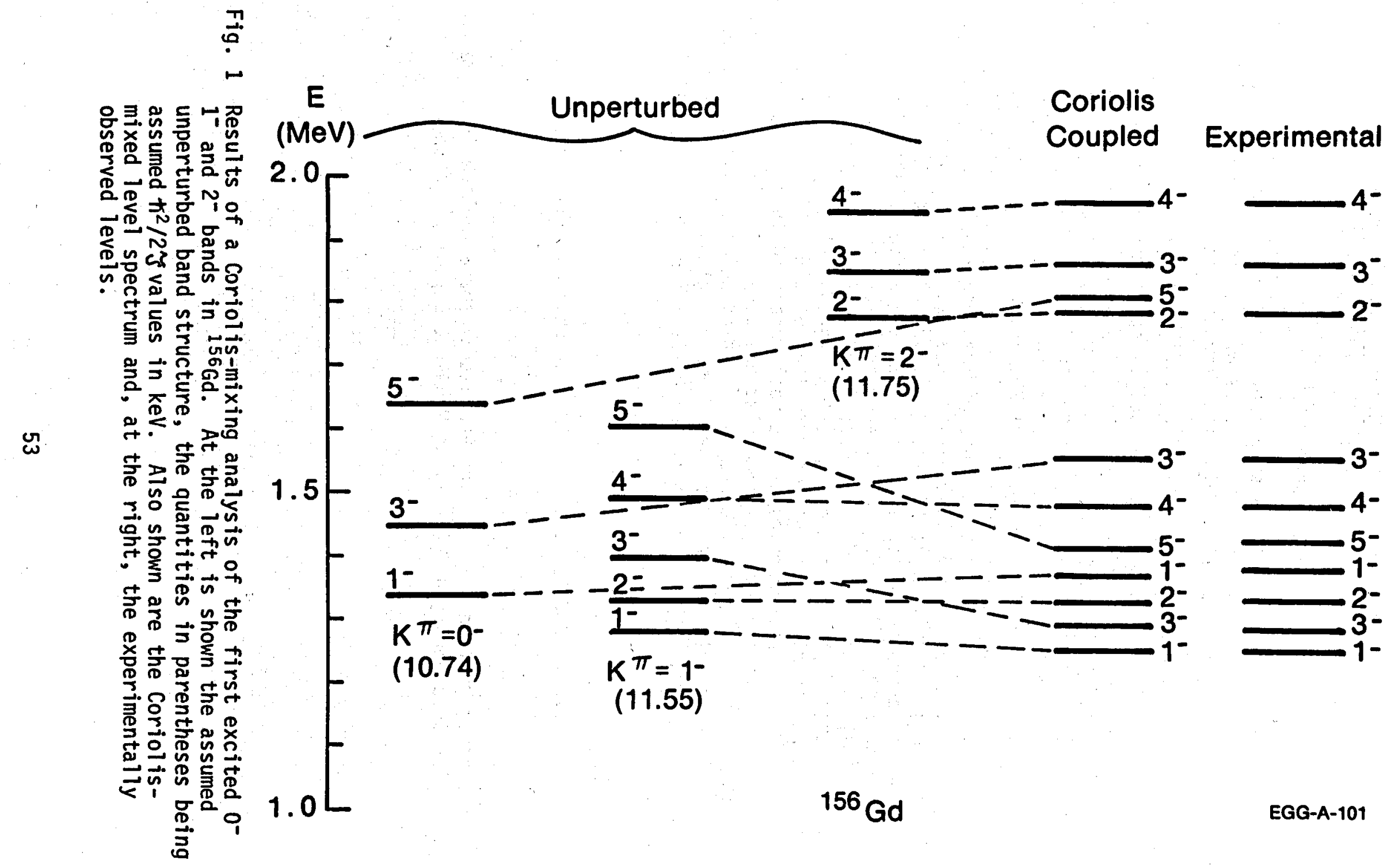


FEATURES OF THE LOW-ENERGY LEVEL SCHEME OF 229Th AS OBSERVED IN THE Q-DECAY OF 233U

L. A. Kroger* and C. W. Reich

The following is the abstract of a paper (1) which has been published in the journal Nuclear Physics A. The level scheme which has been proposed for ${ }^{229}$ Th from this study is shown in Fig. 1.

The $\gamma$ radiation emitted following the a-particle decay of $233 \mathrm{U}$ has been studied using a variety of $\mathrm{Ge}(\mathrm{Li})$ spectrometers and a $\mathrm{Si}(\mathrm{Li})$ spectrometer system. Analys is of $\gamma$-singles and $\gamma \gamma$-coincidence measurements has established the existence of $91 \gamma$ rays which can be definitely established as representing transitions in 229Th. From these data, a level scheme consisting of 28 excited states below $\approx 0.53 \mathrm{MeV}$ in $229 \mathrm{Th}$ is proposed. The well known state at $29.16 \mathrm{keV}$ is shown to have $I^{\pi}=5 / 2$, in contrast with several previous proposals. Additional states which are shown to be members of the rotational band associated with this state are the following (yith the assigned I" values in parentheses): $71.82\left(7 / 2^{+}\right) ; 125.45\left(9 / 2^{+}\right)$; and $195.76 \mathrm{keV}\left(11 / 2{ }^{+}\right)$. Evidence is presented, which indicates that these four states are members of a $K^{\pi}=3 / 2$ rotational band, built on the Nilsson orbital $3 / 2^{+}[631]$. The $I^{\pi}=3 / 2+$ band head is shown from indirect evidence to lie within $\approx 0.1 \mathrm{keV}$ of the $5 / 2+[633]$ ground state of $229 \mathrm{Th}$. Seven negative-parity states are proposed to lie between \& $146 \mathrm{keV}$ and $\approx 237 \mathrm{keV}$; and their energies can be well described in terms of Coriolis coupling among the $\mathrm{j} 15 / 2$-related $\mathrm{Nil}$ sson states $3 / 2-[761], 5 / 2-[752]$, $1 / 2-[770]$ and $7 / 2-[743]]^{2}$ Evidence is presented which strongly suggests that a significant component of the $k^{\pi}=3 / 2^{-}$octupole-vibrational state $\left\{3 / 2+[631], 0^{-}\right\}$is present in these $10 w-1 y i n g$ negative-parity states.

(1) L. A. Kroger and C. W. Reich, Nuclear Physics A259, 29 (1976).

* Associated Western Universities Graduate Research Fellow from the Physics Department, University of Wyoming, Laramie, Wyoming. 


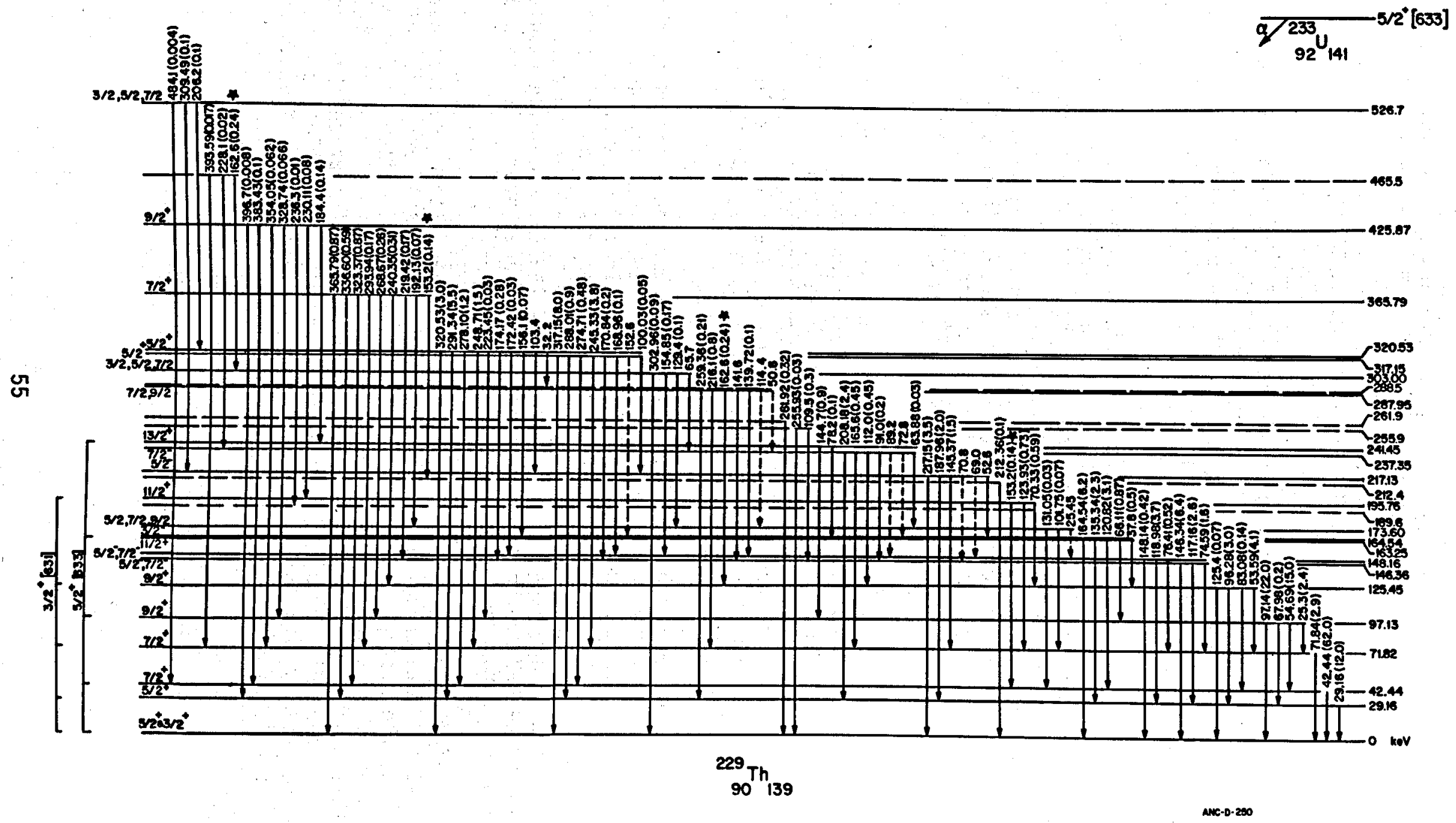

Fig. 1 Level scheme of ${ }^{229} \mathrm{Th}$ proposed from the present study of the ${ }^{233 \mathrm{U} \text { decay. }}$ 


\section{LEVEL STRUCTURE OF ${ }^{23} \mathrm{~T} T \mathrm{~T}$ FROM THE ${ }^{232} \mathrm{Th}(\mathrm{n}, \gamma)$ REACTION USING 2- AND 24-keV NEUTRONS}

R. C. Greenwood, R. E. Chrien*

The level structure of ${ }^{233} \mathrm{Th}$ has previously been studied using both the ${ }^{232} \mathrm{Th}(\mathrm{n}, \mathrm{\gamma})$ reaction $(1,2)$ and the ${ }^{232} \mathrm{Th}(\mathrm{d}, \mathrm{p})$ reaction $(1,3)$. While many levels in ${ }^{233} \mathrm{Th}$ are populated by these two reactions, definitive spin and parity information is lacking for all but the few lowest lying levels (with energies below $\sim 500 \mathrm{keV}$ ).

In order to obtain a more detailed understanding of the structure of the deformed nucleus ${ }^{23}{ }^{3} \mathrm{Th}$, additional $(n, \gamma)$ studies have been undertaken in a cooperative effort both at the Institut Max von Vaue - Paul Langevin (ILL), Grenoble, France, and at Brookhaven National Laboratory. In the present work, the spectra of primary $\gamma$ rays resulting from 2- and 24-keV neutron capture in $232 \mathrm{Th}$ were measured. These data have provided us with more complete information on the occurence and distribution of low lying states in ${ }^{23}{ }^{3}$ Th having $I^{\pi}=1 / 2^{+}$or $3 / 2^{+}, I \pi=5 / 2^{+}$, and $I^{\pi}=1 / 2^{-}$ or $3 / 2^{-}$.

The use of keV neutrons obtained through filters for $(n, \dot{\gamma})$ studies can provide valuable spectroscopic information about the states populated by primary transitions. Generally, such information is not obtainable from conventional thermal-neutron capture measurements. Of importance in obtaining this spectroscopic information are both the finite energy spread inherent in.beams of filtered keV neutrons, and the energy of the principal neutron group in the beam.

A consequence of the finite spread in neutron energies is that the capture $r$-ray spectrum results from an average over many compoundresonance states. Hence, the large statistical fluctuations normally associated with the primary $\gamma$-ray intensities resulting from capture in a single compound-resonance state will tend to be averaged out. For the specific case of the ${ }^{232} \mathrm{Th}(n, \gamma)$ reaction, the spectra which result from 2- and 24-keV neutron capture represent averages over $\sim 42$ and $\sim 119$ s-wave neutron resonance states, respectively.

The energy of the principal group in the filtered neutron beam is also a parameter which is of value in obtaining spectroscopic information about the states populated by primary transitions following neutron capture. The value of neutron energy arises quite simply from the fact that the higher the neutron energy, the more contribution there is from $\ell>0$ capture. To quantify this we can express the relative $p$-to-s wave ratio by the following approximation (ignoring the fluctuation corrections)

$$
\frac{\left\langle\sigma_{\gamma}{ }^{1}\right\rangle}{\left\langle\sigma_{\gamma}{ }^{0}\right\rangle} \sim \frac{3 S_{1}}{S_{0}} \frac{\left\langle\Gamma^{0}(I)\right\rangle}{\left\langle\Gamma^{1}(I)\right\rangle}(k R)^{2}
$$


For the specific case of the ${ }^{232} \mathrm{Th}(n, \gamma)$ reaction we estimate, using parameters $\left\langle\Gamma_{\gamma}\right\rangle=0.0212 \mathrm{eV}, S_{0}=0.84 \times 10^{-4}, S_{1}=1.6 \times 10^{-4}$, $\left\langle D_{0}\right\rangle=16.8 \mathrm{eV}^{\gamma}(k R)_{2}^{2}=0.006$ and $(k R)_{24}^{2}=0.08(4-6)$, that

$$
\begin{aligned}
& \frac{\left\langle\sigma_{\gamma}{ }^{1}\right\rangle}{\left\langle\sigma_{\gamma}{ }^{0}\right\rangle} \sim 2.1 \text { at } E_{n}=24.5 \mathrm{keV} \\
& \frac{\left\langle\sigma_{\gamma}{ }^{1}\right\rangle}{\left\langle\sigma_{\gamma}{ }^{0}\right\rangle} \sim 0.14 \text { at } E_{n}=1.95 \mathrm{keV} .
\end{aligned}
$$

Thus, we anticipate that the 2-keV neutron capture is predominantly s-wave and hence final states with $I \pi=1 / 2^{-}$and $3 / 2^{-}$will be most strong populated by primary $\gamma$-ray transitions. For the 24-keV neutron capture though, the $\mathrm{p}$ - and s-wave contributions are comparable so that primary transitions to final states with $I \pi=1 / 2^{-}, 3 / 2^{-}, 1 / 2^{+}, 3 / 2^{+}$ and $5 / 2^{+}$should all be significant.

Portions of the prompt $\gamma$-ray spectra obtained as a result of 2and 24-keV neutron capture in ${ }^{232} \mathrm{Th}$ are shown, in comparison, in Fig. 1. These spectra each illustrate the double-escape peak structure associated with primary $\gamma$-ray transitions to levels in ${ }^{232}$ Th with energies $\leq 1.1 \mathrm{MeV}$. Since the energies of primary $\gamma$ rays resulting from the $232 T h(n, \bar{\gamma})$ reaction are quite low, $<5 \mathrm{MeV}$, the full-energy peaks are also quite prominent in this spectrum. These full-energy peaks proved to be of particular value in the analys is of these spectra (to obtain $\gamma$-ray energies and intensities) since they were less apt to be contaminated with single- or double-escape peak lines from other $\gamma$-ray transitions.

The energies of the final states in 233 Th populated by primary transitions are listed in Table I together with the $I^{\pi}$ assignments for these states deduced from the 24- and 2-keV capture data alone (columns 3 and 4 , respectively), and from the ratio of their primary $r$-ray transition intensities (column 5). The adopted set of $I^{\pi}$ are shown in column 6 .

1. T. von Egidy, 0.W.B. Schult, D. Rabenstein, J. R. Erskine, 0. A. Wasson, - R. E. Chrien, D. Breitig, R. P. Sharma, H. A. Baader and H. R. Koch, Phys. Rev. C 6 (1972) 266.

2. J. Kern and D. Duc, Phys. Rev. C 10 (1974) 1554.

3. T. Grotdal, J. Limstrand, K. Nybb, K. Skar and T. Thorsteinsen, Nucl. Phys. Al89 (1972) 592.

4. H. I. Liou, H. S. Camarda and F. Rahn, Phys. Rev. C 5 (1972) 1002.

5. F. Rahn, H. S. Camarda, G. Hocken, W. W. Havens, Jr., H. I. Liou, J. Rainwater, M. Slagowit and S, Wynchank, Phys. Rev. C 6 (1972) 1854.

6. S. F. Mughabghab and D. I. Garber, Brookhaven National Laboratory Report BNL-325 (3rd ed.) vol. 1 (1973). 
TABLE I

Assignment of $I^{\pi}$ to final states in ${ }^{233}$ Th based on the 2- and 24-keV neutron capture data

\begin{tabular}{|c|c|c|c|c|c|}
\hline \multirow[b]{2}{*}{$\begin{array}{l}\text { Final-state } \\
\text { energy a) } \\
(\mathrm{keV})\end{array}$} & \multirow[b]{2}{*}{$\begin{array}{l}\text { Previous } \\
\text { work b) }\end{array}$} & \multicolumn{2}{|c|}{ Assignment of $I, \pi^{c, d)}$} & \multicolumn{2}{|c|}{$\cdot$} \\
\hline & & $I_{\gamma}(24) E_{\gamma}^{-3}$ & $I_{\gamma}(2) E_{\gamma}^{-3}$ & $\frac{I_{\gamma}(2)}{I_{\gamma}(24)}$ & Adopted \\
\hline 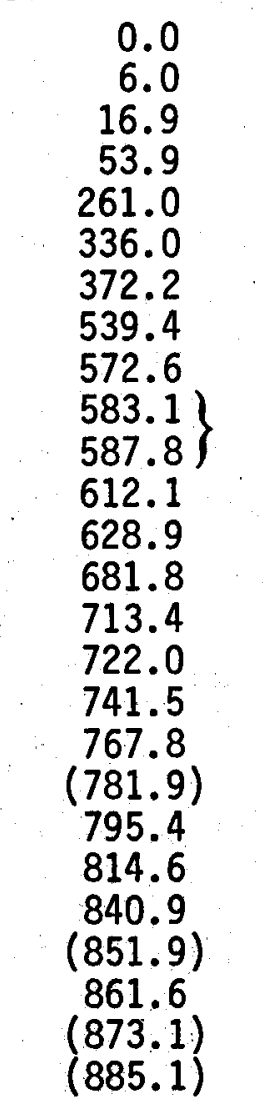 & $\begin{array}{l}1 / 2+ \\
5 / 2+ \\
3 / 2+ \\
5 / 2+ \\
(5 / 2+) \\
3 / 2+ \\
5 / 2+ \\
(1 / 2-) \\
(3 / 2-) \\
(3 / 2-) \\
(3 / 2,5 / 2+) \\
(3 / 2,5 / 2) \\
(3 / 2-)\end{array}$ & $\begin{array}{l}1 / 2-, 3 / 2-, 5 / 2+ \\
1 / 2-, 3 / 2-, 5 / 2+ \\
1 / 2-, 3 / 2-, 5 / 2+ \\
1 / 2-, 3 / 2-, 5 / 2+ \\
1 / 2,3 / 2 \\
1 / 2-, 3 / 2-, 5 / 2+\end{array}$ & $\begin{array}{l}1 / 2,3 / 2+ \\
5 / 2 \\
1 / 2,3 / 2+ \\
5 / 2(+) \\
5 / 2 \\
1 / 2,3 / 2+ \\
5 / 2 \\
1 / 2,3 / 2- \\
1 / 2,3 / 2- \\
1 / 2,3 / 2- \\
1 / 2,3 / 2+ \\
5 / 2 \\
1 / 2,3 / 2- \\
1 / 2,3 / 2+ \\
1 / 2,3 / 2+ \\
1 / 2,3 / 2- \\
1 / 2,3 / 2+ \\
1 / 2,3 / 2+ \\
1 / 2,3 / 2+ \\
1 / 2,3 / 2- \\
1 / 2,3 / 2+ \\
1 / 2,3 / 2+ \\
1 / 2,3 / 2+ \\
1 / 2,3 / 2,5 / 2+\end{array}$ & $\begin{array}{l}1 / 2,3 / 2+ \\
5 / 2 \\
1 / 2,3 / 2+ \\
1 / 2+, 3 / 2+, 5 / 2 \\
1 / 2+, 3 / 2+, 5 / 2 \\
1 / 2,3 / 2+ \\
1 / 2+, 3 / 2+, 5 / 2 \\
1 / 2,3 / 2- \\
1 / 2,3 / 2- \\
\\
1 / 2,3 / 2+ \\
1 / 2+, 3 / 2+, 5 / 2 \\
1 / 2,3 / 2- \\
1 / 2,3 / 2+ \\
1 / 2,3 / 2+ \\
1 / 2,3 / 2- \\
1 / 2,3 / 2+ \\
1 / 2,3 / 2 \\
1 / 2,3 / 2(+) \\
1 / 2,3 / 2- \\
1 / 2,3 / 2 \\
1 / 2,3 / 2(+) \\
1 / 2,3 / 2 \\
5 / 2+, 1 / 2,3 / 2\end{array}$ & $\begin{array}{l}1 / 2,3 / 2+ \\
5 / 2+ \\
1 / 2,3 / 2+ \\
5 / 2+ \\
5 / 2+ \\
1 / 2,3 / 2+ \\
5 / 2+ \\
1 / 2,3 / 2- \\
1 / 2,3 / 2- \\
1 / 2,3 / 2 \\
1 / 2,3 / 2- \\
1 / 2,3 / 2+ \\
5 / 2+ \\
1 / 2,3 / 2- \\
1 / 2,3 / 2+ \\
1 / 2,3 / 2+ \\
1 / 2,3 / 2- \\
1 / 2,3 / 2+ \\
1 / 2,3 / 2+ \\
1 / 2,3 / 2+ \\
1 / 2,3 / 2- \\
(5 / 2)+ \\
1 / 2,3 / 2+ \\
(5 / 2)+ \\
5 / 2+\end{array}$ \\
\hline
\end{tabular}


TABLE I (continued)

\begin{tabular}{|c|c|c|c|c|c|}
\hline \multirow[b]{2}{*}{$\begin{array}{l}\text { Final-state } \\
\text { energy a) } \\
(\mathrm{keV})\end{array}$} & \multicolumn{4}{|c|}{ Assignment of $I, \pi^{c, d)}$} & \multirow[b]{2}{*}{ Adopted } \\
\hline & $\begin{array}{l}\text { Previous } \\
\text { work b) }\end{array}$ & $I_{\gamma}(24) E_{\gamma}^{-3}$ & $I_{\gamma}(2) E_{\gamma}^{-3}$ & $\frac{I_{\gamma}(2)}{I_{\gamma}(24)}$ & \\
\hline $\begin{array}{l}1313.0 \\
(1330.2) \\
1338.5 \\
1350.6 \\
(1389.8) \\
(1394.8) \\
1446.5 \\
(1459.7) \\
1479.1 \\
1497.8 \\
1532.7 \\
1580.2 \\
1587.5 \\
1668.0 \\
1688.7 \\
(1698.0) \\
1717.4 \\
1728.0\end{array}$ & & & $\begin{array}{l}1 / 2,3 / 2- \\
1 / 2,3 / 2 \\
1 / 2,3 / 2- \\
1 / 2,3 / 2- \\
1 / 2,3 / 2- \\
1 / 2,3 / 2- \\
1 / 2,3 / 2 \\
1 / 2,3 / 2(-) \\
1 / 2,3 / 2- \\
1 / 2,3 / 2- \\
1 / 2,3 / 2 \\
1 / 2,3 / 2(-) \\
1 / 2,3 / 2- \\
1 / 2,3 / 2+ \\
1 / 2,3 / 2+ \\
1 / 2,3 / 2(-) \\
1 / 2,3 / 2- \\
1 / 2,3 / 2-\end{array}$ & $\begin{array}{l}1 / 2,3 / 2- \\
1 / 2,3 / 2 \\
1 / 2,3 / 2- \\
1 / 2,3 / 2 \\
1 / 2,3 / 2 \\
1 / 2,3 / 2 \\
1 / 2,3 / 2 \\
1 / 2,3 / 2 \\
1 / 2,3 / 2- \\
1 / 2,3 / 2- \\
1 / 2,3 / 2 \\
1 / 2,3 / 2 \\
1 / 2,3 / 2(-) \\
1 / 2+, 3 / 2 \pm, 5 / 2+ \\
1 / 2,3 / 2+- \\
1 / 2,3 / 2 \\
1 / 2,3 / 2- \\
1 / 2,3 / 2(-)\end{array}$ & $\begin{array}{l}1 / 2,3 / 2- \\
1 / 2,3 / 2 \\
1 / 2,3 / 2- \\
1 / 2,3 / 2- \\
1 / 2,3 / 2- \\
1 / 2,3 / 2- \\
1 / 2,3 / 2 \\
1 / 2,3 / 2(-) \\
1 / 2,3 / 2- \\
1 / 2,3 / 2- \\
1 / 2,3 / 2 \\
1 / 2,3 / 2(-) \\
1 / 2,3 / 2- \\
1 / 2,3 / 2+ \\
1 / 2,3 / 2+ \\
1 / 2,3 / 2(-) \\
1 / 2,3 / 2- \\
1 / 2,3 / 2-\end{array}$ \\
\hline
\end{tabular}

a) Parentheses around a final-state energy indicate that its assignment is tentative.

b) Previous work referred to is that of refs. 1 and 2 .

c) The $I(2)$ and $I(24)$ are the relative $\gamma$-ray intensities obtained from 2- and 24-keV neutron capture, respectively.

d) The $I$ and $\pi$ refer to the spin and parity, respectively, of the final state. 
TABLE I (continued)

\begin{tabular}{|c|c|c|c|c|c|}
\hline \multirow[b]{2}{*}{$\begin{array}{l}\text { Final-state } \\
\text { energy a) } \\
\quad(\mathrm{keV})\end{array}$} & \multicolumn{4}{|c|}{ Assignment of $I, \pi^{c, d)}$} & \multirow[b]{2}{*}{ Adopted } \\
\hline & $\begin{array}{l}\text { Previous } \\
\text { work b) }\end{array}$ & $I_{\gamma}(24) E_{\gamma}^{-3}$ & $I_{\gamma}(2) E_{\gamma}^{-3}$ & $\frac{I_{\gamma}(2)}{I_{\gamma}(24)}$ & \\
\hline $\begin{array}{c}891.6 \\
904.6 \\
917.7 \\
924.7 \\
947.4 \\
(957.6) \\
(968.7) \\
(984.9) \\
1013.4 \\
1031.1 \\
(1039.9) \\
1050.3 \\
1061.3 \\
1074.3 \\
(1087.4) \\
(1101.8) \\
(1115.3) \\
(1133.4) \\
1151.2 \\
1172.5 \\
1184.5 \\
(1212.5) \\
1225.7 \\
1257.1 \\
1262.3 \\
1282.7 \\
1293.4 \\
(1304.8)\end{array}$ & $\begin{array}{l}(3 / 2,5 / 2+) \\
(3 / 2+) \\
(3 / 2+)\end{array}$ & $1 / 2-, 3 / 2-, 5 / 2+$ & $\begin{array}{l}1 / 2,3 / 2+ \\
1 / 2,3 / 2+ \\
1 / 2,3 / 2- \\
1 / 2,3 / 2- \\
1 / 2,3 / 2 \\
1 / 2,3 / 2,5 / 2+ \\
1 / 2,3 / 2+ \\
1 / 2,3 / 2+ \\
1 / 2,3 / 2- \\
1 / 2,3 / 2- \\
1 / 2,3 / 2++ \\
1 / 2,3 / 2- \\
1 / 2,3 / 2+ \\
1 / 2,3 / 2- \\
1 / 2,3 / 2+ \\
1 / 2,3 / 2+ \\
1 / 2,3 / 2+ \\
1 / 2,3 / 2+ \\
1 / 2,3 / 2- \\
1 / 2,3 / 2- \\
1 / 2,3 / 2++ \\
1 / 2,3 / 2,5 / 2+ \\
1 / 2,3 / 2+ \\
1 / 2,3 / 2- \\
1 / 2,3 / 2(-) \\
1 / 2,3 / 2- \\
1 / 2,3 / 2- \\
1 / 2,3 / 2+\end{array}$ & $\begin{array}{l}1 / 2,3 / 2+ \\
1 / 2,3 / 2+ \\
1 / 2,3 / 2- \\
1 / 2,3 / 2(-) \\
1 / 2,3 / 2 \\
1 / 2,3 / 2 \\
1 / 2,3 / 2(-) \\
1 / 2,3 / 2(-) \\
1 / 2,3 / 2- \\
1 / 2,3 / 2- \\
1 / 2,3 / 2- \\
1 / 2,3 / 2- \\
1 / 2,3 / 2 \\
1 / 2,3 / 2- \\
1 / 2,3 / 2(-) \\
1 / 2,3 / 2+ \\
1 / 2,3 / 2 \\
1 / 2,3 / 2 \\
1 / 2,3 / 2- \\
1 / 2,3 / 2- \\
1 / 2,3 / 2 \\
1 / 2 \pm, 3 / 2+, 5 / 2+ \\
1 / 2,3 / 2+- \\
1 / 2,3 / 2(-) \\
1 / 2,3 / 2- \\
1 / 2,3 / 2 \\
1 / 2,3 / 2- \\
1 / 2,3 / 2\end{array}$ & $\begin{array}{l}1 / 2,3 / 2+ \\
1 / 2,3 / 2+ \\
1 / 2,3 / 2- \\
1 / 2,3 / 2- \\
1 / 2,3 / 2 \\
(5 / 2)+ \\
1 / 2,3 / 2+ \\
1 / 2,3 / 2+ \\
1 / 2,3 / 2- \\
1 / 2,3 / 2- \\
1 / 2,3 / 2 \\
1 / 2,3 / 2- \\
1 / 2,3 / 2+ \\
1 / 2,3 / 2- \\
1 / 2,3 / 2(+) \\
1 / 2,3 / 2+ \\
1 / 2,3 / 2+ \\
1 / 2,3 / 2+ \\
1 / 2,3 / 2- \\
1 / 2,3 / 2- \\
1 / 2,3 / 2(+) \\
1 / 2,3 / 2,5 / 2+ \\
1 / 2,3 / 2+ \\
1 / 2,3 / 2- \\
1 / 2,3 / 2- \\
1 / 2,3 / 2- \\
1 / 2,3 / 2- \\
1 / 2,3 / 2+\end{array}$ \\
\hline
\end{tabular}




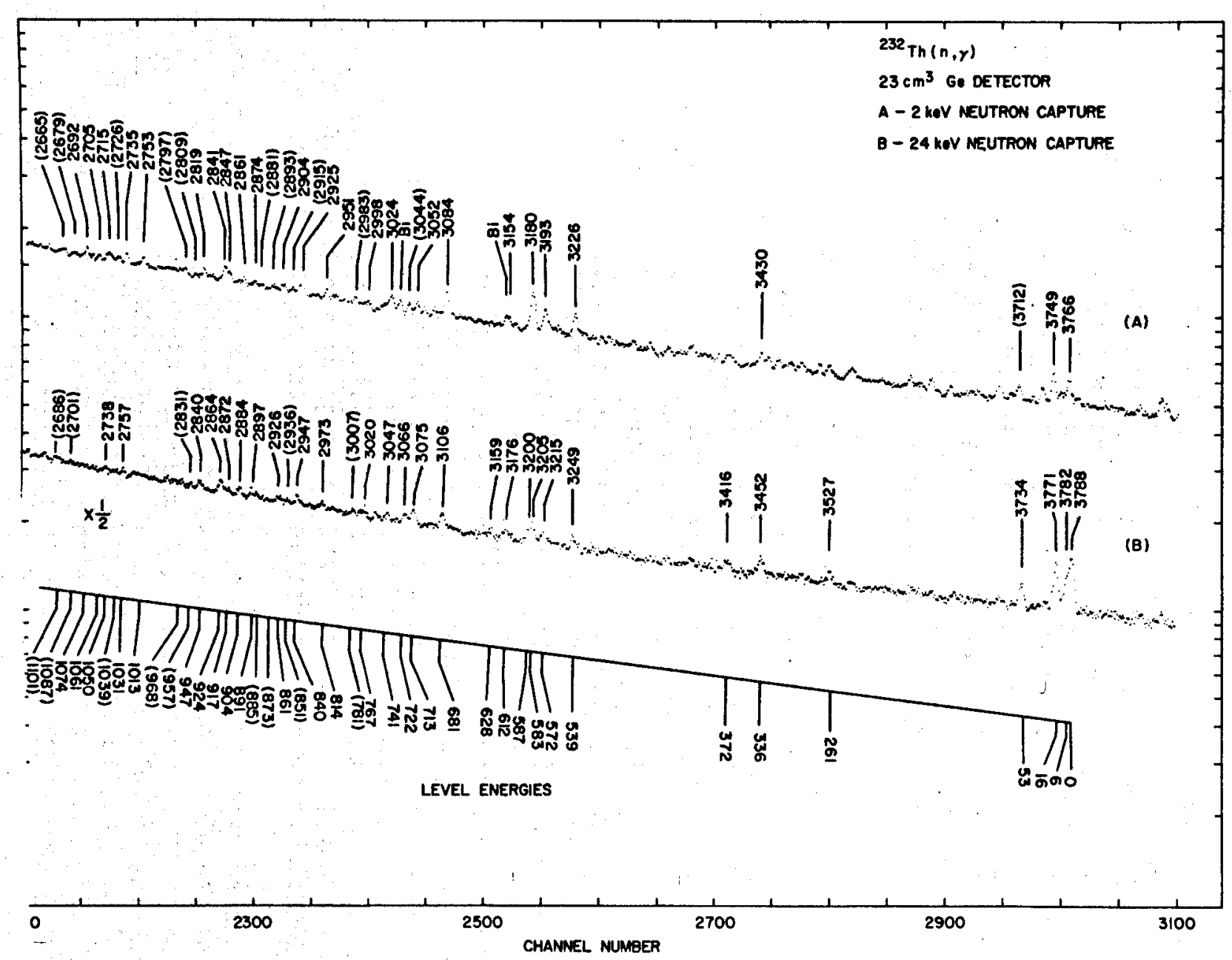

Figure 1. Comparison of the high-energy $\gamma$-ray spectra resulting from capture of 2- and 24-keV neutrons in $232 \mathrm{Th}$. The portions of both spectra illustrate the double-escape peaks resulting from primary $\gamma$-ray transitions to levels in 232 Th with energies below $\approx 1.1 \mathrm{MeV}$. The same $23 \mathrm{~cm}^{3}$ intrinsic Ge detector system was used for both sets of measurements. The energy values shown for lines in this spectrum are those of the double-escape peaks. 


\title{
ABSOLUTE $\gamma$-RAY BRANCHING AND HALF-LIFE OF $250 \mathrm{BK}$
}

\author{
R. J. Gehrke, R. G. Helmer, C. W. Reich, J. D. Baker*
}

As part of an investigation of the decay of $3.2 \mathrm{~h} 250 \mathrm{Bk}(1)$ we have measured the absolute intensity (or branching ratio) of the 989- and the $1028+1031-k e V \quad \gamma$ rays and the ${ }^{25} 0_{B K}$ half-life. These experiments were performed with a $4 \pi \beta-\gamma$ coincidence system and a Ge(Li) spectrometer $(2-3)$
whose absolute photopeak efficiency had been determined previously

The sources were prepared by chemically extracting the ${ }^{250} \mathrm{BK}$ from the ${ }^{254} \mathrm{Es}(275.7 \mathrm{~d})(4)$ parent activity. This was accomplished by oxidation of $\mathrm{Bk}$ to the +4 oxidation state using $10 \mathrm{M} \mathrm{HNO}_{3}-0.1 \mathrm{M} \mathrm{NaBrO}$ and extracting into an equal volume of $0.1 \mathrm{M} \mathrm{HDEHP}^{-}$in heptane. Possible traces of entrained Es were eliminated by washing the HEDHP once with an equal volume of fresh $10 \mathrm{M} \mathrm{HNO}-0.1 \mathrm{M} \mathrm{NaBrO}$. Bk was then back extracted into 6M HNO containing several drops of $30 \% \mathrm{H}_{2} \mathrm{O}_{2}$. The BK source was made relatively massless by using successively anion-exchange and cationexchange techniques.

The ${ }^{250} \mathrm{BK}$ activity was deposited as a small droplet on thin conducting films of VYNS and taken to dryness with a heat lamp. The VYNS films were typically $40 \mu \mathrm{g} / \mathrm{cm}^{2}$ thick with gold layers of $25 \mu \mathrm{g} / \mathrm{cm}^{2}$ thickness deposited on each side by vacuum evaporation. Alpha counting of the residual activity (i.e. ${ }^{250} \mathrm{Cf}$ and $254 \mathrm{Es}$ ) after the $250 \mathrm{Bk}$ decayed indicated that the chemical separation of the ${ }^{254} \mathrm{Es}$ from the ${ }^{250} \mathrm{Bk}$ was extremely good. At the time of separation the activities of ${ }^{250} \mathrm{Bk} /{ }^{254} \mathrm{Es}$ were $>300,000 / 1$. Three sets of experiments were run to determine the absolute $\gamma$-ray,pranch through the 989 and $1028+1031-\mathrm{keV} r$ rays. For each experiment the $25 \mathrm{BK}_{\mathrm{Bk}}$ source was counted with the $G e(L i)$ spectrometer and the $4 \pi \beta-\gamma$ coincidence system.

In the first experiment the ${ }^{250} \mathrm{Bk}$ was counted with the $4 \pi \beta-\gamma$ coincidence system to determine the source disintegration rate. The beta- and $\gamma$-ray counts were corrected for dead time and background. The coincidence counts were corrected for dead time, background and accidental coincidences. The beta chamber efficiency ( $i . e$. , ratio of $\gamma$ ray and coincidence counts) was $95.5 \%$. The $\gamma$-ray branching ratios were calculated from the $G e(L i) \gamma$-ray peak intensities and these disintegration rates.

The second experiment employed two techniques for measuring the sample disintegration rate. The first technique was the same as that used in the first experiment. The chamber efficiency was $96.5 \%$. The second technique involved taking data with beta chamber bias from 2800 to 3300 volts. This range produced beta chamber efficiencies of from 57 to $97 \%$. The source disintegration rate was then determined by extrapolating to a chamber efficiency of $100 \%$.

The third experiment consisted of counting a source with the $4 \pi \beta-\gamma$ system and varying the beta chamber efficiency (from 25 to $94.4 \%$ ) by inserting thin aluminum absorbers over the source. The disintegration rate was determined by extrapolating to a chamber efficiency of $100 \%$. The uncovered source resulted in a chamber efficiency of $94.4 \%$

* Allied Chemical Co. 
For each of these three experiments, the $\gamma$-ray emission rate of the sample was determined for the 989- and 1028+1031-keV transitions. This was done by counting the samples at $10 \mathrm{~cm}$ from a $65-\mathrm{cm}^{3} \mathrm{closed-ended}$ coaxial $\mathrm{Ge}(\mathrm{L} i)$ spectrometer. The sample was counted in two orientations to minimize the uncertainty in the source-detector distance. Since the statistical uncertainty in each of these peaks was $\leq 2 \%$, the ratio of measured values was adjusted to correspond to the much more precise ratio previously determined (from measurements with a ${ }^{254} \mathrm{Es}-{ }^{250} \mathrm{Bk}$ source). The adjustments were $0.0,0.15$ and $0.5 \%$ in the three cases. The resulting branching ratios are given in Table 1. Typical values of the uncertainties considered in the individual values in Table 1 are: statistical error in $\mathrm{Ge}(\mathrm{L}$ i) peak areas, 1-2\%; source position, $0.25 \%$; adjustment to precise relative intensities, $0-0.25 \%$; sample disintegration rate, 0.2-0.3\%; and decay corrections, 0.08-0.50\%. After averaging the three values, an uncertainty of $1.5 \%$ was added from the Ge(Li) detector efficiency curve. The latter is the dominant single error and several of the errors from the $\gamma$-ray counting exceed the error in the disintegration rate.

The system used to measure the ${ }^{250} \mathrm{Bk}$ half-life consists of a $4 \pi \beta-\gamma$ proportional chamber with associated preamplifiers and fast discriminators. Following the fast discriminator, there are two parallel electronic pulseprocessing systems. One system is a conventional $4 \pi \beta-\gamma$ system with fixed (and long) pulse width, to produce a constant dead time $\tau$. The other system is being developed for sources which have very high count rates (e.g. $\beta$ - rates of $100,000 \mathrm{c} / \mathrm{s})$. With this system the pulse widths vary and the associated dead times are measured directly by gating the pulses of an internal $8 \mathrm{MHZ}$ clock.

The ${ }^{250} \mathrm{Bk}$ activity was followed for over 20 half-lives by counting the source for a preset count time $(600 \mathrm{~s})$ alternately with the fixed pulse width and the variable pulse width systems. The time between the end of one count and the start of the next count was $300 \mathrm{~s}$. The counts were corrected for dead time and background. The residual activity in the source, i.e. the background, was determined four days after the last half-life count was made. The data were analyzed by performing a nonlinear least-squares fit to the data with a one-component exponential. Because of the presence of a residual activity, above the background subtracted, the data were also fitted with the sum of two exponential terms. The results of these fits are given in Table 2. Fits were made to the data from the beta chamber for both electronic systems and to the computed disintegration rates, $N_{0}$ from the fixed dead time system.

The $1 / \sigma^{2}$-weighted average of the $N_{0}$ run (1 decay component only), fixed-pulse-width $N_{\beta}$ data $\left(2\right.$ components) and variable pul se width $N_{B}$ data ( 2 components) gives a half-life of $(192.40+0.08) \mathrm{m}$. We assign a systematic error of $\pm 0.2 \mathrm{~m}$ which is intended to include possible errors in background subtraction and fitting of the second component. This gives a final half-life of $(192.40+0.22) \mathrm{m}$ for ${ }^{250} \mathrm{Bk}$. The large discrepancy in the $N_{0}$ half-life and the $\bar{N}_{B}$ (exponentail components) half-life is believed due to the presence of a second component in the $N_{0}$ data. The large errors in these values (due to the very low $\gamma$ count rates in the $4 \pi \beta-\gamma$ system) precluded a meaningful analysis with two components. 
(1) C. W. Reich et. al. this annual report.

(2) R. C. Greenwood, ANCR-1088, 405 (1972).

(3) R. G. Helmer and R. C. Greenwood, ANCR-1088, 396 (1972).

(4) I. Ahmad and J. P. Unik, J. Inorg Nucl. Chem. 37, 1851 (1975). 


\section{TABLE 1}

\section{Experimental results of $\gamma$-ray branching ratio measurements}

\begin{tabular}{|c|c|c|c|c|c|}
\hline${ }^{250} \mathrm{Bk} \gamma$-ray branch & 1st experiment & 2nd experiment & 3rd experiment & $\begin{array}{l}\text { weighted } \\
\text { average }\end{array}$ & $\begin{array}{c}\text { reported* } \\
\text { value }\end{array}$ \\
\hline $989-\mathrm{keV}$ & $0.450_{4}\left(6_{7}\right)$ & $0.446_{4}\left(6_{9}\right)$ & $0.456(10)$ & $0.450(3)$ & $0.450(7)$ \\
\hline $1028+1031-\mathrm{keV}$ & $0.405_{4}\left(5_{5}\right)$ & $0.401\left(5_{4}\right)$ & $0.410_{4}(57)$ & $0.406(3)$ & $0.406(7)$ \\
\hline
\end{tabular}

G

* Heighted average with $1.5 \%$ uncertainty in photopeak efficiency added in quadrature to uncertainty due to $\gamma$-ray peak, source position, normalization to relative $\gamma$-ray intensities and uncertainty in the measured disintegration rate. 


\section{TABLE 2}

$250 \mathrm{Bk}$ half-life measurement with $4 \pi B-\gamma$ system

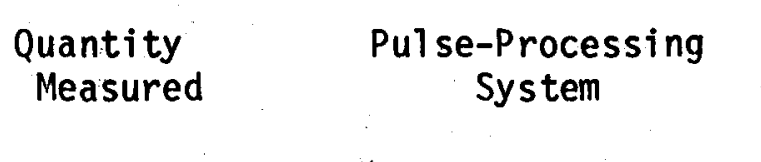

\begin{tabular}{lll}
\hline & & \\
$N_{0}$ & & fixed pulse width \\
$N_{\beta}$ & & fixed pulse width \\
$N_{B}$ & & fixed pulse width \\
$N_{\beta}$ & & fixed pulse width \\
$N_{\beta}$ & & fixed pul se width \\
$N_{\beta}$ & & fixed pulse width \\
$N_{\beta}$ & variable pulse width \\
$N_{\beta}$ & variable pulse width \\
\hline
\end{tabular}

\begin{abstract}
Time Range
Included in

Fit (hours)
\end{abstract}

\begin{tabular}{l}
\hline $0-28$ \\
$0-31$ \\
$2.5-31$ \\
$0-21$ \\
$2.5-23.5$ \\
$0-64.5$ \\
$0.25-32$ \\
$0.25-64.7$
\end{tabular}

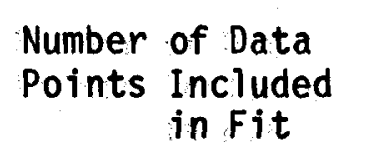

56
62
57
42
42
125
62
123

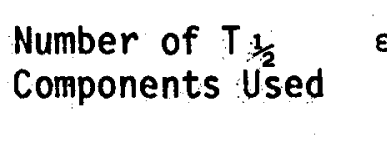

$+2$

$\begin{array}{lll}1 & 0.48 & 193.15+0.18^{*} \\ 1 & 3.66 & 192.71 \pm 0.13 \\ 1 & 3.49 & 192.85 \pm 0.16 \\ 1 & 1.09 & 192.54 \pm 0.04 \\ 1 & 1.11 & 192.67 \pm 0.06 \\ 2 & 0.90 & 192.38+0.04^{\star} \\ 1 & 2.94 & 192.70 \pm 0.10 \\ 2 & 0.83 & 192.39 \pm 0.04^{\star}\end{array}$

*The $1 / \sigma^{2}$ weighted average of the half-lives marked with an asterisk equals $192.40 \mathrm{~m}$ with $\sigma_{\text {internal }}=0.03 \mathrm{~m}$ and $\sigma_{\text {external }}=0.08 \mathrm{~m} . \sigma_{\text {external }}=\varepsilon\left(\sigma_{\text {internal }}\right)$. Uncertainty in the reported half-life, $0.22 \mathrm{~m}$, results from adding in quadrature the $0.08 \mathrm{~m}$ uncertainty and the $0.2 \mathrm{~m}$ systematic uncertainty due to possible errors in the background subtraction and fitting of a 2 nd component. 


\section{DETERMINING THE NEGATIVE ENERGY RESONANCE PARAMETERS FOR}

\section{THE FOUR EXIT CHANNELS OF ${ }^{35} \mathrm{C} \ell$}

\section{Eric H. Ottewitte}

The isotope ${ }^{35} \mathrm{Cl}$ can undergo four interactions at thermal neutrun energies: $(n, n),(n, \gamma),(n, p)$, and $(n ; \alpha)$. Although the latter two are inhibited by the Coulomb barrier, they are still highly significant to a Molten Chloride-Salt Fast Reactor where transmutation leads to corrosive and radioactive impurities. The $p$ and $\alpha$ channels may also be expected to exhibit only one degree of freedom, just like the neutron channel. For this reason a special Breit-Wigner code was written. Fortuitous for analysis, however, the Coulomb barrier in the $p$ and $\alpha$ channels effectively makes $\Gamma_{p}$ and $\Gamma_{\alpha}$ constant with energy. The energy range below $100 \mathrm{eV}$ is entirely dominated by the ${ }^{35} \mathrm{Cl}_{l}$ negative resonance and can be described by an appropriate set of parameters. Contributions from positive resonances are nil. Doppler broadening was determined to be unimportant.

Experimental data include: (1) thermal cross sections for the $\gamma$, $P$, and $\alpha$ exit channels; (2) $\sigma_{\text {tot, nat }}(E) ;(3)$ relative $\sigma_{\gamma \text {, nat }}(E)$; (4) relative $\sigma_{p, 35}(E)$; and (5) a coh, nat and $\sigma_{\text {pot, nat. As }}{ }^{37} \mathrm{Cl}$ is neutron-magic, its contribution is often fortuitously small, thereby enhancing the worth of the natural chlorine cross-section data. Consideration of (1) and (2) determined that $\sigma_{\text {tot }}-\sigma_{\gamma}=16.4 \pm 0.1 \mathrm{~b}$. Evaluation of $a_{\text {coh }}$ and $\sigma_{\text {pot }}$ allows one to derive that $E_{0}=175 .(1 \pm .017) \Gamma_{n}^{\circ}$. With this and $\sigma_{\lambda, 35}$ (thermal) one gets $\Gamma_{\lambda}=0.524(1 \pm 0.038) \Gamma_{n}^{0}$. Now given a $\Gamma_{n}^{0}$ value one has $\Gamma_{\lambda}$ and Eo, complete with their standard deviations. Coupled with evaluated $a_{\text {pot }}=3.22(1 \pm .060) \mathrm{F}$ and $\sigma_{\text {pot, nat }}=1.3 \pm 0.15 \mathrm{~b}$, each of several 
$\Gamma_{n}^{\circ}$ - characterized sets $\left\{r_{n}^{0}, \Gamma_{\lambda}\right.$, Eo $\}$ can be least-squares analyzed to determine which best fits the data (2) and (3) above. Once the best $r_{n}^{0}$ has been found, other parameters can be allowed to vary in order of their sensitivities $\left(r_{n}^{0}\right.$ is the most sensitive). $r_{p}$ and $r_{\alpha}$ can subsequently be deduced by means of their thermal cross section. Alternative to least-squares analysis is the procedure of trialand-eyeball-estimate of error. Results of this effort are $r_{n}^{\circ}=0.76, E_{0}=-134.7 \mathrm{eV}, a_{p o t} 35=3.14, \sigma_{\text {pot }}=1.24 \mathrm{~b}$, and $r_{\gamma}=0.385$ eV. This set gives a much better fit than those previously evaluated and reported in the literature. 


\section{RADIATION MEASUREMENTS}





\title{
FILTERED REACTOR BEAMS FOR FAST NEUTRON CAPTURE $\gamma$-RAY EXPERIMENTS
}

\author{
R. C. Greenwood and R. E. Chrien*
}

The use of thermal neutron beams to induce capture reactions has been extremely effective in nuclear structure studies. The choice of thermal neutron energy is mandated by the ease with which suitably high fluxes of thermal neutrons can be obtained from fission reactors of even a modest power level.

However, the scope of the information about the structure of the nucleus which can be obtained from the capture reaction using thermal neutrons is severely limited by the nature of the capture reaction mechanism itself. More specifically, when one or more compound states of given spin and parity dominate slow neutron capture, the intensities of primary transitions to final states with suitable spin-parity valyes are characterized by a $x^{2}$ distribution with one degree of freedom 11 . Large statistical fluctuations are thus observed in the primary $\gamma$-ray transition intensities following thermal-neutron capture. Because of these fluctuations it is difficult to use primary transitions to deduce spin and parity information for the final states in the reaction; indeed some final state might be missed completely.

One way to overcome this limitation is to use neutrons having a sufficient range of energies to encompass several compound resonance states. For such epithermal neutron capture studies, however, the raw energy spectrum of neutrons obtained from a fission reactor is too broad to be of use. It is desirable to tailor the spectrum of reactor neutrons to achieve a spread of beam energies broad enough to average over several compound states, but narrow compared to the energy resolution of the detector used to measure the primary transition.

A satisfactory method of obtaining such tailored beams of neutrons external to a fission reactor is to use the fact that in many low-Z nuclei with widely spaced compound resonance states, potential-resonance interference can produce dips in the total neutron cross section at neutron energies just below large scattering resonances. A suitably thick filter constructed from one of these materials located in a beam channel of a reactor will preferentially transmit those neutrons with energies corresponding to the interference minima. While such materials generally have more than one such interference minimum in their neutron interaction cross sections, it is usually possible, by use of suitable combinations of two or more different materials in the filter, to suppress the transmission of neutrons with energies corresponding to all but one of these minima. In this manner an intense beam of essentially monoenergetic neutrons, with energies corresponding to that of the selected transmission minimum is obtained.

* Brookhaven National Laboratory 
A flexible filtered neutron beam facility has been installed at the High Flux Beam Reactor at Brookhaven. In this facility, filters, of which the primary components are iron and scandium, are used to provide external beams of 2- and 24-keV neutrons, respectively, with 1 ow $\gamma$-ray and thermal-neutron contaminations. The installation of these filters in a rotary collimator arrangement external to the biological shielding of the reactcrallows for rapid interchange between filters and al so for changes in the individual filter configurations with the reactor operating at full power. The collimator also includes a bismuth single crystal filter for producing a thermal neutron beam. Measurements wi th hydrogenfilled proton recoil detectors have been used to optimize the filter configurations and to deduce the neutron energy distributions in the filtered beams.

These measurements have established the critical importance of the Al in the Fe/Al filter in obtaining the highest beam purity with minimum attenuation of the 24-keV neutron group. The average neutron flux over a beam area of $\sim 7.27 \mathrm{~cm}^{2}$ at the collimator exit is $1.28 \times 10^{6}$ neutron $/ \mathrm{cm}^{2}$. $\mathrm{sec}$ for a filter consisting of $22.86 \mathrm{~cm} \mathrm{Fe}, 36.20 \mathrm{~cm} \mathrm{Al}$ and $6.35 \mathrm{~cm} \mathrm{~S}$. With this filter arrangement, $98.1 \%$ of the transmitted neutrons are in the 24-keV group, and the energy distribution in the group is calculated to have a FWHM $22.0 \mathrm{keV}$.

For the 2-keV filter we studied the effect on beam intensity and beam purity of filter components of $\mathrm{Mg}, \mathrm{Al}, \mathrm{S}, \mathrm{Ti}, \mathrm{V}, \mathrm{Cr}, \mathrm{Fe}, \mathrm{Co}$, and $\mathrm{Ni}$ added to the basic SC filter. The thickness of each of these filter components was chosen such that the transmission of the 2-keV neutron group was approximately 0.5 . For each of these measurements the added filter component was interposed in the beam just at the exit of rotary collimator shield, with the proportional counters being located $-3.4 \mathrm{~m}$ further down the beam. In order that transmission factors might be obtained directly from these data, the live counting time of each run was obtained from the peak area of the precision linear pulser which was recorded simultaneously with each of the proton recoil spectra. A thin $235 \mathrm{U}$ fission chamber located midway between the added filter components and the proton recoil detector was used to monitor the fil tered beam intensity. The transmission factors obtained from these data for each of the major higher energy neutron groups with each of the additional filter components are summarized in Table 1. (In assessing the improvement in beam purity obtained through the added filter components, the transmission factors of Table 1 must be weighted by the relative intensities of each of the higher energy neutron groups transmitted by the Sc filter alone.)

What is immediately obvious from Table 1 is that there is no one added filter component which can dramatically reduce the flux of higher energy neutrons with little reduction in the flux of $2-\mathrm{keV}$ neutrons. For example while it had previously been demonstrated that Ti would severely attenuate the 7.6-, 16.1-, 29.5- and 40.2-keV neturon groups we can see from Table 1 that neutrons with energies $>100 \mathrm{keV}$ are transmitted with little attenuation relative to $\mathrm{Sc}$ alone. On the other hand, while Al severely attenuates neutron with energies of $40.2,48.0$ and $>100 \mathrm{keV}$ there is an increase in the relative transmission of the 
lowest energy neutron groups at $7.6,16.1$ and $29.5 \mathrm{keV}$ since these energies lie in an interference minimum of Al at $-25 \mathrm{keV}$. Similar comments can be made about each of the other materials tested with one exception,.Co. Cobalt was the only material found for which the transmission of all of the higher energy neutron groups was less than of the $~ 2-k e V$ neutron group. Inspection of the Co total neutron interaction cross section curve indicates that this conclusion is reasonable since the 2-keV neutron group energy is close to a broad interference minimum at $2.8-\mathrm{keV}$ neutron energy.

In order that the data presented in Table 1 might be more conveniently used in computations involving different Sc filter configurations, we have reduced them to sets of effective neutron interaction cross sections for each of the major neutron groups tramsitted by the Sc filter alone. These cross sections are given in Table 2. Immediately below our $2-\mathrm{keV}$ neutron cross section value we give the value we would expect from interpolation off the cross section curves of ref. 2. The agreement between each of these two 2-keV neutron interaction cross sections is quite acceptable.

The results of this study thus establish that a filter composed of $\mathrm{Sc}, \mathrm{Ti}$ and $\mathrm{Co}$ has good beam purity with minimum attenuation of the 2-keV neutron group. The average 2-keV neutron flux over a beam area of $7.27 \mathrm{~cm}^{2}$ is $6.54 \times 10^{6}$ neutrons $/ \mathrm{cm}^{2}$. sec for a filter consisting of $188.5 \mathrm{gm} / \mathrm{cm}^{2} \mathrm{Sc}$. The FWHM of the 2-keV neutron group is observed to be $\sim 0.9 \mathrm{keV}$. With this filter arrangement $70 \%$ of the transmitted neutrons are in the 2-keV groups, while for a filter consisting of $188.5 \mathrm{gm} / \mathrm{cm}^{2} \mathrm{Sc}, 8.497 \mathrm{gm} / \mathrm{cm}^{2} \mathrm{Ti}$ and $26.92 \mathrm{gm} / \mathrm{cm}^{2} \mathrm{Co}_{0}$ we estimate that $91 \%$ of the transmitted neutrons are in the 2-keV group. The average 2-keV neutron flux with this latter filter arrangement is estimated as $\sim 2.0 \times 10^{6}$ neutrons $/ \mathrm{cm}^{2} \mathrm{sec}$.

(1) C. E. Porter and R. G. Thomas, Phys. Rev. 104, 483 (1966).

(2) 0. D. Simpson and L. G. Miller, Nucl. Instru. Methods 61, 245 (1968); and $\mathrm{IN}-1218$ (1968).

(3) JW Rogers and 0. D. Simpson, IN-1317 (1970) p.283.

(4) M. K. Drake, BNL 17188. 


\section{TABLE I}

Transmission factors measured for each of the major neutron groups transmitted by the $188.5 \mathrm{gm} / \mathrm{cm}^{2} \mathrm{Sc}$ fil ter with additional filter components.

\begin{tabular}{|c|c|c|c|c|c|c|c|c|c|c|}
\hline \multirow[b]{2}{*}{$\begin{array}{l}E_{n} \\
(k e V)\end{array}$} & \multirow{2}{*}{$\begin{array}{l}\text { Relative } \\
\phi\left(E_{S p}\right) \text { for }\end{array}$} & \multicolumn{6}{|c|}{ Transmission of added filter components ( $T$ ) } & \multirow[b]{2}{*}{$\begin{array}{r}\mathrm{Sc}+\mathrm{Fe} \\
10.1 \mathrm{gm} / \mathrm{cm}^{2}\end{array}$} & \multirow[b]{2}{*}{$\begin{array}{r}S c+C o^{a)} \\
20.3 \mathrm{gm} / \mathrm{cm}^{2}\end{array}$} & \multirow[b]{2}{*}{$\begin{array}{c}\mathrm{Sc}+\mathrm{Ni} \\
2.79 \mathrm{gm} / \mathrm{cm}^{2}\end{array}$} \\
\hline & & $\begin{array}{c}S c+M g \\
8.3 \mathrm{gm} / \mathrm{cm}^{2}\end{array}$ & $\begin{array}{r}\mathrm{Sc}+\mathrm{Al} \\
17.2 \mathrm{gm} / \mathrm{cm}^{2}\end{array}$ & $\begin{array}{c}\mathrm{Sc}+\mathrm{S} \\
31.6 \mathrm{gm} / \mathrm{cm}^{2}\end{array}$ & $\begin{array}{c}\mathrm{Sc}+\mathrm{Ti}^{2} \\
10.3 \mathrm{gm} / \mathrm{cm}^{2}\end{array}$ & $\begin{array}{l}\mathrm{Sc}+V \\
5.6 \mathrm{gm} / \mathrm{cm}^{2}\end{array}$ & $\begin{array}{r}S c+\left(r^{a)}\right. \\
9.4 \mathrm{gm} / \mathrm{cm}^{2}\end{array}$ & & & \\
\hline 2 & 1000 & 0.509 & 0.608 & 0.596 & 0.544 & 0.633 & 0.482 & 0.486 & 0.591 & 0.648 \\
\hline 7.6 & 36.4 & 0.507 & 0.636 & 0.589 & 0.134 & $0.031_{2}$ & 0.075 & 0.208 & 0.074 & 0.689 \\
\hline 16.1 & 19.6 & d) & 0.731 & 0.577 & 0 & 0.114 & 0.584 & 0.685 & 0.528 & 0.143 \\
\hline 29.5 & 96.2 & 0.544 & 0.739 & 0.631 & 0.116 & 0.526 & 0.609 & 0.311 & 0.274 & 0.619 \\
\hline 40.2 & 75.6 & 0.501 & 0.071 & 0.757 & 0.221 & 0.664 & 0.548 & 0.553 & 0.295 & 0.850 \\
\hline 48.0 & & & & & & & & & & \\
\hline 65.5 & 34.3 & 0.415 & 0.556 & 0.878 & 0.285 & 0.361 & 0.594 & 0.700 & 0.348 & 0.482 \\
\hline 76.4 & 28.4 & 0.124 & 0.362 & 0.847 & 0.498 & 0.671 & 0.634 & 0.529 & 0.414 & 0.718 \\
\hline 131 & 36.6 & 0.408 & 0.311 & $0.060_{6}$ & 0.647 & 0.587 & 0.454 & 0.692 & 0.230 & 0.824 \\
\hline 181 & 20.2 & 0.385 & 0.219 & 0.203 & 0.646 & 0.544 & 0.533 & 0.648 & 0.344 & 0.877 \\
\hline 214 & 16.8 & 0.281 & 0.174 & 0.188 & 0.652 & 0.706 & 0.560 & 0.612 & 0.231 & 0.835 \\
\hline 248 & 10.4 & 0.189 & 0.306 & 0.295 & 0.681 & 0.767 & 0.590 & 0.726 & 0.314 & 0.852 \\
\hline 291 & 8.1 . & 0.188 & 0.201 & 0.280 & 0.698 & 0.682 & 0.713 & 0.836 & 0.375 & 0.846 \\
\hline 328 & 14.2 & 0.303 & 0.321 & 0.400 & 0.636 & 0.797 & 0.545 & 0.739 & 0.402 & 0.859 \\
\hline$<350$ & 29.1 & 0.364 & 0.234 & 0.364 & 0.696 & 0.794 & 0.590 & 0.661 & 0.338 & 0.887 \\
\hline$F c^{b)}$ & 1.000 & 0.497 & 0.578 & 0.563 & 0.503 & 0.588 & 0.474 & 0.438 & 0.522 & 0.658 \\
\hline
\end{tabular}

a) There is also $0.429 \mathrm{gm} / \mathrm{cm}^{2} \mathrm{Al}$ in the beam with these samples since they were powdered metal samples contained in Al cans.

b) Relative fission chamber counting rate.

c) Not adequately resolved.

d) Not available. 
TABLE 2

Effective values of total neutron interaction cross sections for the major neutron groups transmitted by the Sc filter, obtained from the measured transmission values for each of the additional filter components.

\begin{tabular}{|c|c|c|c|c|c|c|c|c|c|}
\hline \multirow{2}{*}{$\begin{array}{c}E_{n} \\
(\mathrm{keV})\end{array}$} & \multicolumn{9}{|c|}{ Effective value of $\sigma_{T}\left(E_{n}\right)^{a)}$} \\
\hline & $\mathrm{Mg}$ & Al & $S$ & $\mathbf{T i}$ & $v$ & $\mathrm{Cr}$ & $\mathrm{Fe}$ & $\mathrm{Co}_{0}$ & $\mathrm{Ni}$ \\
\hline \multirow[t]{2}{*}{2} & 3.28 & 1.30 & 0.87 & 4.7 & 6.9 & 6.7 & 6.6 & 2.52 & 15.2 \\
\hline & $(3.5)$ & $(1.5)$ & $(1.2)$ & $(5.3)$ & $(9.0)$ & $(7.0)$ & $(6.6)$ & $(2.0)$ & $(16.5)$ \\
\hline 7.6 & 3.30 & 1.18 & 0.89 & 15.5 & 52.4 & 23.8 & 14.4 & 12.5 & 13.0 \\
\hline 16.1 & & 0.82 & 0.93 & 40.7 & 32.8 & 4.9 & 3.48 & 3.07 & 68.0 \\
\hline 29.5 & 2.96 & 0.79 & 0.78 & 16.6 & 9.7 & 4.5 & 10.7 & 6.2 & 16.8 \\
\hline \multirow[t]{2}{*}{40.2} & & & & & & & & & \\
\hline & 3.36 & 6.9 & 0.47 & 11.7 & 6.2 & 5.5 & 5.4 & 5.9 & 5.7 \\
\hline \multicolumn{10}{|l|}{48.0} \\
\hline 65.5 & 4.28 & 1.53 & 0.219 & 9.7 & 15.4 & 4.8 & 3.28 & 5.1 & 25.5 \\
\hline 76.4 & 10.1 & 2.65 & 0.280 & 5.4 & 6.0 & 4.2 & 5.8 & 4.2 & 11.6 \\
\hline 131 & 4.36 & 3.05 & 4.72 & 3.36 & 8.0 & 7.2 & 3.38 & 7.1 & 6.8 \\
\hline 181 & 4.86 & 3.96 & 2.69 & 3.38 & 9.2 & 5.7 & 3.99 & 5.1 & 4.6 \\
\hline 214 & 6.2 & 4.56 & 2.82 & 3.30 & 5.3 & 5.3 & 4.51 & 7.0 & 6.3 \\
\hline 248 & 8.1 & 3.09 & 2.06 & 2.97 & 4.0 & 4.8 & 2.94 & 5.6 & 5.6 \\
\hline 291 & 8.1 & 4.18 & 2.15 & 2.78 & 5.8 & 3.1 & 1.65 & 4.7 & 5.8 \\
\hline 328 & 5.8 & 2.96 & 1.54 & 3.50 & 3.4 & 5.5 & 2.78 & 4.4 & 5.3 \\
\hline$>350$ & 4.91 & 3.79 & 1.70 & 2.80 & 3.5 & 4.8 & 3.80 & 5.2 & 4.2 \\
\hline
\end{tabular}

a The values given in parenthesis below the cross section value for the 1.9-keV neutron group are those interpolated for a 1.9-keV neutron energy from the curves given in ref. 4. 
DEVELOPMENT OF AN ON-LINE ISOTOPE SEPARATOR SYSTEM FOR FISSION-PRODUCT DECAY STUDIES USING A 252 CF SPONTANEOUS FISSION SOURCE

R. C. Greenwood, R. A. Anderl, R. G. Gehrke and S. T. Croney

The work reported herein is part of a continuing effort to develop a system for on-line isolation of short-lived fission products in order that their decay properties might be measured. Earlier reports on this effort are given in refs. (1) and (2). As noted in these earlier reports, the source of fission fragments for this work has been a 7- $\mu \mathrm{g}$ (now $\sim 4 \mu \mathrm{g}$ ) sample of ${ }^{252} \mathrm{Cf}$ electroplated onto a $\mathrm{Pr}$ foil. In ref. (2) we reported on our earliest efforts to transport the fission fragments emitted by this source over a distance of several meters, with good transport efficiency, using the gas-jet transport system. It is planned to use such a gas-jet transport system to couple the ${ }^{252} \mathrm{Cf}$ spontaneous fission source to the ion source of a mass separator so that on-line mass separation may be performed on a wide range of fission product elements.

During the past year, our effort has concentrated on obtaining, and on the understanding of mechanism for, high (>50\%) efficiency transport of fission fragments from the ${ }^{252} \mathrm{Cf}$ source chamber to a collection chamber through a long capillary tube. It has long been recognized that a negligible transport efficiency was obtained with pure He as the flow gas, but that if impurities (such as $\mathrm{H}_{2} \mathrm{O}$, various organics, oils, etc.) were introduced into the He, heavy mass $\left(-10^{8} \mathrm{amu}\right)$ clusters were formed to which radioactive species attached themselves and good transport efficiencies were obtained (at least with charged particle induced reactions). However, there has been much uncertainty about mechanism for generation of these clusters, and indeed the best cluster materials, for reliable and high efficiency transport, especially of fission products. Our major advance in achieving highly efficient and reliable transport of fission isotopes through the capillary has come with the realization that these clusters were simply aerosols, and that the size of the aerosol particles were of critical importance to efficient transport. If the aerosols are too big ( $>$ few microns diameter) they simply settle out in the sample chamber by sedimentary processes, while if they are too small (< few 1/100 microns diameter) their gas kinetic velocities are such as to cause them to strike (and hence stick to) the capillary wall by the Brownian motion. The important point then, for good transport efficiency is to simply produce the desired cluster types having the correct sizes using standard aerosol generator techniques. In considering transport with various cluster types we will make the distinction between solid aerosols and liquid aerosols; since we believe that this distinction is crucial to the eventual successful coupling of the gas-transport system to the ion source of the mass separator.

Before considering the problem of cluster generation we consider it worthwhile to review some of the basic gas dynamical flow equations as applied to our gas-jet transport system illustrated in Fig. 1. 
Gas Flow Through a Long Capillary Tube

In order that the aerosol particles be transported through the long capillary tube without appreciable loss (due to sticking in the walls, i.e., plate-out) it is essential that laminar flow conditions prevail throughout the flow. This will occur if the Reynolds number, $\mathrm{Re}$, is everywhere <2100;

$$
\operatorname{Re}=\frac{v \rho d}{\mu}=\frac{4}{\pi} \frac{\dot{m}}{\mu d}
$$

where $v, \rho, \dot{m}$ and $\mu$ are the velocity, density, mass flow rate and viscosity of the gas and $d$ is the diameter of the pipe. If the pressure difference between the source chamber $\left(P_{S}\right)$ and the collection chamber $\left(P_{C}\right)$ is sufficient, the gas will exit the capillary at sonic velocity and the flow conditions can be reasonably characterized as adiabatic, and compressible. Defining positions 1 and 2 as the inlet and exit of the capillary, we can characterize an adiabatic laminar flow of a compressible gas (with $M_{2}=1$ ) by the following equation:

$$
f \frac{1}{d}=\frac{1-M_{1}^{2}}{\gamma M_{1}^{2}}+\frac{\gamma+1}{2 \gamma} \ln \left\{\frac{(\gamma+1) M_{1}^{2}}{2\left[1+\frac{(\gamma-1)}{2} M_{1}^{2}\right]}\right\}
$$

where: $M$ is the mach number

1 is the length of the capillary

$\gamma$ is equal to $C_{p} / C_{V}(=1.66$ for $\mathrm{He})$

$f$ is the frictional resistance to flow in the pipe $(f=64 / R e)$.

This equation, which relates $f, l$ and $d$ to the inlet velocity of the gas can be illustrated graphically as shown in fig. 2. Other relationships relating the inlet and outlet pressures and temperatures are:

$$
\begin{aligned}
& \frac{P_{1}}{P_{2}}=\left.\frac{1}{M_{1}} \cdot \frac{(\gamma+1)}{2\left[1+\frac{(\gamma-1)}{2} M_{1}^{2}\right]}\right|^{\frac{1}{2}} \\
& \frac{T_{1}}{T_{2}}=\frac{(\gamma+1)}{2\left[1+\frac{(\gamma-1)}{2} M_{1}^{2}\right]}
\end{aligned}
$$


For the specific case of He flow in the capillary system illustrated in Fig. I we have a volume flow rate of $47 \mathrm{~cm}^{3} / \mathrm{sec}$ of $\mathrm{He}$ (STP) when $\mathrm{P}_{\mathrm{S}}=\mathrm{P}_{1}=37 \mathrm{psia}, \mathrm{T}_{1}=295^{\circ} \mathrm{K}$ and $\mathrm{P}_{\mathrm{C}} \sim 0.2$ Torr. Hence, using an average value of $\mu=1.97 \times 10^{-5} \mathrm{~N}$. $\mathrm{sec} / \mathrm{m}^{2}$ we compute the following.

$$
\begin{aligned}
& R e=630 \\
& f=0.1015 \\
& M_{1}=0.0317 \quad\left(v_{1}=32.1 \mathrm{~m} / \mathrm{sec}\right) \\
& \frac{P}{P_{2}}=36.4 \\
& \frac{T_{1}}{T_{2}}=1.335
\end{aligned}
$$

Hence, $P_{2}=52$ Torr and thus for a collection chamber pressure of $P_{C} \leq 52$ Torr the flow through the capillary will be choked, that is $M_{2}^{C} \equiv 1$ and the mass flow rate will be independent of $P_{C}$. The velocity, pressure and temperature at any points in the capillary with these flow conditions is illustrated in Fig. 3 .

As illustrated in fig. $3, M<0.3$ for $99 \%$ of the flow length, and under these conditions, in order to estimate transit time, it is reasonable to write

$$
f \frac{1}{d} \sim \frac{1-M^{2}}{\gamma M^{2}}
$$

and hence the transit time can reasonably be expressed as

$$
t=\frac{2}{3 a_{1}}\left(\frac{\gamma f}{d}\right)^{1 / 2} 1^{3 / 2}
$$

where $a_{1}$ is the sonic velocity at the capillary inlet $\left(=a_{s}\right)$. In the present case then $t=103 \mathrm{~ms}$.

In the case of $M<0.3$, fluid flow can reasonably be classified as incompressible and hence using the incompressible flow equation for transit time,

$$
t=\frac{32 \mu 1^{2}}{\left(P_{1}-P_{2}\right) d^{2}}
$$

we obtain $t=86 \mathrm{~ms}$, which is in reasonable agreement with the above estimate. 
Gas Transport Using Solid Aerosols

The only solid aerosol which we have used, to date, has been $\mathrm{NaCl}$. The aerosol generator in this case simply consisted of NaCl crystals deposited in a long $\left(-2^{\prime}\right)$ stainless steel tube which was located in a conventional laboratory tube furnace. This arrangement is illustrated schematically in fig. 1. Helium fed into the source chamber can both flow through the aerosol generator, or bypass it. This arrangement allows us to keep the source chamber pressure independent of the flow rate through the aerosol generator. In order to characterize the system transport efficiency we have simply collected the fission fragments on an Al collector foil for 15 min., removed the collection foil from the collection chamber and counted it after a 2 min. delay with a ionization chamber: with $7 / 8$ in. lucite interposed between the collector foil and the detector. An absolute value for the collection efficiency is thus obtained by directly comparing this value to the ionization chamber reading of an $A 7$ foil which had been placed on top of the $\mathrm{Ni}$ window of the ${ }^{252} \mathrm{Cf}$ source for $15 \mathrm{~min}$ and counted under the same conditions as in the above. The absolute collection efficiencies thus obtained are probably less than the true transport efficiencies, since in the latter measurements the $\mathrm{Xe}$ and $\mathrm{Kr}$ gases are included in the ionization chamber reading, (since the fission fragments are driven deep into the $A 1$ foil) whilest these gases are probably not transported by the normal gas-jet transport mechanism.

The results of these measurements are illustrated in Figs. 4 and 5 which show the collection efficiencies as a function of aerosol generator temperature and He flow rate. We conclude from these data that for high transport efficiency the aerosol generator should be operated at a temperature of $-720^{\circ} \mathrm{C}$ with He gas flow rates through the aerosol generator $>20 \mathrm{~cm}^{3} / \mathrm{sec}$ (with the total flow rate sufficient to maintain total thermalization of the fission fragments in the source chamber). The effect of gas flow rate through the aerosol generator on transport efficiency is not well understood at this time.

In an attempt to gain some understanding of the relationship between flow conditions and transport efficiency we have initiated a series of measurements to determine size distributions of the collected aerosols using a scanning electron microscope. The results of one such experiment are illustrated in Fig. 6 which shows $\mathrm{NaCl}$ aerosols ranging in size from $-0.1 \mu \mathrm{m}$ to $-0.5 \mu \mathrm{m}$ sticking to the $\mathrm{A} 1$ collector foil. These experiments are in progress and no definitive conclusions can be drawn from them at this time.

In fig. 5 we show collection efficiency data for $\mathrm{N}_{2}$ carrier gas also. These results, which show reasonable collection efficiencies hold promise, at least for radiochemical separations, of allowing a smaller source chamber volume and thus a smaller hold-up line in the source chamber for the fission isotopes.

Liquid Aerosol Generator

The few experiments we have undertaken to date with liquid aerosols have utilized a standard Collison aerosol generator with methanol. 
A problem with this type of aerosol generator, at least for our application, is that aerosol size is inversely related to flow rate through the generator. Thus, relatively high flow rates were necessary in order to obtain sufficiently small aerosol droplets, and thereby achieve $>50 \%$ transport efficiency.

A promising method for decoupling gas flow rate from aerosol size is to use an ultrasonic nebulizer, in which the aerosol size is dependent on the frequency applied to a piezoelectric transducer. Prel iminary tests are currently being conducted with such a system.

Other Developments

During this past year it was recognized that there were in fact two distinct applications of the gas-jet transport system to the study of short-lived fission products. One of these was the eventual coupling of such a system to a mass separator, for which considerable development effort was still necessary. The other application, however, was tc transport the fission products to a suitable location where fast radiochemical separations could be undertaken. The future developments on this radiochemistry system are of a different nature than those for the on-line mass separator, involving as they do techniques for on-line and rapid radiochemistry. Hence, these two efforts have been separated and a report on the current stakes of the radiochemistry system is given in the following article [ref. 3].

(1) R. C. Greenwood, R. A. Ander1, C. W. Reich, Nuclear Technology Division Annual Progress Report for Period Ending June 30, 1974, ANCR-1177 (1974) p.410.

(2) R. A. Ander1, R. C. Greenwood, R. G. Gehrke, R. L. Bunting and C. W. Reich, Nuclear Technology Division Annual Progress Report For Period Ending June 30, 1975, ANCR-1255 (1976) p 65.

(3) R. J. Gehrke, R. C. Greenwood and J. D. Baker, present report. 


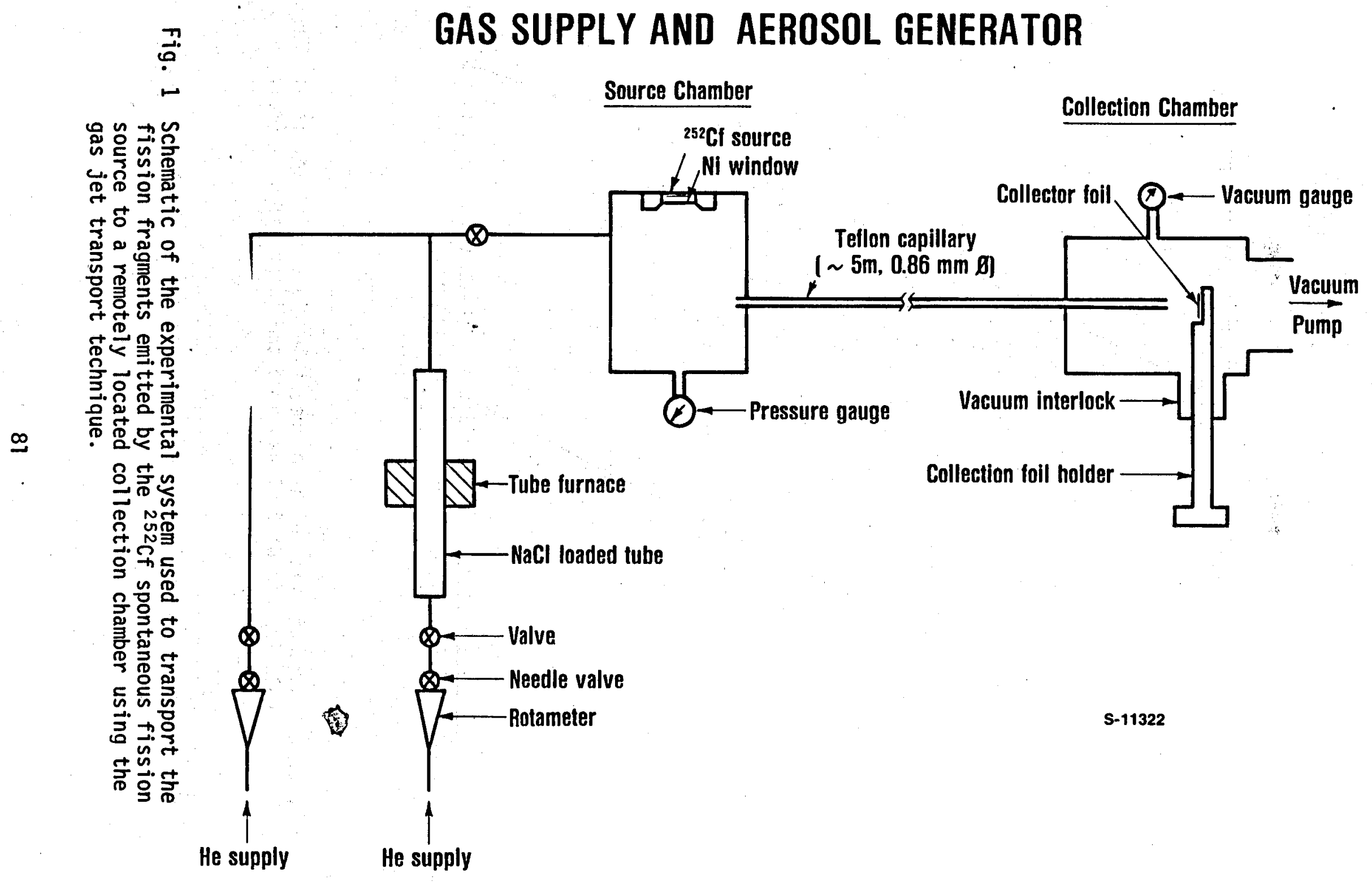




$$
\mathbb{D}
$$




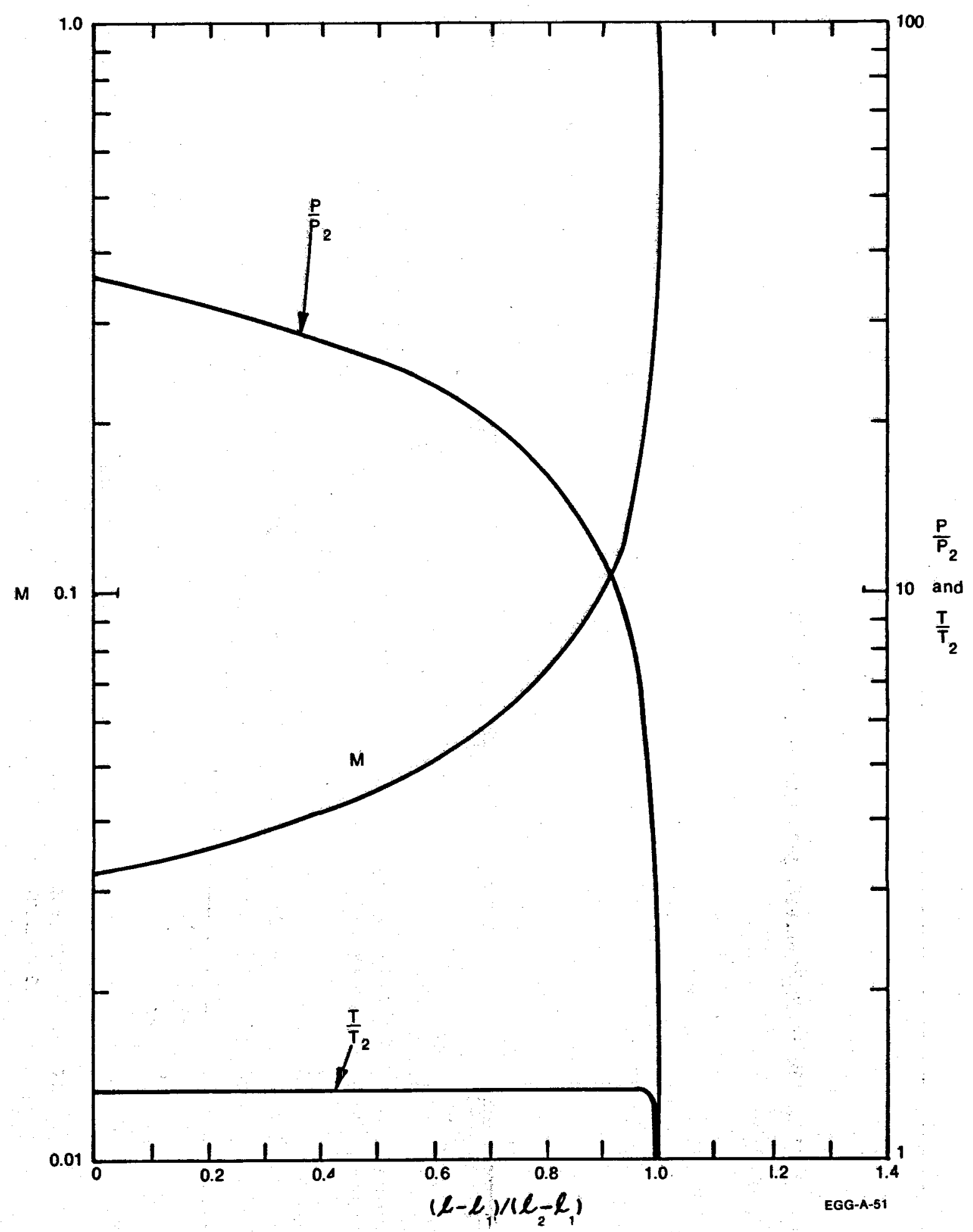

Fig. 3 The calculated velocity, pressure and temperature for He flowing in the gas transport system shown in Fig. 1 when $P_{S}=$ $P_{1}=37$ psi., $T_{1}=295^{\circ} \mathrm{K}$ and $P_{c} \leq 52$ Torr. 


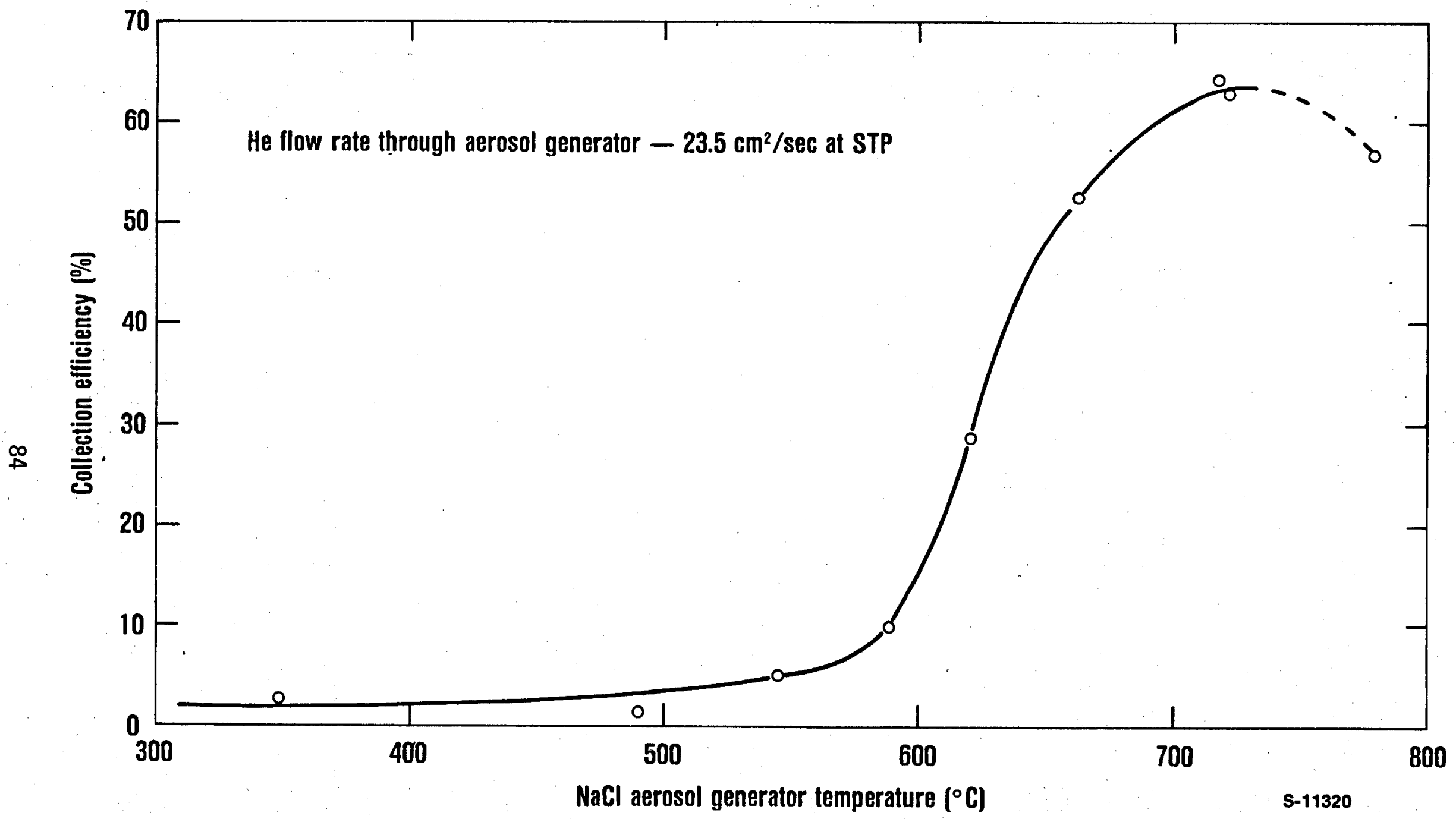

Fig. 4 Dependence of fission collection efficiency on the $\mathrm{NaCl}$ aerosol generator temperature. 


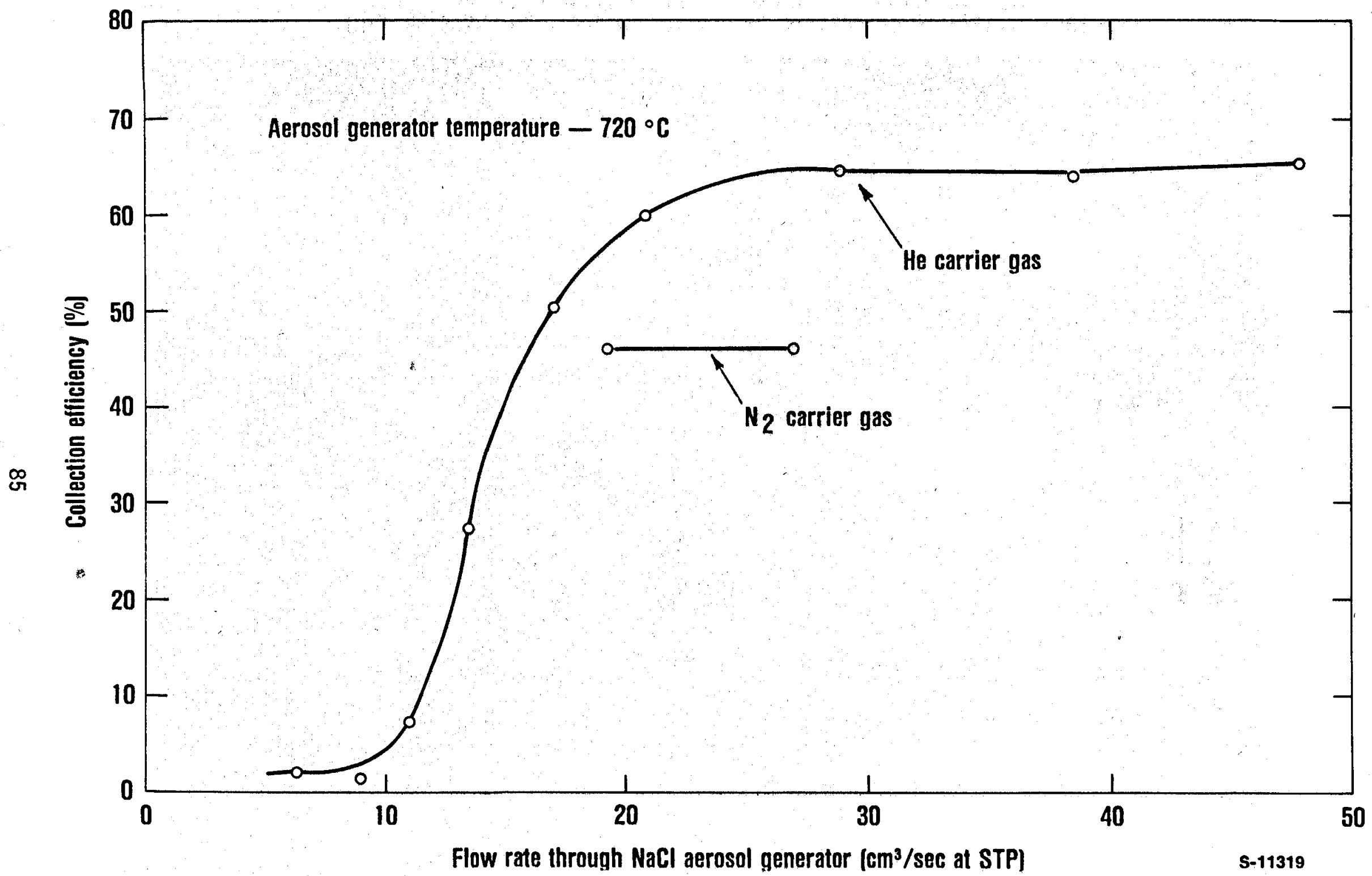

Fig. 5 Dependence of fission collection efficiency on the gas flow rate through the $\mathrm{NaCl}$ aerosol generator. 


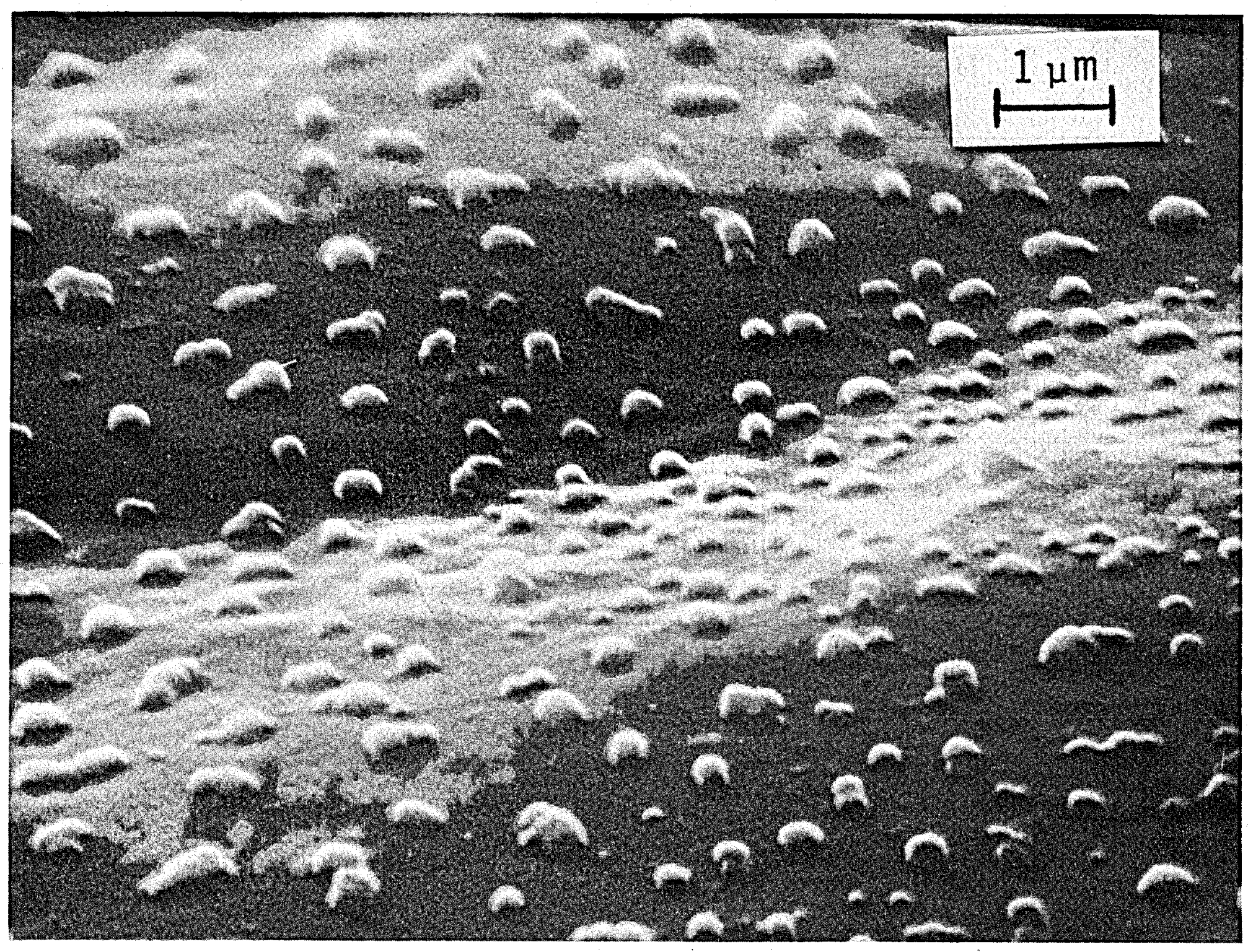

Fig. 6 Scanning electron microscope picture of gas-jetted $\mathrm{NaCl}$ aerosol particles sticking to the Al collector foil. 


\section{wes}

DEVELOPMENT OF A SYSTEM FOR FISSION PRODUCT STUDIES USING A $252 \mathrm{CF}$ SPONTANEOUS SOURCE, HELIUM-JET TRANSFER AND FAST RADIOCHEMISTRY

R. J. Gehrke, R. C. Greenwood, J. D. Baker*

As one facet of our work to develop a capability to study, "on-line," isotopic-separated samples of short-lived fission products from a ${ }^{252} \mathrm{Cf}$ spontaneous-fission source, the associated He-jet transfer system has been used in conjunction with fast chemical-separation techniques to produce chemically pure fission-product samples and in two cases radioisotopically pure sources. These measurements have been carried out to test the feasibility of this technique as a means of providing short-lived fission-product nuclides for $\gamma$-ray spectral studies, and are in parallel with the "on-line" mass separator facility, for those cases where isotope-separation is not crucial to the study. For these measurements the source chamber containing the $-4-\mu \mathrm{g}$ "open" $252 \mathrm{Cf}$ source was maintained at a pressure of $\sim 2 \mathrm{~atm}$. and the collection chamber was evacuated to a pressure of $-2 \mathrm{~mm} \mathrm{Hg}$. A NaCl vapor generator was used to create the aerosol which carried the fission fragments through the capillary to the collection chamber where they are deposited on an a luminum foil located about $1 / 2$ in from the end of the capillary. The $\mathrm{NaCl}$ vapor generator consists of a $3 / 8^{\prime \prime}$ dia $\times 2$ ' long stainless-steel tube with salt crystals lining the inside wall of the tube. The tube extends through a tube furnace which maintains the tube at $600^{\circ} \mathrm{C}$. The He flow rate through the system was $20-30 \mathrm{cc} / \mathrm{sec}$. Under these experimental conditions the fission fragment transport efficiency was $>60 \%$.

\section{Separation of Molybdenum and Cerium Fission Products}

The fission product activity was collected for 3 min on the aluminum collection foil which is affixed to a slot in the side of a stainless steel rod. This rod can be quickly removed from the collection chamber by sliding it through a vacuum interlock at the termination of fission product collection. This eliminates disturbing the steady state of the He jet system. Chemical purification of fission products followed immediately after the foil was removed. The activity was rinsed from the foil into a test tube with approximately $3 \mathrm{cc}$ of $1 \mathrm{M} \mathrm{HNO} \mathrm{H}_{3}$ in the form of a stream from a wash bottle. The test tube contained 1 cc of 0.3 M HDEHP:

Molybdenum was quickly ( $45 \mathrm{sec})$ chemically separated from most fission products by solvent extraction using di-(2-ethylhexyl) orthophosphoric acid, HDEHP. Following 10 seconds of shaking and 15 seconds of centrifugation, most of the organic phase containing molybdenum was removed and transferred to a clean counting vial and counted with $\mathrm{Ge}(\mathrm{L} i)$ spectrometers. Two counts were made of each separated molybdenum sample. The first count was for $2 \mathrm{~min}$ and was started $75 \mathrm{sec}$ after fission product collection was terminated. The second count was for $15 \mathrm{~min}$ and was started 9 min from termination of collection. Five samples were produced and cycled in this manner. The

*Allied Chemical Corporation, Idaho National Engineering Laboratory 
molybdenum samples contained only molybdenum activities and their technetium daughters. We identified $\gamma$-rays emitted from $4.6 \mathrm{~m} 101_{\mathrm{Mo}}$, $1.6 \mathrm{~m} 104 \mathrm{Mo}, 1.9 \mathrm{~m} 105 \mathrm{Mo}, 14.2 \mathrm{~m} 101 \mathrm{Tc}, 4.3 \mathrm{~m} 102 \mathrm{Tc}, 50 \mathrm{~s} 103 \mathrm{Tc}, 18 \mathrm{~m} 104 \mathrm{Tc}$, and $8 \mathrm{~m}{ }^{105} \mathrm{Tc}$ as can be seen from the spectra in figs. 1 and 2.

Cerium was chemically separated $(-100 \mathrm{sec})$ as above, except that all of the organic layer, containing molybdenum, was drawn off and discarded. Two hundred $\mu \mathrm{l}$ of $2.0 \mathrm{M} \mathrm{NaBrO}$ was then added to the $\mathrm{HNO}_{3}$ solution remaining in the test tube along with one $\mathrm{ml}$ of fresh HDEHP. The $\mathrm{Ce}$ (IV) was extracted into the HDEHP by shaking for 10 seconds. Fifteen seconds were allowed for centrifugation and finally most of this organic phase, containing cerium, was transferred to a counting vial and gamma-ray counted with $\mathrm{Ge}(\mathrm{L} i)$ spectrometers. Two counts were also made of each separated cerium sample. The first count was for 2 min and was started 2 min from termination of collection. The second count was for 15 min and was started $9 \mathrm{~min}$ after termination of collection. Five samples were produced and cycled in this manner. The cerium samples contained cerium activities and their praseodymium daughters and possibly ruthenium 107, 108 and 109 and their rhodium daughters. We identified $\gamma$-rays emitted from $3 m^{145} \mathrm{Ce}, 14.2 \mathrm{~m}{ }^{146} \mathrm{Ce}, 70 \mathrm{~s}{ }^{148} \mathrm{Ce}, 24.2 \mathrm{~m}{ }^{146} \mathrm{Pr}$, $12 \mathrm{~m} 147 \mathrm{Pr}$ and $2.0 \mathrm{~m} 148 \mathrm{Pr}$ in addition to the identification of the ruthenium-rhodium activities. The spectra from these runs are shown in figs. 3 and 4 .

\section{Separation of $24.2 \mathrm{~m}{ }^{146 \mathrm{Pr}}$ and of $14.2 \mathrm{~m}{ }^{146} \mathrm{Ce}$}

From studying the spectra in figs. $1-4$ it is obvious from the complexity of these spectra that detailed studies of the shorter half life isotopes can be made only after the $\gamma$-rays from the longer lived radioisotopes and daughter radionuclides have been identified and their energies and intensities measured. In order to determine the feasibility of this approach, we chose to obtain a "clean" spectrum of $146 \mathrm{Pr}$ and of

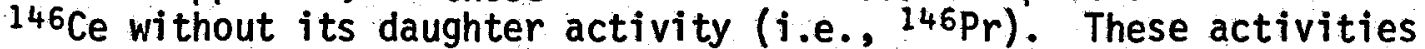
are the longer lived radionuclides which must be completely identified before ${ }^{145} \mathrm{Ce},{ }^{147} \mathrm{Ce},{ }^{148} \mathrm{Ce}$ and their daughters can be studied in detail. In this series of runs the fission product activity was collected for 20-30 min on the aluminum collection foil and the chemical purifications were timed so as to enhance the radioisotope being studied. The activity was washed from the foil into a test tube with approximately $3 \mathrm{cc}$ of $1 \mathrm{M} \mathrm{HNO}_{3}$.

One $\mathrm{mg}$ of $\mathrm{La}\left(\mathrm{NO}_{3}\right)_{3}$ was added as a carrier. The rare earth elements were coprecipitated on $\mathrm{La}(\mathrm{OH})_{3}$ which was formed by addition of excess concentrated $\mathrm{NH}_{4} \mathrm{OH}$. This precipitate was washed once with $\mathrm{H}_{2} \mathrm{O}$ and dissolved in $1 \mathrm{ml}$ of concentrated $\mathrm{HNO}_{3}$. One $\mathrm{ml}$ of HDEHP was added to extract the Mo from the aqueous solution. The organic phase was separated and discarded. Fifty $\lambda$ of $2.0 \mathrm{M} \mathrm{NaBrO}$ was then added to the $\mathrm{HNO}_{3}$ solution along with $1 \mathrm{ml}$ of fresh HDEHP. The Ce(IV) was extracted into the HDEHP by shaking for $\sim 2$ min. The organic phase was removed and placed in a clean test tube and the $\mathrm{Pr}$ activity was allowed to grow in for 20 min. At this time Pr was back extracted into a $10 \mathrm{M} \mathrm{HNO}{ }_{3}-$ $0.1 \mathrm{M} \mathrm{NaBrO}_{3}$ solution and the aqueous phase was removed and counted. Six samples were processed in this way and counted for $2000 \mathrm{sec}$ LT immediately after separation and for $2000 \mathrm{sec}$ LT about $1 \mathrm{hr}$ after separation 
with $\mathrm{Ge}(\mathrm{Li})$ spectrometers at a source distance of $2-1 / 2 \mathrm{~cm}$. Figure 5 shows the $146 \mathrm{Pr}$ spectrum obtained from the six samples immediately after separation.

The cerium was separated as above through the $\mathrm{NaBrO}_{3}$ oxidation step. The $\mathrm{Ce}$ (IV) was placed onto a $1 / 2 \times 1 / 2-\mathrm{cm}^{2}$ stationary phase HDEHP column and the $\mathrm{Pr}$ activity was continuously removed by eluting with $10 \underline{\mathrm{MNO}} \mathrm{HN}_{3}-0.1 \mathrm{M} \mathrm{NaBrO}_{3}$. This column was placed in front of the detector with the eluted activity shielded from the detector. Four samples were processed in this way and counted after the column was operating for a couple of minutes. Half life runs were made on other $\mathrm{Ge}(\mathrm{L} i)$ spectrometers to help in the identification of any contaminating activities. Figure 6 shows the ${ }^{146} \mathrm{Ce}$ spectrum with only a trace of the $146 \mathrm{Pr}$ daughter.

Unfortunately a few contaminating activities were not removed with the chemical separation used above. Improvements to this procedure are being considered.

\section{Optimization of transport and collection conditions}

With the system described above we ran a series of experiments to determine the transport and collection conditions which are, in general, conducive for rapid (i.e., $\leqslant 5 \mathrm{~min}$ ) chemical purification of fission product radioactivities.

First, we investigated the relative merits of collecting the activity on aluminum foil, on various pore sizes of filter paper and in a liquid by blowing the jet onto the surface of the liquid and by bubbling the jet through the liquid. We learned that millipore or nucleopore filters will collect the radioactive aerosols as well as aluminum foil in which collection efficiencies of $>60 \%$ were typical. However, the aerosols will pass right through coarse filter papers (e.g. Whatman 50). As a result the collection efficiency of coarse filter paper is very poor. The aerosols could be collected in a liquid by blowing the jet onto the liquid surface or bubbling the jet through the liquid (see fig. 7).

Second, we studied the effect of changing the pressure in the collection chamber. If fission fragments can be transported into a collection chamber at ambient pressure, the need for a vacuum system is eliminated and chemical separations may be simplified. With a source chamber pressure of $\leq 2-1 / 2$ atomspheres (absolute) and a 5 meter capillary with an inside diameter of $42 \times 10^{-3}$ in. we were not able to obtain transport (i.e., > 10\% efficiency) at an ambient pressure of 225 in of $\mathrm{Hg}$. However, even at a poor vacuum $(-18$ in $\mathrm{Hg}) \mathrm{good}$ collection efficiencies $(260 \%)$ were obtained by collecting the aerosols on aluminum foil. Although, even with a source chamber pressure of i $4 \mathrm{~atm}$ transport was low (i.e.; < 10\% efficiency).

Third, we substituted for the helium carrier gas water pumped and oil pumped nitrogen gas. Both forms of the nitrogen gas were found to provide satisfactory transport ( $\sim 55 \%$ collection efficiency). Because 
nitrogen has a much greater stopping power than helium, a smaller source chamber can be used to stop the fission fragments. As a result, when nitrogen is used as the carrier gas shorter lived activities can then be transported and studied. Also, since nitrogen is more economical than helium, we intend to use nitrogen as the carrier gas for all experiments requiring only chemical purifications. 


\section{C}

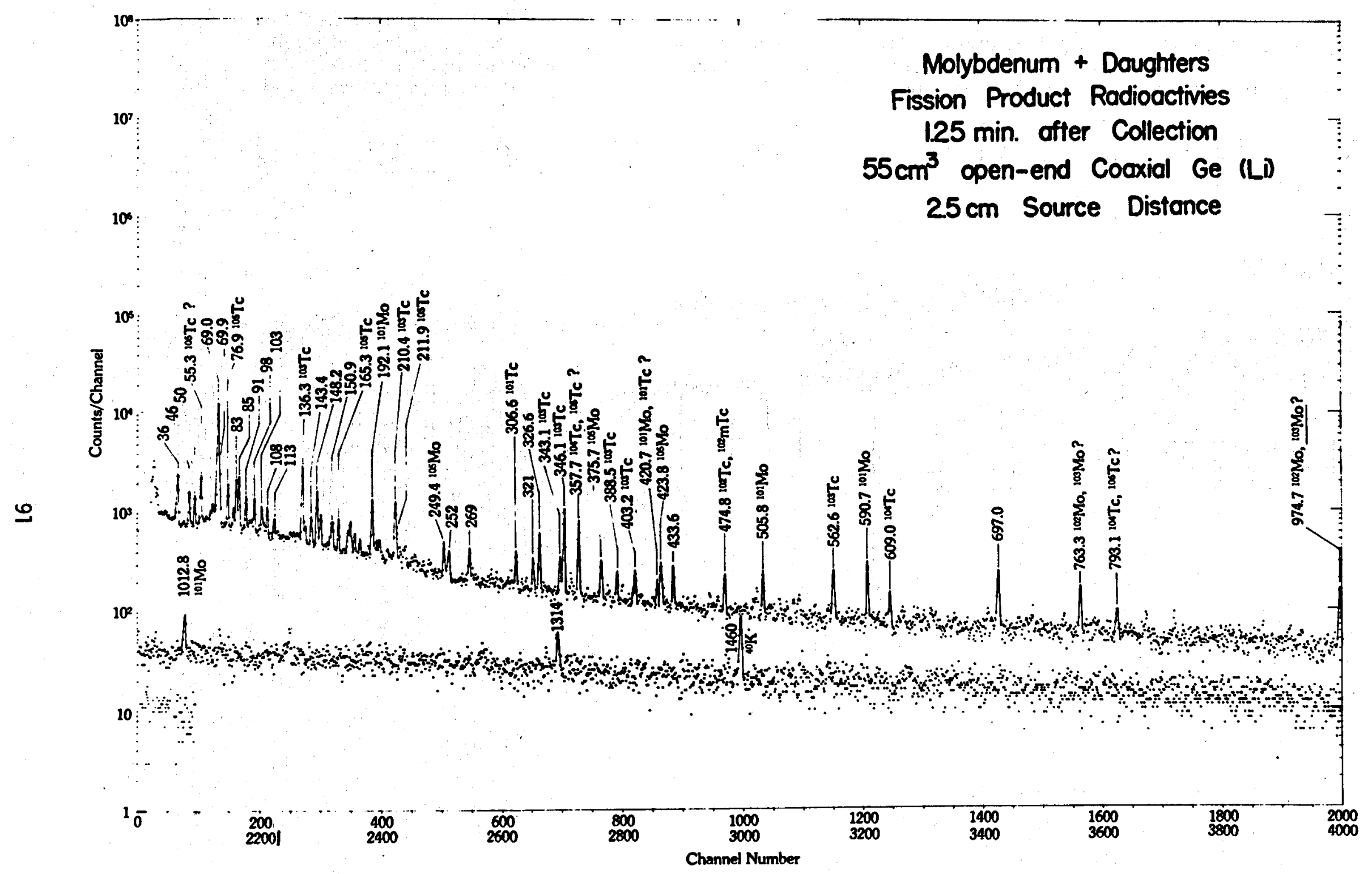

Fig. $1 \gamma$-ray spectrum of chemically separated, short lived, molybdenums fission products and their daughter activities obtained from 5 samples of He jet transported ${ }^{252} \mathrm{Cf}$ fission products collected for $3 \mathrm{~min}$ each. Each sample was counted for 2 min starting 75 sec after collection was terminated. 


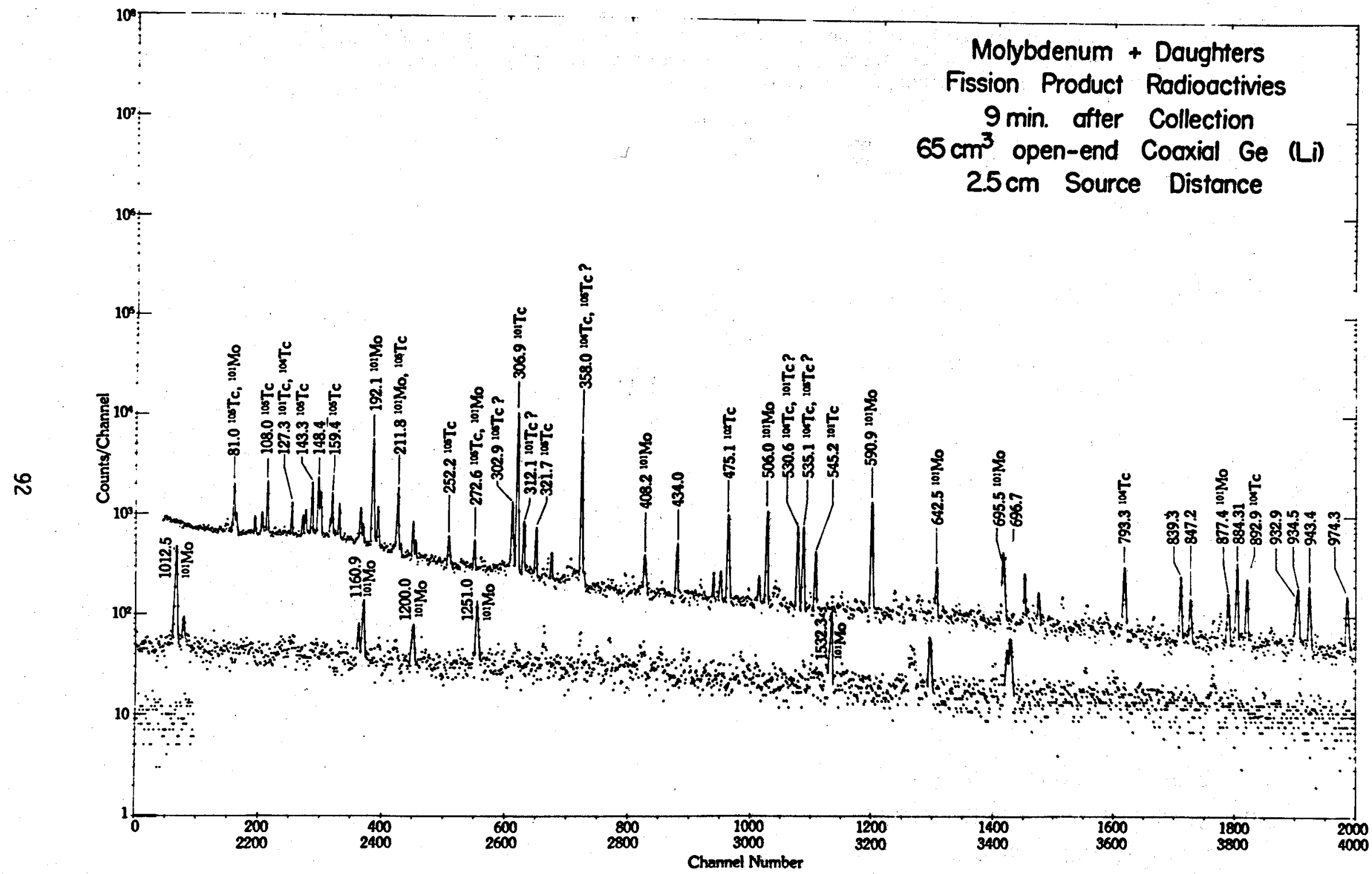

Fig. $2 \gamma$-ray spectrum of chemically separated, short lived, molybdenum fission products and their daughter activities obtained from 5 samples of He jet transported ${ }^{252} \mathrm{Cf}$ fission products collected for 3 min each. Each sample was counted for 15 min starting 9 min after collection was terminated. 

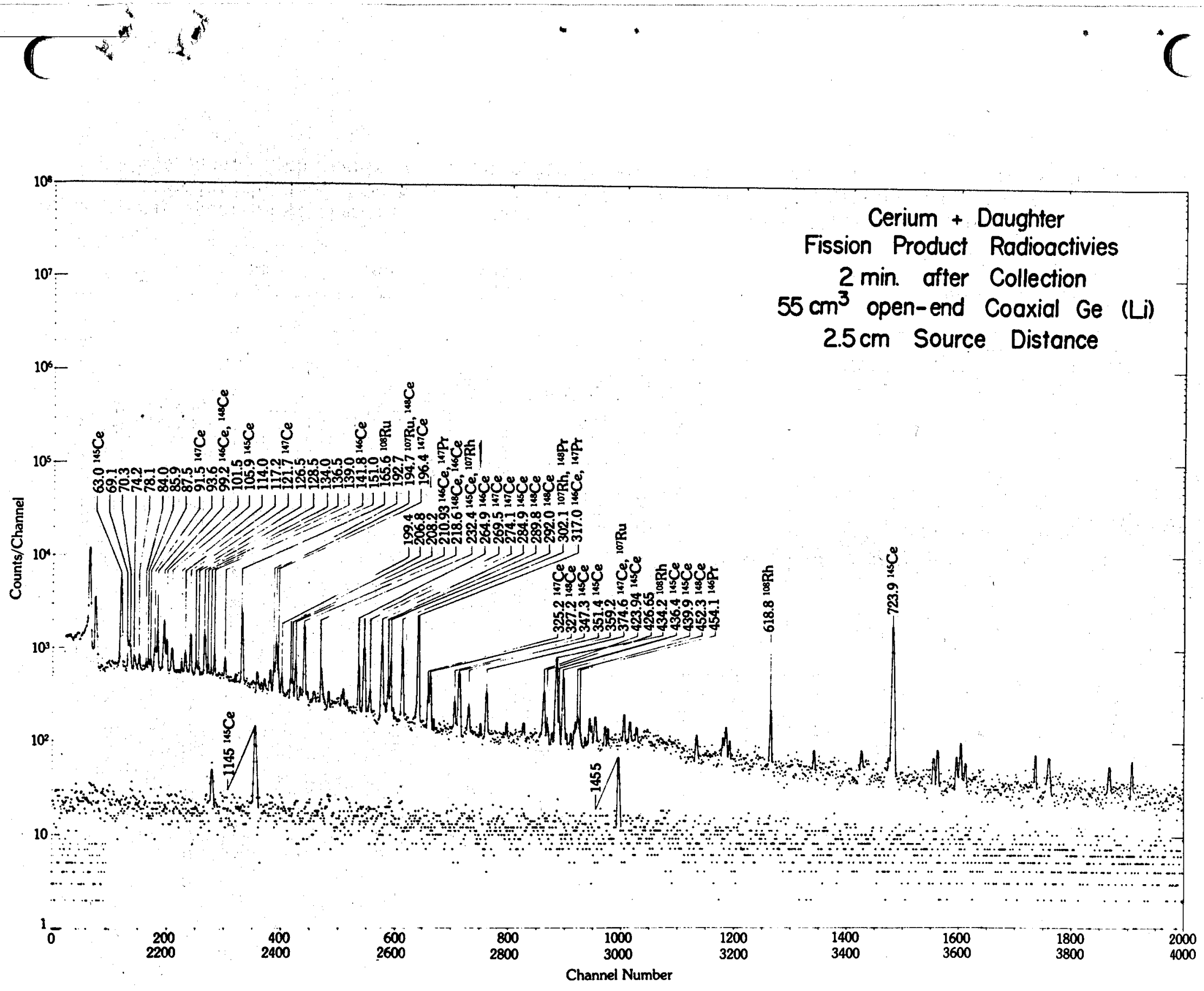

Fig. $3 \gamma$-ray spectrum of chemically separated, short lived, cerium fission products and their daughter activities obtained from 5 samples of He jet transported ${ }^{252} \mathrm{Cf}$ fission products collected for 3 min each. Each sample was counted for 2 min starting 2 min after collection was terminated. 


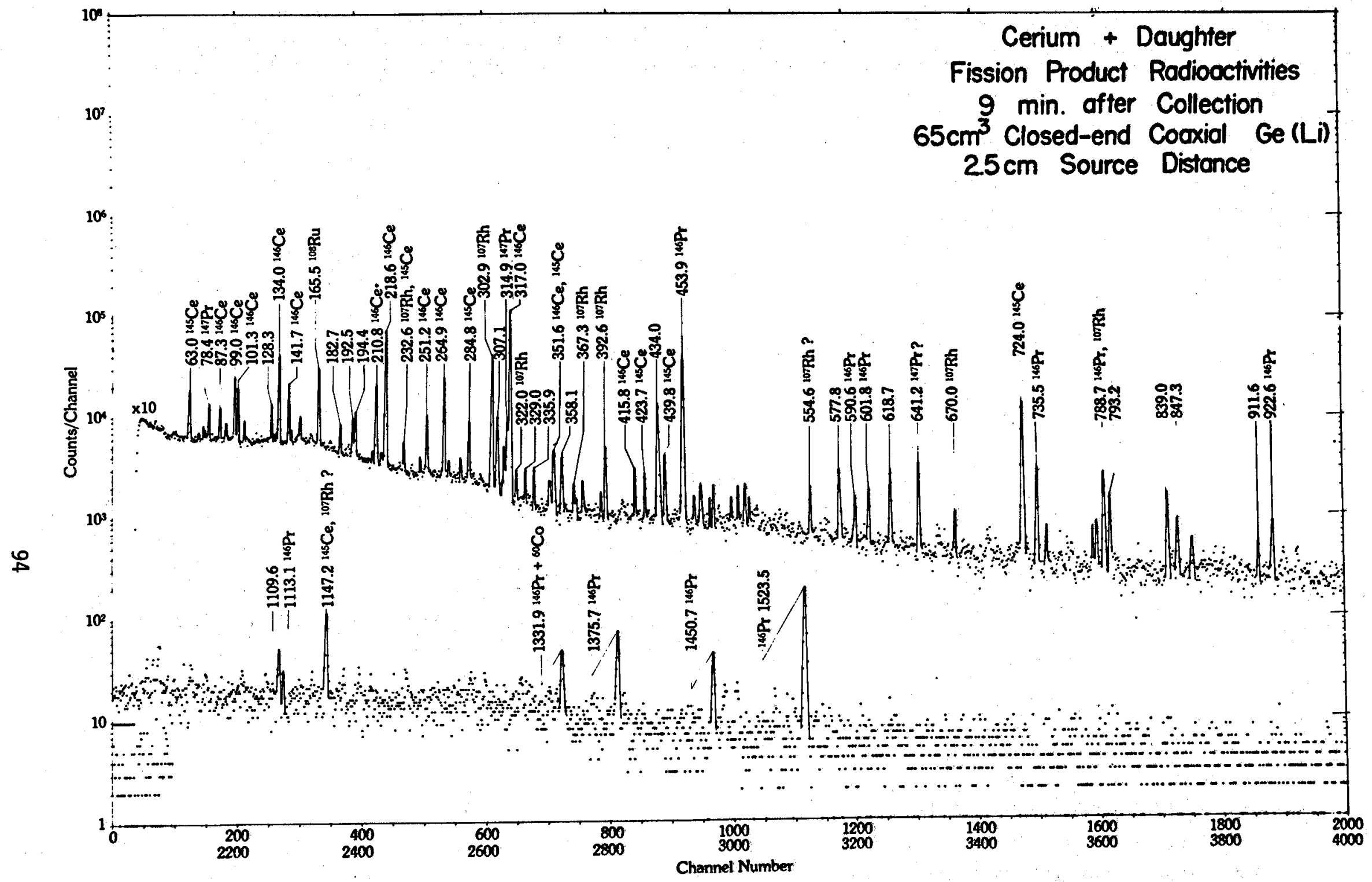

Fig. 4 r-ray spectrum of chemically separated, short lived, cerfum fission products and their daughter activities obtained from 5 samples of He jet transported ${ }^{252} \mathrm{Cf}$ fisston products collected for 3 min each. Each sample was counted for 15 min starting 9 min after collection was terminated. 


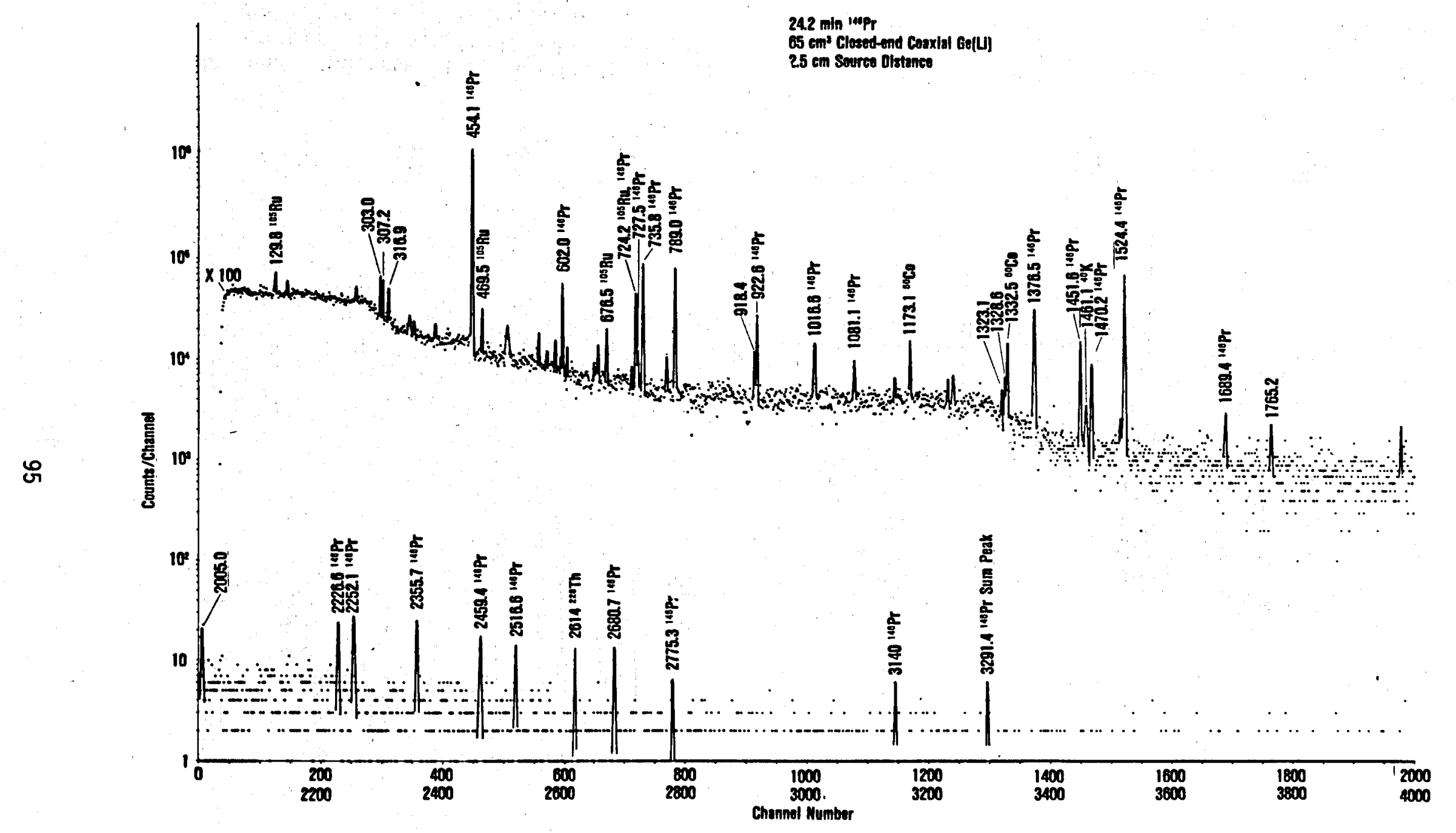

Fig. 5 -ray spectrum of chemically separated $24.2 \mathrm{~min} 146 \mathrm{Pr}$ obtained from 6 samples of He jet transported $252 \mathrm{Cf}$ fission products collected for 20-30 min each. Each sample was counted immediately after chemical purification for $2000 \mathrm{sec}$. 


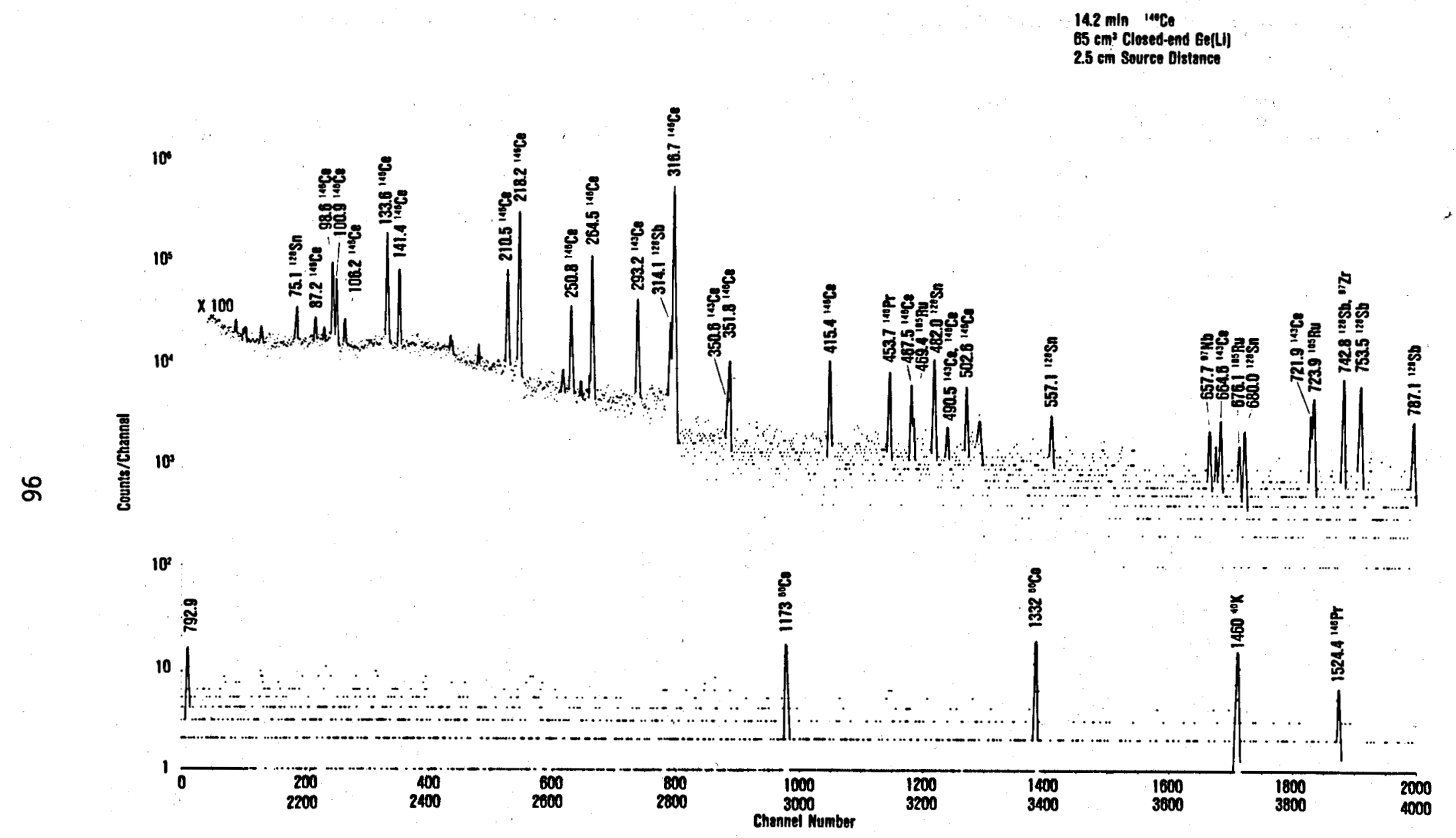

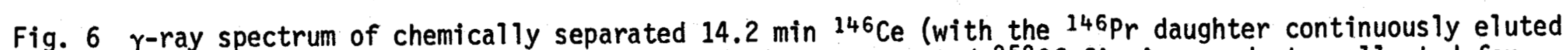
from column) obtained from 4 samples of He jet transported ${ }^{252} \mathrm{Cf}$ fission products collected for 20-30 min each. Each sample was counted after the column was operating a couple of minutes for $2000 \mathrm{sec}$.

$C ;+i$ 


\section{GAS SUPPLY AND AEROSOL GENERATOR}

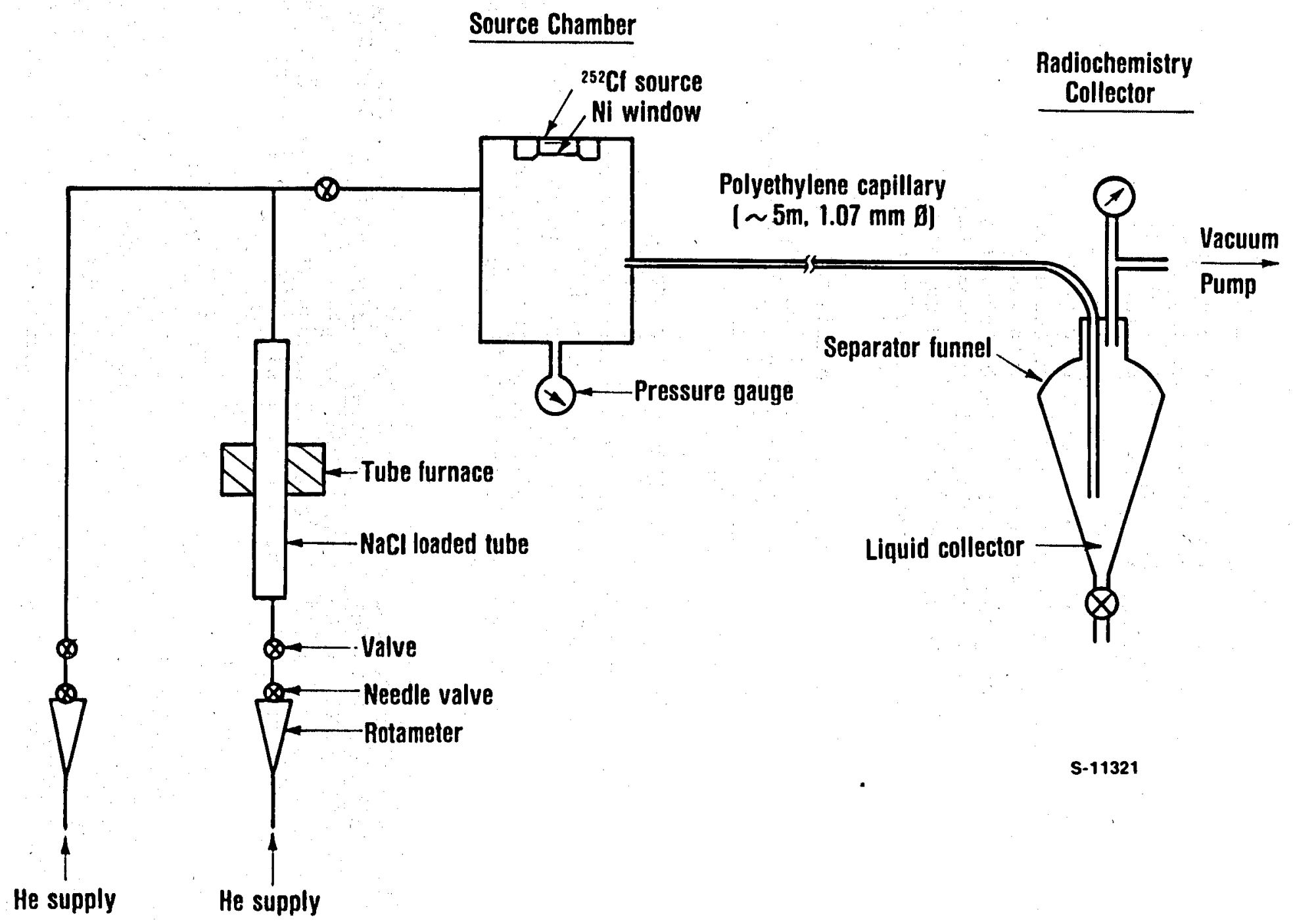

Fig. 7 Diagram of system used to study He jet transport efficiency when collecting in water, when collecting at different pressures and when using nitrogen as the carrier gas. 
PRECISE RELATIVE $\gamma$-RAY INTENSITY MEASUREMENTS

R. J. Gehrke, R. G. Helmer, R. C. Greenwood

Over the past several years most experimentors have used $\mathrm{Ge}(\mathrm{Li})$, and recently intrinsic $\mathrm{Ge}$, detectors for $\gamma$-ray spectrometry. Since each of these detectors is essentially unique, the peak efficiency must be determined experimently for every detector. One standard method of measuring the energy dependence of the absolute efficiency is to make measurements with a series of separate sources, each of which has a known $\gamma$-ray emission rate. A second method is to use a single isotope (or a small number of isotopes) that provides a series of lines of known relative intensity. This technique is much faster, although it is 1 imited to providing the relative detector efficiency. To aid in such calibrations we have made precise relative $\gamma$-ray intensities measurements for a series of isotopes that can then be used for efficiency calibration of other detectors. These measurements have been made on two Ge(Li) detector systems.

The experimental techniques used in this work have been discussed previously. $(1 ; 2)$ Special care has been taken to provide a consistent method of analyzing the $\gamma$-ray peaks including the background determination; errors have been treated in a well defined manner; and corrections for coincidence summing have been made.

Measurements of the $\gamma$-ray spectra have been completed for the ${ }_{12}^{12}$ samples: ${ }^{56} \mathrm{Co},{ }^{75} \mathrm{Se},{ }^{82} \mathrm{Br}, 11{ }^{16} \mathrm{~m}_{\mathrm{Ag}},{ }^{125} \mathrm{Sb},{ }^{13} \mathrm{Ba}, 140 \mathrm{Ba}-\mathrm{La},{ }^{144} \mathrm{Ce}-\mathrm{Pr}$, and the results have been tabulated for over half of the isotopes. Table I gives the results for eight isotopes. With this information, it is expected that the relative efficiency of a Ge(Li) detector can be determined to $\sim 3 \%$ between 300 and $1500 \mathrm{keV}$ and $\sim 5 \%$ outside of this range.

(1) R. G. Helmer, J. E. Cline and R. C. Greenwood, The Electromagnetic Interaction in Nuclear Spectroscopy, edited by W. D. Hamilton (North-Holland, Amsterdam, 1975), Chapter 17.

(2) R. C. Greenwood, R. G. Helmer, JW Rogers, N. D. Dudey, R. J. Popek, L. S. Kellogg and W. H. Zimmer, Nucl. Tech. 25, 274 (1975). 
TABLE I

Relative $\gamma$-Ray Intensities

\begin{tabular}{|c|c|c|c|c|c|}
\hline I sotope & $\begin{array}{r}\text { r-Ray } \\
\text { Energy } \\
(\mathrm{keV}) \\
\end{array}$ & $\begin{array}{c}\text { Relative } \\
\gamma \text {-Ray } \\
\text { Intensity } \\
\end{array}$ & I sotope & $\begin{array}{r}\gamma \text {-Ray } \\
\text { Energy } \\
(\mathrm{keV}) \\
\end{array}$ & $\begin{array}{c}\text { Relative } \\
\text { Y-Ray } \\
\text { Intensity }\end{array}$ \\
\hline${ }^{5} \mathrm{Se}$ & $\begin{array}{r}66 \\
96 \\
121 \\
136 \\
198\end{array}$ & $\begin{array}{c}1.86(9) \\
5.9(3) \\
29.8(9) \\
102.2(30) \\
2.53(8)\end{array}$ & & $\begin{array}{l}706 \\
744 \\
763 \\
818 \\
884\end{array}$ & $\begin{array}{c}17.67(12) \\
4.92(4) \\
23.60(16) \\
7.73(5) \\
76.9(5)\end{array}$ \\
\hline & $\begin{array}{l}264 \\
279 \\
303 \\
400\end{array}$ & $\begin{array}{c}\equiv 100.0(15) \\
42.4(6) \\
2.21(3) \\
19.1(3)\end{array}$ & & $\begin{array}{r}937 \\
1384 \\
1475 \\
1505 \\
1562\end{array}$ & $\begin{array}{c}36.22(25) \\
25.70(18) \\
4.21(3) \\
13.84(12) \\
1.250(9)\end{array}$ \\
\hline \multirow[t]{5}{*}{${ }^{82} 2_{B r}$} & $\begin{array}{r}92 \\
221 \\
273 \\
554 \\
606\end{array}$ & $\begin{array}{c}0.86(4) \\
2.71(8) \\
0.96(3) \\
84.7(6) \\
1.421(13)\end{array}$ & ${ }^{133} \mathrm{Ba}$ & $\begin{array}{r}53 \\
80 \\
160 \\
223 \\
276\end{array}$ & $\begin{array}{l}3.54(18) \\
49.2(26) \\
1.08(4) \\
0.745(24) \\
11.7(4)\end{array}$ \\
\hline & $\begin{array}{l}619 \\
698 \\
776 \\
827 \\
952\end{array}$ & $\begin{array}{c}52.0(4) \\
34.1(3) \\
\equiv 100.0(8) \\
28.8(2) \\
0.454(5)\end{array}$ & \multirow[b]{2}{*}{$140_{B a-L a}$} & $\begin{array}{l}302 \\
356 \\
383\end{array}$ & $\begin{aligned} & 29.76(20) \\
& \equiv 100.0(8) \\
& 14.36(10)\end{aligned}$ \\
\hline & $\begin{array}{l}952 \\
1007 \\
1043 \\
1072 \\
1081 \\
1180\end{array}$ & $\begin{array}{r}1.522(14) \\
32.55(25) \\
0.086(6) \\
0.745(9) \\
0.12(15)\end{array}$ & & $\begin{array}{l}162 \\
304 \\
328 \\
423 \\
432\end{array}$ & $\begin{array}{c}5.83(20) \\
3.89(5) \\
21.4(3) \\
2.80(3) \\
3.07(3)\end{array}$ \\
\hline & 1180 & $0.12(15)$ & & $\begin{array}{l}437 \\
487\end{array}$ & $\begin{array}{c}1.72(2) \\
47.6(5)\end{array}$ \\
\hline & $\begin{array}{l}1317 \\
1474 \\
1650 \\
1779 \\
1956\end{array}$ & $\begin{array}{r}31.74(24) \\
19.53(15) \\
0.889(8) \\
0.136(2) \\
0.047(1)\end{array}$ & & $\begin{array}{l}537 \\
751 \\
815 \\
867 \\
919\end{array}$ & $\begin{array}{c}22.00(23) \\
4.64(5) \\
24.85(25) \\
5.89(6) \\
2.90(4)\end{array}$ \\
\hline $110^{\mathrm{m}} \mathrm{Ag}$ & $\begin{array}{l}446 \\
620 \\
657 \\
677 \\
686\end{array}$ & $\begin{array}{r}3.86(3) \\
2.93(2) \\
\equiv 100.0(7) \\
11.31(8) \\
6.85(5)\end{array}$ & & $\begin{array}{r}925 \\
1596 \\
2347 \\
\\
2521 \\
2547 \\
2899 \\
3118\end{array}$ & $\begin{array}{c}7.41(8) \\
\equiv 100.0(9) \\
0.891(16) \\
\\
3.62(7) \\
0.109(3) \\
0.069(2) \\
0.028(1)\end{array}$ \\
\hline
\end{tabular}


Table I (Continued)

\begin{tabular}{|c|c|c|c|c|c|}
\hline I sotope & $\begin{array}{r}\gamma-\text { Ray } \\
\text { Energy } \\
(\text { keV) } \\
\end{array}$ & $\begin{array}{c}\text { Relative } \\
\gamma \text {-Ray } \\
\text { Intensity } \\
\end{array}$ & Isotope & $\begin{array}{r}\gamma-R a y \\
\text { Energy } \\
(\mathrm{keV}) \\
\end{array}$ & $\begin{array}{c}\text { Relative } \\
\text { r-Ray } \\
\text { Intensity }\end{array}$ \\
\hline $166 \mathrm{~m}_{\mathrm{Ho}}$ & $\begin{array}{r}80 \\
184 \\
215 \\
280 \\
300\end{array}$ & $\begin{array}{c}21.4(11) \\
128 .(4) \\
5.15(16) \\
51.5(16) \\
6.36(20)\end{array}$ & & $\begin{array}{l}116 \\
152 \\
156 \\
179 \\
198\end{array}$ & $\begin{array}{c}1.26(4) \\
20.5(6) \\
7.78(23) \\
9.1(3) \\
4.31(18)\end{array}$ \\
\hline & $\begin{array}{l}365 \\
410 \\
451 \\
529 \\
570\end{array}$ & $\begin{array}{c}4.23(4) \\
19.55(13) \\
5.11(4) \\
16.36(12) \\
9.53(7)\end{array}$ & & $\begin{array}{l}222 \\
229 \\
264 \\
928 \\
959\end{array}$ & $\begin{array}{c}21.9(7) \\
10.6(3) \\
10.5(3) \\
1.80(3) \\
1.00(2)\end{array}$ \\
\hline & $\begin{array}{l}670 \\
691 \\
711 \\
736 \\
752\end{array}$ & $\begin{array}{c}9.43(7) \\
2.31(2) \\
95.3(6) \\
0.673(10) \\
21.21(14)\end{array}$ & & $\begin{array}{l}1001 \\
1044 \\
1121 \\
1157^{\mathrm{a}}\end{array}$ & $\begin{array}{c}5.90(6) \\
0.676(15) \\
\equiv 100.0(8) \\
2.85(4)\end{array}$ \\
\hline & $\begin{array}{l}778 \\
810 \\
830 \\
875 \\
950\end{array}$ & $\begin{array}{c}5.28(4) \\
\equiv 100.0(8) \\
16.97(12) \\
1.253(10) \\
4.71(3)\end{array}$ & & $\begin{array}{l}1189 \\
1221 \\
1230 \\
1257 \\
1273\end{array}$ & $\begin{array}{c}46.6(7) \\
77.6(7) \\
33.0(3) \\
4.25(4) \\
1.86(2)\end{array}$ \\
\hline & $\begin{array}{l}1146 \\
1241 \\
1282 \\
1400 \\
1427\end{array}$ & $\begin{array}{l}0.351(3) \\
1.406(16) \\
0.309(5) \\
0.858(8) \\
0.852(13)\end{array}$ & & $\begin{array}{l}1289 \\
1342 \\
1373 \\
1387 \\
1410\end{array}$ & $\begin{array}{l}3.86(5) \\
0.718(11) \\
0.629(9) \\
0.202(4) \\
0.112(6)\end{array}$ \\
\hline 59 & & & & 1453 & $0.079(3)$ \\
\hline
\end{tabular}

$\begin{array}{crcc}{ }^{169}{ }_{\mathrm{Yb}} & 63 & 116 .(6) & \\ & 93 & 7.1(4) & \text { a) Doublet } \\ & 109 & 48.5(15) & \\ & 118 & 5.31(16) & \\ & 130 & 32.0(10) & \\ & & & \\ & 177 & 62.2(19) & \\ & 197 & \equiv 100.0(30) & \\ & 240 & 0.337(17) & \\ & 261 & 4.69(14) & \\ 182 \mathrm{Ta} & 307 & 27.5(8) & \\ & 65 & 15.4(20) \\ & 67 & 125 .(6) \\ & 84 & 7.6(4) \\ & 100 & 40.9(12) & \\ & 113 & 5.42(17)\end{array}$




\section{A SHIELDED CELL FOR ${ }^{252}$ Cf}

R. A. Anderl, C. W. Reich, R. C. Greenwood, C. H. Cargo*

\section{Introduction}

Two systems for studying the decay properties of short-lived fission products are in various stages of development at the INEL. Current progress on the development of a system which utilizes an electromagnetic isotope separator to provide mass-separated fission product radioactive sources is found el sewhere in this report. (1) This system,called an Isotope Separator "On-line" System (ISOL), util izes the gas-jet technique to transport fission products from an "open" ${ }^{252} \mathrm{Cf}$ fission source to the ion source of the isotope separator. A complementary system for these fission product studies utilizes the gas jet technique to transport fission products from an "open" ${ }^{252} \mathrm{Cf}$ fission-source to fast radiochemistry apparatus which provides chemically pure sources for nuclear radiation measurements.(2) Central to both of these systems is the development of the capabilities and facilities for handling and storing sizable quantities of ${ }^{252} \mathrm{Cf}$. This article presents a summary description of the design of a shielded cell facility for storing and handling, remotely, up to $2 \mathrm{mg}$ of $252 \mathrm{Cf}$. Title II design for this facility is nearly completed and the shielded cell will be fabricated and installed, during the coming fiscal year, in room 124 of Building MTR-661 at the Test Reactor Area of the INEL.

\section{Design Considerations}

In contrast to most of the hot cells at the INEL which are designed for safe-handling of either intense beta-gamma emitters or intense alpha-beta-gamma emitters, the proposed cell is designed for safe-handling of fission sources which are also intense alpha-beta-gamma-neutron emitters. The unique decay and radiation properties(3) of ${ }^{252} \mathrm{Cf}$ most important to shielding and contamination control requirements are summarized in Tables I and II.

TABLE I

DECAY PROPERTIES FOR ${ }^{252}$ Cf

\begin{tabular}{lcccc}
\hline $\begin{array}{l}\text { Decay } \\
\text { Mode }\end{array}$ & $\begin{array}{c}\text { Specific Activity } \\
\text { (dps, } \\
\text { per } \mathrm{mg})\end{array}$ & $\begin{array}{c}\text { Half-1ife } \\
\text { (mei per } \mathrm{mg})\end{array}$ & $\begin{array}{c}\text { Decay Heat } \\
\text { (mW/mg) }\end{array}$ \\
\hline $\begin{array}{l}\text { Alpha decay } \\
\text { Spontaneous }\end{array}$ & $1.92 \times 10^{10}$ & 519.4 & 2.731 & 18.8 \\
$\begin{array}{l}\text { Fission } \\
\text { Total }\end{array}$ & $6.14 \times 10^{8}$ & 16.6 & 85.5 & 19.7 \\
\cline { 2 - 5 } & $1.98 \times 10^{10}$ & 536.0 & 2.646 (eff.) & 38.5
\end{tabular}

*Architect Engineering and Construction Branch, ANC. 
Summarized in Table II is a characterization of the radiations associated with the alpha decay and spontaneous fission of ${ }^{252} \mathrm{Cf}$ and with the decay of the fission products.

TABLE II

CHARACTERIZATION OF THE RADIATIONS FROM ${ }^{252} \mathrm{Cf}$

\begin{tabular}{lcc}
\hline Radiation Type & $\begin{array}{c}\text { Intensity } \\
\text { (n or } \gamma / \mathrm{sec}-\mathrm{mg})\end{array}$ & $\begin{array}{c}\text { Average Energy } \\
(\text { MeV })\end{array}$ \\
\hline Neutrons & $2.3 \times 10^{9}$ & 2.348 \\
Gamma Rays & & \\
- Alpha Decay & $5.2 \times 10^{6}$ & 0.074 \\
- Prompt Fission & $6.6 \times 10^{9}$ & 0.764 \\
- Fission Product & $6.6 \times 10^{9}$ & 0.769 \\
- Total & $1.8 \times 10^{10}$ & 0.767 \\
Alphas & $1.92 \times 10^{10}$ & 6.11 \\
\hline
\end{tabular}

Estimates of the dose rates due to neutron and gamma emission from the ${ }^{252} \mathrm{Cf}$ are useful in evaluating exposure 1 imitations for materials of construction and especially for personnel handling unshielded sources. Summarized in Table III are calculated neutron, gamma and total doserates at various distances from an unshielded ${ }^{252} \mathrm{Cf}$ source weighing $2 \mathrm{mg}$.

TABLE III

DOSE RATES FOR AN UNSHIELDED ${ }^{252} \mathrm{Cf}$ SOURCE

\begin{tabular}{rlcc}
\hline $\begin{array}{c}r \\
(\mathrm{~cm})\end{array}$ & $\begin{array}{c}\mathrm{D}_{\mathrm{n}} \\
(\mathrm{mrem} / \mathrm{h}\end{array}$ & $\begin{array}{c}\mathrm{D} \\
(\mathrm{mrem} / \mathrm{h})\end{array}$ & $\begin{array}{c}\mathrm{D}_{\mathrm{t}} \\
(\mathrm{mrem} / \mathrm{h})\end{array}$ \\
\hline 5 & $1.86 \times 10^{6}$ & $1.31 \times 10^{5}$ & $2.00 \times 10^{6}$ \\
10 & $4.66 \times 10^{5}$ & $3.28 \times 10^{4}$ & $5.00 \times 10^{5}$ \\
20 & $1.16 \times 10^{5}$ & $8.20 \times 10^{3}$ & $1.25 \times 10^{5}$ \\
30 & $5.18 \times 10^{4}$ & $3.64 \times 10^{3}$ & $5.56 \times 10^{4}$ \\
40 & $2.91 \times 10^{4}$ & $2.05 \times 10^{3}$ & $3.12 \times 10^{4}$ \\
50 & $1.86 \times 10^{4}$ & $1.31 \times 10^{3}$ & $2.00 \times 10^{4}$ \\
60 & $1.29 \times 10^{4}$ & 911 & $1.39 \times 10^{4}$ \\
70 & $9.51 \times 10^{3}$ & 669 & $1.02 \times 10^{4}$ \\
80 & $7.28 \times 10^{3}$ & 512 & $7.81 \times 10^{3}$ \\
90 & $5.75 \times 10^{3}$ & 405 & $6.17 \times 10^{3}$ \\
100 & $4.66 \times 10^{3}$ & 328 & $5.00 \times 10^{3}$ \\
120 & $3.24 \times 10^{3}$ & 228 & $3.47 \times 10^{3}$ \\
140 & $2.38 \times 10^{3}$ & 167 & $2.55 \times 10^{3}$ \\
160 & $1.82 \times 10^{3}$ & 128 & $1.95 \times 10^{3}$ \\
\hline
\end{tabular}


of importance to contamination control requirements for the cell is the chemical form, mounting, configuration and physical characteristics of the ${ }^{252} \mathrm{Cf}$ source material. The ${ }^{252} \mathrm{Cf}$ sources will be in the form of $\mathrm{Cf}_{2} \mathrm{O}_{3}$ electro-deposited on platinum discs. Source size will not exceed $500 \mu \mathrm{g}$ per disc.

Because of its fissioning nature and the subsequent interaction of the fission fragments with the source material, ${ }^{252} \mathrm{Cf}$ tends to transfer source material to the enviroment surrounding the source. This property could result in spread of high level contamination throughout the cell interior. To minimize the migration of $252 \mathrm{Cf}$ throughout the cell, each source will be mounted in a holder to which is attached a nickel foil which completely covers the ${ }^{252} \mathrm{Cf}$ source. The nickel window thickness is chosen to permit transmission of the energetic fission fragments but localize migration of the ${ }^{252} \mathrm{Cf}$ to the region enclosed by the nickel foil and the source. In addition, the source holder is mounted in an experimental chamber which further isolates it from the cell interior. Al though these precautionary features will minimize internal cell alpha contamination, operations made to repl ace the nickel window have the potential for cell interior contamination. Hence, the cell will be designed to permit handling of $2 \mathrm{mg}$ quantities of ${ }^{252} \mathrm{Cf}$ with no spread of contamination to the area exterior to the cell.

The fact that ${ }^{252} \mathrm{Cf}$ is an intense gamma and an intense neutron emitter presents a unique problem concerning the design of an effective, compact shield structure around the cell interior. High-density, high$Z$ materials provide the most optimium attenuation factors for gammas of the energies considered here. In contrast, low- $Z$ materials, preferably hydrogenous materials, provide most effective attenuation of fission source neutrons. An additional complicating factor is the production of neutron capture gamma rays in the shield structure material. Unless shielded against by use of a laminated shield of dense and lighter materials or by use of high neutron absorption additives to suppress the production of these secondary gammas, the neutron capture gamma rays can result in unacceptable dose levels exterior to the cell. In summary, the selection of materials for design of the shield structure must give due consideration to neutron and gamma attenuation properties, neutron activation properties, and certain mechanical properties related to structural stability and strength, ease of fabrication, cost, fire resistance, and weight and space limitations.

Also factored into the design of this cell are the operations anticipated for planned and future use. Operations which will be performed with the shielded-cell facility include: (1) transfer of experimental apparatus and ${ }^{252} \mathrm{Cf}$ sources into the cell interior, (2) remote disassembly of ${ }^{252} \mathrm{Cf}$ source shipping canister, (3) remote handling and mounting of ${ }^{252} \mathrm{Cf}$ source holder in experimental apparatus, (4) installation of experimental hardware through service access tubes and remotely-handled connection of such hardware to experiment apparatus within the cell (5) transfer of shipping container and contaminated waste from the cell interior to the operating area for disposal, and (6) periodic replacement of the source transmission window which minimizes 
migration of the ${ }^{252} \mathrm{Cf}$ in the experimental apparatus. - Initial transfer of larger pieces of experimental apparatus will be done through the shield window opening prior to installation of the window. Any subsequent need for transfer of larger equipment will be done by first removing the ${ }^{252} \mathrm{Cf}$ from the cell, decontaminating the cell interior, and removing the shield window. No chemistry or other processing procedures will be done with the ${ }^{252} \mathrm{Cf}$. Because of the nature of the anticipated operation, no service water, air lines, chemical lines, drains, or elaborate firefighting hardware are required in-cell.

Because the principal use for this cell facility is in programs utilizing the isotope separator or fast radiochemistry apparatus, the cell is being designed for installation in room 124 of Building 661 at TRA. This room is adjacent to the isotope separator 1aboratory. Due to size 1 imitations of the room, design of the shield structure requires selection of the material content and shield construction to obtain a compact cell facility.

\section{Description of Cell Design}

Basic components of the shielded cell include an alpha containment box lining the inside walls of the completely enclosed shield structure, a radiation-shielded viewing window in the west wall, a shielded air-lock passageway in the south wal1, two master-slave manipulators through the west wall, internal cell, lighting, service access tubes through the north wall, a completely filtered ventilation system, and appropriate monitor and control instrumentation. Exterior dimensions of the cell facility are: $9^{\prime} 6^{\prime \prime}$ deep, $10^{\prime} 7^{\prime \prime}$ wide, $9^{\prime} 10^{\prime \prime}$ high. Interior dimensions of the al pha containment box are. $4^{\prime}$ deep, $4^{\prime}$ wide, $5^{\prime}$ high. The bottom of the box is located at a height of $36^{\prime \prime}$ from the operating room floor.

The alpha containment box is constructed of stainless steel and all welds are continuous seal welds to form an airtight enclosure. Openings through the stainless liner for the master-slave manipulators and the viewing window are equipped with flanges which form a tight seal with mating booting and gasket material.

The shield enclosing the alpha containment box on all four sides and the top is an integrally constructed structure with a carbon steel structural framework, an outer shell fabricated from $0.25^{\prime \prime}$ carbon steel plate, penetration liners for installation of the viewing window masterslave manipulators and the shielded air-lock passageway, and with the region between the inner stainless liner and the outer carbon steel shell composed of lead sheet and cast-in-place borated gypsum. The 1.5" thick. lead shielding is attached to framework around the alpha containment box. Borated gypsum, which forms the bulk of the radiation shield, is a mixture of 10 parts by weight commercial gypsum $\left(\mathrm{CaSO}_{4} \cdot 1 / 2 \mathrm{H}_{2} \mathrm{O}\right), 7.49$ parts by weight water and 1.51 parts by weight boron frits. Composition of the boron frit includes not less than 16.1 per cent elemental boron in the chemical form $\mathrm{B}_{2} \mathrm{O}_{3}$ and al so includes silicon, aluminum, calcium, magnesium (trace), and iron (trace) as $\mathrm{SiO}_{2}, \mathrm{Al}_{2} \mathrm{O}_{3}, \mathrm{CaO}, \mathrm{MgO}, \mathrm{Fe}_{2} \mathrm{O}_{3}$. 
The as-poured mixture has a density of approximately $1021 \mathrm{bs} / \mathrm{ft}^{3}$ and is approximately $31^{\prime \prime}$ thick in the north, east and west walls and $37^{\prime \prime}$ thick in the south wall. To retain moisture in the cast borated gypsum, the welds in the outer carbon steel shell are continuous seal welds and the shell is sealed to the base concrete slab with a waterproof sealant. All penetrations through the shield walls are of a stepped or spiralled design to minimize neutron and gamma streaming.

Providing a view of better than 80 per cent of the process interior of the cell is a radiation-shielded viewing window of laminated construction. The window assembly consists of a wall liner which is welded into the shield structure and a removable window unit which matches the 1 iner. The window unit consists of a stainless and carbon steel tank with lead cladding on the hot and cold sides of the tank, framework to support lead glass components at the hot and cold sides of the tank and radiation resistant optical oil filling the void region. Approximate dimensions and material composition along the centerline of the window are 1 " of 2.7 density cover glass on the hot side, followed by an air gap and 8" of 3.3 density lead glass, $16.22^{i 1}$ of optical $0 i 1,6$ " of 6.2 density lead glass, and, finally, an air gap and 1 " of 2.5 density cover glass. To facilitate removal and servicing operations, the window unit is equipped with an oil fill system comprised of a reservoir, oil lines, oil drain and fill connections, and vent connections. Attachments for lifting are incorporated to facilitate removal of the unit. At the cell interior the window unit is sealed to the liner by means of a radiation resistant gasket.

Access to the cell interior for transfer of source material and experimental apparatus is provided by the shielded air-lock passageway in the south wall. Comprising this passageway are a remotely operated inner airlock door which makes a tight seal to the alpha containment box, a shield plug of stepped design which provides radiation shielding equivalent to the shield walls, and a transfer tray which can be moved by remote operation into the alpha enclosure and back into the airlock. The shield plug is constructed from carbon steel, lined with 1.5 " of lead at the hot side, filled with borated gypsum and mounted on a ball bushing shaft assembly so that the plug can be easily rolled wi thout interference, into the wall opening.

Two Central Research Model G master-slave manipulators installed above the viewing window provide the capability for remote handling of materials in-cell. The manipulators are equipped with boots and air restriction devices to maintain good contamination control.

Access to the cell interior for experimental lines and signal cables is provided by the eight 1 " service access tubes through the north shield wall. These lines are welded both at the cell interior and at the cell exterior and are easily capped for contamination control. Sealed adapters are used in place of the caps for introduction of an experimental line. To minimize neutron streaming the access tubes are spiralled. 
Two mercury vapor light fixtures, each with a 175 watt 1 amp, are installed in the cell interior to provide adequate lighting for in-cell operations. Ballasts for the fixtures are installed outside the cell. The lamps are so positioned that they can be replaced with the master slave manipulators.

Control of in-cell radioactive contamination is maintained with the filtered ventilation system that provides better than 12 air changes per minute in the cell interior and maintains the cell interior at a 1.0" water negative pressure wi th respect to ambient pressure. The ventilation system consists of two 30 CFM exhaust fans, one for normal operation, the other in a standby condition wired to start automatically in the event the running fan fails; a single HEPA filter and prefilter on the air inlet line; two HEPA filters in each of two parallel exhaust lines, one line for normal air flow, the other for standby operation when the first is being serviced; appropriate shutoff, control and bypass dampers; ducting as required and differential pressure gauges to measure the pressure drop across each filter as well as the pressure differential between the cell interior and ambient and between the airlock interior and ambient. All filters are equipped with DOP test ports. The ventilation system receives supply air from room 124 and exhausts to the building system. In the event of loss of site power, the running fan is picked up on emergency power.

\section{Evaluation of Shield Materials}

Specific materials for construction of the shield structure were made on the basis of neutron and gamma attenuation properties, neutron activation properties, structural stability and strength, ease of fabrication, cost, fire resistant properties, corrosion properties, maintenance requirements and weight and space limitations. A shielding effectiveness versus cost evaluation for several shielding materials was made. Shield materials considered included polyethylene with lead, water-extended polyester with lead, paraffin with lead, borated paraffin with lead, concrete, limonite concrete, water, and borated gypsum with lead. Comparative costs for an equivalent shielding effectiveness were made. Attenuation curves from Stoddard and Hootman(4) were used to evaluate the shielding effectiveness. This evaluation indicated that a shield structure composed of lead and borated gypsum provided the necessary shielding effectiveness at a reasonable cost and was best suited to meet the dimensional constraints on the cell.

A more detailed evaluation of the shielding effectiveness of the lead-borated gypsum shield composition was accompl ished by. means of shielding calculations made by the ORNL Radiation Shielding Information Center. The ANISN transport code(5) was used to perform spherical. geometry calculations for a $2 \mathrm{mg}{ }^{252} \mathrm{Cf}$ point source at the center of a spherical shield composed of different material configurations with $a$. $53 \mathrm{~cm}$ void between the source and the first layer of the shield. The gamma source spectrum was obtained from Stoddard and Hootman (4) and restructured to fit the 18 gamma ray energy groups used. The neutron source spectrum was obtained from a Maxwel1-Boltzmann energy distribution using a neutron temperature of $1.39 \mathrm{MeV}$. A 22 neutron group, 8 
gamma-ray group coupled cross-section library was used for the calculations. These calculations provided specific design parameters and expected dose rates at the outermost edge of the cell shield structure. Expected total dose rates at the edge of the shield wall composed of a layer of lead and borated gypsum were less than .316 $\mathrm{mrem} / \mathrm{hr}$ for the proposed cell design. The calculated total dose rate at the top of the cell shield structure was calculated to be $1.39 \mathrm{mrem} / \mathrm{hr}$.

Similar shielding calculations were made to optimize the design of the radiation-shiel ded viewing window. The total dose rate at the cold side of the window was calculated to be less than $2.50 \mathrm{mrem} / \mathrm{hr}$ for the window design described above.

Summary

The report summarized the design considerations and a description of a proposed cell design for a shielded-cell facility suitable for storing up to $2 \mathrm{mg}$ of ${ }^{25} \mathrm{Cf}$. This facility will be used in programs being developed to study the decay properties of short-lived fission products by an "on-line" i sotope separator system and by a fast radiochemistry apparatus.

(1) R. C. Greenwood, R. A. Anderl, R. J. Gehrke, S. T. Croney, "Development of an On-Line Isotope Separator System for Fission Product Decay Studies Using a $252 \mathrm{Cf}$ Spontaneous Fission Source," this report.

(2) R. J. Gehrke, R. C. Greenwood, J. D. Baker, "Development of a System for Fission Product Decay Studies Using a ${ }^{252} \mathrm{Cf}$ Spontaneous Fission Source, Helium-Jet Transfer and Fast Radiochemistry," this report.

(3) D. H. Stoddard, "Radiation Properties of Californium-252," DP-986 (June, 1965).

(4) D. H. Stoddard and H. E. Hootman, "Cf-252 Shielding Guide", DP-1246 (March, 1971).

(5) W. W. Engle, Jr. "A Users Manual for ANISN," K-1693 (March, 1967). 
ISOTOPE SEPARATOR LABORATORY DEVELOPMENT

R. A. Ander 1

Significant improvements in the capability for collection of massseparated ions were made. These improvements centered on the fabrication and installation of a more versatile collection-beam viewing system and the design, fabrication, installation and testing of a retardation lens. A summary description of this capability development is presented here.

As a result of these developments, different collector configurations can be setup easily with minimum time exposure of the collection chamber interior to atmospheric pressure. The use of the retardation lens provides the capability for direct collection of ions on a variety of backing materials with ion energies variable from $0 \mathrm{keV}$ to $5 \mathrm{keV}$. This latter capability makes possible the direct collection of $1 \mu \mathrm{g} / \mathrm{cm}^{2}$ to $200 \mu \mathrm{g} / \mathrm{cm}^{2}$ deposits of highly-enriched stable and radioactive samples suitable for cross section measurements or accelerator targets. In addition, the use of the retardation capability makes possible the collection of radioactive samples on very thin backing materials to be used as sources for radionuclide metrology applications.

The collection-beam-viewing system was designed and built to provide the following functions:

(1) indirect and direct viewing of the ion beam shape at the focal plane of the beam by use of a vibrating probe beam scanner and a transmission fluorescent screen;

(2) interchangeable mounting of up to three collection frames, each of which can incorporate one or more of the following:

a. a foil for direct collection of small quantities of mass-separated isotopes at full energy,

b. a foil that can be used as a beam stop for all but one or more selected isotopic beams which pass on through apertures to other collection devices,

c. a transmission fluorescent screen for direct viewing of the ion beam shape,

d. a Faraday cup for measuring ion beam currents;

mounting of a platform for installation of individual or multi-isotopic collection devices (retardation lens, pocket collectors).

The 16-inch by 2.4-inch collection frames are attached to two ninesided wheels, each supported in a vertical upright by a stainless shaft and Delrin bearing assembly. One shaft is coupled to a vacuum rotary motion feedthru which permits remote control of the rotation of a collection frame into the focal plane of the ion beam.

Mounted on a separate carriage is a vibrating probe beam scanner unit which locates the probe vertical pin approximately 1.75 inches in front of the ion beam focal plane. Also mounted on this carriage is a set of beam position stabilizing pins. Remote controls are used to position this carriage at any position along a line perpendicular to the ion beam direction. 
An "as-installed" view of the apparatus comprising this system is shown in Figures 1 and 2 which are photos of the collection chamber interior. Shown in the photos is the setup used with the retardation lens. Mounted on the system "wheels" are two collection frames, one supporting a foil used as a beam stop for all ion beams but the one entering the retardation lens, and the second containing a transmission fluorescent screen for viewing the beam shape and a Faraday cup for measuring the isotopic ion current entering the retardation lens.

The design of the retardation lens, as illustrated by the schematic in Figure 3, is based on the study by Dionisio and DeLima (1) and corresponds to their system $D_{5}$. This particular design was selected because it is used easily with line-focussed beams and can provide either sharply-defined narrow deposits when the focus electrodes are used or broader deposits when the focus electrodes are removed. Photographs of the assembled lens, as installed in the collection chamber, are shown in Figures 1 and 2 .

The beam defining electrode, with a .187-inch by 2.0-inch slot aperture, the electron suppression electrodes, with .40-inch by 2.0 -inch slot apertures, and the retardation electrode are each 4-inch by 4-inch square. The focus electrodes are 4 inches high and are separated by a 2-inch gap. With the exception of the beam defining electrode which is constructed from stainless stee1, all other lens elements are fabricated from Type 6061 aluminum alloy. To minimize arcing all corners are rounded and the surfaces of the elements are polished. A foil holder which slips into the T-slot in the retardation electrode is used to collect the isotopically enriched deposit.

The assembled lens is installed as two parts on the platform used in the collection-beam-viewing system. Mounted in one set of Plexiglass supports which provide for electrode alignment and spacing and attachment to the platform are the first three electrodes. Providing alignment and spacing of the focus and retardation electrodes is another set of Plexiglass supports which are mounted to nylon screws which serve as electrical standoff insulators with respect to the grounded platform. Two pins mounted on electrical insulators attached to the beam defining electrode are used to provide, via a difference amplifier, a correction signal to the acceleration supply for beam position stabilization.

As shown by the photographs in Figures 2 and 4 , the retardation voltage is transmitted to the retardation lens through a specially designed high-voltage feedthru located on the top cover-plate of the collection chamber. Fabricated from Plexiglass, the high voltage feedthru insulator is 7 inches long with approximately 4.25 inches extending above the 7.6 -inch $\times 15.7$-inch $\times 2$-inch thick Plexiglass cover plate and .75 inches extending below the cover plate. The insulator body protruding above the cover plate is 1.75 inches in diameter. This system has been successfully tested at $62 \mathrm{kV}$.

It should be noted that although Plexiglass (an acrylic plastic) is not the most desireable material to use in a vacuum environment because of its vapor pressure and outgassing properties, the mass- 
separator vacuum system is able to maintain the vacuum in the collection chamber at less than $3 \times 10^{-7}$ torr. Lexan (a polycarbonate plastic) is a superior material to use in the vacuum environment but is considerably more expensive.

Grounded metal plates are used to shield as much of the collection chamber from the high voltage feedthru, the high voltage lead to the retardation electrode, and the lens itself. These plates are situated as close as possible to the respective high voltage components.

Illustrated in Figure 5 is a schematic of the electrical layout of the power supplies used with the mass separator when operated in the retardation mode. Typical operating voltages are: (1) $50 \mathrm{kV}$, acceleration supply; (2) $12 \mathrm{kV}$, extraction supply; (3) $20 \mathrm{kV}$, focus supply; (4) $200 \mathrm{~V}$, quadrupole supply; (5) $200 \mathrm{~V}$, beam stop supply; (6) $1200 \mathrm{~V}$, electron suppression supply; (7) $300 \mathrm{~V}$, retardation supply.

With the focus electrodes removed, the retardation lens has been used successfully to collect approximately $30 \mathrm{\mu g}$ deposits of massseparated samarium and neodymium samples. These deposits, on nickel and aluminum foil, were approximately $3 \mathrm{~mm}$ wide by $15 \mathrm{~mm}$ high. The deposit mass and sample enrichment were measured by isotope dilution mass spectrometry. Enhancement factors better than 4000 were achieved. Stable operation was achieved with isotopic ion currents as high as $10 \mu \mathrm{A}$ and for collection times of a couple of hours.

In summary, this report summarized efforts to upgrade and make more flexible the capability for collecting mass separated samples. A description of a recently developed versatile collection-beam-viewing system and a retardation lens were presented. A specific application of the retardation lens is described elsewhere in this report (2).

(1) J. S. Dionisio and D. X. DeLima, Nuclear Instruments and Methods, 61 (1968) 260-284.

(2) R. A. Ander I, Y. D. Harker, "EBR-II Irradiation of Highly-Enriched Isotopic Samples," this report. 


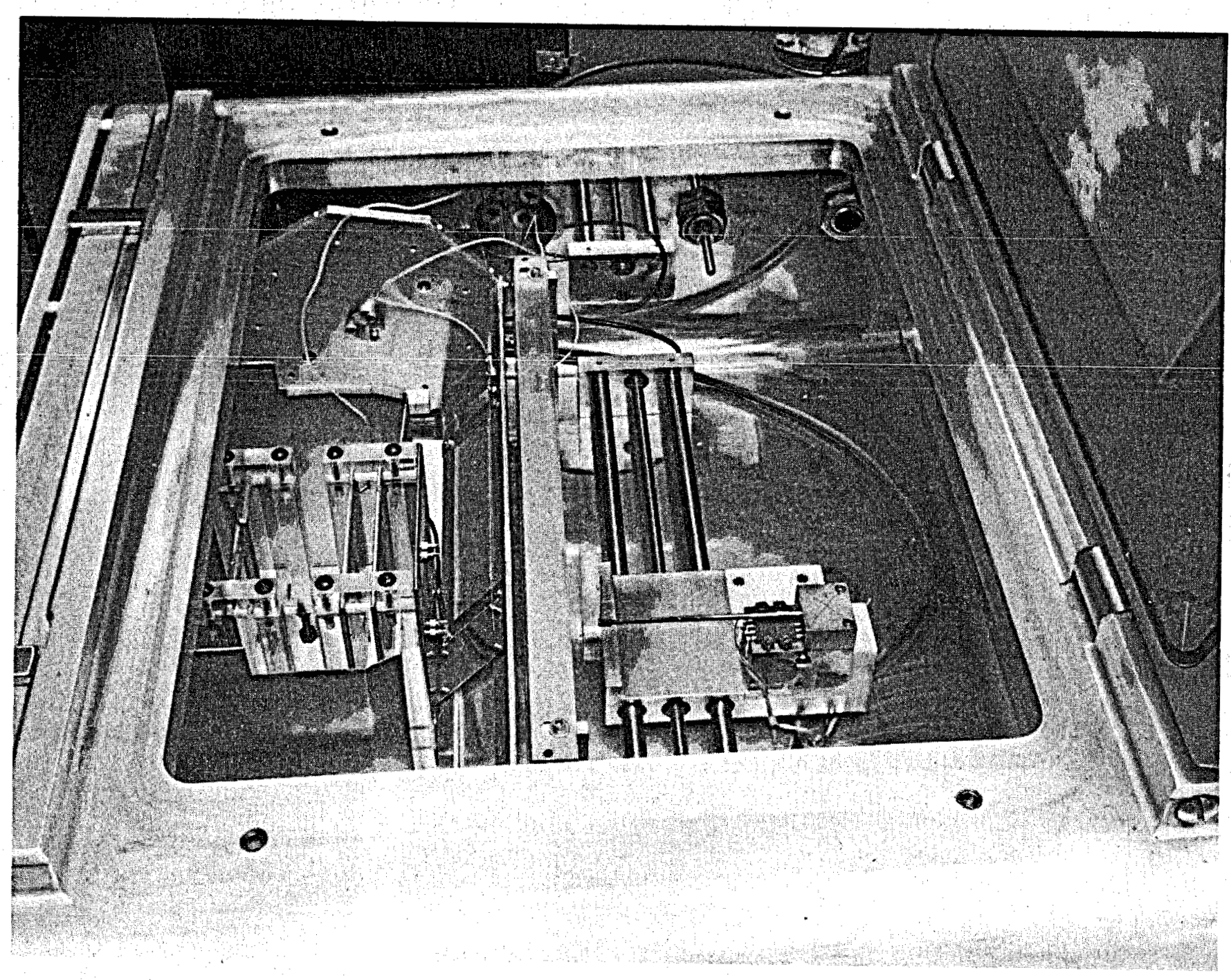

Figure 1. View of the collection-beam-viewing system and the retardation lens through the top opening of the collection chamber. 


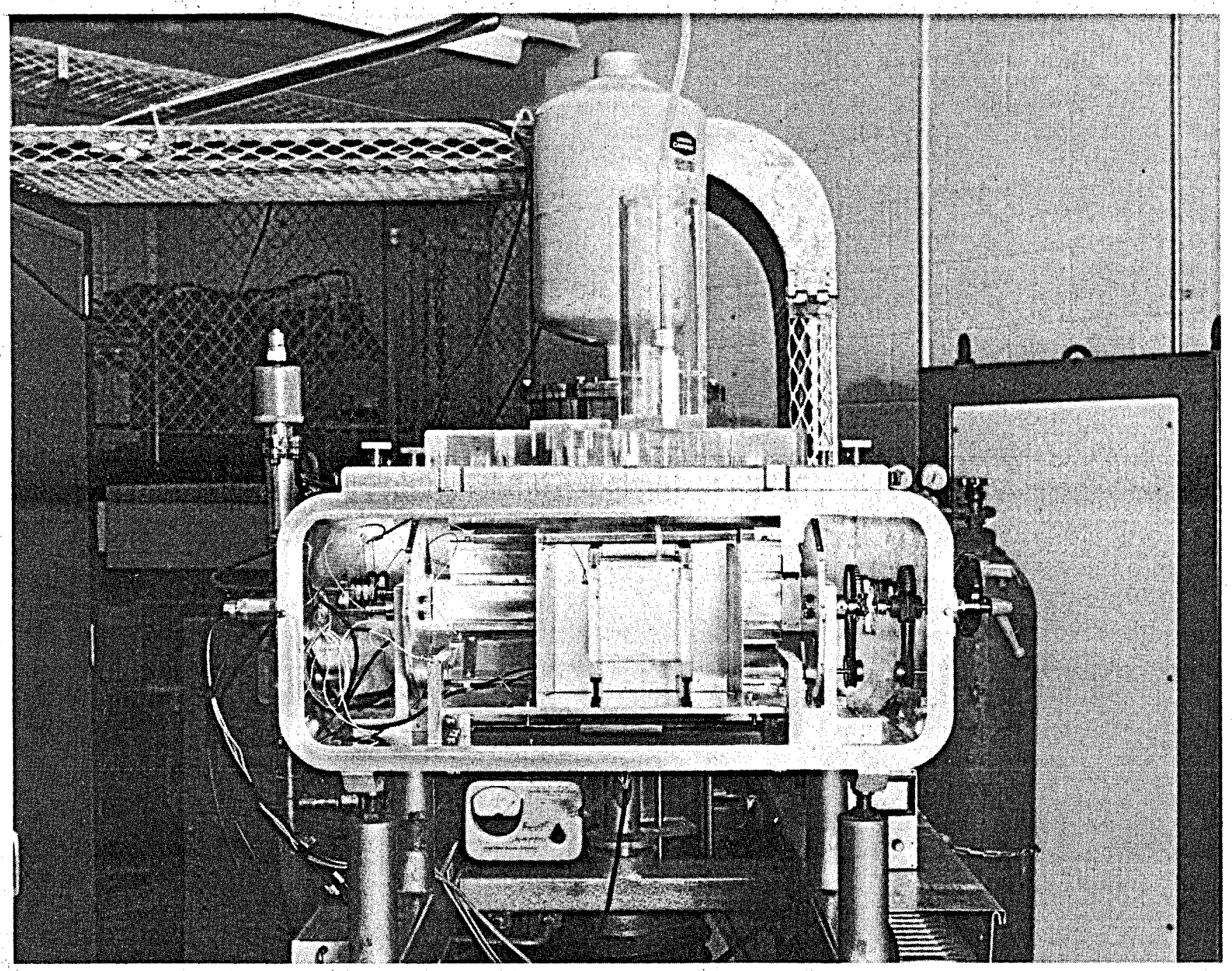

Figure 2. View of the collection-beam-viewing system and the retardation lens through the rear opening of the collection chamber. 


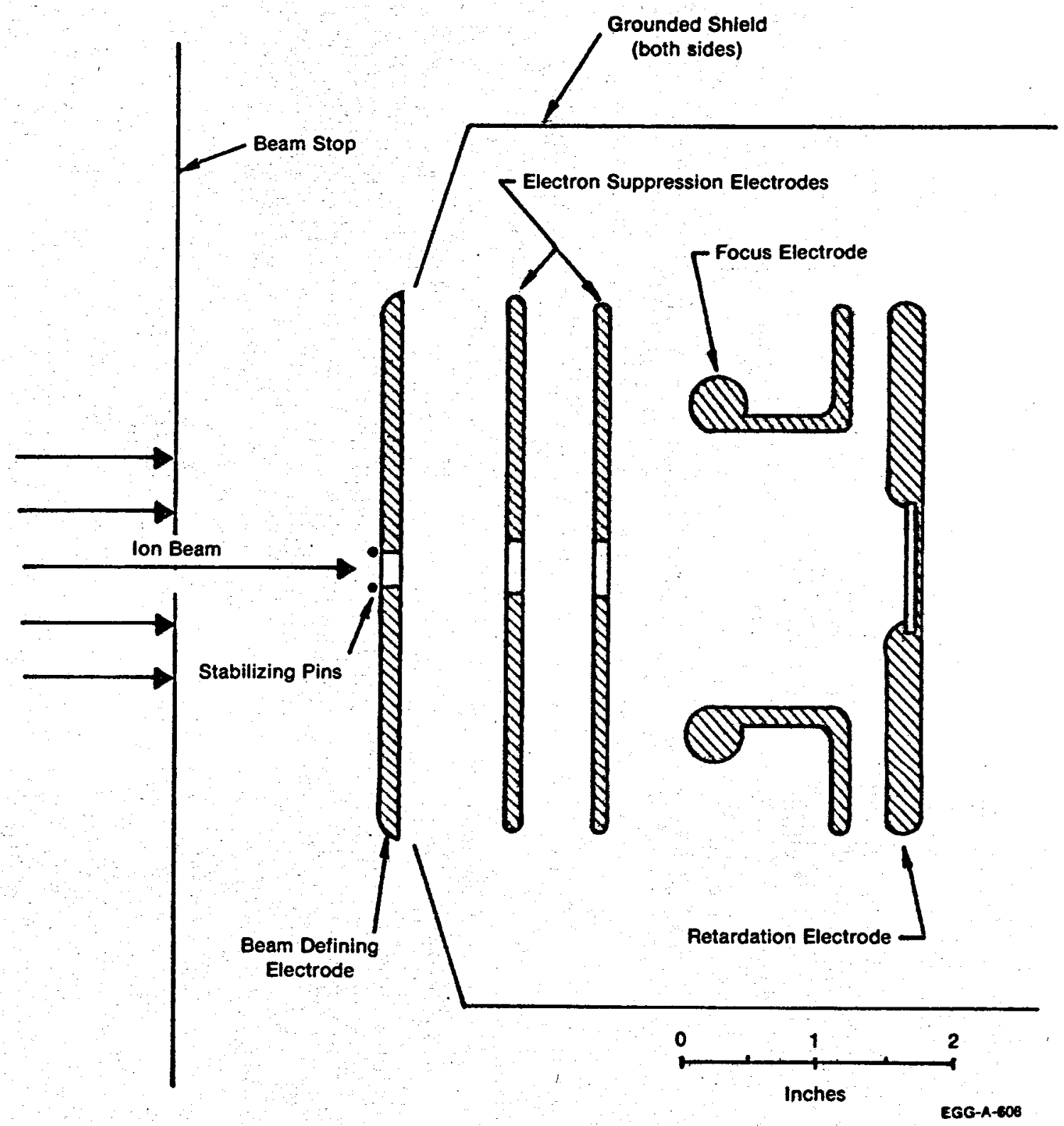

Figure 3. Schematic Illustration of Retardation Lens. 


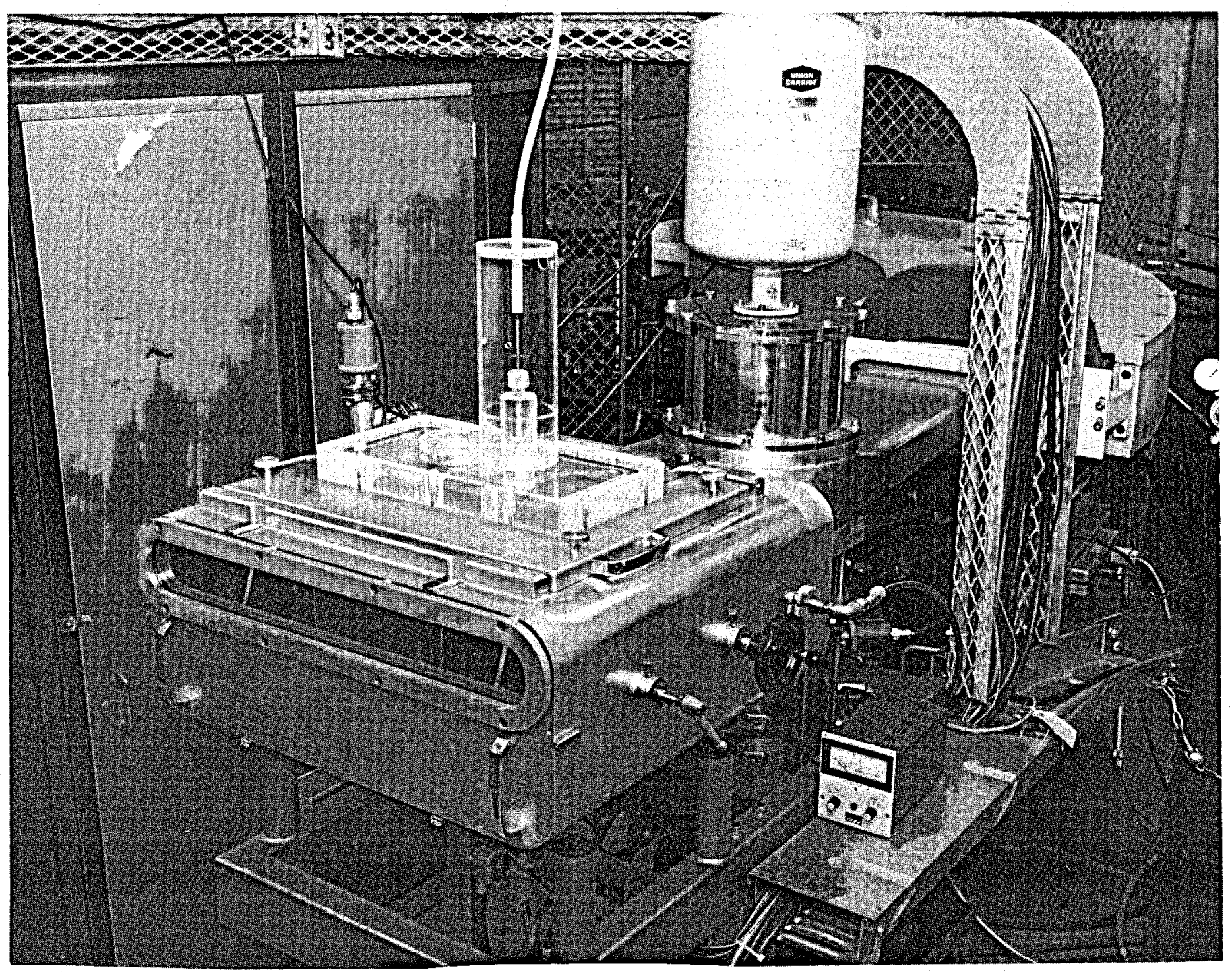

Figure 4. rnotograpn or tne nigh-voltage feedthru as-installea un wie cup ur tne collection chamber. 


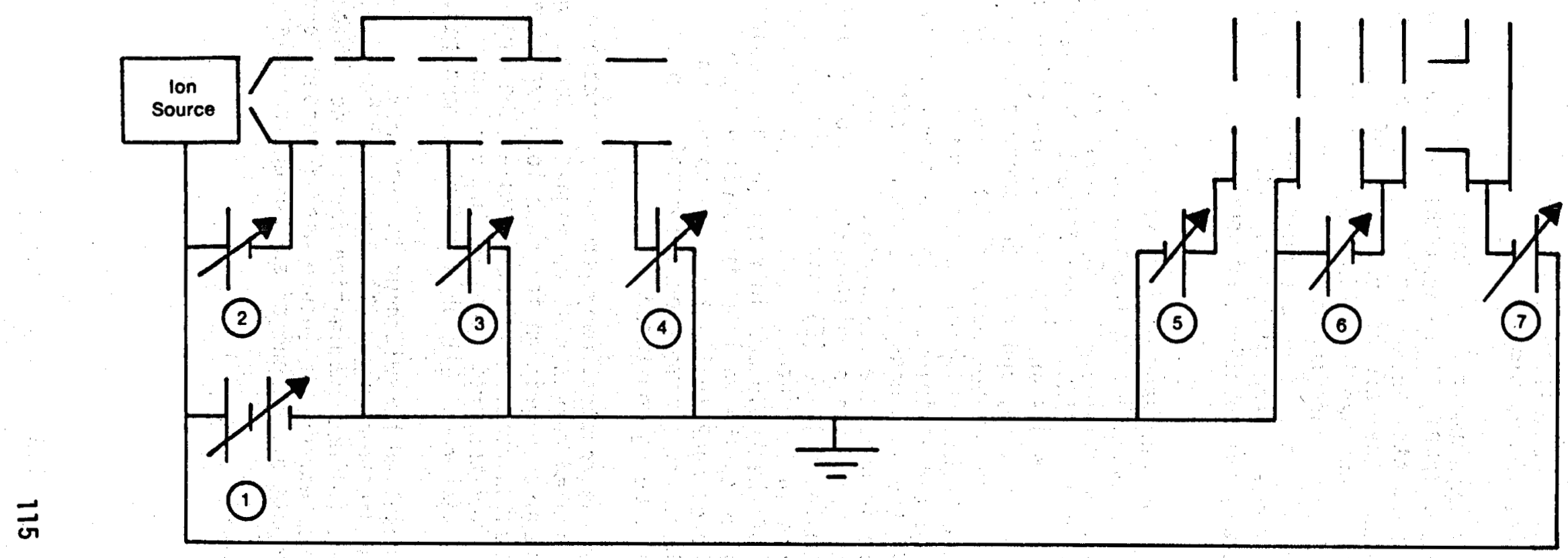

1. Acceleration HV Supply (0-80 kV. positive).

2 - Extraction HV Supply $(0-25 \mathrm{kV}$. negative, floating at acceleration voltage)

3 - Focus HV Supply (0-60 kV $i$ positive).

4- Quadrupole Singlet HV Supply (0-2 kV. positivo).

5 - Beam Stop HV Supply (0-2,kV, positive).

6 - Electron Suppression Electrode HV Supply (0-2 kV, negative).

7 - Focus and Retardation Electrodes HV Supply $(0-5 \mathrm{kV}$, negative. floating at acceleration voltage).

Figure 5. Schematic of Electrical Layout for HV Supplies Used in the Acceleration and Deceleration of an Ion Beam with the Mass Separator. 


\title{
ATR EXPERIMENT LOOP RADIOCHEMISTRY - MONITORING AND REPORTING
}

\author{
J. I. Anderson, E. D. Cadwe 11, P. L. Graham \\ R. A. Morneau, 0. D. Simpson
}

To better understand how an experiment is performing in one of the loops of the Advanced Test Reactor (ATR), it is desirable to know the amounts of the várious fission products, including the gases, and the abundance of the corrosion products that are present. Therefore, state-of-the-art radiochemistry techniques involving $\mathrm{Ge}(\mathrm{Li})$ gamma-ray spectroscopy are now being used to measure the radioactivity in the various loops. Individual isotopic activities are being measured routinely. This new technique replaces the old method which involved chemical separations, gross beta-gamma counting and some $\mathrm{NaI}$ spectrometry.

The new technique involves the analyses of five samples: (1) an unpressurized 540-mi water sample taken before the ion exchange, called BIX, (2) one after the ion exchange, called AIX, (3) a pressurized-water sample before the ion exchange, called PIX, (4) the filtered BIX sample and (5) the filtered AIX sample. The BIX and AIX samples are collected in 1000-ml containers, shaken vigorously, and then transferred to the standard 540-ml bottle. The shaking removes most of the gases making it possible to get closer to the Ge(Li) detector so that the non-gaseous isotopes can be measured with better efficiency. If the samples have too much activity, they are diluted as necessary. Decontamination factors (DF) for the various resin beds are calculated for each radioactive isotope measured by using the simple expression DF = BIX/AIX. The filtered data involving samples (4) and (5) give an indication of the amount of corrosion products in the water. A DF is also calculated which tells the effectiveness of the resin bed in removing particulates. The PIX sample is used for measuring the various radioactive gases. Al1 activities are decay corrected back to the time the sample was drawn. From the above radiochemistry a comprehensive report can be made for each of the experiments being monitored.

While an experiment is in the reactor, many gamma-ray spectra will be measured. All spectra are stored permanently on magnetic tape, and a comprehensive computerized report is written for each loop experiment. The report gives the identification number for each sample, the time and date the sample was drawn, and the activities of the various radioactive isotopes that were measured. The DF's are also reported.

Several advantages have resulted by switching to this new technique for doing the loop radiochemistry: (1) More precise results; (2) fission gases now reported; (3) decontamination factors now obtained for a11 isotopes; (4) permanent records for future use if needed; and (5) published comprehensive report at the end of each reactor cycle for each loop experiment. 


\section{ATR FUEL ELEMENT FISSION LEAK TEST}

\section{D. Simpson}

The economy of operating a nuclear reactor depends significantly on how long a fuel element can be used. One limiting factor on the life of an element is the occurrence of a fission product leak through the fuel plate cladding. When a leak develops, fission products are released into the reactor coolant water and become a potential radioactive effluent. Procedures are needed to establish when a fuel element has become a "leaker." This article summarizes the progress that has been made in establishing fuel leakers and recommends a procedure for determining fuel leakers which might be adapted for ATR fuel elements.

The first radiochemical analysis of soak-test water samples for ATR fue] elements was accomplished about two years ago. A standard 540-ml water bottle, obtained from the water used to soak a fuel element, was counted for gamma-ray activity using a Ge(Li) gamma-ray spectrometer. A study of the data showed that two isotopes, $99 \mathrm{mT} c$ and $132 \mathrm{I}$, produced the majority of the gamma-ray activity. The $99 \mathrm{mT} \mathrm{c}$ is produced from the following reactions:

$$
\begin{aligned}
& 99 \mathrm{Mb} \stackrel{\beta}{\rightarrow} 99 \mathrm{Mo} \stackrel{\beta}{\rightarrow} 99 \mathrm{mTc} \\
& 98 \mathrm{Mo}(\mathrm{n}, \gamma) 99 \mathrm{Mo} \stackrel{\beta}{\rightarrow} 99 \mathrm{mTC}
\end{aligned}
$$

\section{Direct Fission}

In Case (1) the $99 \mathrm{mTC}$ is formed from the fission product ${ }^{99} \mathrm{Nb}$ and in Case (2) the $99 \mathrm{mT} \mathrm{c}$ is formed from the $(n, \gamma)$ reaction on $98 \mathrm{Mo}$, a corrosion product as well as a fission product. Since not all of the $99 \mathrm{mTc}$ is a fission product, it was rejected as a fission leak indicator. The ${ }^{132} \mathrm{I}$ isotope is produced mainly from the following reactions:

$$
\text { Dịrect Fission }
$$

$$
132 \mathrm{Te}+132 \mathrm{I}
$$

The ${ }^{132} \mathrm{Te}$ is a direct fission product and should be a good indication of a fission leak. However, ${ }^{132} \mathrm{Te}$ is not usually observed in the soak test water sample. The half-life of ${ }^{132} \mathrm{Te}$ is 78 hours whereas 132I has a half-life of 2.3 hours. It has been found best to wait several days after reactor shutdown before starting soak tests in order to allow the primary ${ }^{132}$ I to decay away. The ${ }^{132}$ I that is counted in the soak test is then coming from the number (2) reaction above. All indications are that the ${ }^{132} \mathrm{Te}$ remains in the fuel (for all 
the elements tested thus far), or at least attached to the element until it beta decays to the ${ }^{132}$ I at which time the jodine is released into the water.

In order to compare the strengths of the various leakers, the ${ }^{132}$ I activity. is decay corrected back to the time when the water sample is drawn, and then the activity of its parent, ${ }^{132} \mathrm{Te}$, is decay corrected back to the time of the reactor shutdown. If the leakers are counted within a day of one another, these corrections are satisfactory.

A11 evidence shows that the activity of 132 I is a good indicator for a fission leak. However, studies need to be made to verify some of the existing data and to set up test criteria that wili heip isolate fuel elements that are bad leakers. This would basically require that at least half and preferably all of the ATR fuel elements be soak tested at the end of each shutdown. If this were done over a period of about one year, enough data could be collected to establish proper testing procedures. 


\section{NASA COMBINED PULSED NEUTRON EXPERIMENT FOR BULK ELEMENTAL ANALYSIS}

J. W. Mandler, E. B. Nieschmidt, L. O. Johnson, E. E. Owen, E. W. Killian

One objective of the space program is to obtain information concerning the elemental make-up of extraterrestrial bodies. Since samples will not be available in the near future, remote analysis of the surfaces of extraterrestrial bodies is required. The Combined Pul sed Neutron Experiment (CPNE) is currently being developed under NASA contracts to provide elemental information remotely. This development is a joint effort involving several laboratories. INEL's participation includes development of the computer and logic circuits (both hardware and software) and electronics together with integration and testing of the component parts of the system (i.e., neutron generator, detector, electronics, computer) and coordination of a demonstration of the prototype system.

The CPNE utilizes four different neutron analytical methods neutron capture gamma-ray analysis, inelastic neutron scattering, neutron activation analysis, and neutron die-away. The gamma rays produced in the inelastic scattering, capture, and activation processes are emitted in different time domains relative to the time of the incident fast neutrons. It is therefore possible to separate them by time discrimination. The CPNE employs a pulsed source of 14-MeV neutrons and time-gated spectral measurements to separate these radiations. The $A D C$ is gated on for three different intervals: 1) during the neutron burst to collect gamma-rays from the inelastic scattering of neutrons, 2) just after neutron burst (delayed enough to allow the source neutrons time to thermalize) for collection of the capture gamma-ray spectrum, and 3 ) just before each neutron burst (after all the capture gamma rays have decayed away) to collect the activation gamma-ray spectrum. The spectra that result can be used to yield information concerning the bulk elemental composition of a planetary surface.

All the component parts of the protype system were completed during the past year and the components fabricated elsewhere (e.g., neutron generator, detector, probe hardware) were shipped to INEL for integration into the system. The system consists of a neutron generator, NaI (TI) gamma-ray detector and electronics, He neutron detector and associated electronics, a control unit, and a PDP-11 computer.

Software for the CPNE computer was written to allow operation of the neutron generator in real time. Functions implemented by this software include (1) oscilloscope display of selected data regions with linear or semi-log scaling, (2) clock timing of runs, (3) experiment mode selection, and (4) neutron generator control. The software was written in assembly language and operates in $4 \mathrm{~K}$ of core.

The component parts were assembled and tested at INEL and the software was checked out and debugged. Tests were then run to determine the operating characteristics of the system. A series of experiments 
were performed to determine the optimum neutron generator pulse width and replenisher current for each of the two modes of operation. In the high pulse rate mode (for collection of inelastic gamma-ray spectra and epi-thermal neutron die-away information) a low neutron output per pulse is required while in the low pulse rate mode (for collection of capture gamma-ray and activation spectral a high neutron output per pulse is needed. Finally, satisfactory spectra were obtained in all modes of operation using a large iron and concrete sample.

Future plans consist of formally demonstrating the CPNE system to NASA. This demonstration will take place at Goddard Space Flight Center sometime later this year. 
GAMMA-GAMMA COINCIDENCE SPECTROMETER

W. R. Myers, G. 0. Engl ish, V. D. Klingler, C. L. Schull

Gamma-ray two-parameter coincidence measurements are utilized in nuclear structure studies to establish the time dependence of nuclear decay, to correlate the relation of nuclear energy levels, and to produce spectra of high purity. To be of practical value gamma coincidence spectra must be acquired with high resolution, typically 4000 channels per parameter using typical $\mathrm{Ge}(\mathrm{L} i)$ detectors. The storage capacity required for such a spectrum is 16 million words of approximately 20 bits.

The traditional method of acquiring such spectra utilizes magnetic tape to store coincidence events by recording the values of both parameters for each event. A typical nuclear structure experiment will produce from 20 to 50 full (IBM-compatible) magnetic tapes which must eventually be sorted to reconstruct the two-parameter spectrum. This buffer-tape or (channel, channel) recording technique is relatively simple and inexpensive to implement in the data acquisition stage, but becomes very expensive in terms of manpower and computer time in the sorting and analysis stages.

In order to reduce the cost and provide increased convenience to the experimenters, a real-time coincidence sorting system is being constructed utilizing a PDP-8/e minicomputer with 32 kwords of core memory and a 40 Mword moving-head disk. Use of the real-time-sorting coincidence system will obviate the need for large computer system support except in the final stages of data analysis, will lead to improved data quality in enabling live display and timely monitoring of data, and will reduce the time required in attending the experimental system. 
U.

BLANK PAGE

$\mathcal{U}$ 
NUCLEAR DATA COMPILATION AND EVALUATION 


\title{
A COMPUTERIZED FILE OF EVALUATED RADIONUCLIDE DECAY DATA
}

\author{
R. L. Bunting, C. W. Reich, R. G. Helmer \\ M. A. Lee*, M. H. Putman
}

The INEL Decay Data Master File (DDMF) ${ }^{(1)}$ has been expanded to include evaluated decay scheme data for some 190 radionuclides. This master file is now the working data base for several sub-files including ENDF/B $(2,3)$ and NRC $(4)$. The DDMF has al so been used to update our GAUSS-VI (5) nuclide identification library and create similar identification libraries for other computer based gamma-ray spectroscopy systems $(6)$.

The effort to maintain traceability in this file is continuing. New sources of evaluated data from which DDMF entries have been based include recent ORNL $(7,8)$ and $L B L(9)$ communications. A large portion of the DDMF (46 entries) is based on a recent INEL actinide evaluation (10).

Computer processing codes which utilize the evaluated data base to calculate secondary spectra have been completed and are now being tested. These secondary quantities (average beta energy, conversion electron and $x$-ray spectra) are edited into the file at processing time to give a more complete picture of the average energy released per decay.

Foyr fables of $E_{B a v e} / E_{B \max }$ have been calculated using the

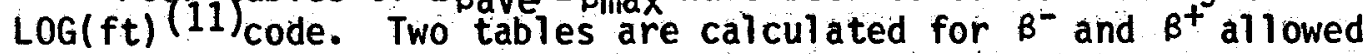
transitions and two additional tables for the first forbidden unique $B^{-}$and $B^{+}$transitions. Each table includes ten $Z$ values and 16 energyvalues for each $Z$. The linear $Z$ range spans $10 \leq Z \leq 100$ in increments of 10 and the $E_{B \max }$ range spans $10 \mathrm{keV} \leq E_{B \max } \leq 1 \overline{0} \mathrm{MeV}$ in equally spaced increments on a logarithmic scale. The correct $\overline{r a t i o}$ of $E_{B a v e} E_{B \max }$ is found by a semi-log cubic-spline interpolation procedure in both $Z$ and $E_{B \max }$.

Conversion electron and $x$-ray spectra are calculated from known gamma-ray multipolarities and electron capture branching ratios. Since the conversion electron (CE) spectra may become quite complex, not all CE data are listed on the file. The CE average energy calculation is done before any intensity selection is applied to the output list. The CE selection criteria now applied require that a CE line be moved to the energy-ordered output list (a) if the CE intensity is greater than $1 \%$ of the most intense CE in the list or (b) if the CE in question contributes at least $1 \%$ to the CE average energy.

The $x$-ray spectra contain energy and intensity data for the $K_{\alpha_{1}}$ $K_{\alpha_{2}}, K_{B_{1}}, K_{B_{2}}$ and $L$ as well as annihilation data from $\beta^{+}$decay. Tables of atomic constants utilized in the $x$-ray calculations were compiled from several references.

\footnotetext{
* Summer Faculty Participant from Wartburg College, Waverly, Iowa
} 
Internal conversion coefficients used in the above calculations can be obtained from the known gamma-ray multipolarities or may be entered in the file as known experimental data. In the case where known multipolarities are used, two-component mixing can be specified. The conversion coefficients are interpolated using standard tables.

All secondary processing is done "in-line" on an IBM $360 / 75$ computer at INEL. The output data consist of an image of the input master with all secondary data merged into the output file. All tabular data (excluding internal conversion coefficient interpolation data) are included in the main processing codes to reduce the number of auxilary input files.

(1) R. L. Bunting, R. G. Helmer, C. W. Reich "Decay Data Master File at INEL", ANCR-1255, UC-2, 27 February 1976.

(2) C. W. Reich, R. G. Helmer, and M. H. Putman, "Radioactive-Nuclide Decay Data for ENDF/B", ANCR-1157 (ENDF-210) August, 1974.

(3) C. W. Reich, R. G. Helmer, and R. L. Bunting, "Expanded Data Content for the Radioactive-Nuclide Decay Data for ENDF/B", ANCR-1255, UC-2, 25, February 1976.

(4) R. L. Bunting, N. C. Dyer, M. A. Lee and M. H. Putman, "A File of Evaluated Decay-Scheme Data for NRC". This report.

(5) J. E. Cline, M. H. Putman, and R. G. Helmer, "Gauss-VI, A Computer Program for the Automatic Batch Analysis of Gamma-ray Spectra from Ge(Li) Spectrometers", ANCR-1113, UC-32, June 1973.

(6) E. W. Killian, Internal Report.

(7) ORNL- Nuclear Data Project, "ENSDF, Evaluated Nuclear Structure Data File" Private Communication, April 1976.

(8) ORNL- Nuclear Data Project, M. J. Martin," A Standard Decimal Format for MEDLIST Output", Private Communication, January 1976. Principally ORNL-5114 computerized file.

(9) C. M. Lederer, Selected evaluations from Table of Isotopes, Private Communication, 1976.

(10) C. W. Reich, "A File of Actinide-Isotope Decay Data for ENDF/B", this report. See also, C. W. Reich, "Status of Beta- and Gamma-Decay and Spontaneous-Fission Data from Transactinium Isotopes", Review paper 87, IAEA Advisory Group Meeting on Transactinium Isotope Nuclear Data, Karlsruhe, FRG November 3-7, 1975. 
(11) N. B. Gove and M. J. Martin, "LOG-f Tables for Beta Decay" Nuclear Data Tables 10, 205 (1971) and associated computer code, Private Communication, January 1976.

(12) M. J. Martin, Atomic Constants from MEDLIST, Private Communication, January 1976.

(13) J. A. Bearden, A. F. Burr, "Reevaluation of X-Ray Atomic Energy Levels", Reviews of Modern Physics, 39 . 125 (1967).

(14) W. Bambynek et al. "X-Ray Fluorescence Yields, Auger, and Coster-Kronig Transition Probabilities", Reviews of Modern Physics, 44, 716 (1972).

(15) S. I. Salem, S. L. Panossian, and R. A. Krause, "Experimental K and L Relative X-Ray Emission Rates", Atomic Data and Nuclear Data Table, 14, 91 (1974).

(16) "Internal Conversion Coefficients" Editor K. Way, Reprinted in Atomic and Nuclear Data Reprints, Academic Press, New York and London 1973. 


\section{A FILE OF ACTINIDE-ISOTOPE DECAY DATA FOR ENDF/B}

\section{W. Reich}

In recognition of the need for a commonly available base of evaluated nuclear decay data for use in a variety of reactor-related applications, the scope of the Evaluated Nuclear Data File (ENDF/B) was expanded several years ago to include such information. The first general category of nuclides whose detailed decay data were incorporated into ENDF/ $B$ were the fission-product nuclides; these data appeared in the Fission-Product File of Version IV of ENDF/B. The increasing importance of the actinides in many areas of reactor technology has led to a decision to produce an Actinide File to be included in ENDF/B-V. We have had the responsibility for preparing the decay data for this file.

The Actinide File contains nuclear data for 46 isotopes and isomeric states. The following is a listing of these 46:

$$
\begin{aligned}
& \text { U }-232,233,234,235,236,237,238,239 \\
& \mathrm{~Np}-236,236 \mathrm{~m}, 237,238,239 \\
& \mathrm{Pu}-236,237,238,239,240,241,242,243,244 \\
& \text { Am - 240, 241, 242, 242m, 243, 244, 244m } \\
& \text { Cm - 241, 242, 243, 244, 245, 246, 247, 248, } 249 \\
& \text { Bk - 249, } 250 \\
& \text { Cf }-249,250,251,252,253 \\
& \text { Es - } 253
\end{aligned}
$$

These decay data have been worked up in the expanded data content adopted for the Version-V ENDF/B decay data.

(1) C. W. Reich, R. G. Helmer and M. H. Putnam, Radioactive - Nuclide Decay Data for ENDF/B, ANCR-1157, (August 1974).

(2) C, W. Reich, R. G. Helmer and R. L. Bunting, Nuclear Technology Division Annual Progress Report for Period Ending June 30, 1975, ANCR-1255 (February 1976), 25-26. 
EVALUATED DECAY-SCHEME DATA FOR ILRR

R. G. Helmer, R. C. Greenwood

The Interlaboratory LMFBR Reaction Rate (ILRR) program was

organized for the purpose of making a series of precise measurements of rates of neutron-induced reactions for a set of well-defined and reproducible neutron spectra. For many of these reactions, the rates are determined by measurements of the gamma-ray spectra from the decay of the radioactive reaction products. A goal of the ILRR program is to measure fission reaction rates by means of gamma-ray spectrometry with accuracies of about $\pm 5 \%$ at the $95 \%$ confidence level.

The computation of the reaction rates depends on two parameters of the nuclear decay scheme; these are the half-life and the absolute gamma-ray intensity. For the ILRR program, it is necessary that these parameters and their errors be as accurate and consistent as possible. Therefore, a series of regular reviews of the decay schemes of interest has been undertaken. Two earlier reviews were noted in Reference 1-3. The results of the most recent evaluation are given in Table I for selected gamma-rays. In this literature review, we have included original references and published evaluations available as of November 1975.

(1) R. G. Helmer and R. C. Greenwood, Nuclear Technology Division Annual Progress Report for Period Ending June 30, 1973, ANCR1129, (1973) p. 20.

(2) R. G. Helmer and R. C. Greenwood, Nuclear Technology Division Annual Progress Report for Period Ending June 30, 1974, ANCR 1177, (1976) p 105.

(3) R. G. Helmer and R. C. Greenwood, Nucl. Tech. 25, 258 (1975) 
TABLE I

Summary of ILRR Decay Scheme Parameters

Half-life

Gamma Energy

(keV)

Gamma Intensity

(\%)

${ }^{24} \mathrm{Na}$

$15.00(2) \mathrm{h}$

$1368.599(29)$

2753.965(56)

99.993(2)

99.873(6)

$27 \mathrm{Mg}$

$9.462(12) \mathrm{m}$

$843.75(3)$

$1014.44(4)$

$71.7(5)$

$28.3(5)$

${ }^{46} \mathrm{Sc}$

$83.9(3) d$

$889.253(16)$

$1120.521(19)$

$99.984(6)$

$99.987(6)$

${ }^{47} \mathrm{SC}$

$3.40(3) \mathrm{d}$

$159.381(15)$

$69.0(25)$

${ }^{48} \mathrm{SC}$

$43.8(1) h$

$983.4(2)$

$1037.4(2)$

$99.987(2)$

$1311.8(4)$

97.5(3)

99.992(2)

${ }^{51} \mathrm{Cr}$

$27.700(10) d$

320.076 (5)

9.83(14)

${ }^{54} \mathrm{Mn}$

$312.5(5) d$

$834.827(21)$

99.97(2)

${ }^{58} \mathrm{Co}$

$70.85(15) d$

$810.753(20)$

$99.44(1)$

${ }^{59} \mathrm{Fe}$

$44.6(1) d$

$1099.228(19)$

$1291.568(23)$

$56.1(10)$

43.6(10)

${ }^{60} \mathrm{Co}$

$5.27(2) \mathrm{yr}$

$1173.208(20)$

$1332.464(28)$

99.86(2)

$99.980(3)$

${ }^{64} \mathrm{Cu}$

$12.702(4) h$

$511.00^{\mathrm{a}}$

$38 .(2)$

$95 \mathrm{Zr}$

$64.1(3) d$

$724.179(13)$

$756.710(18)$

$44.1(5)$

$54.6(5)$

$97 \mathrm{Zr}$

$16.88(6) \mathrm{h}$

743.3(1)

$92.9(3)$

$103 \mathrm{Ru}$

$39.43(10) \mathrm{d}$

497.08(1)

89. (1)

$106 \mathrm{Ru}$

$368.2(12) d$

$106 \mathrm{Rh}$

$30.0(2) \mathrm{s}^{\mathrm{e}}$

$511.8(2)$

$20.5(2)^{b}$

$115 \mathrm{~m}$ In

$4.486(4) h$

$336.23(5)$

$45.9(1)$

$116 \mathrm{~m}_{\mathrm{In}}$

$54.2(1) \mathrm{m}$

$1293.54(15)$

$84.8(5)$

$132 \mathrm{Te}$

$77.9(5) \mathrm{h}$

$228.16(6)$

89. (5)

$30.03(15) \mathrm{yr}$

$661.647(12)$

$85.3(4)$ 
Table I (continued)

$$
\text { Half-life }
$$

$140 \mathrm{Ba}$

$140 \mathrm{La}$

$141 \mathrm{Ce}$

$143 \mathrm{Ce}$

$144 \mathrm{Ce}$

$144 \mathrm{Pr}$

196 Au

$198 \mathrm{Au}$

$239 \mathrm{~Np}$
$12.789(6) d$

$40.26(2) h^{\mathrm{C}, e}$

$32.50(7) d$

$33.0(2) \mathrm{h}$

$284.4(4) d$

$17.28(5) \mathrm{m}^{e}$

$6.1(1) d$

2.6956(10) d

$2.355(4) d$
Gamma Energy

(keV)

537.35 (5)

$1596.18(5)$

$145.440(3)$

$293.26(2)$

$133.53(3)$

696.49(2)

$1489.15(5)$

$2185.70(6)$

$355.73(5)$

411.794(7)

$277.60(3)$
Gamma Intensity

(\%)

$24.4(3)$

$95.40(8)$

49. (2)

47. (4)

$11.0(2)$

$1.342(13)$

$0.279(3)$

$0.700(10)$

$87.7(20)$

$95.52(5)$

$14.3(2)$

${ }^{2}$ This is the value of the electron rest mass, $m_{0} c^{2}$. The observed annihilation photon energy may be lower.

b There may be some experimental problems in the use of this gamma ray due to the presence of $511.0-\mathrm{keV}$ photons from positron annihilation.

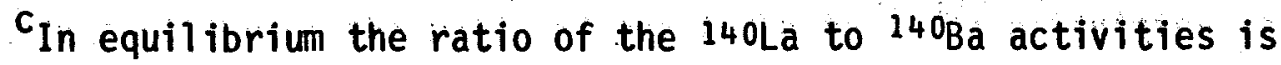

$$
T_{\frac{1}{2}}(\mathrm{Ba}) /\left[T_{\frac{1}{2}}(\mathrm{Ba})\right]-T_{\frac{1}{2}}(\mathrm{La}) 1.15097(12) \text {. }
$$

These isotopes will normally occur with the parent half-life. 


\section{A FILE OF EVALUATED DECAY-SCHEME DATA FOR NRC}

R. L. Bunting, N. C. Dyer, M. A. Lee*, M. H. Putnam

A data file of recently evaluated radionuclide decay-scheme data is being prepared for the Nuclear Regulatory Commission. This file contains data on 111 nuclides and is a subset of the INEL Decay Data Master File (DDMF) $(1)$.

The data file is tailored to satisfy the radionuclide decay data needs for the quantitative monitoring of radionuclides associated with nuclear power $\mathrm{plant}$ operations and the calculation of radiation doses from nuclear power plant effluents.

The report contains the following data classifications.

1. Nuclide Identification

2. Half-life

3. Decay Modes $\left(\beta^{-}, \beta^{+}, \varepsilon, \alpha, I T\right)$

a. Associated branching ratios

4. Average Energy per Disintegration (electron, photon, $\alpha$ )

5. Gamma-ray Intensity Normalization

6. Gamma-ray Listing

a. Energy

b. Relative and/or absolute gamma-ray intensity

c. Assay flags

The associated uncertainties in the reported values are also included.

The average energy per disintegration is calculated for three categories, electron, photon and alpha. The average electron energy includes contributions from the beta spectrum as well as conversion and Auger electrons. The latter two contributions are processed into the file from known $\gamma$-ray multipolarity and electron-capture data. The average photon energy includes the contributions of the $\gamma$-spectrum and $x$-rays and annihilation radiation from other decay processes. Nuclear recoil effects are considered in calculating the average $\alpha$-energy.

\footnotetext{
* Summer Faculty Participant from Wartburg College, Waverly, Iowa
} 
The $\gamma$-spectra from the nuclides of interest for this file are frequently complex. In order to present a meaningful $\gamma$-ray listing, the output list is subjected to the following criteria. The output can be controlled by the value of the $\gamma$-ray intensity code. This code indicates the height of the $\gamma$-ray above background in a single source spectrum. A summary of the intensity code is given below.

\begin{tabular}{|c|c|}
\hline Intensity Code & $\begin{array}{l}\text { Peak-to-Background } \\
\text { Ratio (PBR) } \\
\end{array}$ \\
\hline 1 & $\mathrm{PBR} \geq 10$ \\
\hline 2 & $10>\mathrm{PBR} \geq 5$ \\
\hline 3 & $5>\mathrm{PBR} \geq 2$ \\
\hline 4 & PBR $<2$ \\
\hline
\end{tabular}

Gamma-rays with intensity codes 1 or 2 are output for the NRC file. For each $\gamma$-ray emitting nuclide on the file, one or two $\gamma$-rays have been flagged by the compilers as the "assay $\gamma$-rays." "These "assay $\gamma$-rays" will uniquely identify the presence of the nuclide in question. Consideration has been given to possible interference of assay assignments in known power plant radionuclide samples, e. g., primary coolant samples.

Several summary appendices will be included in this report. These summaries will contain various sorts of the $E_{\gamma}, I_{\gamma}, T_{\frac{1}{2}}$ and Identification records to further aid the user of these data.

(1) R. L. Bunting, C. W. Reich, R. G. Helmer, M. A. Lee, M. H. Putman, "A Computerized File of Evaluated Radionuclide Decay Data", this report. 


\section{B. APPLIED REACTOR PHYSICS}


EXPERIMENTAL MEASUREMENTS 


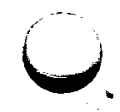

\section{BLANK PAGE}


Measurement of integral-capture cross sections for individual isotopes of selected fission-product, structural-material, controlmaterial, and actinide elements in fast and intermediate energy neutron fields are of importance to the development of advanced fast reactor systems and to safe management of their waste products. These nuclear data are used as a reference data base for direct application in reactor design and analysis, for data-testing differential cross section data files (ENDF/B), and, in conjunction with optical model calculations and the results of differential measurements, for the generation of the ENDF/B energy dependent cross sections. In particular, these data have a direct bearing on assessing the fission product inventory and poison effect in a fast reactor, the capture worth of potential control materials, and the actinide inventory (especially the buildup of spontaneous fission neutron sources). Some of these data are also used to establish a reliable burnup monitor for advanced fastreactor fuels.

This report summarizes the present status of an irradiation experiment which is $\mathrm{pl}$ anned for FY 77. The experiment involves a row 8 EBR-II irradiation of selected highly-enriched fission-product and control-material isotopes to a fiuence of approximately $5 \times 10^{21} \mathrm{n} / \mathrm{cm}^{2}$, the post irradiation measurements of the products of neutron absorption in each sample, and the determination of the capture cross section for each isotope. Multiple samples of the isotopes listed in Table I and suitable dosimetry sets of spectrum monitors as listed in Table II comprise the irradiation package.

TABLE I

ENR ICHED ISOTOPES FOR CAPTURE CROSS-SECTION MEASUREMENTS

\begin{tabular}{lccc}
\hline I sotope & Application & Isotope & Appl ication \\
\hline $143_{\mathrm{Nd}}$ & $\mathrm{FPP}^{1}, \mathrm{BM}^{2}$ & $147_{\mathrm{Sm}}$ & $\mathrm{FPP}, \mathrm{BM}$ \\
$144_{\mathrm{Nd}}$ & $\mathrm{BM}$ & $149_{\mathrm{Sm}}$ & $\mathrm{FPP}, \mathrm{BM}$ \\
$145_{\mathrm{Nd}}$ & $\mathrm{FPP}, \mathrm{BM}$ & $151_{\mathrm{Eu}}$ & $\mathrm{CME}^{3}$ \\
$146_{\mathrm{Nd}}$ & $\mathrm{BM}$ & $152_{\mathrm{Eu}}$ & $\mathrm{CME}$ \\
$148_{\mathrm{Nd}}$ & $\mathrm{BM}$ & $153_{\mathrm{EU}}$ & $\mathrm{CME}$ \\
$150_{\mathrm{Nd}}$ & $\mathrm{BM}$ & $154_{\mathrm{EU}}$ & $\mathrm{CME}$
\end{tabular}

${ }^{1}$ Fission Product Poison

${ }^{2}$ Burnup Method

${ }^{3}$ Control Material Evaluation 
Experiment capsules containing identical sample-dosimetry sets are located at the core midplane and at a position 13.78 in $(35 \mathrm{~cm})$ above the midplane.

TABLE II

MATER IALS PER DOSIMETRY SET

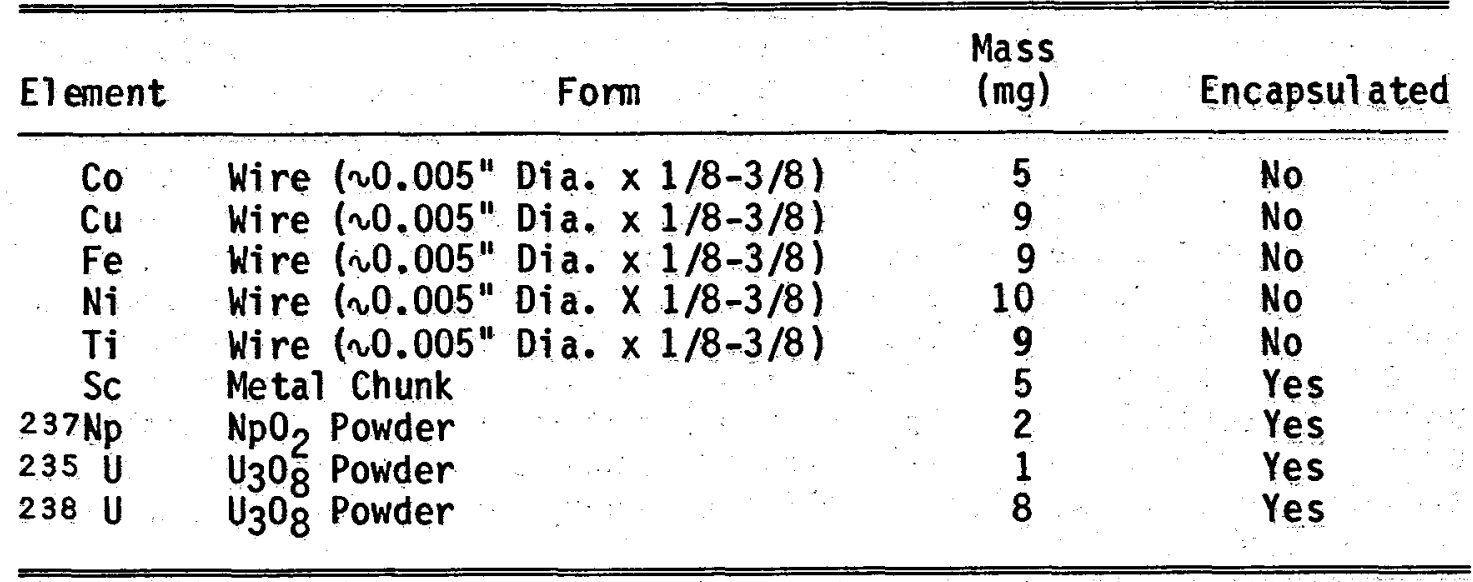

1. Sc, ${ }^{237} \mathrm{~Np}, 235 \mathrm{U}, 238 \mathrm{U}$ materials are encapsulated in stainless steel ampoules $\left(.065^{\prime \prime}\right.$ dia. $\left.\times 5 / 8 "\right)$.

Isotope dilution mass spectrometry and gamma spectrometry will be used for the post-irradiation measurements. These data, in conjunction with the spectral information obtained from measurements of the dosimetry materials, will be used to compute the capture cross sections as a function of spectral index for the various isotopes. These cross sections will contribute to the evaluation of the fission-product poison effect, the evaluation of the long-term capture worth of $\mathrm{Eu}_{2} \mathrm{O}_{3}$ as a control material, and the development of a reliable burnup monitor for advanced fast reactor systems.

$\theta$

Detailed design for this experiment is presented in the drawings listed in Table III. Briefly, the experiment hardware consists of two $B-7 A$ capsules, designated $1-13-1,1-13-2$, wi th five dummy $B-7 A$ pins located in the remaining positions in a Mark $B$ subassembly. Internal components of each capsule are three spacers, a spring, and four experiment capsules containing the samples (specimens) to be irradiated. Each experiment capsule contains five sample capsules. Each sample capsule contains four specimens of the highly enriched isotopes or a dosimetry set. A description of the capsule contents is detailed in Table IV. Illustrated in Figure 1 is the loading configuration for the B-7A capsules and the placement in the Mark B subassembly for irradiation in a-row 8 position in EBR-II.

A data package including a description of the experiment and a safety analysis was prepared and distributed for review as per the EBR-II Guide. The major part of the safety analysis included detailed thermal analyses to determine the anticipated thermal operating characteristics of the experiment for normal and abnormal reactor operating conditions. 
These thermal analyses were made using the SIMIR code which is a twodimensional steady state and transient heat conduction computer code. Internal temperature distributions were computed for a cylindrical model which simulated a representative B-7A capsule.

TABLE III

DRAWING LIST

\begin{tabular}{lll}
\hline DWG No. & Company & \multicolumn{1}{c}{ Title } \\
\hline 406991 & ANC & EBR-II Sample Capsule Assembly and Details \\
406992 & ANC & EBR-I I Experiment Capsule Assembly and Details \\
406993 & ANC & EBR-I I Spacer Detail \\
406994 & ANC & EBR-II FBR Physics Constants B-7 Capsule \\
& & Assembly and Detail \\
$H-3-37403$ & HEDL & B-7A Top Endcap, Vented Type \\
$H-3-37429$ & HEDL & B-7A Top Endcap, Vented Type, Assembly \\
$H-3-37375$ & HEDL & B-7A Bottom Endcap \\
$H-3-38920$ & HEDL & Solid Dummy B-7A pin
\end{tabular}

Fabrication of the sample capsule and experiment capsule hardware has been completed. Arrangements have been made to have fabrication of the final B-7A capsule containment and necessary QA testing with appropriate documentation done at the Hanford Engineering Development Laboratory in Richland, Washington.

Approximately $2 \mathrm{mg}$ lots of highly-enriched $143 \mathrm{Nd}, 145 \mathrm{Nd},{ }^{147} \mathrm{Sm}$ and ${ }^{149} \mathrm{Sm}$ sample material were made with the isotope separator. Source material for the separation was enriched previously at ORNL from $90 \%$ to $98 \%$ in the isotope of interest. Isotope separation with the INEL isotope separator provided sample material for which the $(A \pm 1) / A$ ratio was approximately 1 to $10 \mathrm{ppm}$ as determined by mass spectrometry measurements. All other mass contaminations were less than this. For these separations the highly-enriched material was obtained by first collecting the respective isotopic beams in a pocket collector lined with high purity aluminum foil and then chemically recovering and purifying the material by chemical methods. These procedures were described in a previous annual report(1).

The remaining samples are being prepared by use of a retardation lens (2) which decelerates an individual isotopic beam to approximately $300 \mathrm{eV}$. Direct collection of $10 \mu \mathrm{g}$ to $100 \mu \mathrm{g}$ samples by direct deposition on a foil are possible at ion energies less than $500 \mathrm{eV}$. This method of sample preparation eliminates the chemical processes required by the above technique and reduces considerably separator run time. The collected sample and backing foil are usable directly in the experiment package. Test samples for mass spectrometric analysis have been collected. 
JABLE IV SAMPLES FOR IRRADIATION IN EBR-II

\begin{tabular}{|c|c|c|c|c|c|c|c|c|c|c|}
\hline $\begin{array}{c}\text { B-7 } \\
\text { Capsule }\end{array}$ & $\begin{array}{c}\text { Experiment } \\
\text { Capsule }\end{array}$ & $\begin{array}{l}\text { Sample } \\
\text { Capsule }\end{array}$ & $\begin{array}{l}\text { Sample } \\
\text { Code }\end{array}$ & Isotope & & $\begin{array}{l}\text { Deposit } \\
\text { echnique }\end{array}$ & $\begin{array}{c}\text { Deposit } \\
\text { Foil }\end{array}$ & $\begin{array}{l}\text { Deposit } \\
\text { Mass } \\
(\mu g)\end{array}$ & $\begin{array}{l}\text { Deposit } \\
\text { Activity } \\
\text { (mci) }\end{array}$ & $\begin{array}{l}\text { No. of } \\
\text { Samples }\end{array}$ \\
\hline \multirow[t]{4}{*}{$\begin{array}{l}406994-1 \\
(1-13-1)\end{array}$} & 406992-1 & $\begin{array}{r}406991- \\
- \\
-\end{array}$ & $\begin{array}{l}E U-3 \\
E U-5 \\
E U-9 \\
E U-11 \\
X X-1\end{array}$ & $\begin{array}{l}152 \mathrm{Eu} \\
152 \mathrm{Eu} \\
154 \mathrm{Eu} \\
154 \mathrm{Eu} \\
\text { Dosimetry }\end{array}$ & Set. & $\begin{array}{l}M S \\
M S(R) \\
M S \\
M S(R)\end{array}$ & $\begin{array}{l}\mathrm{V} \\
\mathrm{Ni} \\
\mathrm{V} \\
\mathrm{Ni}\end{array}$ & $\begin{array}{l}.17 \\
2.0 \\
.11 \\
2.0\end{array}$ & $\begin{array}{l}.03 \\
.36 \\
.03 \\
.54\end{array}$ & $\begin{array}{l}3 \\
3 \\
3 \\
3\end{array}$ \\
\hline & $406992-3$ & $\begin{array}{l}- \\
- \\
- \\
-\end{array}$ & $\begin{array}{l}E U-1 \\
\text { EU-7 } \\
\text { ND-9 } \\
\text { ND-11 } \\
\text { ND-13 }\end{array}$ & $\begin{array}{l}151 \mathrm{Eu} \\
153 \mathrm{Eu} \\
144 \mathrm{Nd} \\
146_{\mathrm{Nd}} \\
148_{\mathrm{Nd}}\end{array}$ & & $\begin{array}{l}\text { MS (R) } \\
\text { MS(R) } \\
\text { MS(R) } \\
\text { MS(R) } \\
\text { MS(R) }\end{array}$ & $\begin{array}{l}\mathrm{Ni} \\
\mathrm{Ni} \\
\mathrm{Ni} \\
\mathrm{Ni} \\
\mathrm{Ni}\end{array}$ & $\begin{array}{l}20 \\
20 \\
20 \\
20 \\
20\end{array}$ & $\begin{array}{l}0 \\
0 \\
0 \\
0 \\
0\end{array}$ & $\begin{array}{l}3 \\
3 \\
3 \\
3 \\
3\end{array}$ \\
\hline & $406992-2$ & $\begin{array}{l}- \\
- \\
- \\
-\end{array}$ & $\begin{array}{l}E U-4 \\
E U-6 \\
E U-10 \\
E U-12 \\
X X-2\end{array}$ & $\begin{array}{l}152 \mathrm{Eu} \\
152 \mathrm{Eu} \\
154 \mathrm{Eu} \\
154 \mathrm{Eu} \\
\text { Dosimetry }\end{array}$ & Set & $\begin{array}{l}M S \\
M S(R) \\
M S \\
M S(R) \\
\end{array}$ & $\begin{array}{l}V \\
N i \\
V \\
N i\end{array}$ & $\begin{array}{l}.17 \\
2.0 \\
.11 \\
2.0\end{array}$ & $\begin{array}{l}.03 \\
.36 \\
.03 \\
.54\end{array}$ & $\begin{array}{l}3 \\
3 \\
3 \\
3\end{array}$ \\
\hline & $406992-4$ & $\begin{array}{l}- \\
- \\
- \\
-\end{array}$ & $\begin{array}{l}E U-2 \\
E U-8 \\
N D-10 \\
N D-12 \\
N D-14\end{array}$ & $\begin{array}{l}151 \mathrm{Eu} \\
153 \mathrm{Eu} \\
144 \mathrm{Nd} \\
146 \mathrm{Nd} \\
148 \mathrm{Nd}\end{array}$ & & $\begin{array}{l}\text { MS(R) } \\
\text { MS(R) } \\
\text { MS(R) } \\
\text { MS(R) } \\
\text { MS(R) }\end{array}$ & $\begin{array}{l}\mathrm{Ni} \\
\mathrm{Ni} \\
\mathrm{Ni} \\
\mathrm{Ni} \\
\mathrm{Ni}\end{array}$ & $\begin{array}{l}20 \\
20 \\
20 \\
20 \\
20\end{array}$ & $\begin{array}{l}0 \\
0 \\
0 \\
0 \\
0\end{array}$ & $\begin{array}{l}3 \\
3 \\
3 \\
3 \\
3\end{array}$ \\
\hline \multirow[t]{4}{*}{$\begin{array}{l}406994-2 \\
(1-13-2)\end{array}$} & $406992-5$ & $\begin{array}{l}- \\
- \\
- \\
-\end{array}$ & $\begin{array}{l}\text { SM-1 } \\
\text { SM-3 } \\
\text { SM-5 } \\
\text { SM-7 } \\
\text { ND-15 }\end{array}$ & $\begin{array}{l}147 \mathrm{Sm} \\
147 \mathrm{Sm} \\
149 \mathrm{Sm} \\
149 \mathrm{Sm} \\
150 \mathrm{Nd}\end{array}$ & & $\begin{array}{l}M S(R) \\
E D \\
M S(R) \\
E D \\
M S(R)\end{array}$ & $\begin{array}{l}\mathrm{Ni} \\
\mathrm{Ni} \\
\mathrm{Ni} \\
\mathrm{Ni} \\
\mathrm{Ni}\end{array}$ & $\begin{array}{l}20 \\
20 \\
20 \\
20 \\
20\end{array}$ & $\begin{array}{l}0 \\
0 \\
0 \\
0 \\
0\end{array}$ & $\begin{array}{l}3 \\
3 \\
3 \\
3 \\
3\end{array}$ \\
\hline & $406992-7$ & $\begin{array}{l}- \\
- \\
-\end{array}$ & $\begin{array}{l}\text { ND-1 } \\
\text { ND-3 } \\
\text { ND-5 } \\
\text { ND-7 } \\
X X-3\end{array}$ & $\begin{array}{c}143 \mathrm{Nd} \\
143 \mathrm{Nd} \\
145 \mathrm{Nd} \\
145 \mathrm{Nd} \\
\text { Dosimetry }\end{array}$ & Set & $\begin{array}{l}M S(R) \\
E D \\
M S(R) \\
E D \\
t\end{array}$ & $\begin{array}{l}\mathrm{Ni} \\
\mathrm{Ni} \\
\mathrm{Ni} \\
\mathrm{Ni}\end{array}$ & $\begin{array}{l}20 \\
20 \\
20 \\
20\end{array}$ & $\begin{array}{l}0 \\
0 \\
0 \\
0\end{array}$ & $\begin{array}{l}3 \\
3 \\
3 \\
3\end{array}$ \\
\hline & $406992-6$ & $\begin{array}{l}- \\
-\end{array}$ & $\begin{array}{l}S M-2 \\
\text { SM-4 } \\
\text { SM-6 } \\
\text { SM-8 } \\
\text { ND-16 }\end{array}$ & $\begin{array}{l}147 \mathrm{Sm} \\
147 \mathrm{Sm} \\
149 \mathrm{Sm} \\
149 \mathrm{Sm} \\
159 \mathrm{Nd} .\end{array}$ & $\therefore$ & $\begin{array}{l}M S(R) \\
E D \\
M S(R) \\
E D \\
M S(R)\end{array}$ & $\begin{array}{l}\mathrm{Ni} \\
\mathrm{Ni} \\
\mathrm{Ni} \\
\mathrm{Ni} \\
\mathrm{Ni}\end{array}$ & $\begin{array}{l}20 \\
20 \\
20 \\
20 \\
20\end{array}$ & $\begin{array}{l}0 \\
0 \\
0 \\
0 \\
0\end{array}$ & $\begin{array}{l}3 \\
3 \\
3 \\
3 \\
3\end{array}$ \\
\hline & $406992-8$ & - & $\begin{array}{l}N D-2 \\
N D-4 \\
N D-6 \\
N D-8 \\
X X-4\end{array}$ & $\begin{array}{l}143 \mathrm{Nd} \\
143 \mathrm{Nd} \\
145 \mathrm{Nd} \\
145 \mathrm{Nd} \\
\text { Dosimetry }\end{array}$ & Set & $\begin{array}{l}M S(R) \\
E D \\
M S(R) \\
E D\end{array}$ & $\begin{array}{l}\mathrm{Ni} \\
\mathrm{Ni} \\
\mathrm{Ni} \\
\mathrm{Ni}\end{array}$ & $\begin{array}{l}20 \\
20 \\
20 \\
20\end{array}$ & $\begin{array}{l}0 \\
0 \\
0 \\
0\end{array}$ & $\begin{array}{l}3 \\
3 \\
3 \\
3\end{array}$ \\
\hline
\end{tabular}

1. MS - Deposit formed by direct collection of mass-separated ion beam at full energy.

4. V - Foil of high purity vanadium.

2. MS(R) - Deposit formed by direct collection of mass-separated ion beam at retarded energy.

5. Ni - Foil of high purity nickel.

3. ED - Deposit formed by electrodeposition from stock solution of mass-separated isotope. 
This report summarized the status of an irradiation experiment which will be made in EBR-II during Runs 87A and 87B, January and February, 1977. Highly enriched isotopes of $\mathrm{Nd}, \mathrm{Sm}$ and Eu will be irradiated to a total fluence of approximately $5 \times 10^{21} \mathrm{n} / \mathrm{cm}^{2}$. Integral capture cross sections will be determined by using gamma spectrometric and isotope dilution mass spectrometric techniques. These cross sections will contribute to the evaluation of the fission-product poison effect, the evaluation of the long term capture worth of $\mathrm{Eu}_{2} \mathrm{O}_{3}$, and the development of a reliable burnup monitor for advanced fast reactor systems.

(1) R. A. Anderl, E. H. Turk, Nuclear Technology Division Annual Progress Report for Period Ending June 30, 1975, ANCR-1255 (1975) p 51.

(2) R. A. Anderl, "Isotope Separator Laboratory Development", this report. 


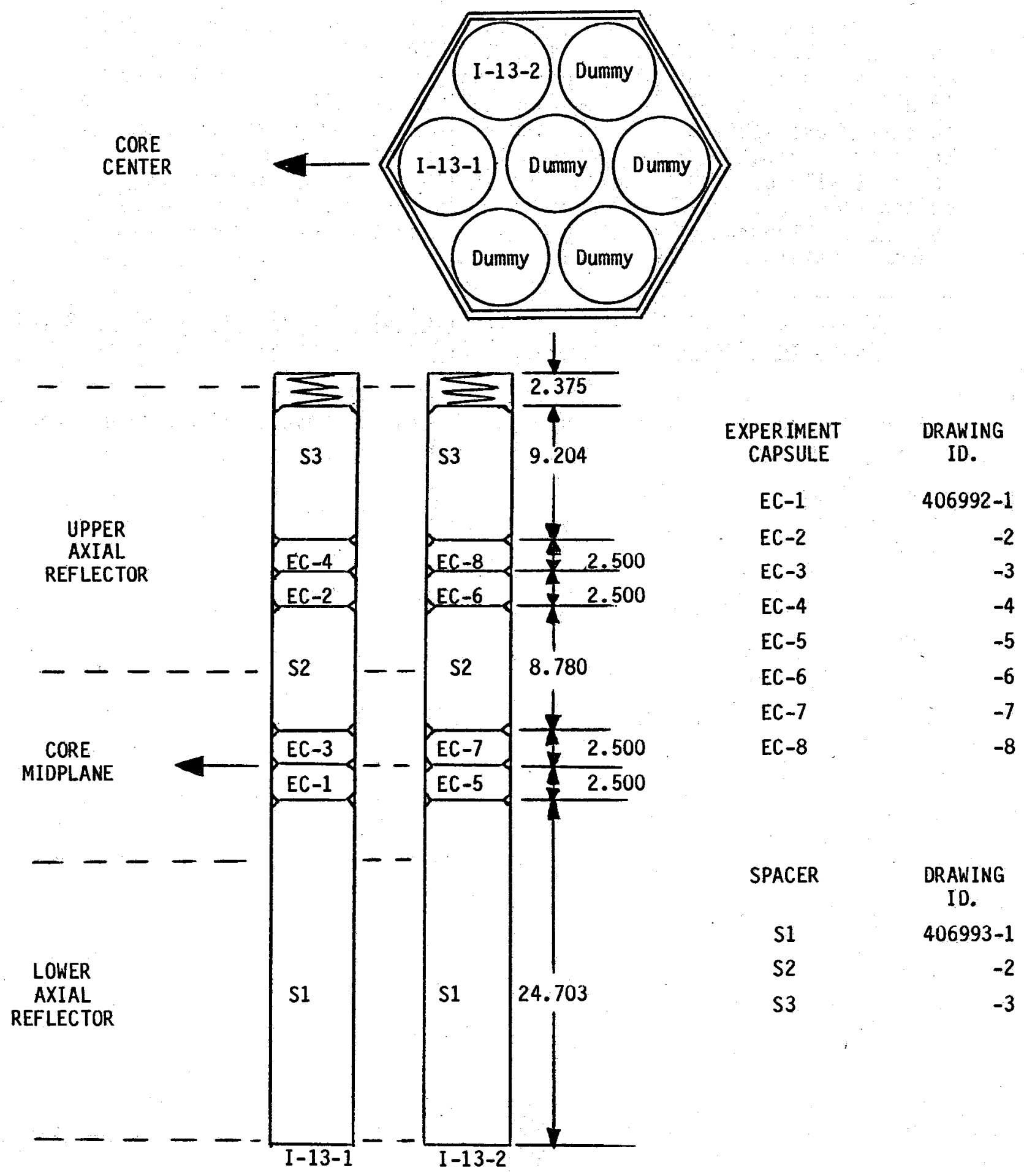

Fig. 1 Loading Configuration for I-13-1, I-13-2 B-7 Capsules in Mark $B$ Subassembly (dimensions in inches) 
MEASUREMENT OF THE CAPTURE CROSS SECTION OF 137 CS IN THE CFRMF

R. A. Anderl, L. 0. Johnson

Measurements of integral-capture cross sections for individual isotopes of selected fission product isotopes in fast and intermediate energy neutron fields have a direct bearing on assessing the fission product poison effect in advanced fast reactor systems. In some cases these measurements are also useful in assessing some waste management problems for advanced fast fuels. Of importance for both of these reasons is the measurement of the capture cross section for the radioactive isotope ${ }^{137} \mathrm{Cs}$. This contribution summarizes efforts made to measure the capture cross section for this isotope in the neutron field of the CFRMF by activation techniques.

Because it is radioactive, ${ }^{137}$ Cs presents unique problems with respect to measurements utilizing activation followed by gamma spectrometry. Consider the following: A $1-\mathrm{mCi}{ }^{137} \mathrm{Cs}$ source, half life $30.147 \mathrm{y}$, is equivalent to $3.7 \times 10^{7} \mathrm{dps}, 5.083 \times 10^{16}$ atoms or $14.5 \mu \mathrm{g}$ of CsCl. Assuming a 100-mb cross section and a neutron flux of $1 \times 10^{11} \mathrm{n} / \mathrm{cm}^{2}-\mathrm{sec}$, typical for CFRMF at $10 \mathrm{~kW}$, the saturation activity for the $32.2-\mathrm{m}^{138} \mathrm{Cs}$ neutron capture product is $508 \mathrm{dps}$. Using a full energy peak efficiency of $2.75 \times 10^{-4}$ and a peak-to-total ratio of .16 (both values measured for ND-5, a $65-\mathrm{cm}^{3}$ closed-end coaxial $\mathrm{Ge}(\mathrm{Li})$ detector), a total input count rate of 54000 counts per second is estimated for the $1-\mathrm{mCi}{ }^{137} \mathrm{Cs}$ source. The corresponding peak count rate for the 661-keV line is approximately 8600 counts per second. For the saturation activity of ${ }^{138} \mathrm{Cs}$, the corresponding peak count rate for the $1435-\mathrm{keV}$ line, the most intense line above $661 \mathrm{keV}$ in the ${ }^{138} \mathrm{Cs}$ decay, is computed to be .05 counts per second. These computations yield a 661-keV peak count rate to $1435-\mathrm{keV}$ peak count rate ratio of $1.6 \times 10^{5}$ to 1 . Considering the random coincidence summing, which is considerable even in the $1435 \mathrm{keV}$ region, and the dead-time losses due to the high ${ }^{137} \mathrm{Cs}$ count rate, one can see that conventional electronics would not be suitable for such a measurement.

To minimize the corrections and spectral effects due to high count rate conditions a prototype-design, state-of-the-art high count rate, $\mathrm{Ge}(\mathrm{L} i)$ spectroscopy system was setup. The specialized spectroscopy system included pileup rejection and base line restoration circuitry, a spectroscopy amplifier with a shaping time of $.7 \mu \mathrm{sec}$, an accurate live-time correction logic circuit and a $100-\mathrm{MHz}$ ADC. Using ${ }^{137} \mathrm{Cs}$ sources of different source strengths, performance tests of the electronics were made to determine the input count rates suitable for optimum thruput with reasonable deadtime and to determine the effectiveness of the pileup rejection circuitry for reducing the randum sum events relative to the true events. An optimum thruput of $15000 \mathrm{cps}$ was measured for an input count rate of $30000 \mathrm{cps}$ and a live-time to count-time ratio of .43. A comparison of ${ }^{137}$ Cs spectra taken with a without pileup rejection for the same live time indicated a reduction of approximately a factor of three in the random sum distribution. 
A selection of ${ }^{137} \mathrm{Cs}$ sources with different source strengths was prepared by dilution of a known stock solution followed by quantitative transfer to quartz ampoules using micropipettes. After evaporation of the liquid carrier, point sources of ${ }^{137} \mathrm{Cs}$ formed in the bottom of the ampoules. To prevent spread of contamination the ampoules were sealed. This source preparation provided sources of approximately $.03 \mathrm{mCi}$, $.06 \mathrm{mCi}, .16 \mathrm{mCi}, .32 \mathrm{mCi}, .63 \mathrm{mCi}$ and $.95 \mathrm{mCi}$.

An attempt to measure the capture cross section of ${ }^{137} \mathrm{Cs}$ was made using the $.63 \mathrm{mCi}$ source and the high count rate $\mathrm{Ge}(\mathrm{Li})$ spectrometry system with detector ND-5. For this experiment, the cesium source was irradiated in the CFRMF, at a power level of $10 \mathrm{~kW}$, for 2.82 hours, a time corresponding to a build-up of ${ }^{138} \mathrm{Cs}$ activity to .97 times the saturation value. After a post-irradiation decay time of 547 seconds the gamma spectrum of the irradiated source was measured with the source located at $25 \mathrm{~cm}$ from the detector. For this measurement the input count rate was approximately $33000 \mathrm{cps}$, the throughput was approximately $15000 \mathrm{cps}$ and the live time and real count time were 2000 seconds and 4793 seconds, respectively. A second spectrum was taken after a post irradiation decay time of approximately 140 minutes.

Nuclear data used in the analysis of the spectra are listed in Table 1. The 604.7-keV, 661.6-keV, 795.8-keV, 1365.2-keV lines and the 1323.3keV sum peak were used to provide an energy calibration for the spectra. Full energy peak efficiencies for sources at $25 \mathrm{~cm}$ from ND-5 are also listed in Table 1.

Neither the 1435-keV line no the 2218-keV line, both prominent in the decay of ${ }^{138} \mathrm{Cs}$, was observed in the first spectrum. Even though the electronics signiticantly reduced the random sum distribution in the vicinity of these lines, the absence of these lines indicated that the neutron capture product ${ }^{138} \mathrm{Cs}$ source strength was not adequate to give visible peaks above the random sum background.

An estimate of the sensitivity of the experiment was made by analyzing the spectrum for the ${ }^{134} \mathrm{Cs}$ lines which were present as an isotopic contamination in the sample material. From a computation using the peak areas of the 604.7, 795.8 and 661.6 lines, the efficiency factors, the branching ratios and live count time, the ${ }^{134} \mathrm{Cs}$ source strength was estimated to be $.04 \%$ that for $13.7 \mathrm{Cs}$. The $1365.2 \mathrm{keV}$ line from the ${ }^{134} \mathrm{Cs}$ decay (with a branching ratio of $3.04 \%$ ) was visible in the spectrum above the same randum sum distribution that the $1435.9 \mathrm{keV}$ line from the ${ }^{138} \mathrm{Cs}$ decay was expected to be observed. For the 1365.2 line, the net area to average background area ratio, the average background, and the uncertainty in the net area determination were estimated to be $.24,730$ and $25 \%$, respectively.

For a neutron flux of $10^{11} \mathrm{n} / \mathrm{cm}^{2}$-sec it was estimated that a capture cross section of $610 \mathrm{mb}$ would be required to produce enough ${ }^{138} \mathrm{Cs}$ activity to give rise to a peak at $1435.9 \mathrm{keV}$ of an area equivalent to that in the 1365.2 peak. An upper limit to the ${ }^{138} \mathrm{Cs}$ capture cross section was estimated by considering the net area in the 1435.9 peak to be equal to the statistical uncertainty in the background in the 
region of the 1435.9 peak. This analysis indicated that the capture cross section for ${ }^{137} \mathrm{Cs}$ was less than $61 \mathrm{mb}$.

In summary, an attempt was made to measure the integral capture cross section for ${ }^{137} \mathrm{Cs}$ in the neutron field of the CFRMF. Irradiation of a $0.63 \mathrm{mCi}$ source followed by Ge(Li) gamma spectrometry utilizing a prototype high count rate system yielded an upper limit of $61 \mathrm{mb}$ for the cross section. This measurement will be repeated with the CFRMF operating at $100 \mathrm{~kW}$.

TABLE 1

DATA FOR ANALYSIS OF CESIUM SPECTRA

\begin{tabular}{rccc}
$E_{\gamma}$ & Isotope & $\begin{array}{c}\text { Branching } \\
\text { Ratio } \\
(\%)\end{array}$ & $\begin{array}{c}\text { Peak } \\
\text { Efficiency } \\
\text { x104 }\end{array}$ \\
\hline 569.4 & Cs-134 & 15.43 & 3.25 \\
604.7 & Cs-134 & 97.6 & 3.08 \\
661.6 & Cs-137 & 85.0 & 2.75 \\
795.8 & Cs-134 & 85.4 & 2.33 \\
801.9 & Cs-134 & 8.73 & 2.30 \\
1009.8 & Cs-138 & 28.4 & 1.90 \\
1038.6 & Cs-134 & 1.00 & 1.86 \\
1168.0 & Cs-134 & 1.80 & 1.68 \\
1365.2 & Cs-134 & 3.04 & 1.46 \\
1435.9 & Cs-138 & 75.0 & 1.40 \\
2218.0 & Cs-138 & 16.0 & .92 \\
\hline
\end{tabular}


INTEGRAL CROSS SECTION MEASUREMENTS ON ${ }^{242} \mathrm{Pu}$ IN THE CFRMF

Y. D. Harker, E. H. Turk

The cross sections of the actinide isotopes produced by neutron capture in the plutonium-uranium fuel are important in various aspects of the nuclear fuel cycle. Therefore, it is important to measure the cross section of these individual isotopes so that their effects can be evaluated. ${ }^{242} \mathrm{Pu}$ is one of these isotopes.

A sample of very pure ${ }^{242} \mathrm{Pu}$ was obtained from Oak Ridge National Laboratory (ORNL). The ORNL isotopic analysis of the sample was:
Pu 239
Pu 240
$0.0092 \%$
Pu 241
$\mathrm{Pu} \cdot 242$
$0.0278 \%$
$0.0518 \%$
$99.911 \%$

The Pu-242 sample was in the oxide form.

A sample containing $30.21 \mathrm{mg}$ Pu-242 was weighed and sealed in a thin-wall aluminum capsule. This capsule wi th gold neutron flux monitors attached above and below the capsule was irradiated at the midplane of the CFRMF for 7.5 hours. The reactor power level for the irradiation was $10 \mathrm{~kW}$.

After irradiation the induced gamma radiation of the sample was measured on a Ge(Li) detector. Two counting periods were used to follow the decay of the induced gamma irradiation. The first period started 8.75 hours after the end of irradiation and continued through 18.1 hours. The second period started 346.3 hours after the end of irradiation and continued for a total of 729.2 hours of decay.

Analysis of the counting data from the first counting period gave information from which the ${ }^{242} \mathrm{Pu}(n, r){ }^{24} \mathrm{Pu}$ cross section was calculated.

Analysis of the counting data from the second counting period followed the decay of $140 \mathrm{La}$ produced in the ${ }^{242} \mathrm{Pu}(\mathrm{n}, \mathrm{f})$ reaction.

The $243 \mathrm{Pu}$ and ${ }^{140} \mathrm{Ba}-14 \mathrm{qa}$ nuclear data used to calculate the $(n, \gamma)$ and $(n, f)$ cross sections are given in Table $I$.

The ${ }^{140} \mathrm{Ba}-149 \mathrm{La}$ chain was at equilibrium when the sample was counted. The disintegration rate of the $140 \mathrm{La}$ was measured and multiplied by 1.151 to obtain the ${ }^{140} \mathrm{Ba}$ disintegration rate.

For the fission cross section measurements a fission yield of $5.98 \%$ for ${ }^{140} \mathrm{Ba}$ chain was used. This value was used since this is the value which has been measured in the CFRMF for $238_{\mathrm{U}}$. Since ${ }^{242 \mathrm{Pu}}$ is an eveneven nuclide it was assumed that its fission yield for $140 \mathrm{Ba}$ would be approximately the same as that measured for $238 \mathrm{U}$. 
The cross sections calculated from the decay data of the $242 \mathrm{pu}$ sample are given in Table II.

The value of $609 \mathrm{mb}$ for the $242 \mathrm{Pu}(\mathrm{n}, \mathrm{f})$ cross section is larger than expected. The contribution of the other Pu isotopes in the sample has been estimated from available cross section data. This contribution is negligible.

TABLE I

\begin{tabular}{lccc}
\hline Nuclide & $T_{1 / 2}$ & Gamma & $\begin{array}{c}\text { Branching } \\
\text { Ratio }\end{array}$ \\
\hline $243 \mathrm{Pu}$ & $4.98 \mathrm{H}$ & $84.16 \mathrm{keV}$ & $23 \pm 2 \%$ \\
$140 \mathrm{La}$ & $1596 \mathrm{keV}$ & $95.33 \%$ \\
$140 \mathrm{Ba}$ & $12.79 \mathrm{~d}$ & & \\
\hline
\end{tabular}

TABLE II

\begin{tabular}{cc}
\hline Reaction & Cross Section (Millibarns) \\
\hline $242 \mathrm{Pu}(n, \gamma)^{243 \mathrm{Pu}}$ & 159 \\
$242 \mathrm{Pu}(n, f)$ & 609 \\
\hline
\end{tabular}


94Nb CROSS SECTION MEASUREMENTS FOR CTR TECHNOLOGY

E. H. Turk, Y. D. Harker

In the Nuclear Technology Division's Annual Progress Report for the Period Ending June 30, 1975 (1) work was reported on measurement of the cross section for the reaction ${ }^{94} \mathrm{Nb}(n, \gamma){ }^{95} \mathrm{Nb}$. This value was reported as 242 millibarns. At the time the report was issued no evidence was available for the reaction ${ }^{94} \mathrm{Nb}(n, \gamma){ }^{95 \mathrm{~m} N b}$. During the past year the early decay data were analyzed by two different techniques to attempt to arrive at a value for ${ }^{94} \mathrm{Nb}(n, \gamma){ }^{95 m_{N b}}$ cross section.

The two techniques used were: (a) production equations for ${ }^{94} \mathrm{Nb}$ and $95 \mathrm{~m}_{\mathrm{Nb}}$ and a two parameter least squares treatment of the early decay data, (b) subtraction of a hand fit background to the $235 \mathrm{keV}$ gamma region of the early decay data to obtain a value for the contribution of the $235 \mathrm{keV}$ gamma of $95 \mathrm{~m}_{\mathrm{Nb}}$ to the gross decay data.

After combining the results from both of the above treatments a value of $5 \pm 16$ millibarns was obtained. The large uncertainty in the cross section value arises from the low activity level of the $95 \mathrm{~m}_{\mathrm{Nb}}$ on top of the large target material background from the ${ }^{94} \mathrm{Nb}$.

(1) R. L. Heath, J. W. Henscheid, and G. L. Smith, Nuclear Technology Division Annual Progress Report for the Period Ending June 30, 1975, ANCR-1255, p. 106. 


\section{ION EXCHANGE SEPARATION OF TRACER AMOUNTS OF ${ }^{244} \mathrm{Cm}$ FROM MILLIGRAM AMOUNTS OF ${ }^{241 \mathrm{Am}}$ \\ E. H. Turk}

Fast neutron cross sections are needed for the trans-plutonium isotopes in the Liquid Metal Fast Breeder Reactor program to evaluate their effect on the breeding capabilities of the reactor. To assist this work a program has been initiated at Aerojet Nuclear Company to use the CFRMF as a fast neutron source to study these reactions. Among the nuclides of interest is ${ }^{241} \mathrm{Am}$. An analytical development program was carried out this year to prepare for the measurement of the ${ }^{241} \mathrm{Am}$ cross section.

The capture product of ${ }^{241} \mathrm{Am}$ is 16 hour ${ }^{242} \mathrm{Am}$ which beta decays to $163 \mathrm{~d}{ }^{242} \mathrm{Cm}$. ${ }^{242} \mathrm{Am}$ has too short a half life to be useful and also its decay scheme is not favorable for using this isotope. However, ${ }^{242} \mathrm{Cm}$ with its long half life $(163 \mathrm{~d})$ and distinctive alpha decay energies (6.12 MeV and 6.07 MeV) makes its selection very attractive. Besides it is possible by ion exchange techniques to chemically separate the capture-decay product $\left({ }^{242} \mathrm{Cm}\right)$ from the target material $\left({ }^{241} \mathrm{Am}\right)$ thus enhancing the sensitivity of this approach.

In the development of the ion exchange separation of $\mathrm{Cm}$ from $\mathrm{Am}$, Dowex $50 \times 8$ resin was selected as the ion exchanger to use. Columns with the following dimensions were constructed using 200-400 mesh resin: $2 \mathrm{~mm}$ I.D. $\times 17 \mathrm{~cm}, 2 \mathrm{~mm}$ I.D. $\times 30 \mathrm{~cm}, 6 \mathrm{~mm} \mathrm{I.D.} \times 53 \mathrm{~cm}$. These columns were run at tracer levels of both ${ }^{244} \mathrm{Cm}$ and ${ }^{241} \mathrm{Am}$, and at tracer level ${ }^{244} \mathrm{Cm}$ with macro amount ${ }^{241} \mathrm{Am}(21 \mathrm{mg})$. The eluant used was $0.4 \mathrm{M}$ alpha hydroxy isobutyric acid with $\mathrm{pH}$ adjusted by conc. $\mathrm{NH}_{4} \mathrm{OH}$ addition to $\mathrm{pH} 3.80$ (17 cm and $30 \mathrm{~cm}$ column runs) and $\mathrm{pH} 4.02$ (53 cm column run). In both the $17 \mathrm{~cm}$ and $30 \mathrm{~cm}$ column run tracer level separation of ${ }^{241} \mathrm{Am}$ and $244 \mathrm{Cm}$ was attained but with macro amount of ${ }^{24} 1_{\mathrm{Am}}$ the separation was poor. This led to construction and testing of the $53 \mathrm{~cm}$ long column which worked very well at both tracer and macro ${ }^{241} \mathrm{Am}$ levels.

Separation efficiency of the column was measured by collecting elution fractions from the column and having them alpha pulse analyzed. Elution of the column was followed by gross alpha counting aliquots of the eluant and reserving fractions from the front of the elution peak for alpha spectrometry for identification of the eluted nuclide.

The elution curve for the $6 \mathrm{~mm}$ I.D. $\times 53 \mathrm{~cm}$ column run using tracer level ${ }^{244} \mathrm{Cm}$ and $1 \mathrm{mg}{ }^{241} \mathrm{Am}$ is shown in Figure 1 .

Identification of the elution peaks are also shown in Figure 1. Alpha spectrometry of the elution peak fractions showed that in the fractions collected for identification complete separation of the ${ }^{241} \mathrm{Am}$ and ${ }^{244} \mathrm{Cm}$ was obtained. The $239 \mathrm{Pu}$ which was the first nuclide to elute from the column was an impurity in the ${ }^{241} \mathrm{Am}$ and amounted to $20.4 \%$ (weight basis). 
The elution of the ${ }^{241} \mathrm{Am}$ in two distinct peaks is probably due to the existence of the Am in two different oxdiation states. At this time identification of the valence states has not been completed. 


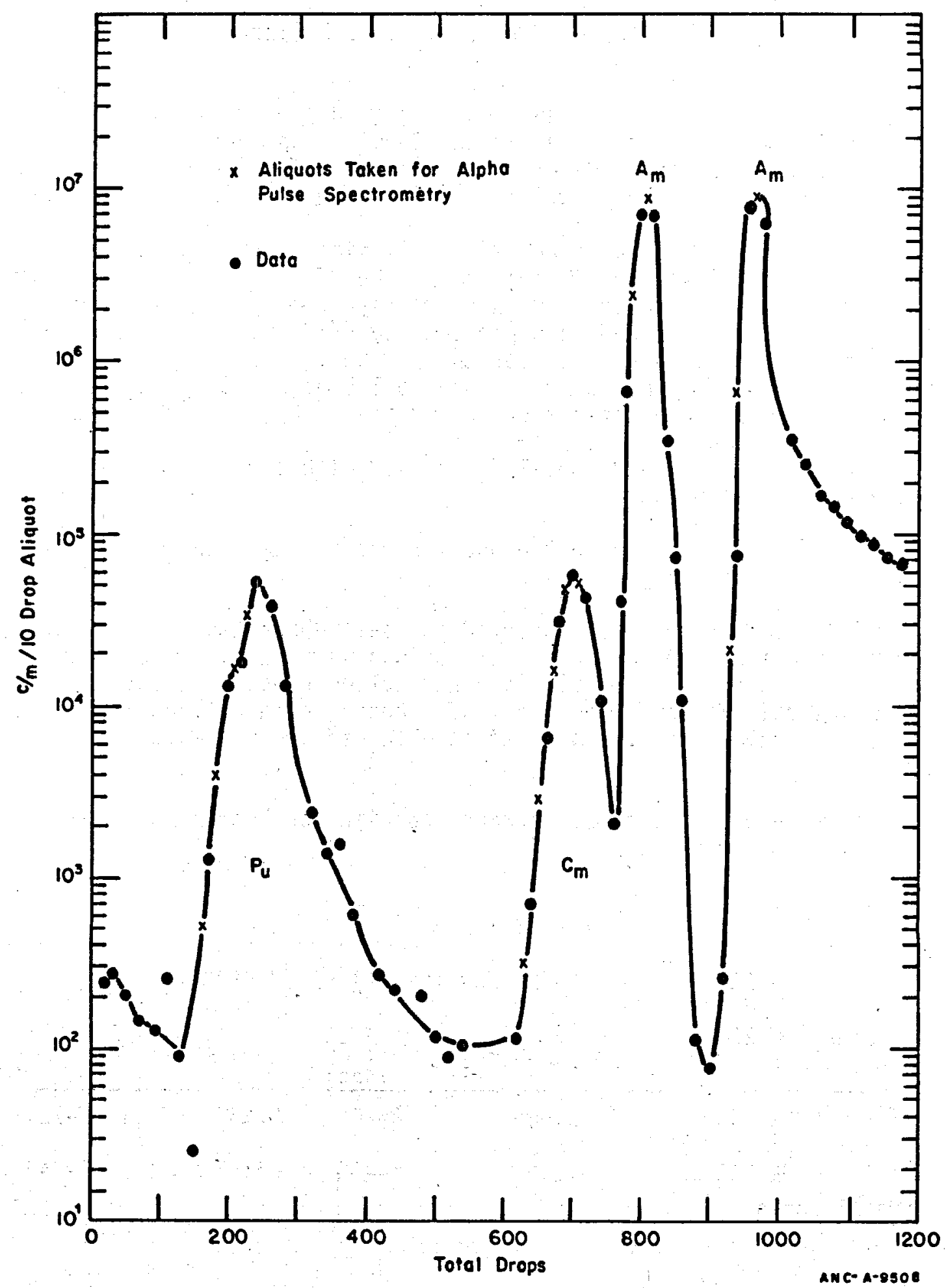

Fig. $1{ }^{244} \mathrm{Cm}-24{ }^{2} \mathrm{Am}$ Elution Spectrum for a solution containing ${ }^{244} \mathrm{Cm}$ Tracer and $1 \mathrm{Mg} 24{ }^{2} \mathrm{Am}$. 
In the Inter-Laboratory Reaction Rate (ILRR) program samples of fissile material were irradiated in the Coupled Fast Reactor Measurement Facility (CFRMF) to study the production rate of fission nuclides using fast neutrons. Among the materials studied were: U-235, Pu-239, Np-237.

In the analysis of the decay of the fission product gamma radiation from the two Np-237 samples irradiated in the CFRMF, it was noted that besides the fission product gamma a gamma ray from $\mathrm{Np}-238$ was also observed. This nuclide had been produced by $237 \mathrm{~Np}(\mathrm{n}, \gamma)^{238} \mathrm{~Np}$ reaction. Therefore, the gamma decay data for the $984 \mathrm{keV}$ gamma of Np-238 was abstracted from the gamma spectrum data collected on the Np-237 samples and used to calculate a cross section for the ${ }^{237} \mathrm{~Np}(n, \gamma)^{238} \mathrm{~Np}$ reaction.

Data on the two Np-237 metal foils used with irradiation history are presented in Table $\mathrm{I}$.

Table II presents the Np-238 nuclear decay data used in the analys is of the $984 \mathrm{keV}$ gamma ray of $\mathrm{Np}-238$.

Al1 counting data were corrected for self absorption in the sample and random summing. After these corrections were made, the corrected counting data were used to calculate a reaction cross section. A cross section was calculated for each set of counting data collected on each sample. The calculated cross sections obtained are presented in Table II.

The average of the cross sections obtained on both samples was $1.12 \pm 0.02$ (Std Error) barns.

TABLE I

DATA ON ${ }^{237} \mathrm{NP}$ SAMPLES* IRRADIATED IN CFRMF

\begin{tabular}{|c|c|c|c|c|}
\hline $\begin{array}{l}\text { Sample } \\
\text { No. }\end{array}$ & $\begin{array}{l}\mathrm{Np}-237 \\
(\mathrm{mg})\end{array}$ & $\left(\mathrm{n} / \mathrm{cm}^{2} / \mathrm{sec} \times 10^{-10}\right)$ & $\begin{array}{l}t_{\text {IRRAD }} \\
(\mathrm{sec}) \\
\end{array}$ & $t_{\text {SHUTDOWN }}$ \\
\hline$N 7-21$ & 310.666 & 7.60 & 25200 & $07: 53$ (MST), 11-6-72 \\
\hline N7-16 & 364.454 & 7.98 & 50400 & $06: 47$ (MST), 1-17-74 \\
\hline
\end{tabular}

TABLE II

${ }^{238} \mathrm{NP}$ NUCLEAR DECAY DATA

Half Life

$984 \mathrm{keV} r$

Branching Ratio 


\section{TABLE III}

CROSS SECTION FOR ${ }^{237} \mathrm{~Np}(n, \gamma)^{238} \mathrm{~Np}$ IN CFRMF

Sample No.

N7-21

N7-21

N7-16

N7-16

$N 7-16$ $\sigma$ (barns)

1.10

1.11

1.14

1.13

1.10

Average 
W. R. Myers, E. E. Owen, G. O. English, E. W. Killian

Equipment currently used for reactivity measurements at the ARMFs consists of hardwired signal averaging devices and multiplexers, and a pair of IBM keypunch machines interfaced as punched card readout devices. Reactivity data are acquired by incrementally moving a sample through an ARMF core, waiting for the reactor to stabilize at each sample position, and manualiy initiating a readout cycle. Typically eight to sixteen sample positions are utilized to measure the integral reactivity of a sample. Punched card data are processed on the INEL CSC computers. to obtain the integral of the regulating rod position curve to obtain the reactivity value.

Increased facilities efficiency in reactivity measurements will be obtained by eliminating reactor settling times incurred by operation in a gross stepping mode. When a sample is slowly and continuously moved through the reactor core, the analog feedback logic will maintain the regulating rod always very close to the required balance position. By operating the reactor in the nearly stable configuration, measurements will be made significantly faster while increasing the accuracy by at least a factor of three.

Faster signal averaging and multiplexing instrumentation with high-speed readout devices enable operation in the continuous mode provided that data are sampled only during intervals of reactor stability. Signals from the neutron linear level channels, the reactor temperature sensors, and the shim rod position indicator will be monitored to verify operation in the critical stable state.

A computerized data acquisition system will perform all of the required functions, and at a much lower cost than hardwired instrumentation. Furthermore, utilization of computerized instrumentation el iminates the need for high-speed hard-copy output devices because the majority of data can be processed on-line in real time. A small microcomputer configuration interfaced to the ARMF and CFRMF signal sources, equipped with alphanumeric display terminals to keep the reactor operators informed of the status of the experiments, and having hardwareimplemented arithmetic instructions to support reasonably fast signals averaging, will adequately support all ARMF measurements. 
NEUTRON ACTIVATION ANALYSIS AT INEL

J. I. Anderson, C. H. Hogg, R. A. Morneau

O. D. Simpson, L. D. Weber*

The Radiation Measurements Laboratory (RML) has extended its capabilities in the characterization and measurement of radiation and in the application of nuclear measurement techniques to problems involving the general environment. Neutron Activation Analysis (NAA) is an analytical tool that can be used to measure trace elements in concentrations as low as a few parts per billion and has been successfully applied in finding many trace elements in materials important to the environment.

NAA is now being used at the RML for trace element detection in non-radioactive effluents. Many environmental type samples have been analyzed, e.g., water, insects, trees, fish, alfalfa, air, and soil (see Table I). With thermal neutron fluxes of $10^{13}$ and $10^{12} \mathrm{n} / \mathrm{sec}-\mathrm{cm}^{2}$ available in the Advanced Test Reactor pneumatic irradiation facility and the Coupled Fast Reactivity Measurement Facility, respectively, identification and quantitative determinations can be made of elements having isotopes with short, intermediate and long half-lives. Data reduction is accomplished with a PDP-15 computer which utilizes state-of-the-art computational techniques in gamma-ray spectrometry.

The computerized analysis program searches for sixty different elements. Where possible, more than one energy peak and/or isotope is used to identify quantitatively each element present in a sample. Table II lists some of the elements that can be observed.

*Ricks College, Rexburg, Idaho 
TABLE I

EXAMPLES OF SAMPLES WHERE TRACE ELEMENTS HAVE BEEN OR COULD BE DETERMINED USING NEUTRON ACTIVATION ANALYSIS

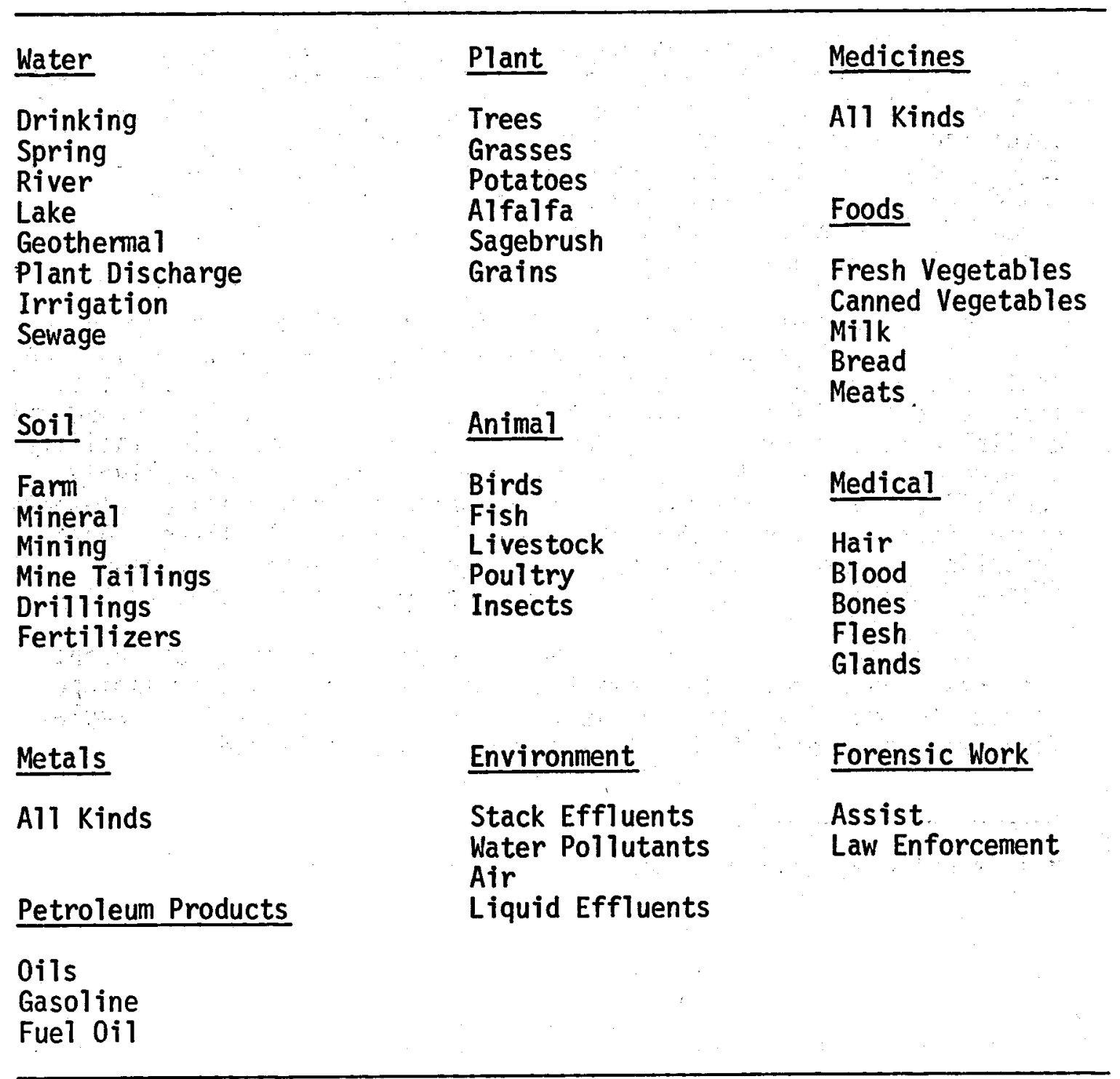


TABLE II

TRACE ELEMENTS THAT CAN BE OBSERVED WHEN USING

LONG, INTERMEDIATE OR SHORT NEUTRON IRRADIATIONS

\begin{tabular}{ll}
\hline Long Irradiation (days) & Intermediate Irradiation (hrs) \\
\hline Antimony & Arsenic \\
Barium & Bromine \\
Cadmium & Cerium \\
Calcium & Copper \\
Cerium & Dysprosium \\
Cesium & Erbium \\
Chromium & Gadolinium \\
Cobalt & Gallium \\
Europium & Germanium \\
Gold & Lanthanum \\
Hafnium & Manganese \\
Iridium & Nickel \\
Iron & Praseodymium \\
Lutetium & Rhenium \\
Mercury & Sodium \\
Molybdenum & Tungsten \\
Neodymium & \\
Niobium & \\
Osmium & Short Irradiation (min) \\
Palladium & Plaminum \\
Platinum & Aluminum \\
Potassium & Rubidium \\
Ruthenium & Indium \\
Samarium & Iodine \\
Scandium & Magnesium \\
Selenium & Silicon \\
Silver & Titanium \\
Strontium & Vanadium \\
Tantalum & \\
Terbium & \\
Tin & \\
Ytterbium & \\
Zinc & \\
Zirconium & \\
& \\
\hline
\end{tabular}




\title{
THE RATIO OF THE HYDROGEN AND MANGANESE CROSS SECTIONS
}

\author{
J. R. Smith, S. D. Reeder,* \\ B. M. Coursey,** V. Spiege $1^{* *}$
}

Axton(1) developed the technique of varying the concentration of the manganese bath and extrapolating measurements to zero hydrogen content. From such measurements the source strength can be derived without reference to separately measured cross sections for hydrogen and manganese. From the same data the hydrogen-to-manganese crosssection ratio can also be derived. It is determined from the ratio of the slope to the intercept of the linear curve fitted to the variation of the reciprocal of the $56 \mathrm{Mn}$ activity observed versus the hydrogento-manganese atomic ratio of the solution.

DeVolpi(2) has also made concentration-dependent measurements, obtaining hydrogen-to-manganese cross-section ratios differing from the Axton data by about $1.4 \%$. This does not necessarily imply a corresponding difference in source strength measurements, but it suggests the existence of unresolved problems in the systematics of the measurement. Because of the implications for ${ }^{252} \mathrm{Cf} \bar{v}$ measurements by the manganese bath technique, it was thought important to make a third $\sigma_{H} / \sigma_{M n}$ determination, using a neutron source for which some of the manganese bath systematic corrections do not apply. Such a source is a neutron beam well below the manganese resonance energies and the thresholds of the $160(n, \alpha)^{13} C$ and ${ }^{32} S(n, p)^{32 p}$ reactions.

Measurements were performed at the National Bureau of Standards Reactor. A Bragg beam of approximately $0.02 \mathrm{eV}$ was obtained by reflection of the reactor beam from a crystal of pyrolytic graphite. The Bragg beam first passed through a manganese-aluminum monitor foil and then into the bath. The ratio of solution to monitor activity provided a measure of source-produced activity independent of reactor fluctuations.

The concentration of the solution was varied over a range from 30 to 530 grams of $\mathrm{MnSO}_{4}$ per liter of solution. Because preliminary investigations indicated that the disagreement between Axton and DeVolpi might be traceable to concentration determinations, the concentration was determined three ways: by evaporation of a sample of solution and weighing of the residual salt, by measuring the density and comparing with a density-vs-concentration curve, and by titration of a sample of solution with EDTA. These methods are discussed in more detail in an accompanying section. (3)

The efficiency of counting ${ }^{56} \mathrm{Mn}$ activity in a solution varies with the concentration. Therefore, counter calibrations were carried out at each concentration. Calibrations were performed by counting in the manganese bath counting system an aliquot of ${ }^{56} \mathrm{Mn}$ sample whose

\footnotetext{
* Allied Chemical Corporation

** National Bureau of Standards
} 
absolute disintegration rate had been determined in the NBS ion chamber.

The results of the measurements are summarized in Table $I$. Presented are the intercepts, slopes, and derived $\sigma \mathrm{H} / \sigma \mathrm{Mn}$ values from weighted fits to the experimental data, using $\mathrm{NH}_{\mathrm{N}} / \mathrm{NMn}_{\mathrm{n}}$ values indicated by the various types of concentration measurements. Comparison of intercepts indicates what differences might be expected in source strength determinations, since the source strength is inversely proportional to the intercept. Only the Counter \#1 data are shown for the intercepts and slopes. The values from Counter \#2 are about $0.5 \%$ lower, because of the difference in geometry for counting the monitor foil. The $\sigma_{H} / \sigma_{\mathrm{Mn}}$ values shown are weighted averages from the two counters.

The agreement between results using volumetric, gravimetric, and densimetric (Reeder) concentration measurements, with corrections for contaminants, indicates that the methods are capable of equal accuracy, provided that adequate care is taken. These three methods yield an average value $\sigma_{H} / \sigma_{n}=0.02503 \pm 0.23 \%$. The results obtained using DeVolpi's densimetric curves are quite different, and demonstrate the necessity of using density-concentration curves prepared for the particular $\mathrm{MnSO}_{4}$ solution matrix to be used in the experiment. It is evident that DeVolpi's curves are not applicable to the solutions we used, particularly at concentrations below 60 grams $\mathrm{MnSO}_{4}$ per liter of solution.

A comparison of the Table I results leads one to suspect that DeVolpi's higher $\sigma_{H} / \sigma_{\mathrm{Mn}}$ value may have been due to underestimation of the $\mathrm{NH}_{\mathrm{H}} / \mathrm{N}_{\mathrm{Mn}}$ ratio, whether due to the effects of solution impurities, densimetric errors, or both. However, it is not clear what part of the difference may have been due to measurement techniques and what part may represent real differences in solution characteristics. We cannot presume to estimate the characteristics of solutions that no longer exist. It is, therefore, not possible to suggest a reasonable quantitative correction to DeVolpi's values for source strength.

The present result agrees well with Axton's revised (4) value of $0.02495 \pm 0.35 \%$ in terms of error overlap, but is about $0.36 \%$ higher. The higher $\sigma_{H} / \sigma_{M n}$ value would raise Axton's source measurements by about $0.15 \%$.

(1) E. J. Axton, et. al., J. Nucl. Energy Parts A/B 19, 409 (1965).

(2) A. DeVolpi and K. G. Porges, Metrologia 5, 128 (1969).

(3) S. D. Reeder and J. R. Smith, "Determination of Manganese Content in Aqueous Solutions", this report.

(4) E. J. Axton, private communication.

(5) A. DeVolpi, R. J. Armani, and K. G. Porges, J. Nucl. Energy Parts $A / B, 19597$ (1965). 


\section{TABLE I \\ $\sigma_{H} / \sigma_{M n}$}

SUMMARY OF FITS TO CURVE

\begin{tabular}{|c|c|c|c|c|c|c|}
\hline \multirow[b]{2}{*}{$\begin{array}{c}\mathrm{NH}_{\mathrm{H}} / \mathrm{NMn} \\
\text { Determination }\end{array}$} & \multicolumn{3}{|c|}{ No Contaminant Correction } & \multicolumn{3}{|c|}{ With Contaminant Corrections } \\
\hline & $\begin{array}{l}\text { SLOPE } \\
\text { (Error) (a) }\end{array}$ & $\begin{array}{l}\text { INTERCEPT } \\
\text { (Error) } \\
\end{array}$ & $\begin{array}{l}\sigma \mathrm{\sigma H} / \sigma \mathrm{Mn} \\
\text { (\% Error) }\end{array}$ & $\begin{array}{l}\text { SLOPE } \\
\text { (Error) } \\
\end{array}$ & $\begin{array}{l}\text { INTERCEPT } \\
\text { (Error) }\end{array}$ & $\begin{array}{l}\sigma H / \sigma M n \\
(\% \text { Error) }\end{array}$ \\
\hline Volumetric & $\begin{array}{l}0.03330 \\
(0.00003)\end{array}$ & $\begin{array}{l}1.38134 \\
(0.00196)\end{array}$ & $\begin{array}{l}0.025040 \\
(0.15)\end{array}$ & $\begin{array}{l}0.03330 \\
(0.00003)\end{array}$ & $\begin{array}{l}1.38231 \\
(0.00194)\end{array}$ & $\begin{array}{l}0.025027 \\
(0.15)\end{array}$ \\
\hline Gravimetric & $\begin{array}{l}0.03341 \\
(0.00003)\end{array}$ & $\begin{array}{l}1.38014 \\
(0.00197)\end{array}$ & $\begin{array}{l}0.025148 \\
(0.15)\end{array}$ & $\begin{array}{l}0.03330 \\
(0.00003)\end{array}$ & $\begin{array}{l}1.38177 \\
(0.00192)\end{array}$ & $\begin{array}{l}0.025039 \\
(0.15)\end{array}$ \\
\hline $\begin{array}{l}\text { Densimetric } \\
\text { (Reeder)* }\end{array}$ & $\begin{array}{l}0.03330 \\
(0.00003)\end{array}$ & $\begin{array}{l}1.38176 \\
(0.00221)\end{array}$ & $\begin{array}{l}0.025034 \\
(0.16)\end{array}$ & $\begin{array}{l}0.03331 \\
(0.00003)\end{array}$ & $\begin{array}{l}1.38251 \\
(0.00218)\end{array}$ & $\begin{array}{l}0.025026 \\
(0.16)\end{array}$ \\
\hline $\begin{array}{l}\text { Densimetric } \\
(\text { DeVolpi, KG)** }\end{array}$ & $\begin{array}{l}0.03344 \\
(0.00004)\end{array}$ & $\begin{array}{l}1.37271 \\
(0.00231)\end{array}$ & $\begin{array}{l}0.025307 \\
(0.21)\end{array}$ & $\begin{array}{l}0.03333 \\
(0.00004)\end{array}$ & $\begin{array}{l}1.37434 \\
(0.00278)\end{array}$ & $\begin{array}{l}0.025197 \\
(0.20)\end{array}$ \\
\hline $\begin{array}{l}\text { Densimetric } \\
(\text { DeVolpi, L)*** }\end{array}$ & $\begin{array}{l}0.03359 \\
(0.0005)\end{array}$ & $\begin{array}{l}1.36739 \\
(0.00343)\end{array}$ & $\begin{array}{l}0.025523 \\
(0.25)\end{array}$ & $\begin{array}{l}0.03349 \\
(0.00004)\end{array}$ & $\begin{array}{l}1.36902 \\
(0.00326)\end{array}$ & $\begin{array}{l}0.025412 \\
(0.24)\end{array}$ \\
\hline \multicolumn{3}{|c|}{$\begin{array}{l}\text { (a) Error estimates are standard deviations } \\
\text { * Reeder density-concentration relation }(3) \\
\text { * DeVolpi density-concentration relation }(5) \\
\text { ** DeVolpi density-concentration relation }(5)\end{array}$} & $\begin{array}{l}\mathrm{kg} \mathrm{MnSO}{ }_{4} / \mathrm{kg} \mathrm{s} \\
\mathrm{kg} \mathrm{MnSO}_{4} / \mathrm{Lite}\end{array}$ & \multicolumn{2}{|c|}{$\begin{array}{l}\text { Average } \sigma_{H} / \sigma_{M n} \\
\text { st } 3 \text { values only) }\end{array}$} & $\pm 0.23 \%^{\dagger}$ \\
\hline
\end{tabular}




\title{
DETERMINATION OF MANGANESE CONTENT IN AQUEOUS SOLUTIONS
}

\author{
S. D. Reeder,* J. R. Smith
}

The authors(1) recently made a careful measurement of the hydrogen-to-manganese cross-section ratio using a manganese bath and a low-energy neutron beam from the National Bureau of Standards Reactor (NBSR). In this experiment the cross-section ratio was derived from the Mn-56 activity observed as a function of the hydrogen-to-manganese atomic ratio. After consideration of many of the analytical methods available for determining manganese content, three methods seemed to be the most practical for this type of experiment, provided highly pure and well characterized manganous sulfate were used. Methods involving chemical separations were considered, but experience showed that the accuracy and precision of these procedures would not give the 0.1 to $0.2 \%$ maximum error desired.

The following sections outline the three analytical methods as they were used in the $\sigma_{H} / \sigma_{\mathrm{Mn}}$ measurement:

I. Volumetric Determination of Manganese

Manganese was determined in this method by a direct compleximetric titration with the disodium salt of EDTA (ethylendiaminetetraacetic acid). Ascorbic acid was used to prevent precipitation of hydroxide. The manganese-ascorbic acid complex is weaker than the manganese-EDTA complex, so the titration reaction with EDTA readily takes place.

Ammonium hydroxide was used to adjust the $\mathrm{pH}$ of the solution while hydroxylamine sulfate acted to keep manganese in a reduced bivalent state.

Eriochrome Black $T$ was used as a metal-ion indicator to detect the equivalence point of the titration. A wine-red solution forms when manganese combines with Eriochrome Black $T$, but when the last trace of manganous ion is strongly complexed by EDTA the indicator sharply changes to a blue color.

The volumetric method presumes that no titratable ions other than manganese are present.

\section{Gravimetric Analysis of Manganous Sulfate}

A weighed sample of solution was gently evaporated to dryness in a platinum dish and then heated at $400^{\circ} \mathrm{C}$ to a constant weight. The high temperature is required to thoroughly dehydrate the sample and to volatilize hydroxylamine sulfate, which is added to prevent formation of $\mathrm{MnO}_{2}$. A comparison of sample analyzed with and without hydroxylamine sulfate showed that the manganous sulfate dehydrated without this reagent present is usually slightly brown due to the presence of a small amount of manganese dioxide.

Non-volatile solution impurities are weighed with manganous sulfate, and therefore the results are inherently high. 
III. Densimetric Determination of Manganous Sulfate

A density-concentration-temperature calibration is prerequisite to this relatively simple analytical procedure. A series of solutions having a range of accurately known manganous sulfate concentration were prepared with the same chemical matrix as used in the operating bath, the densities of the solutions were measured by the volumetric method, and the data fitted to fourth-degree polynomial equations.

The following polynomial equations were obtained on a set of solutions at temperatures of 20 and $30^{\circ} \mathrm{C}$. The calibrating solutions, as was the bath itself, were made from Mallinckrodt "Analytical Reagent" $\mathrm{MnSO}_{4} \cdot \mathrm{H}_{2} \mathrm{O}$ and contain $0.25 \mathrm{~g} / 1$ of $\left(\mathrm{NH}_{2} \mathrm{OH}\right)_{2} \cdot \mathrm{H}_{2} \mathrm{SO}_{4}$. The concentrations are in $\mathrm{g} \mathrm{MnSO}_{4}$ per kilogram of solution.

$$
\begin{aligned}
& C_{20}=-2268.95+4167.56 D-2687.89 D^{2}+914.36 D^{3}-123.97 D^{4} \\
& C_{30}=-2294.28+4221.29 D-2718.03 D^{2}+919.05 D^{3}-124.13 D^{4}
\end{aligned}
$$

These relations give concentrations in agreement with DeVolpi's(2) curves within $0.2 \%$ over most of the concentration range, but diverge widely at concentrations below 60 grams per liter.

The results of these experiments with different methods of determination of manganese content show that the simplest and most accurate system is to use Analytical Reagent $\mathrm{MnSO}_{4}$ for the manganese bath. Then the volumetric method can be accurately used, and the impurity level can be monitored by observing the gravimetric-volumetric difference.

(1) J. R. Smith and S. D. Reeder, "The Ratio of the Hydrogen and Manganese Cross Sections", this report.

(2) A. DeVolpi, R. J. Armani and K. G. Porges, J. Nuclear Energy Parts A/B 19, 597 (1965). 


\section{TRANSIENT REACTIVITY METER}

W. M. Yarbrough

A prototype of a transient reactivity meter (TRM)* has been constructed and successfully tested at the ATR critical facility. This TRM is a combination hard-wired/programmable analog computer that performs a closed-1oop inverse solution of the space-independent reactor kinetics differential equations (See Figure 1). It has several features, compared with those of other reactivity-measuring devices, that provide distinct advantages in performing reactivity measurements: (1) It is a closed-1oop solution (which is inherently stable) wherein the solution $\rho$ is "forced" at the output of a high-gain network. (2) The solution for $\rho$ is performed entirely within the negative feedback 10op, thus minimizing the required number of calculational stages and components. (3) It will recover when reset (to the initialcondition mode) during maneuvering (slow) positive transients, thus accommodating input range-switching during such transients. (4) It is programmable to the extent that delay-group parameters, input and output ranges, and $\tau_{0}$ (or $\ell / B_{\text {eff }}$ ) can be readily changed and/or readjusted to provide for its use with different reactors or to compensate for aging of electrical components. (5) It has a built-in test function, which provides for rapid checkout in the field.

Note, from Figure 1, that this TRM is a 4-delay-group model. From experience it has been learned that a 4-group model, wherein the three fastest groups are coalesced into a single group, provides results virtually identical to those of a 6-group model. This feature provides greater ease of programming than otherwise possible.

The validity of the TRM calculational method is seen from the fundamental relationship of servo theory, $(1)$ as illustrated in Figure 2:

$$
R=\frac{G(s)}{1+G(s) F(s)} E_{\text {in }}
$$

If $E_{\text {in }}=$ 申in and $F(s)=$ forward reactor kinetics operator,

$$
\rho=\lim _{|G(s)|+\infty}=F(s)^{-1} \phi_{\text {in }}
$$

Construction of follow-on models of this TRM, with printed circuitry and other refinements, is planned for the near future. These will be used in physics testing at LOFT, TRA, and wherever appropriate.

*This is based on a design originally developed at the Knolls Atomic Power Laboratory (KAPL) by J. E. Barnes and I. W. Underwood.

(1) J. C. Gille, M. J. Pelegrin, P. Decaulne, Feedback Control Systems, New York: McGraw-Hi11 Book Company, Inc., 1959. 


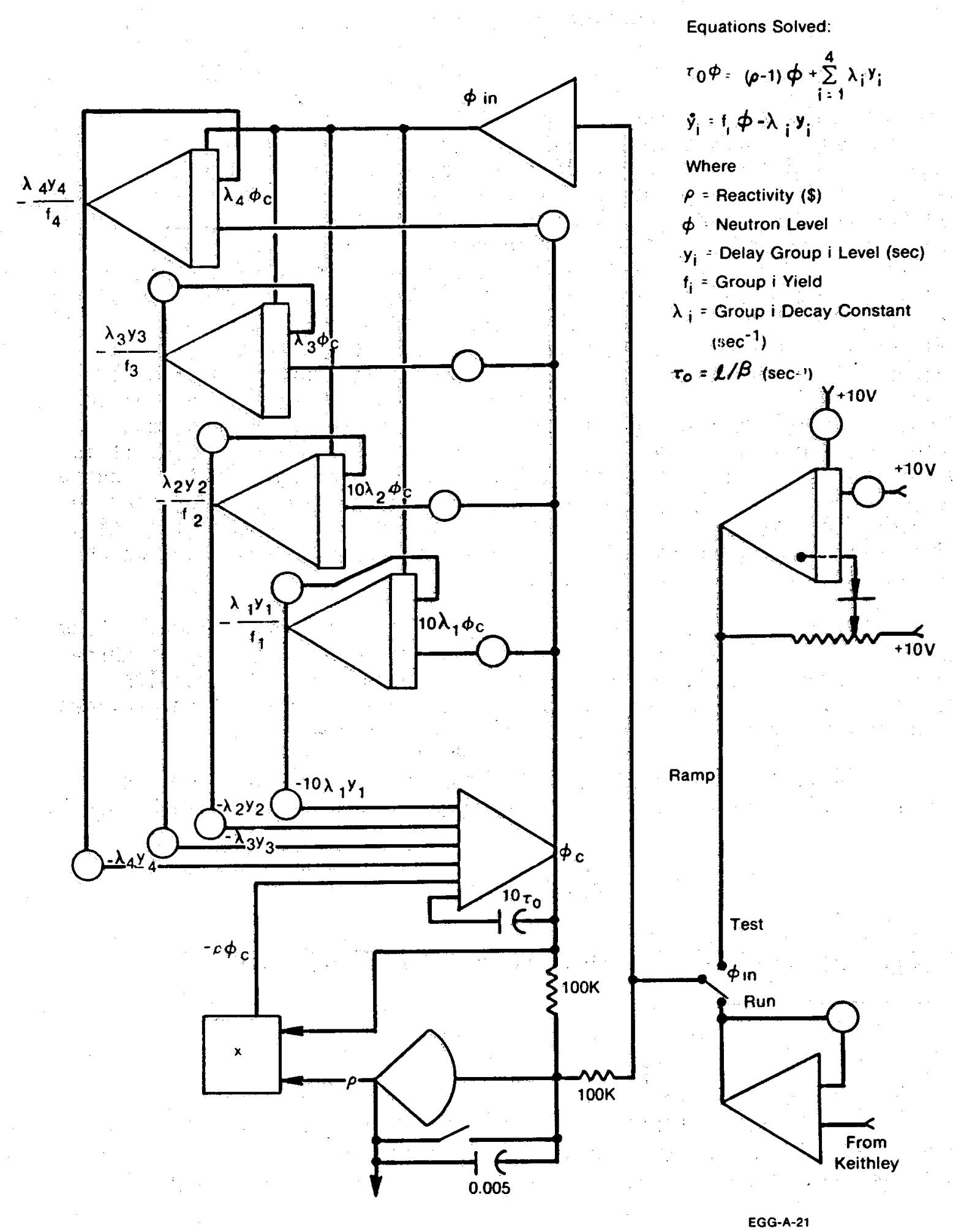

Figure 1. Block diagram of TRM. 


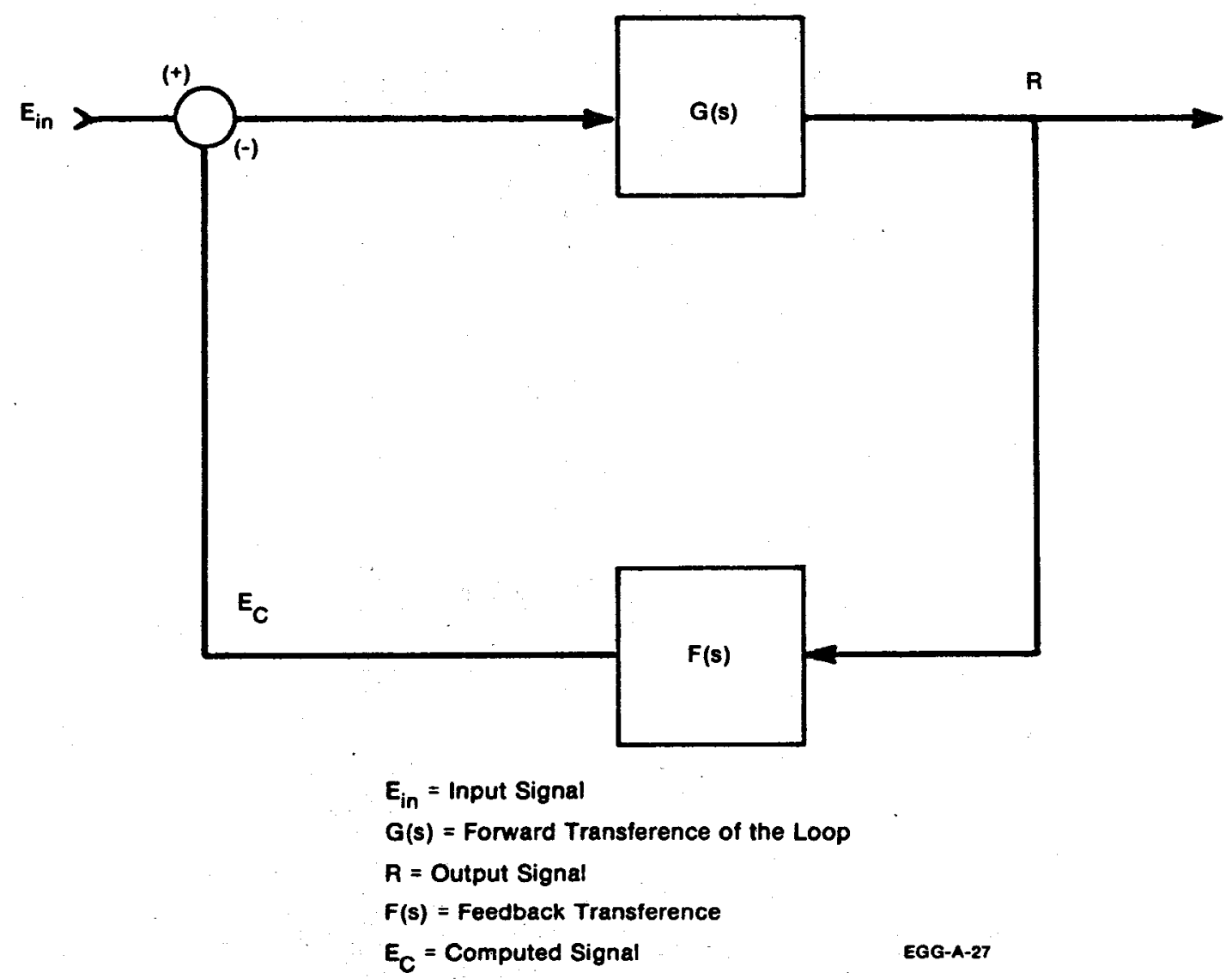

Figure 2. Simplified servo loop. 
$\cup$

\section{BLANK PAGE}


MODEL AND CODE DEVELOPMENT 


\title{
TRANSFORMATION OF HISTOGRAM TYPE DIFFERENTIAL DATA
}

\author{
Y. D. Harker
}

A frequently used method of quantifying energy dependent data in reactor physics is the energy/lethargy group representation. In this approach the representation of the function is characterized in a set of energy/lethargy groups either as integrals over respective groups or as average values. Often in utilizing data tabulated according to different group structures it is necessary to transform one or more sets to obtain group structure compatibility. A FORTRAN program called Group Structure Transformation (GST) has been developed to perform this function and is described in this report. This program was written for the purpose of transforming neutron spectral data in support of research being conducted at the Coupled Fast Reactivity Measurement Facility (CFRMF), however the methodology used is sufficiently general that other applications may be considered.

The intent for developing this transformation program was to provide a mathematical method whereby reproducible transformations could be performed with minimal judgement on the part of the user. To do this it was considered necessary that the mathematical approach have a high degree of stability and that the underlying functional dependence be continuous at least to the first derivative. Transformation of this type will require interpolation at some point in the process and the interpolation method used has considerable bearing on the stability of the whole approach. In this application the cubic spline method was used for interpolation and differentiation, such a method is extremely powerful, simple and fast. Mathematically it is capable of satisfying the continuity requirements and when applied properly satisfies the stability requirement. To prepare the input data set (group representation) for interpolation it was converted to an integral function data set in a pointwise representation. Two integral data sets were defined and evaluated: function I is the integral from each group energy to infinity and 2) function II is the compliment i.e., integral from zero energy to the group energy. Interpolated data for both integral functions are obtained at desired energy/lethargy values via interpolation on the log of each function. The use of $\log ^{\prime} \mathrm{s}$ tends to smooth the data and consequently eliminates those conditions which lead to unstable or non-physical solutions in numerical interpolation. Log's however assume functions which are positive-definite and also tend to reduce the accuracy of the numerical technique. The loss in accuracy is partially compensated for by using higher precision data formats and procedures.

Group internal data in the new energy/lethargy representation are obtained by taking differences between the respective integral functions. Integral function I is used to derive group data at the higher energies and function II at the lower energies; the switch from one function to the other occurs at an energy where the two functions are approximately equal. 
Two integral functions are used in this manner to improve sensitivity and accuracy of the method. Included with the cubic-spline interpolation process is a differentiation routine which is used to obtain a pointwise representation of the differential data in the new energy structure.

The program, therefore, takes group differential data in one group structure and converts it to a new set of group data in another structure and in addition produces pointwise data for the integral function and for the differential function. An example of a data transformation is given for neutron spectral data of the CFRMF. Shown in Figure 1 is the neutron spectrum for this facility as calculated by transport theory. The histogram represents the spectrum as calculated by theory in a 0.25 lethargy group structure and is the input to the transformation program. The solid curve is the output in the form of a pointwise representation evaluated at energy values for the SAND II group structure. Examination of the data indicate that the method is stable and that continuity is maintained.

A check on the accuracy of the methods was performed by taking an output spectrum and regenerating the input spectrum for the case where the output is determined on a finer group structure than that given for the input data. A comparison of the results is tabulated in Table I. The input group structure for this test is the .25 lethargy group structure beginning with an upper energy of $21 \mathrm{MeV}$ and tabulated in ascending order with respect to lethargy (descending in energy). The function used was the CFRMF spectrum shown in Figure 1. The transformation program's output group structure from which the regenerated fluxes were derived consisted of 10 fine groups for each .25 lethargy input group. From Figure 1 it is evident that for groups 1-9 the spectrum is changing rapidly within each group but is monatonic and the regenerated fluxes are in agreement with the input values. Beginning with group 49 resonance effects are more pronounced and variations of the spectrum within each group can be large and not necessarily monatonic. Evidence of the effects of these variations on the limitations of the transformation program are indicated in the small deviations of the regenerated fluxes from the input values. On the whole, however, the regenerated values agree with the originals to at least four significant figures and with a few exceptions better than five significant figures.

This transformation program has proven quite successful for its intended application. There are, however, applications other than conversion of fast neutron spectral data where such a transformation program may prove useful. Clearly any bounded data where the integral over the range covered by the data is defined can be used as input and even non-bounded data may be accommodated when the data given are finite. By extending the actual data bounds with fictitious values which approach zero at the boundaries in way such as to reduce spurious behavior in interpolation, non-bounded data may be made to appear to have defined integrals and therefore the integral interpolation transformation may be applied. Independent of whether the integrals given by the actual data are defined or not, the interpolated points or group values near the bounds of the actual data would tend to be less 
accurately known of the transformed data set. The effective range of the transformed data is therefore somewhat less than the original set. Since this program utilized logarithms it is limited to applications where the data is non-negative.

In summary, a computer program has been written and is briefly described in this report. This program was developed to transform fast neutron spectral data from one group representation to group data in another representation and to generate pointwise date in both differential and integral functions. The results obtained for transforming the CFRMF neutron spectrum from a 71 group 0.25 lethargy representation to the 620 group SAND II representation show that the program is very stable even in regions where large variations in the original data exist. 


\section{TABLE I}

Integral Check of Transformed Data

Group No. Regenerated $\delta /$ Input $\delta \quad$ Group No. Regenerated $\delta / I n p u t \Phi$

1

2

3

4

5

6

7

8

9

10

11

12

13

14

15

16

17

18

19

20

21

22

23

24

25

26

27

28

29

30
1.00006

1.00000

1.00001

.99994

1.00000

1.00000

1.00000

1.00000

1.00000

1.00000

1.00000

1.00000

1.00000

1.00000

1.00000

1.00000

1.00000

1.00000

1.00000

1.00000

1.00000

1.00000

1.00000

1.00000

1.00000

1.00000

1.00000

1.00000

1.00000

1.00000
31

32

33

34

35

36

37

38

39

40

41

42

43

44

45

46

47

48

49

50

51

52

53

54

55

56

57

58

59

60
1.00000

1.00000

1.00000

1.00000

1.00000

1.00000

1.00000

1.00000

1.00000

1.00000

1.00000

1.00000

1.00000

1.00000

1.00000

1.00000

1.00000

.99999

1.00000

1.00000

1.00001

1.00000

1.00001

1.00000 .99998

1.00003

1.00000

1.00001 .99996

1.00007 


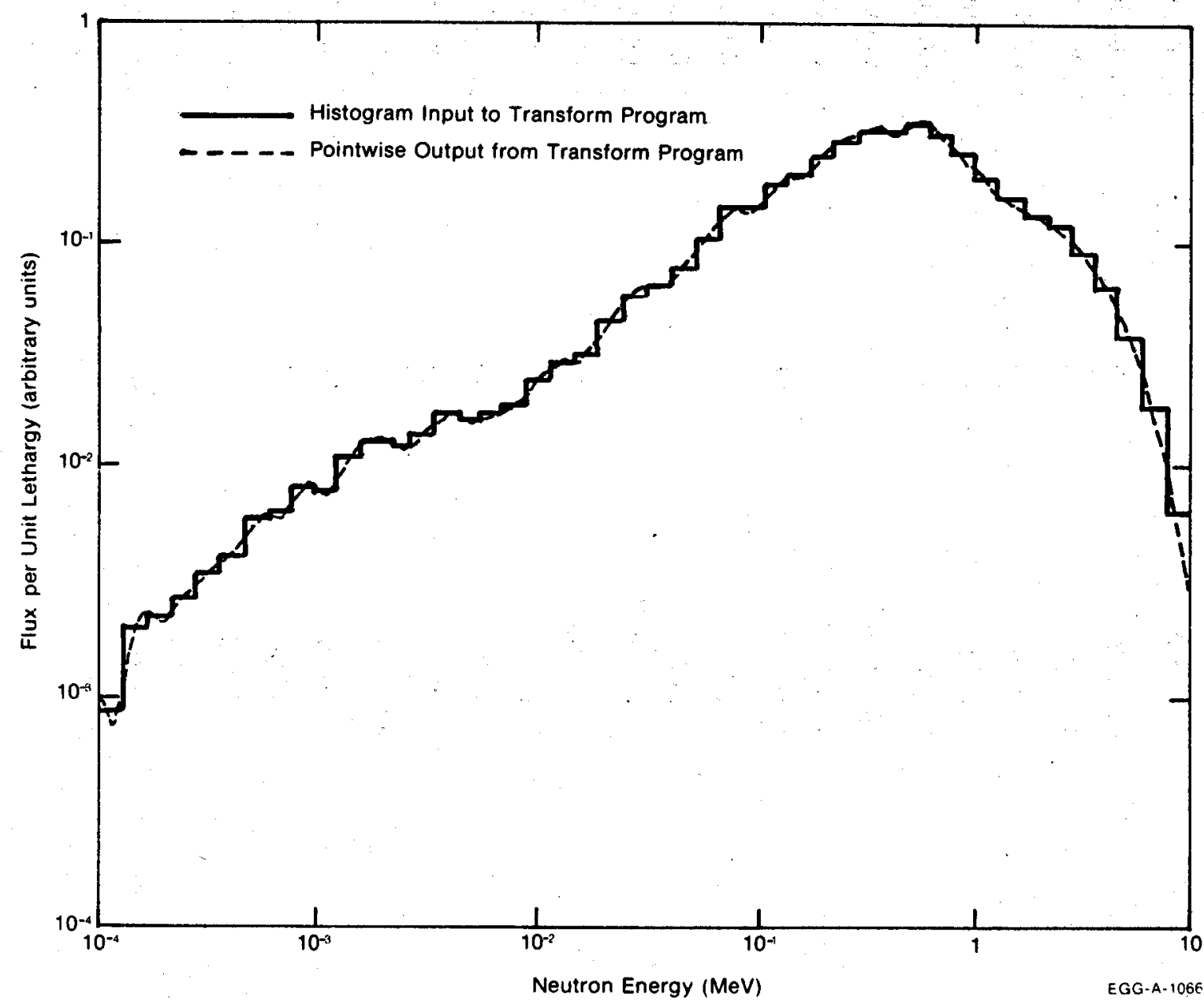

Fig. 1 Comparison of the pointwise spectrum generated by the transform program against the input histogram for the case of the CFRMF Neutron Spectrum. 


\section{WIGNER-SEITZ APPROXIMATION IN PBF RELOAD CORE CELL CALCULATIONS}

D. E. Wessol and F. J. Wheeler

In order to simplify the neutron transport calculation in a cell, it is necessary to replace the usual square or hexagonal cell outer boundary by an equivalent cylindrical outer boundary. This method of using an equivalent cylindrical cell with a zero-current boundary condition is known as the Wigner-Seitz approximation. Numerous discussions on the valjdity of the Wigner-Seitz approximation can be found in the literature $(1,2,3,4)$. The purpose of these investigations was to examine the Wigner-Seitz approximation, in the energy-dependent situation, for a particular cell using Monte Carlo and S6 transport theory. Table I presents pertinent cell parameters of the problem studied. Mirror, isotropic and semi-isotropic neutron return at the cell outer boundary were considered. The semi-isotropic boundary condition is defined as isotropic return about the azimuthal angle, $\Phi$, and mirror return about the polar angle, $\theta$. This is represented in the calculational model by Equation 1 for the angular flux at the outer surface:

$$
\begin{aligned}
& . \phi\left(r_{\mathrm{eq}}, \mu, \phi\right)=\rho \cos \phi^{\prime} \phi\left(r_{\mathrm{eq}}, \mu, \phi^{\prime}\right) \mathrm{d} \phi^{\prime} \\
& \text { where } \mu=\cos \theta \\
& \qquad r_{\mathrm{eq}}=\text { equivalent cylindricalized cell radius }
\end{aligned}
$$

An infinite lattice $y$ using the parameters of Table $I$, was modeled in the RAFFLE ${ }^{(5)}$ and KENO ${ }^{(6)}$ Monte Carlo codes as a three-dimensional square cell with mirror reflection on the six bounding surfaces. The RAFFLE calculation was performed again with an equivalent cylindricalized cell outer boundary coupled with a semi-isotropic return. The one-

dimensional SCAMP ${ }^{7)} S_{n}$ model used the same cell parameters as the Monte Carlo model and the usual equivalent cylindricalized cell outer boundary with either isotropic, semi-isotropic or mirror return. The isotropic and semi-isotropic boundary conditions yielded nearly identical results for this ceil so only semi-isotropic results will be reported.

ENDF/B-III data were used in both the RAFFLE and $S_{6}$ models. In order to eliminate differences due to cross-section representations, it was decided to run a Monte Carlo calculation with KENO and repeat the $S_{n}$ calculation using the identical pre-shielded sixteen-group HansenRoach $^{(8)}$ cross-section set in both calculations.

Comparisons for all of the calculations are shown in Table II. Results show a consistency in the calculation of $k$ for the square cell and equivalent cylindricalized cell. It is also observed from this set of calculations that the Wigner-Seitz approximation yields a markedly different flux distribution, most notably in the thermal region. These results demonstrate that the Wigner-Seitz approximation underpredicts the thermal moderator-to-fuel flux ratio by $12-16 \%$ while also underestimating $k_{e f f}$ by $1 \%$ for the cell investigated. The reasons for this may be more readily understood by examining the sixteen-group 


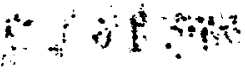

moderator-to-fuel flux ratios shown in Figure 1. Even though the KENO statistical errors are rather large for the sixteen-group fiuxes, the moderator-fuel flux ratios were demonstrably lower in groups $10, \frac{1}{2} \frac{1}{3} 5$ and 12 for $\xi_{8}$ the $S_{6}$ calculation. It is in these same groups where the and ${ }^{238} \mathrm{U}$ resonances are located, and it can be concluded that the Wigner-Seitz approximation underestimates the resonance escape probability for these resonances. It is curious to note from Table II that the $\mathrm{S}_{6}$ calculation with the mirror boundary condition produces improved results with respect to the groups 3 and 4 flux ratios and multiplication factor, but this result seems fortuitous in light of the groups 1 and 2 flux ratios.

We make no attempt to categorize the type of cell problem for which the Wigner-Seitz approximation fails but only to point out to the user that cells of the type given in this paper (tightly packed) should always be checked against a Monte Carlo or two-dimensional transport theory calculations using the precise geometric description.

It is hoped that future studies will reveal which boundary condition works best with Wigner-Seitz approximation or even if the approximation fails entirely, no matter which boundary condition is chosen, for the different cell types.

(1) D. A. Newmarch, Errors Due to the Cylindrical Cell Approximation in Lattice Calculations, AEEW-R34 (1960).

(2) J. A. Thie, "Failure of Neutron Transport Approximations in Small Cells in Cylindrical Geometry", Nucl. Sci. Eng. 9 (1961) 286-287.

(3) H. C. Honeck, "Some Methods for Improving the Cylindrical Reflecting Boundary Condition in Cell Calculations of the Thermal Neutron Flux", Trans. Am. Nucl. Soc. 5, (1962) 350-351.

(4) H. Takahashi, "The Generalized First-Flight Collision Probability in the Cylindricalized Lattice System", Nucl. Sci. Eng. 24 (1966) 60-71.

(5) W. E. Vesley, F. J. Wheeler, R. S. Marsden, The RAFFLE General Purpose Monte Carlo Code, ANCR-1022 (1973).

(6) G. E. Whitesides, N. F. Cross, KENO - A Multigroup Criticality Program, CTS-5 (1969).

(7) G. E. Putnam, private communication.

(8) G. E. Hansen and W. H. Roach, Six and Sixteen-Group Cross Section for Fast and Intermediate Assemb7ies, LAMS-2543 (November 1961). 


\section{TABLE I}

8\% ENRICHED UO 2 PBF FUEL ROD

\begin{tabular}{ll}
\hline Pellet 0.R. & $.83952 \mathrm{~cm}$ \\
Void O.R. & $.85517 \mathrm{~cm}$ \\
Clad 0.R. & $.95682 \mathrm{~cm}$ \\
Pitch & $2.01336 \mathrm{~cm}$ \\
req & 1.13592 \\
\hline
\end{tabular}

NUMBER DENSITIES $\left(\mathrm{bn}-\mathrm{cm}^{-1}\right)$

\begin{tabular}{llll}
\hline Hydrogen & $\frac{\text { Fuel }}{0.0}$ & $\frac{\text { Clad }}{0.0}$ & $\frac{\text { Moderator }}{6.55337-2}$ \\
Oxygen & $4.50631-2$ & 0.0 & $3.27667-2$ \\
Zirc-2 & 0.0 & $4.319-2$ & 0.0 \\
${ }^{235} \mathrm{U}$ & $1.82366-3$ & 0.0 & 0.0 \\
${ }^{238} \mathrm{U}$ & $2.07079-2$ & 0.0 & 0.0 \\
& & & \\
& Fuel $=1010 \mathrm{~K}$ & \\
& Clad $=385 \mathrm{~K}$ & \\
\hline
\end{tabular}




\section{TABLE II}

BOUNDARY CONDITION COMPARISONS FOR THE MULTIPLICATION FACTOR AND MODERATOR TO FUEL FLUX RATIO

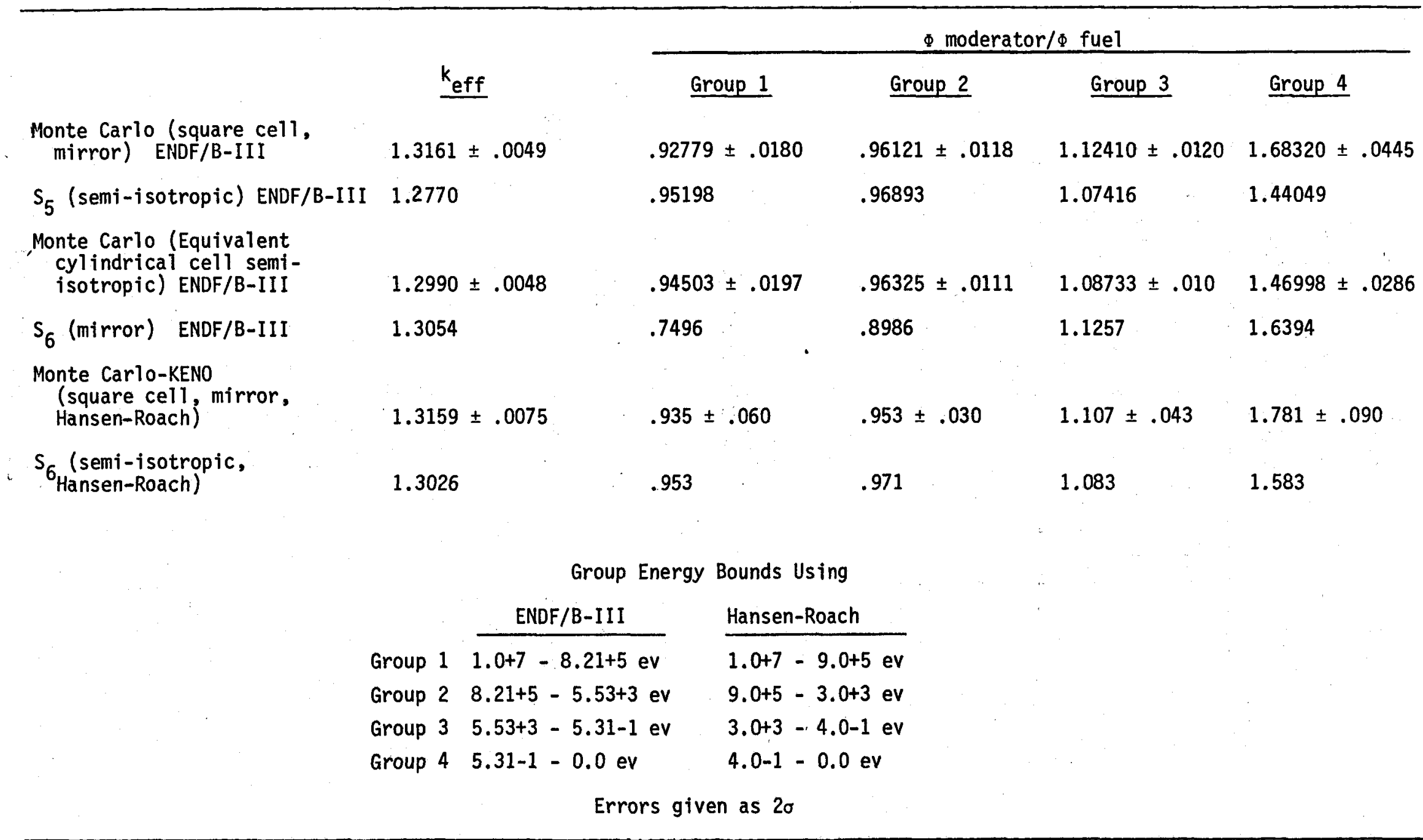




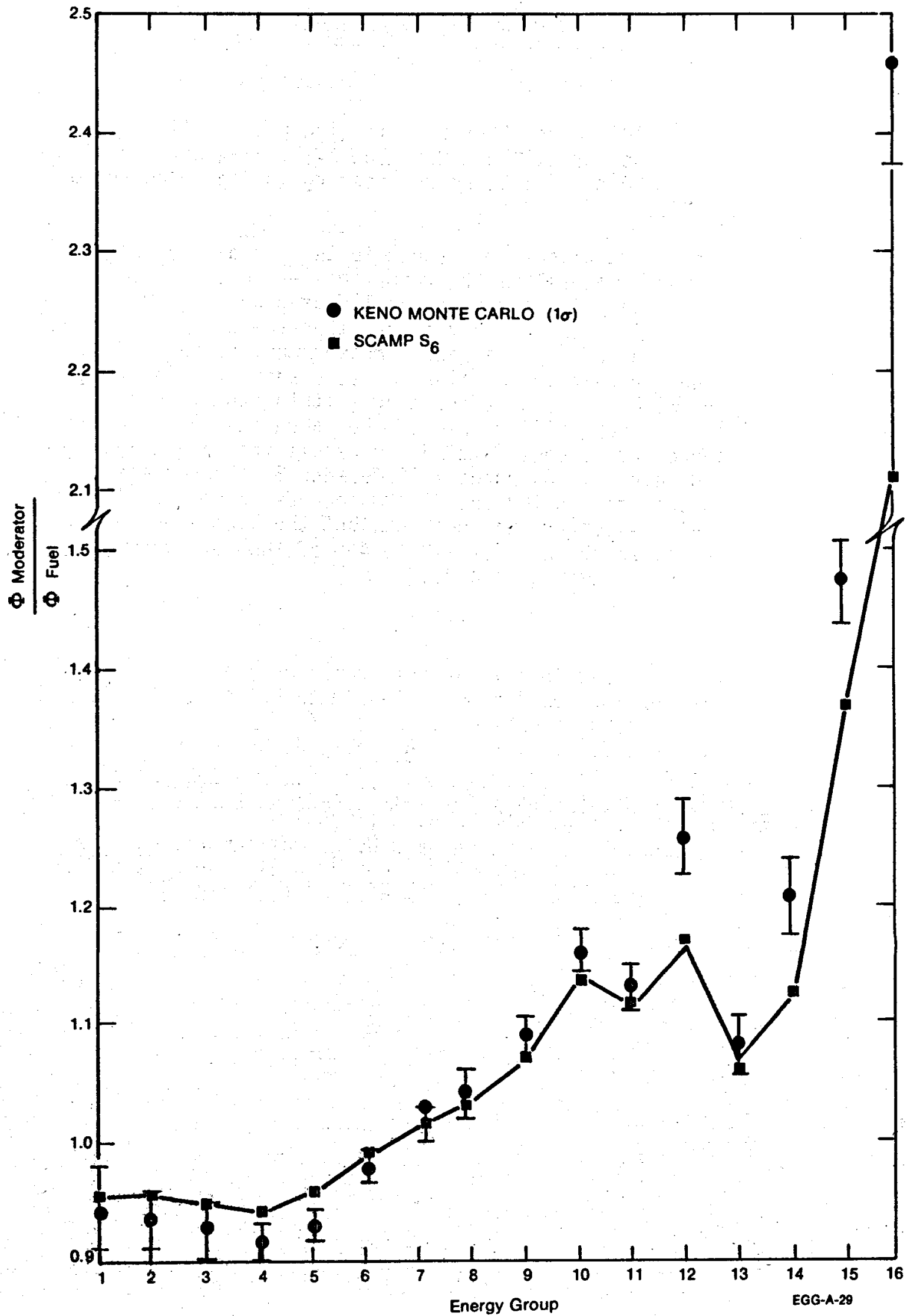

Figure 1. Comparison of Monte Carlo and S6 moderator to fuel flux ratios for Hansen-Roach sixteen group specifications. 


\section{ANS 5.1 DECAY HEAT STANDARD (1)}

\section{T. R. England* and D. E. Wessol}

Using CINDER-10 and the ENDF/B-IV fission-product library, several calculations for $B, \gamma$ and total decay heating were completed and presented at the ANS 5.1 Standards Meeting in San Francisco on November 16, 1975.

Two of the calculations correspond to the irradiation and gammameasurement times in a preliminary report of new measurements at ORNL(2). Tables I and II compare the calculated and measured values. The agreement here is considerably better than the comparison given in Reference 2. In addition, a recent measurement of the $96 \mathrm{y}$ halflife will bring our calculated values at very short cooling times into agreement with the measured heating (within experimental uncertainty). The general increase of the calculated value compared with the measurement vs cooling time may result from a gas loss in the irradiated samples. As noted in Reference 3, the content of radioactive gases between 100 and 20000 seconds drops from $17 \%$ to $9 \%$ of the total fission product content, but the gamma-energy release rate of these gases accounts for $14 \%$ to $43 \%$ of the total gamma energy, with $43 \%$ applying at 20000 seconds.

(1) The work described in this article represents a part of the activities of the Los Alamos Nuclear Data Group for the period October 1 through December 31, 1975.

(2) J. K. Dickens, J. F. Emery, T. A. Love, J. W. McConnel1, and R. W. Peele, Fission Product Beta and Gamma Energy Release Quarterly Progress Report for July - September, 1975, 0ak Ridge National Laboratory, ORNL-TM-5156 (1975).

(3) T. R. England, R. E. Schenter and N. L. Whittemore, Gamma and Beta Decay Power Following $235 \mathrm{U}$ and $239 \mathrm{Pu}$ Fission Bursts, Los A]amos Scientific Laboratory, LA-6021-MS (July 1975).

* Los Alamos Scientific Laboratory 
TABLE I

COMPARISON OF PRELIMINARY ORNL GAMMA MEASUREMENTS WITH CINDER-10 CALCULATIONS

ENDF/B-IV (2.4-S IRRADIATION)

\begin{tabular}{|c|c|c|}
\hline $\begin{array}{l}\text { Cooling } \\
\text { Interval } \\
(\mathrm{s}) \\
\end{array}$ & $\begin{array}{l}\text { Integrated MeV/ } \\
\text { Fission (ORNL) }\end{array}$ & $\begin{array}{c}\% \text { Difference } \\
\text { (Calc-ORNL)/ } \\
\text { ORNL } \\
\end{array}$ \\
\hline $\begin{array}{c}3-5 \\
5-7 \\
7-9 \\
9-14 \\
14-19\end{array}$ & $\begin{array}{l}0.219 \pm 12 \\
0.164 \pm 7.9 \\
0.133 \pm 8.3 \\
0.242 \pm 9.5 \\
0.178 \pm 6.2\end{array}$ & $\begin{array}{l}-15 \\
-13 \\
-11 \\
-5 \\
-2\end{array}$ \\
\hline $\begin{array}{l}19-29 \\
29-39 \\
39-59 \\
59-79 \\
79-99\end{array}$ & $\begin{array}{l}0.260 \pm 5.8 \\
0.190 \pm 5.8 \\
0.278 \pm 5.8 \\
0.195 \pm 5.7 \\
0.149 \pm 5.4\end{array}$ & $\begin{array}{l}-1 \\
0 \\
-2 \\
-0.5 \\
-0.7\end{array}$ \\
\hline $\begin{array}{r}99-149 \\
149-199 \\
199-299 \\
299-600\end{array}$ & $\begin{array}{l}0.254 \pm 5.5 \\
0.163 \pm 5.5 \\
0.206 \pm 5.8 \\
0.307 \pm 6.5\end{array}$ & $\begin{array}{l}+0.8 \\
+3 \\
+5 \\
+8\end{array}$ \\
\hline
\end{tabular}

TABLE II

COMPARISON OF PRELIMINARY ORNL GAMMA MEASUREMENTS WITH CINDER-10 CALCULATIONS ENDF/B-IV (100.4-S IRRADIATION)

\begin{tabular}{ccc}
$\begin{array}{c}\text { Cooling } \\
\text { Interva1 } \\
(\mathrm{s})\end{array}$ & $\begin{array}{c}\text { Integrated MeV/ } \\
\text { Fission (ORNL) }\end{array}$ & $\begin{array}{c}\text { \% Difference } \\
(\text { Calc-ORNL) } \\
\text { ORNL }\end{array}$ \\
\hline $10-30$ & $0.227 \pm 10$ & +4 \\
$30-50$ & $0.161 \pm 8.1$ & +3 \\
$50-100$ & $0.266 \pm 6.8$ & +2 \\
$100-150$ & $0.169 \pm 5.9$ & +3 \\
$150-250$ & $0.212 \pm 5.7$ & +4 \\
$250-350$ & $0.138 \pm 5.8$ & +3 \\
$350-550$ & $0.186 \pm 5.4$ & +2 \\
$550-750$ & $0.129 \pm 5.4$ & +2 \\
$750-950$ & $0.100 \pm 5.9$ & +3 \\
$950-1450$ & $0.185 \pm 5.4$ & +5 \\
$1450-1950$ & $0.132 \pm 5.3$ & +9 \\
$1950-2450$ & $0.100 \pm 6.0$ & +13 \\
$2450-14400$ & $0.636 \pm 6.5$ & +9 \\
& & \\
\hline
\end{tabular}


THE CINDER-10 BURNUP AND FISSION-PRODUCT BUILDUP CODE ${ }^{(1)}$

T. R. England*, D. E. Wessol, and N. L. Whittemore*

CINDER-10 is a general nuclide depletion and fission-product code which computes the temporal composition of coupled nuclides in a time-dependent flux environment.

The basic features of Version-10 of the CINDER code have been completed and are now operational. The code differs from Version-7(2) as described in a previous LASL progress report. Subroutines are now being debugged which provide special edits and options useful in, for example, spectral calculations, and halogen and noble gas edits. An effort is also being made to reduce the running time for this version.

The basic coding is an informal, cooperative effort of LASL and the Bettis Atomic Power Laboratory (BAPL), with some auxiliary work at the Knolls Atomic Power Laboratory (KAPL). Some subroutines and data handling treatments will differ at the three laboratories but the input data formats are identical, and the data libraries are being supplied by LASL.

A complete ENDF/B-IV fission-product library for Version-10 has been completed and copies supplied to BAPL and KAPL. The library consists of 42 linear nuclide chains formed from the data for 825 independent nuclides. The ENDF/B-IV library including corrections is described in Reference 3. The chains are expected to be adequate for many diverse applications including absorption buildup in power reactors, decay heating, $\beta$ and $\gamma$ spectra following any irradiation and cooling interval, radiological hazards, gas content, and waste disposal studies. Libraries for similar studies of actinides, impurities, and structural materials are being developed.

* Los Alamos Scientific Laboratory

(1) The work described in this article represents part of the activities of the Los Alamos Nuclear Data Group for the period October 1 through December 31, 1975.

(2) T. R. England, N. L. Whittemore, and R. Wilczynski, CINDER-7: An Interim Report for Users, LA-5885-M3, (Apri1 1975).

(3) T. R. England and R. E. Schenter, ENDF/B-IV Fission Product Files: Summary of Major Nuclide Data, LA-6116-MS (ENDF-223), (1975). 
MULTI-ISOTOPE RESONANCE LIBRARIES FOR THE PHROG FAST-SPECTRUM CODE

R. A. Grimesey, D. A. Millsap

ETOP $14(1)$ prepares multigroup cross-section libraries for the PHROG(2) fast-spectrum code. Resolved and unresolved resonance parameters are formatted directly for the PHROG cross-section library so cross sections derived from these parameters may be shielded at the correct temperature for fuel pins, etc., by PHROG. Until recently PHROG could calculate shielded cross sections for only a single isotope at a time. Several ENDF/B materials specify resolved resonance parameter libraries for several isotopes of a single naturally occurring element in the same material. Because of the single isotope limitation in PHROG, ETOP 14 would convert these multi-isotope parameter libraries into infinitely dilute multigroup cross sections for PHROG. These materials could not be shielded by PHROG for temperature and heterogeniety effects.

Both ETOP 14 and PHROG have been reprogrammed to produce and process multi-isotope S-wave resonance parameter libraries and shield them according to abundance with PHROG. ENDF/B materials with multi-isotope resonance parameters are being prepared for the PHROG cross-section library. These same modifications are being made in the RAFFLE $(3)$ Monte Carlo code and in ENDRAFL which prepares cross-section libraries for RAFFLE.

(1) R. A. Grimesey, G. L. Singer and D. A. Millsap, ETOP 14, A Fortran Code to ProcesS ENDF/B Data Into the 68-Group PHROG Library Format, ANCR-1322, (July, 1976).

(2) R. L. Curtis et. al., PHROG---A Fortran IV Program to Generate Fast Neutron Spectra and Average Multigroup Constants, IN-1435, (1971).

(3) W. E. Vesley, F. J. Wheeler, R. S. Marsden, The Raffle General Purpose Monte Carlo Code, ANCR-1022, (April, 1973). 


\section{ENDF/B CROSS-SECTION LIBRARIES FOR THE RAFFLE MONTE CARLO CODE}

R. A. Grimesey, T. Watanabe

A limitation for general use of the RAFFLE ${ }^{(1)}$ Monte Carlo code is the absence of a computer code to produce cross-section libraries in RAFFLE format from basic ENDF/B library tapes. RAFFLE at its present state of development is designed to process multigroup cross-section formats with the exception of the resolved resonance range where parameters are converted to pointwise cross sections at the correct temperature internally. Because of the large amount of core storage required by cross-section data during execution of a RAFFLE problem, cross-section libraries for RAFFLE wil l] have a multigroup format, which minimizes core storage. ETOP $14^{(2)}$ is the code which is being modified for production of RAFFLE cross-section libraries. Three sections of ETOP 14 are being reprogrammed to satisfy the requirements of the RAFFLE collision routines in a multigroup format:

1. Treatment of elastic scattering angular distributions.

2. Treatment of inelastic and $n-2 n$ secondary energy and angular distributions.

3. Treatment of the unresolved resonance range.

All other cross-section formats in ENDRAFL will use the present formats for RAFFLE libraries.

A single average elastic angular distribution will be given in tabular form for each lethargy group. The tabular distribution will be generated from Legendre expansion coefficients averaged over each group. Several prescriptions have been studied for representation of the resultant tabular distributions in an accurate and efficient manner. Tabular distributions based on a Gauss-Legendre histogram or a set of discrete abcissa cut points for an equal-area representation in either Laboratory or Center-of-Mass coordinates may be produced for RAFFLE. Short efficient collision routines have been formulated for RAFFLE to use these angular representations.

A large amount of data exists in current ENDF/B files for discrete level non-elastic scattering cross sections. Secondary angular distributions are present with the level data. To formulate the Monte Carlo collision routine in terms of individual level cross sections would require an extremely large amount of core storage to represent nonelastic cross sections and angular distributions. To reduce this storage requirement, $P_{0}$ and $P_{1}$ Laboratory Legendre scattering matrices are computed. This has the merit of convoluting the large amount of data from many levels into a single matrix from which groupto-group scattering properties can be derived within a $\mathrm{P}_{\mathrm{i}}$ approximation. The disadvantage of this formulation is that data from nonelastic angular distributions is limited to a $P_{1}$ representation. This limitation for non-elastic scattering is not nearly as severe as the 
corresponding approximation would have been for elastic scattering. Efficient algorithms to use this non-elastic matrix data have been formulated for RAFFLE.

ENDRAFL has been programed to produce the elastic and non-elastic data discussed above. A new procedure for the treatment of unresolved resonance data is still to be formulated. A Monte Carlo calculation of cross sections directly from unresolved resonance parameters would require a large amount of execution time in the RAFFLE algorithm. Determination of unresolved-range cross sections from probability tables yields the most efficient Monte Carlo algorithm but is a major programing job for ENDRAFL.: As of this date ENDRAFL has not been committed to any particular representation for unresolved resonance data.

1. W. E. Vesley, F. J. Wheeler, R. S. Marsden, The RAFFLE General Purpose Monte Carlo Code, ANCR-1022, Apri1 (1973).

2. R. A. Grimesey, G. L. Singer and D. A. Millsap, ETOP 14, a Fortran Code to Process ENDF/B Data Into the 68-Group PHROG Library Format, ANCR-1322, July (1976). 


\section{AUXILIARY COMPUTER CODES FOR PROCESSING PDQ DATA FILES}

M. W. Holm

The following three codes, operational on the CDC 7600 system, were developed as aids to the analysis and handling of PDQ data files on magnetic tape. A brief description of each follows:

PWRPRNT is a FORTRAN program which causes the four partition power values associated with each mesh point of a two-dimensional core to be printed out for a specified rectangular region. The user may specify a normalization factor as some plus-or-minus integral power of 10. The selected core region can contain up to 900 mesh points. There is no restriction on the number of regions that can be processed in one run of the program.

Printed output consists of monitoring and identifying data followed by pages containing sets of power values. Each page can accommodate up to 10 columns and 13 rows of mesh points. If there is overflow, columns are continued first, then rows on successive pages. The unnormalized partition power values are taken from a PDQ data tape.

CONCABS is a FORTRAN program which prints out normalized values of nuclide concentrations for designated mesh points. The user may specify up to 20 sets of power normalization factors, with each set containing up to seven nuclides. Normalized concentrations are printed out in a format from which data cards may be punched directly for input to LIMFIS, a plotting routine. Unnomalized concentrations are read from a PDQ data tape.

CPYPDQ2 is a FORTRAN program which will copy from one to ten PDQ data tapes onto a single output tape. The PDQ data-tape sentinel structure is preserved so that the resulting composite tape can be used as a source of data files for PDQ-7 problems. Printed output includes a list of all files copies, each being cataloged by its $J O B$ and FILE identifiers and the data-file type number.

The foregoing codes result in a significant saving in time and data handling, plus the elimination of copying errors, in the use of PDQ-7 to model nuclear reactors for design and safety-analys is studies. 
GOPP - A GENERAL ONE-DIMENSIONAL PLOT PROGRAM FOR PDQ FLUX AND POWER FILES

A. W. Brown

The GOPP program plots PDQ-generated data along general traverses specified in the mesh of one-or two-dimensional PDQ7-II (1) problems. This program was converted from the IBM-360 computer to the CDC 7600 computer and was extended and modified in the process. A new user's manual was written and sample problems (including the PDQ problem which generates the data to be plotted) were made up which demonstrate most of the features of the converted code. Values of flux, power, or activation can be plotted along traverses specified by up to 20 discrete spacial points where the distance along each traverse is a function of the cumulative linear distance between the specified traverse points. In addition to the data plotted from PDQ files, point data can also be plotted for comparison with the PDQ data. The data are plotted using the IGS $(2)$ plot routines and each plot on the microfilm output can contain up to four curves.

The printer output consists of a print of the input deck, details of the intermediate calculations, and the plotted data. The amount of data printed is controlled by the user and can vary from the minimum of only the input deck and the data plotted at the specified traverse points up to a complete print of all points plotted and a voluminous print of the intermediate calculations. A special set of input cards can be used to specify specific plot points for data to be printed and punched. The data printed for these special points includes the mesh coordinates and mesh weight values of the points.

The PDQ environmental routines are used to manipulate the PDQ files so these files can be either "tape" files or "disk" files as defined in the PDQ manual. A partition power file is required for power plots and a flux file is required for flux or activation plots. The mesh spacing for the PDQ grid can be supplied by either a PDQ geometry file or by input cards. Both flux and power normalization factors may be input and up to 10 sets of activation cross sections may be included with their application to traverse segments specified by the user.

(1) C. J. Pfeifer, PDQ-7 Reference Manual II, WAPD-TM-947(L), (February 1971).

(2) G. D. Brown, C. H. Bush and R. A. Berman, The Integrated Graphics System for the FR-80: I User's Manual, Memorandum RM-5660-PR (December 1968). 
A MULTI-REGION DANCOFF-GINSBURG

CORRECTION CODE FOR THE IBM 360/75 SYSTEM

M. W. Holm

DCFMR is a FORTRAN program that was developed to calculate Dancoff-Ginsburg correction factors for multi-region fuel pins in rectangular and hexagonal arrays. There is a built-in library of pin configurations containing the more commonly used subsets needed for finite hexagonal arrays, plus libraries for "infinite" rectangular and hexagonal arrays. Provision is also made for reading in from data cards additional subsets of up to 19 pins each, which may be required for cores of unusual shapes. Up to five regions can be accommodated.

Relevant printed output consists of Dancoff-Ginsburg correction factors for the key fuel pin in each library subset elected. These must then generally be appropriately weighted and summed to give correction factors for the actual fuel pins. 


\section{R. A. Dimenna}

The calculations for the reactivity-induced accident analysis for ATR were performed with a RELAP4 (1) mpdel of the core (2). Previous analyses for the ATR used the PARET (3) computer code to perform the kinetics calculations. As benchmark analyses demonstrating the accuracy of RELAP4 for this type of calculation were not available, the use of RELAP4 was partially justified through a comparison with PARET results for many transients of various types. This comparison demonstrated not only the similarity of the results of the two codes, but also the advantage of using RELAP4 to compute especially severe transients. Lesser advantages of using RELAP4, such as the convenience of inserting reactivity tables, were also identified.

Fifteen transients identified as credible faults in the ATR Technical Specifications were analyzed using both RELAP4 and PARET. The calculations compared were those for power vs. time, total energy generated vs. time, energy stored in the fuel vs. time, and maximum reactivity in the system during the transient. The results of these comparisons are given in Table $I$.

It was concluded that, in general:

1. RELAP4 calculates a lower peak power than PARET.

2. RELAP4 calculates a greater total energy released than PARET.

3. RELAP4 calculates a greater energy stored in the fuel than PARET.

4. RELAP4 calculates a slightly greater maximum reactivity than PARET.

(1) K. V. Moore and W. H. Rettig, RELAP4 - A Computer Program for Transient Thermal-Hydraul ic Analysis, ANCR-1127 (December 1973).

(2) R. A. Dimenna, internal report.

(3) C. F. Obenchain, PARET - A Program for the Analys is of Reactor Transients, IDO-17282 (January 1968).

(4) A. H. Bowker and G. J. Lieberman, Engineering Statistics, Prentice Hall, Inc., Englewood Cliffs, NJ (1959). 
TABLE I

COMPARISON OF RELAP4 AND PARET KINETICS CALCULATIONS

\begin{tabular}{|c|c|c|c|c|}
\hline & Power & $\begin{array}{l}\text { Total } \\
\text { Energy }\end{array}$ & $\begin{array}{l}\text { Fuel } \\
\text { Energy }\end{array}$ & Reactivity \\
\hline Average difference, $\bar{\Delta}(\%)^{(a)}$ & -2.74 & 2.50 & 5.72 & 1.06 \\
\hline Sample standard deviation, $\mathrm{s}^{(\mathrm{b})}(\%)$ & 2.71 & 2.32 & 3.89 & 1.16 \\
\hline $99 \%$ confidence interval for $\bar{\Delta}^{(c)}(\%)$ & {$[-4,92,-0.56]$} & {$[0.42,4.58]$} & {$[2.01,9.43]$} & {$[-0.05,2.17]$} \\
\hline
\end{tabular}

$\vec{\infty}$

(a) $\frac{\text { RELAP4 value - PARET value }}{\text { PARET value }} \times 100 \%$

(b)

$$
s=\sqrt{\frac{\Sigma x^{2}-\frac{(\Sigma x)^{2}}{n}}{n-1}}
$$

(c) Confidence intervals are determined using a t-statistic. (4) 
R. A. Dimenna

A RELAP4 ${ }^{(1)}$ kinetics model of the PBF Core I was developed in preparation for the reactivity safety analysis for the PBF Reload Core. The PBF Core I model was used to facilitate the model ing process for the Reload Core, for which detailed specifications and parameters were not available. The two main advantages of RELAP4, spatial weighting of the reactivity feedback and inclusion of moderator feedback effects due to heat transfer, were fully employed.

The RELAP4 model of the Reload Core will be capable of computing reactivity feedback not only from prompt Doppler effects in the fuel, but also from heat transfer into the moderator coolant. This heat transfer capability, which is important when descrjbjng the uninsulated Reload Core fuel rods, is not available in HYDRAX, (2) the neutron kinetics computer code previously used for PBF calculations. In addition, the development effort identified a needed change in the feedback calculation routine. (3) The resulting change allowed a more accurate and more versatile temperature dependence for reactivity feedback. The RELAP4 results for the $3.65 \$$ step insertion gave $26 \%$ lower power and $30 \%$ less energy than HYDRAX.

(1) K. V. Moore and W. H. Rettig, RELAP4 - A Computer Program for Transient Thermal-Hydraul ic Analysis, ANCR-1127 (December 1973).

(2) R. N. Hagen, HYDRAX - A FORTRAN Program to Calculate the Energy Release During a Reactivity Accident in a Water-Moderated Reactor, ID0-172275 (December 1968).

(3) W. R. Carpenter, internal report. 
HEAT TRANSFER MODELS FOR

UPPER HEAD INJECTION

R. C. Young

Westinghouse has designed and is building power reactors in which emergency cooling is delivered to the reactor core partly as a spray into the upper head of the reactor vessel. This technique is known as Upper Head Injection (UHI). To study the probable behavior of such a system in order to qualify the plant for operation, it was desirable to perform some calculations with RELAP4 but using the same, heat transfer correlations as Westinghouse did.

In order to provide such a capabjlity, the Westinghouse documents $(1,2)$ and other literature $(3)$ were reviewed and a procedure was prepared, in coordination with Dr. G. N. Lauben of the Core Performance Branch of NRC, to perform the necessary calculations. A special UHI version, based on the RELAP4 computer code, was generated and delivered to NRC after preliminary testing. In the original plan, the final checkout and all production runs were to be made by NRC personnel at Bethesda, Maryland. However, because of various circumstances, several long runs were also made at INEL. Several modifications were also made to secure agreement with changes by Westinghouse in their model, and to correct model and coding problems which arose during testing of the UHI code.

(1) B. A. McIntyre, R. P. Vijuk, ECCS Heat Transfer Experiments with Upper Head Injection, Volume II, Heat Transfer Data Analysis and Correlations, WCAP-8400 (January 1975).

(2) M. Y. Young, R. P. Vijuk, Westinghouse Emergency Core Cooling System Evaluation Model Application to Plants Equipped with Upper Head Injection, Westinghouse Nuclear Energy Systems \#8479, (January 1975).

(3) V. M. Borishanskii, B. S. Fokin, "Correlation of Heat Transfer Data in Stable Film Boiling on Vertical Surfaces in the Presence of Free Liquid Convection in Large Volumes", International Chemical Engineering, $\underline{5}$, (1965) 666-668. 


\section{DEVELOPMENT OF A RELAP4 MODEL FOR REACTIVITY INDUCED ACCIDENT ANALYSIS IN ATR}

\section{R. A. Dimenna}

A RELAP4 (1) model was developed to perform the kinetics calculations for the ATR Technical Specifications and the accompanying Design Basis Report. The purpose for developing the model was to employ the versatility of the RELAP4 computer code. Previous ATR kinetics analyses used the PARET(2) computer code.

The model used to describe the ATR is shown in Figure 1 . The forty fuel elements were divided into four groups or channels of ten elements each to represent four regions of average core behavior. In addition, a bypass channel was used to model core flow not heated by fuel elements. The channels interfaced at the top of the core with an upper plenum and at the bottom by a lower plenum. Neutronics and feedback parameters and the power distribution used in the model were the same as those in the original PARET model with some modification made to accomodate model differences.

The procedure employed during the analys is was to determine the most severe starting conditions for a transient with the inexpensive and relatively easy-to-prepare PARET model. Final calculations, providing more detailed edits, were then made with the RELAP4 model using the worst starting conditions identified by PARET.

The results obtained with the RELAP4 model were quite gogd. Agreement with PARET was within $5 \%$ for power and energy calculations. In addition, RELAP4 proved to be more capable of computing especially severe transients.

(1) K. V. Moore and W. H. Rettig, RELAP4 - A Computer Program for Transient Thermal-Hydraul ic Analysis, ANCR-1127 (December 1973).

(2) C. F. Obenchain, PARET - A Program for the Analys is of Reactor Transients, ID0-17282 (January 1969). 
$\square$ Control volume

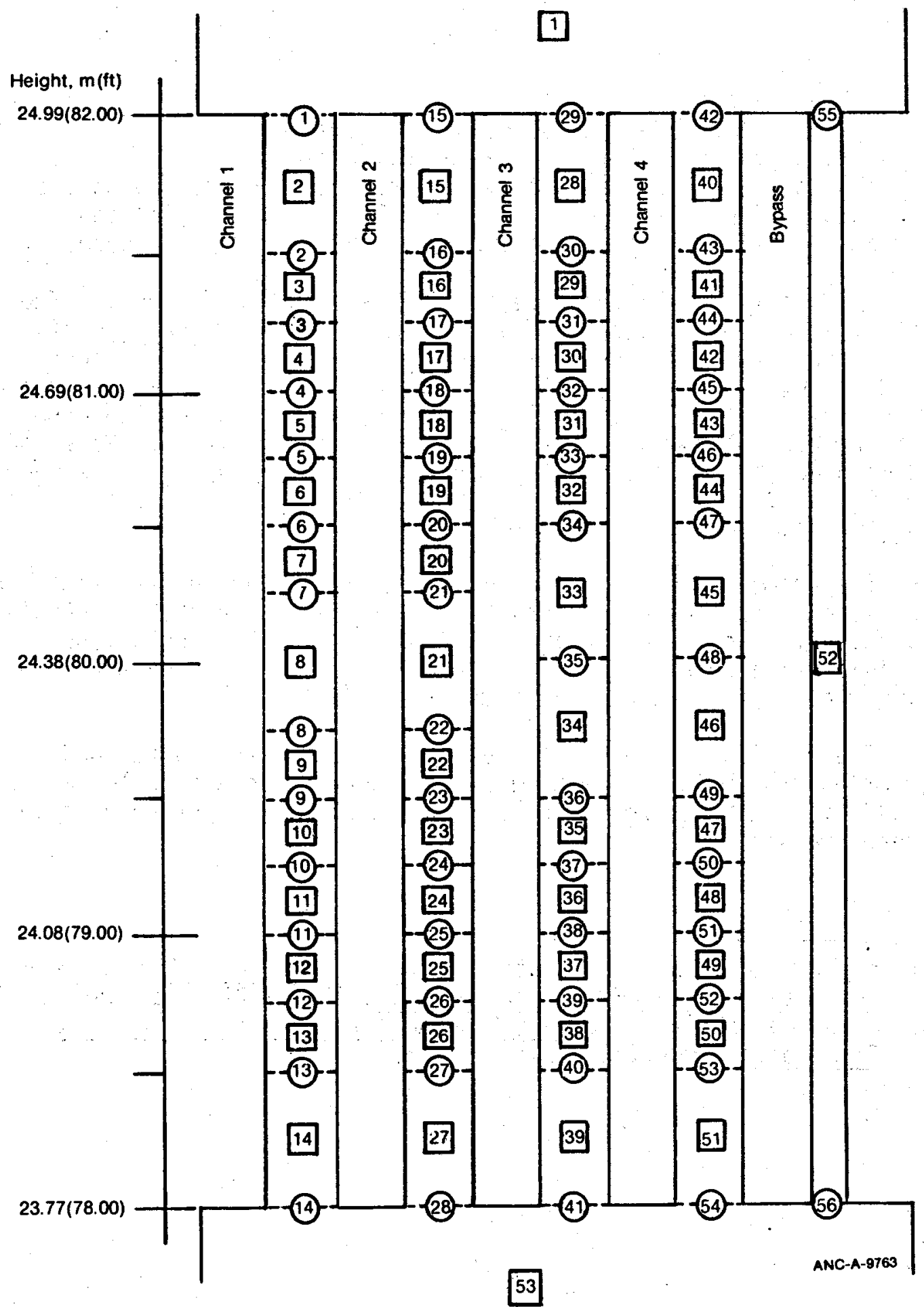

Figure 1. ATR RELAP4 model. 


\section{IMPLEMENTATION OF KENO IV CODE AT INEL}

\section{T. Watanabe}

KENO is a multigroup Monte Carlo criticality code developed at ORNL in 1969. Since its release, several versions under a variety of names have proliferated. This proliferation of the KENO code has become a problem in verification of criticality calculations among criticality evaluators. In order to eliminate the uncertainty in the calculated result which might be attributed to the particular KENO code being used, ORNL released KENO IV. In comparison with the earlier release, KENO IV has been made more flexible and simpler to use, and it was advantageous to implement this version at the INEL.

To make KENO IV code operational at INEL, it was necessary to modify the subroutine TIMFAC at the onset. Subsequently, the subroutine TYPSTART was modified according to instruction received from ORNL.

A cursory checkout of the KENO IV code was made by running Sample Problem No. 1 and Grotesque both taken from the KENO IV manual (ORNL4938) and two INEL criticality calculations. No problem was encountered in this checkout. It is anticipated that further checkout of KENO IV will come with the use of the code, especially the cross-section library.

The corrected load module, the Hansen-Roach cross-section library, the weights and the albedos now reside on tape. For production work, the load module (TEMP. LIBRARY) is moved to temporary storage (DISC) and the tape is used as backup. Because of the sensitivity of criticality calculations, the KENO IV code with the modification will be placed under configuration control. 
A remote hybrid computer simulation of the Gas-cooled Reactor In-pile Safety Test (GRIST) thermohydraulic loop has been developed in parallel with the existing digital simulation of the same mathematical mode1. In addition to providing the two-computer capability desired for GRIST system analysis; the study has yielded a comparison of computer cost and user effectiveness.

As shown in the model schematic, Figure 1, conservation of mass is computed at 15 helium nodes, and conservation of energy is computed at 14 helium nodes, 6 water nodes and 22 metal nodes. Flow inertia is included in helium flow calculations on the hybrid computer and neglected in the digital simulation. The resultant difference in simulations is of too high a frequency to be significant. The model is essentially used to study thermal behavior with a typical approach to a new equilibrium requiring 60 seconds.

The digital simulation utilized the Time And Frequency (TAF) simulation language and the Berkeley CDC 7600 computer. The user codes two subroutines describing his problem in basic Fortran. The code then provides 1) direct steady-state computation by matrix techniques, 2) frequency response and state-matrix calculations for the linearized system of equations and 3) transient response of the non-linear system using a fourth order Runge-Kutta technique.

The hybrid computer program development was underwritten by Martin-Marietta Corporation, Denver Data Center, as a demonstration of their capabilities in providing computation services not available on current INEL equipment. The Martin hybrid computer consists of an EAI 8400 digital computer (32K, 32 bit), three EAI 8800 analog consoles (60 integrators each) and numerous peripherals. The computer is operated from the INEL Hybrid Computer Laboratory using a graphics terminal, an 8-channel strip-chart recorder and a voice link. Additional data are recorded at the Martin computer and mailed to INEL. Computer charges are $750 \$ / \mathrm{hr}$ of time during which the problem is actualiy being modified or operated. Charges are not incurred for data review or run planning with the computer off.

An excellent agreement was found between digital and hybrid calculation of a design steady state and frequency response and two wide-range transients.

Cost and time effectiveness were evaluated by comparison of computer rates and user control and turnaround factors. A 60-second transient cost $\$ 13.00$ on the hybrid and $\$ 465.00$ on the digital at the highest priority (2-8 hour turnaround). Total computation cost for a set of runs could differ from this cost ratio by a factor of two or three in either direction. The resulting comparison indicates that the hybrid solution is one or two orders of magnitude less expensive with a turnaround time measured in minutes vs. hours on 


$$
30
$$

the digital. User control of the hybrid solution is substantially better than that on the digital. Cost and effectiveness would be further improved by an equal-capacity on-site computer. 


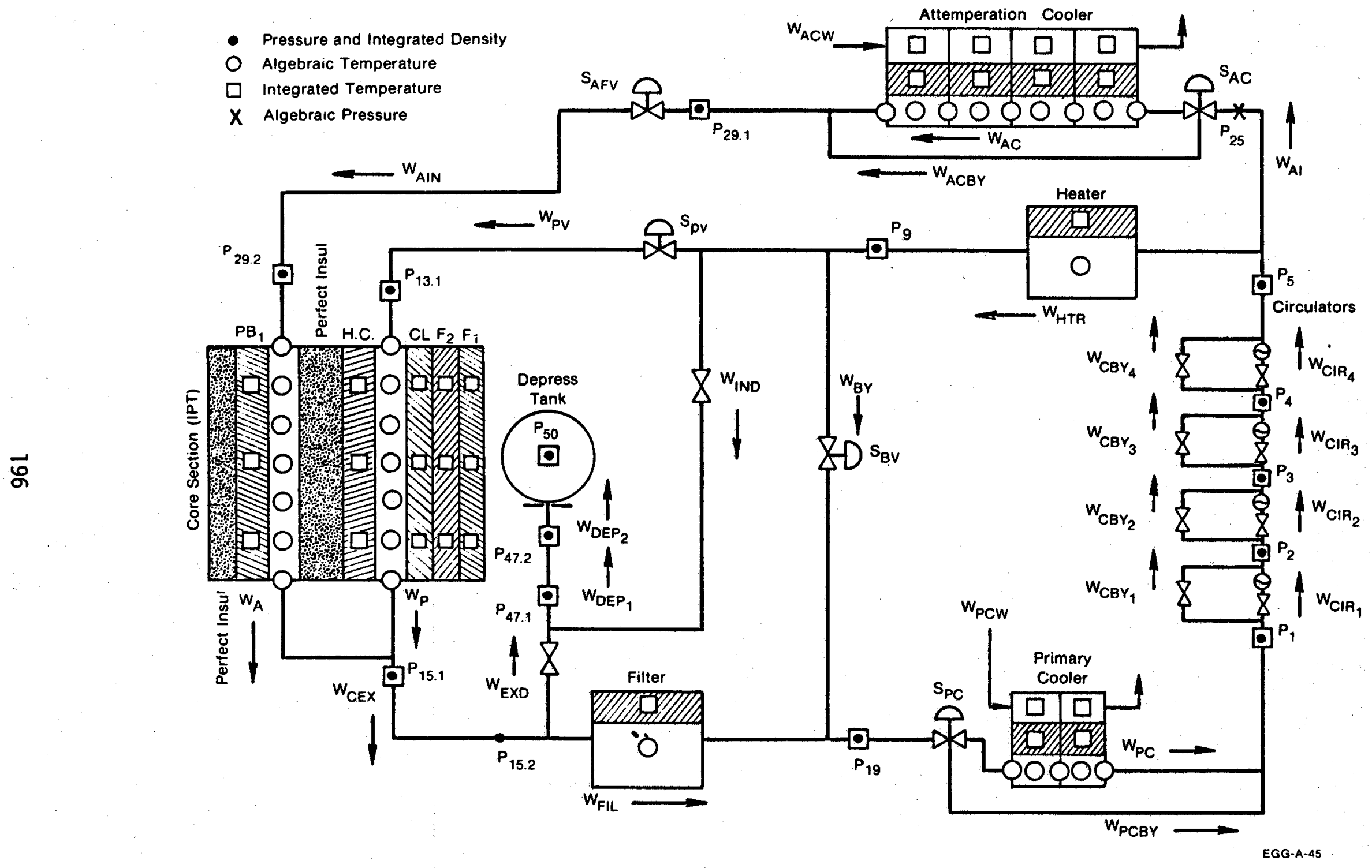

Figure 1. GRIST loop model. 
REACTOR EXPERIMENT DESIGN STUDIES 


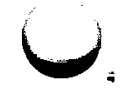

\section{BLANK PAGE}




\section{GRIST CONCEPTUAL DESIGN SYSTEMS ANALYSIS}

J. D. Miller

The Gas-cooled Reactor In-pile Safety Test (GRIST) thermohydraulic model was modified to reflect the change to a $9 \times 9$ reentrant in-pile tube configuration which provided the required maximum ETR test size. In addition, the model was extended to include heat transfer to the axial blanket portion of the fuel rods and the long pipes, and modified to reflect component updates. The Time and Frequency (TAF) simulation code mechanization was updated and used as the basis for a systems analysis study which determined the loop control configuration and adjusted the components and loop characteristics to meet design requirements.

The conceptual design model is shown schematically in Figure 1. The model includes a) the test bundle (average pin) and in-pile tube, b) the test section exit-flow path with shielded filter, primary cooler and circulators, c) the primary supply line with heater and flow-control valve, d) the attemperation flow-path through the attemperation cooler and flow-control valve, 3 ) the test bypass line and flow-control valve, f) the depressurization system and $g$ ) the actuators and closed-loop controllers.

Loop components were reviewed and resized as necessary to provide a design which is realistic and still provides a margin for uncertainty and for erosion during the design development.

The circulators are variations of the designs being used in the Cold Flow Test Loop (CFTL) at Oak Ridge. Blade tip diameter was resized to provide a sufficient head-rise margin with three circulators in series and within motor power limitations.

The sizing of the primary and attemperation coolers and the primary heater was dictated by the following test and loop constraints: 1) Required primary helium temperature at test inlet is 616K 2) Helium temperature at the exit of the third circulator is limited to $645 \mathrm{~K}$ to avoid overheating the helium-cooled circulator motor windings 3) Inner pressure boundary and mixed (primary and attemperation) helium maximum temperatures are limited to $672 \mathrm{~K}$ to avoid high-temperature design. Two coolers, of approximately equal cooling capacity, were chosen to provide a redundancy for gamma heat removal after shutdown. The electric heater, operating at $50 \%$ of its maximum, was sized to allow lowering the circulator exit temperature to provide a substantial winding temperature margin.

Line and valve and depressurization tank sizing provide reasonable operating conditions with significant margins for uncertainty and design change.

The control configuration was evolved to 1) maintain the primary inlet temperature at $616 \pm 5.5 \mathrm{~K}$ and the primary flow rate at $1.13 \mathrm{~kg} / \mathrm{s}$, 2) generate open-loop sequencing to produce the design flow transient 
(bypass $95 \%$ of the primary flow around the test section in 2 seconds) and the design depressurization (reduce loop pressure from $8.96 \mathrm{MPa}$ to $152 \mathrm{KPa}$ in 60 seconds) and 3) control loop operation within all the above discussed 1 imits plus a 60 to $120 \%$ circulator load constraint during the design transients and the anticipated malfunctions.

Closed loop controls were provided to maintain the primary temperature and flow with other loop parameters controlled by design point selection and by open-loop sequencing. The primary flow-control value was selected to control the flow and the primary cooler valve to control the temperature because they presented desirable control characteristics with minimum coupling. The cooler valve was chosen over the heater-power control because it has a greater control range. Proportional plus integral-mode controllers were designed for the two control loops.

Transient simulation included the flow and depressurization design transients, and anticipated malfunctions of a circulator and the attemperation cooler and a Class 2 (ASME Code) line break. In some cases, open-10op sequencing was evolved to be used in conjunction with operation of the two closed loops. Adequate loop performance, in al1 cases, was either demonstrated or shown to be within reach through further development of specific open-loop sequencing. 


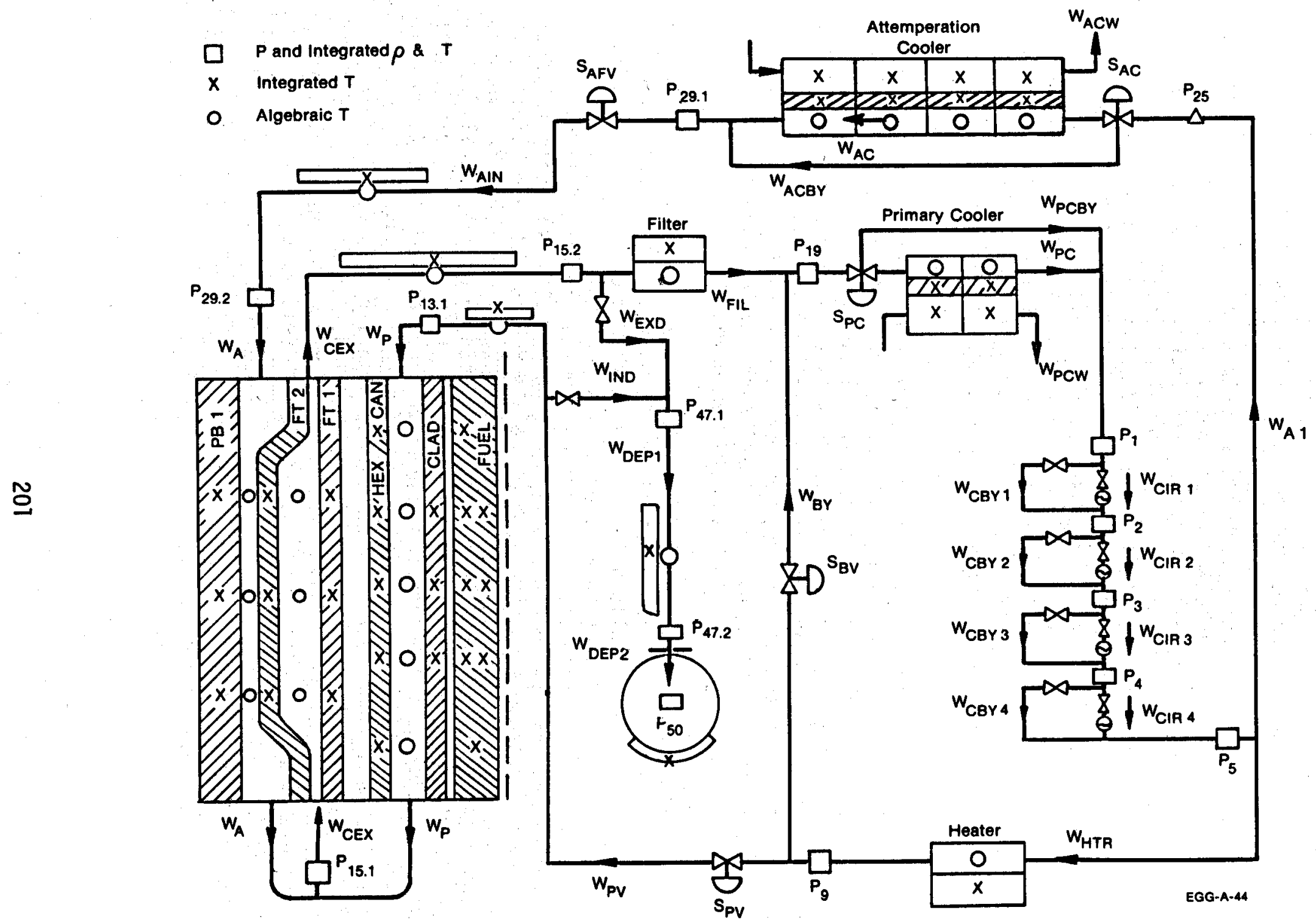

Figure 1. GRIST conceptual design model. 
DESIGN OF THE THERMAL NEUTRON FILTER

ON THE GRIST LOOP IN THE ETR

J. E. Brasier

The Gas Reactor In-Pile Safety Test (GRIST) has the objective of investigating fuel behavior in the unlikely event of cladding and fuel melting. The primary objectives for the GRIST thermal neutron filter (TNF) study were: (1) to provide an ETR core design, within the beryllium reflector boundary, that will drive a reference 37-rod bundle at a "flat" power distribution of $31 \mathrm{~kW} / \mathrm{rod}$; (2) to provide a core design which addresses all parameters required to establish ETR Technical Specifications compliance; (3) to define GRIST fuel rod enrichment requirements; (4) to define the TNF material, thickness, and location; (5) to select the TNF design that provides maximum test bundle space availability; (6) to select materials that are compatible with the test environment and can be fabricated with state-of-the-art techniques; and (7) to obtain the lowest ratio of maximum fuel enrichment-to-minimum fuel enrichment within the fuel bundle. Figures 1, 2 and 3 indicate the GRIST reference physics design used in the analytical work.

It is of prime importance that in-pile-tube materials meet code requirements without incurring high fabrication cost and an extensive preliminary testing program. From a long list of materials suitable for neutron filtering, nickel-base alloys (Inconel's) are favored for the primary and secondary in-pile tubes. Stainless steels are also excellent. Even though more reactor operational history is available for the stainless steel than the nickel-base alloys, many reactors are presently operating with large Inconel components.

Preliminary multigroup diffusion-theory calculations show that the use of cadmium as a filter material on the outside of the secondary inpile tubes may not be allowable because of the power-tilt effect across the core and test fuel bundle. Cadmium also can not be used as a filter material inside the in-pile tubes due to the high helium temperature which would cause melting. These preliminary calculations also show that the most promising materials for the TNF are hafnium, and gadolinium. These materials have high $2200 \mathrm{~m} / \mathrm{sec}$ cross sections, high melting points, small irradiation effects, good fabricability, reasonable cost, and no significant reaction with the test environment. Rare earth oxide dispersions and boron dispersions were considered, but cracking, swelling, and embrittlement of matrix materials presented some problems. Consideration is also being given to a double TNF filter, ie, a thin disposable filter on the outside of the test and an inside filter designed to last 150 full-power days. Final fuel pin enrichments will be determined after the TNF selection. 


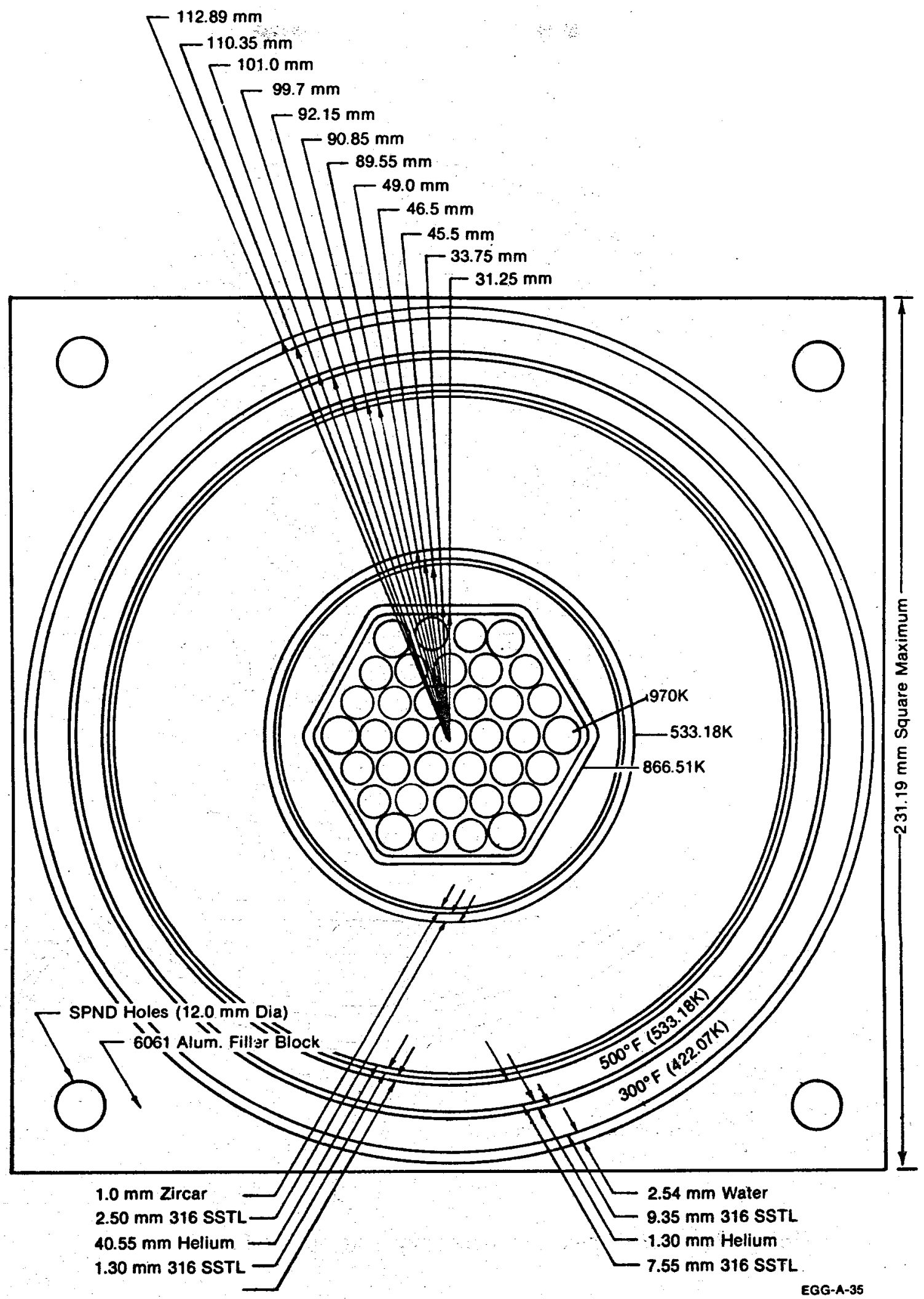

Figure 1. GRIST $9 \times 9$ loop conceptual design. 


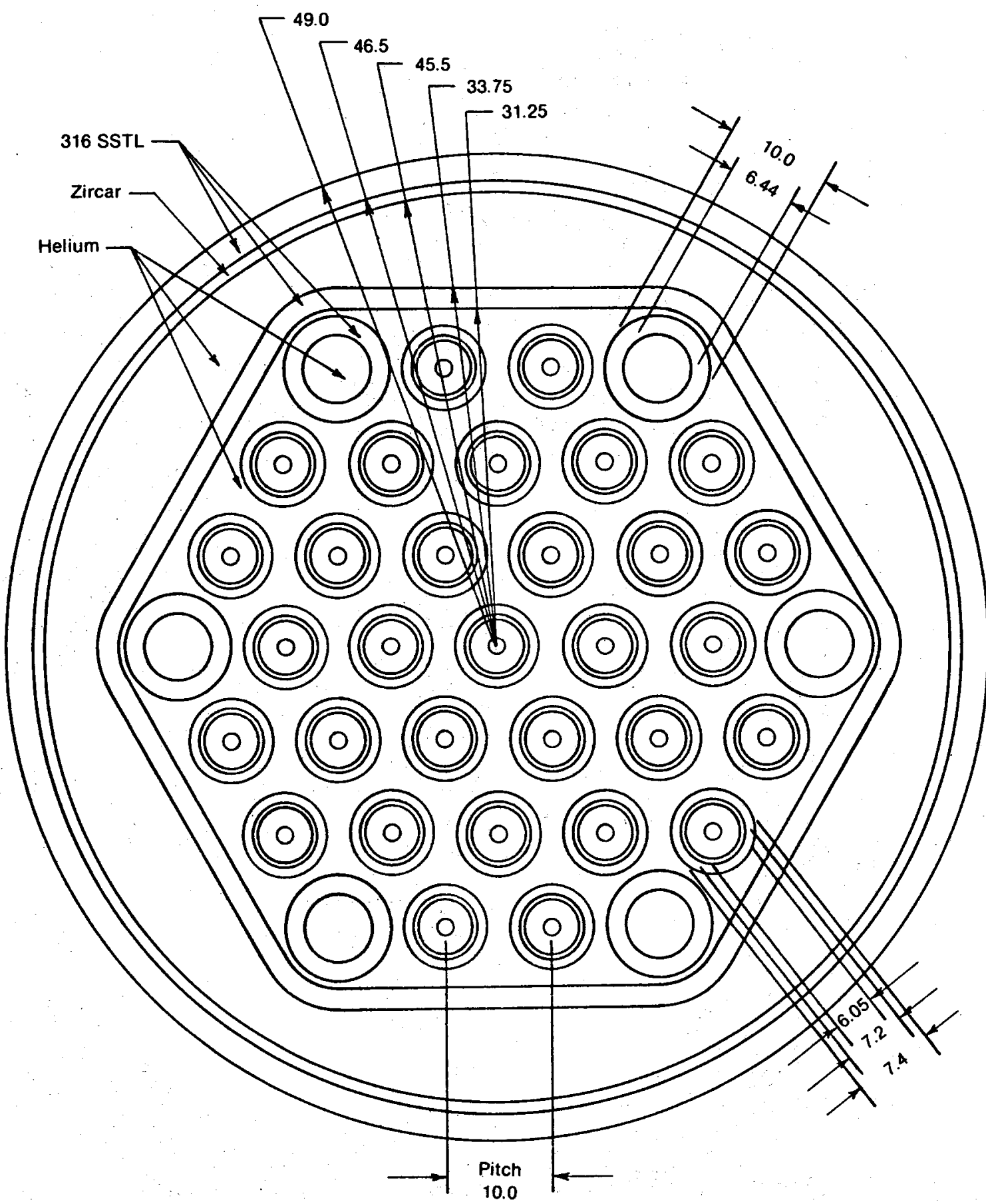

(all dimensions in $\mathrm{mm}$ )

EGG-A-33

Figure 2. GRIST hex can geometry and outer flow duct. 


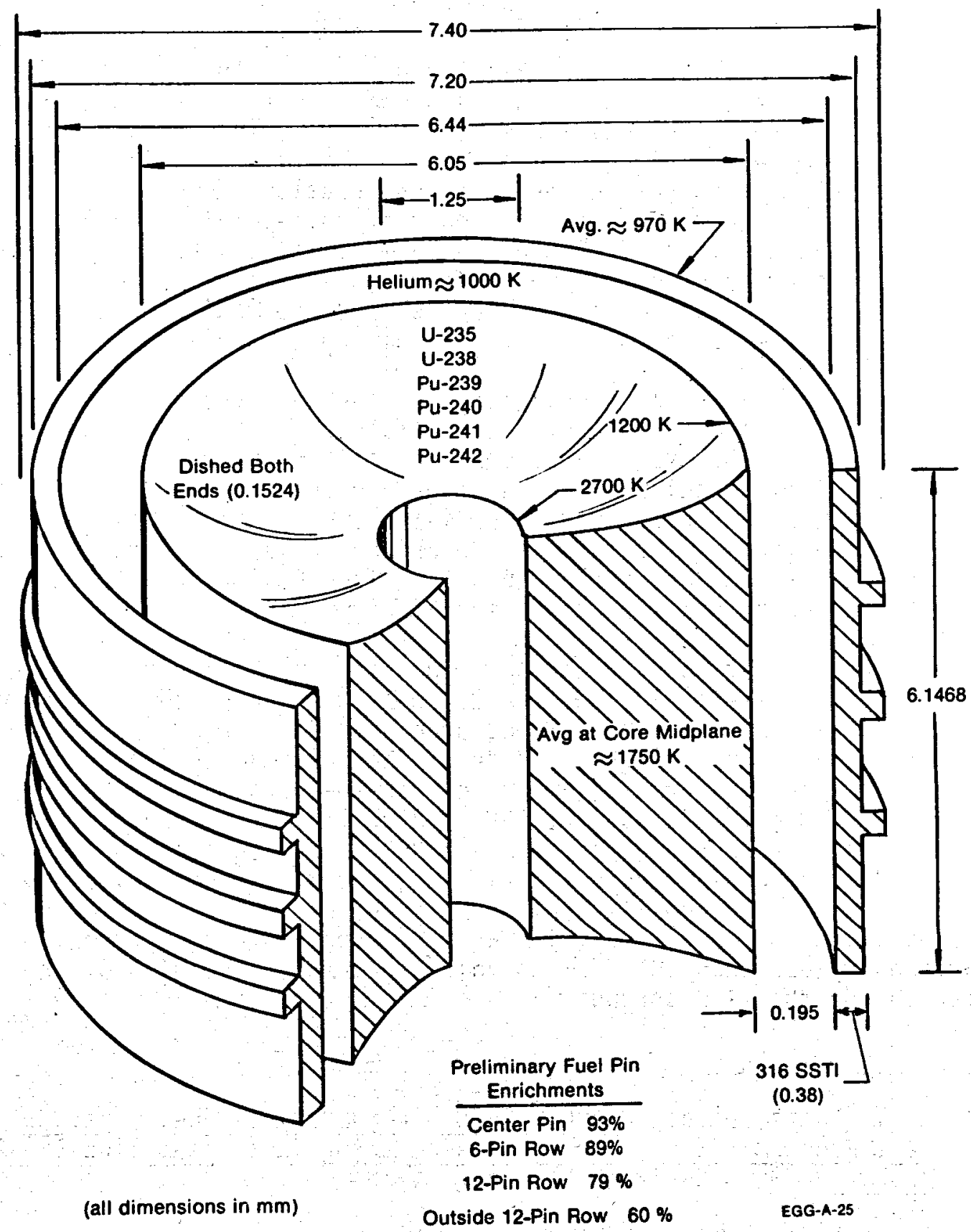

Figure 3. GRIST fuel pellet. 
PHYSICS CALCULATIONS FOR AN INSTRUMENTED FUEL

ASSEMBLY IN HALDEN HBWR

A. J. Scott \& E. Fast

Space-dependent depletion calculations have been completed for a single rod of the Instrumented Fuel Assembly-226 (IFA-226) that was irradiated in the Halden Boiling Water Reactor (1). The calculations were done to determine the pin radial power profile as a function of burnup and to compute the change in the power calibration factor (PCF) with burnup. The PCF correlated the response of self-powered neutron detectors (SPNDS), located in the D20 coolant, to the assembly power that was measured by thermal-hydraulic methods at the start of irradiation. Some calculations were also made to estimate the effect of coolant void fraction on the PCF and pin power profile.

The calculations were done with 90-group $S_{N}$ transport theory coupled with integral transport theory (for resonance shielding) using the SCRABL code. SCRABL is a combination of the SCAMP(2) and RABBLE $E^{(3)}$ programs. A cell model was assumed, ie, one fuel pin with its share of assembly water was represented in radial geometry with zero-current boundary conditions. The model is described in Table I. Using coalesced cross sections and neutron flux from SCRABL results, the depletion calculations were done with SINBAD ${ }^{(4)}$.

The radial regions average power densities as a function of burnup are given in Table II. The IFA-226 pins were composed of $9.5 \mathrm{~W} / 0$ plutonium oxide mixed with depleted uranium oxide. Due to preferential burnup of $\mathrm{Pu}$ toward the outside of the pellets, the power profile flattens out during irradiation. The peak-to-average power density after $41,094 \mathrm{MWd} / \mathrm{tU}$ was about 1.46 compared to 1.62 at the start of irradiation. The PCF dropped by about $30 \%$ as a resuit of the fuel depletion. The coolant void fraction was found to have negligible effect on the power profile. Although a definite answer could not be obtained with the model used, it was concluded that the PCF would increase by less than $10 \%$ from no voids to complete voiding of the water inside the flow shroud.

(1) E. T. Latts, et al, USNRC - OECD HALDEN Project Fuel Behavior Test Program - Experiment Data Report for Test Assemblies IFA-226 and IFA-239, ANCR-1270, (December, 1975)

(2) R. S. Marsden private communication.

(3) P. H. Kier and A. A. Rabba, RABBLE, A Program for Computation of Resonance Absorption in Multiregion Reactor Cells, ANL-7326, (April, 1967)

(4) E. C. Anderson and G. E. Putnam, SINBAD - A Space-Independent Nucl ide Buildup and Depletion Code, ID0-17217, (January, 1969.) 


\section{TABLE I}

SCRABL MODEL

\begin{tabular}{ccc}
\hline Region & Material & Outer Radius $(\mathrm{cm})$ \\
\cline { 2 - 3 } 1 & Fuel & 0.273676 \\
2 & Fuel & 0.387036 \\
3 & Fuel & 0.424263 \\
4 & Fuel & 0.453840 \\
5 & Fuel & 0.474020 \\
6 & Clad & 0.53465 \\
7 & $\mathrm{D}_{2} \mathrm{O}$ & 1.5 \\
8 & $\mathrm{D}_{2} \mathrm{O}$ & 1.6 \\
9 & $\mathrm{D}_{2} \mathrm{O}$ & 3.41276
\end{tabular}

Note: Dimensions are for the hot pin.

TABLE II

RELATIVE REGION AVERAGE

POWER DENSITIES VS BURNUP

$M W d / t U^{*}$

\begin{tabular}{|c|c|c|c|}
\hline Region & 0 & 12,772 & 41,094 \\
\hline 1 & 0.76665 & 0.80774 & 0.91558 \\
\hline 2 & 0.95072 & 0.96343 & 0.97245 \\
\hline 3 & 1.13812 & 1.10993 & 1.01825 \\
\hline 4 & 1.29574 & 1.23171 & 1.08499 \\
\hline 5 & 1.49740 & 1.41664 & 1.30058 \\
\hline
\end{tabular}

Note: Pin Average Power $=1.0$.

* Megawatt-Day per ton uranium 
REACTOR PHYSICS STUDIES FOR AN AHTGR

EXPERIMENT IN THE ETR

\section{J. E. Brasier}

The primary objectives for the Advanced High Temperature Gas Reactor (AHTGR) feasibility study were: (1) review the General Atomic, General Electric, and Westinghouse documents on HTGR fuels and conceptual designs; (2) from the above documents, select a fuel desjgn which would require the highest ETR core power in order to drive the test fuel under design-power-density conditions; (3) from the AHTGR fuel design selected, obtain a loop conceptual design which would utilize GRIST loop components with only slight modifications; (4) obtain cross-section data from the fast and thermal spectrum codes for the special AHTGR loop materials; (5) calculate the ETR core power necessary to drive the AHTGR test fuel at design power density; (6) obtain gamma ray dose rates to within \pm 15 percent as a function of distance from the center of the test loop.

All AHTGR fuels are in the form of coated particles, regardless of whether these coated particles are dispersed in graphite spheres (pebble bed design) or are compacted into tubes or cylinders which are then located in graphite block (prismatic design). Table I compares the three AHTGR fuel options. The General Atomic fuel design was selected for the analytical calculations since it would require the highest ETR core power in order to drive the GA test fuel under design conditions of $4.293 \mathrm{~kW} / \mathrm{g} \mathrm{U}-235$. A calculated ETR core power of $140 \pm 21 \mathrm{MW}$ will be required to drive the General Atomic AHTGR conceptual design at design power density.

The objective in the AHTGR loop design was to use concepts in design, materials, and manufacturing which are feasible and within state-of-the-art for core components operating in a helium environment of $1089 \mathrm{~K}$ to $1255 \mathrm{~K}$ at $6.89 \mathrm{MPa}$ with peak fuel temperatures in excess of $1677 \mathrm{~K}$. Figure 1 shows the AHTGR loop conceptual design and materials used for the analytical calculations. Due to the high helium temperatures inside the AHTGR test loop, special nickel chromium alloys (Incoloy-802) and ceramic materials (Zircar-yttria stabilized Zirconia) were chosen for the loop components. The GRIST loop components can not be used for the AHTGR fuel testing since the available test region is only $83.8 \mathrm{~mm}$ diameter and would not contain a representative sample of the AHTGR fuels. The minimum test region diameter for a representative fuel sample is $127 \mathrm{~mm}$ and requires a core filler block occupying 9 standard ETR fuel element positions $(231.19 \mathrm{~mm} \times 231.19 \mathrm{~mm})$.

The PHROG fast-spectrum code and the INCITE thermal spectrum code were used for obtaining 26-energy-group cross-section data for the AHTGR loop components at a temperature of $1255 \mathrm{~K}$ over a carbon-12 spectrum. ETR core component cross sections were previously obtained at a temperature of $324 \mathrm{~K}$ averaged over an ETR fuel spectrum. The ETR spectrum was unmodified, i.e. no thermal filter was present in the AHTGR test. 
Gamma ray dose rates for the GA loop design at an ETR power of 175 MW were obtained from the center of the test and indicated a gamma heat rate in stainless steel varying from $12.1 \mathrm{~W} / \mathrm{g}$ at test center to $21.7 \mathrm{~W} / \mathrm{g}$ at the outer edge of the core filler piece.

The calculational methods used to obtain the test power-to-core power relationship can not be expected to yield results better than \pm 15 percent. More sophisticated methods are now available if higher accuracy is desired. Calculational techniques indicate that the gamma dose rate cannot be determined to better than \pm 25 percent. 


\section{TABLE I}

COMPARISON OF AHTGR FUEL OPTIONS

\begin{tabular}{llcc}
\hline Parameter & Westinghouse & General Atomic & General Electric \\
\hline Document & WANL-2445-1 & GA-A13158 & GEAP-14018 \\
Fuel Type & Extruded Cylinder & Rod & Pebble \\
Rated Power & $3000 \mathrm{MW}$ & $3000 \mathrm{MW}$ & $3000 \mathrm{MW}(.925 \mathrm{~kW} /$ \\
pebble)
\end{tabular}




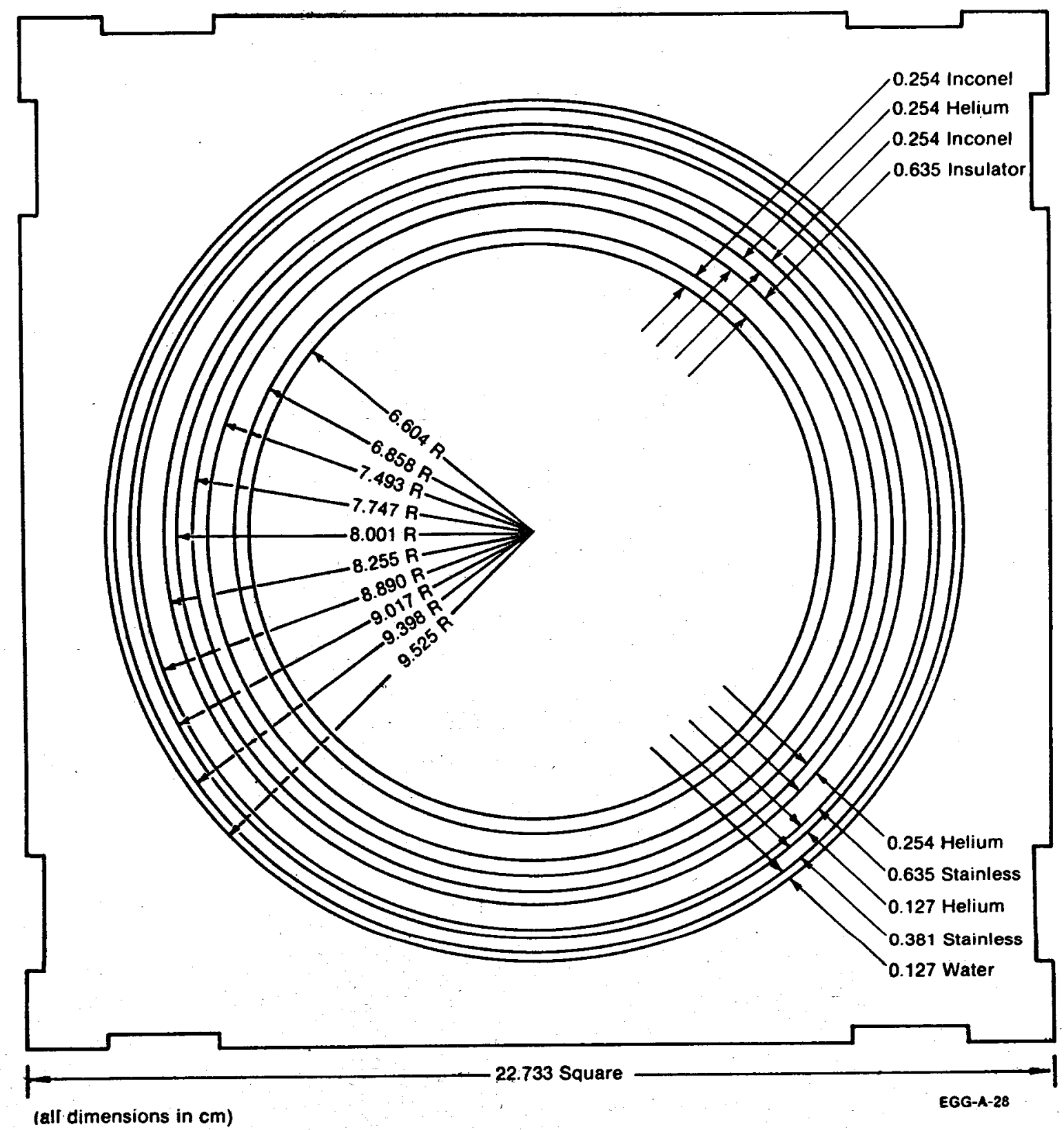
Figure 1. Proposed $9 \times 9$ test facility geometry for the AHTGR
feasibility study. 



\section{STUDY OF EXPANDED XENON AND SAMARIUM DECAY CHAIN INFORMATION}

E. H. Ottewitte, S. J. Seiber

Half-life and fission yield data for nuclides precursor to ${ }^{135} \mathrm{Xe}$ and ${ }^{149} \mathrm{Sm}$ have recently been extended at other laboratories through the development of phenomenological models. A study was initiated to determine the significance of this information to our reactor calculations.

Table I shows how the yield information has expanded in recent years. Note that the yields depend on incident neutron energy. They also vary for other fissionable nuclei. The complexity of the extended decay chains (Figure 1) and associated data constants prompts one to seek differential equation solutions which give "effective" half-life and yields for a simple one- or two-step chain representation without significant loss of accuracy. This should be easily done for times $t \rightarrow \infty$, but may pose problems for time $t \sim$ hours.

Studies to date show that effective decay constants and yields may be defined for a one-step $(\mathrm{Pm} \rightarrow \mathrm{Sm})$ chain which have a decayrate error* of $-3 \%$. Use of effective data with a two-step $(\mathrm{Nd} \rightarrow \mathrm{Pm} \rightarrow \mathrm{Sm})$ chain reduces the error to $1 \%$.

* Maximum error in the $\mathrm{Pm}$ decay rate after significant (to reactor neutronics) $\mathrm{Pm}$ has been accumulated. 


\section{TABLE I}

DIRECT YIELDS OF ${ }^{235} U$ FISSION PRODUCTS IN THE I-135 CHAIN

\begin{tabular}{|c|c|c|c|c|}
\hline FP & Eneutron & $\begin{array}{l}\text { ANL } \\
5800 \\
\end{array}$ & ENDF/B-4 & $\begin{array}{l}\text { Preliminary } \\
\text { ENDF/B-5 }\end{array}$ \\
\hline $50^{S n}$ & $\begin{array}{l}\text { Thermal } \\
0.5 \mathrm{MeV} \\
14 \mathrm{MeV}\end{array}$ & & $1.24-5$ & $\begin{array}{l}1.48-5^{*} \\
1.07-5 \\
4.01-8\end{array}$ \\
\hline $51^{\mathrm{Sb}}$ & $\begin{array}{l}\text { Thermal } \\
0.5 \mathrm{MeV} \\
14 \mathrm{MeV}\end{array}$ & & 0.00197 & $\begin{array}{l}0.00197 \\
0.00228 \\
0.00006\end{array}$ \\
\hline $52^{\mathrm{Te}}$ & $\begin{array}{l}\text { Thermal } \\
0.5 \mathrm{MeV} \\
14 \mathrm{MeV}\end{array}$ & & 0.03077 & $\begin{array}{l}0.03107 \\
0.02917 \\
0.00626\end{array}$ \\
\hline $53^{I}$ & $\begin{array}{l}\text { Thermal } \\
0.5 \mathrm{MeV} \\
14 \mathrm{MeV}\end{array}$ & $\sim 0.061$ & 0.03074 & $\begin{array}{l}0.03081 \\
0.02918 \\
0.03654\end{array}$ \\
\hline $54^{\mathrm{Xe}(g)}$ & $\begin{array}{l}\text { Thermal } \\
0.5 \mathrm{MeV} \\
14 \mathrm{MeV}\end{array}$ & 0.0019 & 0.00093 & $\begin{array}{l}0.00093 \\
0.00117 \\
0.00666\end{array}$ \\
\hline $54^{X e(m)}$ & $\begin{array}{l}\text { Thermal } \\
0.5 \mathrm{MeV} \text {. } \\
14 \mathrm{MeV}\end{array}$ & & 0.00161 & $\begin{array}{l}0.00161 \\
0.00207 \\
0.00961\end{array}$ \\
\hline $55^{\operatorname{Cs}(g)}$ & $\begin{array}{l}\text { Thermal } \\
0.5 \mathrm{MeV} \\
14 \mathrm{MeV}\end{array}$ & & $8.3-6$ & $\begin{array}{l}4.5-6 \\
5.8-6 \\
309-6\end{array}$ \\
\hline $55^{\mathrm{Cs}(\mathrm{m})}$ & $\begin{array}{l}\text { Thermal } \\
0.5 \mathrm{MeV} \\
14 \mathrm{MeV}\end{array}$ & & $6.1-6$ & $\begin{array}{l}3.9-6 \\
5.7-6 \\
308-6\end{array}$ \\
\hline $56^{\mathrm{Ba}(\mathrm{g})}$ & $\begin{array}{l}\text { Thermal } \\
0.5 \mathrm{MeV} \\
14 \mathrm{MeV}\end{array}$ & & $2.2-9$ & $\begin{array}{l}1.6-9 \\
2.3-9 \\
627-9\end{array}$ \\
\hline $56^{\mathrm{Ba}(\mathrm{m})}$ & $\begin{array}{l}\text { Therma } \\
0.5 \mathrm{MeV} \\
14 \mathrm{MeV}\end{array}$ & & $2.4-9$ & $\begin{array}{l}1.5-9 \\
1.3-9 \\
554-9\end{array}$ \\
\hline $57^{\mathrm{La}}$ & $\begin{array}{l}\text { Thermal } \\
0.5 \mathrm{MeV} \\
14 \mathrm{MeV}\end{array}$ & & $3.5-14$ & $\begin{array}{l}2.2-14 \\
3.4-14 \\
1.1-10\end{array}$ \\
\hline 7 & 1.48 & & & 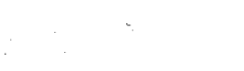 \\
\hline
\end{tabular}




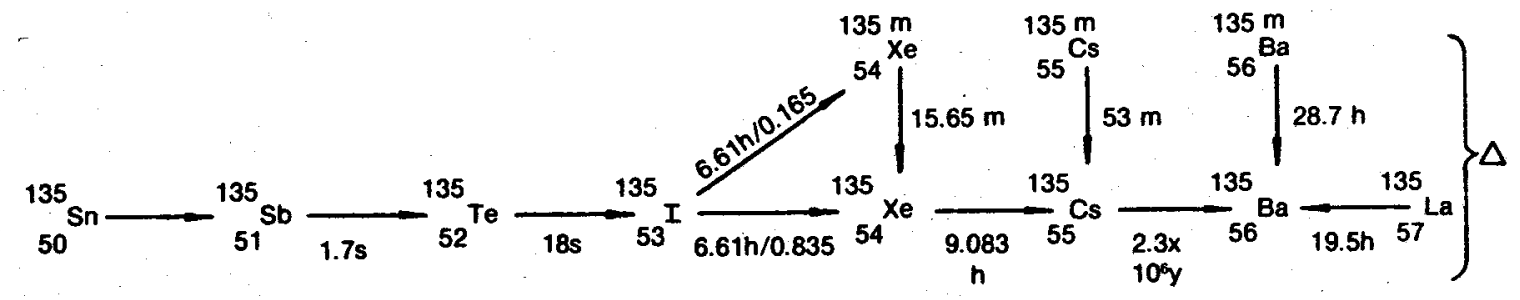

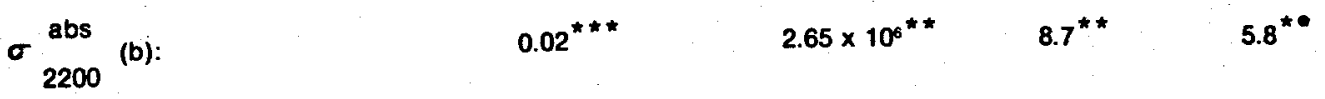

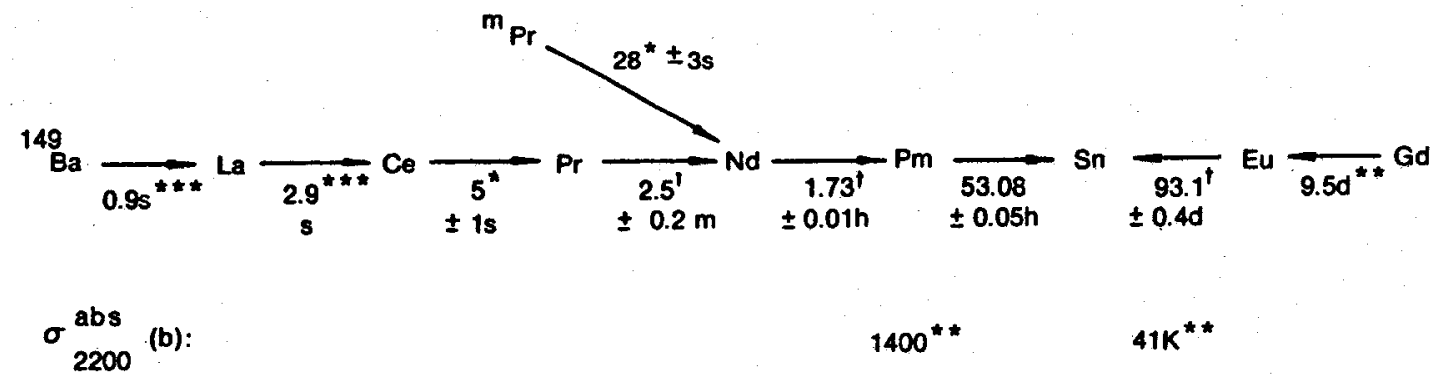

U.Mainz, BMFT-FBK-73-22, pg. 90

* Karlsruher Nuklidkarte, 4. Auflage 1974, Gesellschaft für Kernf., Karisruhe

** * ENDF/B-4 Data Files

1 Murray Martin, Nuclear Data Group, ORNL, Private Comm., 6 Jan 1975

$\triangle$ Nuclear Data Sheets, Vol. 14, No. 2, Feb. 1975

EGG-A-40

Figure 1. ${ }^{135} \mathrm{Xe}$ and ${ }^{149} \mathrm{Sm}$ decay chains. 
Y. D. Harker

Phase II testing of the Evaluated Nuclear Data File/B Version IV (ENDF/B IV) neutron cross section data set for dosimetry applications was updated. The update involved folding energy dependent reaction cross section data with the updated version of the neutron spectrum for the Coupled Fast Reactivity Measurement Facility (CFRMF). The new spectrum was calculated from transport theory using a full core representation of the CFRMF and ENDF/B IV data. The derived integrated reaction rates are then compared with respective measured values to determine the accuracy and limitations of the ENDF/B IV dosimetry data. The CFRMF is designated as a "benchmark" neutron field for data testing not only on dosimetry data but al so fission product and actinide cross section data as well. This work is being done as a part of our programmatic commitment to the evaluation and testing activities carried on by the Cross Sections Evaluations Working Group (CSEWG), a USERDA sponsored multi-laboratory effort to establish a national nuclear data file called ENDF/B.

A comparison of the updated version of the CFRMF spectrum, designated SCAMP 75/271, with the earlier spectrum, designated SCAMP $73 / 008$, is shown in Figure 1. The most pronounced change between the two spectra occurs at energies greater than 1 MeV where the new spectrum is consistently higher than the older version. This change has resulted in a significant improvement in the data testing results for the primary threshold reactions. This is evident in the tabulated reaction rate comparison given in Table I. Calculated reaction rates from three versions of the CFRMF spectrum are compared to the measure values. ENDF/B IV dosimetry reaction data were used in all calculations. The first column contains the results derived from the earlier SCAMP $73 / 008$ version of the CFRMF spectrum. The results contained in this column are the values of the original phase II testing. The second column contains results derived using a version of the CFRMF spectrum calculated using a full core representation but ENDF/B III data was used to derive the spectrum. Comparing columns 1 and 2 would then note the changes in reaction rates due to changes in model representation of the CFRMF. The third column contains results derived using the updated version of the CFRMF spectrum, SCAMP 75/271. Comparing columns 2 and 3 indicates changes resulting from upgrading nuclear data input from ENDF/B III to ENDF/B IV in spectrum derivation. From cross comparisons it is evident that larger changes are derived from the updating of the data set rather than from the expansion of the model representation. There are reactions where the new version ENDF/B does not produce improvement. however it is particylarly noteworthy to focus on the improvements made in reactions: $23 \%(n, f), 23 \pi \mathrm{Np}(n, f), 54 \mathrm{Fe}(n, p), 58 \mathrm{Ni}(n, p)$ and $115 \mathrm{In}\left(n, n^{1}\right)$. For all these reactions considerable improvement is evident. As a result over all improvement is obtained and based on a statistical analysis of the new results it appears that the overall consistency is within $4 \%$. 
Comparison of the capture rate for $238 \mathrm{U}$ shown in Table I show a large discrepancy. These results were derived using infinite dilute cross sections for $238 \mathrm{U}(n, \gamma)$ in folding with the CFRMF spectrum. This approach is an over-simplification due to the resonance selfshielding apparent for this reaction inside a $238 \mathrm{U}$ assembly. such as the CFRMF. This effect is due to the resonance overlap between activation in the ${ }^{238} \mathrm{U}$ sample and dips in the spectrum due to the same resonance absorption. The resolution of the spectrum derivation is not sufficient to provide a proper reaction rate calculation using infinite dilute reaction data for $238 \mathrm{U}(n, \gamma)$. Further investigation using cross section data which accounts of the self-shielding effects was done and the results are given in Table II. These values are in much better agreement with the measured values.

In summary, phase II testing of the ENDF/B IV dosimetry data was updated by using a CFRMF spectrum derived using ENDF/B IV data and also by accounting for self-shielding in ${ }^{238} \mathrm{U}$. Considerable improvement was obtained and at present the dosimetry data set appear to be consistent to within $4 \%$. 


\section{TABLE I}

\section{Reaction Rate Comparison}

SCAMP $73 / 008$ (cel)-ENDF/B III) meas/calc.

\begin{tabular}{|c|c|}
\hline${ }^{6} \operatorname{Li}(n, \alpha)^{3} H$ & 1.001 \\
\hline $10 B(n, \alpha) 7 L i$ & 1.115 \\
\hline $235 U(n, f) f . p . *$ & $1.00(1.588$ \\
\hline $239 p u(n, f) f . p$. & 1.045 \\
\hline $237 \mathrm{~Np}(n, f) f . p$ & 1.035 \\
\hline $238 U(n, f) f . p$. & 1.116 \\
\hline $238 U(n, \gamma)^{239 U}$ & .786 \\
\hline${ }^{45 S C}(n, \gamma)^{46} S c$ & 1.189 \\
\hline${ }^{58} \mathrm{Fe}(\mathrm{n}, \gamma)^{59} \mathrm{Fe}$ & 1.021 \\
\hline${ }^{59} \operatorname{Co}(n, \gamma)^{60} \mathrm{Co}$ & 1.095 \\
\hline${ }^{115} \operatorname{In}(n, \gamma)^{116 m} \operatorname{In}$ & .953 \\
\hline${ }^{197} \mathrm{Au}(\mathrm{n}, \gamma) 198 \mathrm{Au}$ & 1.050 \\
\hline${ }^{27} \mathrm{Al}(\mathrm{n}, \mathrm{p}){ }^{27} \mathrm{Mg}$ & 1.000 \\
\hline${ }^{27} \mathrm{Al}\left(\mathrm{n}, 1{ }^{24} \mathrm{Na}\right.$ & .972 \\
\hline $46 \mathrm{Ti}(n, p) 46 \mathrm{Sc}$ & 1.235 \\
\hline $47 T i(n, p){ }^{47} S c$ & .899 \\
\hline${ }^{48} T i(n, p){ }^{48} S c$ & 1.659 \\
\hline${ }^{54} \mathrm{Fe}(\mathrm{n}, \mathrm{p}){ }^{54} \mathrm{Mn}$ & 1.077 \\
\hline${ }^{58} \mathrm{Ni}(\mathrm{n}, \mathrm{p}){ }^{58} \mathrm{Co}$ & 1.116 \\
\hline${ }^{115} \operatorname{In}\left(n, n^{1}\right)^{115 m}$ In & 1.164 \\
\hline${ }^{63} \mathrm{Cu}(\mathrm{n}, \gamma)^{64} \mathrm{Cu}$ & .980 \\
\hline
\end{tabular}

SCAMP $74 / 153$

(full core-ENDF/B III) (full core-ENDF/B IV) meas/calc.

1.000

1.085

$1.00(1.597)$

1.052

1.048

1.136

.774

1.187

1.012

1.033

.951

1.038

1.023

.994

1.261

.917

1.694

1.097

1.135

1.183

.972
SCAMP 75/271 meas/calc.

1.024

1.122

$1.00(1.595)$

1.047

.983

1.017

.781

1.203

1.022

1.041

.955

1.048

.953

.937

1.176

.834

1.617

1.010

1.040

1.067

.976 


\section{TABLE II}

Foil

Thickness $(\mathrm{cm})$

$\infty$ dilute

$1.22 \times 10^{-4}$

$2.54 \times 10^{-3}$

$1.27 \times 10^{-2}$
${ }^{238} \mathrm{U}$ Capture in CFRMF

$$
\sigma / \sigma_{25 f}
$$

Measured

$.118( \pm 4.0 \%)$

$.114( \pm 3.5 \%)$

$.112( \pm 3.4 \%)$ $\sigma / \sigma_{25 f}$

Calculated

.145

.1198

.1175

.1138
Meas./Calc.

.985

.970

.984 


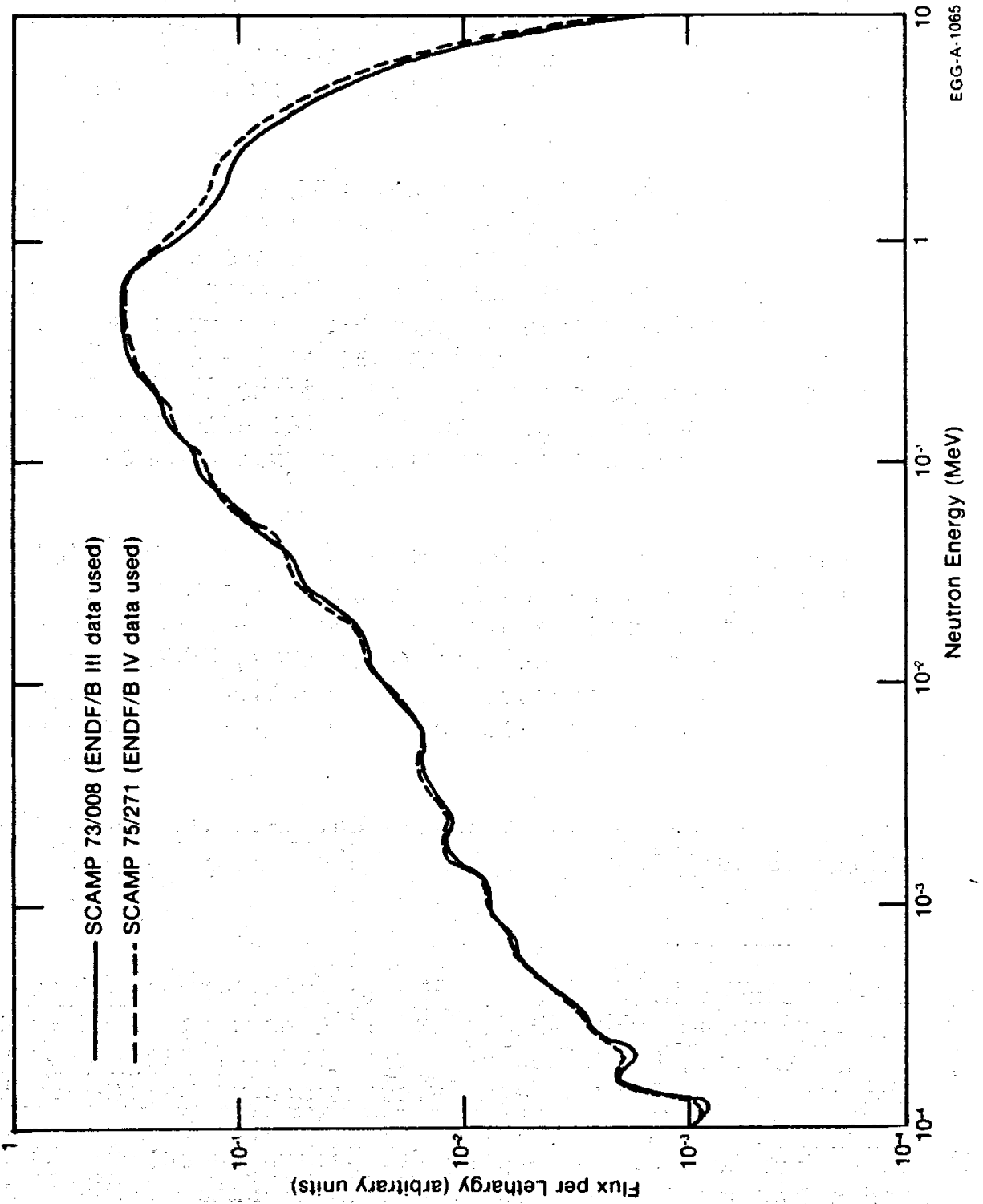

Fig. I CFRMF Neutron Spectrum calculated from transport theory using data from different versions of ENDF/B. 


\section{UPGRADE OF NUCLEAR CRITICALITY SAFETY}

EVALUATION TECHNIQUES

\section{J. H. Lofthouse}

An effort has been made over the past year to upgrade the tools and techniques for performing nuclear criticality evaluations at INEL. This has come about through a nationwide effort by ERDA to develop more complete and better documented criticality analyses for fuel storage and handling facilities.

An upgraded version of the Monte Carlo neutronics code, KENO IV $(1)$, was obtained from the developers at ORNL and made operational on the INEL IBM-360 computer system. This code, along with a "standard" 16-group Hansen-Roach cross-section set which has been rather thoroughly validated $(2,3,4)$, is quite universally used in criticality evaluations because of the ease with which models with complex geometries can be handled.

When it became known that the new INEL computer system would be a CDC CYBER 70/model 76, a version of KENO IV compatible with this system, was requested from ORNL. The actual code conversion was made by CDC and was made operational on their machine at Minneapolis. A copy of the magnetic tape containing this program has been received at INEL and is in the process of being made operational on the CYBER 70 system. The next step is to bring both the IBM 360 and the CYBER 70 versions of the code, along with the cross-section libraries used for criticality calculations, under configuration control so that a calculation is completely traceable.

In addition to the work described above, the upgraded Hansen-Roach cross-section library received on the same tape with KENO IV has been put into a format compatible with the one-dimensional $S_{n}$ transport code, SCAMP, and the one-dimensional diffusion code, MONA. In addition to the conversion, appropriate transport cross sections were generated and included in the library so that non-infinite systems can be handled using SCAMP or MONA.

These tools now provide a consistent base for performing nuclear criticality calculations.

(1) L. M. Petrie, N. F. Cross, KENO IV - An Improved Monte Carlo Criti cality Program, ORNL-4938, Oak Ridge Nat. Lab., (November, 1975).

(2) G. R. Handley, C. M. Hopper, Validation of the "KENO" Code for Nuclear Criticality Safety Calculations of Moderated, Low Enrichment Uranium Systems, Y-1948, (June 13, 1974).

(3) G. R. Handley, C. M. Hopper, Validation Checks of the ANISN and KENO Codes by Correlation with Experimental Data, Y-1858; Union Carbide Corp.; ORNL Y-12 Plant (1972).

(4) S. R. McNeaney, J. D. Jenkins, Comparison of Hansen-Roach and ENDF/B-IV Cross Sections for $233 \mathrm{U}$ Criticality Calculations, ORNL-TM-5113, Union Carbide Corp. (January 1976). 


\section{DETERMINATION OF MINIMUM CRITICAL MASS}

FOR FLOURINEL DISSOLVERS

R. R. Jones, J. H. Lofthouse

Neutron criticality calculations were performed to evaluate the Flourinel Dissolver design for Allied Chemical Company. The evaluation establishes the minimum critical mass and develops a means for specifying a safe loading limit for the system.

The proposed dissolver is an upright cylinder with a $0.4826-m$ (19-inch) inside diameter. During normal operation the dissolving fuel element rests on a series of crash bars forming slots at the bottom of the cylinder, and small undissolved fuel particles collect in a safe cone below the crash bars. The dissolving agent is an acid with $24 \mathrm{~g}$ of cadmium per litre of solution as a neutron poison.

A credible accident is assumed to be an event where large chunks of the dissolving element fall onto the crash bars blocking the entrance to the cone. Eventualiy geometry control of the dissolving element is lost and particles collect in an unsafe geometry above the cone. A conservative calculational model for this accident assumes that the highly enriched fuel and cladding material goes to a homogenized sphere in the dissolver solution with the sphere reflected by the dissolver solution.

A series of calculations on this system, using the one-dimensional $S_{n}$ transport code SCAMP with 16-group Hansen-Roach cross sections, was made to determine the minimum critical U-235 mass at various molar fractions of $\mathrm{UO}_{2}$ and $\mathrm{ZrO}_{2}$. The results of these searches are plotted in Figure 1 and show that the minimum critical mass is $19.0 \mathrm{~kg} \mathrm{U}-235$.

The most conservative situation upon which to base a loading limit for the dissolver is to assume that no cladding material is present. Applying this assumption calculations were performed using various $\mathrm{U}-235$ loadings in the sphere and these results are plotted in Figure 2. Note that for data given in both Figures 1 and 2 , for a given $U-235$ mass and density the radius of the sphere is fixed. The maximum k-effective calculated for each U-235 loading, by searching for the most reactive density (sphere size), is replotted in Figure 3 as a function of the loading. Figure 3 can now be used to select a loading limit with a calculated safety margin. 


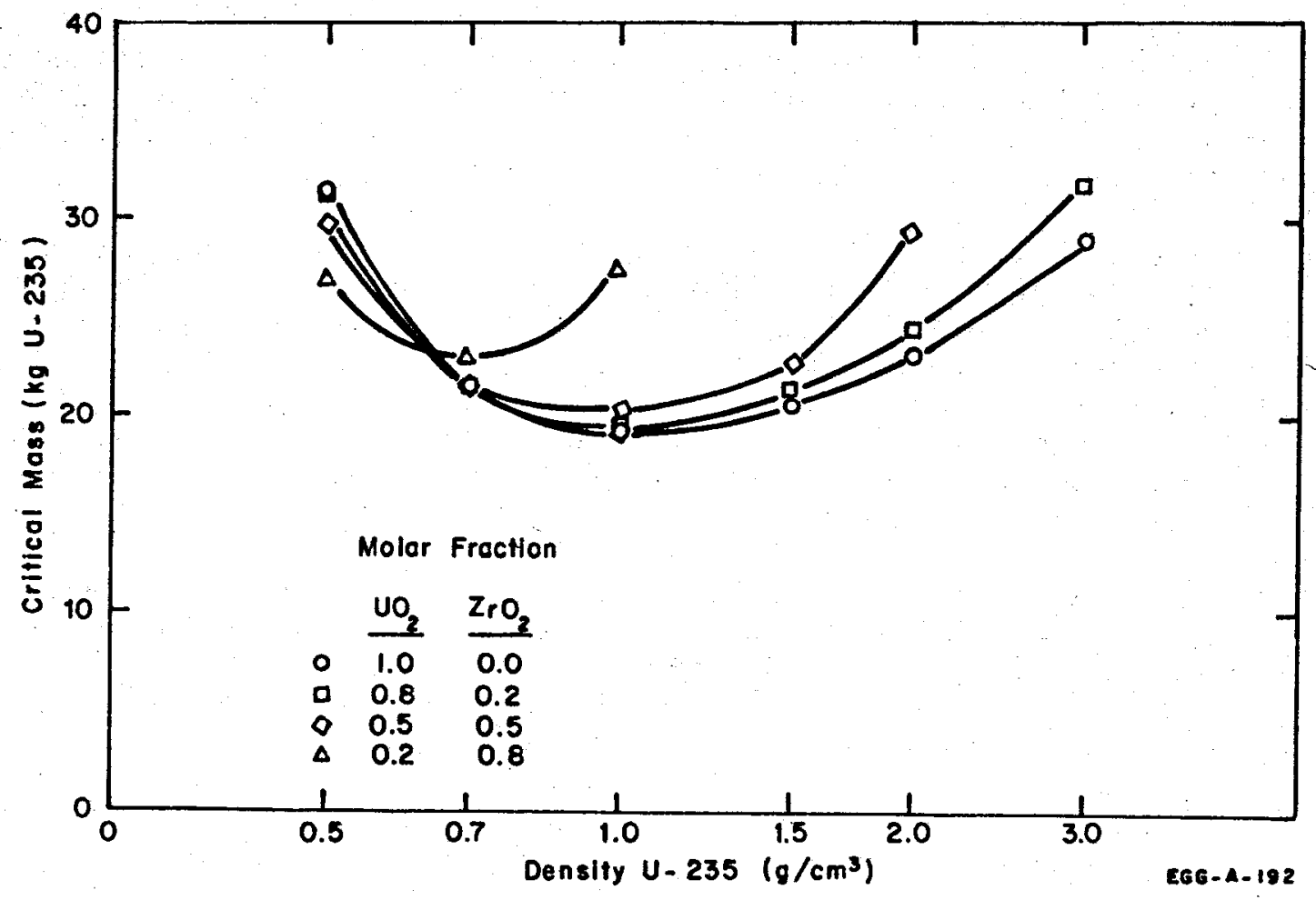

Figure 1. Critical mass versus U-235 density at various molar fractions of $\mathrm{UO}_{2}$ and $\mathrm{ZrO}_{2}$. 


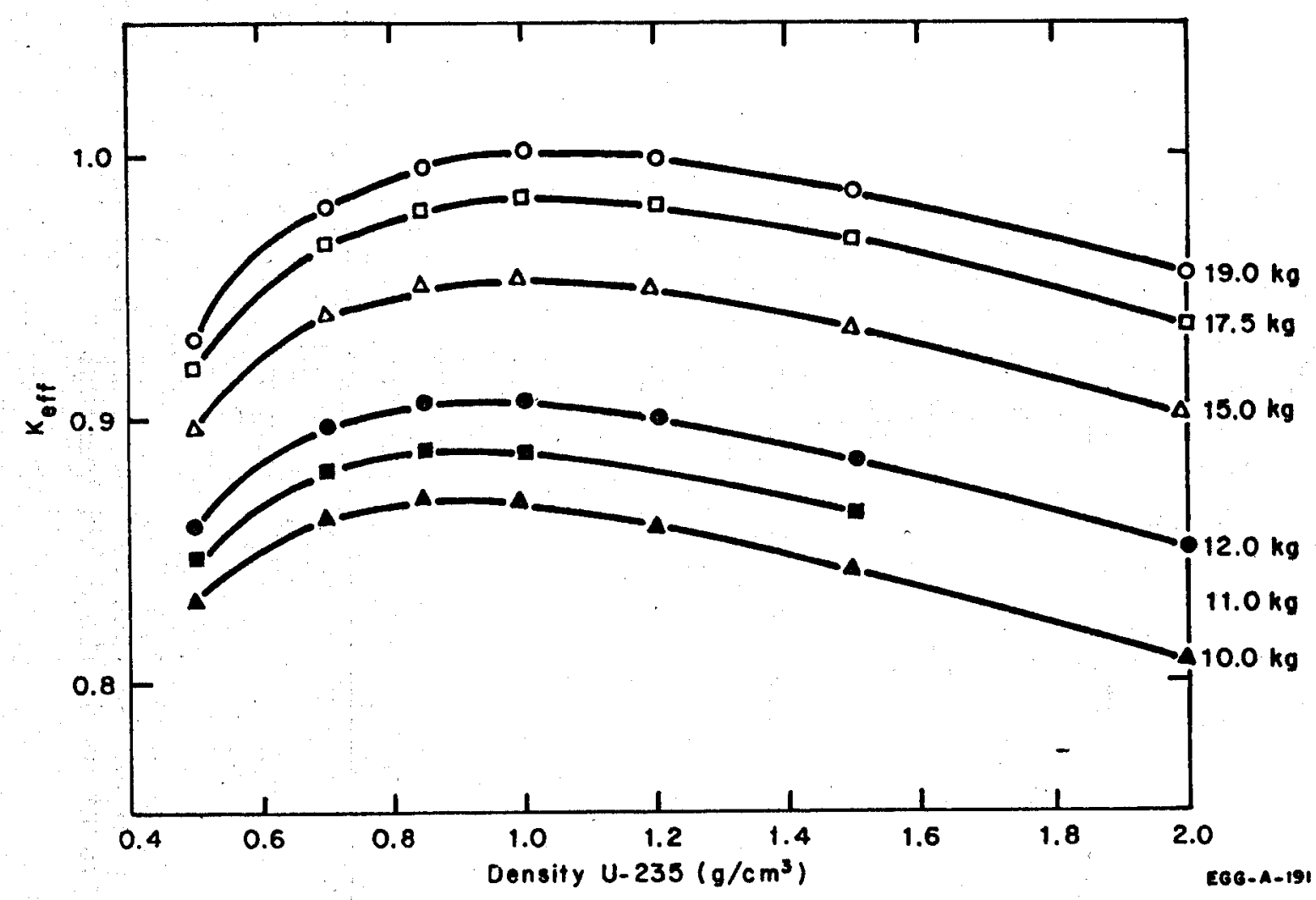

Figure 2. k-effective versus U-235 density at various U-235 loadings. 


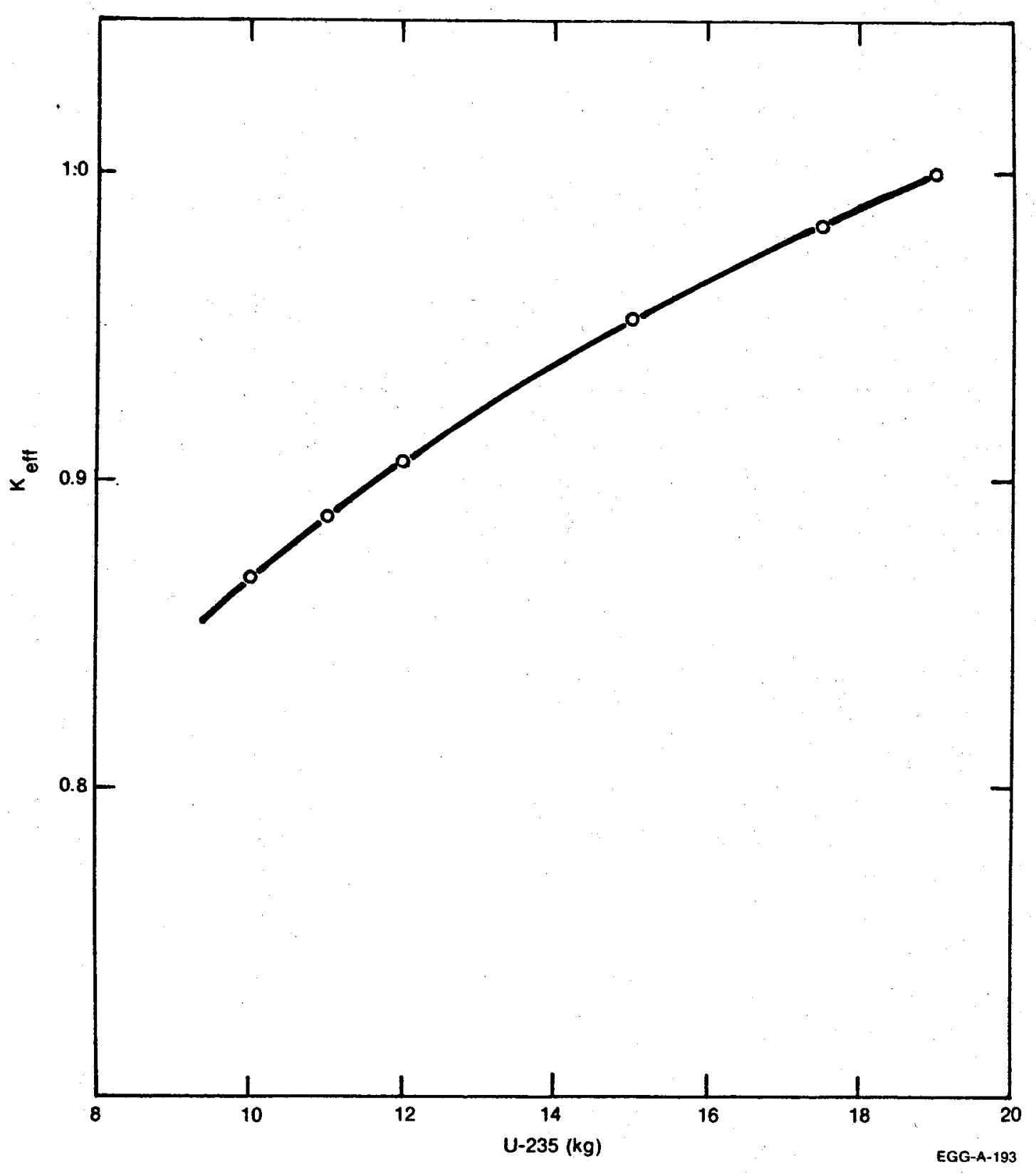

Figure 3. Maximum k-effective from Figure 2 plotted as a function of the U-235 loading. 
CRITICALITY AND SHIELDING EVALUATION OF THE PBF POOL STORAGE RACKS FOR THE PBF RELOAD CORE

J. B. Briggs, J. R. Johnson, J. H. Lofthouse

Temporary storage of the expended PBF Reload Core will be in storage racks in a water-filled basin adjoining the reactor containment vessel. The facility is designed so that personnel have access to areas around the basin wall at basement levels in the building. An extension to the storage basin is planned so that two more storage racks can be installed. An analysis was performed to determine if the shielding is adequate and the storage racks are safe for the Reload Core.

The shielding calculations were performed with computer code QAD P5A and ISOSHLD II. The two codes, both of which are point-source codes, gave excellent agreement. The gamma source was modeled by assuming homogenized cubes of water and fuel modules the size of the racks. The design basis for the calculations was a PBF Reload Core operated at $50 \mathrm{MW}$ steady state for 5000 MWd and cooled for one day. The calculations show that additional shielding material may have to be added to the high-density concrete shield wall to reduce the dose rate to personnel to $0.25 \mathrm{mR}$ per hour. This shielding material will likely be sheet lead a few centimeters thick.

A criticality evaluation was made of the two aluminum storage racks installed in the basin and of the two stainless steel racks for installation in the basin extension. Each rack contains four rows of storage positions. The storage positions within a row are separated by aluminum plates. The sides of the storage positions in a row are constructed of an aluminum/cadmium/aluminum sandwich. The rows are separated by $0.0508 \mathrm{~m}$ ( 2 inches) of water. The rack for the basin extension is to be constructed in the same manner except stainless steel will replace aluminum in the description above.

The evaluation made for storing PBF Reload Core fuel modules at $10 \%$ enrichment in U-235 shows that the racks are safe as long as the assembly has no missing fuel pins. Missing fuel pins from several assemblies render the storage arrangement unsafe.

The Monte Carlo computer code KENO IV with 16-group Hansen-Roach cross sections was used to make the calculations on a conservative model consisting of an infinite number of storage positions in the $x-y$ plane. This model gives a nominal k-effective of 0.92 for the worst case which is the aluminum rack. It was demonstrated that missing pins would increase k-effective. Six missing pins near the center of each module raised k-effective above 0.95 in the stainless steel racks. 


\section{CRITICALITY EVALUATION OF PBF 5-TON CASK}

J. H. Lofthouse

The PBF 5-ton cask is used for on-site shipments of irradiated fuels from the PBF reactor to fuel inspection and storage facilities. Calculations were performed to determine a safe loading limit for the cask. The limit is a general limit which applies to any geometry of fuel or degree of moderation within the cask.

A salient feature of the cask from a criticality standpoint is the $0.1016-\mathrm{m}(4-i n c h)$ by $1.82-\mathrm{m}(\sim 6-\mathrm{ft})$ cylindrical cavity. A schedule 40 stainless steel pipe forms the cavity liner and a $0.457-m-0 D$ (18-inch-OD) schedule 40 iron pipe forms the outer cask shell. The annulus between is filled with lead. The final external dimensions are $0.457 \mathrm{~m}$ ( 18 inches) in diameter by $2.21 \mathrm{~m}$ ( 87 inches) in length.

The general U-235 mass limit for the cask was determined by searching for the most reactive geometry for a water-fuel mixture in the cask cavity, given a U-235 mass limit. The geometry for the water-fuel mixture was $0.1016-m$ (4-inch) diameter cylindrical slugs in the cask cavity. The length of the slug was varied to find the most reactive length. The uranium for this evaluation is $93 \%$ enriched in U-235.

Based on a uranium density of $18.7 \mathrm{~g} / \mathrm{cm}^{3}$, a uranium volume fraction was calculated and the remainder of the fueled volume was assumed to be water. This approaches the highest possible $\mathrm{H} / \mathrm{U}-235$ ratio in the fueled volume. Searches for maximum k-effective were made for $5-\mathrm{kg}, 15-\mathrm{kg}$, and $25-\mathrm{kg}$ loadings using the Monte Carlo computer code KENO with 16-group Hansen-Roach cross sections.

By interpolation this conservative model showed that a $10-\mathrm{kg}$ U-235 mass 1 imit has a safety factor greater than 2.0 , and that double batching will not result in a critical system. The limit was set at $10 \mathrm{~kg} \mathrm{U}-235$. 


\section{CRITICALITY EVALUATION OF \\ OVERLOADED ROCKY FLATS WASTE DRUM}

E. Fast, J. H. Lofthouse

A DOT 17-6 55-gallon waste drum received from Rocky Flats in early 1976 was found to be loaded with Pu-239 above the limits allowed for storage at the Radioactive Waste Management Complex (RWMC) at INEL. A criticality evaluation was made to determine if an exception could be made for storing this one overloaded drum on the storage pad with other drums at or below the storage limit.

The Pu-239 storage limit for drums of this type is 200 grams. Drum RF771-6839 was found to contain $270 \pm 30$ grams Pu-239 by using high-resolution gamma-ray spectrometry. An evaluation was made with a model where this drum contained 330 grams Pu-239, two standard deviations above the measured content. The drum with the Pu-239 moderated in a sphere of polyethyl ne was placed at various positions in an array of close-packed drums each containing 200 grams of Pu-239 in polyethylene. The array was 5 drums high with the drums set on end for all cases calculated.

The calculations were performed with the KENO IV computer code using 16-group Hansen-Roach cross sections. The results given in Table I show that it is safe to store the over-loaded drum on the storage pad in the normal storage manner prescribed by the standard procedures document. 


\section{TABLE I}

RESULTS OF KENO CALCULATIONS FOR STORING WASTE DRUM RF771-6839 AT THE RWMC

\begin{tabular}{cccc}
\hline Case & Neutron Histories & $\frac{k_{\text {eff }} \pm 10}{0.849 \pm .009}$ & $\begin{array}{l}\text { Remarks }(a) \\
\begin{array}{l}\text { RF771-6839 at center of } \\
\text { array infinite in } x-y \\
\text { plane. }\end{array}\end{array}$ \\
3 & 6000 & $0.863 \pm .012$ & $\begin{array}{l}\text { RF771-6839 placed in } \\
\text { lower layer of drums } \\
\text { at center of array } \\
\text { infinite in } x-y \text { plane. }\end{array}$ \\
3500 & $0.766 \pm .010$ & $\begin{array}{l}\text { RF771-6839 placed in } \\
\text { lower layer of drums } \\
\text { at a corner of a very } \\
\text { large array. }\end{array}$
\end{tabular}

(a) An array is composed of drums set on end and stacked 5 high with a $0.019-m$-thick (3/4-inch-thick) plywood sheet separating each layer. The bottom layer is reflected with asphalt and soil.

(b) In this configuration, the array is reflected on the bottom with asphalt and soil and on the sides with soil. 
CRITICALITY EVALUATION OF LOFT FUEL REMOVAL AND INSERTION CASK (FMIRC)

J. H. Lofthouse - E. Fast

The LOFT Fuel Module Insertion and Removal Cask (FMIRC) is designed for handling LOFT fuel modules at both the reactor and in the TAN storage canal. The cask design must prevent criticality in the borated water environment of the LOFT reactor vessel and in the unborated water in the storage canal. A criticality evaluation has been performed to determine if the design is adequate to prevent criticality under the expected conditions of operation.

First, parametric analyses were performed on the fuel module to determine the most reactive condition. The studies considered missing fuel pins, bowed pins, and perforated pins where water has leaked inside the cladding. These calculations, using a detailed model of the fuel module in the Monte Carlo computer code KENO, showed that deforming the module has only a small effect on the calculated $k-$ effective of the water moderated and reflected module.

Next, calculations were performed with a full LOFT module in the FMIRC moderated with room-temperature water. This model included the stainless steel cask liner designed to center and hold the module inside the cask. The results of the analysis shown in Table I demonstrate that the cask design is adequate. The analysis also shows that two LOFT fuel modules placed in close proximity to each other in cold water may not be a safe configuration. 
TABLE 1

RESULTS OF KENO CALCULATIONS ON THE LOFT FMIRC

\begin{tabular}{|c|c|c|c|c|}
\hline Case & Problem Description & $k_{\text {eff }} \pm 1 \sigma$ & 95\% Confidence Limits & $\begin{array}{c}\text { Number of Neutron } \\
\text { Histories }\end{array}$ \\
\hline 1 & $\begin{array}{l}\text { Single LOFT fuel module moderated } \\
\text { and reflected wi th room-temp. water }\end{array}$ & $0.8878 \pm .0081$ & $0.8716-0.9040$ & 14100 \\
\hline 2 & $\begin{array}{l}\text { Two LOFT modules (same as Case 1) } \\
\text { placed } 1.43 \mathrm{~cm} \text { apart }\end{array}$ & $1.0173 \pm 0.0117$ & $0.9938-1.0408$ & 6300 \\
\hline 3 & $\begin{array}{l}\text { LOFT fuel module (same as Case 1) } \\
\text { in FMIRC }\end{array}$ & $0.8795 \pm 0.0114$ & $0.8568-0.9023$ & 8100 \\
\hline 4 & $\begin{array}{l}\text { Same as Case } 3 \text { but with fuel rods } \\
\text { perforated to allow water to } \\
\text { enter gas annulus and pellet end } \\
\text { dish space }\end{array}$ & $0.8755 \pm 0.0128$ & $0.8500-0.9011$ & 4500 \\
\hline
\end{tabular}




\section{CRITICALITY EVALUATION FOR THE UFSF ROVER RACK}

R. R. Jones, C. J. Shaffer

A nuclear criticality safety evaluation was performed on the Unirradiated Fuel Storage Facility (UFSF) Rover rack to demonstrate subcriticality of the loaded rack for all degrees of moderation using Hanford boron splines to reduce the reactivity. The Rover graphite fuel rods are highly enriched in U-235 (conservatively assumed at $93.15 \%)$ and are either $1.91-\mathrm{cm}$-diameter $(3 / 4-i n c h)$ round rods or $1.91-$ $\mathrm{cm}$ (across-flat) hexagonal rods and approximately $132 \mathrm{~cm}$ ( 52 inches) long. Reprocessing burner product (same U-235 enrichment) contained in steel canisters is also stored in the rack. The Hanford aluminum boron splines $1.27 \mathrm{~cm}(.5$ inch) wide and $.13 \mathrm{~cm}(0.50$ inch) thick contain from 1.41 grams to 1.53 grams of natural boron uniformly dispersed in each $30.48 \mathrm{~cm}(1 \mathrm{ft})$ of length. The storage rack consists of cylinders in an array 1 unit deep, 46 units long, and 9 units high. The fuel is stored within the cylinders in bundles of rod or burner product canisters with the highest cylinder loading of 7867 grams $\mathrm{U}-235$ in the burner product form.

The KENO II, Version 5, criticality code with 16-group Hansen and Roach cross sections was used for the calculations.

The fully loaded Rover rack was found to be subcritical for all degrees of moderation with large conservatisms included in the model. The calcuiated maximum k-effectives with optimum moderator for both the most reactive reflector condition and for a reflector condition more representative of the actual reflector (but still conservative) are 0.994 and 0.972 . This analysis of the Rover rack moderated to the most reactive conditions shows a very small safety margin. However, optimum moderation of the entire rack is an inconceivable event as multiple failures of both physical and administrative controls must occur in order to introduce the concentrations of water required to approach an optimumly moderated condition. 


\section{HTGR DISSOLVER CRITICALITY SCOPING CALCULATION}

\section{J. Shaffer}

A criticality scoping calculation was performed for a dissolver designed to dissolve HTGR fuels. This calculation was needed as a validation to a calculation done with the KENO criticality code using the Hansen and Roach cross sections.

Values of k-effective were computed for a 14-inch-diameter reprocessing dissolver for five different $H / x$ values. ( $H / x$ is the hydrogen-to-fuel ratio where $x$ includes the sum of $U-235$ and $U-233$ atom densities). The problem assumes a 14-inch-diameter infinite cylinder containing varying amounts of $U-235, \mathrm{U}-233$ and $\mathrm{Th}-232$ in the oxide form $\left(\mathrm{O}_{2}\right)$ in a homogeneous water solution at room temperature $(298 \mathrm{~K})$. The reflector was assumed to be 8 inches of pure water. The calculations were done using ENDF/B Version 4 cross sections, the cross-sections processing codes PHROG (resonance) and INCITE (thermal), and the one-dimensional $S_{n}$ transport code SCAMP. A group structure of 32 groups was used consisting of 25 fast and epithermal groups processed by PHROG and 7 thermal groups processed by INCITE.

The calculation shows the dissolver to go critical at an $H / x$ of about 34 and peak with a k-effective of 1.18 at an $H / x$ of about 180 . 


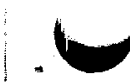

C. PROGRAM SUPPORT 



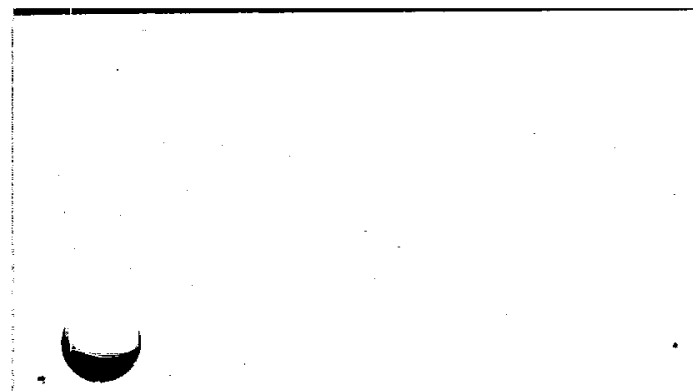

\section{TEST REACTORS}


BLANK PAGE 


\title{
ATR AND SLSF DATA ACQUISITION SYSTEMS UPGRADES
}

\author{
R. C. Davies, G. 0. English, M. S. Cole
}

The ATR System:

A 5 million word disk and 64k of core memory have been added to the ATR PDP-15 computer system. These additions have enabled the PDP-15 to use the RSX-PLUS III operating system supplied by Digital Equipment Corporation. RSX-PLUS III is a general purpose operating system designed to handle both real-time and batch processes in a multiprogramming environment.

The disk which was added to the system was a non-standard PDP-15 peripheral and required modification of RSX PLUS III for RSX and the disk to be compatible. Other modifications were also made to RSX in order to incorporate existing hardware on the PDP-15.

The PDP-15 RSX PLUS III system is now operational at the ATR facility. The upgraded system will allow greater ease of use and greater flexibility for the operations and experimental personnel. The users will be able to run their own Fortran programs simultaneousiy with those of other users and those of the real-time system.

\section{The SLSF System:}

The SLSF computer system has been upgraded to a dual computer system. The original system consisted of an $8 \mathrm{~K}$ PDP-15 computer, DEC tape, two industry compatible magnetic tape units, a $256 \mathrm{~K}$ word fixed head disk and associated data acquisition equipment. The new system consists of dual 24K PDP-15 computers each with two industry compatible magnetic tape units and similar data acquisition equipment. A 5 million. word disk was purchased for the auxiliary PDP-15. A graphics display terminal with hardcopy is connected to the auxiliary PDP-15 for realtime presentation of loop and reactor data.

The two Digital Equipment Corporation PDP-15 computer systems are functionally identical in data acquisition operation and can be used independently if necessary. The computers communicate through a high-speed block transfer interface to perform cooperative operations. During normal loop operation, one system will assume the data acquisition role and the other will be used for on-line data evaluation.

The auxiliary computer system can be utilized as a back-up data acquisition system, monitoring the same sensors as the primary system, or may be used to acquire data from different sensors for increased system capacity. In the back-up mode of operation, the auxiliary system can provide completely redundant data acquisition during critical experimental periods, or it can be operated in a stand-by mode, assuming the data acquisition task on demand or on failure of the primary system. 
The auxiliary computer system supports an IMLAC Corporation PDS-1G Graphic Display Processor. Communication between the PDP-15/78 and the PDS-1G computers is implemented by a 9600 baud direct serial data line.

The Display Processor is a minicomputer-based graphics system with a high-contrast high-resolution large-screen cathode ray tube operated in refresh mode, a programmable alphanumeric keyboard, and an electrostatic hard-copy output device capable of producing reduced copies of displayed data.

The Display Processor provides fast presentation of loop and reactor data in either graphic or alphanumeric modes. With the hard-copy output, it can substitute for a system line printer. 


\title{
ON-LINE TESTING OF THE ADVANCED TEST REACTOR PLANT PROTECTION SYSTEM
}

\author{
W. R. Myers, E. E. Owen, V. D. Kl ingler
}

The Control System Upgrade for the Advanced Test Reactor (ATR) includes installation of modern Plant Protection System (PPS) logic. The PPS is designed to incorporate on-line surveillance and testing utilizing specially designed isolating interfaces and a conventional minicomputer real-time data system.

The ATR PPS monitors numerous process variables and experimental loop status in the reactor facility, with the purpose of initiating automatic shut-down of the reactor when unusual or abnormal conditions are detected. Proper functioning of the PPS is a prerequisite for operation of the reactor. All of the PPS instrument subsystems and instrument logic will be tested periodically to determine that the PPS is operable, or if not operable, to identify faulty cards or modules so that they may be quickly replaced. The Surveillance and Test System (SATS) will inject programmed analog and binary voltages into selected PPS test points and will monitor PPS response to the stimuli. In addition, the SATS will monitor PPS status to observe actual levels and logic states, temporarily storing the values of all monitored parameters. These data will be automatically transmitted to the ATR primary data system for permanent storage and proeessing in the event of a reactor shutdown or unusual or abnormal condition.

The SATS will not inhibit the ability of the PPS to respond to an event requiring reactor shutdown and will not contribute to the initiation of a reactor shutdown. The PPS logic and the interface logic between the PPS and the SATS computer have been designed to meet these requirements. The system has been designed so that SATS can respond within 200 microseconds to special conditions in the PPS to completely withdraw from all active tests and allow the PPS to proceed in its intended function. 
A SUMMARY OF ATR SHIM PREDICTIONS

W. D. Gregory

One area of technical support for ATR is predicting shim positions for criticality at beginning-of-1ife as well as midcycle restarts. Successful startup for each ATR cycle is highly dependent on the ability to make reliable shim position predictions. The ATR fuel loading, which consists of many combinations of new and recycled fuel elements, complicates the calculations of the spatial distribution of fuel, burnable boron poison and fission product poisoning.

A summary of the standard deviations (expressed in dollars) of reactivity differences between predicted and actual ATR shim positions for criticality from Core II through Cycle $32 . \mathrm{A}-1$ startup are listed below:

\begin{tabular}{|c|c|}
\hline Fuel Loading & $\underline{1 \sigma}(\$)$ \\
\hline New-fuel startups & 0.28 \\
\hline Recycled-fuel startups & 0.53 \\
\hline Mixed-fuel startups & 0.4 \\
\hline
\end{tabular}

A comparison of the standard deviations presented here and in Reference 1 shows improvement in the predicted ATR shim positions for both the recycled-fuel startups and mixed-fuel startups. The differences are well within acceptable limits.

A description of the methods used in calculating the predicted ATR shim positions for criticality is given in the reference.

(1) W. D. Gregory, "A Summary of ATR Shim Predictions", Nuclear Technology Division Annual Progress Report for Period Ending June 30,1975 , ANCR-1255, p. 291. 


\title{
ATR POWER DIVISION CALCULATION WITHOUT THE CENTER ${ }^{16} \mathrm{~N}$ DETECTOR
}

\author{
W. Serrano, P. R. Napper
}

The power division for the five lobes in the ATR is determined using data obtained from ten locations near the active core. The data source consists of ten tubes passing vertically through the core through which demineralized water flows and is activated by the $160(n, p)$ ${ }_{16} \mathrm{~N}$ fast neutron reaction. The ${ }^{16} \mathrm{~N}$ activity is monitored and the ATR power division is computed by a complex algorithm relating these activities and relative positions of the ten ${ }^{16} \mathrm{~N}$ detectors around the reactor core.

A leak occurred in the center water tube allowing activated primary coolant water to enter the tube and contaminate the data from the center detector. This resulted in an erroneous ATR power division calculation. A modified power division calculation was devised as a temporary solution. The modified calculation required data input only from the nine remaining detectors. The reliability of the modified power division calculation was assured by comparing calculated results to measured power divisions.

This temporary solution provided ATR Operations with the necessary power division information until a plumbing modification el iminated the problem. The power division calculating has reverted to the original algorithm requiring input from ten detectors. However, the plumbing modification required an adjustment to some of the original coefficients in the power division calculation. 


\section{VOID ANALYSIS FOR THE ATR EXPERIMENTS}

P. R. Napper, W. Serrano, W. A. Peeler

Prior to each ATR operating cycle, a void analys is is performed on each of the eight in-pile tube experiments in the ATR in support of the ATR's Core Safety Assurance Package (CSAP). The analys is is made to determine the magnitude of the potential positive reactivity that could be inserted into the reactor from an accident resulting in the partial or complete voiding of the in-pile experiments. Partial and complete voidings have been identified as the limiting accidents in the unlikely and extremely unlikely fault categories, respectively.

The analysis follows an accepted empirical process which includes hand calculations and the computer codes MXPWR and ATRVOID. The analysis iterates on certain parameters to restrict the calculated void reactivity within acceptable limits. Results of the analysis usually require restrictions or limitations on the operation of the reactor and/or the experiments.

A significant accomplishment in the streamlining of the void analysis was the combining of the MXPWR and ATRVOID programs into a single program called MAXVOID. This version automatically iterates in its search for an acceptable solution. Formerly several timeconsuming hand iterations were required. This modification to the void analysis has served the purposes of efficiency and accuracy. The MAXVOID program is in the checkout phase as additional modifications are being considered before final acceptance. 


\section{REACTIVITY-INDUCED ACCIDENT ANALYSIS FOR THE ATR}

W. R. Carpenter

ATR plant modifications and system upgrading which will take place during the Reflector III installation requires an updated safety analysis of the ATR including a core kinetics analysis for reactivityinduced transient accidents. The analysis was conducted primarily using the RELAP4 (I) computer code with supporting calculations made using the PARET (2) computer code. The use of the newer RELAP4 code alleviated the thermo-hydraulic shortcomings of PARET. PARET had been widely used in past ATR core safety analysis.

With regard to the core kinetics, the most notable change from previous analyses is the incorporation of the new ATR Wide Range Scram (WRS) system. The PPS-grade scram system, which will be installed during Reflector III installation, provides semi-continuous period protection from about $10^{-5} \mathrm{~N}_{\mathrm{F}}$ to $\mathrm{N}_{\mathrm{F}}$. The updated kinetics analysis shows this WRS system virtually eliminates the damage potential from unl imited control rod withdrawal accidents initiated at very low power levels. Before the incorporation of the WRS, calculated damage parameters for some postulated control rod withdrawal accidents were unacceptably severe.

The present analysis showed that the damage parameters calculated for the postulated faults all fell within established criteria and that the ATR can be safely operated to power levels of $250 \mathrm{MW}$ pressurized and to $2.5 \mathrm{MW}$ unpressurized.

(1) K. V. Moore and W. H. Rettig, RELAP4 - A Computer Program for Transient Thermal-Hydraulic Analysis, ANCR-1127 (December 1973).

(2) C. F. Obenchain, PARET - A Program for the Analys is of Reactor Transients, ID0-17282 (January 1969). 
FAST FLUENCE OF ATR SAFETY RODS

W. Serrano, P. R. Napper

During ATR operation, the five hafnium safety rods are withdrawn such that four inches of the hafnium remains in the top of the active core. Thus, the lower ends of the safety rods experience exposure to material-damaging fast flux ( $E>1 \mathrm{MeV})$. A fluence value of

$1.2 \times 10^{22} \mathrm{n} / \mathrm{cm}^{2}$ had been recommended as an upper exposure 1imit. A more accurate fluence calculation was made because the estimated fluence was approaching the recommended limit. The calculation was al so made to determine if safety rod replacement (if necessary) could be put off until the forthcoming ATR extended shutdown required for bery11 ium replacement.

The fluence was calculated using the expression:

$$
\text { fluence }=\underset{\mathbf{i}}{\bar{\phi}} \sum_{\mathbf{i}}
$$

where

$$
\begin{aligned}
& \bar{\phi}=\text { average fast flux obtained from sampling data } \\
& \text { taken at the bottom of each safety rod } \\
& \left(\mathrm{n} / \mathrm{cm}^{2} . \mathrm{sec}\right) \\
& t_{i}=\text { effective time at the average flux level (sec) } \\
& i=\text { cycle number }
\end{aligned}
$$

The fast flux data were obtained from activated nickel wires irradiated every cycle. The reactor operates predominantly in an unbalanced power division, and the $\bar{\phi}$ used in the calculations reflected both the balanced and unbalanced modes of operation.

The calculation resulted in updated values of fast fluence for each safety rod. The updated values indicated that four of the five safety rods were rapidly approaching the recommended fluence limit. 


\title{
ATR NUCLEAR SURVEILLANCE PROGRAM
}

\author{
P. R. Napper, W. Serrano
}

The ATR nuclear surveillance program is the principal method for detecting incipient reactor physics problems at the ATR. The surveillance program is core oriented al though the source of a problem could be traced to some mechanism external to the core. The primary purpose of the program is to ensure safe operation by recognizing and identifying possible problem areas and bringing these to the attention of cognizant management. A secondary function of the program is to generate a trend-setting data base such that deviations from the data base can flag that a change may have occurred.

A standard routine initiates the daily surveillance, the outcome determining the extent of further investigation. The initiating surveillance includes:

(1) Comparison of initial critical conditions with the expected conditions.

(2) Comparison of the actual with the expected power division.

(3) Analysis of neutron level signal recordings and comparison with trend-setting data.

(4) Reading of Operations log book.

(5) Feedback from Operations personnel.

(6) Feedback from experiment sponsor personnel.

(7) Verification of multiplier settings in power division calculation.

(8) Comparison of the thermal quadrant power division and a pseudo quadrant power division determined from ${ }^{16} \mathrm{~N}$ data.

(9) Comparison of flow to no-flow signals in ${ }^{16} \mathrm{~N}$ monitors to detect leakage.

The surveillance is then extended depending upon the outcome of this initiating surveillance. If a problem exists, data from all instrumentation are used to identify the source or to support recommendations for the solution of the problem. The results of the daily surveillance are recorded in a daily log and a summary of the surveillance is reported in a letter following the cycle.

In addition to the daily surveillance, less frequent surveillance and periodic measurements are made. The surveillance extends to the tracking and reporting of the fast fluence on all hafnium components within the ATR core. The measurements include neutron signature analysis at various power holds and measured reactivity worths of shim and safety rods. 
DECAY HEAT SOURCE INFORMATION

FOR ATR SAFETY ANALYSIS

\section{T. E. Young}

Heating-rate data were calculated for use in ATR in-core and in-canal accident analyses that support development of new ATR Technical Specifications. Calculated values of heat generation permit the determination of maximum heating rates in the ATR core for times up to $10^{7}$ seconds (120d) after initiation-of-shutdown. Also, calculated values of heating in the ATR canal storage racks permit determination of maximum heating rates at times between 10 and 300 days after shutdown when the storage racks are voided of cooling water.

Heating-rate data were obtained from previous PARET-SINBAD calculations, and from SINBAD and QAD-P5A calculations made specifically for the present study. (PARET is a reactor kinetics code; SINBAD is a fission-product buildup and decay code; and QAD-P5A is a pointkernal shielding code used for calculating heating by gamma rays.)

Procedures were described that are to be used to calculate heating of fuel meat, aluminum (side plates and cladding), and water in the ATR core after initiation of shutdown. Equations for heating rates were given for each of these components. Graphs of heating rate as a function of time after initiation of shutdown were furnished for three types of fuel meat, aluminum, and water. Data from these graphs are to be used in the equations to calculate heating rates for the desired values of power, operating time and decay time.

Procedures and data recommended for determination of maximum heating rates in the ATR core were used to calculate total heating in the fueled region of the core. The heating obtained for the case of reactor operation at $250 \mathrm{MW}$ was $240.6 \mathrm{MW}$, or $96 \%$ of reactor power. An alternate determination, which did not depend on these procedures and data, gave $239.8 \mathrm{MW}$ for the heating of the fueled region plus all materials enclosed by the fueled region. This check case indicates that the procedures and data furnished here should give reasonable, but somewhat conservative, values of heating in the ATR core. 
USE OF THE DWARF CODE TO

PREDICT ETR ROD POSITIONS AT STARTUP

\author{
M. W. Holm
}

A study was initiated to determine whether or not DWARF ${ }^{(1)}$ could be used to predict reactor rod positions at startup. The first phase consisted of using DWARF to model the ETR Cycle-122 run of OctoberNovember 1975. Initial nuclide concentrations were those of a typical ETR fuel rod; time ranges and power profile closely followed those of the actual run. From these input data, DWARF calculated a sequence of "generation factors" that were then converted into excess reactivities.

ETR control rod excess reactivities were calculated from rod positions as recorded in the operating log at hourly intervals. These and the DWARF values were plotted as a function of time for the full ETR Cycle 122. The two curves were observed to have very similar shapes, with very good agreement at the start of the cycle, but gradually diverging as the megawatt days accumulated.

There were 25 shutdowins during the cycle. It was possible to obtain reasonably good values of the rod excess reactivities at the start and finish of 18 of these down times by extrapolating the rod worth curves forward from the last log entry to the shutdown time, and backwards from the first log entry after startup to the time of startup.

By adding the change in excess reactivity calculated from DWARF data to the excess rod reactivity at shutdown, the rod excess reactivity at the following startup could be predicted. The mean absolute error for the 18 down times for which reasonably good rod worth data could be determined was $0.38 \$$, and the start of Cycle 123A was predicted with an error of $0.36 \$$. When the core power for each time range of the DWARF simulation was reduced by $25 \%$, the mean absolute error for the 18 shutdowns was $0.20 \$$ and the error in predicting the start of Cycle $123 \mathrm{~A}$ was $0.32 \$$. The large errors in the rod worth data for Cycle 122 discouraged further refinement of this technique.

Nevertheless, it does appear feasible to use DWARF to predict ETR rod worths at startup following a shutdown of a few days, or from the end of one cycle to the start of the next. The time required to prepare input data cards following a shutdown, to run the problem, and interpret the data generated is too long for DWARF to be of value in its present form for shutdowns of an hour or so within a cycle.

(1) G. E. Putnam and E. C. Anderson, DWARF, A Few Group, OneDimensional, Diffusion Theory Program for Reactor Depletion with Adjustable Reactivity Feedback, ANCR-1083, (November 1972). 
ETR SPND COMPUTER PROGRAM

D. R. Chick, M. S. Cole

Rhodium self-powered neutron detectors (SPND's) installed at various locations in the ETR core are used to monitor the neutron flux adjacent to an ETR experiment and at several other locations in the reactor core. A train of SPND's is located in each of four columns at the corners of the experiment's core filler piece and in each of the remaining three quadrants of the reactor core. There are five SPND's in each train vertically positioned $\pm 35.6 \mathrm{~cm}$ (14 in.), $+17.8 \mathrm{~cm}(+7 \mathrm{in}$.$) and 0 \mathrm{~cm}, 0$ in. from the core midplane. A platinum detector is positioned at the midplane of each corner in the filler piece. The platinum detectors respond rapidly to neutron flux change and are used to detect fuel movement and measure gamma heating.

Using the current signals generated by the SPND's, programs were written for the PDP-15 computer to:

1. Calculate the flux tilt across the experiment.

2. Calculate the peak-to-average ratio of the vertical flux profile.

3. Determine possible fuel movement and alert the experiment operator.

4. Alert the operator in the event any of these calculations exceed preset limits.

The operator can request more detailed output for evaluation. The program compensates for burn-up of the rhodium SPND's.

To date, the system has been used primarily for monitoring the flux tilt across the experiment. Also, reported flux levels have been used in computing the loop's power. 


\section{W. Serrano}

Measurements were made in the ETR critical facility to determine the reactivity worth of fuel rods used in an ETR experiment. The results from the measurements were used to predict the rod positions for criticality in the ETR. The ETR experiment contained $\mathrm{UO}_{2}$ fuel rods of varying enrichments whereas the fuel rods in the ETRC were mixed-oxide rods (25 wt \% $\mathrm{PuO}_{2}-75$ wt $\% \mathrm{UO}_{2}$ ) of varying uranium enrichments. The ETRC measurements were corrected to estimate the reactivity worth of the 19 fuel rods in the ETR test.

Three different measurements were made to determine the reactivity worth of 7,12 , and 19 mixed-oxide fuel rods in the ETRC experiment mock-up. The reactivity was determined by comparing to a base case measurement which had aluminum filler rods in the mock-up. A summary of the experimental results is contained in Table I.

\section{TABLE I}

REACTIVITY WORTHS OF ETRC MIXED-OXIDE FUEL RODS

\begin{tabular}{ccccc} 
Measurements & $\begin{array}{c}\text { Number of } \\
\text { Fuel Rods }\end{array}$ & $\begin{array}{c}\text { Pu wt } \\
(\mathrm{g})\end{array}$ & $\begin{array}{c}\text { U-235 } \\
(\mathrm{g})\end{array}$ & Reactivity (\$) \\
\cline { 2 - 2 } & 0 & 0 & 0 & -1 \\
2 & 7 & 270 & 659 & 0.24 \\
3 & 12 & 472 & 913 & 0.37 \\
4 & 19 & 780 & 1572 & 0.53
\end{tabular}


The contribution of the gamma heating from the mixed-oxide fuel in the ETR mixed-oxide fuel experiment to the total gamma heating in the ETR core filler piece (CFP) was required for experiment analysis. An approximation of this contribution has been made from measurements made in the ETR Critical Facility.

The gamma heating measurements were made using $\mathrm{CaF}_{2}: \mathrm{Mn}$ thermoluminescent detectors (TLD's). The $1 \times 1 \times 6 \mathrm{~mm}$. TLD's were positioned in slots milled in the aluminum filler rods in the four corners of the CFP. Two TLD's were placed at the core center line of each rod and at $\pm 457 \mathrm{~mm}$ ( \pm 18 inches) from the midplane.

The experiment was conducted in three steps:

(1) During Step 1 the gamma heating in the CFP was measured with 19 fuel pins loaded in the ETRC experiment.

(2) During Step 2 the gamma heating in the CFP was measured with 13 fuel pins in the ETRC experiment (inner ring of fuel pins removed).

(3) During Step 3 the gamma heating in the CFP was measured with all fuel removed from the ETRC experiment. Table I.

The experiment fuel loadings during the three runs are shown in

An analysis of the data shows the following:

(1) Gamma heating from Step 1 is approximately $4.0 \%$ greater than the gamma heating from Step 3.

(2) Gamma heating from Step 2 is approximately $2.7 \%$ greater than the gamma heating from Step 3.

(3) Gamma heating from Step 7 is approximately $1.3 \%$ greater than the gamma heating from Step 2.

\section{TABLE I}

FUEL LOADING DURING GAMMA-HEAT MEASUREMENTS

\begin{tabular}{lccc}
\hline & $\frac{U-235(\mathrm{~g})}{1571.8}$ & $\underline{\mathrm{Pu}(\mathrm{g})}$ & $\frac{\text { Total }(\mathrm{g})}{731.5}$ \\
Run 1 & 1571.8 & 7303.3 \\
Run 2 & 1018.0 & 500.5 & 1518.5 \\
Run 3 & 0 & 0 & 0 \\
\hline
\end{tabular}


The results of the above comparison indicate the experiment fuel contributes a minimal amount to the overall gamma heating in the CFP. 
REACTOR GAMMA-HEATING MEASUREMENTS USING THERMOLUMINESCENT DOSIMETERS

B. L. Hall

Ways to accurately measure gamma heating in reactor materials have been investigated at the ETR critical facility (ETRC). Several experiments involved the use of thermoluminescent dosimeters (TLD's) as gamma detectors:

(1) Contrasting experimental techniques of attaching the bare TLD to a material with tape as opposed to encapsulating the TLD in the same material.

(2) Comparison of absorbed doses in aluminum-encapsulated TLD's with that of stainless steel-coated TLD's in otherwise identical environments.

(3) Determining the effects of sealing the TLD capsules from a water environment compared with allowing small amounts of water to enter the capsule.

The TLD's used are manganese-activated calcium fluoride rods of dimensions $1 \times 1 \times 6 \mathrm{~mm}$. They are the most widely used TLD's that approximate aluminum in effective atomic number.

From these initial measurements, some observations can be made:

(1) In each case compared, the encapsulated TLD responded with a significantly higher dose than the taped TLD, consistently on the order of $22 \%$.

(2) Taking under consideration the approximate $\pm 5 \%$ uncertainty for each data point, the absorbed doses for aluminum and stainless steel at the same position in the reactor are approximately equal.

(3) Sealing the TLD's from water seems to result in a higher dose response in the aluminum capsules and a lower response in stainless steel. This might be explained on the basis of lower neutron and gamma energies if enough water were present. Although both aluminum and stainless steel exhibit some dose response dependence upon the gamma energy, it is not as significant for aluminum as for steel at low gamma energies.

Experimentation with gamma-heat measurements will continue at ETRC, including obtaining gamma spectrum data for predicting what the absorbed doses should be in a given material relative to another material. These doses are dependent upon the ratios of the materials' mass-absorption coefficients which are dependent upon the effective energy of the gamma spectrum. Other data-gathering will involve repeats of previous rieasurements for substantiation of observations and investigation into the effect of charge particle equilibrium conditions upon the absorbed dose. 


\section{REVIEW OF PHYSICS PARAMETERS USED FOR ATR/ETR}

\section{BURNUP CALCULATIONS}

\section{E. H. Ottewitte}

The purpose of this work was to review the methods and data used by nuclear fuel accountability personnel for ATR and ETR fuel burnup. This included re-derivation of equations, review of data, and estimation of uncertainties. The goal is to achieve accountability of $U$ and ${ }^{235} \mathrm{U}$ within one gram $U$ for each $200-300 \mathrm{~g}$ consumed.

The method of approach was first to search out all possibly significant burnup chain members. Then equations were defined, solved, and the resulting terms examined using typical nuclear constants. The net results were

$$
\begin{aligned}
& \text { Burnup }\left(g^{235} U\right)=C_{1} \frac{P}{\gamma} \quad(1+\alpha) \\
& \text { Burnup }(g \quad U)=C_{2} \frac{P}{\gamma} \\
& \text { Where } C_{1}=2.10 \mathrm{~g} 235 \mathrm{U} \times \frac{\mathrm{MeV}}{\mathrm{MWd}} \\
& C_{2}=2.10 \mathrm{~g} U \\
& P=\frac{\mathrm{MeV}}{\mathrm{MWd}} \\
& \alpha=0.21 \pm 10 \% \quad \frac{235 \mathrm{U} \text { absorptions }}{\text { fission }} \text { for ATR and ETR } \\
& \gamma=201+0.5 \% \quad \frac{\text { MeV }}{-2.0 \%} \text { fission after a typical 15-day operation. }
\end{aligned}
$$

Uncertainty estimates of $P$ for ETR and ATR were 3 and $5 \%$, respectively. Coupled with other uncertainty estimates above, this gave an uncertainty in burnup (U or ${ }^{235} U$ ) of $4 \%$ for ETR and $6 \%$ for ATR. 
U. 
EVALUATION OF CURRENT MEV-PER-FISSION DATA:

DEPENDENCE ON FISSIONING ISOTOPE AND REACTOR COMPOSITION

E. H. Ottewitte

The calculational methods currently being used to maintain accountability records of nuclear fuel in the test reactors (ETR,ATR) at INEL have been reviewed. Of particular interest was a re-evaluation of the energy release per fission.

The energy "available" from a nuclear reaction is generally comprised of:

(1) The energy balance, $Q$, for the reaction leaving the products in excited states. This is determined from the masses of the particles and excited nuclei involved. It will appear as kinetic energy (KE) of the final products which include charged particles, $v$ neutrons, and prompt photons.

(2) The non-neutrino portion of the energy release in beta decay of a radioactive product, as $\mathrm{KE}$ of the beta particle, and the recoiling atoms.

(3) All the energy release in gamma decay of product nuclei.

(4) All the energy release in alpha decay of product nuclei, as kinetic energy. For fission reactions this is negligible.

(5) The energy release in subsequent nuclear captures by the $(v-1)$ secondary neutrons.

For the fission reaction, Items 1-4 above are commonly grouped together and referred to as the energy release in fission, $E_{f j}$. Table I shows some current values in Columns 2-4. Most of these assume thermal incident neutrons.

Item 5 really depends on the composition of a reactor. Some material-dependent values for capture reactions are shown in Table II.

Consider a reactor average value:

$$
E_{c a p}=\frac{\sum_{i g} E_{i, c a p} \sigma_{i g} \phi_{g}}{\sum_{i g} \sigma_{i g} \phi_{g}}
$$

where $i$ material index

$$
\begin{aligned}
& g=\text { group index } \\
& \sigma=\text { macroscopic capture cross section } \\
& \phi=\text { neutron flux }
\end{aligned}
$$


As the $\left\{E_{i}^{C a p}\right\}$ from Table II are all of similar magnitude, several authors have determined average $\bar{E}_{\text {an }}$ values using different reactors. Multiplied by $(v-1)$, one then 8 BRains the entries in Columns 5 and 6 of Table I. Adding results of Columns 2 and 4 to those of 5 and 6 gives an estimate of the "total energy absorbed in a reactor" (Columns 7-9 of Table I). Note, however, that this approach has allowed no neutron leakage from the system, nor is it limited to the core boundaries. Thus, $(v-1)$ should at least be multiplied by a non-leakage (from the calorimetric boundary) probability, $P_{n \ell}$.

A more accurate approach would be to compute the capture activities from reactor calculations in one or more dimensions, and apply Table II.

A final item worthy of note is the time dependence of the $\left\{E_{\text {fiss }}\right\}$. This is due to the variation in half-life for the various fiss $f(t)$ fragments. This may be represented as $E_{\text {fiss }}(t)=E_{\text {fiss }}$ +
$\delta E(t)$.

Suggested values for $\delta E(t)$ are $^{2}$ :

Length of Prior
Reactor Operation

30 days

1 year

100 years

$\infty$ (saturation)

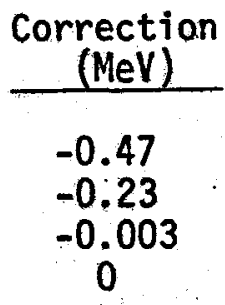

Thus if the reactor has been at full power for only 30 days prior to time $t$, then $E_{\text {fiss }}$ should be reduced by $0.47 \mathrm{MeV}$. Values for shorter operations are not immediately available, but could be calculated.

(1) Evaluated Nuclear Data Files/B Version 4, ("Q" values for $\overline{M F}=3, M T=18), N N C S C / B N L$, New York $(-1974)$. Note: Reference (1) does not have a literature reference; it is the latest "black box" product of the US Cross Section Evaluation Working Group (CSEWG). This committee includes specialists such as Walker of reference (4), and is very reliable. References (2) and (3) are a bit older and well documented. They closely (within error bars) substantiate and form the basis for ENDF/B-4 (4).

(2) J. P. Unik, J. E. Gindler, A Critical Review of the Energy Released in Nuclear Fission, ANL 7748 (March 1971).

(3) M. F. James, Energy Released in Fission, AEEW-M-863 (May 1969); J. Nucl. En. 23, (1969) 517 . 
(4) W. H. Walker, Mass Balance Estimate of the Energy Released per Fission in a Reactor, AECL-3109 (April 1968); Errata (August 1969).

(5) C. M. Lederer, J. M. Hollander and I. PerIman, Table of Isotopes, 6th Edition, John Wiley (1967).

(6) M. F. James, B. G. Steel, J. S. Story, "Average Electron Energy in Beta Decay," AERE-M640 (1960).

(7) C. Maples, G. W. Goth, J. Cerny, "Nuclear Reaction Q-Values", Nuclear Data, 2, (1966) 429.

(8) P. F. Rose, Improved Methods and Data for Calculating Control Rod Heating, Atomics Internationa1, T1-095-21-060 (March 4, 1970) 28. 
TABLE I

CURRENT EVALUATED VALUES FOR ENERGY RELEASE IN FISSION**

\begin{tabular}{|c|c|c|c|c|c|c|c|c|}
\hline \multirow[t]{2}{*}{1} & 2 & 3 & 4 & \multirow{2}{*}{\multicolumn{2}{|c|}{$\begin{array}{l}5 \\
\text { Energy Release in Sub- } \\
\text { sequent Capture of } \\
\text { Fission Neutrons } \\
\text { (vi-l) Ecap } \\
\text { (MeV per Fission) }\end{array}$}} & 7 & 8 & 9 \\
\hline & \multicolumn{3}{|c|}{$\begin{array}{l}\text { Energy Release in Fission, } E_{\text {fiss }} \\
\text { (MeV per Fission) }\end{array}$} & & & \multicolumn{3}{|c|}{$\begin{array}{l}\text { Total Energy Absorbed in a } \\
\text { Reactor (MeV per Fission) }\end{array}$} \\
\hline Isotope & ENDF/B-4 (1) & ANL (2) & James $(3)$ & ANL $(2)$ & James (3) & ANL (2) & James (3) & Walker $(4)$ \\
\hline $232 \mathrm{Th}$ & 184.4 & $\overline{183.7 \pm 0.9 *}$ & & $8.88 \pm 0.5^{*}$ & & $192.0 \pm 1.0^{*}$ & & $193.0 \pm 1.2$ \\
\hline${ }^{233} \mathrm{U}$ & 190.3 & $190.2 \pm 0.5$ & & $9.71 \pm 0.3$ & & $200.0 \pm 0.6$ & & $199.5 \pm 0.9$ \\
\hline $235 U$ & 192.5 & $192.4 \pm 0.6$ & $192.9 \pm 0.5$ & $9.29 \pm 0.3$ & $8.8 \pm 0.4$ & $201.7 \pm 0.7$ & $201.7 \pm 0.5$ & $200.8 \pm 0.6$ \\
\hline $238 U$ & 194.0 & $191.2 \pm 1.0^{*}$ & $193.9 \pm 0.8$ & $11.82 \pm 0.5^{*}$ & $11.7 \pm 0.4$ & $203.0 \pm 1.7 *$ & $205.0 \pm 0.9$ & $204.6 \pm 1.2$ \\
\hline${ }^{239} \mathrm{Pu}$ & 198.6 & $198.4 \pm 0.5$ & $198.5 \pm 0.8$ & $12.26 \pm 0.4$ & $11.5 \pm 0.5$ & $210.6 \pm 0.7$ & $210.0 \pm 0.9$ & $209.3 \pm 1.0$ \\
\hline $240 \mathrm{Pu}$ & 195.0 & $196.3 \pm 1.7^{*}$ & & $14.17 \pm 1.4^{*}$ & & $210.5 \pm 2.2^{\star}$ & & \\
\hline $241 \mathrm{Pu}$ & 200.5 & $199.4 \pm 0.7$ & $200.3 \pm 0.8$ & $12.63 \pm 0.4$ & $12.7 \pm 0.5$ & $212.0 \pm 0.8$ & $212.9 \pm 7.0$ & $212.5 \pm 1.1$ \\
\hline${ }^{242 \mathrm{Pu}}$ & 200.0 & $197.9 \pm 3.3^{*}$ & & $14.24 \pm 2.6^{*}$ & & $212.1 \pm 4.2^{*}$ & & \\
\hline \multicolumn{9}{|c|}{$\begin{array}{l}\text { * Specified to be for incident neutrons with a fission spectrum distribution of energies. } \\
\text { ** All values exclude the kinetic energy of antineutrinos and the decay energy of neo-stable (very } \\
\text { long-lived) fission products. }\end{array}$} \\
\hline
\end{tabular}


TABLE II

MeV/REACTION ESTIMATES*

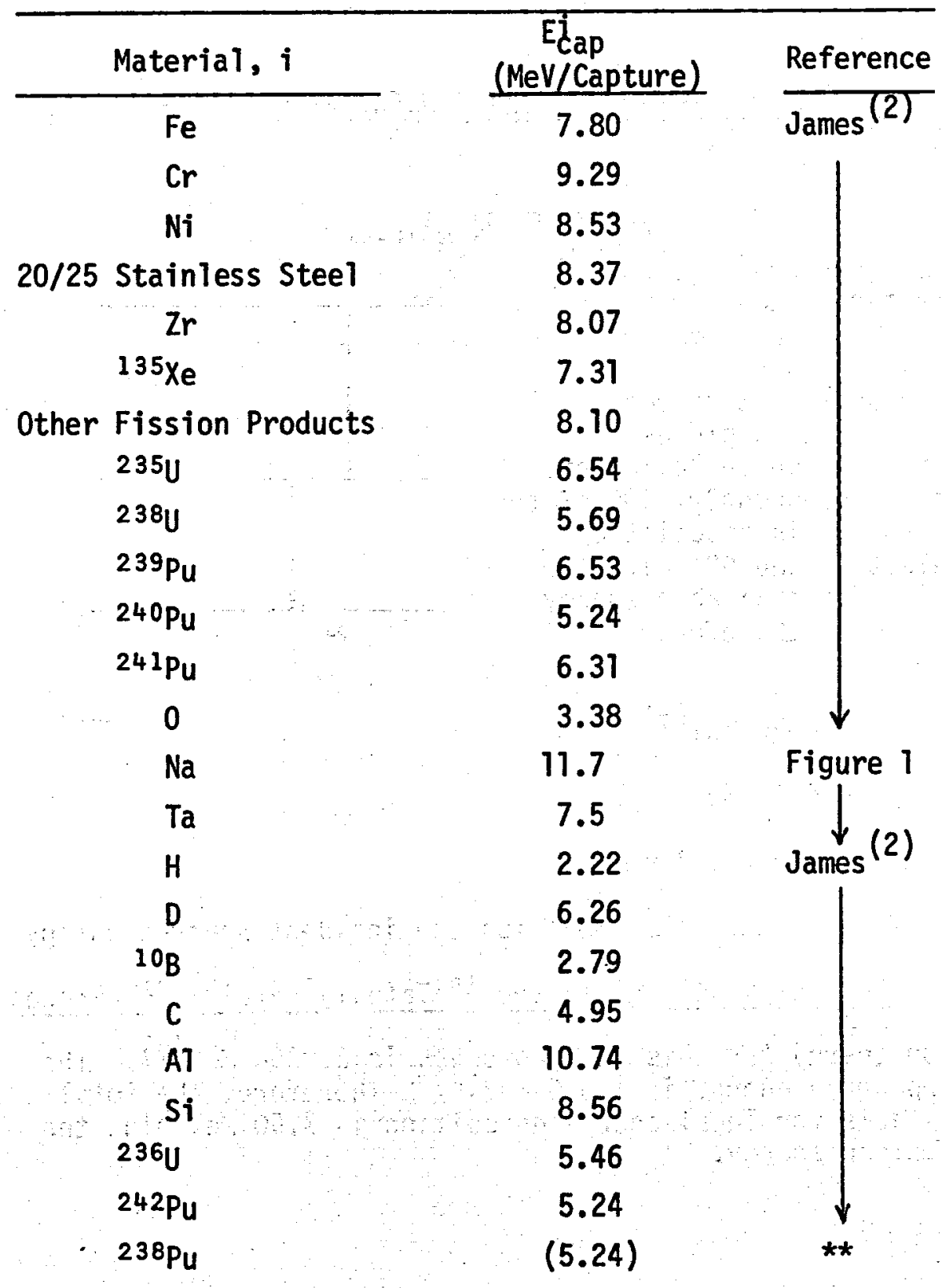

* Additional estimates can be developed as needed (ex. Figure 1). ** Guess 
Estimate of the Energy Release in the ${ }^{23} \mathrm{Na}(n, \gamma)^{24} \mathrm{Na}(4+)^{\beta} \mp 24 \mathrm{Mg}(4+) \chi$ Mg(0+) Process

The diagram shows the appropriate defay scheme for this process $(5)$. The beta decay transition is $4^{+}+$ 4+. This, plus the measurement $\log f t=6.2$ for this process indicates this to be an "allowed" transition. Consequently, $40 \%$ of the beta decay energy is associated with the electron and $60 \%$ with the neutrino( 6 ). The available energy from this process therefore includes:
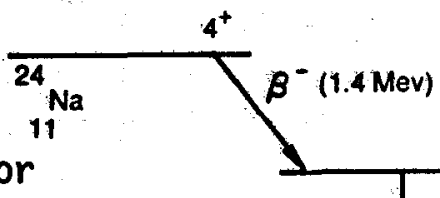

$$
\begin{aligned}
Q_{\text {reaction }} & =6.96 \mathrm{MeV}(7) \\
\text { Total } E_{Y} & =4.12 \mathrm{MeV} \\
E_{e-} & =.40 \times 1.4=.64
\end{aligned}
$$

The total energy is then $11.7 \mathrm{MeV}$ plus the incident neutron energy. Estimate of the Energy Release in the $181 \mathrm{Ta}(n, \gamma)^{182} \mathrm{Ta}^{15}+182 \mathrm{~W}$ Process

The reaction energy for this $(n, \gamma)$ process is $5.9854 \mathrm{MeV}(7)$. The non-neutrino decay energy is $1.516 \mathrm{MeV}(8)$. Therefore, the total energy available for local energy deposition is $7.50 \mathrm{MeV}$ plus the indident neutron energy.

Figure 1. Estimation of energy releases. 
The reactor physics parameter $\beta / \ell$ was computed from neutron noise signals in support of the ETR requalification. Two methods were used, one neglecting background noise which gave an unsatisfactory result and another which considered the background noise and gave a value of $181.6 \mathrm{sec}^{-1}$ for $\beta / \ell$.

Neutron noise signals from two in-core neutron detectors were recorded during the ETR NT-4 tests at low power. The cross-power spectral density (CPSD) of the two signals was computed and the resulting data were fitted to the equation:

$$
y(f)=\frac{A}{1+\left(f / f_{B}\right)^{2}}
$$

where

$$
\begin{aligned}
y(f)= & \text { amplitude of CPSD function } \\
A= & \text { amplitude of } y(f) \text { at zero } \\
& \text { frequency } \\
f= & \text { frequency in hertz } \\
f_{\beta}= & \frac{1}{2 \pi} \frac{\beta}{\ell}
\end{aligned}
$$

A least squares fit gave a value of $192.0 \mathrm{sec}^{-1}$ for $B / \ell$; however, a lower value was expected. The above equation (model) is valid when the detectors are located near each other and near the center of the reactor core. For this condition, the signals from the detectors are largely correlated as they see a common flux with little disturbance from independent sources. However, in the ETR measurements, the detectors could not be ideally located. Additionally, the lower grid was activated, emitting uncorrelated gamma radiation at the two detector locations. The uncorrelated signals appear as low-level white noise on CPSDs from finite record lengths of data. The above model does not account for this added signal and results in increased errors in the computed parameters, the amount of additional error being dependent on the magnitude of the added noise. The added noise is modeled by an added parameter B giving:

$$
y(f)=\frac{A}{1+\left(f / f_{\beta}\right)^{2}}+B
$$

This model resulted in a value of $181.6 \mathrm{sec}^{-1}$ for $\beta / \ell$. The value was closer to computer-calculated values, indicating that uncorrelated noise should be considered.

$$
263-264
$$




\section{BLANK PAGE}

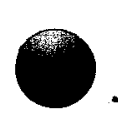


POWER BURST FACILITY 
IRRADIATED FUEL ROD SCANNING

J. W. Mandler, R. A. Coates

Gamma-ray scanning of PBF fuel rods using a Ge(Li) detector at the TRA hot cell scanning facility is approaching a routine procedure. Each rod is examined at 13 locations in the fuel stack plus two background locations outside of the fuel stack fone at the top of the rod and one at the bottom). In order to obtain adequate statistics count times are varied from 300 seconds up to 2000 seconds when viewing the fuel and from 4000 seconds to 10,000 seconds when obtaining backgrounds. Currently, the intensities of selected gamma rays from $95 \mathrm{Zr}$, $95 \mathrm{Nb}, 103 \mathrm{Ru}$, and $140 \mathrm{La}$ are measured. The intensity of the $137 \mathrm{Cs}$ gamma ray is of importance, but cannot currently be obtained due to the presence of $137 \mathrm{Cs}$ in the background. In addition, the location of each end of the fuel stack is determined by stepping the appropriate end of the rod past the collimator and observing the intensity of the $1596 \mathrm{keV} 14 \mathrm{Oa}$ gamma ray.

Up to now rod scanning is done manually - the scanner bed is mannually run to the position of interest on the rod and then a spectrum is obtained using a Ge(Li) detector and a multichannel pulse-height analyzer. However, a computer based scanner system with $x$-and $y$-axis incremental control has been designed and is being assembled. The basic sytem consists of a $\mathrm{Ge}(\mathrm{Li})$ detector, 12 bit $\mathrm{ADC}, 2$ peak precision pulser, Fabri-Tec 12 bit micro-computer with $4 \mathrm{k}$ of RAM memory and $8 \mathrm{k}$ of memory for data storage, 1200 baud data set, 110 baud acoustic coupler, TTY for local control and local copy, display scope, and $x$ - and $y$-axis stepping motor control.

The stepping motor control allows positioning of the rod to be scanned, within the $x$ - and $y$-axis limits of travel of the scanner bed, to a maximum of 6.5 inches to an accuracy of 0.001 inch. The system can be controlled locally via the TTY or remotely from a PDP-15 llocated in the Radiation Measurement Laboratoryl via the 110 baud acoustic coupler. The 1200 baud data set is used for transmitting the pul se-height spectra to the PDP-15. Upon command the system will move the rod to a predetermined position, take a 4096 channel spectrum for a present time, and then output either the entire spectrum and/or regions of interest to the TTY for local hard copy or the PDP-15 for analysis, storage, and/or graphic hard copy.

The computer based system will improve the rod scanning capability of the TRA hot cell facility in several ways. Since it can be computer controlled, rod scanning can be accomplished unattended and on off-shift hours. Data can be analyzed quickly using the PDP-15 thereby reducing turn-around time. The computer (PDP-15) can be programmed to analyze each spectrum immediately after it is obtained and to made decisions concerning rod position and count times. 


\title{
PHYSICS CALCULATIONS FOR IRRADIATION EFFECTS TEST IN PBF
}

\author{
A. J. Scott \& E. Fast
}

Monte Carlo calculations have been performed to predict the power density and distribution in test fuel rods for the Irradiation Effects Tests to be conducted in the Power Burst Facility in-pile tube (IPT). The test rods will be located in widely spaced, individual flow shrouds and have $\mathrm{UO}_{2}$ enrichments of $5.7 \mathrm{w} / 0,9.5 \mathrm{w} / \mathrm{o}$ and $12.5 \mathrm{w} / \mathrm{o} \mathrm{U}-235$. The $5.7 \mathrm{w} / 0$ rods were irradiated in Saxton to an average burnup of $6000 \mathrm{MWd} / \mathrm{t}$. Some of the $9.5 \mathrm{w} / \mathrm{o}$ and $12.5 \mathrm{w} / \mathrm{o}$ rods were depleted to about 16000 MWd/t and some were fresh rods. Assuming a PBF core power of $28 \mathrm{MW}$ and a control rod position of $0.61 \mathrm{~m}$ (24 inches), the results predict axial peak power densities varying from $44.6 \mathrm{~kW} / \mathrm{m}(13.6 \mathrm{~kW} / \mathrm{ft})$ for the $5.7 \mathrm{w} / \mathrm{o}$ rods to $71.5 \mathrm{~kW} / \mathrm{m}(21.8 \mathrm{~kW} / \mathrm{ft})$ for the fresh $12.5 \mathrm{w} / 0$ rods. Table I summarizes these results.

The calculations were done in general three-dimensional geometry with the RAFFLE $(1)$ code using 97 energy groups. Only the test space inside the in-pile tube was represented with RAFFLE; i.e., neutron histories were followed through the test space from an external neutron source input at the IPT boundary. The external source was taken from the results of previous radial $S_{N}$ transport-theory reactor calculations.

Figure 1 shows a RAFFLE model of the four-rod experiment in the PBF in-pile test space. The larger diagram is typical of the subdivisions considered for each of the fuel rods. Inscribed numbers represent the power relative to the average rod power for this case, and the fractional errors at the $50 \%$ confidence level. These may be combined to give radial or azimuthal power distributions.

(1) W. E. Veseley, F. J. Wheeler, and R. S. Marsden, The RAFFLE General Purpose Monte Carlo Code, ANCR-1022, (April 1973). Also a supplement to the above: R. S. Marsden, F. J. Wheeler and D. E. Wessol, A Mixed Zone Geometry for the RAFFLE General Purpose Monte Carlo Code, ANCR-1206, (February 1975). 


\section{TABLE I}

COMPUTED ROD POWERS FOR THE IPT SERIES AT

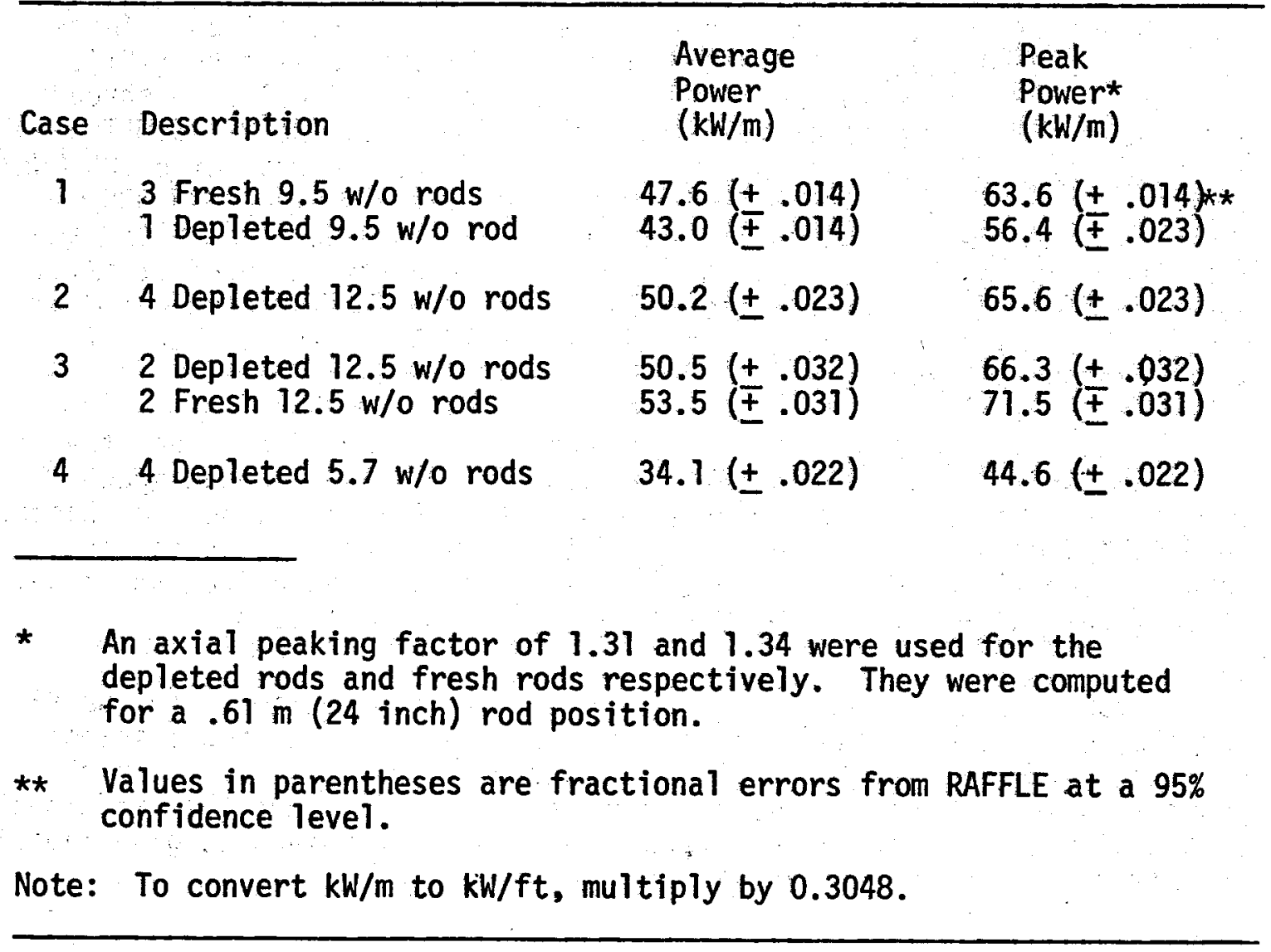




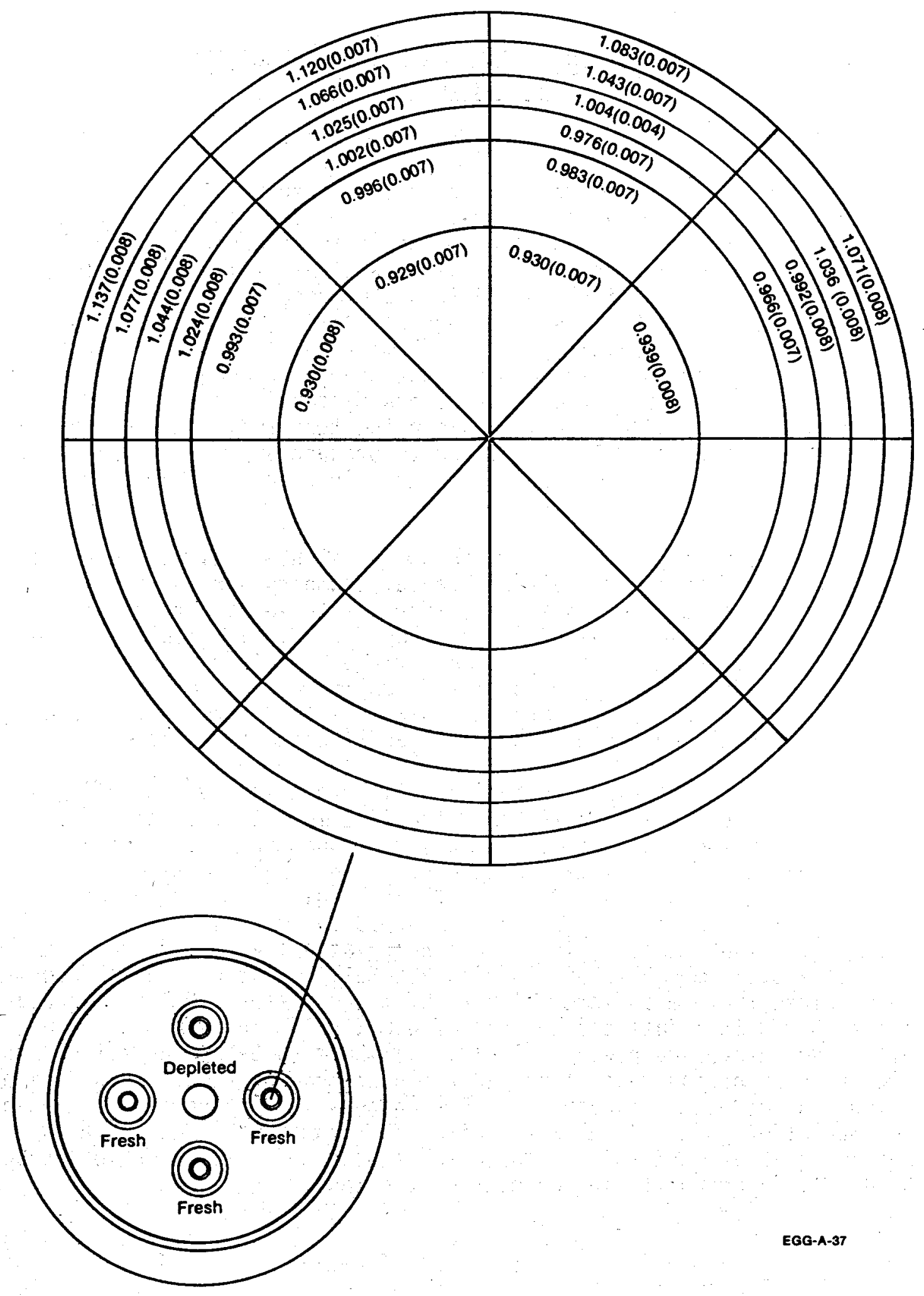

Figure 1. Relative power distribution in fresh $9.5 \mathrm{w} / 0$ rod. (Values given are ratios of region average power density-to-pellet average power density followed by fractional errors at a 50\% confidence level.) 
PHYSICS CALCULATIONS FOR A 16-PIN LOCA TEST DESIGN IN THE PBF

T. E. Young and A. J. Scott

Several experiments are planned in the Power Burst Facility (PBF) to investigate the behavior of PWR-type fuel under postulated loss-ofcoolant accident (LOCA) conditions. One of these experiments will simulate decay heating that would follow a LOCA by fission heating of a 16-pin cluster of test fuel in PBF in a steam environment. The experiment requires that the heat generation within the cluster be uniform radially and axially over a $457-\mathrm{mm}(18-i n)$ segment. Physics calculations were made to determine what change from an axially uniform containment will be required to produce a flat axial power distribution for approximately $457 \mathrm{~mm}_{2}(18 \mathrm{in}$. $)$ along the test fuel. Also determined were the enrichments in ${ }^{235}$ required to produce a power of $9.84 \mathrm{~kW} / \mathrm{m}$ $(3.00 \mathrm{~kW} / \mathrm{ft}$.) at the axial center of each pin when the PBF is operated at approximately $22.1 \mathrm{MW}$.

Thirty-two-group SCAMP ( $S_{n}$ transport) calculations were used to determine the proper materials and radial geometry for the test hardware. These calculations also gave approximate values of test fuel enrichments required. RZ-PDQ (four-group diffusion) calculations were used to determine the axial variation of the containment which will produce a uniform axial power distribution for $457 \mathrm{~mm}$ (18 in.) near the axial center of the test. Final calculations to determine the powers in the test pins were made with the RAFFLE (Monte Carlo) code.

A flat axial power distribution will be obtained by attaching a 316 SStl "shaper" to the outside of the innermost test containment vessel, beginning $294.1 \mathrm{~mm}$ (11.58 in.) from the top of the active PBF fuel. This shaper consists of two parts. The inner portion is 373.2 $\mathrm{mm}$ (14.693 in.) long and $1.91 \mathrm{~mm}$ (.075 in.) thick. The outer portion is attached to the inner portion, and is $190.1 \mathrm{~mm}$ (7.484 in.) long and $.51 \mathrm{~mm}(.020 \mathrm{in}$.) thick. The shaper calculations were made for the case of a 3.175-mm (0.125-in.)-thick 316 SStl canister. The shaper may be machined to form an integral part of the canister. RAFFLE calçulations showed that fuel enrichments of $4.0 \%, 4.35 \%$ and $4.97 \%$ in $\mathrm{U}$ will give an average power of $11.12 \pm .13 \mathrm{~kW} / \mathrm{m}(3.39 \pm .04 \mathrm{~kW} / \mathrm{ft})$ in the central part of the test if the PBF is operated at $25 \mathrm{MW}$. This design allows for moderate (probably 10-15\%) changes in test power by changing the operating power of the PBF. With the geometry and enrichments specified above, the PBF will need to be operated at $22.1 \mathrm{MW}$ to produce $9.84 \mathrm{~kW} / \mathrm{m}(3.00 \mathrm{~kW} / \mathrm{ft})$ in the pins of the test. 


\section{COMPUTED GAMMA-RAY ENERGY DEPOSITION DURING PCM-20 POWER CALIBRATION TEST IN PBF}

T. E. Young

In support of the NRC!s Thermal Fuels Behavior Program, calculations were made of the gamma-ray energy deposited within the test rod flow shroud of PBF during the PCM-20 power calibration test conducted on January 30,1975 . The computed energy deposition values were required for use in converting measured fission rates in the test rod to power level.

Calculation of the energy deposition rates required solving neutron and gamma-ray distribution problems separately. The spacedependent neutron flux distributions and the fission rates for operation at $16 \mathrm{MW}$ were computed with 4-group $S_{N}$ transport theory. These flux and fission-rate distributions were used to determine the intensity of gamma-ray sources from various components of the reactor and test. The gamma-ray sources were then used in a 15-group $\mathrm{S}_{4}$ transport calculation to determine the energy deposited in the various components of the reactor and the PCM-20 test.

The axial average gamma-ray energy deposition in the test (defined as including one-half of the test rod flow shroud) at the end of the 16-MW operation was calculated to be 56.6 watts per foot. This corresponds to a total of $1.72 \mathrm{~kW}$ deposited over the fuli length of the test. For an assumed axial peak-to-average power ratio of 1.38 , the maximum rate of energy deposition by gamma rays will be 781 watts per foot. 


\section{PBF GAP CONDUCTANCE EXPERIMENTS AND DATA ANALYSIS}

\section{J. W. Sielinsky, D. R. Chick}

The Power Burst Facility (PBF) program for the measurement of the fuel pin gap conductance is continuing.(1) The gap conductance is computed using the phase angle between the fuel pin cladding temperature and the reactor power oscillations. The phase angle is determined using a Boonshaft and Fuchs Transfer Function Analyzer (TFA) for the on-line measurement of the phase angle.

The TFA computes the phase angle by analyzing data over one full cycle of reactor power oscillation. This phase angle measurement is repeated up to 66 times and recorded. The mean phase angle and its variance are then computed from the recorded measurements. The phase angle is determined at several different frequencies to permit a more complete comparison between the measurements and the mathematical model. The frequency range is selected to give maximum sensitivity of gap conductance to phase angle. The cladding temperature and reactor power signals are recorded for more detailed analysis, but the on-line analysis allows for timely indication and subsequent correction of measurement problems.

(1) J. W. Siel insky, "PBF Gap Conductance Measurements," Nuclear Technology Division Annual Progress Report For Period Ending June 30, 1975, ANCR-1255 (1975) 335-338. 


\section{ENERGY DEPOSITION CALCULATIONS FOR LEAD ROD TESTS IN PBF}

T. E. Young

Calculations were made of the energy deposited in fuel rods of PBF design in the PBF IPT and in the core during the Single-Lead-Rod-to Centerline-Melting Test (Run \#95). Calculations of energy deposition rates were made for the end of the test, since the contributions by betas and gammas were greatest at that time. Results were used to convert measured fission rates to heat generation rates and aid in determining PBF steady-s tate power level capability.

The space-dependent neutron flux distributions and fission rates were computed with 32-group and 4- group $\mathrm{S}_{4}$ transport theory. These flux and fission rate distributions were used to determine the intensity of gamma-ray sources from various components of the reactor and test. The gamma-ray sources were then used in a 20-group $S_{4}$ transport calculation to determine the gamma-ray energy deposited in the various components of the reactor and test space.

The axial average energy deposition in the test pin was calculated to be $179.8 \mathrm{MeV} /$ fission, including $167.9 \mathrm{MeV} / \mathrm{fission}$ by fission fragments, $5.79 \mathrm{MeV} /$ fission by gamma rays, and $6.15 \mathrm{MeV} /$ fission by beta particles. The axial average energy deposition in an average core pin is $190.6 \mathrm{MeV} /$ fission, including $167.9 \mathrm{MeV} /$ fission by fission fragments, $16.59 \mathrm{MeV} /$ fission by gamma rays, and $6.15 \mathrm{MeV} /$ fission by beta particles. 
COMPUTED REACTIVITY REQUIREMENT FOR OPERATION

OF PBF WITH A LARGE, REACTIVE EXPERIMENT

D. W. Nigg \& A. J. Scott

It has been proposed, as part of the Thermal Fuels Behavior Program, to conduct loss-of-coolant (LOCA) tests in the Power Burst Facility (PBF). One such test would involve preliminary irradiation of a 4 × 4 array of highly enriched PWR fuel rods for a period of 48 hours at a maximum PBF core power of $30 \mathrm{MW}$. A study was undertaken to complete the reactivity requirements associated with this type of operation, and to determine whether core shutdown requirements would be satisfied with the large reactive LOCA experiment array in place.

A three-dimensional, 4-energy-group, $P D Q-7^{(1)}$ diffusion-theory model of the PBF, based on flux synthes is techniques, was developed for use in this study. Thermal-hydraulic calculations were performed in PDQ-7 for each fuel rod coolant channel to account for spacedependent temperature distributions during simulated power operation. Space-dependent buildup of xenon and samarium in the core and experiment during the 48-hour irradiation period was computed with the HARMONY depletion routines incorporated into PDQ-7. Group constants for PDQ-7 were generated primarily from ENDF/B Versions 3 and 4 data using multigroup transport-theory calculations. Calculations showed that the cold, clean core excess reactivity would be about $\$ 7.00$ with the pressurized $\left(600^{\circ} \mathrm{F}\right)$ LOCA experiment in place and with the present shim pin configuration. Further calculations showed that there would be a total reactivity requirement (power defect and fission product poisoning) of about $\$ 6.50$ associated with $30-\mathrm{MW}$ operation of the PBF for 48 hours with the LOCA experiment installed. Thus, sufficient excess reactivity would be available for the planned irradiation. No reactivity shimming would be necessary unless additional control rod bite is desired after 48 hours.

Since the highly enriched experiment array was found to contribute significantly to the core reactivity and was expected to reduce control rod worth, it was necessary to investigate whether adequate shutdown margins could be maintained in the PBF during the LOCA test. Twodimensional $X-Y$ PDQ-7 calculations predicted a shutdown margin of $\$ 3.05 \pm .50$ for the cold reactor with the $600^{\circ} \mathrm{F}$ experiment in place and all control rods inserted (transient rods withdrawn). Reducing the temperature of the experiment array and coolant inside the in-pile tube was found to improve this shutdown margin; thus the calculation cited above is conservative. If only seven control rods are inserted (representing the stuck rod accident condition) the shutdown margin was computed to be $\$ 1.29 \pm .40$ again with the $600^{\circ} \mathrm{F}$ LOCA experiment in place. These results apply to the reactor at startup. At any later time the presence of xenon and samarium will make the core more subcritical after shutdown. It should be pointed out that a blowdown of the test loop would probably result in a small positive reactivity insertion $(<50 \phi)$. If this were to occur at the beginning of operation, the above shutdown margins would be reduced. Detailed calculations of this effect were not made in this study. 
The results of this study indicate that, from a reactivity standpoint, a 16-pin PWR-LOCA test could be conducted in the present PBF core. Reactivity requirements would be met without altering the present shim pin configuration. Assuming that the reported shutdown margins are considered adequate, control requirements for such a test would be met with the present control system consisting of the 8 outer rods. Although it is expected that the reactivity effect of voiding the experiment array would be small, this should be confirmed by calculation prior to conducting LOCA experiments in the PBF.

(1) A. W. Brown, et al, Summary of PDQ-7 (IBM-360-370 Version) Input Data Requirements and Operating Procedures, ANCR-1061 (March, 1972).

(2) J. R. Breen, et a1, HARMONY, A System for Nuclear Reactor Depletion Computation, WAPD-TM-478 (Jan. 1965). 
PBF RELOAD CORE PHYSICS DESIGN

F. J. Wheeler

A replacement core for the PBF facilities has been specified to provide increased capability for steady-state testing of reactor fuels as part of the NRC reactor safety program. Preliminary physics calculations for this reload core have been completed and included in a Preliminary Proposal submitted to ERDA in June 1976.

The reload core will provide enhanced test powers for steadystate testing of commercial fuels and is being designed such that maximum geometric compatibility with core 1 is maintained and minimum replacement time between the two cores can be accomplished. Major differences between the reload core and Core 1 are: (1) use of $\mathrm{UO}_{2}$ fuel pellets with no insulator rather than the dilute fuel pellet and $\mathrm{ZrO}_{2}$ insulator used in the core 1 fuel rod, (2) use of a thin-wall in-pile-tube, (3) reduction in core volume by about $25 \%$ and (4) redesign of the control system including poison sections.

The primary goal of the PBF Reload Core is the steady-state testing of PWR/17 x 17 and BWR-6 commercial fuel rods having prior irradiation. As many as 49 PWR fuel rods or 25 BWR fuel rods will be tested under conditions simulating power-coolant mismatch (PCM) accidents, loss-of-coolant (LOCA) blowdown accidents, gap conductance tests, irradiation effects tests and BWR flow blockage accidents.

The neutronics of the reload core are quite unique with respect to any other operating light-water reactor. The core is very highly under-moderated; hence, most of the fissions occur above the cadmiumcutoff energy. The prompt neutron lifetime in the system is very short for a water reactor; about eight microseconds.

The physics design of the core is proceeding using ENDF/B Version IV data. The model has been checked by comparison with critical experiments, comparison with other cross-section data sets and calculations using very accurate methods. It is estimated that the design model underestimates the neutron multiplication of the system by about one-and-one-half percent. It is felt that the cause of this underestimation is the high background resonance capture cross section in the ENDF/B data for uranium-238 and use of the Wigner-Seitz outer boundary approximation in unit-cell calculations.

Final design of the reload core has been initiated with core availability scheduled for about mid-year 1978. 


\section{PBF RELOAD CORE REACTIVITY EVALUATION}

R. T. McCracken

A study was done during the preliminary phase of the PBF Reload Core design effort to obtain estimates of several reactivity effects making up the excess reactivity requirement for the Reload Core. The magnitudes of the power defect, moderator heating, fission-product reactivity, fuel depletion, accidental test-space voiding, and the reactivity worths of several proposed solutions to power-peaking problems were determined. The results of this scoping study were used to determine areas in which more detailed calculations would be required as the design effort progressed.

The calculations were done with the $\mathrm{PDQ}^{(1)}$ code in conjunction with a one-dimensional radial model of the Reload Core. Four-group constants for use in PDQ were obtained with the LEOPARD ${ }^{(2)}$ code. The fission-product reactivity and fuel depletion were computed with the PDQ depletion option which represented the spatial variation of the poisoning and depletion processes. As a check a LEOPARD burn-up calculation was done and the resultant constants were used in a series of PDQ calculations: This in effect assumes fission products are built-up and fuel is depleted uniformly throughout the core. The average concentrations of the depleting isotopes in the core predicted by the radial calculation agreed well with those predicted by LEOPARD, adding assurance that errors were not made in setting up the PDQ depletion chains.

Large voids can not be handled well with diffusion theory. Thus, the in-pile-tube voiding calculations were done using methods for simulating large voids outlined in Reference (3).

Large power peaks have been calculated in the innermost fuel elements of the Reload Core. The effects of three proposed solutions to this problem on the excess reactivity requirement were examined. These solutions were 1) borating the walls of the innermost fuel canisters, 2) lowering the enrichment of the inner row of fuel pins (40 pins), and 3) using low-enrichment fuel in the inner three rows of fuel pins (140 pins). It was found that the use of low-enrichment fuel in the inner row of fuel pins provided a satisfactory decrease in the power peak with little effect on test performance and voiding characteristics.

Table I gives the calculated reactivity values contributing to the excess reactivity requirement. These were computed using the formula given in the Table with $k_{1}$ and $k_{2}$ equal to the eigenvalues of the PDQ calculations for the initial and final conditions, respectively.

The combination of LEOPARD and PDQ in radial geometry proved to be a fast and reasonably accurate method for use in scoping studies, especially those involving a large number of core calculations. The results of these calculations have been found to be in good agreement with $S_{n}$ transport calculations done in subsequent phases of the 
core design.

(1) C. J. Pfeifer, PDQ-7 Reference Manual, WAPD-TM-947(L), (February 1971).

(2) R. F. Barry, LEOPARD - A Spectrum Dependent Non-spatial Depletion Code, WCAP-3269-26 (September 1963).

(3) B. L. Rushton, Physics Methods Used to Compute Reactivity $\lambda$ B , and Rod Worth for the PBF Critical Experiments, 100-17276 (0ctober 1968).

TABLE I

Computed Reactivity Values

REACTIVITY EFFECT

$\Delta p=\frac{k_{2}-k_{1}}{k_{2} k_{1}}$

Power Defect

$-0.02037$

Moderator Heating

$-0.00327$

48-Hr. Fission Product Reactivity

$-0.00824$

Fuel Depletion (5000 MWd)

$-0.03875$

Addition of Borated-Canister Wal1

$-0.01489$

Addition of 40 Low-Enriched Fuel Pins

$-0.00254$

Addition of 140 Low-Enriched Fuel Pins

$-0.00840$ 
EVALUATION OF PHYSICS METHODS AND DATA FOR THE

PBF RELOAD CORE USING PBF CRITICAL EXPERIMENTS

W. 0.01 son

The original PBF core was designed for transient type tests. Since that time the reactor has proven useful for steady state operation. The PBF Reload Core is being designed specifically for this mode of operation, with a requirement of interchangeability with the original core. Included in the design work is an evaluation of physics methods and nuclear data. The methods used are similar to those used in the original design $\left({ }^{2}\right)$ except that greater calculational detail is available with larger computers. The ENDF/B cross-section data set has also become available in the period since the original design.

Critical experiments were performed in connection with the design of the original cord ${ }^{2}, 3,4,5$. These use an aluminum annulus to simulate the fuel pin insulator of the original core. While the insulator is not to be retained in the Reload Core, the large non-moderator-tomoderator ratio (2.46) will be used. The criticals also mock up many of the details which will be retained in the Reload Core, namely, the large central test area, the large-diameter poison rods and their air followers, and the shim pins at the periphery of the core. Thus, the criticals provide a useful test for the methods and data to be used in the present design task.

The ENDF/B Version 4 data were processed into a 68-fast-energy-

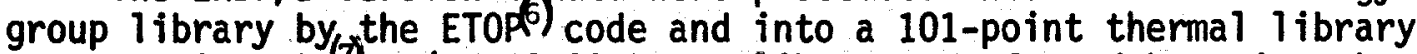
by the FLANGE-I $(7)$ code. A 90-group library was formed by using the PHROG ${ }^{(9)}$ code for the fast group and INCITE for the thermal groups. SCRABBLE( 10 ) was used to process the resonance data. A 90-group pin-cell calculation made with SCRABBLE using an equivalent cylindrical cell produced homogenized fuel cell constants. These were used to produce 33-and 4-group constants for the various takks. In general, it was necessary to use fairly realistic models and transport theory for the collapsing due to the heterogeniety of the system being modeled.

Criticality calculations were made using the CORA ${ }^{(1)}$ diffusion code using 4 groups in the automatic buckling-iteration mode. In this mode the code calculates in one direction using the perpendicular leakage calculated for the other directions, with iteration between directions continuing until convergence is achieved.

Diffusion-theory constants for the ${ }^{2} \mathrm{~B}_{4} \mathrm{C}$ poison sections were obtained with the use of a "super cell" which has the poison rod at its center surrounded by an annulus of fuel and a reflective boundary. Diffusion constants for the poison section are adjusted to force a fit between diffusion theory and a 33-group $S_{6}$ calculation for the same cell. Cylindrical voids to represent test spaces and poison rod followers were treated using a method developed by Putnanf 2), who numerically evaluated the probability a neutron entering the void has of re-entering the material. The prompt neutron generation time $(\Lambda)$ and effective delayed neutron fraction (Beff) are evaluated using the 
methods of Reference 13 .

The critical experiments include two sets by Phillips Petroleum $(2,3)$ with 1.9 and 2.3 non-moderator-to-moderator ratios, respectively. A set performed by G. E. $(4,5)$ was used to verify control rod calculations. This has a 1.9 non-moderator-to-moderator ratio and $96 \%$ of the fuel density used in the other calculations. All use a 4.8 weight percent enrichment.

The criticality calculations yield eigenvalues which are $1.3 \%$ low for the 1.9 ratio cells and $1.7 \%$ low for the 2.3 ratio cells. The standard deviation in a single value is $0.2 \%$, which is good internal consistency considering the variety of things modeled (voids, control rods etc.).

Control rod worths are well wi thin the experimental uncertainties, both for CORA and two-dimensional PDQ $\left.{ }^{4}\right)$ runs. The latter are necessary for cases where the control rod mock-up is not at the center of the core. The ratio $\Lambda / \beta_{\text {eff }}$ also agrees well with experiments.

(1) B. L. Rushton and W. R. Carpenter, Methods used for Physics Analysis of the Power Furst Facility and Computed Results for the Design Core, ANCR-1008 (July 1971).

(2) B. L. Rushton, Physics Methods Used to Compute Reactivity, $\Lambda / \beta_{\text {eff }}$, and Rod Worth for the PBF Critical Experiments, ID0-17276 (October 1968).

(3) A. H. Spano, Editor, Quarterly Technical Report SPERT Project, ID0-17030 (September 1964).

(4) J. F. Kunze, et. a1., PBF Critical Experiment Control Rod Measurement, GEMP-560 (October 1960).

(5) F. L. Sims, Test Program and Standard Reactor Operating and Maintenance Procedures for the PBF Critical Experiment Reactor at the Low Power Test Facility, GEMP-522 (June 1967).

(6) R. A. Grimesey et al, ETOP 14, A FORTRAN code to Process ENDF/B Data into the 68-group PHROG Library Format, ANCR-1322 (July 1976).

(7) H. C. Honeck and D. R. Finch, FLANGE-II (Version 71-1), A Code to Process Thermal Neutron Data from an ENDF/B Tape, DP-1278 (0ctober 1971).

(8) R. L. Curtis et. aI., PHROG--A FORTRAN IV Program to Generate Fast Neutron Spectra and Average Multigroup Constants, IN-1435 (Apri1 1971). 
(9) R. L. Curtis and R. A. Grimesey, INCITE -- A FORTRAN IV Program to Generate Thermal Neutron Spectra and Multi-group Constants Using Arbitrary Scattering Kernals, IN-1062 (1967).

(10) G. E. Putnam, personal communication.

(11) E. C. Anderson and G. E. Putnam, CORA - A few Group Diffusion Theory Code for One-Dimensional Reactor Analysis, IN-1416 (August 1970).

(12) G. E. Putnam, The Simulation of Axial Cylindrical Voids in Neutron Reactor Computer Programs, PTR-794 (May 1966).

(13) G. E. Putnam, Some Methods of Calculation of the Parameters in the Reactor Kinetics Equations, ID0-16983 (February 1965).

(14) A. W. Brown, et. al., Summary of PDQ-7 (IBM-360-370 Version) Input Data Requirements and Operating Procedures, ANCR-1061 (March 1972). 
TEST LOOP AXIAL POWER SHAPING FOR THE PBF RELOAD CORE

G. S. Gill

Studies were performed to try to shape the axial power distribution in the test space of the PBF Reload core. Previous calculational results for Core I showed that by manipulating materials within the inpile tube (i.e., aluminum inserts), the power distribution could be shaped to give an acceptably flat power distribution $( \pm 5 \%)$ over a $.457-m$ (18-in.) region in the test space. Investigated was the feasibility of utilizing an axially distributed borated region in the inner canister walls (outside the in-pile-tube) as a power shaper for the core.

Using the PDQ-7 Version 2 computer program and an R-Z model, axial power distribution calculation studies were made in the core and in the test space using various $\mathrm{B}_{4} \mathrm{C}$ poison densities in the shaper region and a 36-pin PWR conmercially enriched test inside the in-pile tube. The $\mathrm{B}_{4} \mathrm{C}$ volume fraction in the canister wall was varied axially from 0.0 to 0.15 . The driver core was composed of $8 \%$ enriched $\mathrm{UO}_{2}$ with Zircaloy-2 cladding, and the feasibility studies were made with the reactor operating at $50 \mathrm{MW}$ with all control rods withdrawn. Figure 1 illustrates the axial power distributions with no $\mathrm{B}_{4} \mathrm{C}$ present in the shaper canister and the two distribution curves representing the best flattening of the power profiles in the core and in the test.

Results indicate that some flattening of the test and core power profile does occur with a $B_{4} C$ shaper in the inner canister; however, the requirements of a flat axial power distribution in the central region of the test space cannot be met using the $B_{4} C$ shaper alone. The best flattening occurred when $0.307 \mathrm{~m}$ (12.1 in.) of $15 \% \mathrm{~B}_{4} \mathrm{C}$ was centered relative to the core with $0.152 \mathrm{~m}(6.0 \mathrm{in}$.$) of 10 \% \mathrm{~B}_{4} \mathrm{C}$ located directly above and below the $15 \% \mathrm{~B}_{4} \mathrm{C}$ in the shaper canister. 


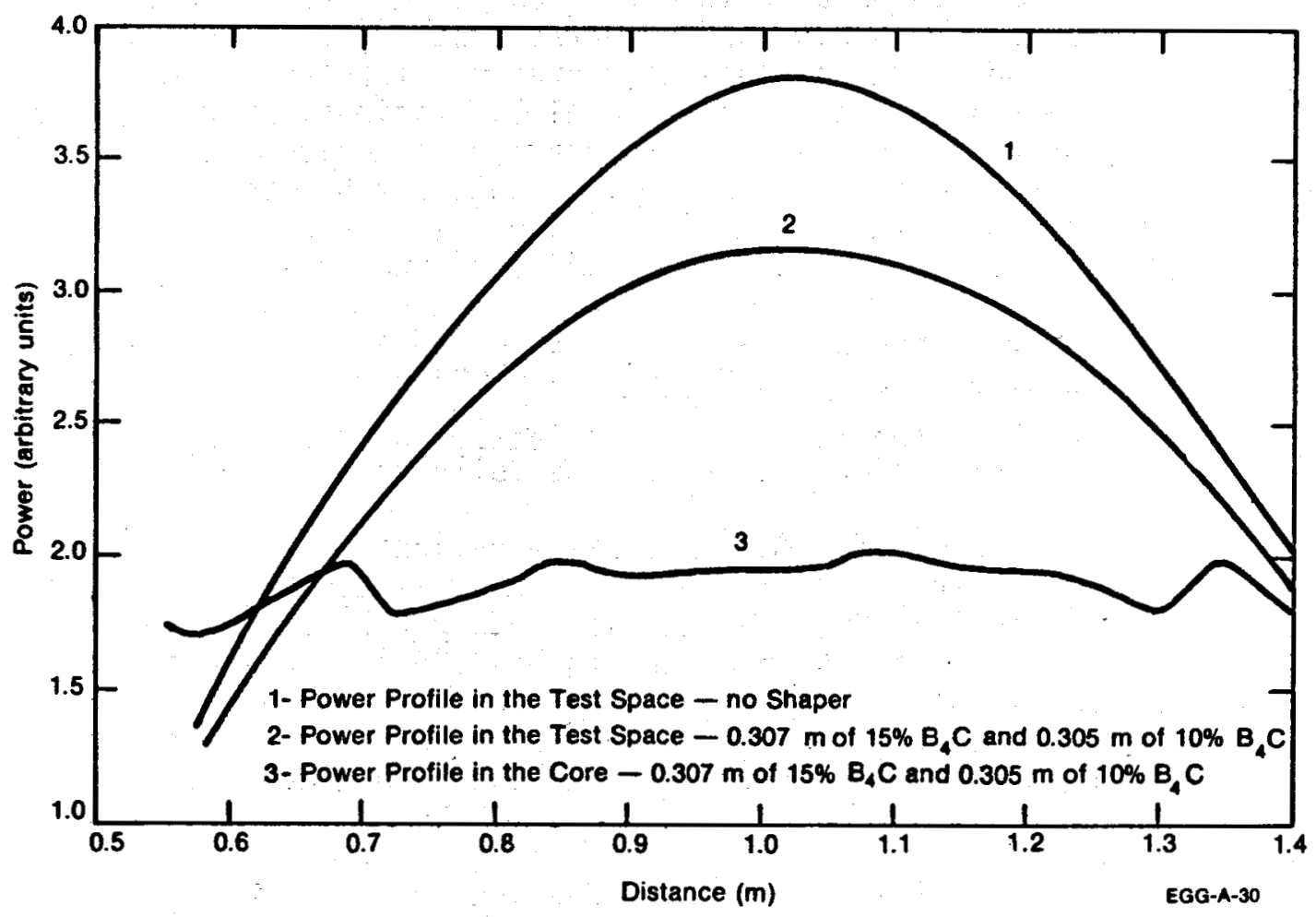

Figure 1. Axial power distribution (through the maximum power peak level). 
D. E. Wessol

RAFFLE (1) Monte Carlo calculations were performed using the design concept of the $8 \%$ enriched PBF reload core oxide fuel rod. Under the assumption that fuel rod bowing has occurred, both reactivity changes and fuel pellet power distributions were examined for the hot case. Fuel rod bowing was simulated, due to model limitations, by moving the entire axial length of the fuel rod to nearly the maximum lateral displacement in an infinite reactor environment, a truly conservative representation of the actual situation. Figure 1 represents the RAFFLE geometric model for both the unbowed and bowed cases.

The reactivity change betweep the unbowed and fully bowed representation was calculated to be $\Delta \rho=\frac{k_{2}-k_{1}}{k_{1}}=0.00319 \pm 0.0019$ at $90 \%$ confidence where $k_{1}$ is the multiplication factor for the unbowed case and $k_{2}$ is the multiplication factor for the fully bowed case.

The fuel portion was divided into sixty regions of equal volume for power edits. As shown in Figure 2 the fuel was divided into five radial zones with twelve $30^{\circ}$ azimuthal segments per zone. Maximum azimuthal power variation was predicted to be $4.9 \%$ for the unbowed or centered case and $17.5 \%$ for the fully bowed case. Figures 3 and 4 present a visual interpretation of the power variation for each azimuthal, segment in each radial zone for the unbowed and bowed rods respectively.

The model used in this calculation was chosen so as to exaggerate the effects of rod bowing not only for conservatism but to decrease the variance since small changes are difficult to observe in the standard non-perturbation Monte Carlo calculation. Reactivity and power effects that apply to this model overestimate that which would occur in the actual core but the trends should remain the same. The changes in power distribution predicted by this model could conceivably be attained and even exceeded in localized areas of the actual core.

(1) F. J. Wheeler, et. a1., The RAFFLE General Purpose Monte Carlo Code, ANCR-1022 (Apri1 1973). 


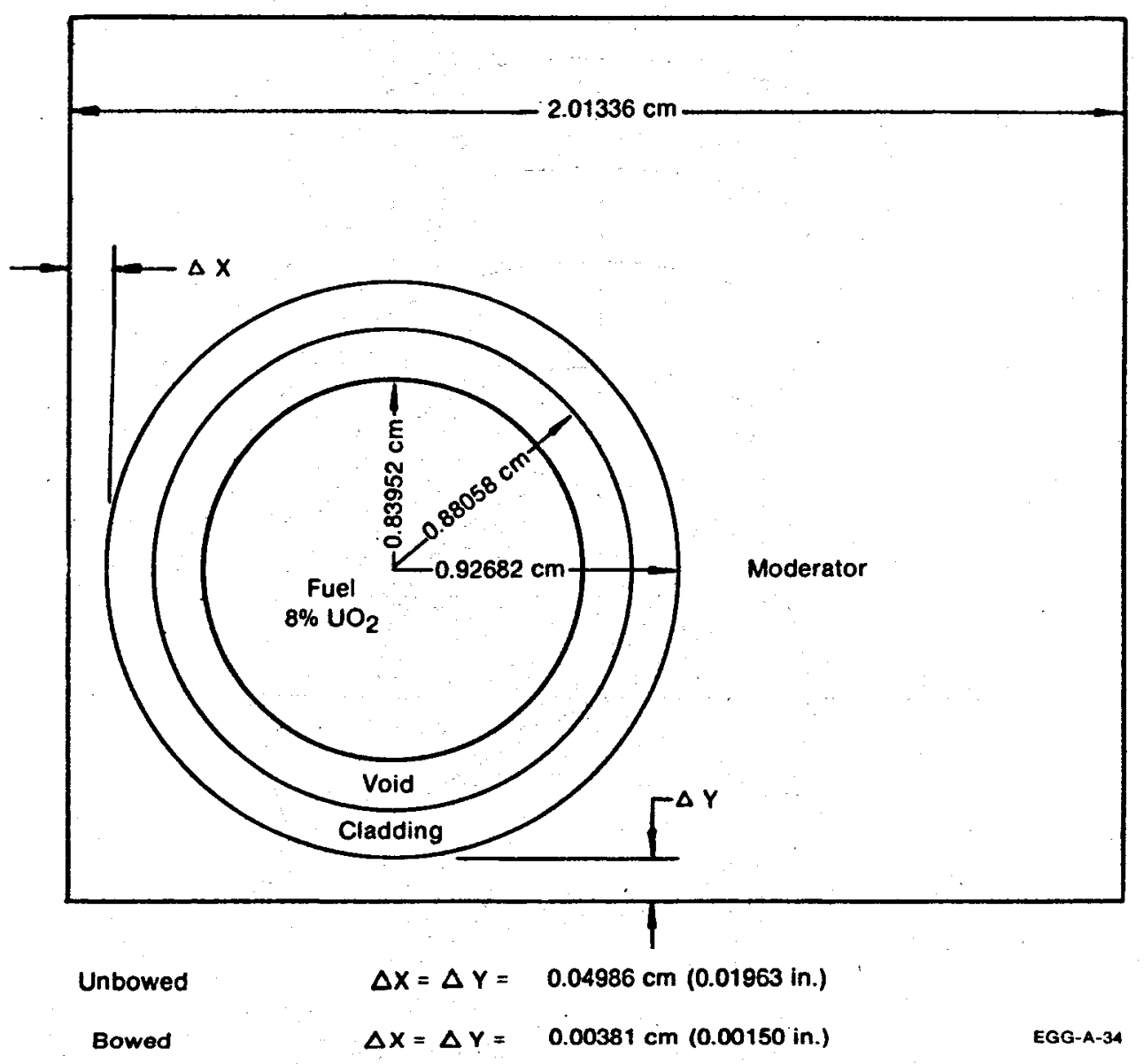

Figure 1. Cell model for RAFFLE Monte Carlo calculations. 


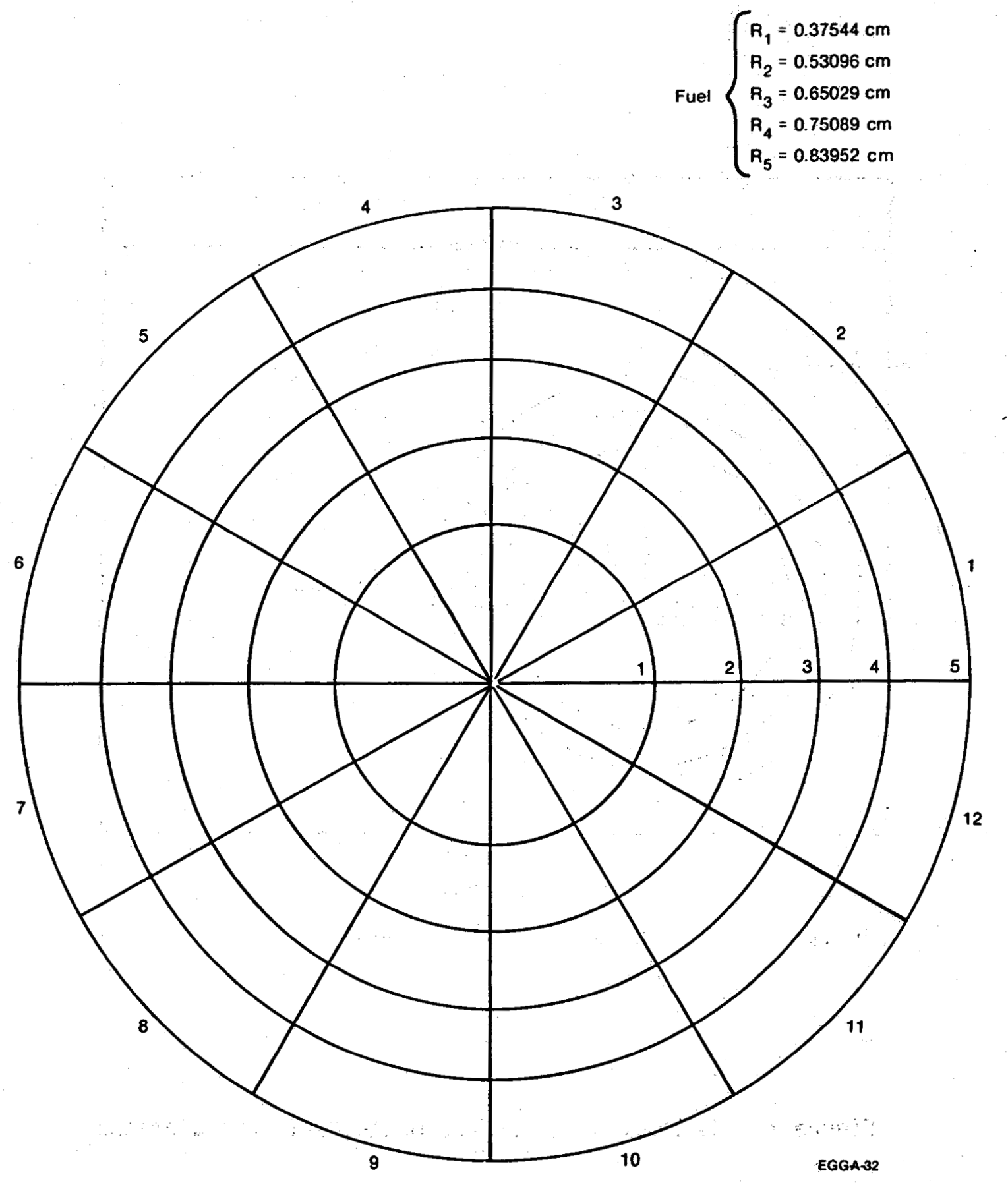

Figure 2. Division of fuel pellet into radial and azimuthal edit regions. (Internal numbers designate radial regions and external numbers designate azimuthal regions.) 


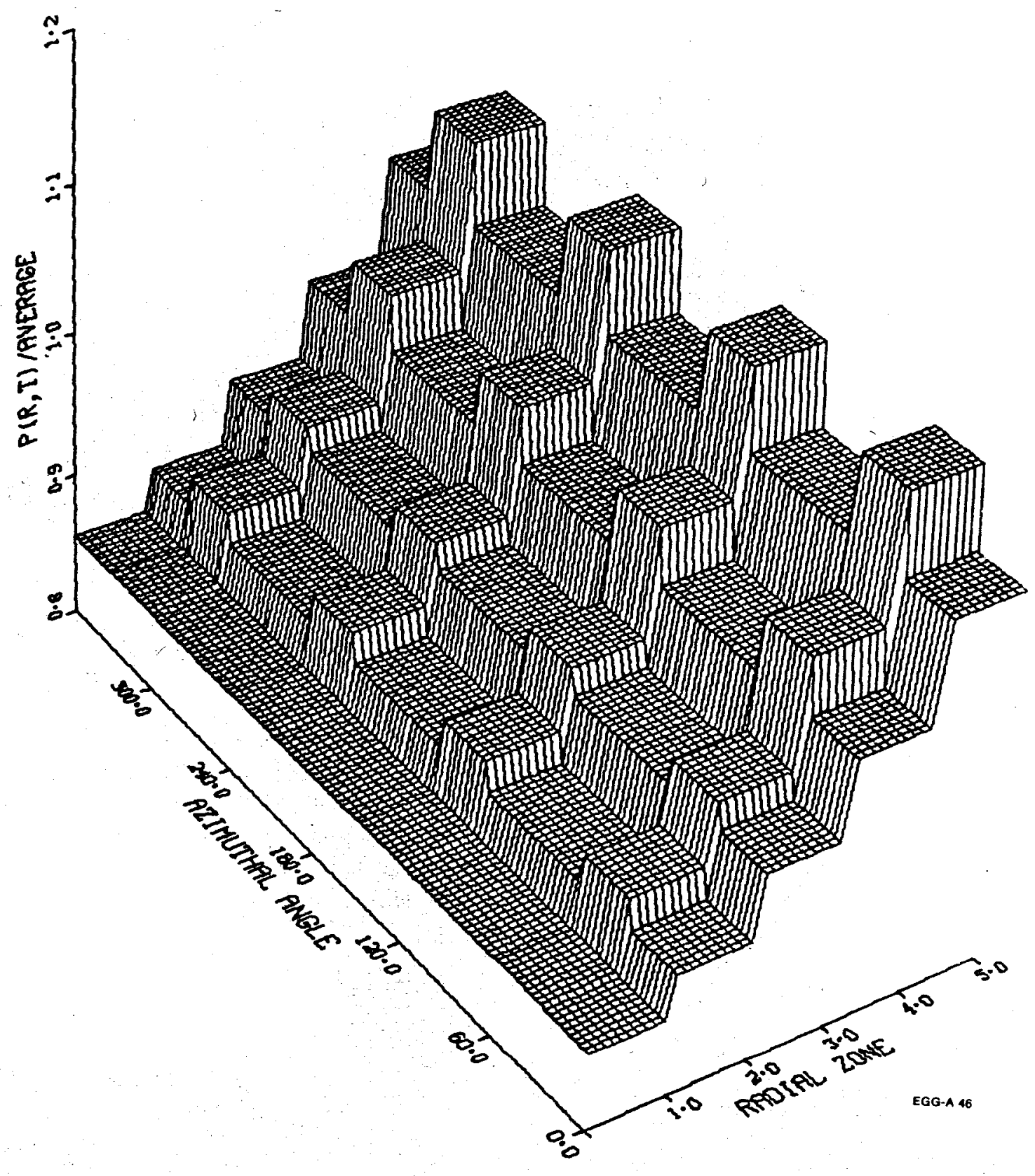

Figure 3. RAFFLE power distribution PBF centered pin. 


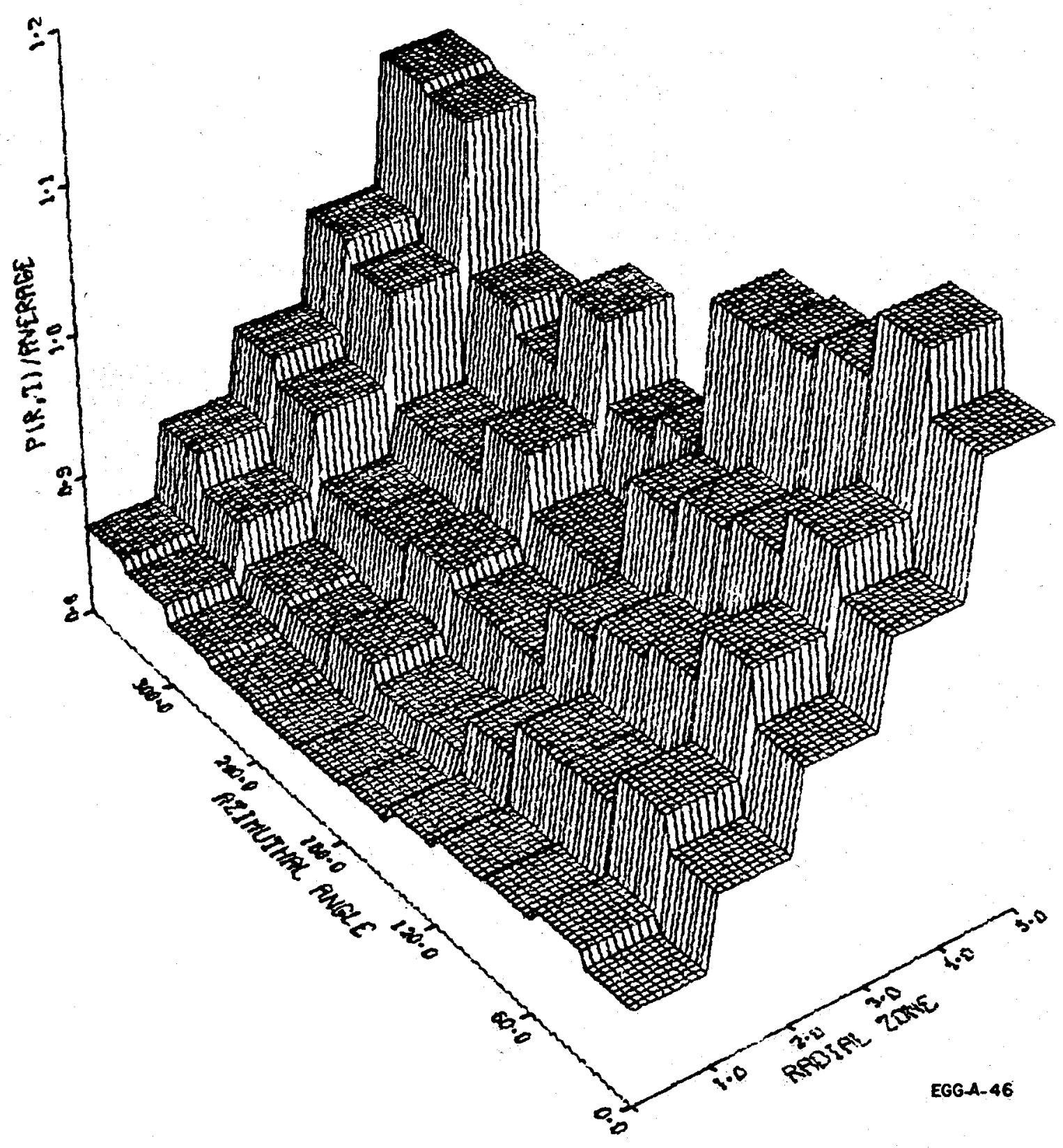

Figure 4. RAFFLE power distribution PBF bowed pin. 


\section{ALTERNATE CONTROL ROD DESIGN FOR THE PBF RELOAD CORE}

W. 0. 01son and G. S. Gill

Various calculations have demonstrated a loss of control rod reactivity worth if the present PBF control rod system is used in the envisioned PBF reload core (3/4 present core size, oxide fuel, thinwall in-pile tube). This loss of worth is primarily due to a hardening of the neutron spectrum caused by replacing the insulated ternary fuel pin with a $\mathrm{UO}_{2}$ pin of the same dimensions. A study has been made to examine the feasibility of enhancing this worth by an alternate control rod design which incorporates a moderator within the poison rods. The large diameter of the present poison rods is to be retained to make interchange of the two cores possible.

Comparative calculations for tentative designs were made using a cylindrical "super cell" which has the poison rod at its center surrounded by an annulus of homogenized fuel and a reflective boundary. The transport code SCAMP was used, with a 4-group model used for optimization and 32- and 33-group models used to compare the optimal moderated rod to the present all-B $\mathrm{B}_{4} \mathrm{C}$ poison section.

Preliminary calculations established that a moderator annulus interior to the $B_{4} C$ (replacing part of the present $B_{4} C$ ) was superior to a mixture of $\mathrm{B}_{4} \mathrm{C}$ and moderator. The $\mathrm{B}_{4} \mathrm{C}$-to-moderator ratio was also optimized.

In comparing various poison rods in the same cell there is an approximate proportionality between the worth of a given rod and the fraction of the cell absorption which takes place in the poison rod. Table I compares the optimal $\mathrm{TiH}_{2}$-moderated rod with the present all$\mathrm{B}_{4} \mathrm{C}$ rod for the $8 \%$ oxide fuel and with the all $-\mathrm{B}_{4} C$ rod in the old $\mathrm{PBF}$ fuel. This comparison for the inner rods indicated that the absorption for the all-B $\mathrm{B} C$ rod in the oxide fuel is $65 \%$ of that for the same rod in the old fuel. The $\mathrm{TiH}_{2}$-moderated rod in oxide fuel has an absorption which is $82 \%$ of that for the old fuel $-B_{4} C$ combination, thus gaining back about half of the worth lost in going to the oxide fuel.

TABLE I: INNER ROD ALTERNATIVES

\begin{tabular}{|c|c|c|}
\hline Fuel & Transient Rod & Abs. Fraction in Trans: Rod \\
\hline old PBF & 2.14-cm. annulus of $B_{4} C$ & .2056 \\
\hline $8 \%$ Oxide & 2.14-cm. annulus of $B_{4} C$ & .1330 \\
\hline $8 \%$ Oxide & $\begin{array}{l}.5-\mathrm{cm} \text {. annulus of } \mathrm{B}_{4} \mathrm{C} \text { outside } \\
\text { a } 1.64-\mathrm{cm} \text {. annulus of } \mathrm{TiH}_{2}\end{array}$ & .1695 \\
\hline
\end{tabular}

A similar calculation was made for the outer poison rods using a $\mathrm{B}_{4} \mathrm{C}-\mathrm{TiH}_{2}$ rod which incorporated a cooling annulus between the $\mathrm{B}_{4} \mathrm{C}$ and $\mathrm{TiH}_{2}$. With this model an estimate of $40 \%$ of the control rod 
worth lost in going to oxide fuel is recovered by going to a $\mathrm{TiH}_{2}$ $\mathrm{B}_{4} \mathrm{C}$ rod.

Constants for a two-dimensional diffusion-theory model were developed by adjusting the poison constants for a diffusion-theory representation of the "super cel1" to cause agreement with a 33-group, $S_{6}$ representation. These constants were then used in a two-dimensional reactor model. This yielded a worth for the $\mathrm{B}_{4} \mathrm{C}-\mathrm{TiH}_{2}$ rods which is 1.2 times that for the all- $B_{4} C$ rods in the $8 \%$ oxide fuel. This agrees with the predictions made above using the super cells. 


\section{LOSS OF FLUID TEST}




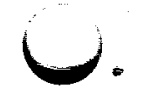

\section{BLANK PAGE}




\section{A HYBRID COMPUTER SIMULATION FOR THE ANALYSIS

OF A MAIN STEAM LINE BREAK IN THE LOFT PLANT

$$
\text { F. K. Hyer }
$$

A hybrid computer simulation of the LOFT primary loop and the study of the thermal-hydraulic response of the LOFT plant to a break in the steam line from the steam generator secondary have been performed as required for LOFT plant safety analysis.

The hybrid computer used was the EAI-PACER 600 system of the Electronics Associates, Inc. Computation Center at West Long Branch, New Jersey.

The implementation was accomplished for an absolute pressure range of 0.6895 to $16.55 \mathrm{MPa}$ (100.0 to $2400 \mathrm{psi}$ ) and a temperature range of 385.4 to $621.5 \mathrm{~K}\left(234\right.$ to $\left.659^{\circ} \mathrm{F}\right)$. Analytical runs were performed with the following parametric vlaues:

(1) Power: 55 and 8 megawatts.

(2) Moderator density feedback reactivity: 22.13 to $1.771 \$ / \mathrm{kg} \mathrm{m}^{-3}$ (1.377 to $\left..1102 \$ / 1 \mathrm{~b} . \mathrm{ft}^{-3}\right)$.

(3) Feedwater flow (post transient): 68.04 to $0.0 \mathrm{~kg} / \mathrm{s}$ (150 to $01 \mathrm{~b} . / \mathrm{s})$.

(4) Initial post-transient secondary heat transfer coefficient: 1.897 to $4.553 \mathrm{MW} / \mathrm{K}(1000$ to $2400 \mathrm{Btu} / \mathrm{s}$ OF $)$.

Worst-case combination of the above parameters resulted in recriticality. However, for low values of feedback, no feed flow, and the low coefficient of secondary heat transfer, recriticality was not obtained. Therefore, the range of parameters selected for the sensitivity study did indeed cover the range of response desired.

The results indicate that without the cool-down effect of feed flow, recriticality would occur only with feedback reactivity values which are greater than the best-estimate values.

Features of the hybrid computer program are as follows:

(1) Steady state was obtained with adequate precision of mass, energy and momentum balances.

(2) A 1:i probiem to machine time scaling requires filtering of the analog circuits to eliminate the higher sonic frequencies. This filtering is permissible in this study since the primary transients are thermally induced. Therefore, in the frequency spectrum of the transients, the magnitude of the sonic range of frequencies is expected to be considerably reduced in. comparison to the lower frequencies, i.e., <10 hertz.

(3) Floating-point arithmetic resulted in a digital processor frame time of 101 milliseconds. The frame time should be reduced by a factor of from two to four by using fixed-point arithmetic. 
(4) Time-dependent output is obtained from strip chart only. Time functions of selected variables could be listed from data inherently resident in the digital processor.

This simulation is adaptable for utilization in the analysis of two-phase phase phenomena in power plants where the frequency content of the transients does not extend into the sonic region, i.e. 50 to $500 \mathrm{~Hz}$. The secondary blow down, small break analysis, and anticipated transients without scram are examples of these relatively slow transients.

The advantages of performing these analyses on a hybrid computer system such as the PACER 600 are:

(1) Fast computing time.

(2) User interaction.

(3) Continuous real time output (strip chart) in addition to the usual digital output, such as numerical listing and post-run plotting. 
AN ANALOG COMPUTER SIMULATION OF THE LOFT BLOWDOWN SUPPRESSION TANK AND PRESSURE CONTROL SYSTEM

\section{J. J. Feeley}

An analog computer simulation of the LOFT blowdown suppression tank and pressure control system has been developed. Given initial conditions on tank temperature, pressure, and water level, and transient input data on primary coolant and nitrogen mass flow rate and energy content, tank conditions during a blowdown can be predicted. Various modes of automatic pressure control may also be simulated. The pressure control system model consists of a pump with bypass flow control, spray valves, controllers, actuators, and interconnecting piping.

The Loss of Fluid Test (LOFT) facility is designed to conduct loss-of-coolant experiments to simulate loss-of-coolant accidents on large pressurized water reactors (LPWRs). An experiment is initiated by rapidly opening two valves in the reactor coolant system's blowdown loop allowing the pressurized coolant to discharge into the relatively low-pressure suppression tank. During the initial phase of reactor blowdown the flow through the break is choked, and the flow rate is independent of the suppression tank pressure. As the primary coolant system depressurizes and the back pressure generated in the suppression tank plays an increasingly important role in determining the break flow rate and it is important, therefore, that this pressure be similar to the expected containment building pressure during an actual loss-ofcoolant accident. Since the various differences between the LOFT suppression tank and a typical LPWR containment building preclude a naturally occurring similarity in back pressure, a spray system has been installed in the suppression tank to achieve the desired pressure response.

A physical representation of the suppression tank is shown in Figure 1. For modeling purposes the tank is considered to consist of a liquid region and a vapor region separated by a distinct interface. The vapor region consists of a homogeneous mixture of water vapor and nitrogen gas. The tank is considered a perfect insulator, and no heat storage in the tank walls or associated piping is accounted for.

Effluent from the primary coolant system enters the tank via downcomer piping whose outlet is considered to always be submerged. Primary coolant is assumed to be completely condensed in the water region so that no steam enters the vapor space directly. Furthermore, the turbulence of the condensation process and the tank geometry are such that perfect mixing is assumed to occur and the water region is taken to be isothermal. In addition, the nitrogen pressure in the vapor space is always large enough so that no bulk boiling of the liquid occurs.

Others have shown that it is valid to assume that the water vapor in the vapor space is saturated throughout the blowdown. Therefore, evaporation and condensation as well as sensible heat transfer from (and to) the nitrogen and across the liquid-vapor interface are allowed to occur such that the vapor pressure of the water is always equal to the saturation pressure corresponding to the actual vapor temperature. 
Nitrogen, from the blowdown header and the accumulators, is added directly to the initial nitrogen stored in the tank and heat is transferred between it and both the liquid water and the water vapor. The transient temperatures of the nitrogen, the water vapor, and the liquid water are assumed to be uniform, but not necessarily equal.

Water from the pressure control system is sprayed into the vapor space to condense water vapor and, thereby, reduce pressure. No attempt is made to rigorously model the complex condensation process. Instead, the condensation rate is based on a simple heat balance on a typical spray droplet and an experimentally determined spray effectiveness factor.

The simulation has been used to study various control schemes and controller settings and to recommend initial controller setpoints for future LOFT experiments. 


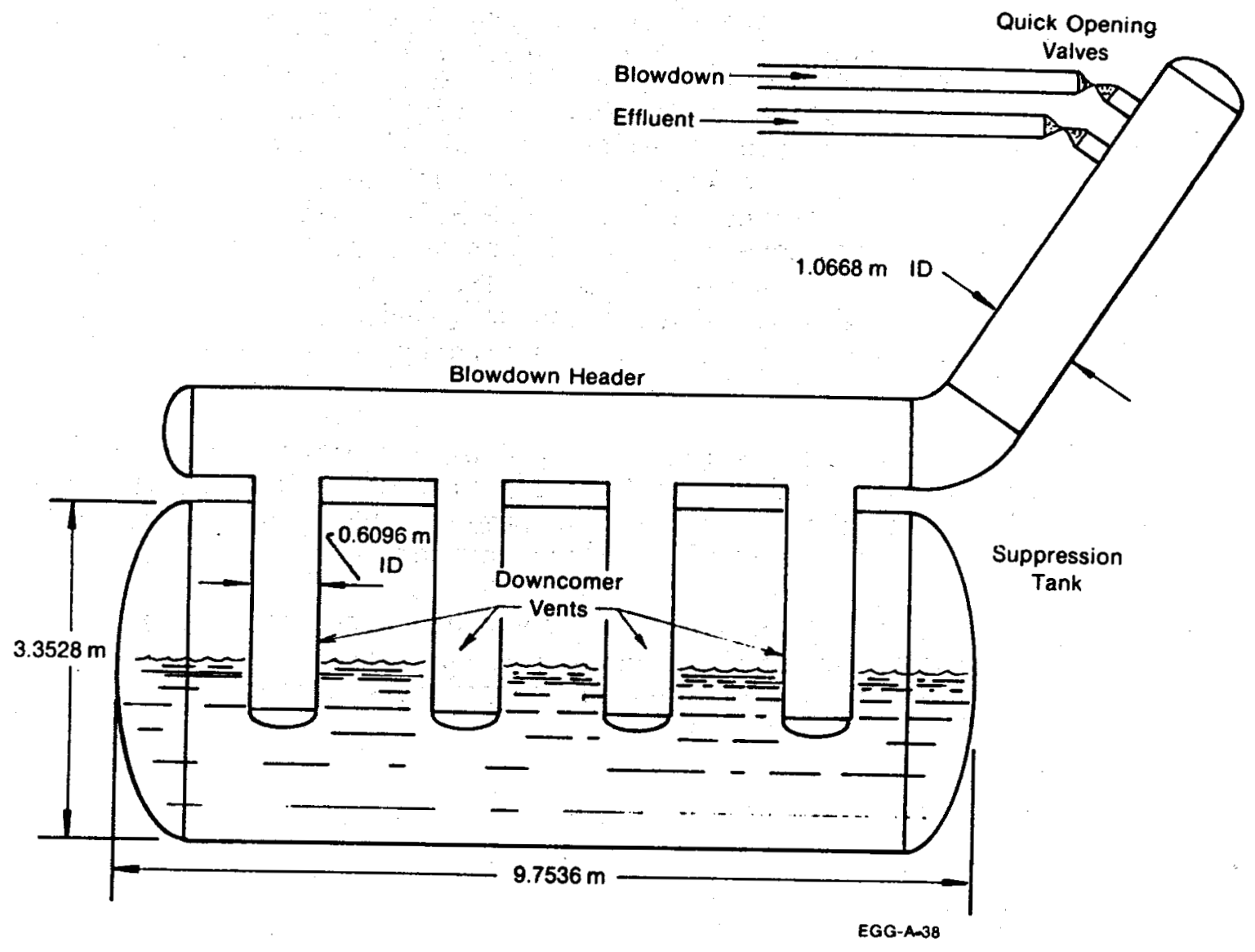

Figure 1. LOFT blowdown suppression system (conceptual). 
CALCULATION OF CRITICAL BORON CONCENTRATION

VERSUS CONTTROL ROD POSITION USING XYZ SYNTHESIS IN PDQ-7

T. E. Young

The boron concentrations required to make LOFT critical (critical boron concentrations) for several control rod positions were cal culated at uniform reactor temperatures of $293^{\circ} \mathrm{K}\left(68^{\circ} \mathrm{F}\right)$ and $579^{\circ} \mathrm{K}\left(583^{\circ} \mathrm{F}\right)$ using the PDQ-7 three-dimensional diffusion-theory code and XYZ-synthes is techniques. These computations were done to verify a more extensive set of calculations made using the one-dimensional "windowshade" technique. The windowshade calculations are much less expensive than the XYZ-synthesis calculations, but they use a less detailed description of the reactor and therefore may be less accurate. Critical boron concentrations computed with the 3-D model are 23 to $68 \mathrm{ppm}$ lower than those obtained with the windowshade technique, indicating that the rods must be withdrawn slightly more than predicted by the windowshade calculations before criticality occurs.

Critical boron concentrations computed with control rods fully withdrawn are $1967 \mathrm{ppm}$ at $68^{\circ} \mathrm{F}$ and $1473 \mathrm{ppm}$ at $583^{\circ} \mathrm{F}$. With control rods fully inserted the critical boron is $845 \mathrm{ppm}$ at $68^{\circ} \mathrm{F}$. The reactor cannot be made critical at $583^{\circ} \mathrm{F}$. with the control rods fully inserted.

Results of the one-dimensional windowshade calculations are adequate to estimate the critical control rod position for a given boron concentration and uniform reactor temperature during LOFT's initial critical and low-power tests. 
CALCULATED WORTHS FOR THE LOFT-1 Ag-ALLOY CONTROL ROD CLUSTERS

\author{
D. R. Cooper
}

LOFT-1 control rod cluster differential and integral worths are required input for the LOFT-1 Technical Specifications Report, for rod cluster ejection analysis, and for initial physics input for the LOFT-1 startup. The rod cluster worths reported earlier were for $\mathrm{B}_{4} \mathrm{C}$ poison pins while this study was done for the as-built Ag-alloy rod clusters. Rod cluster worths reported earlier were for the $\mathrm{B}_{4} \mathrm{C}$ poison pins. This study reports rod cluster worth for the Ag-alloy poison pins.

The current analysis computed for the as-built cluster design consisted of three phases:

1. Differential and integral worths were computed for rod cluster drop or scram conditions.

2. Differential and integral worths were computed for various cluster ejection conditions.

3. Three-dimensional PDQ-7 calculations were made to check the onedimensional "window shade" technique used to generate the above data.

The differential and integral rod cluster worth calculations were performed using the PDQ-7(1) and MONA $(2)$ codes and the "window shade" technique. Radial bucklings are first computed for the rod clusters fully withdrawn and fully inserted. The configurations are computed with $\mathrm{PDQ}-7$ in the $X-Y$ geometry for the required temperatures and boron concentrations. These resulting radial bucklings provide leakage terms when input to the MONA calculation in the $Z$ direction. This approximation to the three-dimensional solution provided a means for computing the large number of configurations economicaliy. A comparison of the onedimensional approach in LOFT was made using the three-dimensional flux synthes is technique.

Control rod cluster worths are presented in Table I for the two conditions examined: $579.8 \mathrm{~K}\left(584^{\circ} \mathrm{F}\right)$ with $1232 \mathrm{ppm}$ boron and $293.2 \mathrm{~K}$ $\left(68^{\circ} \mathrm{F}\right)$ with $1960 \mathrm{ppm}$ boron.

The comparison of the three-dimensional results with the onedimensional indicates the maximum difference exists at the point where the reactivity change with rod movement is greatest (the peak of the differential reactivity curve). This maximum difference is $0.65 \%(\$ 0.903)$.

The agreement between the one-dimensional calculations and the three-dimensional flux synthesis calculation is considered good. The differential and integral rod cluster worths computed with the onedimensional method are adequate for the low-power physics tests and rod ejection analysis. 
(1) W. R. Cadwel1, et. al. , PDO-7 Reference Manual, WAPD-TM-698 (January, 1963).

(2) G. E. Putnam, MONA - A Multigroup One-Dimensional Neutronics Analys is Code, ANCR-1051 (March 1972). 


\section{TABLE I}

\section{CONTROL ROD CLUSTER WORTHS}

\begin{tabular}{lccc}
$579.8 \mathrm{~K}\left(584^{\circ} \mathrm{F}\right)-1232 \mathrm{ppm}$ Boron & $\lambda$ & $\Delta \rho$ \\
\hline A11 rod clusters out of core & 1.02033 & - \\
One rod cluster in core, three out & 0.99257 & 0.02741 \\
Two opposite rod clusters in core, two out & 0.95396 & 0.06819 \\
Three rod clusters in core, one out & 0.92227 & 0.10420 \\
A11 rod clusters in core & 0.86862 & 0.17117 \\
One rod cluster in core, three in core & 0.86862 & 0.06697
\end{tabular}

$291.5 \mathrm{~K}\left(68^{\circ} \mathrm{F}\right)-1960 \mathrm{ppm}$ Boron

All rod clusters out of core 1.00181 --

One rod cluster in core, three out

$0.98288 \quad 0.01923$

Two rod clusters in core, two out

$0.95622 \quad 0.047589$

Three rod clusters in core, one out

0.93403

0.072432

All rod clusters in core

0.89601

0.11786

One rod cluster in core, three in core

0.89602

0.045426 
REACTIVITY TRANSIENT FOLLOWING SHUTDOWN

AND ON RECOVERY AT FULL POWER IN LOFT-I

B. L. Rushton

The reactivity transient due to fission product poisoning following shutdown and the reactivity transient due to xenon absorption on recovery at full power ( $55 \mathrm{MW}$ ) were required to provide input to the LOFT Technical Specifications.

The calculations were performed using the $\mathrm{PDQ}-f^{(1)}$ diffusion-theory code in XY geometry. The core was depleted for 100 hours at a power level of $55 \mathrm{MW}$ and a zero power level was then assumed. Fission product poisoning reached the maximum 8.5 hours later, and the initial concentration was attained approximately 28 hours after shutdown. The reactivity loss due to maximum poisoning was 3.1 percent.

The maximum rate that reactivity increases due to xenon burnup on recovery was computed by assuming a 55-MW power level with the maximum xenon concentration ( 8.5 hours after shutdown). The maximum slope or rate of reactivity increase was approximately $\$ .04$ per minute. The mechanisms for following the reactivity increase are control rod insertion and/or boron addition to the reactor coolant, and the inherent overall temperature coefficient (as power increases). The reactivity transients are shown on Figure 1.

LOFT can recover from a scram at any time after shutdown including the peak fission product poisoning that occurs 8.5 hours after scram. On recovery, the reactivity increase due to xenon burnup can be followed with the control mechanisms.

(1) W. R. Cadwe11, et al., PDQ-7 Reference Manual, WAPD-698 (January 1967.) 


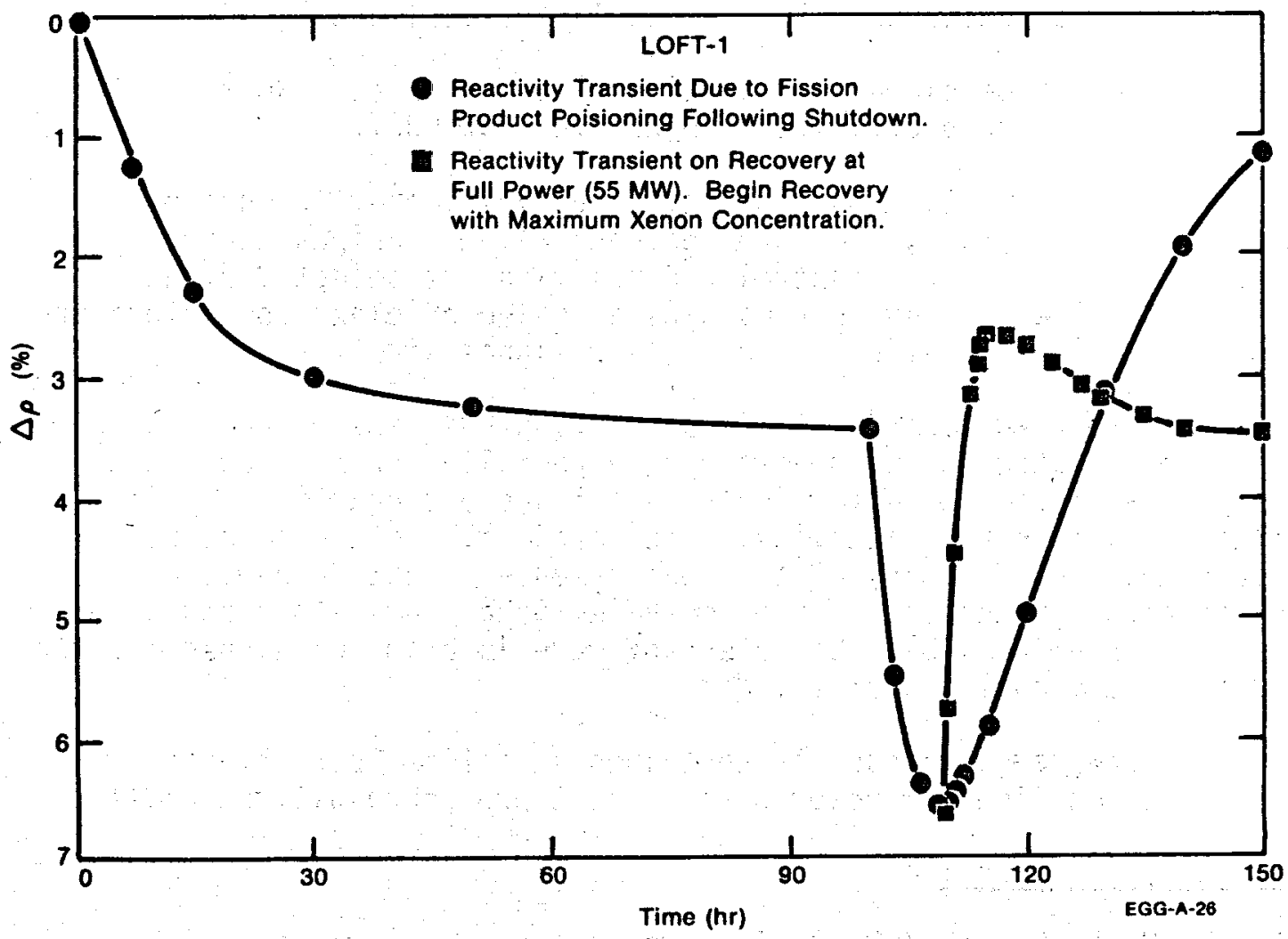

Figure 1. Reactivity transient after scram and on recovery at fu11 power (55 MW). 


\section{LOFT LOW-POWER PHYSICS PROGRAM}

W. M. Yarbrough, R. J. Talbert, D. R. Chick

A low-power physics testing program has been developed for the Loss-0f-Fluid Test (LOFT) reactor. The objectives for this program are:

(1) To safely achieve initial criticality of the LOFT reactor;

(2) To measure zero-power reactor physics parameters to support confirmation of the design bases;

(3) To obtain zero-power physics measurements for baseline information to support the requalification of the LOFT core after subsequent loss-of-coolant experiments (LOCE).

The program was developed within the framework of the NRC Regulatory Guide 1.68 and the programmatic objectives for LOFT.

The measurements will include: reactivity coefficients of temperature, pressure, and boron concentration; various control rod sensitivities; critical control rod positions at selected plant conditions; reactor shutdown margins at selected plant conditions and control rod configurations; and nuclear signal signatures.

This testing program utilizes existing LOFT instrumentation and additional instrumentation developed especially for this application. The special instrumentation includes a Transient Reactivity Meter which monitors core reactivity. The meter will be the principal instrument used for many of the measurements. Most data will be collected and processed on the installed Data Acquisition and Visual Display System (DAVDS). Computer software is being developed for the reduction and analysis of the data.

The program developed is contained in a Test Specification( $(i)$ from which the detailed low-power physics test procedure will be written.

(1) W. M. Yarbrough \& R. J. Talbert, internal report. 
POWER DISTRIBUTION CALCULATIONS TO SUPPORT LOFT-I

LOW-POWER REACTOR PHYSICS MEASUREMENTS

E. F. Aber

Power distributions were calculated in support of the LOFT-I lowpower reactor physics measurements. During control rod worth measurements, severe skewing of the control rod configuration is expected; therefore, predictions of power distributions for cases where large flux tilts occur were required by LOFT support groups for subsequent safety analysis.

Control-rod configurations and core temperatures were selected to provide a safety analysis envelope. The calculations were performed using the XYZ synthes is in PDQ-7. In the envelope studied, the most severe flux tilting occurred for the case of a dropped-rod' (with three rods remaining balanced at $711 \mathrm{~mm}$ ) and $38^{\circ} \mathrm{C}$ Uniform Core Temperature. A maximum peak-to-core average power of 4.7 resulted, and this peak power was located $355 \mathrm{~mm}$ from the bottom of the core in the center assembly.

During some LOFT-I zero-power reactor physics measurements, large flux tilts will occur as a result of expected skewing of the control rod clusters. For instance, in the measurement for the worth of a single control rod cluster, the remaining clusters will compensate for the one rod moving out. This study provided power distributions for an envelope of expected control rod configurations and the data have been provided to other support groups for required safety analys is.

(1) W. R. Cadwell, et. al., PDQ-7 Reference Manual, WAPD-TM-698 (Jan. 1967). 


\section{LOFT CORE LOAD REACTIVITY SURVEILLANCE PROGRAM}

W. M. Yarbrough

The initial, or specification, stage of developing a reactivity surveillance program for loading fuel assemblies into the LOFT reactor vessel has been virtually completed. The purpose of this surveillance program is to ensure that adequate shutdown margin is maintained throughout the loading process. The surveillance will also include monitoring of count rates on the normal nuclear instrumentation (SR-1 and $(\mathrm{R}-2)$ near the end of the loading procedure.

Because of large expected changes in fuel/detector geometry as loading progresses, which could give false indications of impending criticality, it was decided to replace the conventional " $1 / M$ " method. Following the loading of each fuel assembly,* precise count rates will be obtained from each of four special in-vessel source-range detectors (CLD-1,... CLD-4), which are connected to instrumentation channels as shown in Figure 1. For each channel, selected count-rate ratios determined from measurement will be compared with corresponding ratios determined from diffusion-theory calculations performed using techniques developed especially for this application. (1)

Based on inferred values of keff and pre-specified upper 1 imits, (1) the measurement-versus-calculational information will be used to ensure the safe loading of the next fuel assembly.

Throughout the loading process, those variables that could affect shutdown reactivity margins (such as primary coolant temperature and boron concentration) will be closely monitored and controlled within pre-specified 1 imits.

The end-product of this stage of the LOFT Core Load Reacfivity Surveillance Program is a comprehensive test specification, $(2)$ from which a detailed operating procedure (DOP) will be written.

* Loading sequence of fuel assemblies is to be $3,5,6,2,8,9,4,1,7$ as numbered in Figure 1 .

(1) G. E. Putnam, internal report.

(2) W. M. Yarbrough, internal report. 


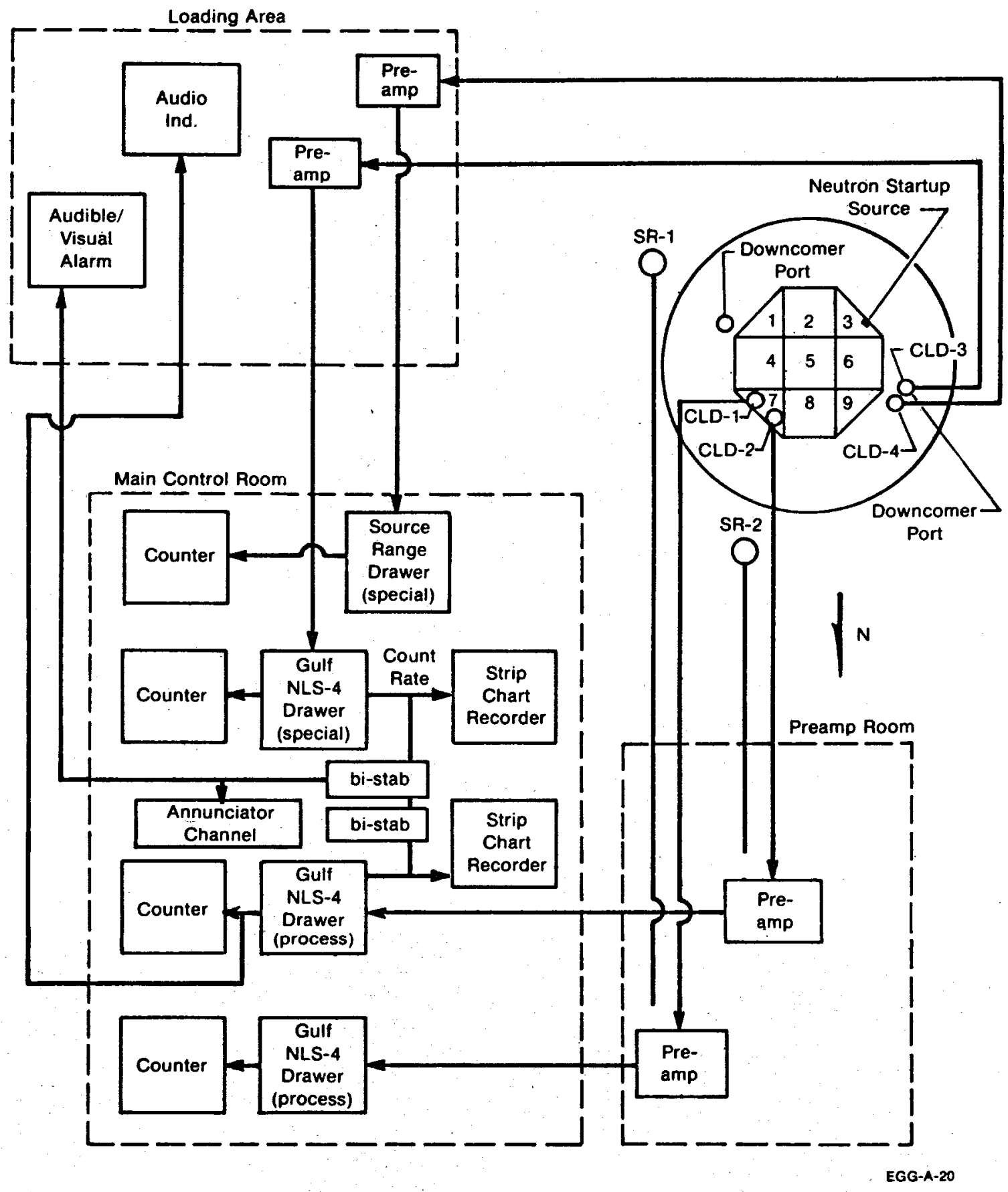

Figure 1. Instrumentation for core-load reactivity surveillance. 
BLANK PAGE 


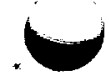

SEMISCALE 


\title{
COMPUTERIZED CORE POWER CONTROL SYSTEM
}

\author{
D. L. Rittenhouse, J. R. Venhuizen
}

A control system has been developed that drives the Semiscale electrically heated core in a manner that emulates a nuclear core. The need for this power control system can best be explained by briefly summarizing the operation of the Semiscale Facility.

Electrically heated rods are used at the Semiscale Facility as the heat source simulating uranium dioxide $\left(\mathrm{UO}_{2}\right)$ fuel rods typical of those used in light-water reactor cores. These experiments are designed to provide data for development and evaluation of analytical models that describe the thermodynamic and hydraulic behavior of the reactor coolant and core, and mechanical behavior of the structural system during blowdown in a postulated Loss-of-Coolant Accident (LOCA). The experiments are conducted to gain information on the coolant behavior in the primary system and on the rate of heat transfer to the coolant.

Since the coolant behavior is affected by the heat source used to simulate the $\mathrm{UO}_{2}$ fuel rod, the design and operation of the electrical heater rod should be such that the heater rod behaves similarly to the $\mathrm{UO}_{2}$ fuel rod. The effect of the heater rod on the coolant will be the same as that of the $\mathrm{UO}_{2}$ fuel rod if the cladding surface temperature and surface heat flux for the heater rod are the same as for the nuclear rod.

The conditions of fluid flow, pressure, enthalpy, and quality produced during a transient experiment cannot be known precisely before an experiment is conducted. Thus, the heater rod should be designed and controlled independent of the fluid conditions, thereby avoiding the need for a number of experiments followed by repeated changes in the method of operation to optimize the response of the heater rod.

To achieve the above goals, a control technique was developed that utilizes a small digital computer for on-line power control. The basic control scheme and information flow is shown in Figure 1. The digitial program gets as inputs, from analog-to-digital converters, the measured value of heater rod power, heater clad temperature, and coolant temperature. It then must calculate the heat transfer coefficient, the surface heat flux, and the temperature profile of the heater rod, then apply the calculated heat transfer coefficient to a model of a nuclear fuel rod and calculate its surface heat flux and temperature profile. The difference in heater and nuclear rod surface heat flux or surface temperature is then applied to a control algorithm, and the resulting power command is sent to the power supply via a digital-to-analog converter. The resulting power profile forces the heater rod to emulate a nuclear rod which is operating in a Semiscale environment.

The finite-element method for calculation of the temperature 
field in both the nuclear and heater rods was selected for the digital computer models. The rods may consist of an arbitrary number of cylindrical shells with or without heat generation. This heat generation can be a function of either the radius for the nuclear fuel rod, or temperature for the electrically heated rod. In the first case the radial power profile is an input; in the second case power is given.

All material properties can be temperature dependent with the exception of the gap conductance associated with the fuel rod. Heat is conducted inside the shells, and there is thermal resistance between the shells.

Since the finite-element method is used, the rods must be divided into an arbitrary number of nodes in which the whole mass is concentrated. The radial location of these nodes is specified by input data, the volumes and thermal resistances associated with each node are calculated.

The digital program, which consists of a model of the nuclear rod and heater rod in addition to the necessary control compensation, must cycle fast enough to allow stable closed-loop power control. Consequently, to keep the cycle time within bounds, the heater rod was modeled with nine nodes, and the fuel rod was modeled with seven nodes.

The control concept has been developed using the INEL HYDAC hybrid computer. The digital portion of the hybrid computer was assumed to be the control computer, while the analog portion contained a model of the Semiscale heater rod. This apportioning of equipment is very appropriate for this study because it nicely simulates the control environment (i.e., the analog and Semiscale heater rods are continuous systems, while the digital computers have a one-to-one relationship). The hybrid computer study has shown the digital control system to be stable and, when installed at the Semiscale Facility, will provide superior power control. 


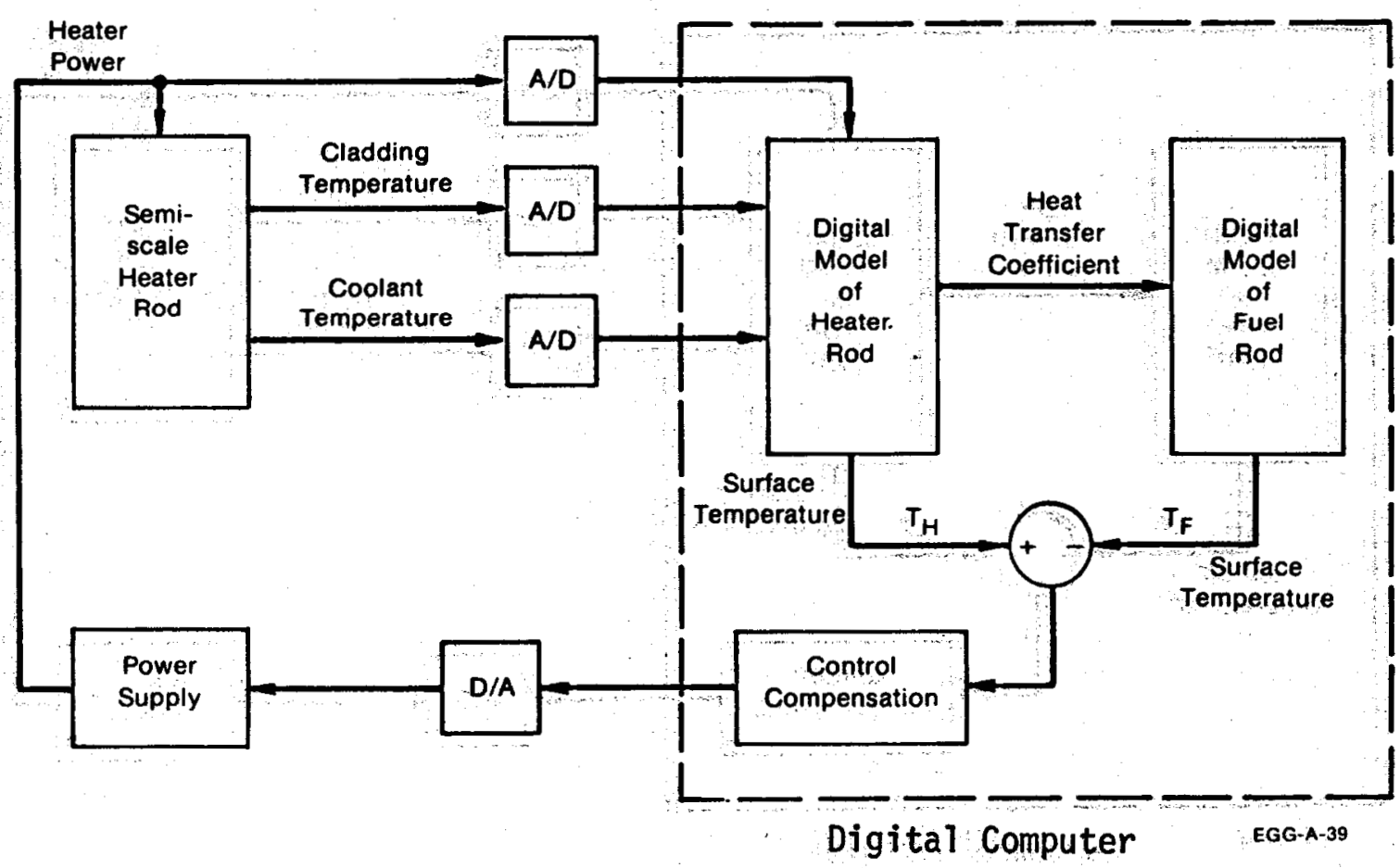

Figure 1. Semiscale core power control system. 
NON-NUCLEAR PROGRAMS 
こ

BLANK PAGE 
THE CLARENDON COMMUNITY: MIXED ENERGY APPLICATION FOR HUMAN LIVING

F. B. Simpson, C. J. Shaffer

The objective of the Clarendon Community is to approach energy independence through geothermal, solar and wind applications, as well as full utilization of energy conservation. In order to determine the wind energy potential for a typical mountain terrain, wind data are being collected in the area of the Clarendon Community.

Clarendon is in the Sawtooth National Recreation Area near Sun Valley. Information obtained from such a development could be of regional and national importance in establishing benchmark data base for a variety of energy systems. A proposal was submitted to ERDA for the INEL to give technical supervision in the development of the energy systems and monitoring the efficiencies of the different systems. 
A GUIDE FOR THE CONVERSION TO AND MAINTENANCE OF HYDROGEN-FUELED, SPARK-IGNITED ENGINES

F. B. Simpson, D. R. Swope, J. H. Lofthouse, D. H. Henriksen ${ }^{\dagger}$

In recent years there has been much research on the operation of internal combustion engines on hydrogen fuel. Many different paths have been explored in attempts to eliminate the problems found by early researchers and so devise a workable engine and fuel storage system. In the 1930 's there were many engines putting out useful work on hydrogen, either as the fuel or as a fuel supplement, in Germany and possibly elsewhere. However, due to various problems, including the destruction of Germany in World War II, hydrogen as a fuel fell into disuse. A paper has been written $(1)$ as a guide to one approach to the conversion of an internal combustion engine to hydrogen fuel. It includes knowledge gained from the authors' own experiences and from the study of the work of others. It discusses the safety aspects, conversion, operation, and maintenance of the engine. It is hoped that this guide will be useful to those beginning work in the field of hydrogen engines, and that it might suggest areas for further research.

When making a choice of engines to convert to hydrogen fuel, one should carefully consider the characteristics of hydrogen. Four of these characteristics which are probably most important to the choice of an engine are (1) low ignition energy, (2) high ignition temperature, (3) significant displacement of air by hydrogen fuel, and (4) high heating value (see Table 1). The first characteristic manifests itself in a tendency for pre-ignition and backflashing, but may be partially compensated by the second. The third characteristic results in reduced power which is partially balanced by the increased power due to the fourth characteristic.

TABLE I

COMPARISON OF HYDROGEN WITH ISO-OCTANE

\begin{tabular}{lcc}
\hline & ISO-OCTANE & HYDROGEN \\
Ignition Energy $\times 10^{-5}$ Joule & 135 & 2.0 \\
Ignition Temperature ${ }^{\circ} \mathrm{C}$ & 447 & 610 \\
Volume Air & 1.00 & 0.70 \\
$\begin{array}{l}\text { Volume (air + fuel)* } \\
\text { Lower Heating Value } \\
\text { J/g } \\
\text { (Btu/lbm) }\end{array}$ & 44261 \\
*Stoichiometric Mixture & $(19029)$ & 120010 \\
\hline
\end{tabular}

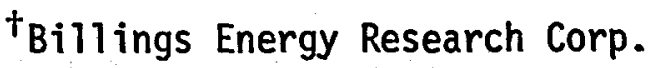


The surface and materials of the combustion chamber, the compression ratio, and the valve timing influence the power and efficiency of an engine and its tendencies for preignition, backflashing, and emission of NOx. It is possible to exercise some control of these tendencies by modifying appropriate features of an engine. However, any gains in one parameter must be balanced against losses in others, the cost of the modification, and alternative methods of achieving the same gain.

(1) F. B. Simpson, D. R. Swope, J. H. Lofthouse, D. L. Henriksen A Guide for the Conversion to and Maintenance of Hydrogen Fueled Spark Ignited Engines, Eleventh Intersociety Energy Conversion Engineering Conference, State Line, Nevada (Sept. 1976). 
MODIFICATION TECHNIQUES AND PERFORMANCE CHARACTERISTICS OF HYDROGEN-POWERED IC ENGINES

F. B. Simpson, J. H. Lofthouse, D. R. Swope and R. L. Wooley*

Over the past quarter of a century there has been a significant amount of work on modification of internal combustion (IC) engines to operate on hydrogen. However, comparisons are difficult because of large variations in designs, engine parameters, and test conditions. A paper has been written (1) which gives a comparative summary of the recent work on hydrogen-powered engines. This includes material extracted from published reports and obtained by private communication. It is intended that this summary be of value to those beginning work in the field, and will provide some guidance in determining the direction of future research. The report includes information on: engine parameters, modifications, operating conditions, running characteristics, performance, and related subjects. More detailed discussion is included on the manpower and material costs associated with the modification of one of the engines listed.

The following are a few conclusions and recommendations arrived at from this survey:

(1) Several good demonstrations involving conversion of modern automobiles to operate on hydrogen engines and actual vehicle road tests have been accomplished. These demonstrations show, in general, that hydrogen-air systems can be designed which easily meet government pollution emission standards. Safety can be readily designed into automobile systems, especially with use of metal-hydride storage tanks. There is a substantial increase in thermal efficiency with hydrogen fuel. Some designs have about one-third less power.

(2) There is a need for improved documentation and publication of test results as well as a lack of some important measurements. Whenever possible data should include curves of power and torque as a function of engine RPM for different fuels. This would enable engineers to make choices for specific applications.

(3) Specialty carburetors using "computer" control would be advantageous for variable mixture designs.

(4) Very little long-term endurance testing has been done on hydrogen engines. Hydrogen is so clean burning that conversion of new ergines is usually preceded by a break-in period on gasoline which produces enough abrasive action to "seat" piston rings and close-tolerance engine parts. However, questions arise about hydriding of engine parts under normal and extreme operating conditions over the lifetime of the engine. If hydriding effects are small, engine life may be extended considerably, resulting in large national resource savings in steel and lubrication oil. This should be a high priority effort.

* Billings Energy Research Corporation (BERC). 
(5) The authors feel that the state-of-the-art is advanced to the point where fleet demonstrations should be initiated. This demonstration should include an efficient production system, refueling, handling, bulk storage system; and a sufficiently large fleet operation within a limited area to produce comparative overall system efficiency. The existence of government fleets at national laboratories presents a good opportunity for the federal government to fund such a demonstration with participation by industrial research organizations.

(6) High quality system modeling using computer simulation is warranted to study parametric tradeoffs and establish the relative importance of various reasons for efficiency improvement and peak temperature $\left(\mathrm{NO}{ }_{\mathrm{X}}\right)$ reduction.

(7) The metal-hydride form of fuel storage in conjunction with an internal combustion engine shows definite advantages in performance (acceleration and range), weight, total cost, and refueling time when compared to electric vehicles with battery storage $(2,3,4)$. Furthermore, if the production and distribution of hydrogen or electricity is taken into consideration, as it should be, the advantage electric vehicles may have in emissions and overall efficiency may be significantly reduced. An operational comparison between two urban vehicles with identical payload is warranted.

(1) F. B. Simpson, J. H. Lofthouse, D. R. Swope, R. L. Woolley, Modification Techniques and Performance Characteristics of HydrogenBowered IC Engines--State of the Art, 1975, 1st World Hydrogen Energy Conference, Miami Beach, Florida (March 1976).

(2) Metal Hydrides as a Source of Fuel for Vehicle Propulsion, SAE Publication No. 690232, (1969).

(3) Daniel L. Cummings, Gary L.. Powers, "Storage of Hydrogen as Metal Hydrides", Ind. Eng. Chem. Des. Develop., 13, No. 2, (1974).

(4) Herbert Shuldiner, "Battery Powered Cars", Popular Science, (June 1975) 61. 


\title{
ENERGY SYSTEMS NETWORK SIMULATOR
}

\author{
J. A. Close, J. R. Johnson
}

The Energy System Network Simulator (ESNS) is being made available for INEL users. Developed at Brookhaven National Laboratory, ESNS is quite useful for scoping studies which compare alternative energy systems and perturbed cases of a given energy system.

ESNS assembles information taken from its own data file using "energy trajectories". An "energy trajectory" is a series of processes that takes an energy resource and converts it into a form that satisfies a particular end use. For example, a quantity of coal may be extracted from the ground via strip-mining, be cleaned and processed, transported via railroad, stored, burned in a steam-electric power plant, the electricity transmitted and distributed into homes to power color television sets. This is an energy trajectory. The information ESNS displays includes efficiency, costs, energy use, occupational deaths and injuries, land use and pollutants per end use energy demand. One trajectory or an entire network with several end uses and several energy resources may be analyzed. The network can be as large as desired, even encompassing the entire U.S. energy supply and demand system. The total energy demanded for each end use may be input by the programmer or called from the ESNS file, which contains historical and projected demands for each end use.

Energy supply resources available in the data file include coal, oil, natural gas, nuclear fission and hydro-electric. Data for other resources such as solar and geothermal, and new technologies such as synfuels from coal which are not yet available in the data file may be included by the user. The Energy Model Data Base, also developed at Brookhaven, is available and contains the data required to include these latter resources and technologies. 


\title{
THE USE OF RELEVANCE TREES IN GEOTHERPAL PLANNING
}

\author{
J. A. Close, J. C. Shepherd, I. E. Stepan
}

INEL has been asked by ERDA's Division of Geotherma1 Energy (DGE) to assist its national and regional planning contractors in developing relevance trees to aid planning for geothermal development. These relevance trees provide a graphic display of all elements of the geothermal program including the other agencies involved. Further, these trees will provide for a systematic evaluation of alternative courses of action by identifying the potential impact of each program on the national goals.

Relevance trees are of two types: descriptive and quantitative. The descriptive version shows interaction among its components and assists in identifying areas where technology transfer is possible. The quantitative version provides a measure of the relative value of each component. The construction of these trees js best described by combining the comments of Martino(1) and Gordon $(2)$ :

Relevance trees are used to describe and analyze systems or processes in which distinct levels of "causation", complexity, or hierarchy can be identified. They are developed by carrying out successive identification of increasingly finer components at progressively lower levels.

At each level in the structure, mutually exclusive elements are displayed which, in the aggregate, completely define the next higher level.

To demonstrate this technique, a simple tree is presented. Figure 1 shows a three-level tree which is used to determine what factors influence geothermal energy use. The individual components of each level are obtained by answering specific questions about that level. The questions used for this tree are:

Level I - What is the potential for geothermal energy use?

Level II - What industries are potential users of geothermal energy?

Level III - What influences industries in their choice of energy sources?

There is a logic requirement that all elements on a given level be mutually exclusive and form a complete or closed set. The completeness requirement is met by either an exhaustive list or by definition. The latter approach is more common, but it assumes that those items. which are omitted are insignificant and would not alter the relative value of the others. To acknowledge this prerequisite, the block "all others" is included on Levels II and III.

To use a tree for an evaluation tool, the contribution of each element on a level to a specific element on the level above is quantified by use of criteria. That is, each element is "graded" against some predetermined standard. These scores are then normalized to unity at each level; the product of the normalized values along, a path 
gives the relative contribution of the lower element to the upper. A more complete description of construction of quantitative relevance trees is given in References 1 and 3.

The relevance trees being developed for DGE have several themes: organizational, resource characterization, programatic and regional structure. These themes are briefly illustrated in Figure 2, which also shows the levels of inquiry used to develop the trees. Notice that the Organizational, Regional and Programmatic Trees have a commonality at the "problems, needs, and deficiencies" level; the items on this level are the same in each tree but they are aggregated differently. This feature allows a user of the trees to extract a great deal of information from them with relative ease as shown in the following example:

Suppose a DGE manager is considering a specific problem for a given sub-region of the country. The Regional Tree will provide him with information about other sub-regions having the same problem. By transferring to the Programmatic Tree at the "problem" level, he can determine what projects are under way or are being considered to alleviate the problem. He can also determine what the status of complementary projects must be before the projects addressing his problem can be completed. If the manager would like to know who is involved in this particular problem and how, he needs only find the problem on the Organizational Tree. All participants and how they interact are listed there. This includes those aggravating the problem as well as those trying to relieve it. Returning to the Programmatic Tree and using it in conjunction with the Resource Characterization Tree, the manager can determine how resolution of the problem can affect power on line.

The Resource Characterization Tree provides a structure for aggregating many reservoirs having common characteristics into "reservoir types." This tree is used to determine the energy content available from each reservoir type. Applying this information and the results of a study by the national planning contractor on the number of reserviors available, the manager can determine how much energy can potentially be brought "on line" by solving the problem.

Two other trees are also contemplated: one will identify the components of a utility executives decision-making process and the relative importance of each from the utility executives perspective. The other will identify the cost components of a fluid suppliers price and the importance of each component from the supplier's viewpoint.

(1) Joseph P. Martino, Technological Forecasting for Decision Making, American Elseview Publishing Company, Inc., New York, (1972). Chapter 9.

(2) T. J. Gordon, et. a1., "A Technology Assessment of Geothermal. Energy Resource Development" prepared for Nationa'l Science Foundation by the Futures Group, April 15, 1975, NSF-RA-X-75-011. 
(3) James R. Bright, A Brief Introduction to Technology Forecasting, The Permaquid Press, Austin, 'Texas, (1974) Chapter 7. 


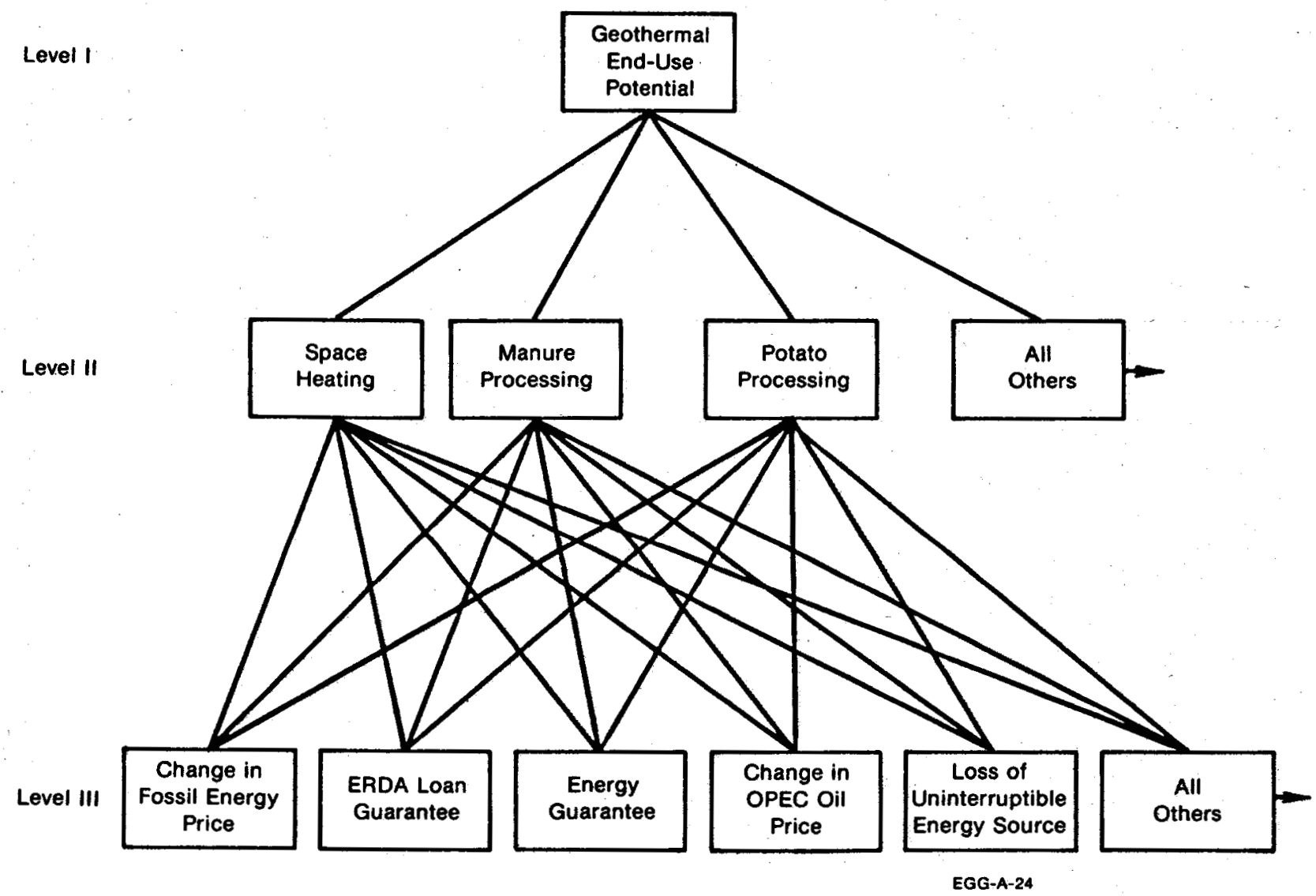

Figure 1. Example of a simple descriptive relevance tree. 


\section{Organizational Theme}

Level

1. What are the participant categories?

II. What are the participant sub-categories?

III. Who are the participants having authority over, responsibility to, or exerting influence on other participants?

IV. What modes of interaction occur between the participants in Level III and Level V?

v. Who are the participants subject to the authority, responsibility, or influence of the participants of Level III?

VI. What are the problems of geothermal development with which the above participants (Level V) are associated?

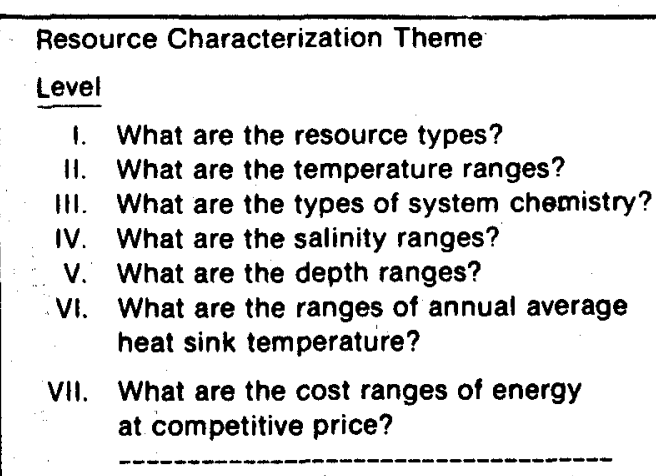

A path traced through an element on each level in the above tree down to Level VII characterizes a reservoir type.

\section{Programmatic Theme}

Level

1. What parameters affect power on line?

II. What are specific problems, needs, and deficiencies affecting the above parameters?

III. What are the projects (completed, existing, proposed, and potential) addressing the above problems, etc.?

IV. What are the relationships with other projects?

V. What are the projects related to those on Level III?

\section{Regional Theme}

Level

1. What are the regions?

II. What are the sub-regions?

III. What are the problems needs, and deficiencies for each sub-region?

Figure 2. Schematic representation of four relevance trees showing the inter-relationship between them. 
WIND-AUGMENTED GEOTHERMAL POWER PLANT CONCEPTUAL STUDY

C. J. Shaffer

An analytical evaluation was performed for the geothermal-wind hybrid concept. The potential power of the concept comes from three basic potentials: (1) expansion of pressurized geothermal brine by a Hero engine, (2) wind using a vortex wind-energy system, and (3) a hydrostatic pressure differential created by a heated air-vapor column which encloses the Hero engine and by heating the tower air-flow. The condjtjons of the evaluation assumed $0.075 \mathrm{~m}^{3} / \mathrm{s}(1200-\mathrm{gpm})$ of $422^{\circ} \mathrm{K}$ $\left(300^{\circ} \mathrm{F}\right)$ geotherma 1 brine and an effective wind velocity of $6.7 \mathrm{~m} / \mathrm{s}$ (15 mph).

The vortex wind-energy system consists of a stationary tower with vertical vanes which open on the windward side and close on the leeward side to form a vortex in the tower. A low pressure is created at the core which draws air up through the bottom driving a turbine. The vortex flows vertically out of the top of the tower with the core still at reduced pressure. As the vortex breaks up after exiting, the low pressure is built back up by the slowing down of the free air stream.

The results of this brief study are:

(1) The vortex wind-energy system could be one of the most viable wind concepts today because of its ability to convert pressure to kinetic energy and use it.

(2) The Hero engine concept is highly efficient under ideal conditions, but the actual use of such an engine will require further data and design into the material stress, flow separation, drag, and corrosion areas.

(3) The hydrostatic-head power cycle, which is the mainstay of this geothermal augumentation concept, is a highly inefficient cycle requiring large, high towers. 
TRANSIENT MODELING AND SIMULATION OF THE GEOTHERMAL ELECTRIC PLANT

J. D. Miller

A transient model of the Raft River Geothermal Electric Plant has been developed for parellel implementation on digital and hybrid computers. The simulation is to be used for systems analysis work supporting preliminary design and procurement activities.

The mathematical model is shown schematically in Figure 1 . The transient thermo-hydraulic behavior of the brine, working fluid (isobutane) and coolant (water) loops is represented with emphasis on the thermal response to atmospheric and controller variations and the anticipated class of malfunctions.

The brine loop is shown on the right side of the figure. The boost pump raises the pressure and temperature from the boundary conditions at node 52 to the bypass tee at node 53 . The brine normally remains subcooled as it proceeds to the boundary pressure at node 66 through both the bypass and the heat exchanger tube (high pressure boiler, high pressure preheater, low pressure boiler, low pressure preheater) paths.

The isobutane flow, beginning at node 9, is normally subcooled as it passes through the low-pressure preheater to node 17A where it splits into high- and low-pressure side flows. The high-pressure flow proceeds to the left to node 20, either directly or though the optional pump. After further subcooled preheating, it enters the high-pressure boiler where a thermal equilibrium between buoyancy separated saturated liquid and saturated vapor phases is assumed to exist. The saturated vapor flows to node 27 and then through either of two al ternative components: a turbine stage or a throttling valve. The 1 ow-pressure side flow leaves node $17 \mathrm{~A}$ and is throttled if there is no high-pressure pump between $17 \mathrm{~A}$ and 20 , i.e., the entire pressure rise is accomplished in the pump between nodes 8 and 9. Flow then proceeds through the low-pressure boiler and turbine or throttling valve, mixes with the high-pressure flow and proceeds to the condenser. The initial portion of the condenser provides desuperheating to saturation. The remainder of the condenser is assumed to contain saturated liquid and saturated vapor phases in thermal equilibrium. Saturated liquid is stored in the hot well at node 6 and then pumped to either of two pressure levels depending on the use of a high-pressure pump between nodes $17 \mathrm{~A}$ and 20 .

Subcooled cooling water proceeds from node 49 , through the initial pass of condenser tubes and then through both second-pass condenser and desuperheater tubes and through the spray nozzles to node 43 at the top of the cooling tower. A portion of the cooling water is evaporated; the balance, together with the makeup water, enters the cooling tower sump and then moves through two parallel pumps.

Heat exchange from the center of each tube wall node to each of 
the fluids incorporates a film coefficient for the appropriate fluid phase, a fouling resistance and a tube wall conductivity. Cooling tower heat exchange is based on the assumption that the leaving air is saturated and that the water-to-air overall heat transfer coefficient remains constant at the design value.

The simulation accepts variable input pump and turbine speeds and valve positions and brine and cooling tower air boundary conditions. Closed-loop controllers will be developed and incorporated.

The digital implementation using the Time and Frequency (TAF) simulation is currently being debugged. A parallel hybrid computer implementation will be programmed at the Martin-Marietta Corporation, Denver Data Center, as the primary computation tool. The hybrid computer will be operated from the INEL Hybrid Computer Laboratory using a graphics terminal, a strip-chart recorder and a voice link. The TAF code will be used as a check and to provide a steady-state mapping, frequency response and model change study capability. 


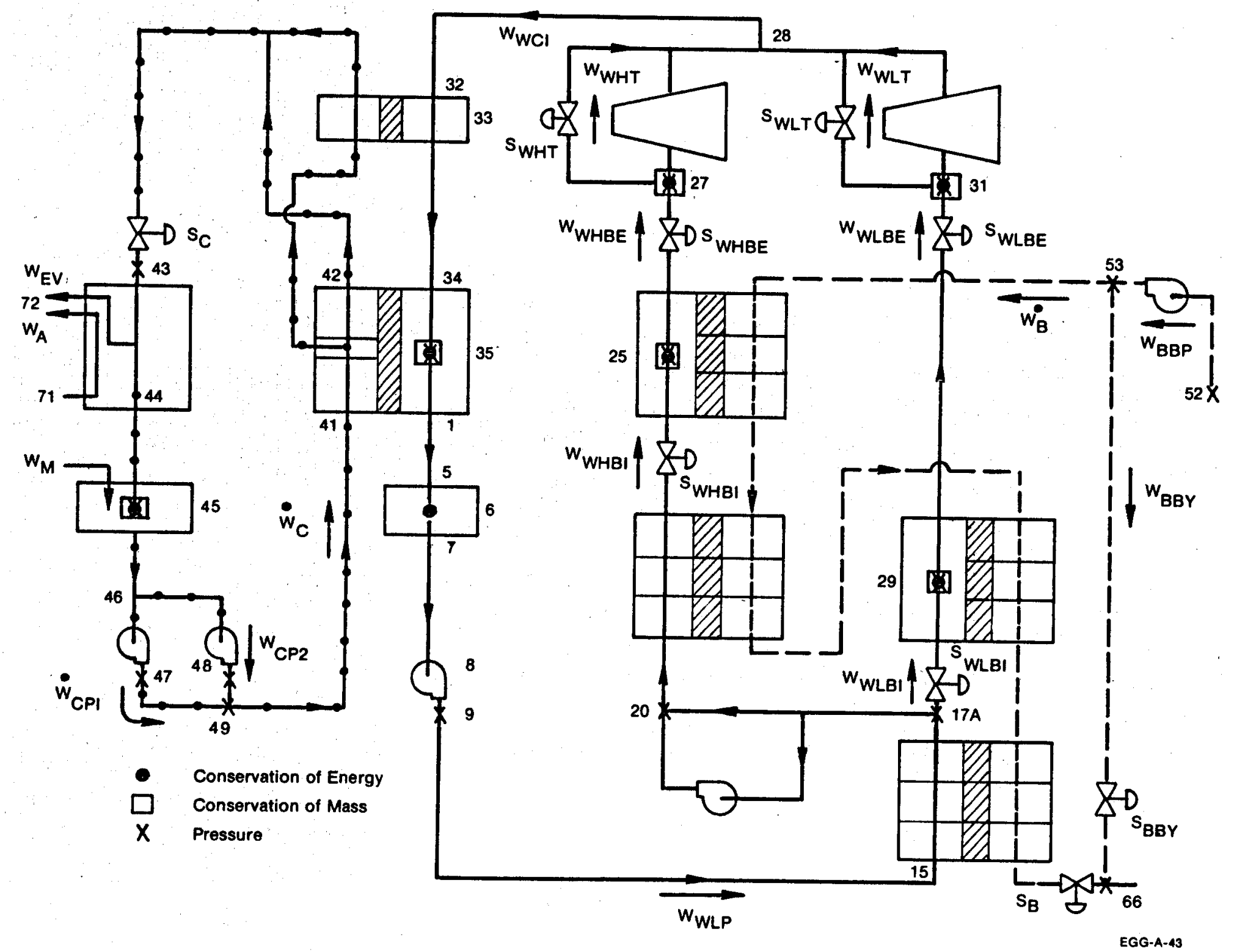

Figure 1. Geothermal electric transient model. 
$\mathcal{U}$

$\smile$ 
GEOTHERMAL STEAM PLANT MODELING AND POWER

TRADE-OFF STUDIES

C. J. Shaffer

Geothermal steam plant modeling and power trade-off studies were finished and the final report published completing this analytical support for the geothermal project. This model will provide calculational capability to plant designers and provide insight as to the more sensitive and significant parameters.

The model contains the flashers (up to 4 stages), pre-flasher, turbine, condensers, air ejectors, cooling tower, and pumps. The model is capable of tracking nitrogen and carbon-dioxide (which are initially dissolved in the geothermal brine) throughout the plant in both the dissolved and free states. It then computes the steam flow necessary to drive the air ejectors to remove the gases from the condensers. The model also computes the significant frictional pressure drops throughout the plant piping. The model has been programmed for the IBM- 360 and is fully operational.

Sensitivity studies were performed on significant parameters such as the initial dissolved gas concentrations, air ejector effectiveness, condenser pressure, brine temperature, number of flashes, flash pressures, relative humidity and atmospheric air temperature. The results are correlated according to the brine flow rate necessary to generate $10 \mathrm{MW}$ net electricity. These sensitivity studies shed light on power trade-off options such as the optimum number of flash stages, uses of a pre-flasher, and condenser pressure which will be useful in an actual plant design.

(1) C. J. Shaffer, Geothermal Steam Plant Modeling and Power Tradeoff Studies, ANCR-1295 (January 1976). 
GEOTHERMAL FLOATING POWER PLANT

AND PHASE COOLING STUDIES

C. J. Shaffer

Analytical support was supplied to the geothermal project for floating power plant and phase cooling studies. The floating power plant is a concept wherein the plant power varies as the changing atmospheric conditions reduce or increase the cooling tower effectiveness. The term, phase cooling, refers to the storage of cold cooling water from times of good cooling conditions to times of poor conditions in order to reduce the size of the power fluctuations of a floating power plant.

The analytical model includes (1) a power plant model to compute the net electrical power output as a function of the atmospheric conditions, (2) a source of atmospheric data and (3) a statistical model to evaluate the plant performance over a given period of time. The power plant model simulates a dual boiling isobutane Rankine cycle geothermal plant which produces 10 MW net electrical power from $300^{\circ} \mathrm{F}$ geothermal brine with an atmospheric wet bulb temperature of 650F. The atmospheric surface observations for the Pocatello airport were obtained from NOAA's National Climatic Center in Asheville, N.C. and used as a source of data.

Preliminary results of the floating power plant and phase cooling studies show an increase in yearly averaged net power of the order of $25 \%$ to $30 \%$ by allowing the power plant output to float with atmospheric conditions. 
DEVELOPMENT OF AN EVALUATION METHODOLOGY FOR THE

GEOTHERMAL LOAN GUARANTY PROGRAM

\section{J. A. Close, J. C. Shepherd}

The Geothermal Loan Guaranty Program was conceived by ERDA to accelerate the utilization of geothermal energy by minimizing the financial risk to lenders associated with underwriting new geothermal resource technology. INEL has begun developing a methodology for evaluating loan guaranty applications for non-electric geothermal projects. The evaluation categories have been broken down into five areas: a) financial, b) environmental, c)technical, d) marketing/management, and e) legal. Thus far, emphasis has been placed on the technical evaluation al though some preiliminary work has been done with the other evaluations.

Each evaluation is divided into two parts: the first is a rough investigation to determine if the loan applicant's project meets certain minimum requirements, and the second part involves a figure-of-merit analysis for the project. Figure 1 shows some of the considerations which are a part of the figure-of-merit analys is for the technical evaluation.

In addition to the methodology development, INEL will perform the technical evaluations as applications are received. 


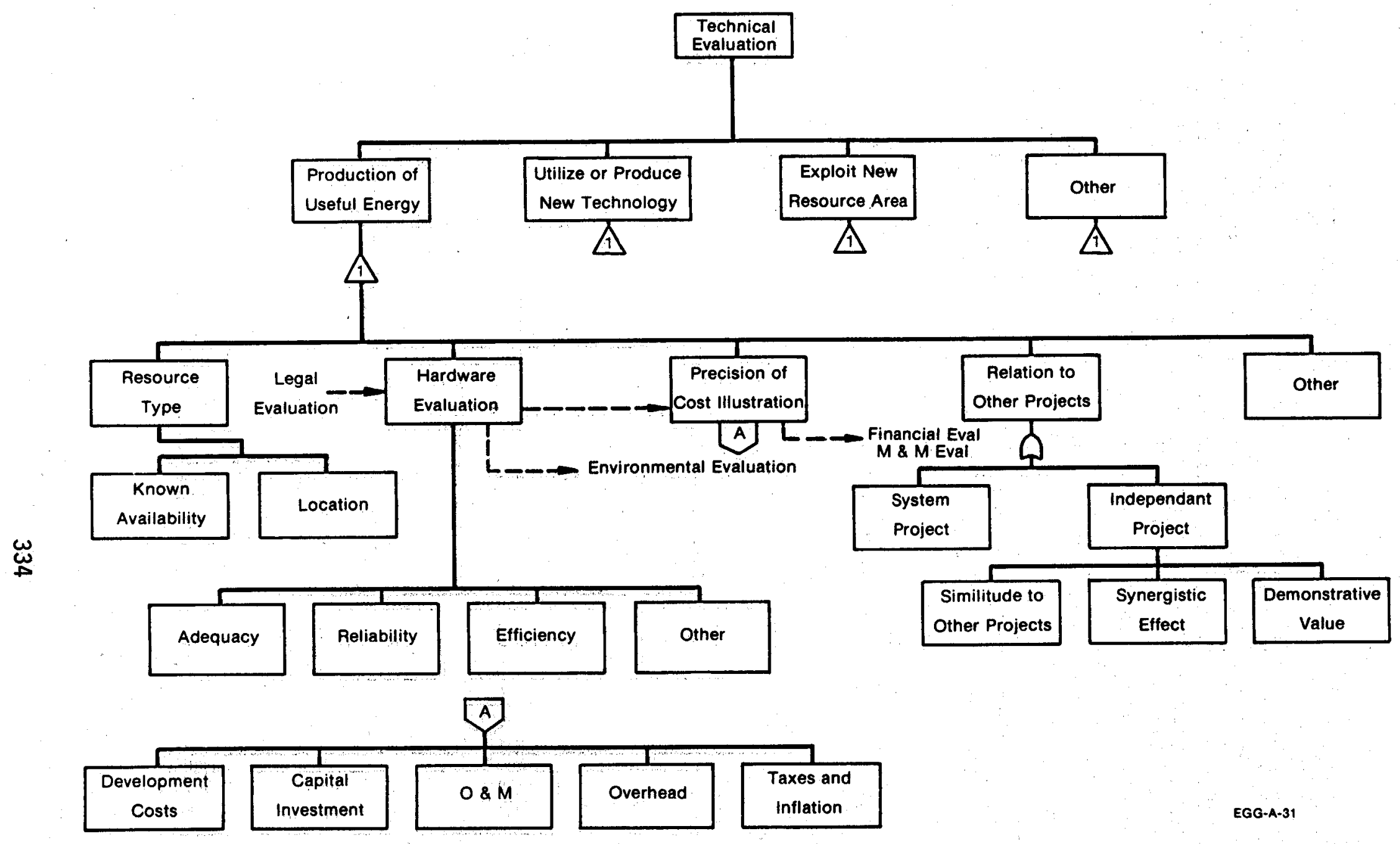

Figure 1. Components of the figure of merit analysis for the technical evaluation. 


\section{J. Shaffer}

An evaluation for solar systems application in new and existing buildings at the INEL was done at the request of the Architect Engineering and Construction Division. This study evaluates the economje viability of solar heating at INEL for both $130^{\circ} \mathrm{F}$ (54.40 $\mathrm{C}$ ) and $180^{\circ} \mathrm{F}\left(82.2^{\circ} \mathrm{C}\right)$ hot water over a 25 -year life-cycle period. A proposed laundry would use both $130^{\circ} \mathrm{F}(75 \%)$ and $180^{\circ} \mathrm{F}(25 \%)$ hot water in the laundering. Several buildings at three different areas of the INEL site would use $130^{\circ} \mathrm{F}\left(54.4^{\circ} \mathrm{C}\right)$ water for space heating. The $130^{\circ} \mathrm{F}$ $\left(54.4^{\circ} \mathrm{C}\right)$ energy requirement peaks in the winter and drops to near zero in the summer, whereas the $180^{\circ} \mathrm{F}\left(82.2^{\circ} \mathrm{C}\right)$ energy requirement is constant throughout the year.

The solar energy costs have been related to an equivalent cost of energy at today's prices in terms of dollars per 1000 cubic feet of gas. The results show the cost sensitivity to the discount rate, energy growth rate, and percent of the total annual energy requirement provided by solar heating. The assumptions of this analysis are:

(1) An estimated solar panel system cost of $\$ 20$ per square feet. ( $0 \& M$ costs are not considered).

(2) Gas-fired steam plant efficiency with piping distribution of $60 \%$.

(3) Available annual solar insolation of $658,000 \mathrm{Btu} / \mathrm{ft}^{2}$-year $\left(7.47 \times 10^{5} \mathrm{~J} / \mathrm{m}^{2}\right.$-year). Based upon horizontal monthly solar insolation data for nearby Pocatel10, Idaho converted to a tilted collector sloped with an angle of latitude plus $10^{\circ}$.

(4) A solar panel system conversion efficjency from solar insolation to useful heat of $50 \%$ and $30 \%$ for $130 \%\left(54.4^{\circ} \mathrm{C}\right)$ and $180^{\circ} \mathrm{F}$ $\left(82.2^{\mathrm{C}}\right)$ hot water, respectively.

This cursory cost benefit analys is shows, with the current cost of gas of $\$ 2.40 / 1000$ cubic feet, that solar heating could be competitive. For example, the break-even points (assuming an $8 \%$ discount rate and a $10 \%$ energy growth rate) are $85 \%$ and $98 \%$ of the total yearly energy requirements for the $130(54.4)$ and $180^{\circ} \mathrm{F}\left(82.2^{\circ} \mathrm{C}\right)$ hot water temperatures, respectively. 
A LOOK AT THE STATUS

OF THE TOPOLOTRON PROJECT AND INEL'S ROLE

R. C. Young, E. H. Ottewitte

The Topolotron project is an effort to apply some recent

mathematical developments to the problem of magnetic confinement for fusion power generation. Despite intense national efforts for many years, the magnetic confinement of a high-temperature plasma has remained an elusive goal. The toroid shape is desirable for magnetic confinement because it permits a closed path for flow of the plasma with no ends causing loss of the plasma. However, uncontrollable instability has accompanied the toroidal experiments with very rapid loss of the plasma to the sides. The toroid is unfamiliar enough that intuition soon fails to be useful in understanding systems with toroidal geometry. When the complications of the vector magnetic fields are added to the odd behavior of a plasma, it is clear that very powerful mathematical techniques are required to provide any real understanding of the situation.

Some advances in the topology field of mathematics $(1,2)$, in particular the specialty of catastrophe theory, were noticed by professors at the Brigham Young University. The new work appeared to have application to magnetic confinement of a plasma in a toroidal shape. (Note that catastrophe theory here refers not to disasters, but to sudden changes in the response of a system which is normally continuous.) Further study and extension of the mathematics seemed to suggest that a plasma configuration could be specified that would avoid some of the more severe instabilities that limit all the present. toroidal experiments for magnetic confinement of plasmas.

With a hint from the new theory, a group was formed at the University for further study, and an experimental device was proposed and named Topolotron. The design was refined from numerous calculations and preliminary experiments, and construction begun early in: 1976 for a small hydrogen experiment (SHX). Meanwhile, an analytical effort to investigate the stability of the Topolotron continues to be encouraging. Through the summer of 1976, the group was enthusiastic and pressing forward, making extensive use of Scyllac technology from LASL. However, their funds are very limited, and they have not yet been able to obtain any funding from ERDA. Some limited analytical. support has been provided by INEL through a smal1 participation in the stability analysis.

It was recommended that INEL increase substantially its particjpation in this nearby project. Experts in the field consider the concept promising, and this appears to be a situation where the risk of a small amount of resources might provide great return.

(1) R. Thom, Structural Stability and Morphogenesis (W. A. Benjamin: Reading, Mass., 1975; French edition 1972).

(2) M. M. Peixoto, "Structural Stability on Two-dimensional Manifolds", Topology, 1 (1962) 101-120. 


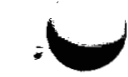

WASTE MANAGEMENT 


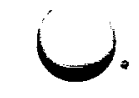

\section{BLANK PAGE}


TECHNICAL FEASIBILITY STUDIES OF BULK SOIL MONITORING FOR TRANSURANIC CONTAMINATION AT $10 \mathrm{nCi} / \mathrm{g}$

J. W. Mandler, P. D. Randolph

Retrieval of formerly buried containers of transuranic contaminated wastes involves removal of the over burden of soil, removal of the containers, repackaging, and retention in retrievable storage prior to shipment of permanent storage. Since the containers of wastes have been buried for long periods (up to about 20 years), it can reasonably be expected that some of these, especially the older ones, have lost their integrity, allowing partial content escape or entrance of water. As a result it is expected that some of the soil in the vicinity of such containers may be contaminated with transuranics.

From the standpoint of disposal, solid materials contaminated with transuranics at a level of $10 \mathrm{nCi} / \mathrm{g}$ or greater must be treated as low level waste. Thus large amounts of soil removed during the retrieval operation will have to be treated as low-level waste unless this soil can be surveyed and shown to contain less than $10 \mathrm{nCi} / \mathrm{g}$ of transuranics. Since packaging and storage of large amounts of soil will be costly, it was worthwhile. to see if it is technically feasible to assay bulk quantities of soil at the $10-\mathrm{nCi} / \mathrm{g}$ level so that the amount of soil that must be repackaged would be reduced.

A large fraction of the transuranic contaminated wastes came from weapons production and is composed of $239 \mathrm{Pu}, 240 \mathrm{Pu}, 241 \mathrm{Pu}$, and $241 \mathrm{Am}$, of which the largest contributor is $239 \mathrm{Pu}$. All are strong alpha emitters and, with the exception of $241 \mathrm{Am}$, only weakly gamma emitting. From the standpoint of bulk assay, the only radiations that show promise of being useful for monitoring low levels of transuranics are gamma rays and $x-$ rays. Alpha counting is restricted to small samples due to the very short range of alpha particles. Neutron counting is not feasible because of the long spontaneous-fission half life of $240 \mathrm{Pu}$.

Plutonium-239 emits a spectrum of gamma rays whose energies range from $42 \mathrm{keV}$ to $769 \mathrm{keV}$. Al though all the gamma rays have low intensities, several (e.g., $129.3 \mathrm{keV}, 375.0 \mathrm{keV}, 413.7 \mathrm{keV}$ ) have high enough intensities to be detectable from bulk samples containing plutoni um at the 10 $\mathrm{nCi} / \mathrm{gm}$ level. In addition, high-energy $\mathrm{x}$-rays ( $100 \mathrm{keV}$ ) emitted upon decay of plutonium are also visible in the spectrum and have higher intensities than the gamma rays.

Initial investigations consisted of determining which radiations (x-rays, $129 \mathrm{kel}$ gamma rays, or higher-energy gamma rays) would be the most useful for monitoring soil in bulk quantities. Samples of uncontaminated soil were obtained and gamma counted to insure that only naturally occurring isotopes were present. A small plutonium source was placed at various locations on and below the surface of the se soil samples and gamma-ray spectra were obtained using a 3-inch $\times 3$-inch NaI(TI) detector. The data indicated that the spectral region encompassing the $x$-rays and 129-keV gamma ray exhibited the highest intensity. Based 
on this information, a 5-inch $\times 1 / 2$-inch $\mathrm{NaI}(\mathrm{TI})$ detector was obtained. This detector has the advantage compared to the 3-inch $\times 3$-inch detector of having a much lower efficiency for high-energy gamma rays but only a slightly lower efficiency for 90-130-keV gamma rays.

Small sealed $239 \mathrm{Pu}$ and $241 \mathrm{Am}$ standards that could be used to approximate various soil contamination levels were fabricated. Using those sources, contamination levels of approximately 5,10 and $50 \mathrm{nCi} / \mathrm{gm}$ $239 \mathrm{Pu}$, and $1,2,6$, and $11 \mathrm{nCi} / \mathrm{g} 2{ }^{41} \mathrm{Am}$ could be realized in a 12 -inch $\mathrm{x}$ 12-inch $\times 3$-inch-thick dirt sample. Using these sources and the 5-inch $x$ 1/2-inch detector located 2 inches above the surface of the 12-inch $x$ 12-inch $\times 3$-inch-thick dirt sample, the best energy regions for assay were determined to be a window from $45 \mathrm{keV}$ to $70 \mathrm{keV}$ encompassing the 59-keV ${ }^{241} \mathrm{Am}$ gamma $\mathrm{line}$ and a second window from $82 \mathrm{keV}$ to $138 \mathrm{keV}$ encompassing the $x$-rays plus the 129-keV $239 \mathrm{pu}$ gamma ray.

Measurements of detector response to plutonium and americium were made by obtaining spectra with a source (239pu or ${ }^{241} \mathrm{Am}$ ) located various distances from the detector's axis on the surface of the soil, 1-1/2 inches below the surface, and 3 inches below the surface. It was found that the response drops rapidly as the source is moved past the edge of the detector. Moreover, the response decreases rapidly as the depth at which the source is located. As a result, a sample thickness of no more than 1-1/2 inches is recommended.

Using the results of the detector response measurements, the responses of different arrangements of 5 -inch $\times 1 / 2$-inch detectors to various source configurations (e.g., point sources, uniformly distributed planar sources) were evaluated. This was done for both a continuously moving sample (i.e., a sample on a conveyor) and a stationary sample. Figure 1 shows the detector arrangements considered. Geometries $A$ and $D$ were evaluated for both stationary and moving samples while geometries B and C were evaluated only for moving samples.

Our evaluation consisted of obtaining a calibration by using a planar array of small sources (both plutonium and americium) located midway between the top and bottom of the soil layer. Then, using the detector response measurement results, the response ( $\mathrm{nCi} / \mathrm{g} \mathrm{Pu}$ and $\mathrm{Am}$ ) was calculated for each of the various detector arrays for the following source geometries: (1) uniform planar source lying on the surface, (2) uniform planar source located at the bottom of the 1-1/2-inch-thick soil sample, (3) point source at the location which gives the highest response, (4) point source at the location which given the lowest response. In all cases the source strength was such that the transuranic contamination level was $9 \mathrm{n} \mathrm{Ci} / \mathrm{g}$. Pu plus $1 \mathrm{nCi} / \mathrm{g}$. Am and the density of the soil was $1.19 \mathrm{~g} . / \mathrm{cm}$ (measured density of our soil samples). Table 1 contains the results of these calculations.

The results in Table 1 indicate that al though all detector geometries give almost identical results for planar sources (they should because they were all calibrated using a planar source), for point sources located at the highest and lowest response locations, the moving sample geometries give far better results than do the stationary geometries. As a figure 
of merit for dynamic range we obtained the ratio of the highest to the lowest response values for each detector geometry. These ratios are given in Table 2. Ideally, a ratio of unity indicates a perfectly uniform response to all locations in the sample. Using the information from Tables 1 and 2 together with relative responses to source positions across the conveyor belt (perpendicular to direction of belt motion) obtained for the various detector geometries, we concluded that 1) geometries $B$ and $C$ yield the most uniform response and 2) a more uniform response is attained if the sample is moving continuously rather than if counted in discrete batches.

We conclude that using a multiple detector array and sampling continuously, soil uniformly contaminated with $5-\mathrm{nCi} / \mathrm{g}$ of $239 \mathrm{Pu} \mathrm{plus}$ $241 \mathrm{Am}$ (and nothing else) can be assayed on a high (about $1 \mathrm{yr} / \mathrm{hr}$ ) throughput basis. For nonuniform contamination there will always be some soil that cannot definitely be said to be contaminated of uncontaminated at the $10-\mathrm{nCi} / \mathrm{g}$ level. This is due to the variation in response to a given bulk contamination level as the source geometry is altered (ff. Table I). Moreover, the presence of gamma-ray emitters other than ${ }^{\mathrm{Pu}}$ and ${ }^{24} \mathrm{Am}$ will tend to decrease our ability to assay at the 10$\mathrm{nCi} / \mathrm{gm}$ level. Future studies will investigate this in greater detail. 
Table 1

Summary of Responses for Various Detector Arrays

\begin{tabular}{l}
$\begin{array}{c}\text { Detector } \\
\text { Geometry }\end{array}$ \\
\hline A - Moving \\
B - Moving \\
C - Moving \\
D - Moving \\
D' - Moving \\
A - Stationary \\
D - Stationary \\
D' - Stationary \\
\hline Detector Geometry A \\
Detector Geometry B \\
Detector Geometry C \\
Detector Geometry $D$ \\
Detector Geometry $D^{\prime}$ \\
Source Geometry 1 \\
Source Geometry 2 \\
Source Geometry 3 \\
Source Geometry 4 \\
D
\end{tabular}

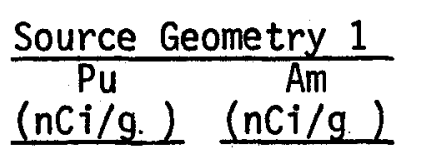

$11.6 \pm 4.8 \quad 1.71 \pm .17$

$11.6 \pm 5.1 \quad 1.71 \pm .18$

$11.6 \pm 3.9 \quad 1.71 \pm .18$

$11.6 \pm 2.6 \quad 1.70 \pm .17$

$11.6 \pm 2.9 \quad 1.70 \pm .17$

$11.6 \pm 4.8 \quad 1.71 \pm .17$

$11.6 \pm 2.6 \quad 1.70 \pm .17$

$11.6 \pm 2.9 \quad 1.70 \pm .17$

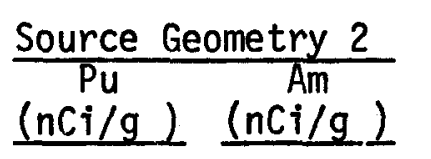

$6.37 \pm .84 \quad 0.29 \pm .03$

$6.42 \pm .87 \quad 0.29 \pm .03$

$6.40 \pm .89 \quad 0.29 \pm .03$

$6.5 \pm 1.2 \quad 0.29 \pm .03$

$6.4 \pm 1.5 \quad 0.30 \pm .03$

$6.36 \pm .87 \quad 0.29 \pm .03$

$6.5 \pm 1.2 \quad 0.29 \pm .03$

6.41 .5

$0.30 \pm .03$

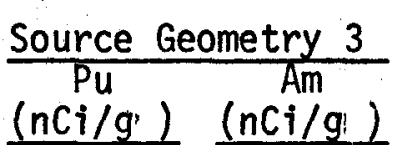
$\left(\mathrm{nCi}^{\left.\mathrm{u} / \mathrm{g}^{\prime}\right)} \quad \begin{array}{c}\mathrm{Am} \\ (\mathrm{nCi} / \mathrm{g})\end{array}\right.$

$12.9 \pm 6.0 \quad 1.95 \pm .20$

$11.8 \pm 5.5 \quad 1.72 \pm .18$

$12.0 \pm 4.1 \quad 1.80 \pm .18$

$20.4 \pm 6.0 \quad 3.15 \pm .32$

$15.8 \pm 4.1 \quad 2.41 \pm .25$

$14.4 \pm 8.1 \quad 2.32 \pm .24$

$43 \pm 23$

$7.42 \pm .76$

$22.5 \pm 7.3 \quad 3.82 \pm .40$
Source Geometry 4 $\mathrm{Pu}$
$(\mathrm{nCi} / \mathrm{g})(\mathrm{nCi} / \mathrm{g})$

$3.73 \pm .56 \quad 0.13 \pm .02$

$4.34 \pm .66 \quad 0.23 \pm .02$

$4.66 \pm .73 \quad 0.22 \pm .02$

$3.5 \pm 1.1 \quad 0.07 \pm .01$

$3.1 \pm 1.2 \quad 0.12 \pm .01$

$2.69 \pm .50 \quad 0.09 \pm .01$

$1.28 \pm .710 .03 \pm .01$

$1.6 \pm 1.2 \quad 0.07 \pm .01$

12-inch $\times 14$-inch sample size, $37.5 \mathrm{ft}^{3} / \mathrm{hr}$ throughput 12 -inch $\times 13$-inch sample size, $37.5 \mathrm{ft}^{3} / \mathrm{hr}$ throughput 12-inch $\times 14$-inch sample size, $37.5 \mathrm{ft}^{3} / \mathrm{hr}$ throughput 12 -inch $\times 12$-inch sample size, $37.5 \mathrm{ft}^{3} / \mathrm{hr}$ throughput 8 -inch $\times 8$-inch sample size, $25.0 \mathrm{ft}^{3} / \mathrm{hr}$ throughput

$9 \mathrm{nCi} / \mathrm{g}$. Pu plus $1 \mathrm{nCi} / \mathrm{g}$ Am in uniform planar distribution on dirt surface

$9 \mathrm{nCi} / \mathrm{g}$ Pu plus $1 \mathrm{nCi} / \mathrm{g}$ Am in uniform planar distribution at bottom of dirt sample

$9 \mathrm{nCi} / \mathrm{g}$ Pu plus $1 \mathrm{nCi} / \mathrm{g}$ Am point source at highest response location

$9 \mathrm{nCi} / \mathrm{g}$ Pu plus $1 \mathrm{nCi} / \mathrm{g}$ Am point source at lowest response location 


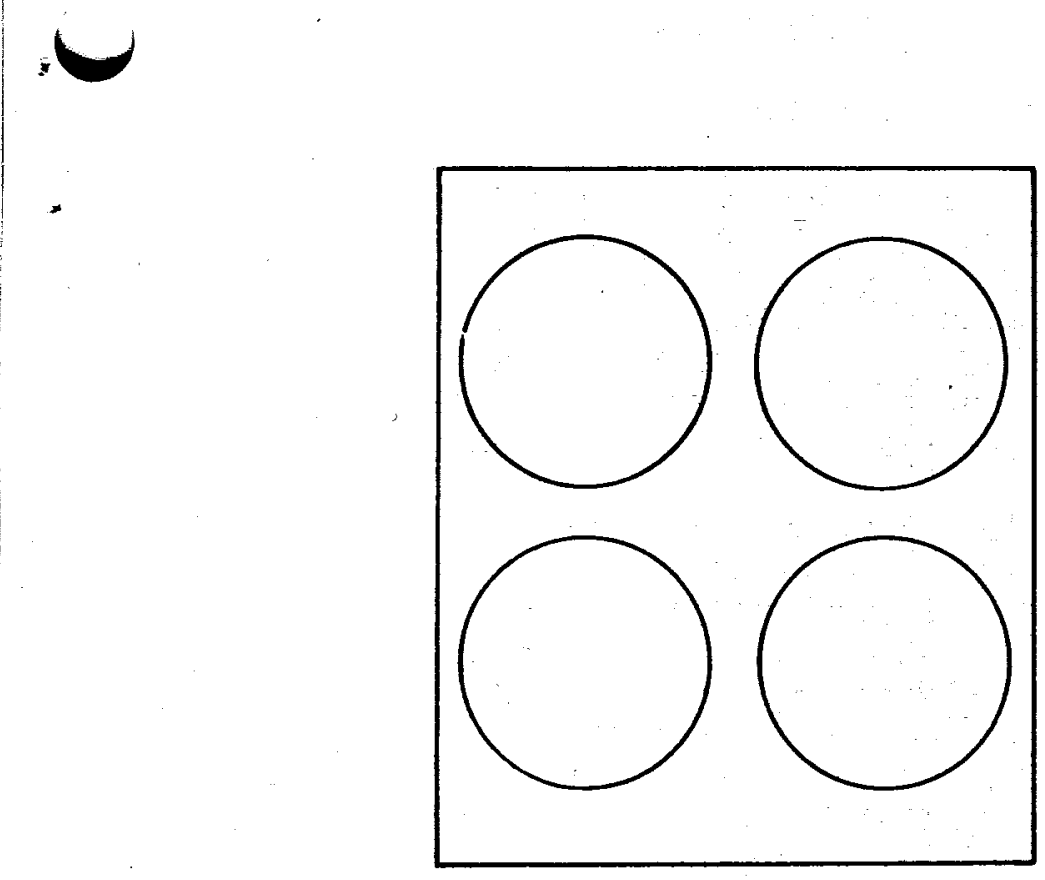

Geometry A

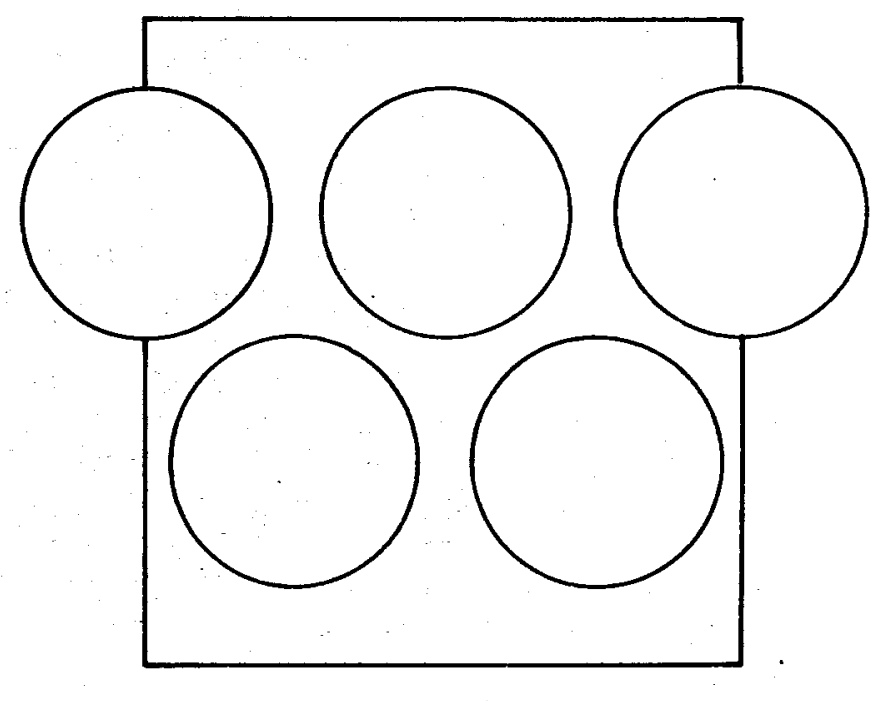

Geometry B

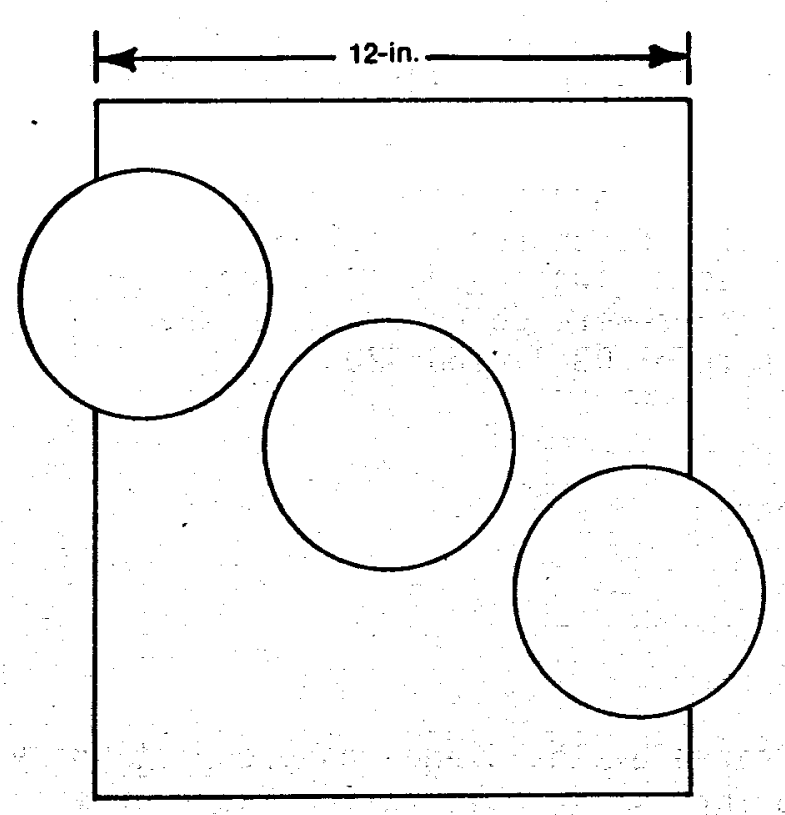

Geometry $\mathbf{C}$

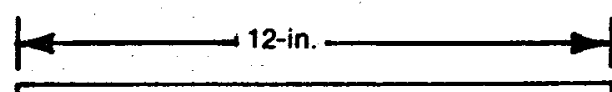

5-in. $\times 1 / 2$-in

Detector

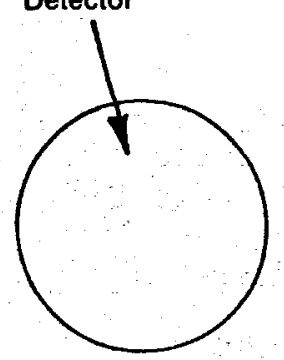

Geometry D

EGG-A-423

Fig. 1 Detector arrangement. 


\section{EXPERIMENTAL STUDIES FOR LARGE BOXES OF TRANSURANIC WASTE}

$$
\text { J. W. Mandler, P. D. Randolph, L. K. Seymour* }
$$

Initial studies on conceptual design of a monitor for large Waste Boxes $\left(4 \times 4^{\prime} \times 7^{\prime}\right)$ containing Pu-contaminated wastes were discussed in the Annual Report for the period ending June 1975(1). the proposed method uses passive counting of neutrons emitted from the spontaneous fission of ${ }^{240 \mathrm{Pu}}$ (present to about $5 \%$ of $\mathrm{Pu}$ ) and $(\alpha, n)$ neutrons from $239 \mathrm{Pu}$ oxide. It involves passing the box horizontally parallel to its 7. foot length through an annular rectangle of fast neutron detectors.

The present report discusses a laboratory prototype used to evaluate the concept and presents a summary of some of the results. Funding limitations precluded fabrications of a complete prototype; however enough was obtained to evaluate the neutron counting concept. Two polyethylene moderated slabs capable upholding $15 \mathrm{BF}_{3}$ detectors were fabricated. There are 48 inches high by 40 inches 10 ng and 5 inches thick. The slabs can contain up to 152 inch diameter 36 inch length counters. The slabs were initially completely covered with 0.020 inches of cadmium, so that they are primarily responsive to fast neutrons. They were mounted in a vertical plane 54 inches apart. Tests consisted of evaluation of a single slab efficiency, effect of varying the number of counters in the slab, and count rates for various geometries of plutonium standards. Mechanical motion was simulated by moving standards between the detectors and later by putting the detector mounts on castors and moving them past a waste box containing standards and added matrix material. Fig. 1 shows the geometry of the latter arrangement.

Initial tests showed that adequate count rates could be obtained by 8 detectors 6 inches apart in each slab.

Overall slab detector efficiency was measured using a $\mathrm{Pu}$ Be source of known strength, and by calculation of the neutron emission rate-from the Pu wastes standards. Efficiency was defined as neutrons detected divided by neutrons emitted. All detectors were Cd covered and the sl ab held 15 detectors, Reuter-Stokes model RS-P1-1636-203.

The result was:

$$
\begin{array}{lc}
\text { Source } & \text { Efficiency } \\
\text { Pu Be } & 1.76 \% \\
\mathrm{PuO}_{2} & 2.50 \%
\end{array}
$$

The Waste Standards used were preared by the Standards group at Rocky $\mathrm{Fl}$ ats $\mathrm{Plant}$, were originally obtained for use in cal ibrating Waste Drum Systems. These consist of double metal contained one-gallon cans each containing 20 grams of $\mathrm{Pu}$ in the form of high fired $\mathrm{PuO}_{2}$. This is described in Table 1.

\footnotetext{
*Energy Research Development Administration
} 
The amount of waste matrix material in the standards is too small to represent the contents of a waste box for which typical weights are 3000 to 5000 1bs. Thus the amount of matrix involved in the standards can be considered as contributing only small effects.

An indication of the count rate sensitivity of the laboratory prototype is shown in Figure 2. This shows a graph of count rate versus $\mathrm{Pu}$ content obtained with the standards centered between the detector slabs. No additional matrix was used.

Two features are worth noting. The first is that the graph is quite linear out to the full amount of $\mathrm{Pu}$ indicating that the mixture of matrix types in the standards introduce very little absorption or scattering. Secondly the count rate is quite adequate for monitoring purposes.

Although count rates appear adequate if no additional waste matrix is present, a useful monitor cannot be built until the effects of 1 arge amounts of matrix material are examined. The approach used here was to evaluate the best and worst cases. The best case is no matrix at all. The worst case from a neutron standpoint was considered to be a box with large amounts of moderating material. That is, the performance of such a monitor would be no better than the no-matrix case, and no worse than the highly moderated case.

Since concrete is not uncommon in actual waste boxes, solid concrete blocks (4" $\left.\times 8^{\prime \prime} \times 16^{\prime \prime}\right)$ was used to simulate a hydrogeneous matrix. To assess the matrix effects $420 \mathrm{gm}$ low $Z$ standards, located as shown in Fig. 1, were centered in an otherwi se empty waste box and surrounded on all sides by a layer of blocks.

Measurements were performed by recording $200 \mathrm{sec}$. counts of the slab pair at successive positions along the box lengths. This simulated motion of the waste box relative to the monitor. Due to space limitations the traverse extended primarily in only one direction from the box center and went out to 120 inches. Fig. 3 shows a set of these profiles of background-corrected count rate versus distance (d in Fig 1) for various thicknesses of concrete, each 1 ayer being $31 / 2$ inches thick. Measurements of this type were made both with (Fig. 3) and wi thout cadimum on the slab detector surface facing the box. As expected the concrete has a very large effect. Since the integral of the count rate along a traverse (for a moving box, or moving monitor system) is what would be used to assay the amount of $\mathrm{Pu}$ in a box, these curves were approximately integrated from 0 to 120 inches using a planmeter. The integrated results are shown in Fig. 4 as total counts expected for a traverse made in 200 seconds, versus total weight of concrete and box ( 400 lbs). This is shown for both $C d$ covered and bare faced slabs. The effect of the concrete is very large, causing a factor of as much as 16 reduction with 4100 ibs of concrete. The response for both Cd-covered and bare detectors is not linear, but both curves have similar shapes. The ratio of response bare/Cd covered is $\leq 1.20$ over the weight range. This range of ratios is rather small to be useable for reliable assessment of matrix effects in a routinely operating monitor. 
The general conclusions are:

1) Neutron counting method using BF counters in moderated slabs gives adequate count rates for $80 \mathrm{gms}$ of $\mathrm{Pu}$ and higher.

2) The presence of concrete is significant amounts of concrete must be corrected for.

3) Correction made on knowledge of Waste category in the box together with box weight should be adequate. 
TABLE 1

MEASURED AND CALCULATED ATTRIBUTES

of $\mathrm{PuO}_{2}$ (Aerojet P.0. 42970)

\begin{tabular}{|c|c|c|c|c|c|c|c|c|c|c|c|}
\hline $\begin{array}{c}\text { Pu Assay } \\
g / g\end{array}$ & $\begin{array}{c}\text { To1 } 95 \mathrm{C.L} . \\
\mathrm{g} / \mathrm{g}\end{array}$ & $\begin{array}{l}238 \mathrm{pu} \\
\text { wt } \%\end{array}$ & $\begin{array}{l}\text { To1. } 95 \mathrm{C.L} . \\
\text { wt } \%\end{array}$ & $\begin{array}{l}239 p u \\
\sim w t \%\end{array}$ & $\begin{array}{c}\text { Tol } 95 \text { C.L. } \\
\text { wt } \%\end{array}$ & $\begin{array}{c}240 p u \\
w t \%\end{array}$ & $\begin{array}{l}\text { Tol 95.C.L. } \\
\text { wt } \%\end{array}$ & $\begin{array}{l}24 \mathrm{Pu} \\
\text { wt } \%\end{array}$ & $\begin{array}{c}\text { Tol } 95 \text { C.L. } \\
\text { wt } \%\end{array}$ & $\begin{array}{r}242 \mathrm{Pu} \\
\text { wt \% }\end{array}$ & $\begin{array}{c}\text { Tol.95 C.L. } \\
\text { wt\% }\end{array}$ \\
\hline 0.8778 & 0.00016 & 0.012 & 0.001 & 93.661 & 0.02 & 5.887 & 0.022 & $\begin{array}{c}0.414 \\
(4-7-75\end{array}$ & 0.004 & 0.027 & 0.0003 \\
\hline
\end{tabular}

\begin{tabular}{|c|c|c|c|c|}
\hline$\stackrel{\omega}{ \pm}$ & & & & \\
\hline $\begin{array}{l}\text { Am } \\
\mathrm{ppm}\end{array}$ & $\begin{array}{c}\text { To1. } 95 \text { C.L. } \\
\text { ppm }\end{array}$ & $\begin{array}{l}\text { Atm } \\
\text { wt/ }\end{array}$ & $\begin{array}{c}\mathrm{Ci} / \mathrm{g} \\
\text { (alpha) }\end{array}$ & $\begin{array}{c}\text { Total Imp. } \\
\text { ppm }\end{array}$ \\
\hline $\begin{array}{l}112 \\
(-7-75)\end{array}$ & 5 & 239.12 & 0.074 & $\sim 550$ \\
\hline
\end{tabular}

Plutonium Waste Standards

\begin{tabular}{|c|c|c|}
\hline $\begin{array}{c}\text { \# of } \\
\text { Standards }\end{array}$ & $\begin{array}{l}\text { Nominal } 239 \mathrm{Pu} \\
\text { Content (each) } \\
\end{array}$ & Description \\
\hline 2 each & $10 \mathrm{gms}$ & Puo Powder in sealed vials \\
\hline 5 each & $20 \mathrm{gms}$ & Low atomic number matrix \\
\hline 4 each & $20 \mathrm{gms}$ & High atomic number matrix \\
\hline 2 each & $20 \mathrm{gms}$ & Borated glass matrix \\
\hline 4 each & $20 \mathrm{gms}$ & Medium atomic number matrix \\
\hline
\end{tabular}

Form of Pu: High fired PuO Powder 


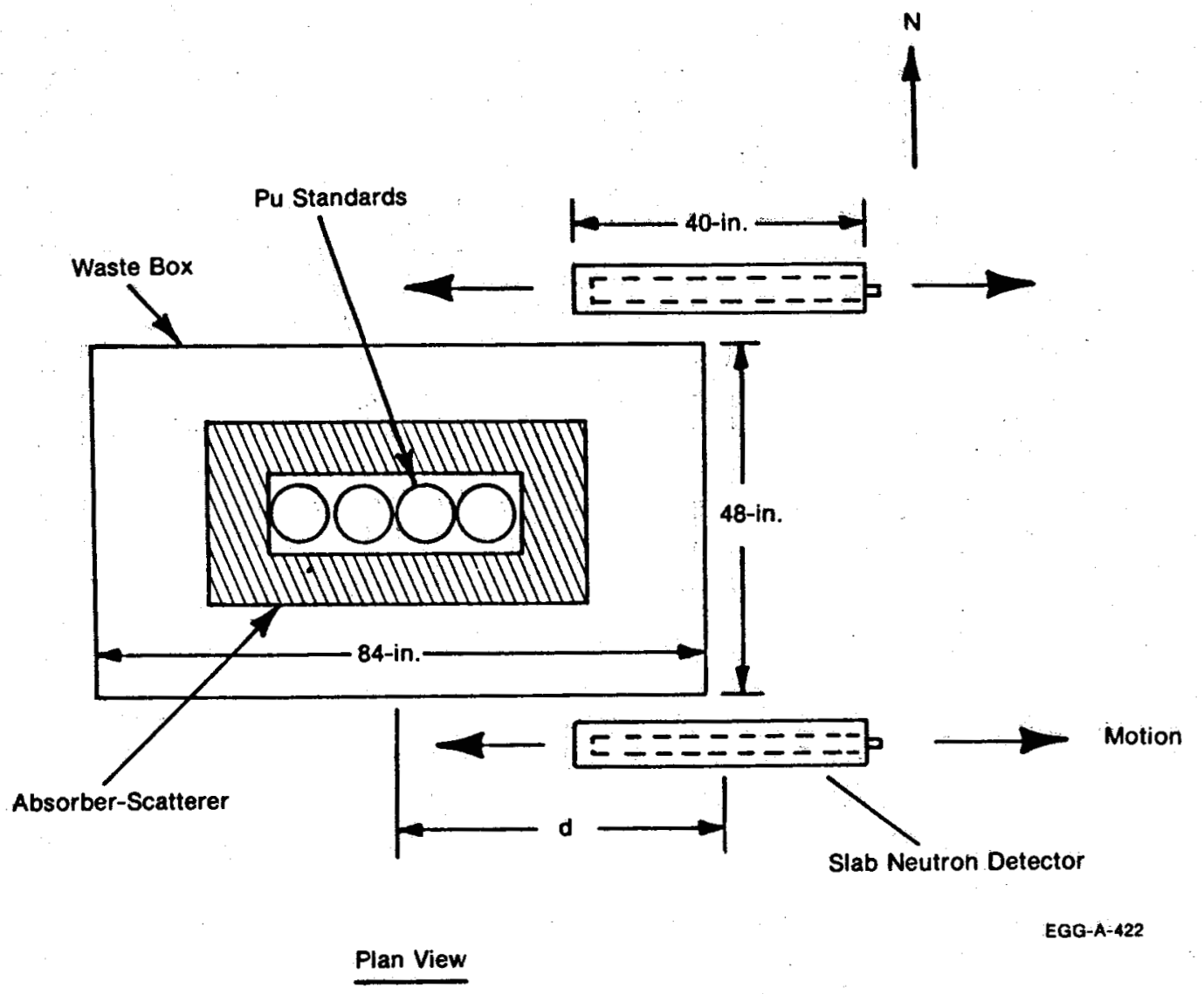

Fig. 1 Geometry of box monitor laboratory prototype experiments. 


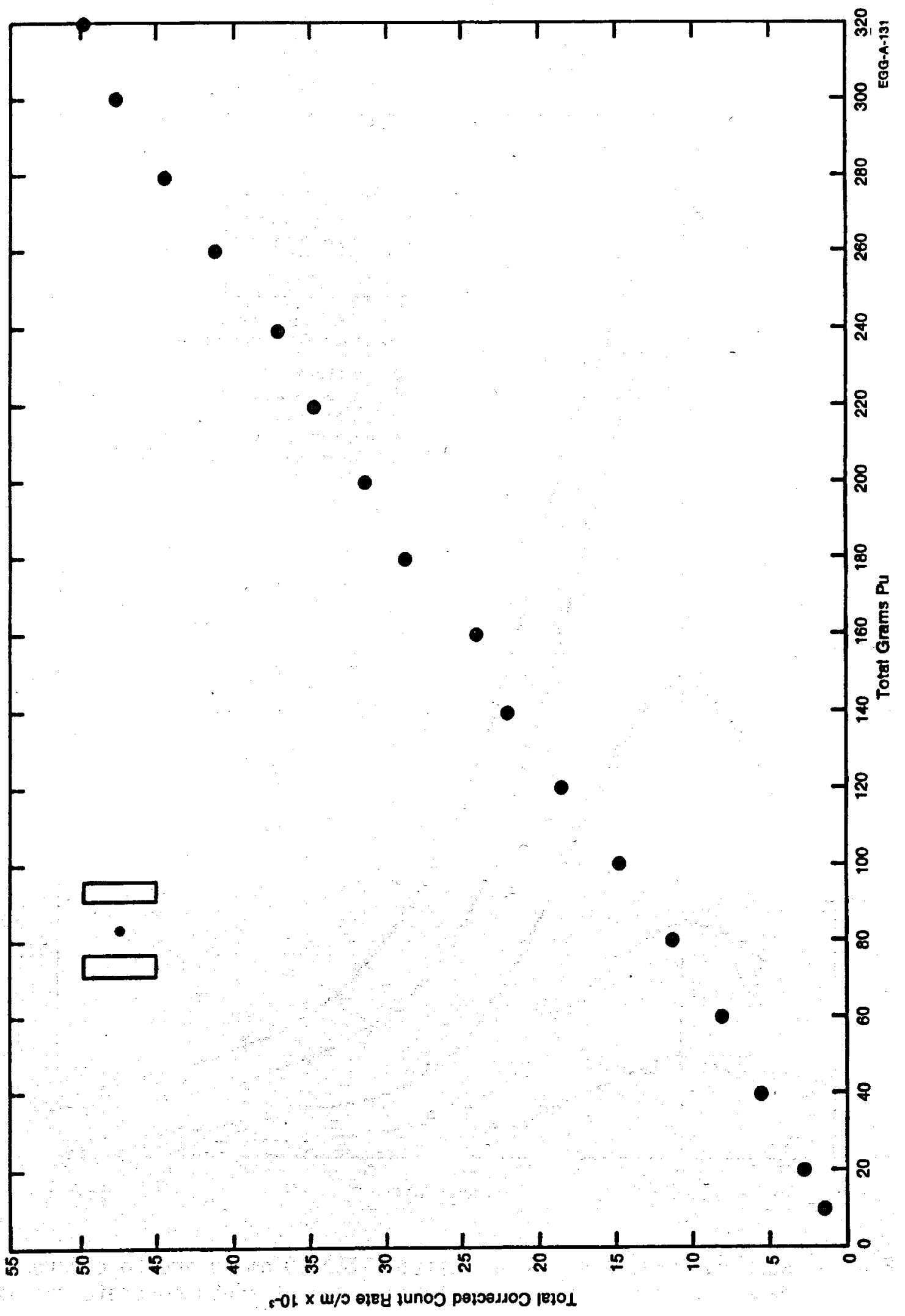

Fig. 2 Prototype count rate vs. plutonium mass with no added matrix. 


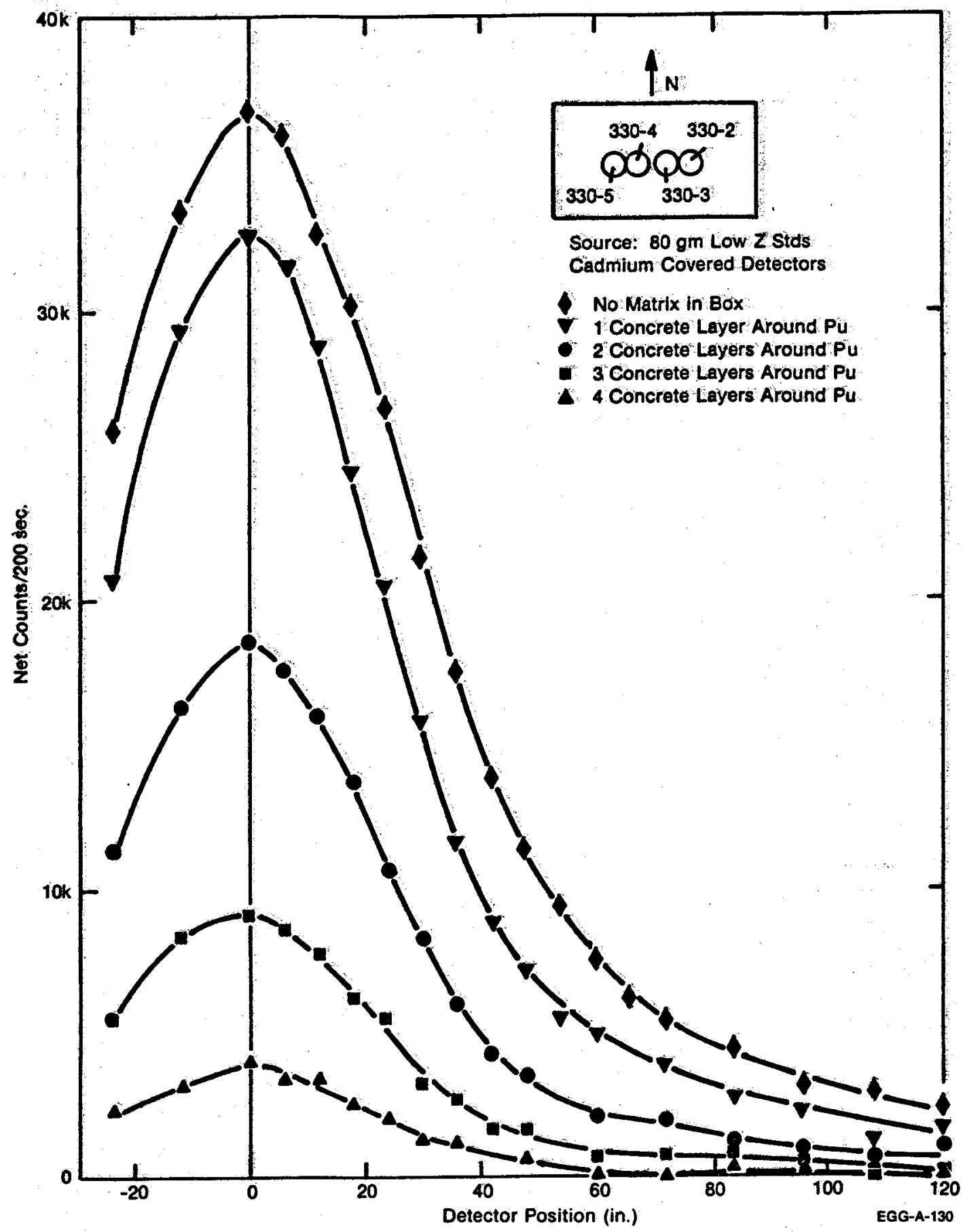

Fig. 3 Response versus detector position for $80 \mathrm{gm} \mathrm{Pu}$ and $\mathrm{Cd}$ covered detector faces for vairous thicknesses of added concrete matrix. 


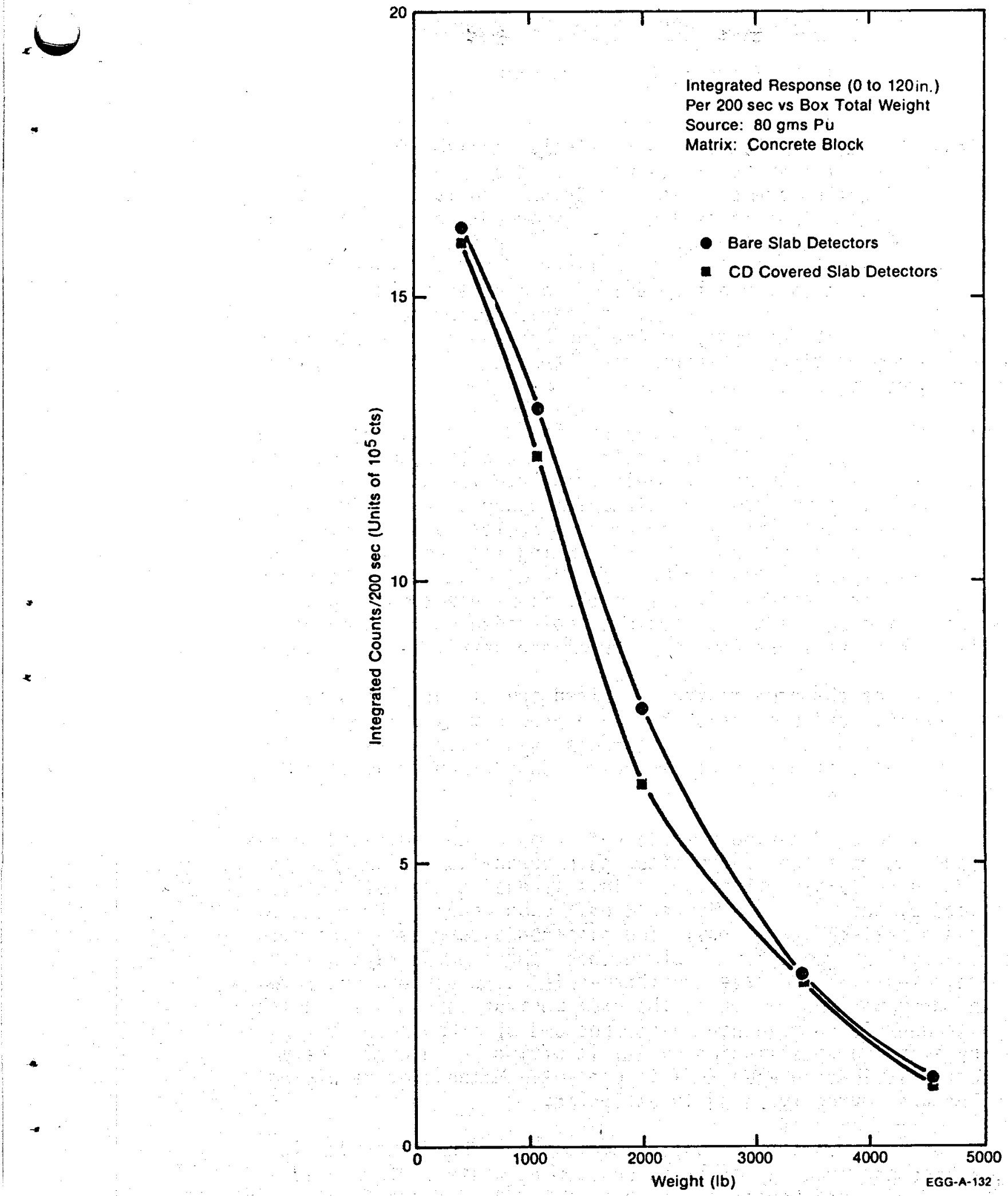

Fig. 4 Total response integrated over detector position for $80 \mathrm{grms}$ $\mathrm{Pu}$ and various thicknesses of added concrete matrix. 
TRANSURANIC WASTE MONITOR FOR LARGE DRUMS

J. W. Mandler, P. D. Randolph

Low level transuranic wastes are routinely received for interim recoverable storage at the INEL waste management complex. The waste is packaged in 55-gallon drums, 83-gallon drums, and large (4 ft. $x$ $4 \mathrm{ft} . \times 7 \mathrm{ft}$.$) boxes. These containers are stored in a large array on$ an asphalt pad which is located above ground. The top of the array is then covered with about an 18-inch-thick layer of soil. Since it is important that no criticality hazard exists, a monitor is required to assay the containers for transuranics. An assay device for the drums is considered here while an assay device for large boxes is described in a companion report titled "Experimental Studies for Monitoring Large Boxes of Transuranic Contaminated Waste."

A commercial drum assay system was purchased from National Nuclear Corporation. This system is totally self-contained (including detectors, electronics, shielding, and programmable calculator) and was designed for rapid, routine, non-destructive plutonium assay of waste drums by personnel relatively untrained in the area of nuclear assay instrumentation. It is based on the coincidence counting for spontaneous fission neutrons and gammas from $238 \mathrm{Pu}, 240 \mathrm{Pu}$, and $242 \mathrm{Pu}$. Ratios of the various coincidence count rate combinations from the four detectors (e.g., coincidences in two of the four detectors, coincidences in three of the four detectors, etc) are used to correct for matrix attenuation.

Calibration of the drum monitor required the use of plutonium standards. We obtained standards that were prepared by mixing known amounts of $239 \mathrm{Pu}$ with various waste matrices, simulating $10 w-Z$, medium- $Z$, high- $Z$ and borated wastes. Total plutoni um masses from $20 \mathrm{gs}$ to $300 \mathrm{gs}$ were used in the calibrations.

In the course of assaying a series of drums of contaminated wastes it was discovered that the drum monitor gave erroneously high results for older drums containing fluorides. This is due to the buildup of ${ }^{22} \mathrm{Na}$ produced by the $19_{\mathrm{F}}(\alpha, n)^{22} \mathrm{Na}$ reaction. Upon decay, ${ }^{22} \mathrm{Na}$ emits a positron and a 1274-keV gamma ray. The positron undergoes annihilation emitting two 511-keV gamma rays. Since these three gamma rays are in coincidence, they will increase the three-fold coincidence count rate seen by the drum monitor. Because the drum monitor cannot distinguish between a three-fold coincidence event produced by $22 \mathrm{Na}$ from one produced by a spontaneous fission event, it will give an anomalously high plutonium indication when ${ }^{22} \mathrm{Na}$ is present. Methods of resolving this problem are currently being investigated.

Since the drum monitor was calibrated using $239 \mathrm{Pu}$ standards, it cannot yet be used for assay of drums containing waste contaminated with $238 \mathrm{pu}$. The spontaneous fission rate for $239 \mathrm{Pu}$ differs from that of ${ }^{240} \mathrm{Pu} /{ }^{239} \mathrm{Pu}$ always contains some $240 \mathrm{Pu}$ and it is the $240 \mathrm{Pu}$ that the drum monitor uses to indicate the presence of $239 \mathrm{Pu})$. Therefore, $238 \mathrm{Pu}$ waste matrix standards will be required to enable the drum monitor to assay for ${ }^{238} \mathrm{Pu}$. 


\section{CALIBRATION OF A STABILIZED ASSAY METER FOR WASTE DRUM ASSAY}

$$
\text { P. D. Randolph }
$$

This report deals with an evaluation and calibration for 55 gallon Waste Drums of an Eberline Instrument Corporation "Stabilized Assay Meter" which contains two single channel analyzers, (abbreviated SAM-2), having a 2-inch by 2-inch detector crystal. It is an outgrowth of earlier work(1) which dealt with another SAM-2 having a 2 " $\times 1 / 2$ " detector crystal. In that report a calibration could be done only up to 21 grams of $\mathrm{Pu}$ due to lack of $\mathrm{Pu}$ standards. The report also recommended that, for use with $\mathrm{Pu}$, a thicker crystal would give a better response. As a result of this recommendation a new SAM- 2 was obtained which has the thicker crystal. In addition a set of Pu-contaminated waste standards has been obtained from Rocky Flats (320 gms Pu total) and these allowed calibration out to 280 gms Pu.

The Stabilized Assay Meter (SAM) built by Eberline Corp. is a portable device meant to be used for certain limited types of assay. It uses a scintillation detector to count gamma (or $x$-ray) radiation. This is an energy sensitive detector whose range of useful energies is largely determined by the crystal thickness. The usefulness of an energy sensitive system such as this for field use depends on having a stable system gain. The SAM provides for this by incorporating an $241 \mathrm{Am}$ seed in the scintillation crystal. The a radiation from this $(\sim 3.5 \mathrm{MeV})$ is used to cause the high-voltage supply to change as required to maintain a constant pulse height from the a particles. The SAM-2 incorporates two pul se height single-channel analyzers (or windows). These are individually adjustable for both threshold (energy) and window width (energy band).

The purpose for which this instrument is to be used and toward which the calibration is directed, is as a hand-held field instrument for rough assessment of the quantities of $\mathrm{Pu}$ in a 55-gallon drum. Since the matrix of waste materials in a drum is usually not well known, and the instrument is to be hand held, it cannot be expected to yield an accurate assay.

1) Calibrate the SAM-2 for ${ }^{239} \mathrm{Pu}$ assay.

2) Determine a combination of absorbers that would reduce the lowenergy contribution (primarily $60 \mathrm{keV}$ from ${ }^{241} \mathrm{Am}$ ) with as 1 ittle sacrifice of intensity of the Pu gamma energy $(370-400 \mathrm{keV})$ as possible so that the system can be used for high ${ }^{241}$ Am barrels without large count-rate pile up effects.

3) Adjust the gain settings so that if PuF or fission products (137 Cs) are present, this can be determined, since their presence can reduce assay accuracy, or if enough is present give rise to misleading results. 
The energy region used to assay $\mathrm{Pu}$ is the series of gamma rays in the region around $385 \mathrm{keV}$ in ${ }^{239} \mathrm{Pu} .{ }^{241} \mathrm{Am}$, a prolific emitter of $60-\mathrm{keV}$ gamma rays is always present to some extent in Pu. It is desirable to reduce its count rate so that pile up and count rate losses are as small as practical. For this reason an absorber cap for the detector was evaluated using $\mathrm{Pb}$ and $\mathrm{Cd}$ absorbers. The best cap was one using 0.060 inches of $\mathrm{Cd}$ and 0.041 inches of $\mathrm{Pb}$. Fig. 1 shows a single-channel analyzer spectrum obtained with the SAM-2 with and without the cap. The $385-\mathrm{keV}$ region is at a threshold value of $V=4.6$ and the $241 \mathrm{Am}$ and $x$-ray contribution is (for the bare detector) at $v=0.8$. The cap reduces this by a factor of $\sim 30$, while the $385-\mathrm{keV}$ peak is reduced by $\sim 32 \%$.

For use in assay a window width $\Delta V=1.0$ and a threshold at $V=4.6$ is used for Channel 1. Automatic background subtraction is obtained using the Second Channel at $V=7.0, \Delta V=1.0$.

Calibration was done using Waste Standards in one-gallon doublemetal contained cans. These Standards are described more fully in another part of this report (Experimental Studies for Monitoring Large Boxes of Transuranic Waste). This was done by putting the standards in a DOT 17-C Drum on a turntable. Measurements of Pu response were made at 36 and 60 inches from the drum surface. Figure 2 shows the cal ibration curve at both distances plotted as count rate versus mass of Pu. Nonlinearity is observed in both plots. This is due to self shielding of the Standards. However the calibration is adequate for its purposes.

The particular gain settings used are such that if ${ }^{137} \mathrm{Cs}$ is present, or ${ }^{22} \mathrm{Na}$ from $(\alpha, n)$ reactions in $\mathrm{PuF}_{4}$ is suspected this can be checked simply by changing window threshold settings.

(1) P. D. Randolph, E. B. Nieschmidt, Internal Report. 
$\cup$

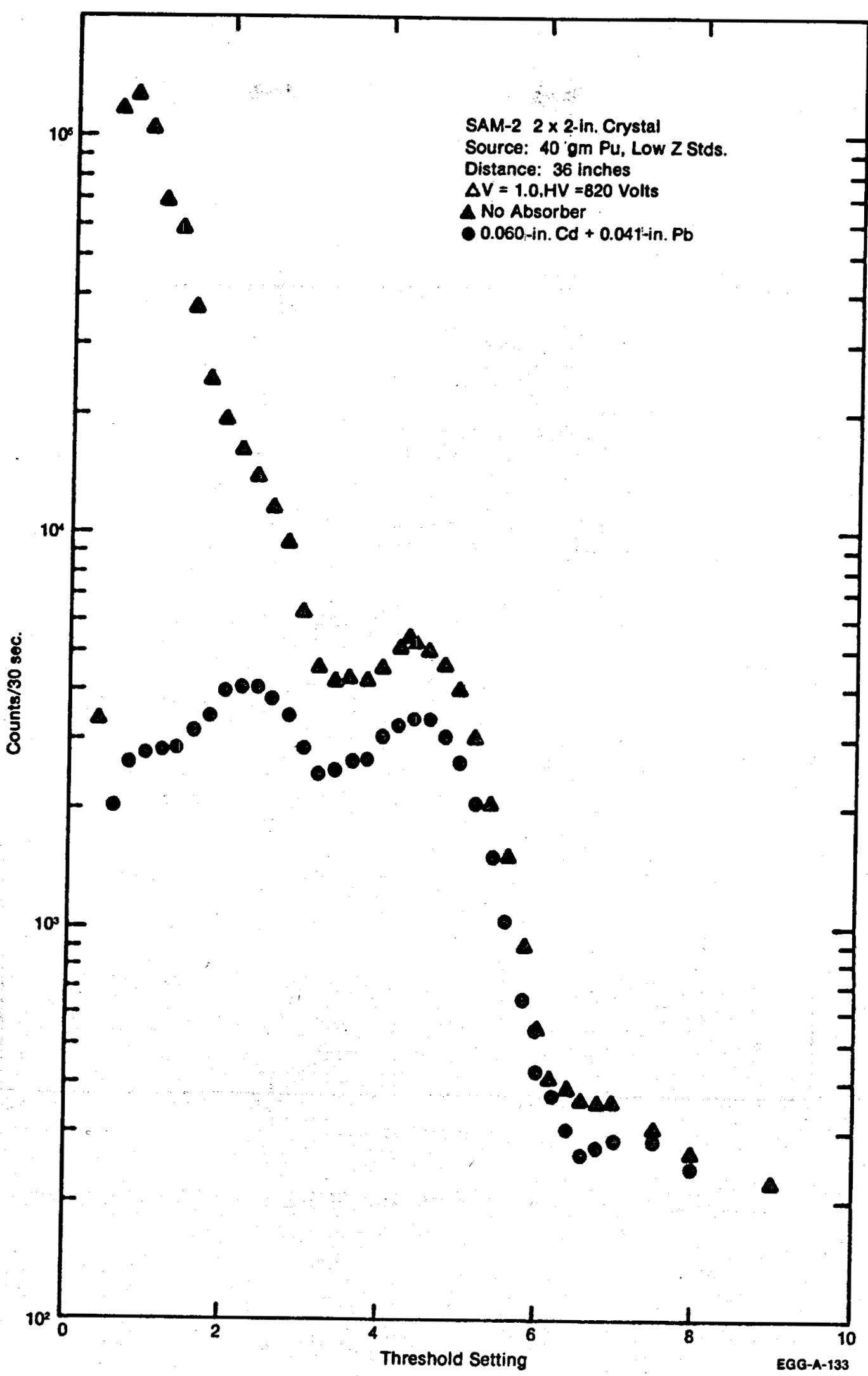

Fig. 1 Single Channel Spectrum of ${ }^{239} \mathrm{Pu}$ taken with the SAM-2 with and without absorber cap. 

NRC SPECIAL SAFEGUARDS STUDY

S. H. Vegors, J. W. Mandler, E. B. Nieschmidt, P. D. Randolph

The purpose of this study was to obtain background information on the highly enriched uranium (HEU) fuel cycle for the Nuclear Regulatory Commission so that this information could be used in a generic environmental impact statement. More specifically this information was to be used in writing a chapter in a revised GESMO (1) report, the bulk of which dealt with problems associated with the recycling of plutonium (Pu) as a power reactor fuel.

The work on this project began in the first half of June 1975. Two final reports on our activities were submitted at the end of September 1975 . $(2,3)$ Intermediate reports were also presented to NRC in August of $1975(4,5)$.

This study was a joint project between Aerojet Nuclear Corporation and Allied Chemical Corporation. In addition to being a joint project between these two organizations it was also a multidiscipline project involving chemists, metallurgists, physicists and statisticians.

There were several main areas of investigation in this study. These areas were:

1) A comparative study of the highly enriched uranium and $\mathrm{Pu}$ and/or mixed oxide (MO) fuel cycles.

2) Isotopic separation and enrichment.

3) Fuel fabrication.

4) Nuclear reactors.

5) Fuel reprocessing.

6) Scrap recovery.

7) Spiking.

8) Non-destructive analysis (NDA) techniques.

9) Reactivity NDA methods.

10) High Temperature Gas Cooled Reactor (HTGR). A11 aspects of its fuel cycle.

In order to obtain first hand information on these various aspects of the HEU fuel cycle visits were made to the following installations:

1) Goodvear Atomic gaseous diffusion plant near Portsmouth, Ohio.

2) Atomics Internation fuel fabricating plant at Canoga Park, California.

3) University of Missouri research reactor (which uses highly enriched $U$ fuel) at Columbia, Missouri.

4) United Nuclear Corporation scrap recovery plant at Wood River Junction, RI and the Oak Ridge National Laboratory scrap recovery plant $(Y-12)$ at Oak Ridge, Tennessee.

5) General Atomics at San Diego, California for information on the HTGR.

In order to present our information to NRC and to coordinate our efforts with other laboratories engaged in various other aspects of 
this project (6) participants in this study at INEL attended a meeting, sponsored by NRC, at Livermore, California late in August 1975.

At the present time a small follow up study on the fabrication of HEU fuel is in progress by personnel at INEL.

This information gathered in this study was used by NRC; see for example "Working Paper C....Special Safeguards Study...Chapter 8....Material Control and Accounting" by the office of Special Studies, US Nuclear Regulatory Commission, November 1975. are:

A brief summary of some of the general conclusions of this study

1) HEU with U235 isotopic content of $90 \%$ or greater is more attractive than $\mathrm{Pu}$ for fabricating a nuclear weapon.

2) Due to the expected low level of activity and the associated small quantities of Special Strategic Nuclear Material (SSNM) relative to $\mathrm{Pu}$ recycle, the research and test reactor and power reactor fuel cycles are not expected to pose a significant safeguards threat in the near future.

3) Relative to $\mathrm{Pu}$ recycle, the only existing fuel cycle which currently involves, and which is expected to involve, a significant quantity of SSNM is the Navy fuel program.

4) The only method capable of significantly upgrading Material Control and Accounting (MC\&A). at HEU facilities is "rapid" or "real time" MC\&A.

5) HTGR fuel is less atractive from a diversion standpoint than other HEU fuels.

(1) "Generic Environmental Statement Mixed Oxide Fuels," WASH-1327, by USAEC, August 1974.

(2) Internal Report.

(3) "Special Safeguards Study....INEL Summary for Chapter 8... Material Control and Accounting Systems....Extension of Real-time Material Safeguards Concepts to Highly-Enriched Uranium Fuel Cycles", by the INEL Special Safeguards Study Group, September 25, 1975.

(4) "Safeguards Report for NRC from the INEL Special Safeguards Study Group", by INEL Special Safeguards Study Group, August 25, 1975.

(5) "Special Safeguards Study.... INEL Summary for the Uranium Fuel Cycle", by INEL Special Safeguards Study Group, August 26, 1975.

(6) Internal Study. 


\section{URANIUM MEASUREMENTS ASSESSMENT}

E. B. Nieschmidt

The nuclear material accountability system presently used at the Atomics International fuel fabrication facility is being studied. The study is restricted to only fabrication of Advanced Test Reactor (ATR) fuel.

Facets of the program include;

1. Description of the measurements standards program.

2. Description of the sampling program.

3. Description of the measurement program.

4. Analysis of the statistical treatment of the measurement data.

5. Evaluation and suggestions based upon these analyses and descriptions.

All of the information from this study will be used to model the ATR fuel production process. Computer programs based upon this model will be written to describe the distribution of nuclear material in the plant as a function of time. The effects of various proposed regulations may be studied by use of the program as well as diversion sensitivity studies. 
SOURCE TERM MEASUREMENT PROGRAM AT OPERATING LWR'S

N. C. Dyer, R. L. Bunting; S. T. Croney

R. L. Kynaston, E. W. Killian, R. L. Heath

A program of inplant measurements at operating nuclear power plants is being conducted for the Nuclear Regulatory Commission (NRC). The measurements will provide a qualitative and quantitative determination of the radionuclide distribution within the various reactor system components. The main objective of the program is to provide NRC with the information necessary for evaluating licensing applications from the background of realistic performance and operating radionuclide data. The several specific objectives are:

1. Provide realistic and actual radionuclide source term data for input to the NRC effluent and radiation dose calculational models.

2. Provide actual operating data on rad-waste equipment performance for use in NRC evaluations of rad-waste treatment systems.

3. Provide actual operating information on equipment performance so as to indicate directions for design improvements.

The inplant source term measurement program will study six pressurized water reactors (PWR's) during the next 2 to 3 years. The study will identify and quantify the sources of radioactive waste effluents from operating PWR's during the different modes of plant operation. That is, during power operations on a relatively old core prior to refueling, during refueling operations, and during power operations with a relatively new core following refueling. Gaseous, liquid, and solid samples will be taken from the operating system of a PWR and the radionuclides within these samples will be determined using a computer-based, gamma-ray spectrometer system. The gamma-ray spectrometer will be in a mobile laboratory that will be moved to the PWR being studied. Certain samples will be returned to INEL for measurement of the beta emitting radionuclides.

The main effort on the source term program during FY 1976 has been on the procurement and outfitting of the mobile laboratory. Figure 1 is a picture of the mobile laboratory which is in a 29-foot, fifth-wheel travel trailer. A one-ton, crew-cab pickup provides the motive power for the trailer plus transportation for personnel and supplies at the reactor site. The trailer, parked at the reactor site, provides a radionuclide counting laboratory plus fabrication and storage facilities for the equipment needed to obtain the reactor samples.

The radionuclide counting equipment is a $G e(L i)$ gamma-ray spectrometer system which is based on a NOVA 1200 computer system. Figure 2 is a picture of the computer-spectrometer system within the mobile laboratory. Gamma-ray spectral data are collected from each reactor sample and stored on magnetic tape along with the pertinent sample and measurement information. The computer-spectrometer system 
in the mobile laboratory has the capability for prel iminary analysis of the gamma-ray spectral data to check the validity of the sample at the reactor site. The 200 to 300 gamma-ray spectra collected during a measurement period will be batch processed on the large INEL computer using a program, Gauss VI $(1)$, developed at INEL.

An automatic sample changer has been developed for use with the gamma-ray spectrometer system in the mobile laboratory. Figure 3 is a picture of the sample changer in the mobile lab. The sample changer enables the automatic counting of up to twenty samples at any distance from zero to 30 centimeters from the $\mathrm{Ge}(\mathrm{Li})$ detector. The positioning of the proper sample, the count time, plus the recording of the spectral data and pertinent sample information are controlled by the computerspectrometer from a specified counting sequence setup by the operator. This enables the mobile laboratory counting system to continue to collect data when personnel are occupied with in-plant measurements and sample collection or off-site. The sample changer, hardware interfaces, and the computer software $(2)$ were developed and constructed at INEL.

(1) J. E. Cline, M. H. Putnam, R. G. Helmer. "Gauss VI, A Computer Program for the Automatic Batch Analys is of Gamma-Ray Spectra from Ge(Li) Spectrometers", ANCR-1113 (1973).

Interna] Report. 


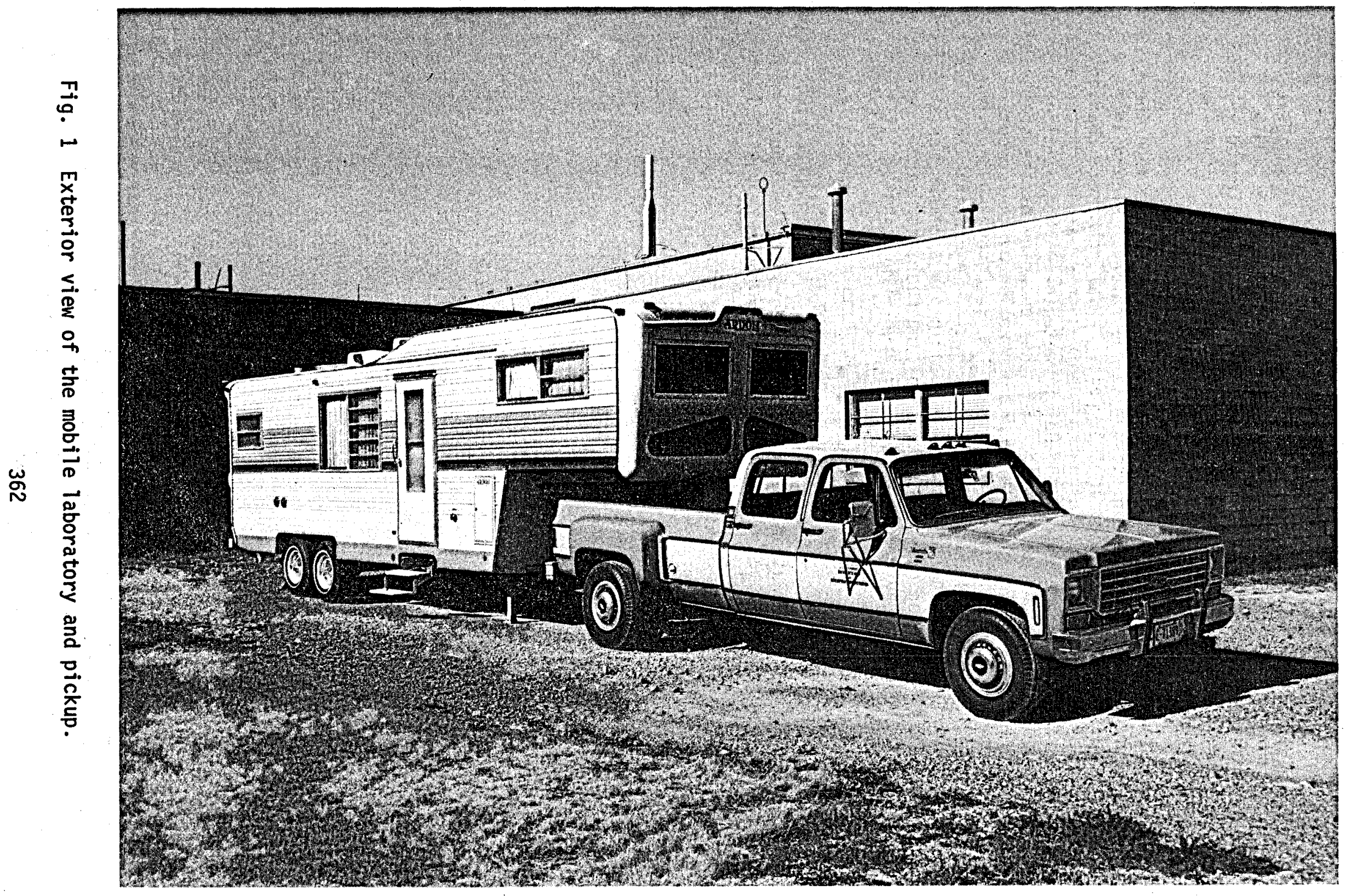

$0 \ldots$ 


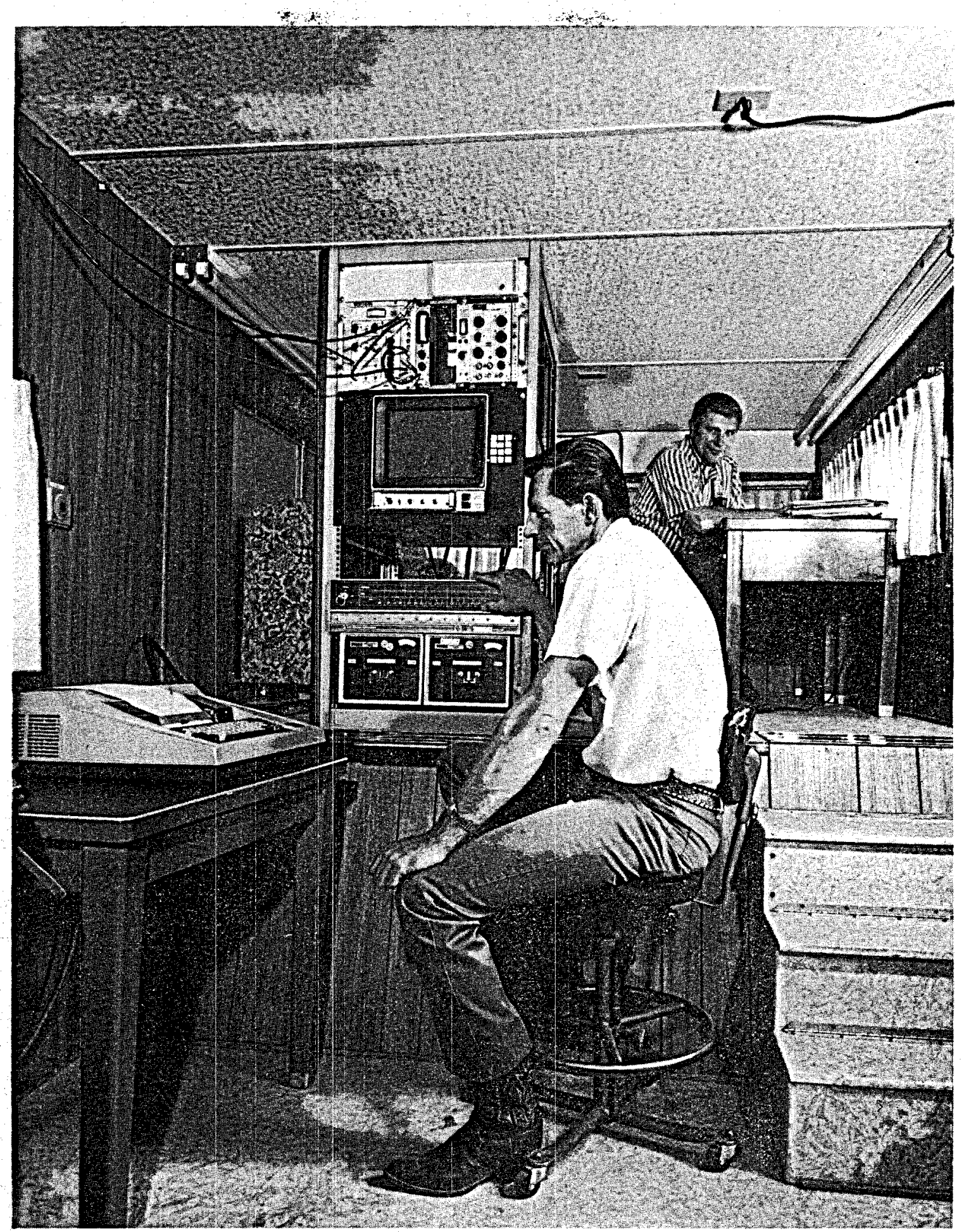

Fig. 2 Interior view of the mobile laboratory showing the computer and data read-out portions of the gamma-ray spectrometer. 


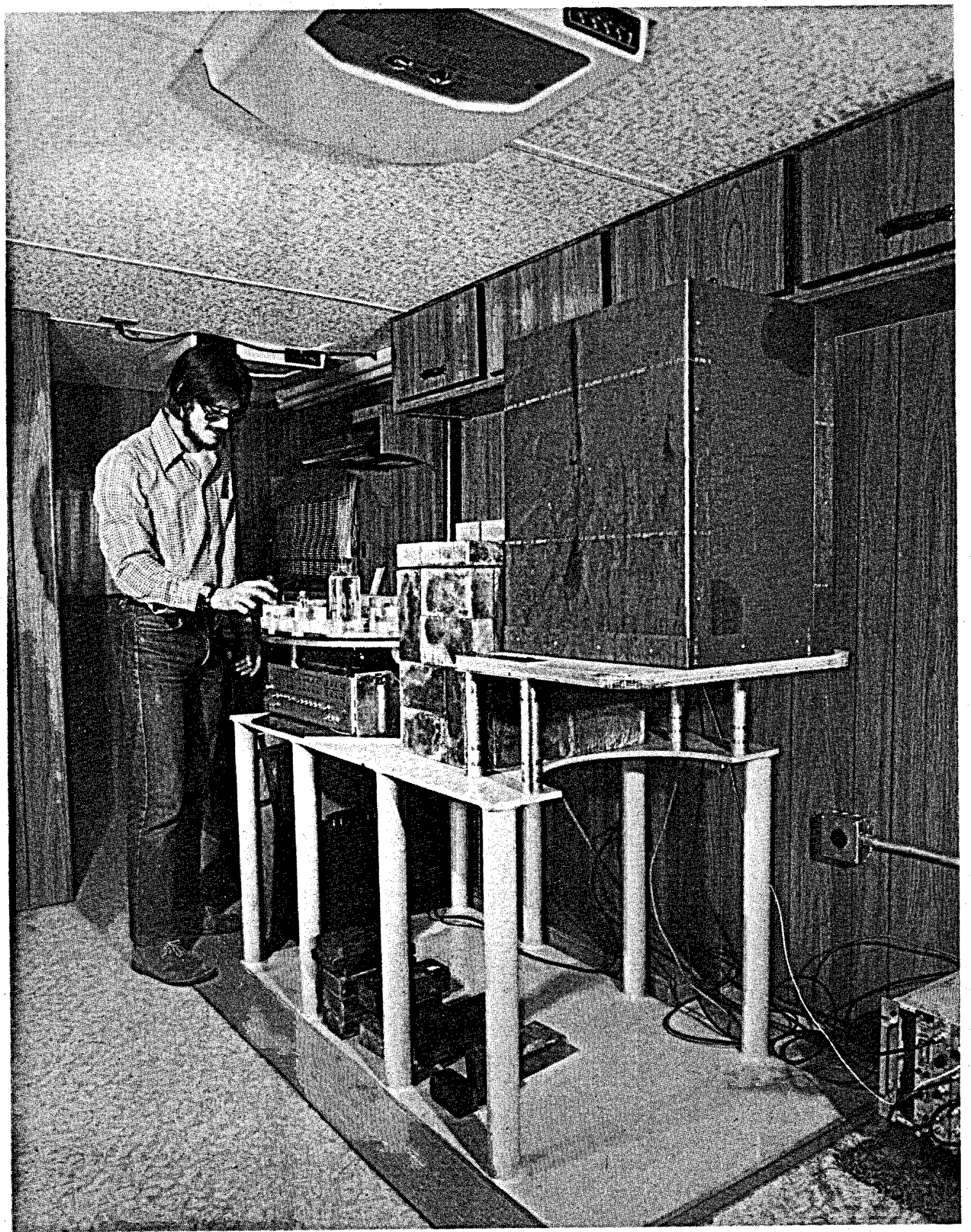

Fig. 3 Interior view of mobile laboratory showing the sample changer, lead shielding cave, and $\mathrm{Ge}(\mathrm{Li})$ detector portions of the gamma-ray spectrometer. 


\title{
REMOTE GAMMA-RAY SPECTROMETER SYSTEMS FOR FACILITY MONITORING
}

\author{
R. L. Heath, E. W. Killian, R. A. Coates, \\ L. 0. Johnson, R. G. Helmer, W. R. Myers
}

Effective monitoring of process streams from nuclear plants is essential to assure safe operation and minimal impact on the environment. For several years, the Nuclear Physics Branch of Aerojet Nuclear Company has been developing systems to enable the quantitative assay of radionuclides through gamma ray spectroscopy with $G e(L i)$ detectors. In the last few years, a program to develop remotely-controlled spectrometer systems for effluent monitoring has evolved through several stages with an ultimate goal of achieving a reliable minimal-cost design suitable for commercial production on release of the design reports to the general public.

The first version of the remotely-controlled analyzer was based on a commercially available PHA system, with special hard-wired communications interfaces, to demonstrate the feasibility of remote control from a central station, with centralized on-line data reduction. The second version was developed on a PDP-8/e as a prototype programable system to implement self-calibration with a precision pulse generator, several self-testing functions such as ramp generation and transmission, study of communication techniques, and to provide preliminary operating experience to refine the hardware, software, and functional requirements.

At this time, work is proceeding in two directions: The development effort is continuing to produce the ultimate microprocessor-based version, and, meanwhile, the current technology is being put to work utilizing an interim version based on the Fabritek MP12 microcomputer configuration.

The operation of large nuclear installations involves significant inventories of radioactive materials. Effective monitoring of effluent streams to permit evaluation of potential hazards which might result from discharge of radioisotopes is a requirement for safe operation of such facilities. Programs are being conducted at the Idaho National Engineering Laboratory (INEL) to apply gamma-ray spectrometry to realtime isotopic monitoring of nuclear installations.

The development of such a system requires a reliable method of data accumulation remote from a larger computer system which has the responsibility for verification, analysis, and interpretation of the data. In designing such a system, three goals were established:

1. The data accumulation and transfer of data must be completely automated and under control of a central processor. The automation of the data transfer is essential for routine monitoring of a source. Manual manipulation of the large amounts of data is time consuming and error prone. 
2. The remote system must be able to monitor and verify its own. operation. Operation of gamma-ray spectrometers is subject to several types of problems which effect the quality of the data being acquired. Among them are power failures, gain and zero shifts, and other effects due to wide variation in the intensity of radiation incident upon the detector. The remote system should be able to detect these problems and report them upon command.

3. The remote system must be able to control external devices. Several applications of the remote system in an industrial environment require control of the source of data that is being acquired, such as the routing of gas or liquids from various parts of the plant to the detector. Moreover, if quantitative analysis is to be performed, the remote system has to be able to provide information from flow meters, pressure gauges, etc.

To accomplish these goals, a multiphase development schedule was established. The first hardware configuration served as a development and feasibility prototype in the effort to design an inexpensive integrated monitoring device. The system consists of a stripped commercial 4096-channel pulse-height analyzer, a detector system and an in-house developed remote control logic and modem interface. The control logic for the remote analyzer performs the same functions as the manual control switches on a multi-channel pulse-height analyzer. It receives and executes commands, transmits data or status by command from the central processor via telephone using a 1200 Baud asynchronous, half-duplex, auto-answer modem. The capability of this system was demonstrated by using it to collect about 100 spectra over a period of 800 hours of ATR operation.

The phase II spectrometer design includes a programmable (minicomputer) system at the remote end to provide extended capability in data reliability monitoring, optimized data formatting and communication, and additional remote functions. An existing PDP-8/e was utilized on a temporary basis to construct the prototype system to reduce the developmental costs. The standard PDP-8/e minicomputer is programmed for operation from read-only memory. The spectrometer is implemented as a 4096-channel analyzer with a capacity of over a million counts per channel. A 1200-baud telephone interface for transmission of data and receipt of control information, a precision multi-pulse generator for energy calibration and performance monitoring, and general-purpose digital interfaces for detection and control of auxiliary external events are included. Both hardware and software are modular to facilitate continued optimization. The Phase II remote analyzer is completely controlled from a central installation. Primary functions of the central controller include erase, start, stop and transmit, and read and set external devices. Transmitted data include elapsed and live time clocks, analyzer status, up to five windowed calibration pulse peaks, and the 4096-channel spectrum. All data will be transmitted in ASCII suppressed-zero format to provide a favorable compromise between data transmission time and a universally recognized data format. 
Pending completion of the design of the microprocessor-based version (phase III), the Fabritek MP-12 microcomputer is being utilized in the interim field version "production" models. To date, five of these configurations have been installed in the Test Reactors Area.

The third phase in the development effort is to design a relatively inexpensive and reliable device, based on LSI and microprocessor technology, that is suitable for commercial production. Design of a remotely-controlled analyzer based on the Intel 8080 microprocessor has been nearly completed. LSI-11 and microNOVA microcomputers are being acquired to evaluate various system configurations. 


\section{DISTRIBUTION RECORD FOR TREE-1116}

\section{Internal Distribution}

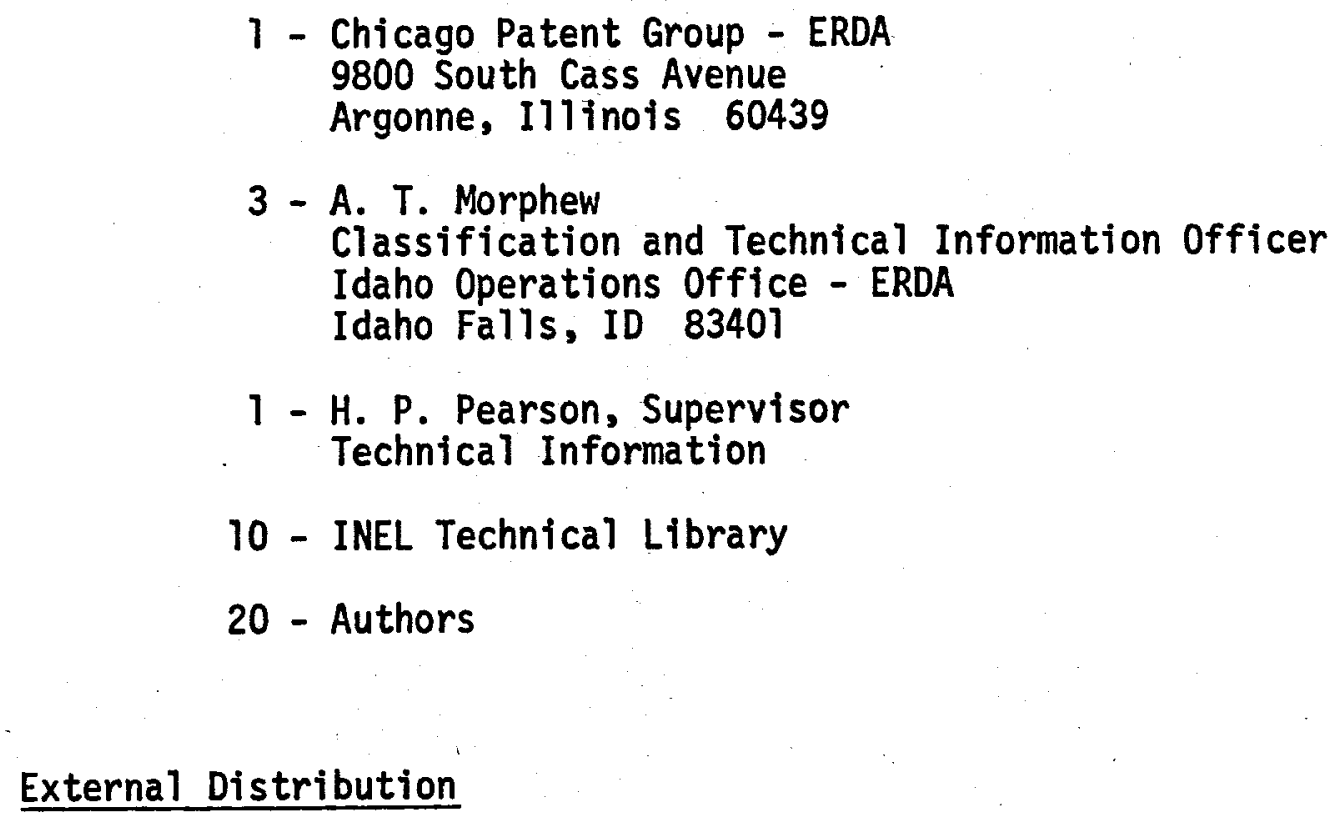

External Distribution

40 - Special External

213 - UC-2, Genera1, Miscellaneous, and Progress Reports (Nuclear), TID-4500, R65 